\title{
Regulation of Arteriogenesis by Mechanosensitive MicroRNAs and DNA Methylation: A Potential Epigenetic Approach for Treating Peripheral Arterial Disease
}

\author{
A Dissertation \\ Presented to \\ the faculty of the School of Engineering and Applied Science \\ University of Virginia
}

in partial fulfillment

of the requirements for the degree

Doctor of Philosophy

by

Joshua L. Heuslein

December 2016 


\section{APPROVAL SHEET}

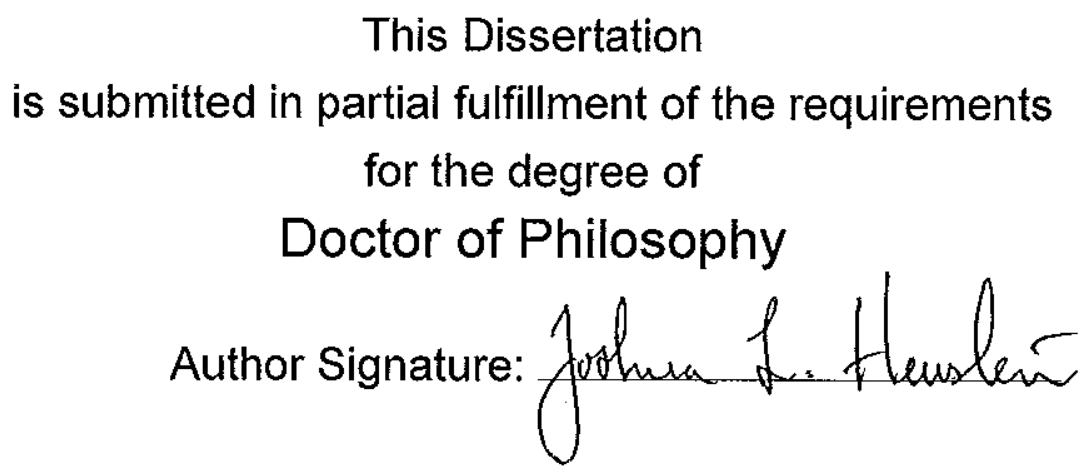

This Dissertation has been read and approved by the examining committee:

Advisor: Richard J. Price, Ph.D.

Committee Member: Brian P. Helmke, Ph.D.

Committee Member: Brian H. Annex, M.D.

Committee Member: Gary K. Owens, Ph.D.

Committee Member: Shayn M. Peirce, Ph.D.

Committee Member:

Accepted for the School of Engineering and Applied Science:

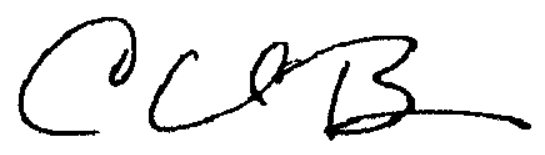

Craig H. Benson, School of Engineering and Applied Science

December 2016 


\title{
Regulation of Arteriogenesis by Mechanosensitive MicroRNAs and DNA Methylation: A Potential Epigenetic Approach for Treating Peripheral Arterial Disease
}

\author{
A Dissertation \\ presented to the faculty of the School of Engineering and Applied Science \\ in partial fulfillment of the requirements for the degree of
}

Doctor of Philosophy

by

Joshua L. Heuslein

December 2016 


\title{
Regulation of Arteriogenesis by Mechanosensitive MicroRNAs and DNA Methylation: A Potential Epigenetic Approach for Treating Peripheral Arterial Disease
}

\author{
A Dissertation \\ presented to the faculty of the School of Engineering and Applied Science \\ in partial fulfillment of the requirements for the degree of \\ Doctor of Philosophy \\ by \\ Joshua L. Heuslein \\ December 2016
}




\section{Table of Contents}

Table of Figures .................................................................................................................... viii

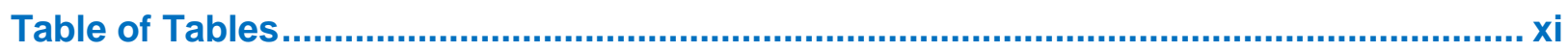

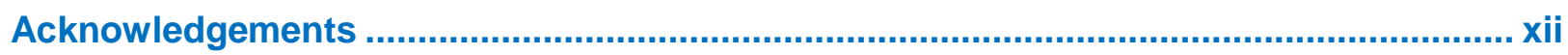

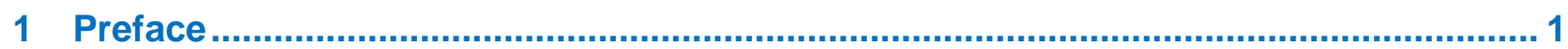

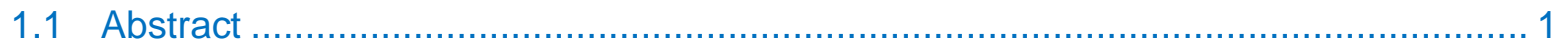

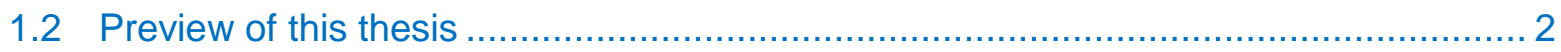

2 Peripheral arterial disease (PAD) and arteriogenesis................................................ 2

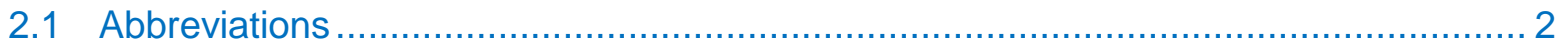

2.2 Clinical background and motivation................................................................... 3

2.2.1 Peripheral arterial disease - definition and epidemiology …......................... 3

2.2.2 Clinical manifestations of PAD ................................................................ 4

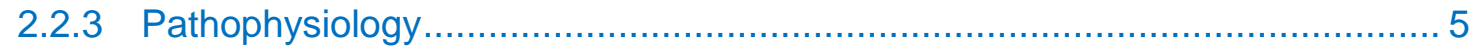

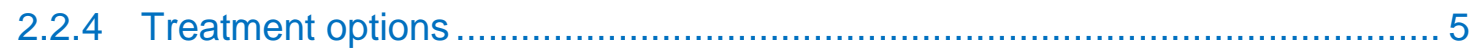

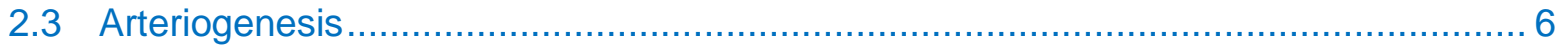

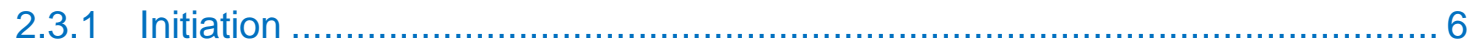

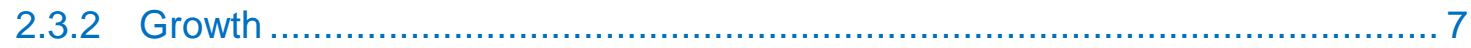

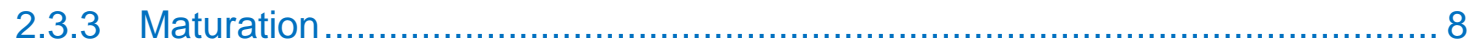

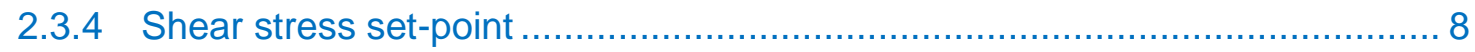

3 Epigenetics in peripheral arterial disease (PAD) …...................................................11

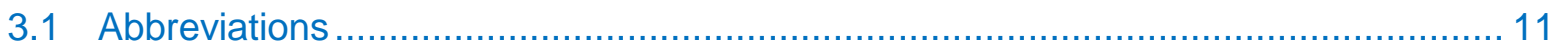

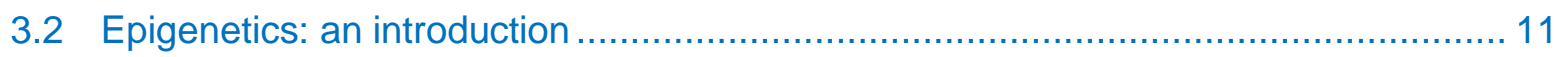

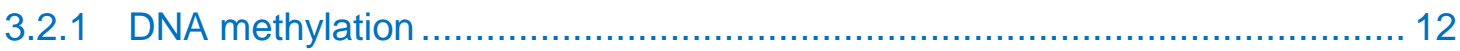

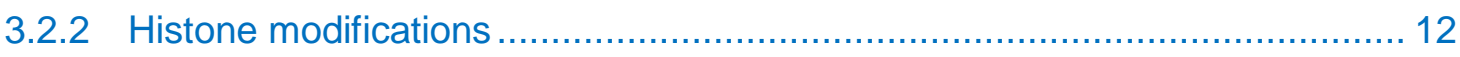

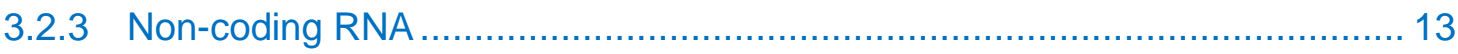

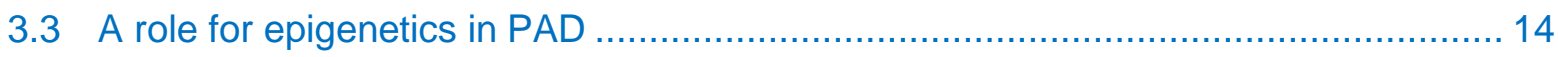

3.3.1 Histone modification and DNA methylation patterns and are likely to be altered

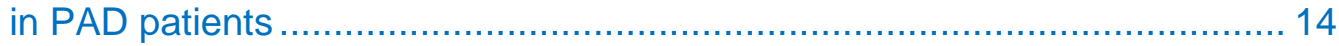

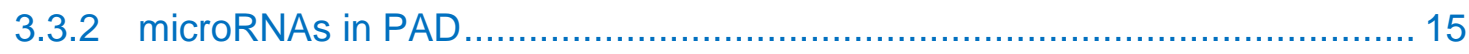

3.4 Epigenetic regulation in response to hindlimb ischemia ..................................... 16

3.4.1 Histone post-translational modifications and DNA methylation ..................... 16

3.4.2 Non-coding RNAs ............................................................................. 17 


\section{Mechanisms of amplified arteriogenesis in collateral artery segments exposed to}

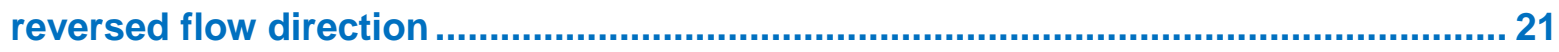

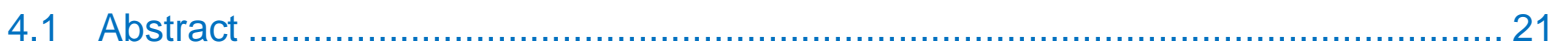

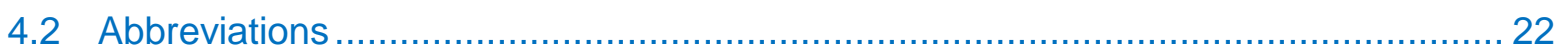

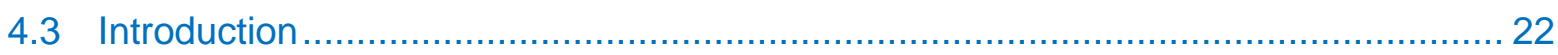

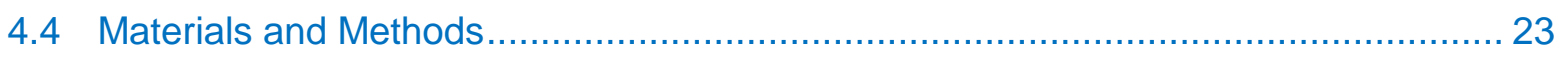

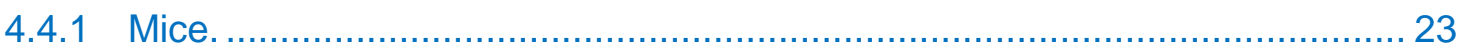

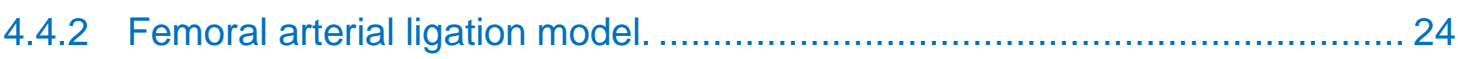

4.4.3 Muscular branch ligation model............................................................. 24

4.4.4 Laser Doppler perfusion imaging.......................................................... 25

4.4.5 Tissue harvesting for cross sectional and collateral structure analysis by

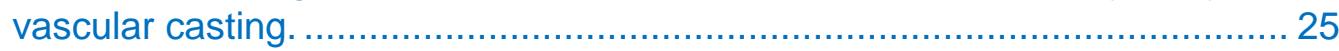

4.4.6 Cross sectional analysis of collateral artery structure ................................. 26

4.4.7 Cross sectional analysis for regional capillary density and muscle fiber

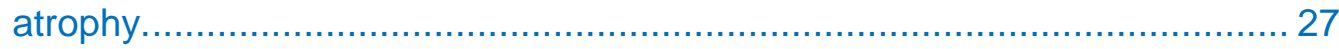

4.4.8 Immunofluorescence labeling of pericollateral $\mathrm{Mac}^{+}$cells............................ 27

4.4.9 Tissue harvesting for in-vivo collateral artery endothelial planar cell

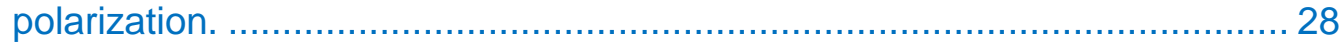

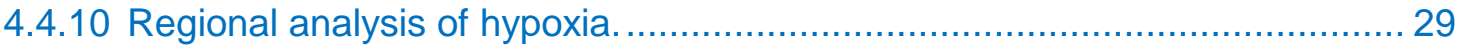

4.4.11 Human umbilical vein endothelial cell culture. ............................................... 30

4.4.12 In-vitro exposure of endothelial cells to simulated ligation shear stress......... 31

4.4.13 HUVEC RNA isolation, qRT-PCR, and microarray gene expression profiling.31

4.4.14 Immunofluorescence labeling for HUVEC planar cell polarity ........................ 33

4.4.15 Data analysis of endothelial cell planar polarity. ........................................... 33

4.4.16 HUVEC western blot and NF-KB activity assays....................................... 34

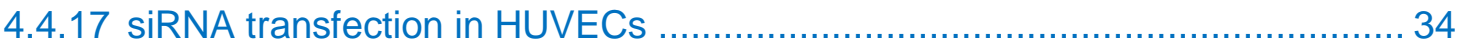

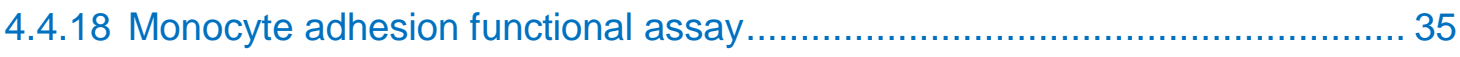

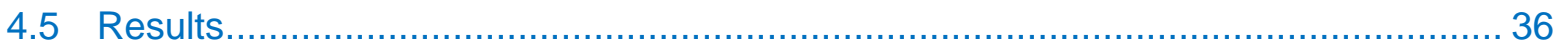

4.5.1 Planar polarization confirms endothelial cell responsiveness to predicted flow

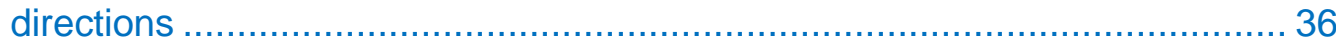

4.5.2 Arteriogenesis is enhanced in collateral artery segments exposed to reversed

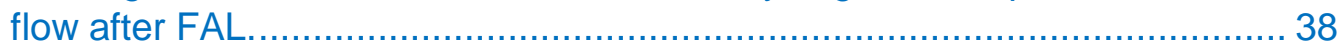

4.5.3 FAL does not elicit regional differences in hypoxia in gracilis adductor muscles.

4.5.4 Amplified arteriogenesis in flow-reversed segments occurs independent of position. 
4.5.5 HUVECs exhibit directional responsiveness to simulated femoral artery ligation.

4.5.6 Reversed flow broadly enhances the arteriogenic transcriptional profile 43

4.5.7 NFKB as a predicted upstream regulator of gene expression patterns in flowreversed conditions. 45

4.5.8 Amplified arteriogenesis in flow-reversed collaterals depends on ICAM-1 .... 47

4.6 Discussion 50

4.6.1 Reversed flow as an independent stimulus leading to amplified arteriogenesis. 50

4.6.2 Endothelial cell sensing of shear stress magnitude and reversed direction. .. 52

4.6.3 Endothelial cell repolarization and signaling for amplified arteriogenesis.......53

4.6.4 Complex role of eNOS signaling in arteriogenesis. ................................. 54

4.6.5 Implications for understanding shear stress set-point............................. 54

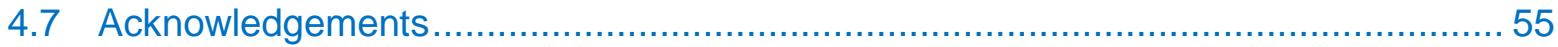

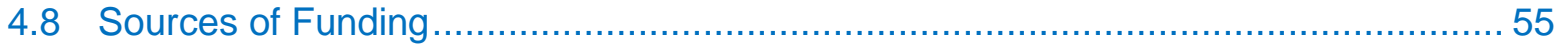

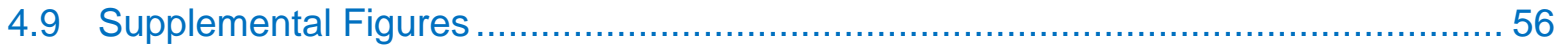

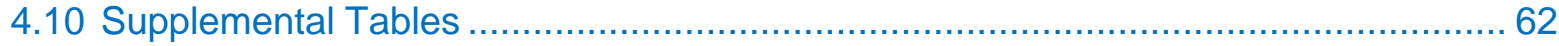

5 DNMT1-dependent DNA hypermethylation constrains arteriogenesis by augmenting

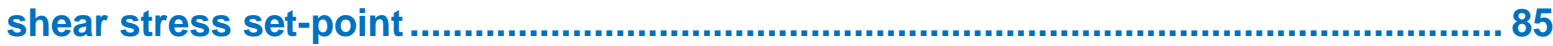

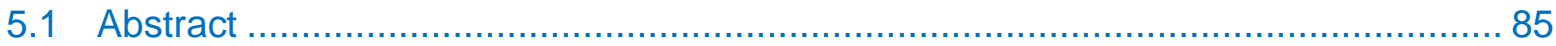

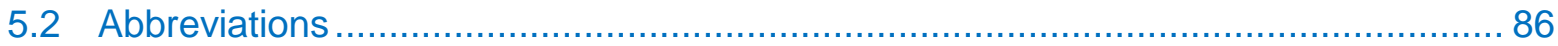

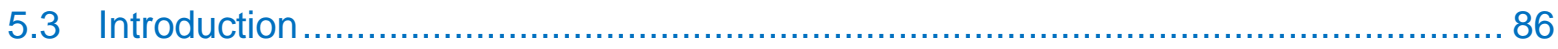

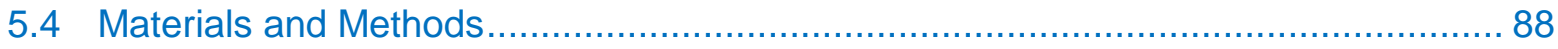

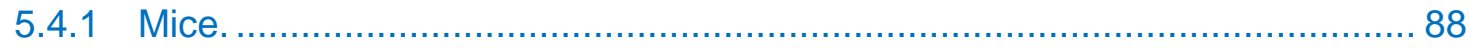

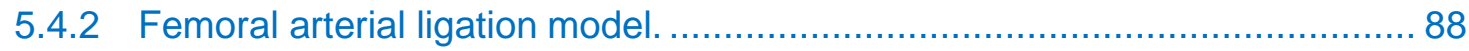

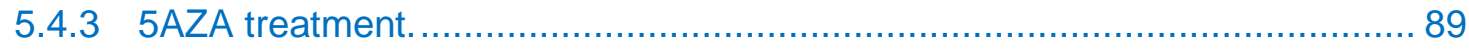

5.4.4 Quantification of global DNA methylation by high resolution melting (HRM).. 89

5.4.5 Tissue harvesting for whole mount vascular casting and cross sectional

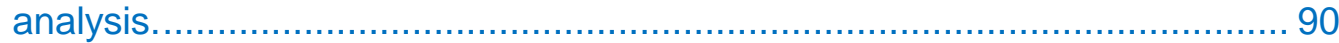

5.4.6 Immunofluorescence labeling of 5-methylcytidine. .................................... 91

5.4.7 Cross sectional analysis of collateral artery structure ................................ 92

5.4.8 Human umbilical vein endothelial cell culture. ........................................... 92

5.4.9 In vitro exposure of endothelial cells to biomimetic shear stress waveforms. 93

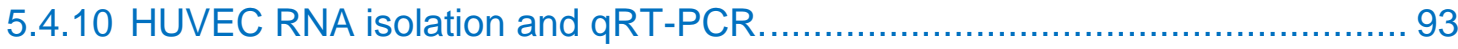

5.4.11 Reduced representation bisulfite sequencing (RRBS) and mRNA sequencing. 


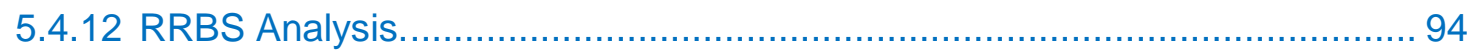

5.4.13 Transillumination laser speckle imaging and shear stress analysis. ............. 95

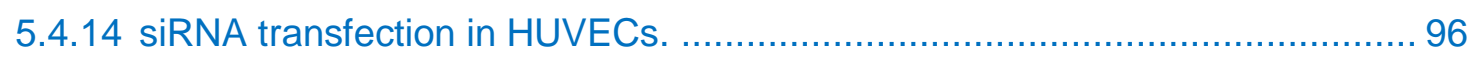

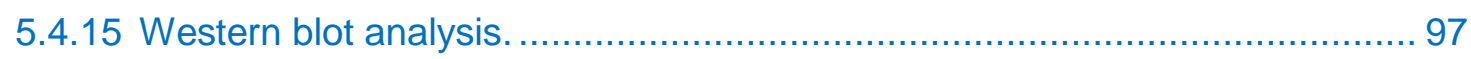

5.4 .16 Monocyte adhesion functional assay ............................................... 98

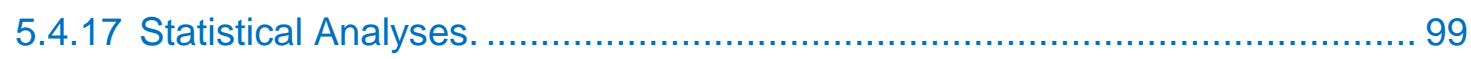

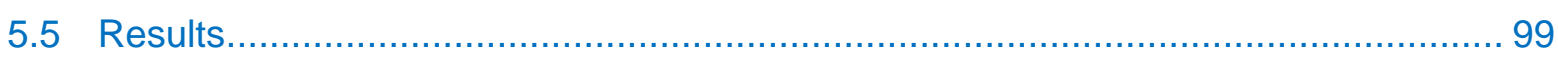

5.5.1 Collateral artery segments exhibiting limited arteriogenic capacity exhibit hypermethylated DNA 12-weeks after FAL. ......................................... 99

5.5.2 ECs exposed to a non-reversed increase in shear stress magnitude exhibit augmented DNMT1 expression.......................................................... 100

5.5.3 Altered genome-wide DNA methylation patterns in ECs exposed to a arteriogenic shear stress waveforms in-vitro ...................................... 101

5.5.4 DNMT1 regulates the adhesion of monocytes to endothelial cells exposed to a non-reversed increase in shear stress magnitude in-vitro. ......................... 104

5.5.5 The arteriogenic capacity of non-reversed collateral artery segments is restored by DNMT1 inhibition......................................................... 105

5.5.6 Shear stress set-point is re-established in non-reversed collateral artery

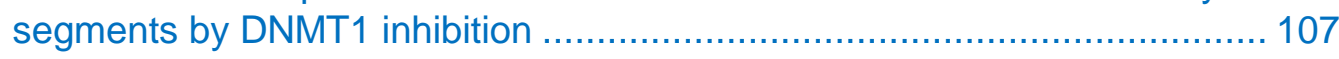

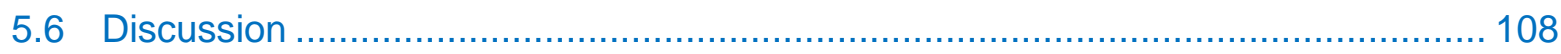

5.6.1 Mapping the EC mechanosensitive DNA methylation to differential arteriogenic capacity 109

5.6.2 The role of DNMT1 in flow-mediated endothelial inflammation is dependent on hemodynamic context ............................................................. 110

5.6.3 Molecular regulation of collateral artery shear stress set-point .................. 111

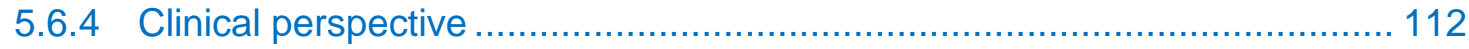

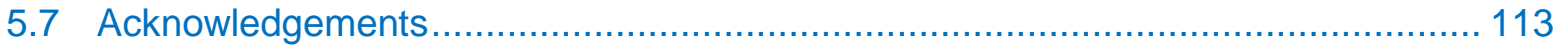

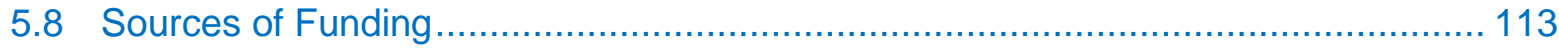

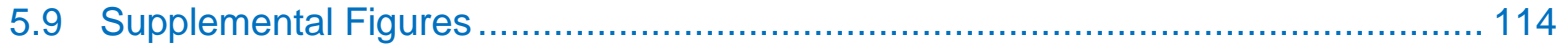

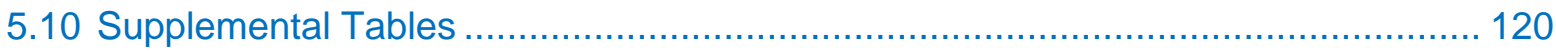

6 Mechanosensitive microRNA-199a regulates perfusion recovery and collateral arteriogenesis in Balb/c mice following femoral arterial ligation ............................ 151

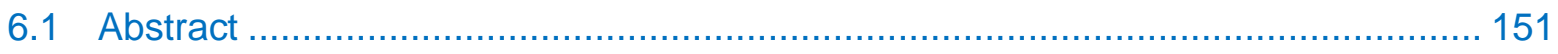

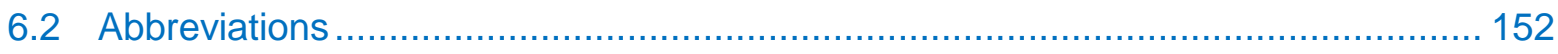

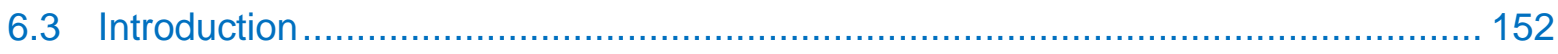

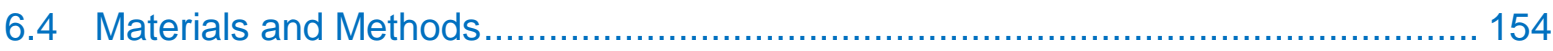


6.4.1 Human umbilical vein endothelial cell culture...................................... 154

6.4.2 In vitro exposure of endothelial cells to biomimetic shear stress waveforms. ............................................................................ 154

6.4.3 HUVEC microarray gene expression profiling. ................................... 155

6.4.4 Transfection of miRNA antagomirs and mimics in HUVECs. ...................... 155

6.4.5 HUVEC RNA isolation and qRT-PCR ............................................ 155

6.4.6 Monocyte adhesion functional assay ............................................... 156

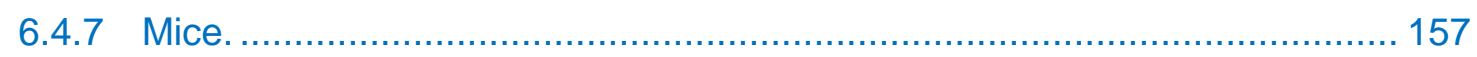

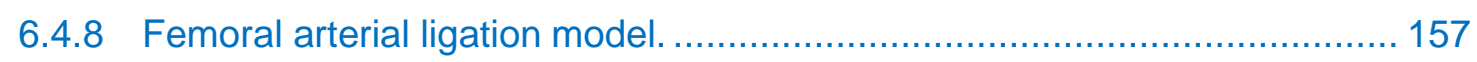

6.4.9 In vivo miR-199a antagomir treatment............................................... 158

6.4.10 In vivo miR-199a mimic treatment................................................... 158

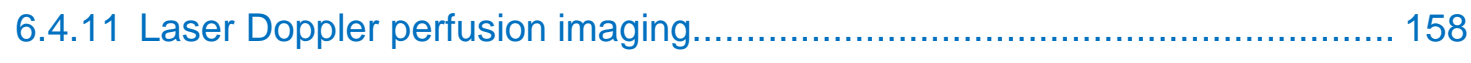

6.4.12 Tissue harvesting for miRNA and target expression.............................. 159

6.4.13 RNA isolation from tissues and qRT-PCR ........................................ 159

6.4.14 Tissue harvesting for whole mount vascular casting and cross sectional

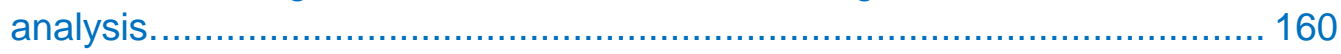

6.4.15 Cross sectional analysis of collateral artery structure............................. 161

6.4.16 Immunofluorescence labeling of pericollateral Mac3 ${ }^{+}$cells....................... 161

6.4.17 Cross sectional analysis for gastrocnemius muscle morphology. ............... 162

6.4.18 Cross sectional analysis for gastrocnemius capillary density..................... 163

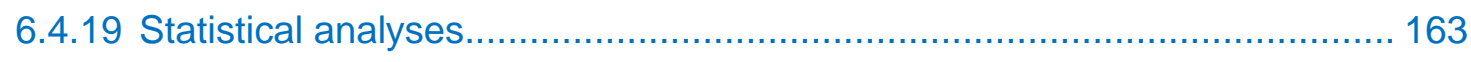

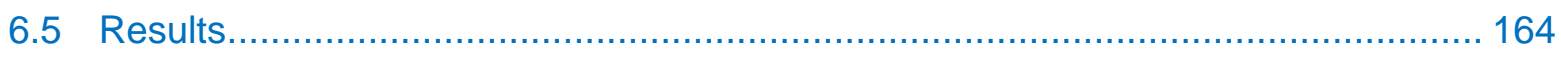

6.5.1 Endothelial cell miRNA expression is regulated by shear stress waveforms biomimetic of collateral artery segments exhibiting differential arteriogenic responses.

6.5.2 MicroRNA-199a negatively regulates pro-arteriogenic endothelial gene expression and monocyte adhesion to flow-exposed ECs in-vitro.

6.5.3 Overexpression of miR-199a limits foot reperfusion following FAL while miR199a inhibition elicits complete perfusion recovery in Balb/c mice.............. 168

6.5.4 Overexpression of miR-199a inhibits arteriogenesis in Balb/c mice............ 170

6.5.5 Inhibition of miR-199a amplifies arteriogenesis in Balb/c mice following

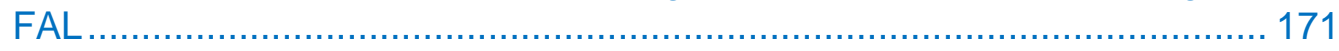

6.5.6 Pericollateral macrophage recruitment is modulated by miR-199a............ 172

6.5.7 MicroRNA-199a inhibition improves gastrocnemius muscle composition in FAL-operated Balb/c mice........................................................ 173

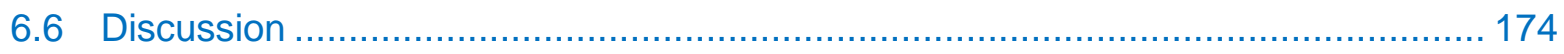


6.6.1 Identification of microRNA-199a as a novel mechanosensitive regulator of proarteriogenic gene expression ....................................................... 175

6.6.2 Previous studies implicating a role of miRNA-regulated arteriogenesis ....... 177

6.6.3 MicroRNA-199a as a regulator of arteriogenesis leading to enhanced

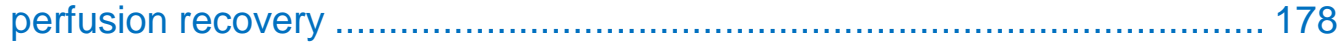

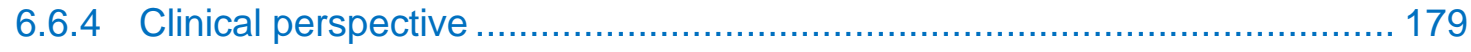

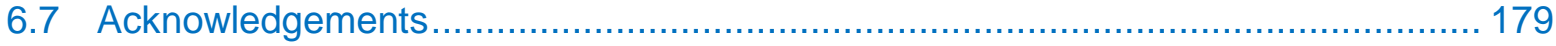

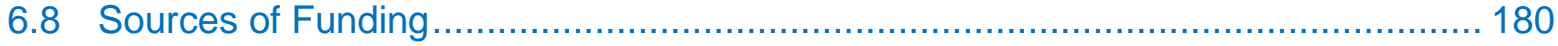

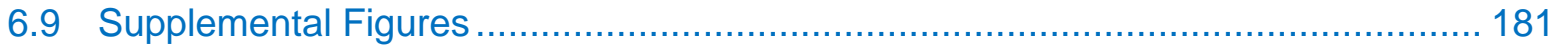

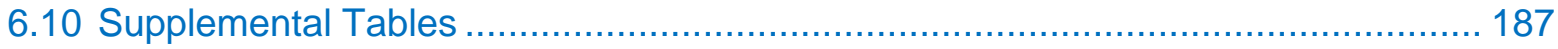

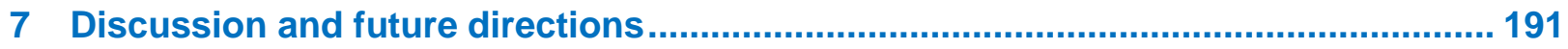

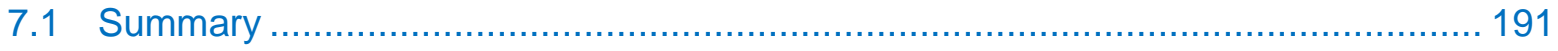

7.2 Thoughts and future studies: DNMT1, DNA methylation, and regulation to the shear

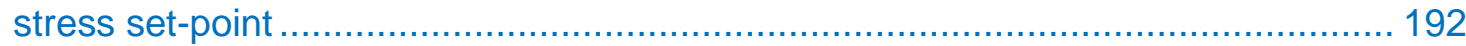

7.3 Thoughts and future directions: miR-199a and mechanosensitive miRNA as regulators of arteriogenesis and as therapeutic targets ................................................. 193

7.4 Thoughts and future studies: arteriogenesis, angiogenesis, and beyond ............... 194

7.4.1 Promise for pro-arteriogenic and pro-angiogenic combination therapy ........ 194

7.4.2 Need to consider muscle composition and metabolism ........................... 195

7.5 Thoughts and future directions: applications to other disease models ................... 197

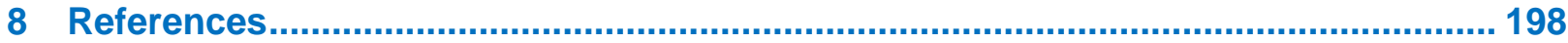




\section{Table of Figures}

Figure 2.1. Overview of arteriogenesis and regulation by shear stress set-point............. 9

Figure 4.1. Collateral artery endothelial cells repolarize in response to reversed shear stress after FAL.

Figure 4.2. Gracilis collateral artery regions exposed to reversed flow exhibit enhanced arteriogenesis after FAL.

Figure 4.3. Genome-wide analysis indicates that reversed flow broadly enhances the arteriogenic transcriptional profile.

Figure 4.4. Reversed flow enhances monocyte adhesion in-vitro in an ICAM-1 dependent manner.

Figure 4.5. ICAM-1 is necessary for enhanced macrophage recruitment and amplified arteriogenesis in collateral segments exposed to a reversed flow. 48

Figure 5.1. Gracilis collateral arteries exposed to a non-reversed increase in shear stress magnitude exhibit limited arteriogenic capacity and hypermethylated

DNA. 100

Figure 5.2. ECs exposed to a non-reversed increase in shear stress magnitude exhibit augmented DNMT1 expression and altered genome-wide DNA methylation patterns in-vitro. 102

Figure 5.3. DNMT1 regulates the adhesion of monocytes to endothelial cells exposed to a non-reversed increase in shear stress magnitude in-vitro. .105

Figure 5.4. The arteriogenic capacity of non-reversed collateral artery segments is restored by DNMT1 inhibition. 106

Figure 5.5. Shear stress set-point in non-reversed collateral artery segments is restored by DNMT1 inhibition. 108

Figure 5.6. Summary of DNMT1-dependent regulation of arteriogenic capacity and shear stress set-point in gracilis adductor collateral arteries following FAL.........112

Figure 6.1. Endothelial cell miRNA expression is differentially regulated by shear stress waveforms biomimetic of collateral artery segments exhibiting varied arteriogenic responses.

Figure 6.2. MicroRNA-199a negatively regulates the expression of CD44, CCND1, and IKK $\beta$ in endothelial cells exposed to biomimetic shear stress waveforms invitro. 166 
Figure 6.3. Monocyte adhesion to HUVECs exposed to shear stress waveforms biomimetic of arteriogenic collaterals is modulated by miR-199a.

Figure 6.4. Overexpression of miR-199a limits foot reperfusion following FAL while miR199a inhibition leads to complete perfusion recovery in Balb/c mice. 169

Figure 6.5. Overexpression of miR-199a inhibits arteriogenesis in Balb/c mice. 170

Figure 6.6. Inhibition of miR-199a amplifies arteriogenesis in Balb/c mice following FAL.

Figure 6.7. Pericollateral macrophage recruitment is modulated by miR-199a. 173

Figure 6.8. MicroRNA-199a inhibition improves gastrocnemius muscle composition in FAL-operated Balb/c mice. 174

\section{Supplemental Figures}

Supplemental Figure 4.1. Laser Doppler perfusion recovery curve. 56

Supplemental Figure 4.2. Regional characterization of hypoxia, muscle fiber size, and capillary density in mouse gracilis muscle.

Supplemental Figure 4.3. Arteriogenesis in gracilis collateral artery segments exposed

to a reversed flow after muscular branch ligation. .58

Supplemental Figure 4.4. HUVECs repolarize in response to directional change in shear stress induced by simulated FAL 59

Supplemental Figure 4.5. Gene ontology clustering architecture of microarray expression in non-reversed and reversed flow waveforms. 60

Supplemental Figure 4.6. Amplified arteriogenesis in flow reversed segments does not require eNOS.

Supplemental Figure 5.1. Similar degree of methylation coverage in HUVECs exposed to biomimetic shear stress waveforms. .114

Supplemental Figure 5.2. DNA methylation across different gene regions. .115

Supplemental Figure 5.3. DNA methylation negatively correlates to mRNA expression in gene promoter regions. 116

Supplemental Figure 5.4. Genome-wide mRNA expression patterns in HUVECs exposed to arteriogenesis biomimetic shear stress waveforms.

Supplemental Figure 5.5. Top 50 most significantly overrepresented gene ontology (GO) biological processes. 
Supplemental Figure 5.6. DNMT1 inhibition also restores the arteriogenic capacity of non-reversed collateral artery segments in Balb/c mice

Supplemental Figure 6.1. Regional analysis of Balb/c mice treated with miR-199a mimic after FAL.

Supplemental Figure 6.2. Regional analysis of Balb/c mice treated with anti-miR-199a after FAL 182

Supplemental Figure 6.3. Regional pericollateral macrophage recruitment is modulated by miR-199a. 183

Supplemental Figure 6.4. Gastrocnemius muscle composition is not further impaired with miR-199a overexpression following FAL .184

Supplemental Figure 6.5. Sigmoidal response of CD44, IKK $\beta$, CCND1 mRNA expression and THP-1 monocyte adhesion to alterations in miR-199a expression in endothelial cells exposed to biomimetic shear stress waveforms in-vitro. . 185 Supplemental Figure 6.6. MicroRNA-199a inhibition does not affect capillary density in the gastrocnemius muscle of FAL-operated Balb/c mice. 


\section{Table of Tables}

Table 2.1. Comparison of microarray genes to RT-PCR analyses for selected genes known to mediate arteriogenesis .44

Table 2.2. NFKB is a predicted upstream regulator of gene expression patterns seen with flow reversed increased shear stress.

\section{Supplemental Tables}

Supplemental Table 2.1. Gene ontology analysis for over-representation of molecular function

Supplemental Table 2.2. Predicted upstream regulators of gene expression patterns seen with reversed and non-reversed waveforms.

Supplemental Table 2.3. Ingenuity Pathways Analysis significant cannonical pathways 64

Supplemental Table 2.4. All differentially regulated genes between reversed versus control conditions with FDR $<0.10$

Supplemental Table 2.5. All differentially regulated genes between non-reversed versus control conditions with FDR $<0.10$

Supplemental Table 5.1. Summary of total number of reads, mapping ratio, and CpG coverage in RRBS datasets.

Supplemental Table 5.2. Summary of total number of reads, mapped reads, and mapping ratio for mRNA-seq datasets.

Supplemental Table 5.3. All DMRs corresponding to the promoter regions of genes with a methylation ratio difference $\geq|0.10|$ and FDR $<0.1$ between nonreversed and reversed conditions

Supplemental Table 5.4. All significantly hypermethylated promoter regions corresponding to genes that are down-regulated in non-reversed conditions compared to reversed conditions.

Supplemental Table 5.5. All significantly hypomethylated promoter regions corresponding to genes that are up-regulated in non-reversed conditions compared to reversed conditions.

Supplemental Table 5.6. Top 50 significantly overrepresented biological process $\mathrm{GO}$ terms for genes with expression patterns corresponding to differential promoter methylation under arteriogenic shear stress waveforms.

Supplemental Table 6.1. Differential microRNA gene expression in ECs exposed to arteriogenesis-biomimetic shear stress waveforms. 


\section{Acknowledgements}

Through my graduate studies, I have received a tremendous amount of support from many students, faculty, and staff. Thank you for letting me come to your labs and offices to ask questions, for providing your expertise, and for your dedication to a collaborative environment.

Thank you to my dissertation committee - Brian Helmke, Shayn Peirce-Cottler, Gary Owens, and Brian Annex. Our discussions, though they have challenged me at times, have ultimately shaped much of this work have helped me improve as a scientist and as a thinker.

I am also thankful for the mentorship of my advisor, Rich Price. He has continually provided guidance and support that have helped me develop both scientifically and professionally. He has encouraged me to think independently and has allowed me the freedom to pursue my own ideas. Thank you for your patience and for the chance to succeed, as well as the chance to fail.

Thank you to all the past and present members of the Price lab. I would especially like to thank Josh Meisner, for his mentorship, friendship, and essential contributions to this work, as well as Ji Song, for all her technical expertise. I am also thankful to Devin Weinberg, Lauren Boerboom, and Christen Hamilton of the Blackman Lab for their guidance during my first two years of graduate school.

Thank you to all the undergraduate students that have trusted me as an advisor. Their questions have helped me grow as a mentor, teacher, and communicator, and their hard work has been essential to this work.

I am deeply grateful to my family for their unconditional love and support, not just during graduate school, but throughout my life. You have always encouraged me to pursue my interests, curiosities, and for me to always do the best I can.

Finally, I am incredibly grateful to my fiancée, Anna. Thank you for your incredible patience, encouragement, advice, friendship, love, and support. You keep me laughing and constantly remind me that the most important things are not in lab. 


\section{Preface}

\section{$1.1 \quad$ Abstract}

Peripheral arterial disease (PAD) is the leading cause of lower limb amputation and is estimated to affect over 202 million people worldwide. PAD arises when atherosclerotic plaques block arteries in the lower limbs, thereby limiting blood flow to the distal tissue. The lumenal expansion of pre-existing collateral arteries bypassing the occlusion(s) (i.e. arteriogenesis) remains a promising therapeutic option. However, large clinical trials have had limited success to date, highlighting the critical need to better understand the basic mechanisms regulating arteriogenesis.

In this thesis, we report that collateral artery segments in the mouse hindlimb exhibit either "moderate" or "amplified" arteriogenesis, depending on the initiating hemodynamics to which they are exposed (i.e. non-reversed or reversed flow waveforms, respectively) following femoral arterial ligation (FAL). We first determined this reversed flow-mediated amplification of collateral artery growth to be dependent on ICAM-1 mediated macrophage recruitment. Moreover, we were able to apply flow waveforms biomimetic of those quantified in-vivo, to endothelial cells (ECs) invitro, and perform genome-wide analyses to comprehensively map EC mechanosensitive signaling to sustained, differential arteriogenesis responses.

We next sought to uncover molecular regulators of arteriogenic capacity and collateral artery maturation. We determined that ECs exposed to a non-reversed flow waveform exhibit increased DNMT1 expression and DNA hypermethylation. Moreover, we determined that DNMT1-dependent EC DNA hypermethylation regulates arteriogenic capacity via adjustments to shear stress set-point in-vivo, identifying a novel role for DNA methylation in arteriogenesis.

Finally, we interrogated our genome-wide analysis of EC mechanosensitive signaling mapped to differential arteriogenesis responses to identify potentially novel microRNA regulators of arteriogenesis. Using this unique approach, we discovered microRNA-199a to be a potent 
mechanosensitive regulator of perfusion recovery and arteriogenesis after arterial occlusion. To this end, miR-199a inhibition elicited complete foot perfusion recovery, markedly augmented collateral arteriogenesis, and improved gastrocnemius muscle tissue composition following FAL.

Overall, these studies demonstrate the critical roles of EC mechano-signaling, flow mediated inflammation, and epigenetic mechanisms in regulating endogenous shear stress-mediated arteriogenesis. These results may also have important bearing on the development of therapeutic arteriogenesis strategies in patients with peripheral arterial disease.

\subsection{Preview of this thesis}

In Chapter 2, this thesis will begin with an introduction to the definition, epidemiology, and pathophysiology of peripheral arterial disease, the primary clinical motivation for this work. Chapter 2 will also include an introduction to the individual stages of arteriogenesis and the concept of shear stress set-point. Chapter 3 will review epigenetic mechanisms, their relevancy to $P A D$, and recent work investigating epigenetic regulation of the response to hindlimb ischemia, the pre-dominant animal model used to study PAD. Chapters 4-6 will report our recent studies investigating the mechanisms of amplified arteriogenesis in collateral artery segments exposed to reversed flow direction, the regulation of arteriogenic capacity and shear stress set-point via DNMT1-dependent DNA hypermethylation, and the role for mechanosensitive microRNA-199a as a novel regulator of perfusion recovery and arteriogenesis, respectively. Finally, Chapter 7 will provide discussion for the significance and future directions of this work.

\section{Peripheral arterial disease (PAD) and arteriogenesis}

\subsection{Abbreviations}

PAD peripheral artery disease

CAD coronary artery disease

$\mathrm{ABI} \quad$ ankle brachial index 


$\begin{array}{ll}\text { IC } & \text { intermittent claudication } \\ \text { CLI } & \text { critical limb ischemia } \\ \text { EC } & \text { endothelial cell } \\ \text { SMC } & \text { smooth muscle cell }\end{array}$

\subsection{Clinical background and motivation}

\subsubsection{Peripheral arterial disease - definition and epidemiology}

Peripheral arterial disease (PAD) is a chronic disease that is caused by blockage(s) of the arteries, typically in the lower limbs, due to atherosclerosis ${ }^{1}$. PAD is the leading cause of lower limb amputations and is estimated to have a total cost exceeding $\$ 21$ billion annually in the United States alone ${ }^{2}$. It is estimated that $>200$ million people worldwide, in high-, middle, and low-income countries, have PAD, indicating that it is truly a global problem ${ }^{3}$.

PAD has a total prevalence that is nearly equal to that of coronary artery disease (CAD) ${ }^{4}$. Moreover, PAD prevalence increases with age, where total PAD prevalence is $\sim 6-12 \%$ of people $50-60$ years old and increases to $20-25 \%$ in people over 70 years ${ }^{1,5-9}$. In addition to age, smoking is also a strong risk factor for $P A D^{10}$. Smokers have been shown to develop symptoms of PAD almost 10 years earlier than non-smokers and are twice as likely to develop complications necessitating lower limb amputation ${ }^{11}$. Smoking also appears to be more strongly associated with PAD than CAD and increases the likelihood of graft failure 3 -fold ${ }^{11}$. Diabetes mellitus has also been shown to be a strong risk factor for PAD; $26-33 \%$ of individuals with PAD also have concomitant diabetes ${ }^{6,12,13}$. Patients with both diabetes and PAD have a 5-fold higher rate of lower limb amputation and 3-fold higher mortality than non-diabetic PAD patients over a 4.5 year followup period ${ }^{14}$. Other risk factors for PAD include hypertension, dyslipidemia, race, chronic kidney disease, c-reactive protein levels, and vitamin $\mathrm{D}$ deficiency. To date, searches for genetic susceptibility variants, such as GWAS, have had limited success for PAD ${ }^{15}$. 


\subsubsection{Clinical manifestations of PAD}

PAD is clinically defined as an ankle-brachial index $(A B I)$, the ratio of the highest systolic blood pressure in the dorsalis pedis or posterior tibial artery to the highest systolic blood pressure in the left or right brachial artery, of $\leq 0.90^{5,7}$. Whereas a normal $A B I$ is $1.0-1.4$, an $A B I \leq 0.90$ is indicative of hemodynamically significant occlusive disease between the heart and the ankle ${ }^{1}$. An $A B I \leq 0.90$ is associated with between a 3- to 4-fold increase in 10-year cardiovascular mortality ${ }^{16}$. While $A B I$ diagnoses PAD, it does not predict or correlate with disease severity 4 .

There are two distinct symptomatic clinical manifestations of PAD - intermittent claudication (IC) and critical limb ischemia (CLI). Intermittent claudication is defined as calf or buttock pain with walking that is relieved with rest ${ }^{4}$. Approximately $10-35 \%$ of PAD patients present with classic claudication symptoms and $20-40 \%$ present with atypical leg pain ${ }^{11}$. Patients with intermittent claudication have annual risk of amputation or death of $1-2 \%{ }^{1}$. Critical limb ischemia, in contrast, is defined as reduced blood flow to the lower limb resulting in pain, even at rest, with or without associated ulcers or gangrene ${ }^{4}$. These different clinical manifestations appear to be quite distinct as only $1-2 \%$ of IC patients actually progress to CLI per year and many CLI patients do not report previous $\mathrm{IC}^{7}$. In total, only $1-2 \%$ of PAD patients present with critical limb ischemia, though their prognosis is much more severe ${ }^{4}$. CLI patients have an annual mortality of $20-25 \%$ and a $25-40 \%$ 6-month risk of major amputation ${ }^{1,17}$.

Interestingly, $20-50 \%$ of individuals with PAD report as "asymptomatic" in that they don't typically experience leg symptoms, with walking or at rest, despite an $A B \mid \leq 0.90^{18}$. Despite the lack of self-reported symptoms, "asymptomatic" PAD patients have been shown to have poorer functional performance (6-minute walk test), poorer quality of life, and more adverse calf muscle characteristics (higher calf muscle fat percentage, smaller calf muscle area) than sedentary, normal individuals ${ }^{18}$. Moreover, "asymptomatic" patients still have an increased mortality ${ }^{19}$. Indeed, patients with PAD but with no clinical evidence of CAD have the same relative risk of 
death from cardiac or cerebrovascular cause as those patients whose main diagnosis is $C A D^{17}$. These studies demonstrate that there is no truly "asymptomatic" manifestation of PAD.

\subsubsection{Pathophysiology}

Peripheral arterial disease is a pathophysiological "manifestation of systemic atherosclerosis"17, whereby the underlying mechanisms for the functional limitations of PAD are hemodynamic in origin $^{20}$. Atherosclerosis is a progressive disease of the large arteries characterized by the accumulation of lipids and fibrous elements in the sub-endothelial space (intima), in arterial regions experiencing disturbed blood flow ${ }^{21,22}$. Advanced lesions can grow sufficiently large, with the continued intimal accumulation of cholesterol-laden macrophages (i.e. foam cells), to block blood flow ${ }^{22}$. As a consequence of these atherosclerotic blockages, reduced blood flow to the lower extremities leads to an insufficient oxygen supply (i.e. ischemia) for meeting the metabolic demands of these distal tissues. The majority of patients with symptomatic PAD have a complete occlusion in the iliac or femoral arterial system between the aorta and the foot ${ }^{1}$. In addition, PAD patients exhibit endothelial dysfunction ${ }^{23,24}$ and microvascular impairments (i.e. reduced capillarity ${ }^{25}$ and increased arteriolar rarefaction ${ }^{26}$ ) that further lead to distal tissue ischemia.

\subsubsection{Treatment options}

Surgical and catheter based revascularization methods are currently the preferred treatment for patients presenting with claudication ${ }^{4}$. In correctly chosen patients, these revascularization methods have been shown to have preserved life and limb of $>75 \%$ of patients at 1 year following revascularization ${ }^{27}$. However, many PAD patients are not amenable to surgical revascularization options and many that do undergo surgery receive little to no long term benefit ${ }^{4}$. This has led to new therapeutic strategies that have sought to use biochemical, molecular, and cellular-based approaches to induce endogenous revascularization (i.e. angiogenesis and/or arteriogenesis) to restore lower limb perfusion. The stimulation of angiogenesis, or the growth of new capillaries from pre-existing vessels, is important as capillary density is reduced in PAD patients ${ }^{4,25,28}$. 
However, it is also imperative to restore the driving pressure to the distal tissue via lumenal expansion (i.e. arteriogenesis) of collateral arteries bypassing the occlusion(s) ${ }^{26,29,30}$. Yet, largescale therapeutic arteriogenesis clinical trials have been largely unsuccessful to date due to insufficient and/or impermanent collateral artery growth ${ }^{31-35}$. These failures have highlighted our incomplete understanding of the basic mechanisms of arteriogenesis and underscore the critical need for the continued study of the endogenous regulation of this complex, highly orchestrated process in order to achieve eventual therapeutic success.

\subsection{Arteriogenesis}

It has been known since the 1780s that there is a pre-existing network of collateral arteries capable of compensating for a major arterial occlusion ${ }^{36}$. Arteriogenesis, or the lumenal growth of endogenous collateral arteries that provide alternate paths for blood flow around arterial occlusions, can prevent ischemic injury in downstream tissue. This collateral growth has been shown to occur in the human arterial systems of the lower limbs, heart, and brain ${ }^{36-38}$. Altogether, arteriogenesis is a multi-faceted, highly coordinated signaling cascade involving recruitment, migration, and proliferation of multiple cell types, as well as the re-organization of the extracellular matrix. The progression of this complex process of arteriogenesis (or collateral growth) can be broken down into three stages: initiation, growth, and maturation ${ }^{39}$.

\subsubsection{Initiation}

As early as 1893, Thoma observed a correlation between vessel diameter and blood flow ${ }^{40}$. Biomechanical forces exerted by blood flow on the endothelium are now known to be critical in the regulation of cell phenotype and blood vessel remodeling ${ }^{37,41-48}$. Indeed, a key initiating stimulus for arteriogenesis is an increase in shear stress ${ }^{37,49}$.

Upon occlusion of a major artery, the downstream vascular network experiences a drop in pressure. This steep pressure gradient is further sustained by the higher resistance of the collateral network due to the increased vascular path length and small initial diameters of these 
arteries. The resulting pressure gradient drives blood flow along any pre-existing collateral arteries that bridge the occlusion(s), resulting in an increased shear stress exerted on the endothelium ${ }^{39}$. Endothelial cells (ECs) can sense and transduce changes in the wall shear stress into biophysical, biochemical, and gene signaling responses (i.e. mechanotransduction) ${ }^{43}$, via mechanosensory complexes (e.g. CD31 / VE-Cadherin / VEGFR2) ${ }^{50}$, that can initiate the growth response ${ }^{51}$.

\subsubsection{Growth}

Altered shear stress activates the ECs lining collateral arteries, inducing an inflammatory response that begins the collateral growth phase of arteriogenesis ${ }^{49}$. Both in-vivo and in-vitro studies have shown increased shear stress promotes the expression of a wide array of inflammatory cytokines, chemokines, and adhesion molecules ${ }^{52-54}$. Shear-stress mediated upregulation of adhesion molecules, particularly ICAM-155, are of particular importance as they are known to be critical for collateral growth, primarily through their role in the recruitment of leukocytes ${ }^{56}$. Additionally, the upregulation of a variety of chemokines (e.g. CCL2, GM-CSF, CXCL1) helps to induce the recruitment of bone-marrow derived cell populations that propagate the growth process ${ }^{57-59}$. While multiple leukocyte populations play a role initiating collateral artery growth (see review by Meisner and Price ${ }^{39}$ ), monocytes are the most-widely studied. The recruitment of monocytes and subsequent pericollateral accumulation of macrophages has been shown to be necessary for collateral artery growth ${ }^{56,60-68}$. Recruited monocytes and macrophages act a "point-sources" of necessary paracrine growth factors (e.g. INF $\beta^{69}, \mathrm{TNFa}{ }^{70}, \mathrm{TGF} \beta^{71}, \mathrm{FGF}{ }^{72}$ ), which promote the proliferative activity of ECs, smooth muscle cells (SMCs), and adventitial fibroblasts ${ }^{73-75}$. Additional growth factors are simultaneously released from a coordinated breakdown of basement membrane components (e.g. desmin and laminins) and of the internal elastic lamina by matrix metalloproteases (e.g. MMP-2 and MMP-9) ${ }^{39}$. Furthermore, this 
inflammatory signaling induces the differentiation of collateral artery smooth muscle cells from a contractile to a synthetic phenotype ${ }^{52}$.

\subsubsection{Maturation}

As vascular cells proliferate and collateral vessels grow, there is a corresponding expansion of the media and the formation of a neointima ${ }^{52}$. Over time, as lumenal diameter increases, the signaling environment shifts from pro-inflammatory to anti-inflammatory ${ }^{53}$. The number of perivascular macrophages begins to decrease, paralleling a reduction in vascular cell proliferation ${ }^{52,62}$. SMCs return to a contractile phenotype ${ }^{52}$ and there is a reestablishment of a mature basement membrane ${ }^{76,77}$. Ultimately, the largest, most developed collateral vessels tend to mature and stabilize, but at the expense of the smaller, less developed collateral vessels which undergo eventual regression ${ }^{52,78,79}$. Outward lumenal growth is hypothesized to continue until normalizelation to the original shear stress level (i.e. the shear stress "set-point") has been achieved $^{39,41,80,81}$.

\subsubsection{Shear stress set-point}

Arteries adapt to chronic changes in blood flow by undergoing adjustments of their internal diameters $^{41,46,47,49,82-84}$. The concept of an arterial homeostatic wall shear stress magnitude (i.e. shear stress set-point) at which vessels maintain a steady-state lumenal diameter ${ }^{85}$ arises from Murray's principle of minimum work ${ }^{86}$. In 1926, Murray ${ }^{86}$ proposed that the vascular system is optimally configured such that there is a compromise between the frictional cost to drive blood through vessels and the metabolic cost of maintaining blood volume, given by:

$$
H_{t}=\frac{8 \eta l Q^{2}}{\pi r^{4}}+k \pi r^{2} l
$$

2.1

where $\mathrm{H}_{t}$ is the total power requirement, $Q$ is volumetric flow rate, $\eta$ is blood viscosity, $r$ is vessel radius, $l$ is vessel segment length, and $k$ is a constant. By minimizing the total power requirement, 
this compromise occurs at an optimal diameter $\left(\mathrm{d}_{\mathrm{opt}}\right)^{87}$. Therefore, for an optimized vasculature, there ought to be a shear stress $(\tau)$ set-point for any given blood vessel segment such that:

$$
\tau\left(d_{o p t}\right)=\frac{32 \eta Q}{\pi d_{o p t}{ }^{3}}
$$

\section{2}

According to this theory, deviations of shear stress from the set-point initiate a negative feedback process that acts to return shear stress to the original set-point by chronically adapting the arterial diameter $^{84}$ (Figure 2.1).

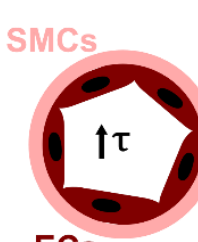

ECs

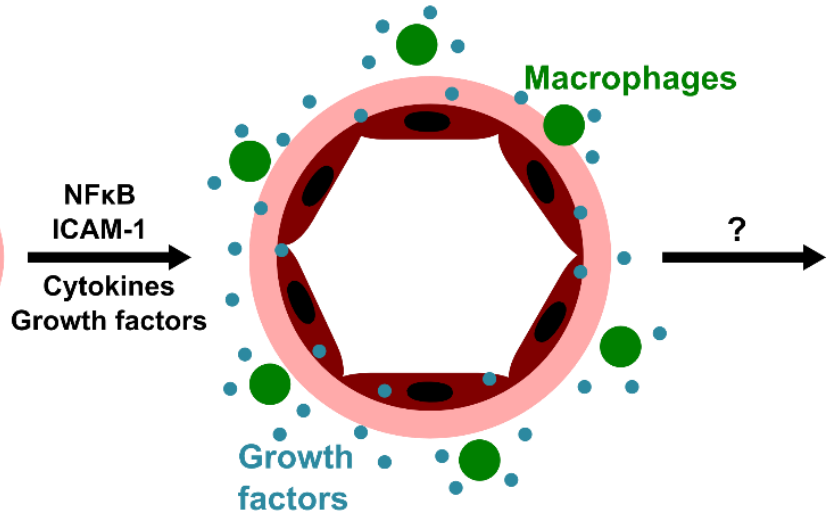

Growth

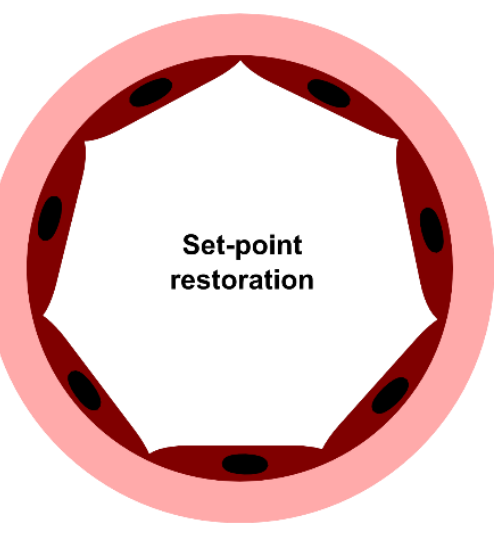

Maturation

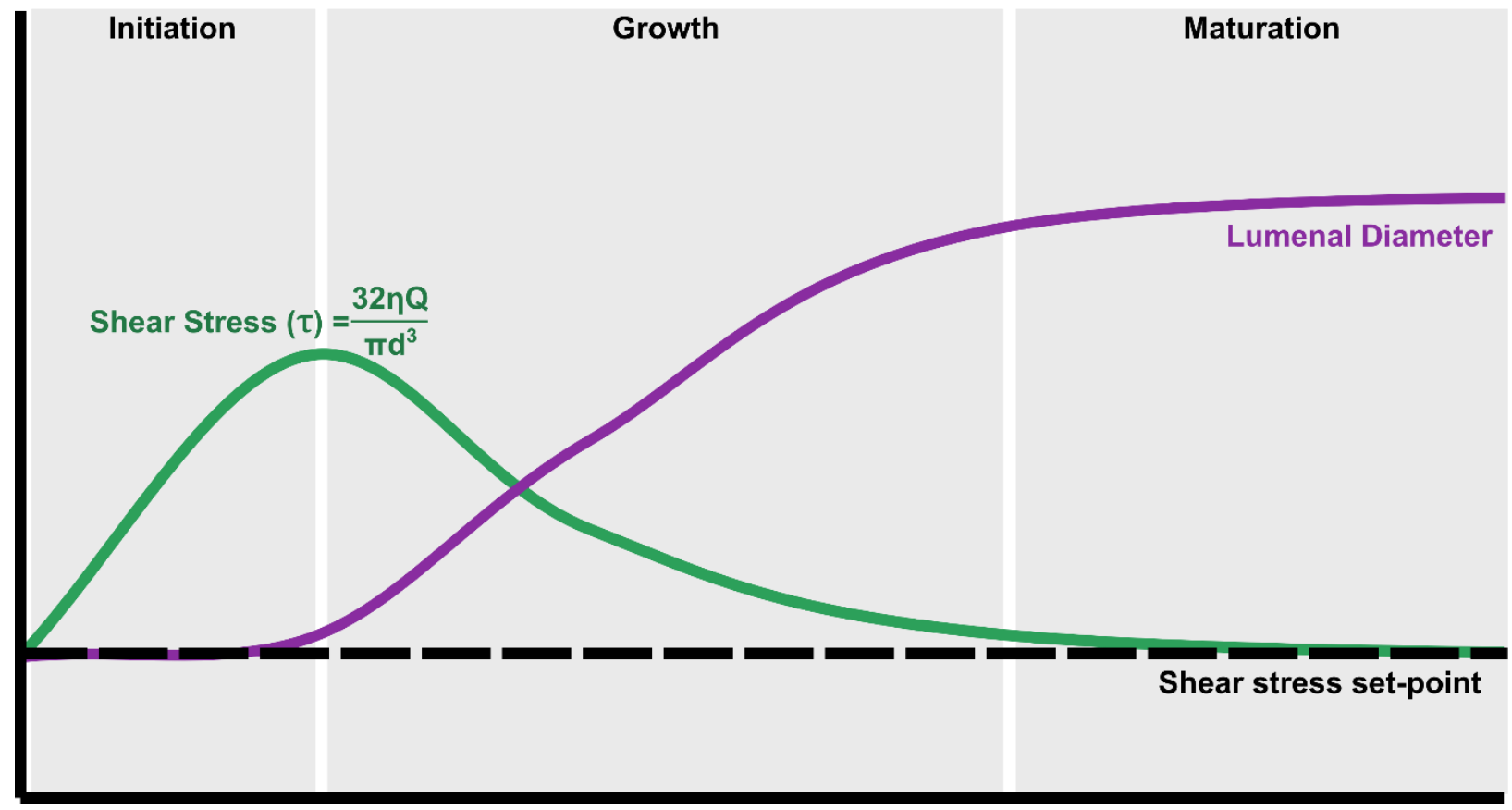

Figure 2.1. Overview of arteriogenesis and regulation by shear stress set-point. 
Though its magnitude can vary in different vascular beds ${ }^{88,89}$, numerous studies have either experimentally or theoretically supported the concept of a shear stress set-point $t^{41,47,48,83,85,90-93}$. However, there are several studies reporting an altered set-point in arteries following adaptation. To this end, following remodeling of the common carotid artery in a canine arterio-venous shunt model, the wall shear rate returned to within $15 \%$ of baseline after $6-8$ months, except for those arteries exposed to the greatest relative flow increases $(>4 x \text { control })^{47}$. In another study in which the carotid artery blood flow of mature rabbits was acutely increased by $60 \%$, the lack of compensatory diameter enlargement caused shear stress to remain elevated two months later ${ }^{83}$. Weanling rabbits, however, exhibited significant diameter enlargement, enabling for shear stress to normalize to the set-point ${ }^{83}$. This age-dependent remodeling was also observed in rats in which ligation of the left internal and external carotid arteries increased right carotid flow by $46 \%$. After 4-weeks, the right carotid outer diameter increased and shear stress returned to initial values in juvenile but not adult rats ${ }^{94}$.

Though critical in determining ultimate arteriogenic capacity, the molecular mechanism(s) involved in establishing and maintaining the shear stress set-point remain unknown. A previous study recently demonstrated that VEGFR3 expression regulates differences in shear stress sensitivity (i.e. cell orientation) between ECs and lymphatic ECs and that the loss of VEGFR3 reduces the diameter of the descending aorta in mice ${ }^{95}$. However, whether VEGFR3 regulates shear set-point in-vivo remains unclear, as in-vivo hemodynamics were not explicitly determined in this study ${ }^{95}$. The overall paucity of data on the mechanism(s) regulating vessel maturation and shear stress set-point highlights both a critical need to enhance our basic understanding of this fundamental vascular growth response and an opportunity to discover new therapeutic targets for treating arterial occlusive diseases. As such, epigenetics are currently an area of intense research focus that could be a way to regulate long-term gene expression in response to local hemodynamic factors. 


\section{Epigenetics in peripheral arterial disease (PAD)}

Epigenetic regulation of cardiovascular disease ${ }^{96,97}$, vascular gene expression ${ }^{98}$, and biomechanical signaling ${ }^{99-101}$ have been previously reviewed elsewhere. Here, we will focus on the epigenetic mechanisms and regulation specifically relevant to peripheral arterial disease.

\subsection{Abbreviations}

PAD peripheral artery disease

CAD coronary artery disease

EC endothelial cell

SMC smooth muscle cell

DNMT DNA methyltransferase

HAT histone acetyltransferase

HDAC histone deacetyltransferase

IncRNA long noncoding RNA

miRNA microRNA

FAL femoral arterial ligation

\subsection{Epigenetics: an introduction}

Epigenetics is "a bridge between genotype and phenotype" - it is the modification to gene expression and phenotype in a sequence-independent manner ${ }^{102}$. Epigenetic mechanisms, such as DNA methylation, histone modifications, and non-coding RNAs, can regulate gene expression by altering DNA accessibility and chromatin structure ${ }^{102}$. The significance of epigenetics in vascular biology, as regulators of molecular signaling and as potential therapeutic targets to treat disease, is now well-recognized ${ }^{98,99,103}$. 


\subsubsection{DNA methylation}

DNA methylation is the addition of a methyl group to a cytosine base pair at the 5' carbon-position, (i.e. 5-methylcytosine), occurring most often at $\mathrm{CpG}$ dinucleotide site ${ }^{103}$. Methylation occurs through the activity of DNA methyltransferases (DNMTs), including DNMT1, DNMT3a, and DNMT3b ${ }^{98,104,105}$. DNMT1 is thought to pre-dominate post-development, preferentially methylating hemi-methylated DNA, whereas DNMT3a and DNMT3b preferentially target fully unmethylated DNA during embryogenesis and gametogenesis ${ }^{104}$. DNMT1, however, is also capable of de novo methylation ${ }^{104}$. Demethylation can occur passively or actively, the latter thought to be mediated by the ten-eleven translocation enzyme family (TET1, TET2, TET3) and by oxidation of 5methylcytosine to 5 -hydroxymethylcytosin ${ }^{106,107}$. Approximately $40-60 \%$ of human genes are associated with dense regions of $\mathrm{CpG}$ sites with higher than expected $\mathrm{CG}$ content called $\mathrm{CpG}$ islands ${ }^{98,108,109}$. In healthy cells, CpG islands are typically hypomethylated thereby allowing for an open chromatin structure and transcriptional activity ${ }^{108}$. However, DNA methylation of gene promoter regions is associated with repression of gene expression ${ }^{110-112}$. Inhibition of gene expression is thought to be achieved by physically impeding transcription factor binding to the gene promoter and/or to methyl-CpG-binding domain proteins ${ }^{100}$.

\subsubsection{Histone modifications}

Chromatin, is a "beaded chain" DNA-protein complex consisting of a string of nucleosomes (i.e. DNA nucleotides wrapped around an octamer of histone proteins) connected by linker DNA ${ }^{113}$. The higher order packing of chromatin can be dynamically regulated by post-translational modifications to histone proteins ${ }^{113}$. Typically, more open chromatin enables for transcription factor binding whereas more condensed chromatin represses gene expression ${ }^{98}$. There are numerous (>100) post-translational histone modifications, though the most well characterized types are acetylation and lysine methylation ${ }^{103,114}$. Histone acetylation is governed by histone acetyltransferase (HAT) and deacetyltransferase (HDAC) activity ${ }^{103,105}$. Histone acetylation tends 
to correlate with chromatin accessibility and transcriptional activity ${ }^{114}$. Histone lysine methyltransferases and demethyltransferases regulate histone lysine methylation ${ }^{105}$. Importantly, histone lysine residues can be mono-, di-, or trimethylated and both the specific lysine residue and number of methyl groups on that residue determine the specific effect on transcription ${ }^{103}$.

\subsubsection{Non-coding RNA}

In addition to DNA methylation and post-translational histone modifications, non-coding RNA can regulate gene expression. Non-coding RNA can be broadly classified as long noncoding RNAs (IncRNAs) and small noncoding RNAs (e.g. microRNAs). Long noncoding RNAs are arbitrarily defined as >200-nucleotide long, non-coding transcripts that may be 5'-capped, spliced, or polyadenylated, and are typically not well-conserved ${ }^{103,115}$. Long noncoding RNAs are typically generated by the same transcriptional machinery as mRNAs ${ }^{116}$. Regulation of gene expression can be mediated by IncRNAs via RNA-, protein-, and DNA-binding interactions ${ }^{116}$, with several IncRNAs having been shown to regulate chromatin remodeling, splicing, and by acting as microRNA sponges ${ }^{115}$. In contrast to IncRNAs, small noncoding RNAs are well-conserved, $<200-$ nucleotides long, and are endogenously processed by endonucleoases ${ }^{115}$. There are a number of different classes of small noncoding RNAs, including microRNAs (miRNAs), PIWI-interacting RNAs (piRNAs), short interfering RNAs (siRNAs), tRNA-derived RNA fragments (tRFs), and small nucleolar RNAs (snoRNAs). Of these, miRNAs have been the most extensively studied to date. MicroRNAs are 22 nucleotide non-coding RNAs that are endogenous regulators of gene expression $^{117}$. Mature miRNA incorporates into the RNA-induced silencing complex (RISC) and then binds to a target mRNA, usually in the $3^{\prime}$ untranslated region (UTR) of the mRNA ${ }^{118}$. MicroRNA binding acts to suppress target gene expression by inhibiting mRNA translation to protein or by promoting mRNA degradation ${ }^{119}$, depending on miRNA-target complementarity ${ }^{120}$. 


\subsection{A role for epigenetics in PAD}

PAD pathophysiology is likely governed by both genetic and epigenetic mechanisms. Though there has been limited success to date in identifying specific genetic variants (e.g. GWAS), it is clear that genetics are associated with PAD. To this point, people with a family history of PAD have a 2 -fold greater risk of developing PAD than those without ${ }^{121}$. Moreover, the odds ratio of having PAD between monozygotic twins is 17.7 and 5.7 for dizygotic twins, with genetic effects accounting for $58 \%$ of the phenotypic variance ${ }^{122}$. However, a potential role for epigenetics in PAD is supported by the strong association of "environmental" risk factors (e.g. age, smoking, and diabetes) with PAD and the fact that the remaining $42 \%$ of phenotypic variance is due to environmental factors.

\subsubsection{Histone modification and DNA methylation patterns and are likely to be altered in PAD patients}

To date, however, there has been a paucity of reports investigating epigenomic changes in PAD. Though it has not yet been explicitly determined, PAD patients are likely to exhibit altered histone modifications and DNA methylation patterns. In support of this concept, altered chromatin structure (i.e. histone modifications) has been observed in patients with common risk factors of PAD such as aging ${ }^{123}$, smoking ${ }^{124}$, and diabetes ${ }^{125}$. Additionally, global and gene-specific DNA methylation patterns are also altered with aging ${ }^{123}$, smoking ${ }^{124,126-128}$, and diabetes ${ }^{129}$, promoting the likelihood of altered DNA methylation patterns in PAD patients. Furthermore, in CAD patients, there is a significant positive correlation of global DNA hypermethylation with elevated plasma levels of homocysteine ${ }^{130}$, a non-protein forming amino acid that can alter DNA methylation patterns $^{131}$. Interestingly, elevated homocysteine levels are also present in PAD patients ${ }^{132}$ and are negatively correlated with functional outcomes for critical limb ischemia $(C L I)^{133,134}$. Moreover, hyperhomocysteinemia (elevated homocysteine) has also been shown to alter the expression of key vascular genes (e.g. eNOS, VEGF, HIF1 $\alpha)^{135-137}$ and to suppresses perfusion recovery ${ }^{137-139}$, 
angiogenesis $^{137-139}$, and collateralization ${ }^{139}$ following arterial occlusion. Together, these results are be consistent with the hypothesis that elevated homocysteine in PAD impairs revascularization and functional outcomes via altered DNA methylation.

\subsection{2 microRNAs in PAD}

MicroRNAs have been the most studied epigenetic modification in PAD, mostly as circulating biomarkers. To this end, miRNA transcriptome profiling identified 53-differentially expressed miRNAs in the peripheral blood of white, male, PAD patients presenting with intermittent claudication from non-symptomatic controls ${ }^{140}$. This study further determined 12-miRNAs, including let-7e, miR-15b, -16, -20b, -25, -26b, -27b, -28-5p, -126, -195, -335, and -363, as diagnostic biomarkers of $\mathrm{PAD}^{140}$. Several additional studies have also reported differential expression of miRNAs in PAD patients with concomitant diabetes. In diabetic patients, lower circulating miR-126 expression correlated with decreased $A B I(A B \mid<0.9)^{141}$. Elevated serum expression of miR-15a, $-15 b,-16,-18 a,-23 a,-24,-27 b,-103,-107$, and -503 was seen in diabetic patients with CLI, while miR-222 and miR-497 were reduced compared to non-symptomatic controls $^{142}$. Additionally, miR-15a and miR-16 expression predicted amputation occurrence in these diabetic patients with CLI ${ }^{142}$. Another study also identified elevated expression of miR-503 in calf muscle biopsies of diabetic patients with $\mathrm{CLI}^{143}$.

Future studies are needed to more comprehensively determine potential biomarkers and functional targets in larger cohorts of PAD patient subpopulations. It is also important to note that while circulating miRNA expression is useful for diagnosis, disease monitoring, and outcome predictions, it does not necessitate a functional role for these miRNAs in disease pathophysiology. Differential miRNA expression in human muscle biopsies and in pre-clinical models may provide the best clues toward identifying specific miRNAs as potential therapeutic targets. Additionally, there is the potential that IncRNAs may play a role in PAD as the 9p21 locus, which is associated with several vascular disease phenotypes, has polymorphisms within the ANRIL IncRNA, a 
directly silencer of the atheroprotective gene, CDKN2B ${ }^{15}$. However, a definitive association between PAD and IncRNAs has not yet been determined.

\subsection{Epigenetic regulation in response to hindlimb ischemia}

\subsubsection{Histone post-translational modifications and DNA methylation}

Studies exploring the role of the epigenetic mechanisms of histone post-translational modifications and DNA methylation in experimental models of PAD are limited. To date, histone acetylation is the only histone modification to be studied in this context. Transcriptional coactivator p300-CBP-associated factor (PCAF) is a HAT that promotes histone ( $\mathrm{H} 3$ and $\mathrm{H} 4)$ acetylation and the transcriptional activation of multiple pro-inflammatory genes ${ }^{144}$. Both genetic and pharmacological inhibition of PCAF impaired blood flow recovery and arteriogenesis following hindlimb ischemia via suppression of inflammatory signaling and leukocyte recruitment ${ }^{144}$. Additionally, though HDAC inhibition is a promising treatment for several ischemic diseases, it has been shown to have variable effects on the response to hindlimb ischemia ${ }^{145}$. To this end, in a femoral arterial excision model, male C57BL/6 mice treated with a class I specific HDAC inhibitor (MS275) exhibited significant muscle atrophy and increased fibrosis. In contrast, treatment with the class Ila specific HDAC inhibitor (MC1568) increased the number of regenerating muscle fibers, though it delayed their terminal differentiation. Moreover, the class lla inhibitor also modestly increased the arteriolar density in ischemic tissues, though neither class I or class Ila HDAC inhibitor affected capillary density or reperfusion ${ }^{145}$. In another study ${ }^{146}$, HDAC9 specific inhibition was shown to reduce EC tube formation, sprouting, and retinal vessel outgrowth. Defects in angiogenesis were rescued by both HDAC9 overexpression and by inhibition of miR17-20a. Additionally, perfusion recovery was reduced $~ 50 \%$ in HDAC9 knockout mice compared to littermate controls, following induction of hindlimb ischemia. Interestingly, there appeared to be sex-differences in HDAC9-/- mice, as female HDAC9 ${ }^{-/-}$mice trended toward improved perfusion recovery ${ }^{146}$ 
Only two previous studies to date have investigated the regulation of the ischemic response by DNA methylation. Both of these studies focused on a conserved family of methyl-CpG-binding proteins (MBD1, MBD2, MBD3, MBD4, and MeCP2) which are responsible for reading alterations to the DNA methylome and for enacting subsequent changes in transcriptional activity ${ }^{147}$. ECspecific MeCP2-null mice were protected against a TGF- $\beta$ induced impairment in angiogenesis ${ }^{148}$. In-vitro, knockdown of MBD2 significantly enhanced angiogenesis and provided protection against peroxide-induced apoptosis ${ }^{149}$. In vivo, $\mathrm{Mbd2}^{-/-}$mice were protected against hindlimb ischemia demonstrating a significant improvement in perfusion recovery, along with increased capillary and arteriole densities following femoral arterial ligation (FAL) compared to littermate controls $^{149}$. MBD2 binds to hypermethylated $\mathrm{CpG}$ regions in the gene promoters of eNOS and VEGFR2, therefore loss of MBD2 increases eNOS and VEGFR2 expression ${ }^{149}$. These studies demonstrate that DNA methylation is likely a crucial, though under-studied, mechanism regulating vascular growth following arterial occlusion.

\subsubsection{Non-coding RNAs}

Non-coding RNAs have been much more widely studied in experimental models of PAD than any other epigenetic mechanism. The vast majority of these studies have focused on microRNAs, which have been primarily identified by one of three methods: miRNA expression levels in isolated cells, miRNAs with functions known to be important for the response to hindlimb ischemia, and by examining altered miRNA in response to hindlimb ischemia in-vivo.

Several studies have identified relevant miRNAs by examining miRNA expression levels in isolated cells, namely ECs. To this end, the miR-17 92a cluster was found to be highly expressed in HUVECs and to negatively regulate angiogenesis in-vitro ${ }^{150}$. Furthermore, systemic delivery of miR-92a antagomirs enhanced perfusion recovery in C57BL/6 mice following hindlimb ischemia $^{150}$. In another study, endothelial-specific deletion of the miR-17 92 cluster was also seen to increase foot perfusion 14 days following FAL, as well as arteriole density in FAL-operated 
$\operatorname{limbs}^{151}$. Within this miR-17 92 cluster, antagonism of miR-19a/b in aged mice improved the perfusion recovery after ischemia by targeting key aspects of the WNT signaling pathway ${ }^{151}$. MicroRNA-223 was also found to be highly expressed in freshly isolated human, murine, and porcine ECs and to abrogate EC migration and sprouting in-vitro, via regulation of $\beta 1$ integrin ${ }^{152}$. Compared to littermate controls, miR-223 ${ }^{-1 y}$ mice exhibited enhanced perfusion recovery (nearly 2-fold), increased $\alpha-\mathrm{SMC}^{+}$staining in adductor muscles, and increased capillary density in gastrocnemius muscles following hindlimb ischemia ${ }^{152}$.

Other studies have sought to determine if miRNAs with known functions important for vascular growth also regulate the response to hindlimb ischemia. To this end, miR-15a had been previously shown to be overexpressed in oxygen-glucose deprived cerebral ECs and to regulate Bcl-2153. Following hindlimb ischemia, EC-specific miR-15a overexpressing transgenic mice exhibited reduced capillary density in ischemic limbs and reduced perfusion recovery compared to littermate controls by suppression of FGF2 and VEGF ${ }^{154}$. Another study identified candidate miRNAs bioinformatically through reverse target prediction of 197 genes known to be involved in neovascularization ${ }^{155}$. This analysis identified $14 q 32$ as an enriched cluster of miRNAs with targets involved in neovascularization ${ }^{155}$. Systemic inhibition of 4 different $14 q 32$ cluster miRNAs (miR-320, -487b, -494, and -495) increased perfusion ( 25-40\%), arteriolar diameter, and capillary densities following hindlimb ischemia ${ }^{155}$. Another highly abundant, EC-specific miRNA that has been shown to have a role in vascular development is miR-126 ${ }^{156-158}$. Following FAL in C57BL/6 mice, miR-126a [-3p] antagomirs impaired angiogenesis, both in-vivo and in-vitro, but had no effect on overall reperfusion in-vivo ${ }^{156}$. However, overexpression of miR-126-3p through use of a non-invasive ultrasound-targeted microbubble destruction method, increased perfusion recovery and microvessel density in a rat hindlimb ischemia model ${ }^{159}$. The miR-126 25 cluster, which includes 3 mature miRNAs (miR-106b, miR-93, and miR-25) and is a paralogue of the miR17 92 and miR-106a 363 clusters, had been previously shown to be highly expressed in HUVECs and to promote tumor angiogenesis ${ }^{160}$. This cluster also appears to be necessary for 
normal reperfusion following FAL, as blood flow recovery was impaired in miR-126 25 knockout mice compared to controls, but was rescued by miR-126 25 overexpression ${ }^{160}$.

Finally, several studies have quantified altered miRNA expression in the ischemic tissue following arterial occlusion in-vivo to identify potential regulators of reperfusion. To this end, miR100 was found to be down-regulated in the adductor muscle of C57BL/6 mice 3 days post-FAL ${ }^{161}$. Subsequent inhibition of miR-100 enhanced perfusion recovery and capillary density through regulation of mTOR signaling ${ }^{161}$. Similarly, miR-155 was also down-regulated during hindlimb ischemia in C57BL/6 mice ${ }^{162}$. Inhibition of miR-155 increased EC proliferation and angiogenic tube formation in-vitro and promoted angiogenesis in the ischemic tissue of FAL-treated mice invivo $^{162}$. Despite promoting angiogenesis, miR-155 deficient mice exhibited attenuated blood flow recovery and leukocyte recruitment, thereby indirectly implying miR-155 impairs arteriogenesis, though collateral diameters were not explicitly reported ${ }^{162}$. Another study identified miR-132/212 to be significantly up-regulated in the adductor muscles 4 and 7 days after femoral arterial occlusion ${ }^{163}$. Perfusion recovery after arterial occlusion was slower in miR-132/212 knockout mice compared to wild-type mice ${ }^{163}$. Hazarika et al. incorporated a variation on this approach by comparing differential genome-wide miRNA expression between C57BL/6 and Balb/c ${ }^{164}$, two mouse strains known to exhibit widely different baseline vascular network structures and reperfusion capacities ${ }^{165-168}$. From this approach, they identified miR-93 and determined that it enhanced perfusion recovery and capillary density by modulation of multiple genes involved in the regulation of cell proliferation and apoptosis ${ }^{164}$.

Recently, other non-coding RNAs have been shown to play a role in the response to

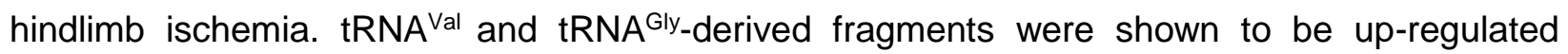
following hindlimb ischemia in mice and to inhibit EC proliferation, migration, and tube formation in-vitro ${ }^{169}$. Several IncRNAs, including H19, MIR210HG, MEG9, MALAT1, and MIR22HG, were found to be up-regulated both in hypoxic ECs and in the gastrocnemius muscle of mice following femoral artery dissection ${ }^{170}$. Moreover, $\mathrm{H} 19$ knockdown impaired capillary tube formation in- 
vitro $^{170}$. In another study ${ }^{171}$, MALAT1 inhibition enhanced EC migration but impaired proliferation, leading to discontinuous sprouts in the presence of VEGF in-vitro. Pharmacological inhibition of MALAT1 reduced blood flow recovery and capillary density following hindlimb ischemia in-vivo ${ }^{171}$. These studies represent only very recent discoveries of functional roles for these non-coding RNAs. The regulation of vascular growth by IncRNAs and other non-coding RNAs will likely be an area of intense research focus in the near future. 


\section{Mechanisms of amplified arteriogenesis in collateral artery}

\section{segments exposed to reversed flow direction}

Heuslein JL, Meisner JK, Li X, Song J, Vincentelli H, Leiphart RJ, Ames EG, Blackman BR, Price RJ. Mechanisms of amplified arteriogenesis in collateral artery segments exposed to reversed flow direction. Arterioscler Thromb Vasc Biol. 2015;35:2354-2365.

\subsection{Abstract}

Objective: Collateral arteriogenesis, the growth of existing arterial vessels to a larger diameter, is a fundamental adaptive response that is often critical for the perfusion and survival of tissues downstream of chronic arterial occlusion(s). Shear stress regulates arteriogenesis; however, the arteriogenic significance of reversed flow direction, occurring in numerous collateral artery segments after femoral artery ligation (FAL), is unknown. Our objective was to determine if reversed flow direction in collateral artery segments differentially regulates endothelial cell signaling and arteriogenesis.

Approach and Results: Collateral segments experiencing reversed flow after FAL in C57BL/6 mice exhibit increased pericollateral macrophage recruitment, amplified arteriogenesis $(30 \%$ diameter and 2.8-fold conductance increases), and remarkably permanent (12 weeks post-FAL) remodeling. Genome-wide transcriptional analyses on HUVECs exposed to reversed flow conditions mimicking those occurring in-vivo, yielded 10 -fold more significantly regulated transcripts, as well as enhanced activation of upstream regulators (NFkB, VEGF, FGF2, TGF $\beta$ ) and arteriogenic canonical pathways (PKA, PDE, MAPK). Augmented expression of key proarteriogenic molecules (KLF2, ICAM-1, eNOS) was also verified by qRT-PCR, leading us to test whether ICAM-1 and/or eNOS regulate amplified arteriogenesis in flow-reversed collateral segments in-vivo. Interestingly, enhanced pericollateral macrophage recruitment and amplified arteriogenesis was attenuated in flow-reversed collateral segments after FAL in ICAM-1/- mice; however, eNOS ${ }^{-/-}$mice showed no such differences. 
Conclusions: Reversed flow leads to a broad amplification of pro-arteriogenic endothelial signaling and a sustained ICAM-1-dependent augmentation of arteriogenesis. Further investigation of the endothelial mechanotransduction pathways activated by reversed flow may lead to more effective and durable therapeutic options for arterial occlusive diseases.

\subsection{Abbreviations}

LSF laser speckle flowmetry

FAL femoral arterial ligation

MBL muscular branch ligation

EC endothelial cell

HUVEC human umbilical vein endothelial cells

NvC non-reversed vs. control

RvC reversed vs. control

PCP planar cell polarization

MTOC microtubule organizing center

GA Golgi apparatus

\subsection{Introduction}

The importance of adequate remodeling of pre-existing arterial interconnections to form endogenous collateral bypasses - i.e. arteriogenesis - is highlighted by the extensive link between adequate collateral development and improved outcomes in patients with arterial occlusive disease ${ }^{36,172}$. However, few clinical trials designed to therapeutically stimulate collateral development have proven successfu| $\left.\right|^{34,35,173,174}$. This is likely related to the fact that these interventions do not necessarily recapitulate the complex sequence of processes that must be coordinated to achieve functional collateral development ${ }^{37,39,172}$. These clinical results have led to a re-examination of the basic mechanisms underlying collateral remodeling in the hope of identifying central signaling pathways for better therapeutic development. 
As early as 1893 , Thoma observed a correlation between vessel diameter and blood flow ${ }^{40}$. We now know the biomechanical forces exerted by blood flow on the endothelium are critical in regulating cell phenotype and blood vessel remodeling $37,41-45$. Indeed, a key initiating stimulus for arteriogenesis is a change in shear stress ${ }^{37}$. Upon occlusion of a major artery, downstream pressure is reduced, causing an increase in pressure gradient, blood flow, and shear stress along pre-existing collateral arteries that bypass the occlusion. Both the magnitude ${ }^{15}$ and duration ${ }^{16}$ of increased shear stress determine maximal collateral outgrowth. Collateral artery growth is hypothesized to eventually resolve as the increased outward remodeling reduces shear stress magnitude to a homeostatic "set-point"86.

Nonetheless, the topological arrangement of collateral arteries in skeletal muscle also dictates that shear stress will vary on a segment-to-segment basis along any given collateral pathway after the occlusion of a major artery. Recently, we applied transillumination based laser speckle flowmetry (LSF) to quantify, for the first time, the in-vivo "segment-to-segment" spatial distribution of collateral artery hemodynamics before and after femoral artery ligation in the mouse ischemic hindlimb, the most widely used model of peripheral arteriogenesis. While shear stress magnitude increased $\sim 2$-fold along the length of the collateral vessels in the gracilis adductor muscle, some pre-existing collateral artery segments also experienced reversed flow direction after femoral artery ligation ${ }^{176}$. In the present study, we tested the hypothesis that a change in flow direction has a profound influence on both arteriogenesis and mechanotransductive endothelial cell signaling.

\subsection{Materials and Methods}

\subsubsection{Mice.}

All animal protocols were approved by the Institutional Animal Care and Use Committee at the University of Virginia and conformed to all regulations for animal use outlined in the American Heart Association Guidelines for the Use of Animals in Research. C57BL/6 mice were purchased 
from Charles River Laboratory (Wilmington, MA). For ICAM-1/- and eNOS ${ }^{-/}$studies, wild-type

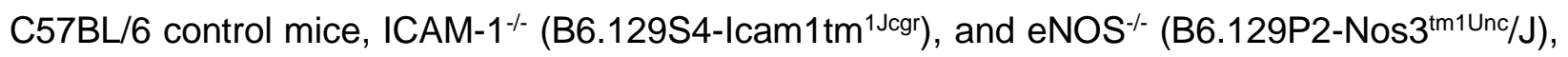
were purchased from Jackson Laboratory (Bar Harbor, ME). All animals were housed in the animal facilities at the University of Virginia.

\subsubsection{Femoral arterial ligation model.}

To produce uniform hemodynamic changes in the collateral arteries in a superficial adductor muscle, we used a previously detailed femoral artery ligation scheme ${ }^{167}$. This particular ligation pattern has been previously shown to produce consistent arteriogenesis in the collateral arteries of the gracilis adductor muscles ${ }^{177-180}$ with minimal heterogeneity in the baseline collateral structure and with the predicted changes in flow direction from baseline. Male mice, 9-13 weeks of age, were anesthetized (i.p $120 \mathrm{mg} / \mathrm{kg}$ ketamine, $12 \mathrm{mg} / \mathrm{kg}$ xylazine, and $0.08 \mathrm{mg} / \mathrm{kg}$ atropine), depilated, and prepped for aseptic surgery. On the left leg, an incision was made directly above and along the femoral artery, which was gently dissected from the femoral vein and nerve between the bifurcation of the superior epigastric artery and popliteal artery. Two 6.0 silk sutures were placed immediately distal to the epigastric artery, which served as the origin of the muscular branch artery in all mice, and the artery segment between the two ligatures was then severed with micro dissecting scissors. The surgical site was then closed with 5.0 prolene sutures. A sham surgery, wherein the femoral artery was exposed but not ligated, was performed on the right hindlimb (i.e. on the other leg). Animals received one injection of buprenorphine for analgesia at the time of surgery and a second dose 8-12 hours later.

\subsubsection{Muscular branch ligation model.}

Male mice were anesthetized (i.p $120 \mathrm{mg} / \mathrm{kg}$ ketamine, $12 \mathrm{mg} / \mathrm{kg}$ xylazine, and $0.08 \mathrm{mg} / \mathrm{kg}$ atropine), depilated, and prepped for aseptic surgery. On the left leg, an incision was made directly above and along the femoral artery. The muscular branch artery which was gently dissected from the paired vein and ligated with one 6.0 silk suture just distal to the epigastric artery. The surgical 
site was then closed with 5.0 prolene sutures. A sham surgery, wherein the muscular branch artery was exposed but not ligated, was performed on the right hindlimb (i.e. on the other leg). Animals received one injection of buprenorphine for analgesia at the time of surgery and a second dose 8-12 hours later.

\subsubsection{Laser Doppler perfusion imaging.}

For monitoring blood flow recovery and post-surgical ischemia, mice were anesthetized (i.p 120 $\mathrm{mg} / \mathrm{kg}$ ketamine, $12 \mathrm{mg} / \mathrm{kg}$ xylazine, and $0.08 \mathrm{mg} / \mathrm{kg}$ atropine) then placed prone on a surgical heating pad for 5 minutes to minimize temperature variation. The soles of the feet were scanned (Lisca PIM laser Doppler imager), and mean voltage of foot perfusion was used to calculate relative perfusion ratio (ligated/unligated).

\subsubsection{Tissue harvesting for cross sectional and collateral structure analysis by vascular casting.}

For analysis of lumenal diameters in the gracilis collateral arteries and to enable sectioning at specific regions, vascular casting was performed using an opaque polymer that allows for accurate lumenal diameter measurements ${ }^{179}$. After femoral artery ligation, mice were anesthetized (i.p $120 \mathrm{mg} / \mathrm{kg}$ ketamine, $12 \mathrm{mg} / \mathrm{kg}$ xylazine, and $0.08 \mathrm{mg} / \mathrm{kg}$ atropine), euthanized via an overdose of pentobarbital, and then the abdominal aorta was cannulated. The lower body was then perfused with $7 \mathrm{~mL}$ of $2 \%$ heparinized saline with $2 \mathrm{mmol} / \mathrm{L}$ adenosine $(16404$, Fisher Scientific, Pittsburg, PA) and 0.1mmol/L papaverine (P3510, Sigma Aldrich, St Louis, MO) to clear and vasodilate the downstream vasculature at a constant rate of $1 \mathrm{~mL} / \mathrm{min}$ (PHD2000, Harvard Apparatus). Once perfused, we waited 5 minutes to enable vasodilation. Tissues were then perfused with $3 \mathrm{~mL}$ of $4 \%$ paraformaldehyde solution (19943, Affymetrix, Cleveland, OH) at $1 \mathrm{~mL} / \mathrm{min}$ and allowed to fix for 10 minutes. The lower body was then perfused with $0.8 \mathrm{~mL}$ of Microfil@ casting agent (FlowTech, Inc, Carver, Massachusetts) at a constant pressure of $100 \mathrm{mmHg}$. Viscosity of Microfilß was adjusted to minimize transport across capillaries. After 
curing for 1.5 hours at room temperature, gracilis muscles were dissected free and then cleared in $50 \%$ glycerol in phosphate buffered saline (PBS) overnight. Cleared tissues were mounted between two coverslips using $500 \mu \mathrm{m}$ thick spacers (645501, Grace Bio-Labs Inc) to keep constant thickness between muscles. Muscles were imaged using transmitted light at $4 \mathrm{x}$ magnification on a Nikon TE200 inverted microscope with a CCD camera (Quantifier, Optronics Inc). Individual fields of view were montaged together (Photoshop CS2, Adobe Systems Inc).

For analysis of lumenal diameter from intact gracilis collateral whole mounts (i.e. vascular casting), collateral entrance regions were defined according to the following method. A cropped portion $(560 \mu \mathrm{m} \times 560 \mu \mathrm{m})$ of the montaged image (previously randomized and de-identified) was taken of the collateral artery at the first visible branch point of a terminal arteriole from the primary collateral as it extended from either the saphenous or muscular branch artery. This was done for each primary collateral running through the anterior and posterior heads of the gracilis muscle, yielding 4 total image regions per tissue whole mount. After each cropped image region was taken, all images were randomized and de-identified. The mean diameter was then taken from three separate diameter measurements along the length of cropped portion of the collateral artery.

After imaging, muscles were rehydrated, cut, and then paraffin embedded for cross sectional analysis at the muscular branch and saphenous artery entrance regions to the collateral arteries. Resulting cross sections were re-hydrated and immunolabeled for the macrophage marker Mac3 (day 3 post-FAL), H\&E stained for collateral artery structure analysis (day 7 and day 84 post$\mathrm{FAL}$ ), or fluorescently labeled with isolectin-B4 for capillary density analysis (day 7 post-FAL).

\subsubsection{Cross sectional analysis of collateral artery structure.}

Sections ( $5 \mu \mathrm{m}$ thickness) of paraffin embedded muscle from the muscular and saphenous regions were labeled for H\&E. Individual fields of view encompassing the collateral vessels were imaged with a 20x oil objective on a Nikon TE200 inverted microscope with a CCD camera (Quantifier, 
Optronics Inc). All images were randomized and de-identified prior to analysis. Lumenal diameter, wall area, and wall thickness were determined using Fiji ${ }^{181}$.

\subsubsection{Cross sectional analysis for regional capillary density and muscle fiber atrophy.}

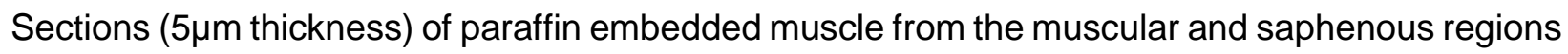

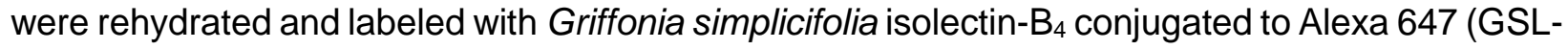
I-B $10 \mu \mathrm{g} / \mathrm{ml}$, I32450 Life Technologies Inc) and 10nmol/L SYTOX green nuclear stain (S7020, Life Technologies Inc). The entire cross-section for each region (encompassing both the anterior and posterior heads of the gracilis muscle) was imaged at 20x magnification on a Nikon TE2000 C1 confocal microscope and individual fields of view were stitched together (Photoshop CS2, Adobe Systems Inc). All GSL-I-B 4 positive vessels $<7 \mu \mathrm{m}$ in diameter and all muscle fibers (identified by autofluorescence) were counted in each section using Fiji ${ }^{181}$. Total muscle area was determined by semi-automated threshold analysis for calculation of average muscle fiber area.

\subsubsection{Immunofluorescence labeling of pericollateral Mac3 ${ }^{+}$cells.}

Sections (5 $4 \mathrm{~m}$ thickness) of paraffin embedded muscle from the muscular and saphenous regions were rehydrated and subjected to heat mediated antigen retrieval for 10 minutes in a citrate based antigen retrieval buffer (Vector Laboratories, Burlingame, CA; H-3300). Slides were then quenched of endogenous peroxidase activity with a 30 minute incubation in $3 \% \mathrm{H}_{2} \mathrm{O}_{2}$, blocked, and labeled with rat-anti-Mac3 (1:100, M3/4 clone, 550292; BD Biosciences, San Jose, CA) overnight at $4^{\circ} \mathrm{C}$. Slides were washed and incubated with a biotinylated sheep-anti-rat secondary antibody (Abcam, Cambridge, MA, ab6851, 1:500) for 1 hour at room temperature. Slides were washed and incubated with an avidin-biotin complex (Vectastain ABC solution, Vector Laboratories) for 30 minutes at room temperature. Slides were washed and incubated with a Tyramide Signal Amplification (TSA) reagent (Perkin Elmer, Waltham, MA; 1:50) for 10 minutes at room temperature. Slides were washed and incubated with streptavidin-488 (Life Technologies 
Inc, 1:500), Cy3-anti-SMA (1A4 clone, Sigma, 1:1000) and DRAQ5 (Thermo Scientific Inc, 1:1000). Slides were then mounted with Prolong Gold (Life Technologies Inc) to minimize photobleaching, allowed to cure overnight, and imaged using a Nikon TE2000 C1 laser scanning confocal microscope with a $20 x$ oil objective. Cropped fields of view (512x512 pixels) encompassing the collaterals in each region were randomized and de-identified. The pericollateral region was outlined ( 25 microns around the vessel) and pericollateral Mac3 ${ }^{+}$nuclei were counted in Fiji ${ }^{181}$.

\subsubsection{Tissue harvesting for in-vivo collateral artery endothelial planar cell polarization.}

For analysis of the spatial variation of endothelial cell planar polarization in the gracilis collateral arteries, whole gracilis muscles were immunolabeled for smooth muscle alpha-actin, the Golgi apparatus, and the nucleus. The Golgi apparatus was chosen over the more widely reported microtubule organizing center (MTOC) $)^{182-184}$ due to its larger size in order to aid identification in the situation of decreased resolution and clarity when imaging thick $(>100 \mu \mathrm{m})$ tissues using confocal microscopy. The Golgi apparatus, which colocalizes with the MTOC ${ }^{185}$, has previously been shown to serve as a functional marker of endothelial cell planar polarization ${ }^{186}$. Pre-ligation and 24 hours after femoral artery ligation, mice were euthanized, and the abdominal aorta was cannulated. The lower body was then perfused with $2 \%$ heparinized saline with $2 \mathrm{mmol} / \mathrm{L}$ adenosine and $0.1 \mathrm{mmol} / \mathrm{L}$ papaverine to clear and vasodilate the downstream vasculature at a constant rate of $1 \mathrm{~mL} / \mathrm{min}$ (PHD2000, Harvard Apparatus) and then fixed with $2 \%$ paraformaldehyde solution for 30 minutes. Tissues were then flushed with PBS and perfused with an antibody and blocking solution consisting of PBS, $2 \%$ BSA, 10\% NGS, and $0.015 \%$ saponin and containing anti-smooth muscle $\alpha$-actin-FITC (1:400 1A4 clone, A5691, Sigma Aldrich), rabbit anti-giantin (1:500, ab24586 Abcam Inc), and 5 $\mathrm{mol} / \mathrm{L}$ Draq 5 (62251, Thermo Scientific Inc). This perfusion step was found necessary to ensure adequate penetration of the giantin antibody to the 
collateral artery endothelial cells. After 60 minutes, muscles were removed and further incubated overnight at $4{ }^{\circ} \mathrm{C}$ in an antibody solution consisting of PBS, $2 \%$ BSA, $10 \% \mathrm{NGS}$, and $1 \%$ TritonX100 and containing anti-smooth muscle alpha actin-FITC (1:400 1A4 clone, SigmaAldrich), rabbit anti-giantin (1:500, ab24586, Abcam Inc), and 5 $\mu \mathrm{mol} / \mathrm{L}$ Draq 5. Muscles were then washed and incubated overnight at $4^{\circ} \mathrm{C}$ in a secondary antibody solution consisting of PBS, $2 \%$ BSA, and 1\% TritonX100 and containing goat anti-FITC Alexa 488 (1:200 A11090, Life Technologies Inc), goat anti-rabbit IgG Alexa 555 (1:200, A21428 Life Technologies Inc), and $5 \mu \mathrm{mol} / \mathrm{L}$ Draq 5. Muscles were then washed and cleared in a series of $50 \%$ glycerol in PBS and then $90 \%$ glycerol in PBS overnight at $4^{\circ} \mathrm{C}$. Cleared muscles were then mounted between two coverslips using $500 \mu \mathrm{m}$ thick spacers (645501, Grace Bio-Labs Inc). Muscles were then imaged on a Nikon TE2000 C1 laser-scanning confocal microscope with a 20x oil lens immediately upstream of the first visible transverse arteriole branch off of the anterior and posterior collateral arteries extending from the muscular branch and saphenous arteries and at the midpoint of the anterior and posterior collateral arteries.

\subsubsection{Regional analysis of hypoxia.}

A femoral arterial ligation and sham procedure were performed as previously described on C57BL/6 mice. 24 hours post ligation, mice were anesthetized (i.p $120 \mathrm{mg} / \mathrm{kg}$ ketamine, $12 \mathrm{mg} / \mathrm{kg}$ xylazine, and $0.08 \mathrm{mg} / \mathrm{kg}$ atropine) then injected i.p. with $60 \mathrm{mg} / \mathrm{kg}$ pimonidazole- $\mathrm{HCl}$ (Hypoxyprobe, Burlington, MA). After 20 minutes, mice were euthanized with an overdose of pentobarbital. The heart was cannulated and perfused with cold $2 \%$ heparinized saline with $2 \mathrm{mmol} / \mathrm{L}$ adenosine (16404, Fisher Scientific) to clear and vasodilate the downstream vasculature at a constant rate of $4 \mathrm{~mL} / \mathrm{min}$ (PHD2000, Harvard Apparatus) and then fixed with cold $4 \%$ paraformaldehyde solution (19943, Affymetrix). Gracilis muscles and liver sections were removed and further fixed in $4 \%$ PFA $+0.1 \%$ saponin for 20 minutes at room temperature. Tissues were then washed in PBS with 1\% Triton-X overnight. Tissues were then cut and paraffin embedded 
for cross sectional analysis at the muscular branch and saphenous artery entrance regions to the collateral arteries. Sections (5 4 m thickness) of paraffin embedded muscle from the muscular and saphenous regions were rehydrated and subjected to heat mediated antigen retrieval for $20 \mathrm{~min}$ in a citrate based antigen retrieval buffer (H-3300; Vector Laboratories). Slides were blocked and then incubated with anti-pimonidazole-HCl-FITC primary antibody (1:200, Hypoxyprobe) for 45 minutes at room temperature. Slides were washed then incubated with an 633 labeled anti-FITC secondary antibody (clone 1F8-1E4, Sigma, 1:400), Cy3-anti-SMA (1A4 clone, Sigma, 1:1000), and 10nmol/L SYTOX green nuclear stain (S7020, Life Technologies Inc) for 45 minutes at room temperature. Slides were washed and then sealed with Prolong Gold (Life Technologies). The entire cross-section for each region (encompassing both the anterior and posterior heads of the gracilis muscle) was imaged at 20x magnification on a Nikon TE2000 C1 laser-scanning confocal microscope and individual fields of view were stitched together (Photoshop CS2, Adobe Systems Inc). Integrated fluorescence intensity (mean gray value $x$ area) was determined in cropped (256x256 pixels) fields of view encompassing the collateral vessels in each region from previously randomized and de-identified montaged images.

\subsubsection{Human umbilical vein endothelial cell culture.}

Human umbilical vein endothelial cells (HUVECs) purchased from Cell Applications Inc., VEC Technologies Inc. (Rensselaer, NY), and Lonza were unthawed and maintained on $0.1 \%$ gelatin coated flasks in M-199 medium (Lonza), supplemented with 10\% fetal bovine serum (Life Technologies Inc, Grand Island, NY), 100U/mL penicillin-G + 100ug/ml streptomyocin (Life Technologies Inc) , 2mmol/L L-glutamine (Life Technologies Inc), 5ug/ml endothelial cell growth supplement (Biomedical Technologies, Stoughton, MA), and 10ug/ml heparin (Sigma Aldrich Inc). For each set of experimental comparisons, cells were used from the same company and cell line, in their third subculture passage. 


\subsubsection{In-vitro exposure of endothelial cells to simulated ligation shear stress.}

HUVECs were plated on cell culture grade plastic dishes coated with $0.1 \%$ gelatin and grown to confluence. A cone and plate flow apparatus ${ }^{187}$, which maintains cells at $5 \% \mathrm{CO}_{2}$ and $37^{\circ} \mathrm{C}$, was used to induce a shear stress protocol. The applied shear stress protocol consisted of a 24 hour preconditioning period at a steady of $15 \mathrm{dyne} / \mathrm{cm}^{2}$ and then either increased to $30 \mathrm{dynes} / \mathrm{cm}^{2}$ (non-reversed), increased to 30 dynes $/ \mathrm{cm}^{2}$ and reversed in direction (reversed), or held constant at 15 dynes $/ \mathrm{cm}^{2}$ (control). Fresh culture media consisting of M199 with 4\% dextran from Leuconostoc spp (Sigma Aldrich, $\mathrm{M}_{\mathrm{r}} \sim 500,000$ ), $2 \%$ fetal bovine serum, 100U/mL penicillin-G + $100 \mathrm{ug} / \mathrm{ml}$ streptomyocin, $2 \mathrm{mmol} / \mathrm{L}$ L-glutamine, $5 \mathrm{ug} / \mathrm{ml}$ endothelial cell growth supplement, and $10 \mathrm{ug} / \mathrm{ml}$ heparin was added to cells before exposure to shear stress and was continuously exchanged throughout the duration in the cone and plate apparatus.

\subsubsection{HUVEC RNA isolation, qRT-PCR, and microarray gene expression profiling.}

For RT-PCR and CDNA microarray assays, total RNA was extracted using the PureLink total RNA purification system (Life Technologies Inc). RNA concentration and purity was confirmed with a NanoDrop spectrophotometer for RT-PCR and by Agilent Bioanalyzer. Each replicate consisted of three plates split from the same culture. The three plates were then run in tandem, under one of the three separate shear conditions.

For microarray analysis, 4 replicates were collected for each condition at 6 hours after simulated FAL. RNA amplification, labeling, hybridization with Human Gene 1.0ST oligo microarrays (Affymetrix), quality control, and raw data acquisition were performed by the University of Virginia Biomolecular Research facility and data analysis by the University of Virginia Bioinformatics Core facility. All preprocessing and analysis was done using $\mathrm{R}$ version 2.15.0. CEL files were imported using the affy package, version 1.34.0. Expression intensities were summarized, normalized, and transformed using Robust Multiarray Average (RMA) algorithm ${ }^{188}$. Probesets were annotated using the bioconductor annotation package 
hugene10sttranscriptcluster.db version 8.0.1, using the annotate package, version 1.34.0. Probesets not mapping to an Entrez gene were excluded (probesets mapping to RIKEN genes were not excluded). For examining differential gene expression, a linear model with empiricalBayes moderated standard errors was fit using the limma package in $\mathrm{R}$. The BioConductor package arrayQualityMetrics was used to perform quality assessment on the preprocessed, summarized, normalized, transformed, filtered data. The expression dataset is publically available at GEO (http://www.ncbi.nlm.nih.gov/geo/ (GSE 46248)). Heat maps were created using the gplots package in R (http://CRAN.R-project.org/package=gplots). Gene ontology was assessed through the gene ontology enrichment analysis and visualization tool (GOrilla) ${ }^{189}$. Biological context was analyzed by Ingenuity Pathway Analysis (http://www.ingenuity.com/) for functional gene clustering and function.

For quantitative reverse transcriptase PCR (qRT-PCR), total RNA was reverse transcribed using the iScript cNDA synthesis kit (Bio-Rad, Hercules, CA). RT-PCR was performed on CCL2 (forward 5'-CCAGCAGCAAGTGTCCCAAAG -3' reverse 5'-TGCTTGTCCAGGTGGTCCATG-3'), eNOS (forward 5'-CTCCATTAAGAGGAGCGGCTC-3', CTAAGCTGGTAGGTGCCTGTG-3'), E-selectin $\quad$ (forward 5'AATCCCAGTTTGTGAAGCTTTCCA-3', reverse 5'-GCCAGAAGCACTAGGAAGACAATT-3'), ICAM (forward 5'-TCGCTATGGCTCCCAGCAGC-3', reverse 5'TTCCGGTTGTTCCCAGGCAGG-3')， KLF2 (forward 5'-GCTGAGTGAACCCATCCTGCC-3', reverse 5'-CGCTGTTGAGGTCGTCGTCG-3'), $\quad$ KLF4 (forward 5'GGCCAGAATTGGACCCGGTGTAC-3', reverse 5'-CTGCCTTTGCTGACGCTGATGA-3'), and VCAM (forward 5'-GTTTGTCAGGCTAAGTTACATATTGATGA-3' reverse 5'GGGCAACATTGACATAAAGTGTTT-3'), with SYBR Green (Roche Applied Sciences) on a CFX96 Real Time Detection System (Biorad). Expression was normalized to $\beta 2$-microglobulin (forward 5'-AGCATTCGGGCCGAGATGTCT-3', CTGCTGGATGACGTGAGTAAACCT-3') which is endogenously expressed and is not altered by 
many stimuli including shear stress ${ }^{44}$. Normalized expression was quantified using the comparative $2^{\Delta \Delta \mathrm{Ct}}$ method.

\subsubsection{Immunofluorescence labeling for HUVEC planar cell polarity.}

After exposure to our shear stress protocol, cells were fixed in 4\% PFA for 10 minutes at room temperature, blocked with PBS $+0.1 \%$ saponin $+2 \%$ BSA $+5 \%$ normal goat serum for 45 minutes at room temperature, and then incubated in primary antibody solution containing Vibrant Dyl (1:200, Life Technologies Inc) or CD31 (1:200, 89C2, Cell Signaling Technology, Danvers, MA), Draq5 (5 $4 \mathrm{~mol} / \mathrm{L}$, Thermo Scientific Inc), and either rabbit-anti-giantin (1:1000, ab24586, Abcam Inc) or rabbit-anti-pericentrin (1:500, ab4448, Abcam Inc). Cells were washed three times in PBS $+0.1 \%$ saponin then incubated in goat-anti-rabbit Alexafluor 488 (1:500, Life Technologies) secondary antibody solution for 45 minutes. Cells were washed twice in PBS, then twice in 50:50 PBS: glycerol, and then in 10:90 PBS: glycerol prior to cover slip mounting with Prolong Gold (Life Technologies). Plates were allowed to cure overnight then were imaged using a Nikon TE2000 C1 laser scanning confocal microscope. The relative location of the nucleus to the Golgi apparatus or MTOC from three independent sets of experimental conditions, each an average of three representative fields of view (for a total of nine images per experimental condition), was determined by a blinded, independent observer.

\subsubsection{Data analysis of endothelial cell planar polarity.}

For analysis of endothelial orientation with respect to flow, maximum intensity projections of collateral arteries (for whole mount analysis) or fields of view (for HUVEC plates) were indicated with flow direction then de-identified for blinded analysis. Peri-nuclear location of the Golgi apparatus or MTOC was determined with respect to flow direction and nuclear position (Figure 4.1B-C and Supplemental Figure 4.4). 


\subsubsection{HUVEC western blot and NF-KB activity assays.}

HUVECs were lysed in MAPK buffer (63.5mM Tris-HCL pH 6.8, 2\% w/v SDS, 10\% glycerol, 50mM DTT, $0.01 \%$ bromophenol blue). Total protein was collected and lysates resolved on a $7.5 \%$ SDSPAGE gel and blotted on a nitrocellulose membrane. Primary antibodies [ICAM-1 (R\&D Systems, 1:750), pS1177-eNOS (BD Biosciences, 1:750), a-tubulin (Sigma, 1:1000) were incubated overnight at 4C. A LICOR Odyssey imager was used for blot image acquisition and densitometry analysis.

To determine NF-kB activity by luciferase reporter activity, endothelial cells were infected with adenovirus containing NF-kB luciferase reporter (Vector Biolabs, Philadelphia, PA; Cat. No. 1740, 1:5000) 24 hours prior to shear stress. Luciferase reporter activity was assessed using the Promega Luciferase Assay System per manufacturer's instructions.

\subsubsection{7 siRNA transfection in HUVECs}

Twenty-four hours prior to exposure of HUVECs to flow conditions, HUVECs were plated without antibiotics on $0.1 \%$ gelatin coated plates in serum-free M199 (Life Technologies) supplemented with $2 \mathrm{mmol} / \mathrm{L}$ L-glutamine. After cells were allowed to adhere for 2 hours after plating, cells were transfected with either 120pmol of ON-TARGETplus SMARTpool human ICAM-1 siRNA (L003502-00-0005, GE Dharmacon, Lafayette, CO) or 120pmol of ON-TARGETplus non-targeting

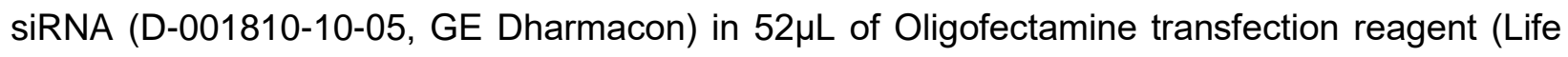
Technologies) and 6.8mL Opti-MEM media (Life Technologies) for 5 hours at 37C. After 5 hours, plates were flooded with $8 \mathrm{~mL}$ of M199 media without antibiotics supplemented with $10 \%$ fetal bovine serum + L-glutamine $+5 \mathrm{ug} / \mathrm{ml}$ endothelial cell growth supplement (Biomedical Technologies, Stoughton, MA), and 10ug/ml heparin (Sigma Aldrich Inc). 24 hours posttransfection this solution was aspirated off and normal flow media was applied. Validation of transfection was performed on HUVEC plates not exposed to shear stress but in culture for the same duration (54 hours post-transfection) via ICAM-1 western blotting. 


\subsubsection{Monocyte adhesion functional assay}

Human derived monocytes (THP-1 cell line) were purchased from the ATCC. Monocytes were unthawed and maintained in RPMI 1640 (11875-093, Life Technologies Inc) $+10 \%$ fetal bovine serum (Life Technologies Inc) $+0.05 \mathrm{mM} \beta$-mercaptoethanol per ATCC culture instructions. Monocytes sub-cultured once cell density approached 800,000 cells $/ \mathrm{mL}$. Cells were used between passages $2-6$.

Prior to the adhesion assay, cells were counted to obtain $3,000,000$ cells / plate of HUVECs. Cells were pelleted, washed with PBS, pelleted, and then re-suspended in serum-free RPMI media at 1,000,000 cells $/ \mathrm{mL}$. Thawed calcein AM was added at $1 \mathrm{ug} / \mathrm{ml}$ and incubated with cells for 15 minutes at $37^{\circ} \mathrm{C}$. After 15 minutes, the reaction was stopped by adding excess serum-free RPMI to the cell solution then pelleted. Cells were washed once with serum-free M199 media, pelleted, and then re-suspended in serum-free M199 at 500,000 cells/mL. Immediately following completion of flow exposure to HUVECs, flow media was removed by aspiration. HUVECs were quickly washed with serum-free M199 media. This media was then aspirated off and $6 \mathrm{~mL}$ of serum-free M199 + monocytes (3,000,000 / plate) were added to and incubated with HUVECs for 30 minutes at $37^{\circ} \mathrm{C}$. Following the 30 minutes, cells were washed twice with PBS to remove unbound monocytes. Adhered monocytes and HUVECs were fixed with 4\% PFA for 10 minutes followed by two washes with PBS. Cover slips were mounted with Prolong Gold (Life Technologies Inc). Plates were then imaged using a Nikon TE2000 C1 laser scanning confocal microscope. Nine randomly selected FOVs per condition for 4 independent experiments were obtained. Images were de-identified and randomized in MATLAB. Images were converted to 8bit images, set to an equivalent threshold, and bound monocytes were quantified using Fijis "Analyze Particles" tool $\left(20 \mu \mathrm{m}^{2}\right.$ minimum particle size). Results were centered on the mean of all conditions within each independent experiment. 


\subsection{Results}

\subsubsection{Planar polarization confirms endothelial cell responsiveness to predicted flow directions}

Femoral arterial ligation (FAL), performed as previously described ${ }^{176}$, was used to induce arteriogenesis in the gracilis adductor muscle collateral arteries. In agreement with previous studies $^{179,180}$, perfusion measurements of the plantar surface of the foot indicated moderate ischemia immediately post-FAL, followed by a return to full perfusion by day 7 post-FAL (Supplemental Figure 4.1). The position of the ligation along the femoral artery was chosen to yield a change in blood flow direction and magnitude along the length of the two collateral arteries in this muscle. Pre-ligation, blood flows toward a convergence point in the center of the gracilis muscle via the muscular branch artery (also known as the lateral caudal femoral artery) and via the saphenous artery (Figure 4.1A). FAL causes a decrease in downstream pressure, resulting in increased flow through the gracilis collaterals and reversed flow direction in those collateral segments near the saphenous artery (Figure 4.1A). Using transillumination laser speckle flowmetry (LSF), we recently determined that shear stress magnitude increases 2-fold in both the muscular and saphenous collateral entrance regions 30 minutes post-FAL. Additionally, we confirmed the predicted flow directions in this ligation scheme through observation of circulating fluorescent microspheres during intravital imaging ${ }^{176}$.

To further confirm endothelial cell responsiveness to the change in flow direction, we characterized endothelial cell planar polarization (PCP) in the gracilis collateral arteries by examination of the perinuclear position of the Golgi apparatus (GA) with respect to predicted flow direction both pre-FAL and 24hrs post-FAL (Figure 4.1B-E). At baseline, endothelial cells at the collateral entrance regions were primarily polarized toward their respective upstream directions (Figure 4.1F, D), while the central regions of the gracilis collateral arteries showed no significant polarization. By 24 hours post-FAL, however, most of the endothelial cells in both the central and 
saphenous entrance regions repolarized toward the muscular branch artery, instead of the saphenous artery (Figure $4.1 B, E$ ).

A

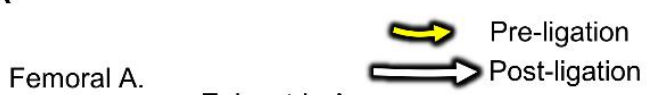

B
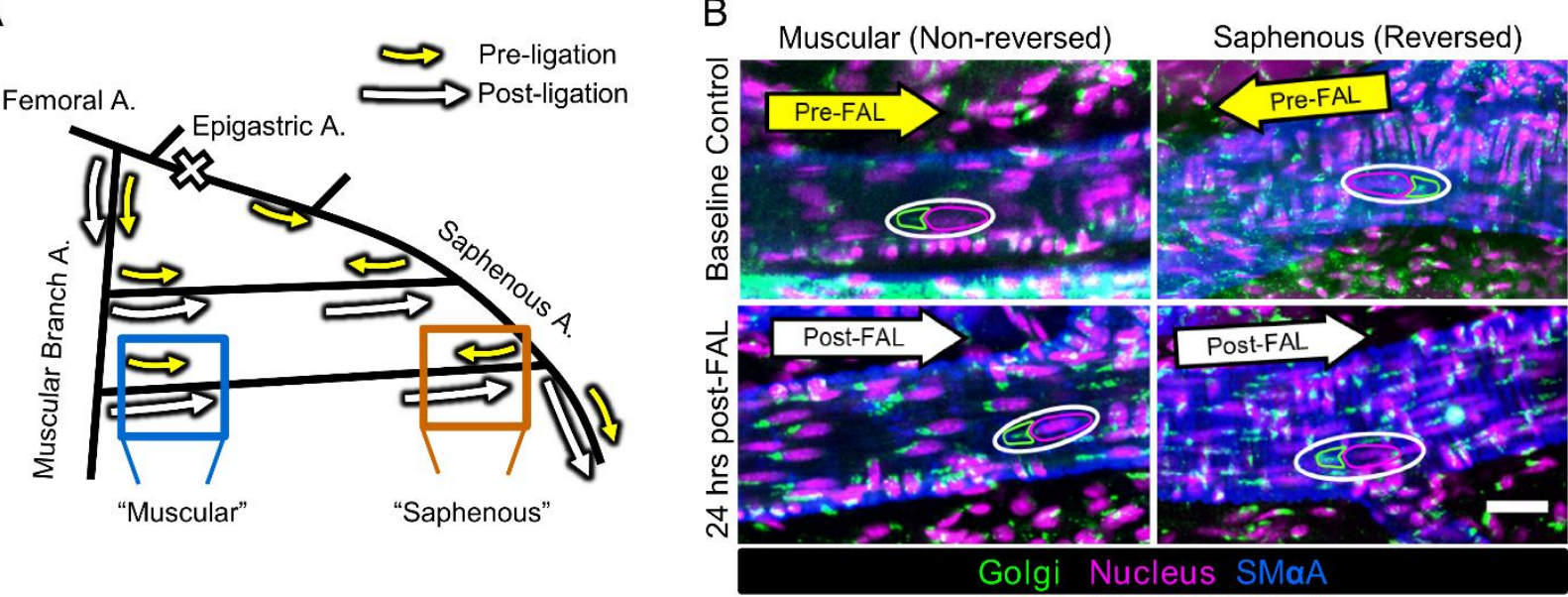

C

Flow
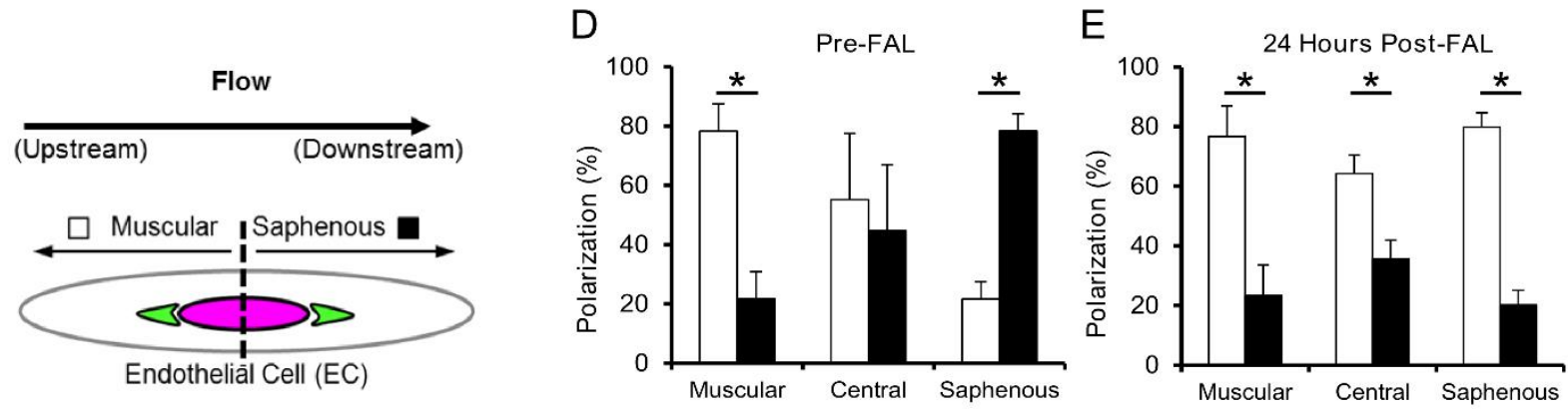

Figure 4.1. Collateral artery endothelial cells repolarize in response to reversed shear stress after FAL.

A) Schematic of the primary gracilis adductor collateral flow pathways after femoral arterial ligation (FAL). The femoral artery is ligated just distal to the epigastric artery thereby creating a change in flow direction at the saphenous branch entrance region. Arrows indicate the direction and magnitude of blood flow pre(yellow) and post- (white) FAL. Flow magnitude increases in both the muscular branch and saphenous entrance regions, while flow direction also changes in the saphenous region. B) Representative images of collateral regions immunolabeled for the Golgi apparatus (anti-giantin, outlined in green), the position of which with respect to the nucleus (Draq5, outline in magenta) was used to determine planar polarization, a marker of endothelial response to flow direction. Images are oriented such that the muscular branch is toward the left and the saphenous artery is toward the right. Arrows indicate blood flow direction. (Scale bar=25 $\mu \mathrm{m}$ ). C) Diagram depicting the method used to quantify of polarization. Golgi apparatus (green) position with respect to the nucleus (magenta) was used to classify EC polarization as toward either the saphenous (black bars) or muscular branch (white bars) arteries. D) Bar graph of pre-FAL EC planar polarization. ECs in the entrance regions show upstream polarization, while those central region show no directional bias $(n=4)$. E) Bar graph of 24 hour post-FAL EC planar polarization. ECs in all regions show upstream polarization toward the muscular branch entrance region $(n=4)$. ${ }^{*} p<0.05$ between muscular and saphenous polarization within the given region. Data are mean \pm SEM. 


\subsubsection{Arteriogenesis is enhanced in collateral artery segments exposed to reversed flow after FAL.}

To determine if reversed flow affects arteriogenesis, we measured lumenal collateral diameter in the muscular branch and saphenous artery entrance regions using vascular casting (Figure 4.2A). After 24 hours, neither region demonstrated significant lumenal expansion compared to the unligated control limb. By day 3, however, both the saphenous (reversed) and muscular (nonreversed) regions underwent significant ( $p<0.001$ and $p=0.04$ respectively) lumenal expansion compared to unligated controls ( $58.8 \pm 8.5 \%$ and $19.9 \pm 4.7 \%$ increases respectively). Furthermore, the saphenous (i.e. flow-reversed) segment began to exhibit a significant $(p=0.004)$ enhancement in lumenal diameter when compared to the non-reversed muscular branch. Lumenal diameter in both reversed and non-reversed regions increased further by day 7 postligation $(93.7 \pm 9.1 \mu \mathrm{m}$ and $70.7 \pm 6.1 \mu \mathrm{m}$ within saphenous and muscular branch regions respectively), but thereafter remained constant through 12 weeks post-FAL $(91.6 \pm 3.3 \mu \mathrm{m}$ and $75.2 \pm 5.2 \mu \mathrm{m})$. At both 1 and 12 weeks post-FAL, lumenal diameter was significantly greater in the flow-reversed saphenous region when compared to the non-reversed muscular branch region $(p=0.01)$ (Figure 4.2B). The time course of arteriogenesis matched well with the laser Doppler reperfusion measurements (Supplemental Figure 4.1), where resting foot perfusion returned to baseline around 5 days post-ligation, with no further increases in reperfusion.

Cross-sectional analysis of the collateral artery entrance regions was used to confirm wholemount diameter measurements and determine whether wall mass was also increased in flow reversed segments (Figure 4.2C). Both reversed and non-reversed regions showed significantly increased lumenal diameter, wall area, and wall thickness versus their respective unligated controls at 7 days post-FAL (Figure $4.2 C-F$ ). In confirmation of the whole mount vascular cast 

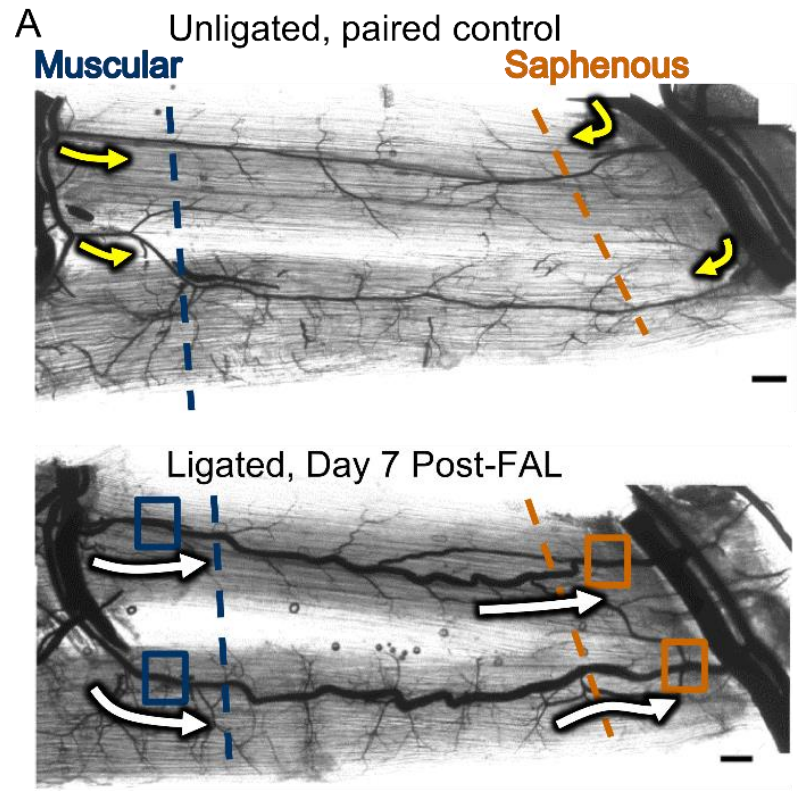

C

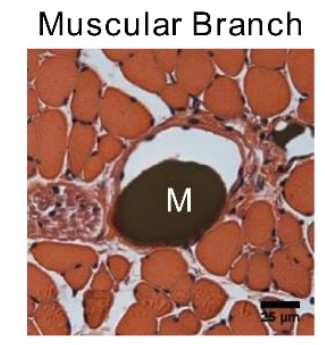

Ligated

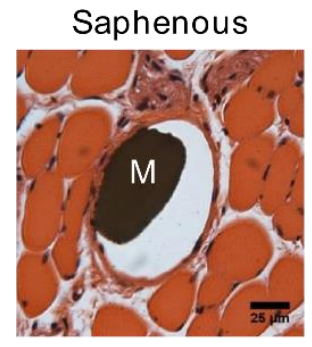

B

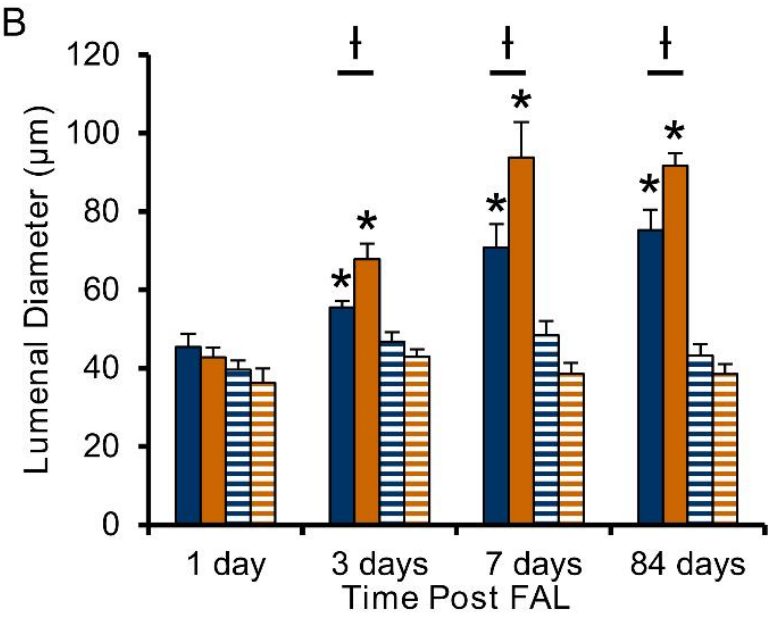

Muscular Ligated 目 Muscular Unligated (Non-reversed)

Saphenous Ligated

(Reversed)
Saphenous Unligated

Unligated

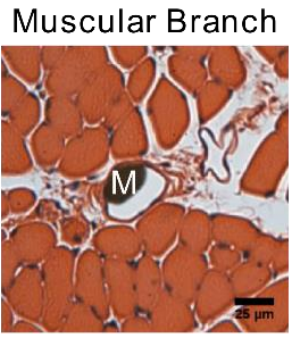

Saphenous

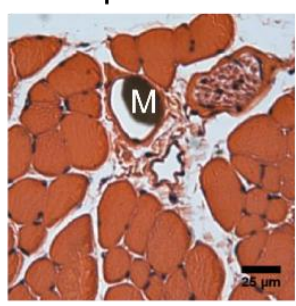

D

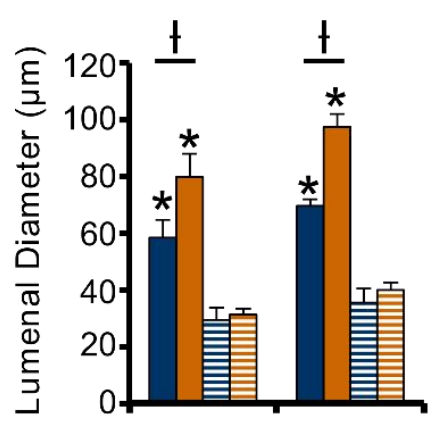

Day 7 Day 84

E

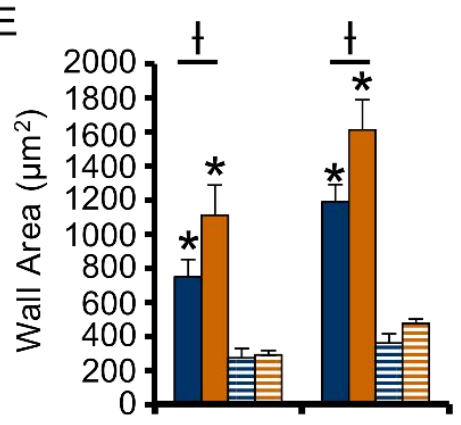

Day 7 Day 84

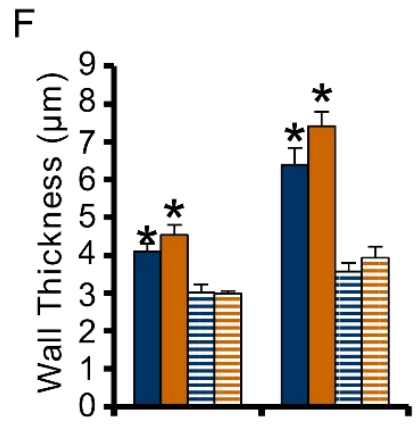

Day 7 Day 84

Figure 4.2. Gracilis collateral artery regions exposed to reversed flow exhibit enhanced arteriogenesis after FAL.

A) Representative vascular cast images of gracilis collateral arteries from unligated sham control (top) and FAL-treated (bottom) mice. Arrows indicate the direction and relative magnitude of blood flow pre- (yellow) and post- (white) FAL. Dashed lines indicate the collateral artery region (muscular=blue; saphenous=orange) and approximate location where muscles were cut for regional cross-sectional analysis. Boxes indicate location at which lumenal diameters were measured B) Bar graph of collateral artery lumenal diameter at both the non-reversed (muscular branch, blue) and flow-reversed (saphenous, orange) entrance regions. ( $n=6-7$ mice per day). C) Representative H\&E stained cross-sections of gracilis muscles. Microfil casting material (M) is evident in artery lumens. D-F) Bar graphs of cross sectional lumenal diameter, wall area, and wall thickness ( $n=6-7$ mice per day). ${ }^{*} p<0.05$ between ligated and unligated within the given region at the specified time-point; $t p<0.05$ between regions (i.e. saphenous vs. muscular) at the specified time-point. Data are mean \pm SEM. 
analysis, we observed an enhancement of lumenal diameter in the flow-reversed saphenous region $(36.7 \pm 2.3 \%$ vs. non-reversed muscular region, $p<0.01)$ at day 7 . Wall area in the flow reversed region was also significantly increased at day 7 when compared to the non-reversed region $(47.9 \pm 5.8 \%, p<0.05)$. These significant increases in lumenal diameter and wall area in flow-reversed regions were maintained up to 12 weeks after FAL (Figure 4.2D-E).

\subsubsection{FAL does not elicit regional differences in hypoxia in gracilis adductor muscles.}

We next examined whether hypoxia in the gracilis adductor muscle could be contributing to the observed spatial differences in arteriogenesis between the flow reversed and non-reversed regions. Twenty-four hours post-FAL, we observed no regional differences in gracilis muscle hypoxia as determined by pimonidazole-HCl immunolabeling (Supplemental Figure 4.2A-C). Other indicators of tissue hypoxia, namely muscle fiber atrophy (Supplemental Figure 4.2E) and angiogenesis (Supplemental Figure 4.2F), were also found to be unchanged in the flow-reversed saphenous region when compared to both non-reversed muscular branch regions and unligated controls.

\subsubsection{Amplified arteriogenesis in flow-reversed segments occurs independent of position.}

To determine if enhanced arteriogenesis in response to reversed flow can occur in other collateral regions, we employed a muscular branch ligation (MBL) scheme that creates reversed flow in the muscular branch collateral region, instead of in the saphenous region (Supplemental Figure 4.3A). Reversed flow direction was confirmed via reorientation of the perinuclear position of the Golgi apparatus toward the saphenous entrance region (Supplemental Figure 4.3B-C). Flow-reversed collateral segments in the muscular branch region exhibited significant lumenal growth when compared to non-reversed segments in the saphenous region at 14 days post-MBL $(36.2 \pm 4.9 \%$ vs. non-reversed, $\mathrm{p}<0.01$ ) (Supplemental Figure 4.3D-E). 


\subsubsection{HUVECs exhibit directional responsiveness to simulated femoral artery ligation.}

To investigate the influence of FAL-elicited hemodynamic changes on EC signaling, HUVECS were exposed to biomimetic changes in relative shear stress magnitude and/or direction, as previously by determined by transillumination $\operatorname{LSF}^{176}$ (Figure 4.3A). A value of 15 dynes $/ \mathrm{cm}^{2}$ shear stress was chosen as a baseline shear stress ${ }^{190}$. Preconditioning for 24 hours at this baseline shear stress was used to establish endothelial cell alignment and PCP, thereby mimicking the in-vivo baseline state. FAL was then simulated by a step-wise $100 \%$ increase in shear stress, in either the same direction or in the opposite direction, to mimic shear stress changes occurring in the muscular branch (non-reversed) and saphenous artery (reversed) entrance regions, respectively (Figure 4.3A). Consistent with in-vivo observations, HUVECs aligned with the flow direction and showed an upstream polarization of the GA with respect to the nucleus in control plates maintained at $15 \mathrm{dynes} / \mathrm{cm}^{2}$. Upstream polarization was maintained (at 2 hours) or enhanced (at 6 hours) with a non-reversed, step-wise increase in shear stress (Supplemental Figure 4.4). However, when shear direction was reversed, endothelial cells transitioned from the PCP induced by preconditioning toward the new upstream direction within 6 hours (Supplemental Figure 4.4). Using HUVECS, we confirmed that GA perinuclear position

matches well with MTOC perinuclear position (Supplemental Figure 4.4). These data demonstrate sensitivity to directional change in shear stress in HUVECs similar to that seen invivo, with complete re-orientation of PCP toward the new upstream direction within 6 hours after simulated FAL. 
A

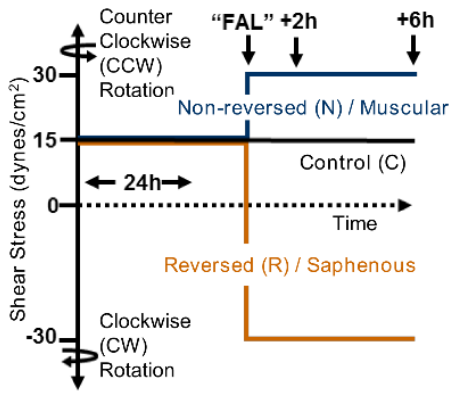

D

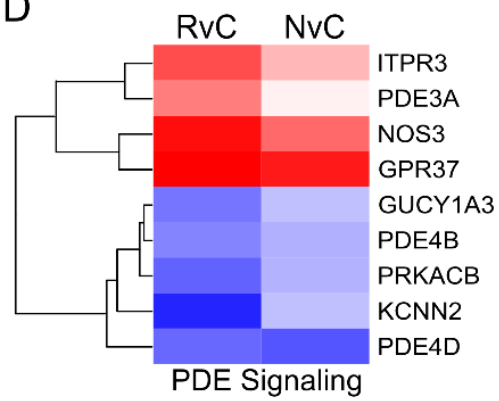

G

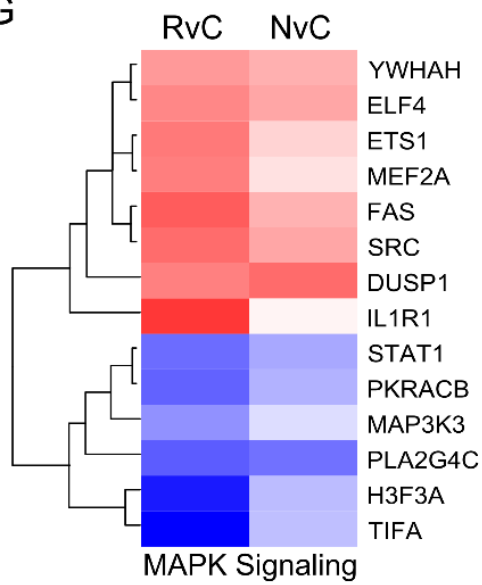

B

All Transcripts FDR $<0.1$

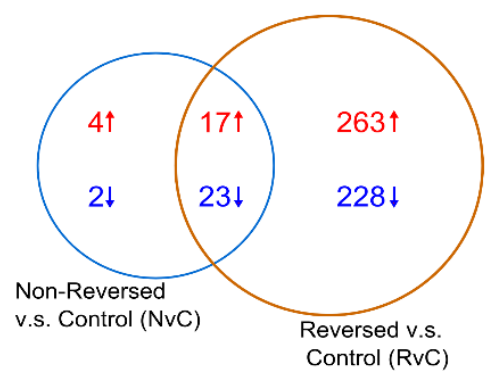

$\mathrm{E}$

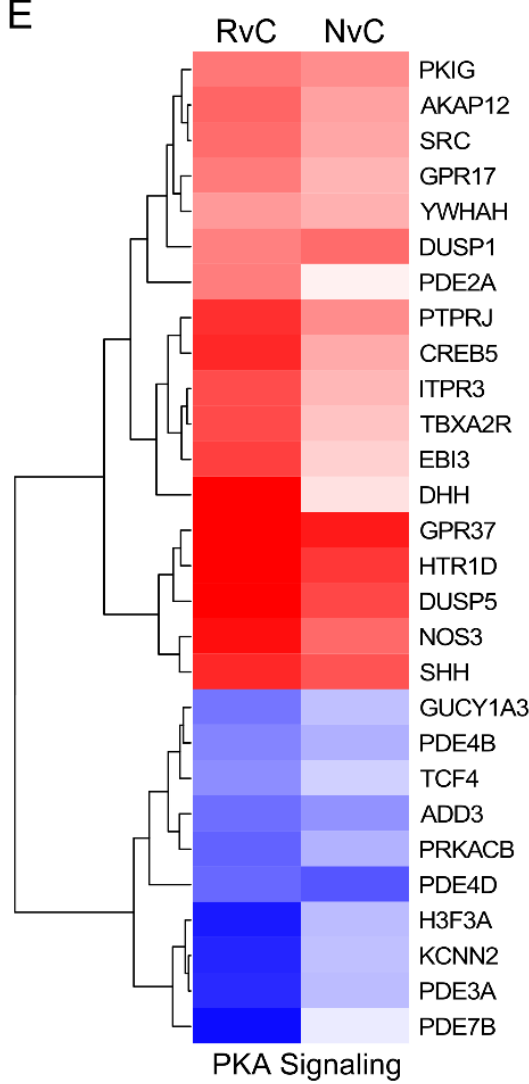

C

All Transcripts FDR $<0.1$

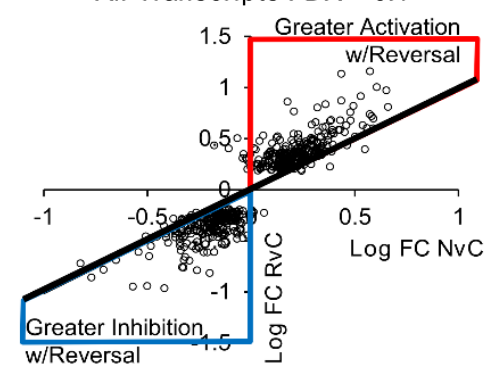

F
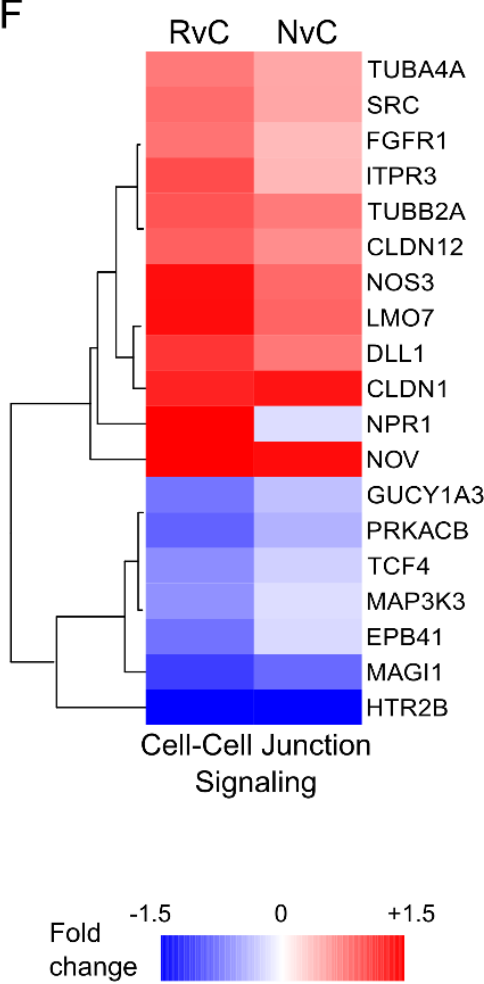

Figure 4.3. Genome-wide analysis indicates that reversed flow broadly enhances the arteriogenic transcriptional profile.

A) Schematic depicting shear stress conditions applied to HUVECs to simulate reversed (saphenous) and non-reversed (muscular) regions. HUVECs were preconditioned for 24 hours at 15 dynes $/ \mathrm{cm}^{2}$. Shear stress was then increased (or kept constant in the Control group) to $30 \mathrm{dynes} / \mathrm{cm}^{2}$ in the same or opposite direction. B) Venn diagram of Affymetrix ST 1.0 human cDNA array data $(n=4)$ comparing significantly altered transcripts $(F D R<0.1)$ in non-reversed vs. control $(\mathrm{NvC})$ conditions to those significantly altered with reversed direction vs. control $(\mathrm{RvC})$. Red text and arrows=upregulated; Blue text and arrows=downregulated. C) Scatterplot showing gene expression changes between RvC and $\mathrm{NvC}$ conditions, red region=greater up-regulation in RvC; blue region=greater down-regulation in RvC; black line=equal $R v C$ and $N v C$ ). D-G) Heatmaps grouped along significantly altered canonical pathways (cell-cell junctional signaling, protein kinase A signaling, MAP kinase signaling, and phosphodiesterase signaling) activated in both RvC and NvC conditions. Similar patterns of activation were observed between the conditions, but to a greater degree in the reversed flow condition. 


\subsubsection{Reversed flow broadly enhances the arteriogenic transcriptional profile.}

To then comprehensively determine how a change shear stress magnitude, coupled with a change in flow direction, activates pro-arteriogenic endothelial cell mechanosignaling pathways, we performed genome-wide, microarray transcriptional analysis on HUVECs exposed to the simulated femoral arterial ligation (FAL) shear stress protocol (Figure 4.3A). The 6 hour post-FAL time-point was chosen because PCP reorientation is complete and robust transcriptional changes in response to a stepwise shear stress increase have been previously reported ${ }^{191}$. Using a false discovery rate (FDR) for significance of $<0.10$ obtained through the Robust Multiarray Average (RMA) algorithm, HUVECs exposed to reversed flow showed an $\sim 10$-fold greater number of transcripts (reversed versus control, RvC, Supplemental Table 4.4) compared to HUVECs exposed to increased shear stress magnitude only (non-reversed versus control, $\mathrm{NvC}$, Supplemental Table 4.5) (Figure 4.3B). While reversed flow induced significant changes in more transcripts, the genes altered by a shear stress increase alone $(\mathrm{NvC})$ demonstrated a similar expression pattern as those also exposed to reversed shear stress direction (RvC). When the expression of the 537 genes with $\mathrm{FDR}<0.1$ (in NvC and/or RvC comparisons) was compared in both $\mathrm{NvC}$ and $\mathrm{RvC}$ groups, $97.4 \%$ were altered in the same expression direction; i.e. genes that were up-regulated in reversed conditions were also up-regulated in non-reversed conditions and vice versa (Figure $4.3 \mathrm{C}$ ). Interestingly, within this overlap, $93.9 \%$ of genes were altered to a greater degree in the reversed condition than in the non-reversed condition (Figure 4.3C). Of the $2.6 \%$ of genes exhibiting regulation in opposing directions between reversed and non-reversed conditions, all had a fold change $<0.1$ and an FDR $>0.88$ in the non-reversed group. With the changes of expression occurring in similar directions from the control state, there were no significant differences between reversed and non-reversed expression levels with an FDR $<0.20$. An a priori selection of 7 genes was used to validate the microarray results through real-time quantitative PCR (Table 4.1). All genes showed similar expression patterns and significance between microarray and RT-PCR measurements. However, the increased sensitivity of RT-PCR 
yielded significant differences between reversed and non-reversed conditions for ICAM-1, eNOS, and KLF2, all of which are known to play key roles in arteriogenesis ${ }^{56,180,192}$ and are established as being sensitive to arterial levels of shear stress $52,55,187,193-201$.

Table 4.1. Comparison of microarray genes to RT-PCR analyses for selected genes known to mediate arteriogenesis

\begin{tabular}{|c|c|c|c|c|c|c|}
\hline \multirow{2}{*}{$\begin{array}{l}\text { Entrez } \\
\text { Symbol }\end{array}$} & \multirow[b]{2}{*}{ Gene Name } & \multirow[b]{2}{*}{ Comparison } & \multirow{2}{*}{$\begin{array}{l}\text { RT-PCR } \\
\text { Log }_{2} \\
\text { Change }\end{array}$} & \multicolumn{3}{|l|}{ Microarray } \\
\hline & & & & $\begin{array}{ll}\log _{2} & \text { Fold } \\
\text { Change } & \\
\end{array}$ & $\mathrm{p}$-value & FDR \\
\hline \multirow{2}{*}{ NOS3 } & \multirow{2}{*}{$\begin{array}{l}\text { nitric oxide synthase } 3 \\
\text { (endothelial cell) }\end{array}$} & $\mathrm{RvC}$ & $1.17 \pm 0.49$ * & $0.47 \pm 0.13$ & $2.03 e-5$ & 0.011 \\
\hline & & $\mathrm{NvC}$ & $0.30 \pm 0.44$ & $0.29 \pm 0.13$ & 1.09 e-3 & 0.207 \\
\hline \multirow{2}{*}{ ICAM1 } & \multirow{2}{*}{$\begin{array}{l}\text { intercellular adhesion } \\
\text { molecule } 1\end{array}$} & $\mathrm{RvC}$ & $0.92 \pm 0.25 *$ & $0.15 \pm 0.12$ & 0.031 & 0.331 \\
\hline & & $\mathrm{NvC}$ & $0.49 \pm 0.44$ & $0.10 \pm 0.12$ & 0.114 & 0.640 \\
\hline \multirow{2}{*}{ VCAM1 } & \multirow{2}{*}{$\begin{array}{l}\text { vascular cell adhesion } \\
\text { molecule } 1\end{array}$} & $\mathrm{RvC}$ & $0.23 \pm 0.78$ & $-0.22 \pm 0.29$ & 0.161 & 0.641 \\
\hline & & $\mathrm{NvC}$ & $-0.67 \pm 0.43$ * & $-0.66 \pm 0.29$ & 9.86 e-4 & 0.203 \\
\hline \multirow{2}{*}{ KLF2 } & \multirow{2}{*}{$\begin{array}{l}\text { Kruppel-like factor } 2 \\
\text { (lung) }\end{array}$} & $\mathrm{RvC}$ & $0.84 \pm 0.31 * t$ & $0.33 \pm 0.11$ & $1.30 \mathrm{e}-4$ & 0.025 \\
\hline & & $\mathrm{NvC}$ & $0.43 \pm 0.25$ * & $0.26 \pm 0.11$ & $7.47 \mathrm{e}-4$ & 0.176 \\
\hline \multirow{2}{*}{ KLF4 } & \multirow{2}{*}{$\begin{array}{l}\text { Kruppel-like factor } 4 \\
\text { (gut) }\end{array}$} & $\mathrm{RvC}$ & $0.95 \pm 0.35$ * & $0.47 \pm 0.13$ & 1.86 e-5 & 0.010 \\
\hline & & $\mathrm{NvC}$ & $0.53 \pm 0.33$ * & $0.26 \pm 0.13$ & 2.06 e-3 & 0.261 \\
\hline \multirow{2}{*}{ SELE } & \multirow{2}{*}{ selectin $\mathrm{E}$} & $\mathrm{RvC}$ & $-0.86 \pm 0.63$ * & $-0.86 \pm 0.32$ & $2.57 \mathrm{e}-4$ & 0.032 \\
\hline & & $\mathrm{NvC}$ & $-0.94 \pm 0.51$ * & $-0.77 \pm 0.32$ & $6.35 \mathrm{e}-4$ & 0.157 \\
\hline \multirow{2}{*}{ CCL2 } & \multirow{2}{*}{$\begin{array}{l}\text { chemokine (C-C motif) } \\
\text { ligand } 2\end{array}$} & $\mathrm{RvC}$ & $-0.69 \pm 0.37$ * & $-0.19 \pm 0.11$ & 5.53 e-3 & 0.153 \\
\hline & & $\mathrm{NvC}$ & $-0.38 \pm 0.32$ * & $-0.08 \pm 0.11$ & 0.168 & 0.706 \\
\hline
\end{tabular}

Data represents RT-PCR ( $n=8$ /group) and microarray analysis ( $n=4 /$ group). RvC, reversed versus control. NvC nonreversed versus control. *, indicates $p<0.05$ significance versus control RT-PCR, $t$, indicates significance $p<0.05$ of reversed $v$ non-reversed by RT-PCR expression levels. Uncertainty in PCR is mean $+/$ - standard deviation for microarray mean $+/-95 \%$ confidence

Initial assessment of activated molecular functions was conducted through gene ontology analysis $^{189}$ to assess over-representation of molecular pathways. The 500 most significantly regulated genes based on $p$-value ranking for increased shear stress, with and without reversed flow, as compared to control were used to investigate the broad functional processes involved in both conditions (Supplemental Table 4.1). Oxidoreductase activity was the only significantly enriched molecular function in the case of the non-reversed increased shear stress (Supplemental Figure 4.5A). However, with the addition of a change in shear stress direction, there was activation of multiple molecular functions including: reelin receptor activity, RNA pol II 
promoter transcription factors, cAMP phosphodiesterase activity, and GTPase regulation (Supplemental Figure 4.5B, Supplemental Table 4.1).

\subsubsection{NFKB as a predicted upstream regulator of gene expression patterns in flow-reversed conditions}

Additional function annotation, clustering, and analysis of predicted upstream regulators were performed with the Ingenuity Pathways Analysis software. Analysis of predicted upstream transcriptional regulators for all genes with FDR $<0.1$ identified many of the known signaling pathways involved in arteriogenesis including activation of the NFKB pathway ${ }^{202-204}$ (Table 4.2) as well as growth factors [e.g. VEGF ${ }^{205,206}, \mathrm{HGF}^{207,208}$, TGF $\beta^{71}$, FGF2 ${ }^{209,210}$ ], MAPK signaling ${ }^{49,211,212}$, and PI3K signaling ${ }^{213}$ (Supplemental Table 4.2). Across all of these signaling pathways and upstream regulators, a clear activation was only apparent under the reversed flow condition.

Further clustering of expression changes of all genes with FDR $<0.1$ along canonical pathways showed activation of several key pathways known to be involved in arteriogenesis, including cGMP signaling ${ }^{180,214}$, protein kinase A signaling ${ }^{215,216}$, MAPK signaling ${ }^{49,211,212}$, and cellcell junction signaling ${ }^{50,51}$ (Figure 4.3D-G, Supplemental Table 4.3). HUVECs exposed to a reversed flow showed much stronger activation of these canonical pathways versus those exposed to non-reversed conditions (Supplemental Table 4.3).

Table 4.2. NFKB is a predicted upstream regulator of gene expression patterns in HUVECs exposed to flow reversed conditions

\begin{tabular}{|c|c|c|c|c|}
\hline \multirow[b]{2}{*}{ Upstream Regulator } & \multicolumn{2}{|c|}{ Reversed v Control } & \multicolumn{2}{|c|}{ Non-reversed v Contro } \\
\hline & $\begin{array}{c}\text { Activation } \\
\text { Z-Score }\end{array}$ & $\begin{array}{l}p \text {-value } \\
\text { overlap }\end{array}$ & $\begin{array}{c}\text { Activation } \\
\text { Z-Score }\end{array}$ & $\begin{array}{l}p \text {-value } \\
\text { overlap }\end{array}$ \\
\hline \multicolumn{5}{|l|}{ I. NFKB Pathway } \\
\hline $\begin{array}{l}\text { phorbol myristate acetate } \\
\text { (activator PKC and NFKB) }\end{array}$ & 2.767 & 1.35E-03 & & \\
\hline NFKBIA & 2.273 & 2.56E-02 & & 4.95E-02 \\
\hline RELA & 1.85 & $1.54 \mathrm{E}-02$ & & \\
\hline NFkB (complex) & 1.47 & 3.99E-03 & & \\
\hline IKBKB & 1.272 & 6.94E-06 & & $1.22 \mathrm{E}-02$ \\
\hline
\end{tabular}


A

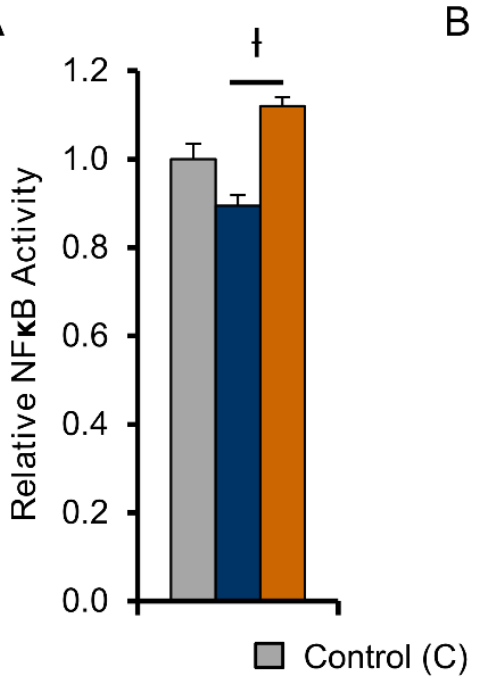

B

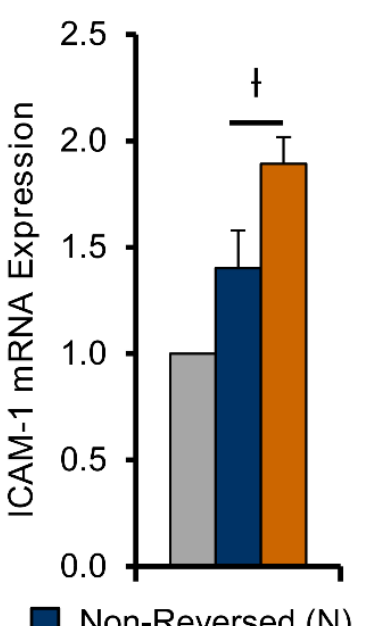

C
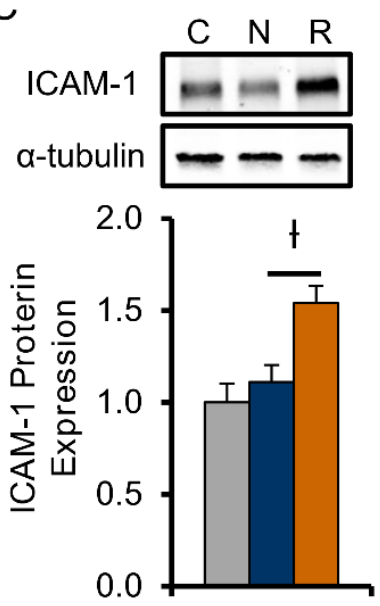

Reversed (R)

$\mathrm{E}$

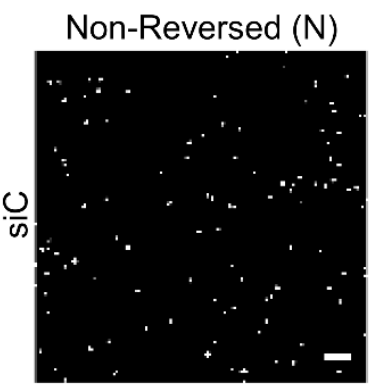

Reversed (R)

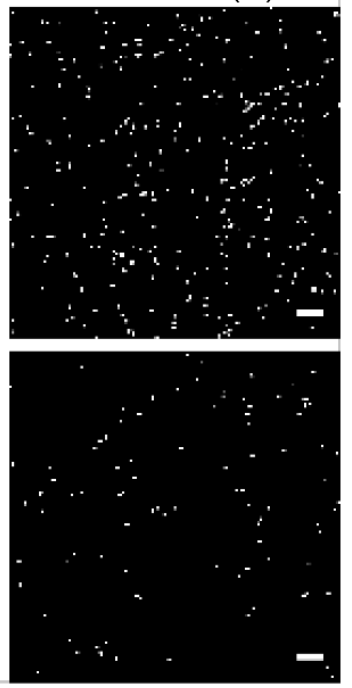

D
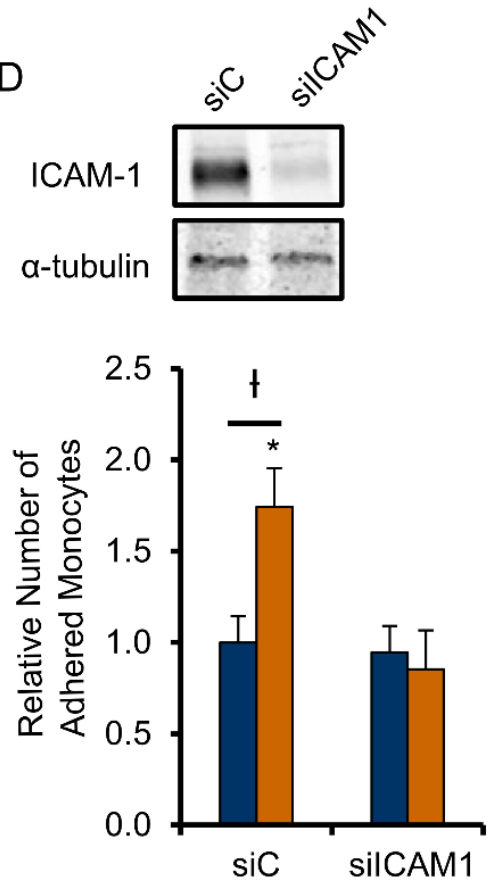

Figure 4.4. Reversed flow enhances monocyte adhesion in-vitro in an ICAM-1 dependent manner. A) NFKB activity $(n=3)$ relative to control ( $C$, gray) in HUVECs exposed to reversed ( $R$, orange) and nonreversed ( $\mathrm{N}$, blue) shear conditions 1 hour after simulated FAL in-vitro (Figure 4.3A). B, C) ICAM-1 mRNA expression by RT-PCR $(n=8)$ and protein expression $(n=4)$ in HUVECs relative to control 6 hours after simulated FAL. D) Bar graph quantifying relative number of adhered monocytes in each condition E) Representative FOVs of THP-1 monocytes adhered to HUVECs exposed to reversed and non- conditions transfected with either ICAM-1 siRNA (silCAM-1) or non-targeting control (siC) (Scale bar=100 $\mu \mathrm{m}$ ). $t \mathrm{p}<0.05$ between reversed $(R)$ and non-reversed $(N) ;{ }^{*} p<0.05$ between siC and silCAM-1 for given flow condition. Data are mean \pm SEM. 


\subsubsection{Amplified arteriogenesis in flow-reversed collaterals depends on ICAM-1.}

One pathway of particular interest from our Ingenuity Pathway Analysis of upstream regulators (Table 4.2) was the NFKB-ICAM-1 pathway. NFKB is a mechanosensitive transcription factor that regulates arteriogenesis ${ }^{204,217}$ through ICAM-155,218 dependent monocyte/macrophage recruitment ${ }^{56}$. To further investigate the NFKB- ICAM-1 pathway, we confirmed increased NFKB activity in HUVECs 1 hour after exposure to our in vitro simulated FAL, using a luciferase reporter assay (Figure 4.4A). Moreover, ICAM-1 mRNA expression was already shown to be increased (35\%) under reversed flow conditions as determined from our a priori RT-PCR screen of known pro-arteriogenic genes (Table 4.1, Figure 4.4B). Western blotting confirmed its up-regulation $(38.5 \%)$ at the protein level 6 hours after (Figure $4.4 C$ ). Additionally, reversed flow led to increased pro-arteriogenic function in-vitro as determined by increased monocyte adhesion to flow-conditioned HUVECs (Figure 4.4D-F). Furthermore, using a siRNA knockdown of ICAM-1, this increase in monocyte adhesion was determined to be ICAM-1 dependent (Figure 4.4D-F). We also determined there was over a 2-fold increase in pericollateral $\mathrm{Mac}^{+}$macrophages 3 days post-FAL in collateral segments of C57BL/6 mice that experienced reversed flow $(6.53 \pm 1.00$ and $3.12 \pm 0.47$ cells in reversed and non-reversed pericollateral regions, respectively), as seen in (Figure 4.5A-B. This increase in pericollateral macrophage density was attenuated in flowreversed segments of ICAM-1/ mice as there was no significant difference in reversed vs. nonreversed collateral segments $(2.33 \pm 0.44$ and $1.67 \pm 0.31$ cells in reversed and non-reversed pericollateral regions, respectively, $\mathrm{p}=0.34$ ) (Figure $4.5 A-B$ ). 

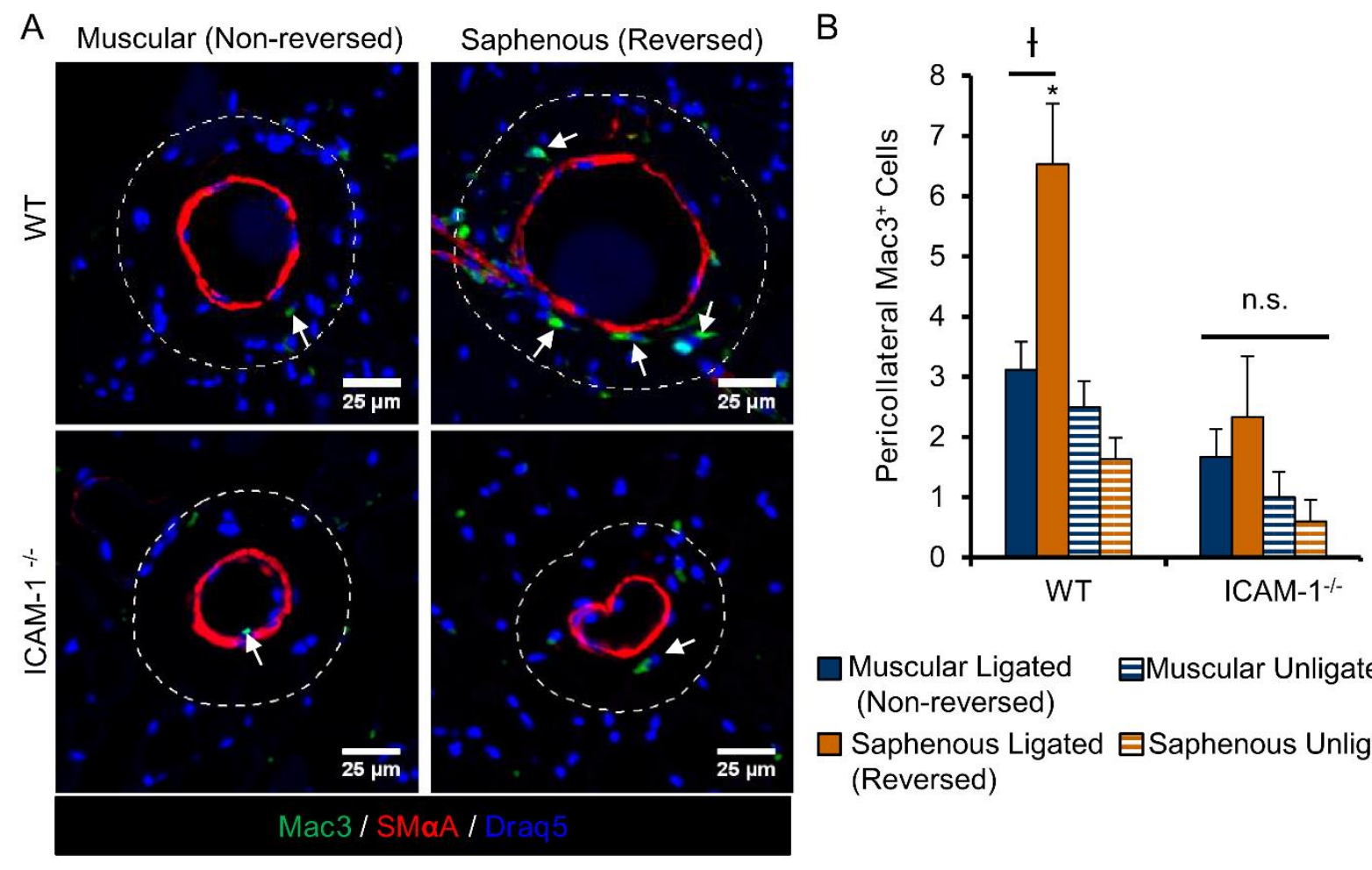

C

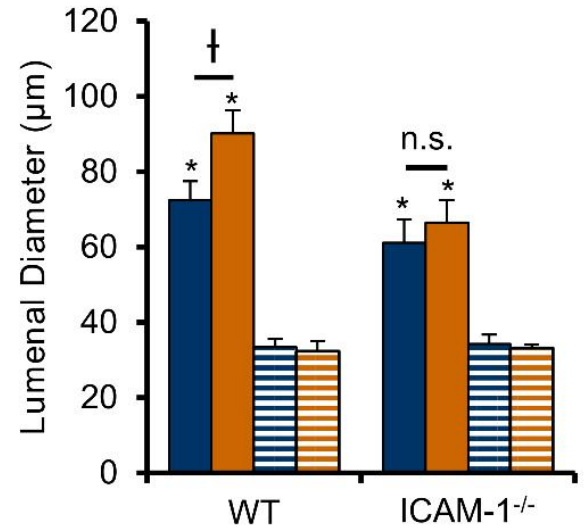

Figure 4.5. ICAM-1 is necessary for enhanced macrophage recruitment and amplified arteriogenesis in collateral segments exposed to a reversed flow.

A) Representative cross-sections of gracilis collateral artery regions in WT C57BL/6 and ICAM-1/- mice 3 days post-FAL immunolabeled for macrophage marker, Mac3 (green) and smooth muscle alpha actin (red). Dotted line indicates pericollateral region $(25 \mu \mathrm{m}$ from vessel wall) used for quantification. Arrows indicate

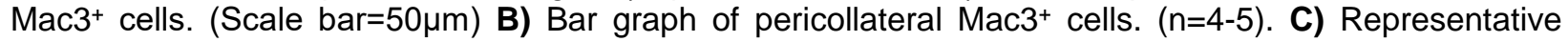
vascular cast images from ICAM ${ }^{-/-}$mice 14 days post-FAL from both muscular and saphenous regions. $($ Scale bar $=100 \mu \mathrm{m})$. Arrows indicate the measured collateral artery while arrowheads indicate transverse arterioles. D) Bar graph of regional lumenal diameter for both WT and ICAM-1/- mice $(n=6)$. ${ }^{*} p<0.05$ between ligated and unligated within the given region; $t p<0.05$ between regions (saphenous versus muscular) within the given treatment (ligated or unligated). Data are mean \pm SEM. 
Based on these findings, we next tested whether ICAM-1 was necessary for amplified arteriogenesis in flow-reversed collateral segments in-vivo by applying FAL to ICAM-1\% mice. There were no significant differences between ICAM-1 null mice and WT in diameters of collateral segments in unligated limbs. The muscular, non-reversed collateral segments in ICAM-1/ mice were not significantly different than those in WT mice $(p=0.11)$. However, deletion of ICAM-1 reduced the amplified collateral growth that occurs in collateral segments of WT mice exposed to a reversed flow $(p=0.017)$. Importantly, in these ICAM-1/ mice, we also observed no significant differences in diameter between reversed and non-reversed collateral segments at 14 days postFAL (66.5 \pm 5.95 and $61.2 \pm 6.05$ respectively, $p=0.48)$. Therefore the amplified arteriogenesis that occurs in flow reversed segments of WT mice $(1.24 \pm 0.12$ fold-change, reversed vs. non-reversed) is attenuated in ICAM-1 null mice (1.08 \pm 0.14 fold-change, reversed vs. non-reversed), demonstrating that ICAM-1 is necessary for the amplified response in segments exposed to reversed flow direction (Figure 4.5C-D).

Our transcriptional profiling data and a priori RT-PCR screen also suggested a potential role for the KLF2/eNOS pathway. We first determined that KLF2 and eNOS mRNA expression were enhanced by $33 \%$ and $83 \%$, respectively, in HUVECs exposed to FAL-simulated reversed flow (Supplemental Figure 4.6A-B). Phosphorylated (S1177) eNOS protein expression was also increased under reversed flow conditions (Supplemental Figure 4.6C). To then test whether eNOS was required for amplified arteriogenesis in flow-reversed collateral segments in-vivo, we applied FAL to eNOS ${ }^{-1-}$ mice. When all regional measurements of collateral growth are binned together, we observe a $17 \%$ decrease in arteriogenesis in eNOS $^{-/}$mice compared to wild-type

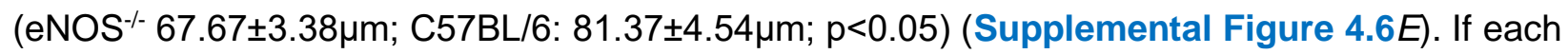
collateral region is analyzed separately, lumenal diameter in non-reversed, muscular collateral artery segments was significantly reduced in $\mathrm{eNOS}^{-/-}$mice compared to wild-type mice (eNOS ${ }^{-/-}$ $52.4 \pm 4.1 \mu \mathrm{m}$; C57BL/6: $72.5 \pm 5.0 \mu \mathrm{m}, \mathrm{p}<0.05$ ), but not in the flow reversed, saphenous segments $\left(\mathrm{eNOS}^{-/-} 82.6 \pm 3.6 \mu \mathrm{m} ; \mathrm{C} 57 \mathrm{BL} / 6: 90.2 \pm 6.2 \mu \mathrm{m}, \mathrm{p}=0.463\right)$ (Supplemental Figure 4.6F). However, 
when each region was normalized to its respective unligated control, amplified lumenal growth in flow reversed segments was maintained in $\mathrm{eNOS}^{-/-}$mice, indicating that this enhanced remodeling response is independent of eNOS (Supplemental Figure 4.6G).

\subsection{Discussion}

We report here a comprehensive, genome-wide, and direct mapping of mechanotransductive endothelial cell signaling pathway activation to a uniquely amplified and sustained in-vivo arteriogenesis response. We first used endothelial cell planar polarization as a marker to confirm endothelial cell responsiveness to the change in flow direction in the gracilis adductor collateral arteries following femoral arterial ligation (Figure 4.1). We then determined that collateral artery segments that are exposed to both an increase in shear stress magnitude and a reversed flow direction exhibit markedly amplified arteriogenesis when compared to collateral artery segments exposed to increased shear stress magnitude alone (Figure 4.2). Genomewide transcriptional profiling of HUVECs exposed to a biomimetic "reversed-flow + increased shear stress magnitude" waveform yielded a $\sim 10$-fold increase in significantly regulated transcripts when compared to HUVECs exposed to increased shear-stress alone. Indeed, this stimulus acts as a broad amplifier of transcriptional activation (Figure 4.3), including a set of potent arteriogenesis regulators (eNOS, ICAM-1, and KLF-2) that were then confirmed by RTPCR (Table 4.1). Further, Ingenuity Pathways Analysis indicated activation of a number of important canonical arteriogenesis pathways and upstream regulators, notably NFkB (Table 4.2). After confirming that the NFKB-ICAM-1 pathway was activated in HUVECs exposed to reversed flow, we showed the increased monocyte adhesion to HUVECs exposed to reversed flow was abrogated by knockdown of ICAM-1 (Figure 4.4). Finally, we demonstrated that enhanced pericollateral $\mathrm{Mac3}^{+}$macrophage density and amplified arteriogenesis in flowreversed collateral segments of WT mice was attenuated in ICAM-1/- mice, indicating that this amplified arteriogenic response is ICAM-1 dependent (Figure 4.5) 


\subsubsection{Reversed flow as an independent stimulus leading to amplified arteriogenesis.}

Our ability to link amplified arteriogenesis to a unique hemodynamic stimulus (i.e. reversed flow with increase shear stress magnitude) was facilitated by the development of a laser speckle flowmetry approach for mouse hindlimb collaterals ${ }^{176}$. We developed that approach because, despite the known importance of hemodynamic stimuli in driving collateral development, there was a surprising lack of quantitative data on the hemodynamic changes within these arteries. This is likely due to both the small size of the arteries $(<100 \mu \mathrm{m})$ and the fact that different surgical models elicit arteriogenesis along different collateral pathways ${ }^{36}$. Using laser speckle flowmetry ${ }^{176}$, we determined the occurrence of at least three distinct hemodynamic conditions in these collateral vessels: a non-reversed increase in shear stress near the feeding entrance to the collateral loop, an increase in shear stress from low/oscillating flow to sustained high shear stress at the central anastomotic region, and an increase in shear stress but in a reversed direction at the downstream outlet back into the occluded arterial tree. In this study, we only considered the two entrance regions because they experience the same step increase in relative shear stress magnitude after FAL, thereby permitting isolation of the influence of reversed flow.

Nonetheless, we also had to consider that hypoxia in flow reversed regions could be contributing to amplified arteriogenesis. Indeed, previous studies using saphenous artery excision models that elicit severe ischemia have suggested a role for hypoxia and metabolic signaling in the arteriogenic response ${ }^{219,220}$ and observed spatial differences in capillary density in the adductor muscles as a marker of ischemic response ${ }^{221}$. Thus, we examined the potential role of hypoxia in eliciting spatial differences in arteriogenesis between the flow reversed and nonreversed regions. In our study, pimonidazole- $\mathrm{HCl}$ immunolabeling revealed no signs of regional differences in hypoxia at 24 hours post-FAL (Supplemental Figure 4.2A-C). Consistent with previous findings for the gracilis muscle using a similar, milder (i.e. far less ischemic) FAL 
scheme $^{168}$, there was no evidence of angiogenesis in either the muscular branch or saphenous artery regions (Supplemental Figure 4.2F). As another indirect measure of tissue ischemia, muscle fiber size in the gracilis muscle showed no evidence of atrophy in either the muscular branch or saphenous artery regions (Supplemental Figure 4.2E). Thus, our results are consistent with previous studies using similar hindlimb models in which arteriogenesis proceeds independent of a hypoxic stimulus ${ }^{168,222,223}$.

Additionally, to determine if reversed flow can elicit amplified arteriogenesis independent of longitudinal position along a given collateral, we employed the MBL model to induce reversed flow in the muscular branch entrance region, instead of in the saphenous region (Supplemental Figure 4.3). With this ligation scheme, we observed both endothelial cell repolarization and amplified arteriogenesis in the muscular branch region of the collaterals, thereby establishing that this amplified arteriogenesis response is indeed not unique to the saphenous collateral region. In all, we conclude that reversed flow is a novel, independent stimulus for arteriogenesis.

\subsubsection{Endothelial cell sensing of shear stress magnitude and reversed direction.}

One motivation for the current study is that the endothelial response to increased shear stress from a pre-conditioned baseline state is not well-studied. In perhaps the most closely related study, a nearly $20 x$ reduction in the number of genes that are sensitive to a step-wise increase in shear stress from a pre-conditioned baseline was observed when compared to a step change from static conditions (i.e. 86 versus 1838) ${ }^{191}$. Our data matches this previous study well, as only 48 genes where significantly altered $($ FDR $<0.1)$ at 6 hours after a non-reversed $100 \%$ step increase in shear stress. This implies that gene expression changes induced by the application of laminar shear stress from static culture are not wholly predictive of gene expression changes occurring in the more physiological scenario of a step increase in shear stress from a non-zero baseline ${ }^{191}$. 
Studies incorporating non-reciprocating directional flow changes indicate that shear stress reversal from baseline has a disproportionate impact on responses such as permeability ${ }^{224}$, which is a hallmark in the initiation of arteriogenesis ${ }^{37}$, and intracellular calcium ${ }^{225}$. Because preconditioned, flow-adapted endothelial cells align with flow direction and incline their cell-cell junctions to reduced tension, they experience a dramatic increase in cell to cell tension when faced with a sudden reversed flow. This leads to a disproportionate response in endothelial cell signaling ${ }^{225}$. Interestingly, a step change in shear stress magnitude from a preconditioned level of 15 dyne $/ \mathrm{cm}^{2}$ to a very low level of 2.5 dynes $/ \mathrm{cm}^{2}$ with reversed flow direction elicits a disproportionately larger transcriptional response (of a similar pattern) than a step increase in shear stress $\left(+10\right.$ dynes $\left./ \mathrm{cm}^{2}\right)$ in the same direction ${ }^{226}$. Our data demonstrate that adding reversed flow to a $2 x$ increase in shear stress magnitude elicits a 10 -fold increase in transcriptional activation. Moreover, in addition to broadly modulating the endothelial shear stress response, a change in flow direction relative to an endothelial cell's morphological and cytoskeletal axes can stimulate activation of distinct pathways ${ }^{227}$. Together, these data suggest that $E C$ responsiveness to altered shear stress is highly dependent on initial conditions. For the specific case of shear signaling for arteriogenesis, consideration of the initial hemodynamic conditions across a given collateral network is especially significant.

\subsubsection{Endothelial cell repolarization and signaling for amplified arteriogenesis.}

In this study, endothelial cell planar polarization was used simply as a marker of endothelial cell responsiveness to a change in flow direction. To date there has been no direct functional link between endothelial planar (re)polarization and arteriogenesis; however our results offer an opportunity to explore this linkage. Indeed many molecular pathways that are activated by reversed flow in our study, such as small GTPase signaling (Supplemental Figure 4.5) and ICAM-1 (Figure 4.4), regulate both planar cell polarization ${ }^{182,228-230}$ and arteriogenesis ${ }^{49,56,231}$, raising the hypothesis that endothelial cell polarization may play a significant role in collateral 
artery remodeling. However, the functional consequence(s) of endothelial cell repolarization remains to be determined and future work could investigate the mechanistic role(s) of endothelial planar polarization in arteriogenesis.

\subsubsection{Complex role of eNOS signaling in arteriogenesis.}

Our transcriptional profiling and qRT-PCR studies led us to hypothesize that ICAM-1 and eNOS are important regulators of amplified arteriogenesis in flow-reversed collaterals. Here, we demonstrated ICAM-1 is necessary for enhanced monocyte/macrophage recruitment and augmented arteriogenesis in flow-reversed collateral segments. Together, these results are consistent with the previously demonstrated vital role for ICAM-1 in arteriogenesis through its regulation of monocyte/macrophage recruitment ${ }^{56}$.

While our ICAM-1/- studies show attenuation of this amplified arteriogenic response, our eNOS $^{-/-}$studies do not. The role of eNOS and/or NO in arteriogenesis is fairly well-studied, yet still difficult to interpret. While eNOS and NO regulate arteriogenesis in more severe excision hindlimb models ${ }^{232,233}$ and in training models ${ }^{234}$, no significant defects in arteriogenesis were reported for $\mathrm{eNOS}^{-/-}$mice three weeks after induction of a less ischemic femoral ligation model ${ }^{235}$. For our data set, if lumenal diameters at either end of the collateral are averaged, we observed a moderate decrease in arteriogenesis in eNOS ${ }^{-/-}$mice compared to wild-type. Given that eNOS can affect the baseline network configuration and maintenance ${ }^{180}$, we normalized the diameters of the ligated muscular branch and saphenous regions to their respective unligated controls (Supplemental Figure 4.6G). Using this analysis, we observe that the enhanced arteriogenesis caused by reversed flow is eNOS independent potentially due to the ability to compensate for the loss of eNOS with other NOS subtypes.

\subsubsection{Implications for understanding shear stress set-point.}

Finally, our results may have important implications for our understanding of the so-called homeostatic "set-point" for constant shear stress in the arterial circulation. In 1926, Murray put 
forth his "principle of minimum work", a consequence of which is that shear stress is maintained at a relatively constant level throughout the arterial vasculature ${ }^{86}$. In support of this concept, there appears to be a homeostatic wall shear stress magnitude (i.e. set-point) at which endothelial cells become quiescent and vessels maintain a steady state lumenal diameter ${ }^{236}$. Little is known, however, about how this set-point is achieved or maintained at the molecular signaling level. In our FAL model, if we assume Poiseuille flow, apply mass conservation, and consider that the lumenal diameter at the distal end (i.e. saphenous region) of the collateral is $22 \%$ greater than the lumenal diameter in the proximal end (i.e. muscular branch region) at 12 weeks post-FAL (Figure 4.2B), we estimate that the new steady-state shear stress in the saphenous region is reduced by at least $45 \%$ from pre-FAL levels. Thus, in essence, the reversed flow stimulus effectively resets the set-point for constant shear stress. We posit that further examination of this response could uncover molecular regulators of the shear stress set-point and/or provide clues for generating sustained therapeutic arteriogenic responses via set-point adjustment(s).

\subsection{Acknowledgements}

The authors would like to thank Dr. Stephen Turner (University of Virginia Bioinformatics Core) for help in processing and analysis of the microarray data and the University of Virginia Research Histology Core (under the direction of Sheri VanHoose) for histological tissue processing. The authors would also like to thank Dr. Brett Blackman for material support and guidance.

\subsection{Sources of Funding}

This material is based upon work supported by the National Science Foundation Graduate Research Fellowship Program under Grant No. NSF DGE-1315231 (JLH). Additional funding sources: American Heart Association awards 09PRE2060385 (JKM) and 10GRNT3490001 (RJP), and NIH grants T32-GM007267 (JKM), T32-HL007284 (JKM and JLH), R21-HL098632 (RJP). 


\subsection{Supplemental Figures}

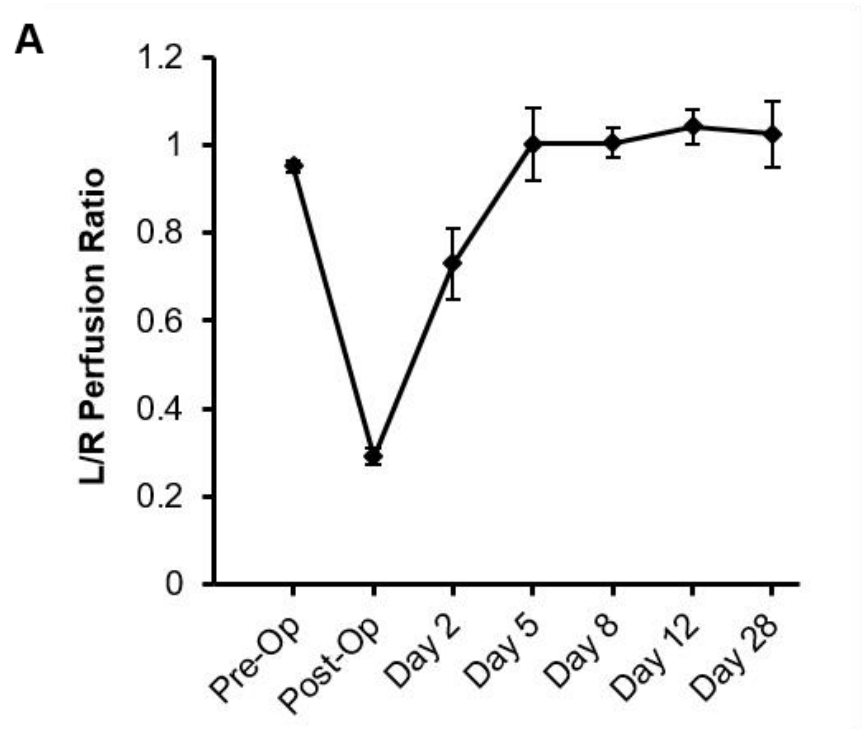

B

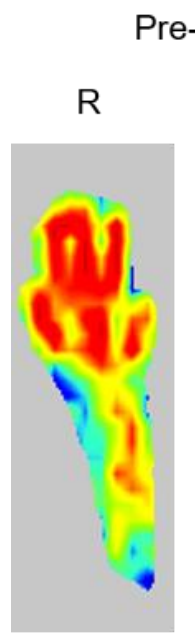

Pre-Op

Post-Op

Day 2

Day 5

L

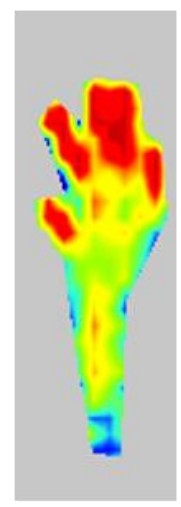

$\mathrm{R}$

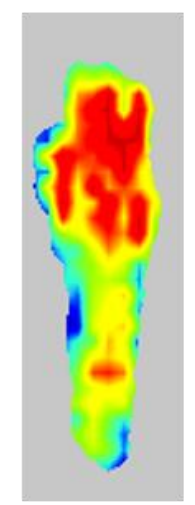

L

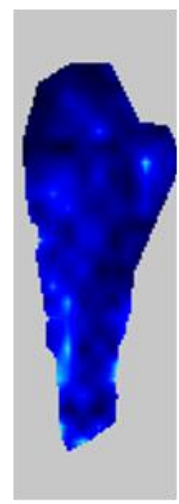

L

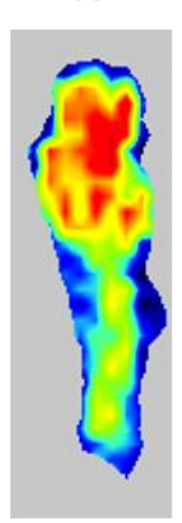

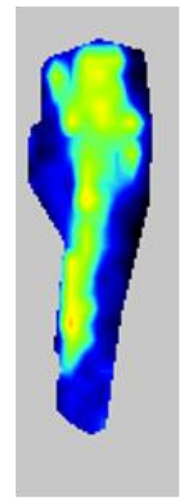

$\mathrm{R}$

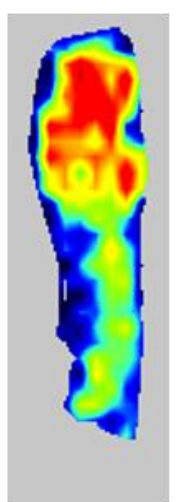

L

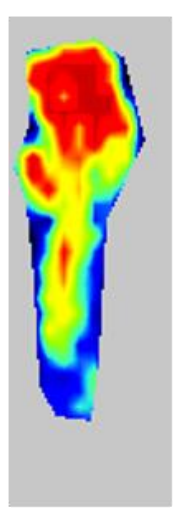

Max

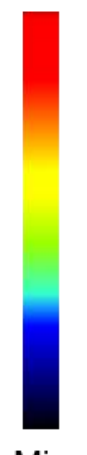

\section{Min}

Supplemental Figure 4.1. Laser Doppler perfusion recovery curve.

A) Laser Doppler perfusion measurements show mild ischemia in distal hindlimb immediately post-FAL. Perfusion is restored by day 5 post-FAL $(n=5)$. Data are mean \pm SEM. B) Representative images of measured foot perfusion in the ligated $(L)$ and unligated $(R)$ legs. Data are mean \pm SEM. 
A
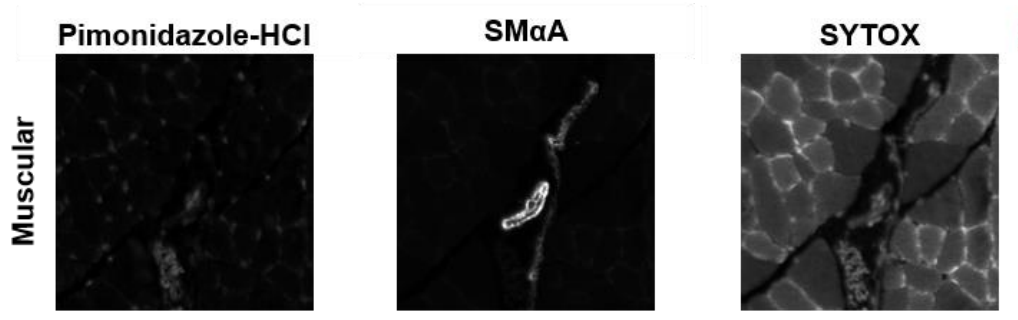

Pimonidazole/SMaA/SYTOX
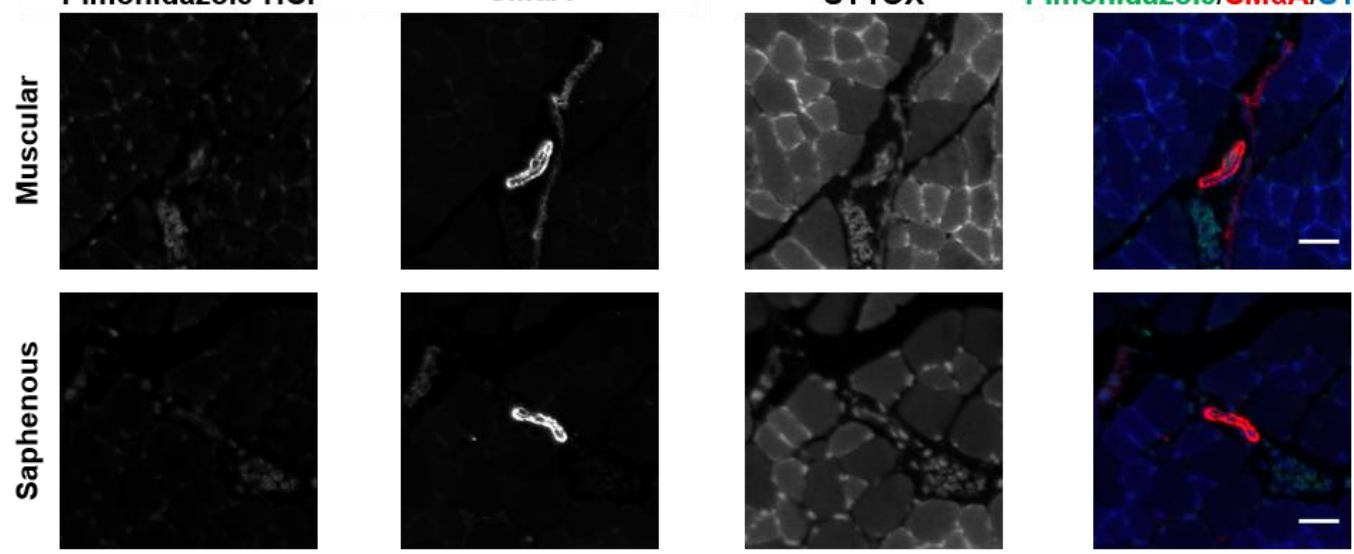

B
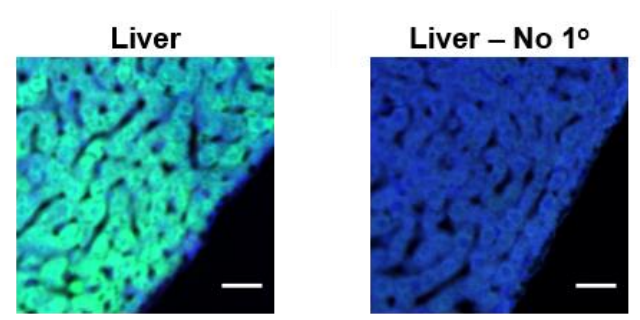

C
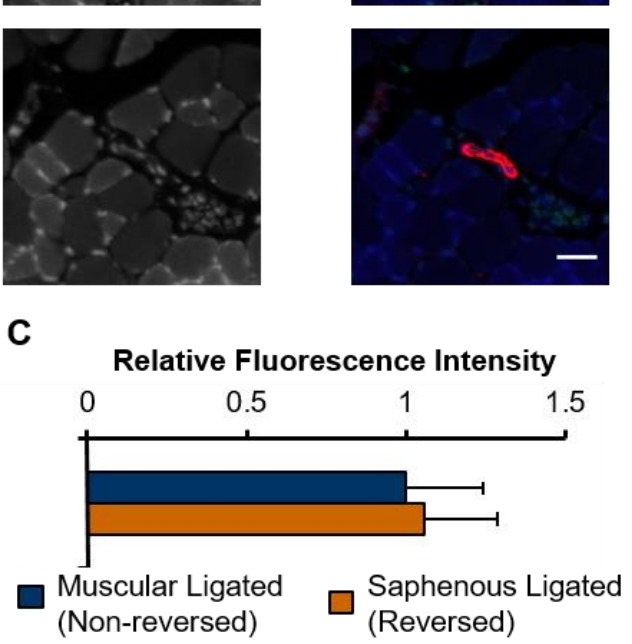

D
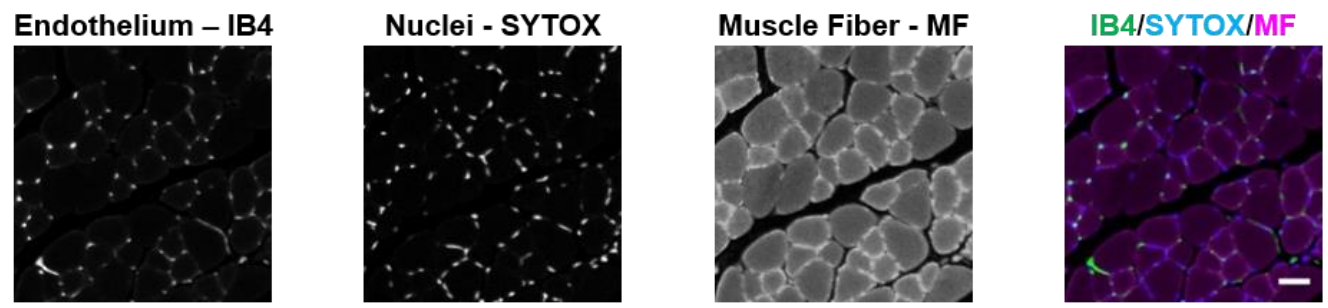

E

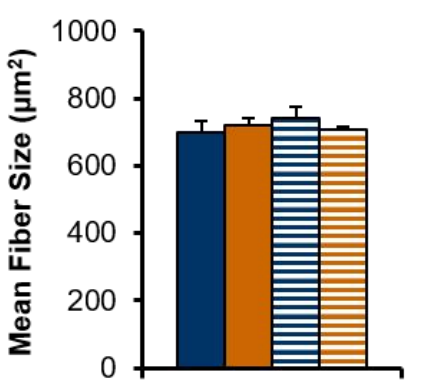

$\mathbf{F}$

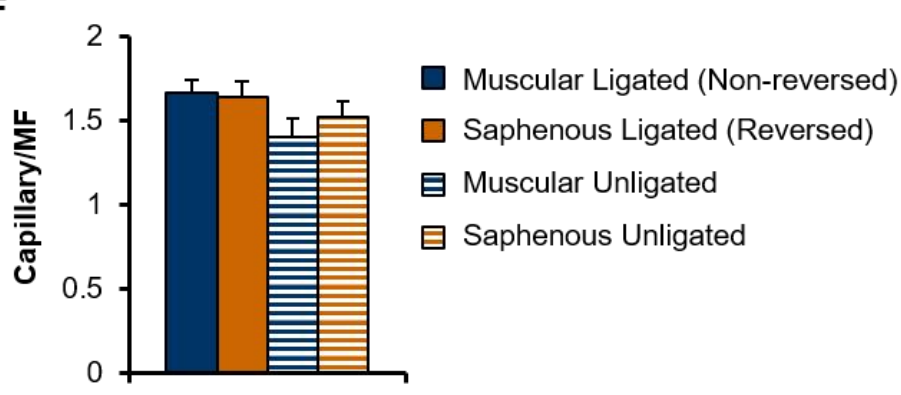

Supplemental Figure 4.2. Regional characterization of hypoxia, muscle fiber size, and capillary density in mouse gracilis muscle.

A) Representative paired fields of view of the gracilis muscles immunolabeled for pimonidazole- $\mathrm{HCl}$ to detect regions of hypoxia $\left(\mathrm{pO}_{2}<10 \mathrm{mmHg}\right) 24$ hours post-FAL. Scale bars $=25 \mu \mathrm{m}$. B) Liver cross-sections with and without primary anti-pimonidazole- $\mathrm{HCl}$ antibody were used as positive and negative immunolableing controls. Scale bars $=25 \mu \mathrm{m}$. C) Bar graph of relative fluorescence intensity 24 hours postFAL indicates no significant differences in regional hypoxia $(n=4)$. D) Representative FOV of a gracilis muscle cross-section stained for capillaries (isolectin B4). Scale bar $=25 \mu \mathrm{m}$. Tissue auto-fluorescence provided endogenous contrast for identifying individual muscle fibers. E) At 7 days post-FAL, there was no evidence of muscle atrophy or F) capillary to muscle fiber (MF) ratio to between ligated and unligated limbs in either region $(n=8)$. Data are mean \pm SEM. 
A

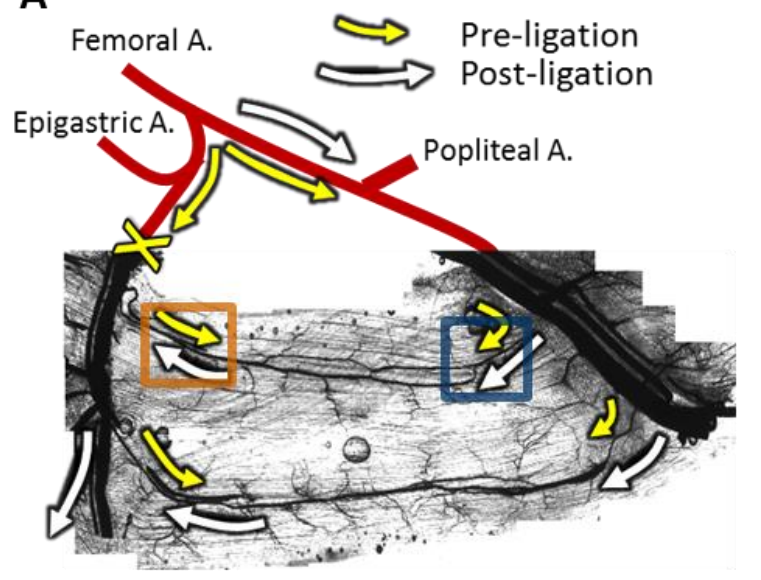

C

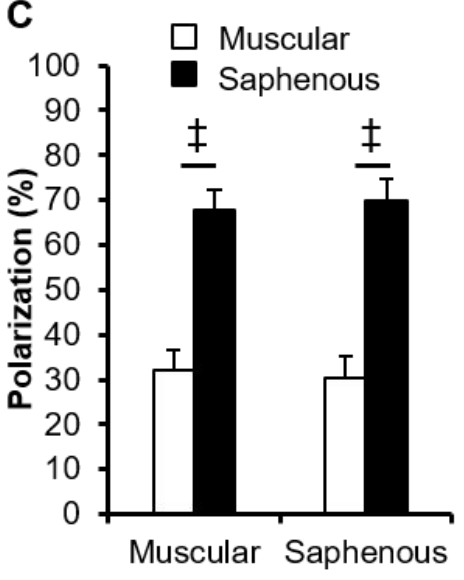

D
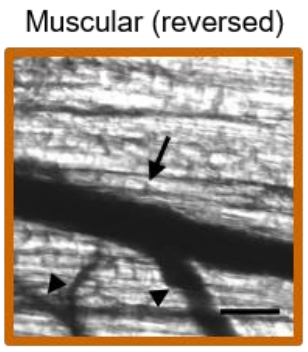

Muscular Ligated (Reversed)

Saphenous Ligated (Non-reversed)
B

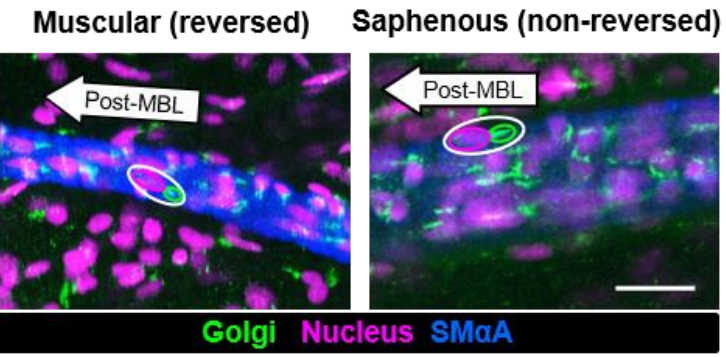

\section{Supplemental Figure 4.3. Arteriogenesis in gracilis collateral artery segments exposed to a reversed flow after muscular branch ligation.}

A) Schematic of the primary gracilis adductor collateral flow pathways after muscular branch ligation (MBL). The muscular branch feed artery is ligated just distal to the epigastric artery bifurcation thereby creating a change in flow direction at the muscular branch entrance region. The femoral and popliteal arteries are indicated for reference. Arrows indicate the direction and magnitude of blood flow both pre- (yellow) and post- (white) MBL. Boxes indicate location at which lumenal diameters were measured (reversed muscular branch entrance region=orange; non-reversed saphenous entrance region=blue). B) Representative images of each collateral region immunolabeled for the Golgi apparatus to determine planar polarization. Images are oriented such that the muscular branch is toward the left and the saphenous artery is toward the right and arrows indicate blood flow direction post-FAL. (Scale bar $=25 \mu \mathrm{m}$ ). C) Bar graph of EC planar polarization 24 hours post-MBL. ECs in all regions show upstream polarization toward the saphenous branch entrance region $(n=3)$. D) Representative vascular cast images from remodeled gracilis collateral arteries 14 days post-MBL (Scale bar $=50 \mu \mathrm{m}$ ). Arrows indicate measured collateral artery while arrowheads indicate transverse arterioles. E) Bar graph of regional lumenal diameter 14 days post-MBL $(n=6)$. ${ }^{*}<<0.05$ between ligated and unligated within the given region; $t p<0.05$ between regions (saphenous versus muscular) within the given treatment (ligated or unligated). $\neq \mathrm{p}<0.05$ between total upstream versus downstream polarization within the given flow condition. Data are mean \pm SEM. 
A

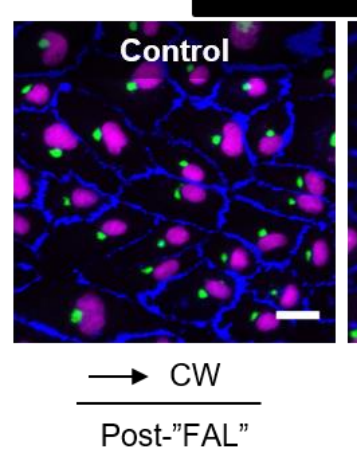

Golgi Nucleus CD31

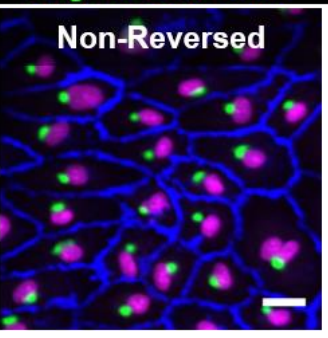

$\longrightarrow$ CW

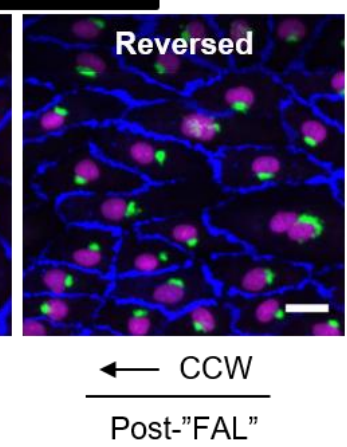

B

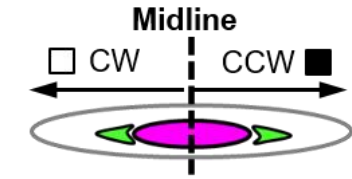

Counterclockwise (CCW)

Clockwise (CW)

Dispersed

Peri-nuclear Golgi / MTOC position with respect to upstream flow direction
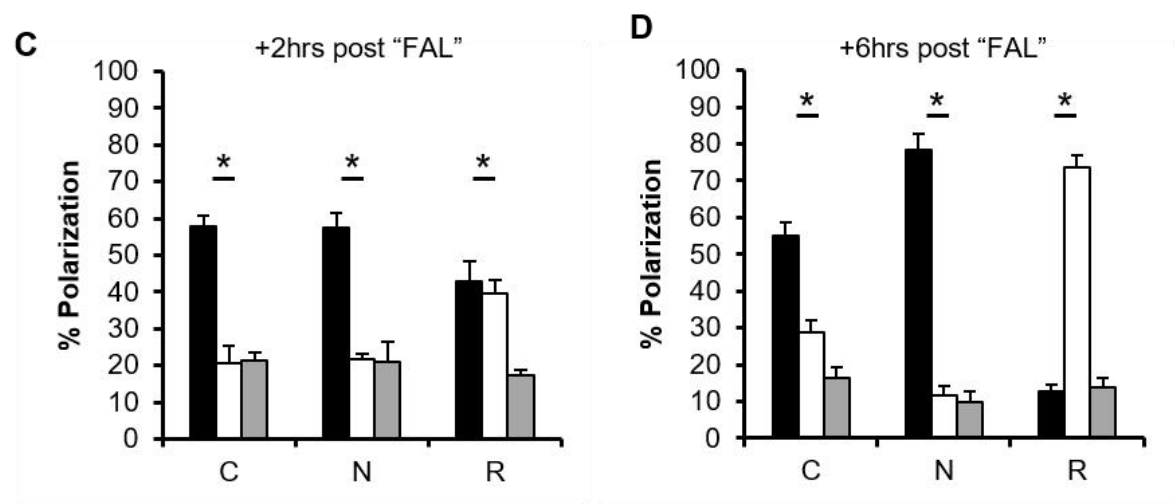
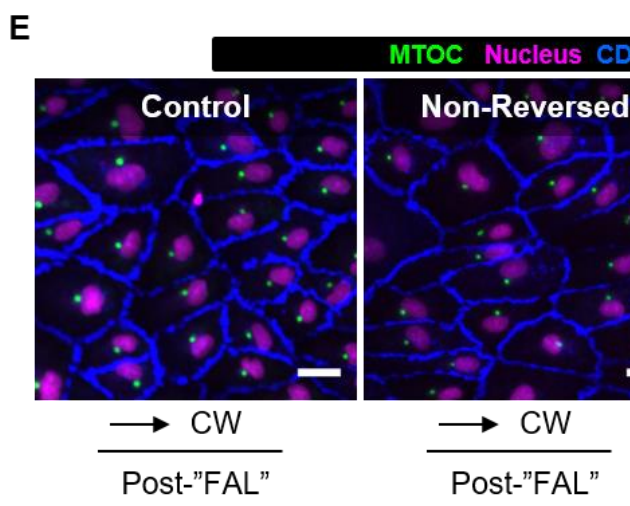

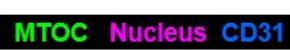

F
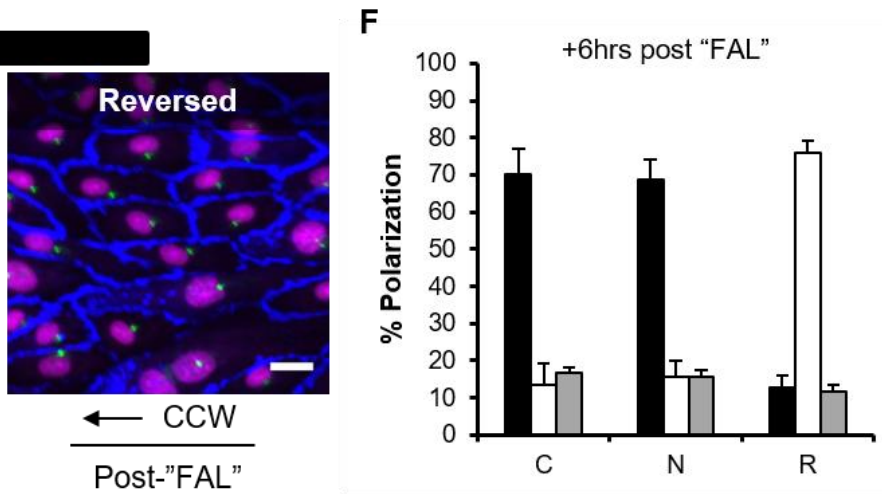

Supplemental Figure 4.4. HUVECs repolarize in response to directional change in shear stress induced by simulated FAL.

A) Representative images of HUVEC planar polarization assessed by the perinuclear position of the Golgi apparatus with respect to the pre-conditioning shear direction (see Fig $3 A$ ) at 6 hours after simulated FAL (Scale bar=25 $\mu \mathrm{m})$. Arrows indicate flow direction after simulated FAL $(\mathrm{n}=3)$. B) Diagram depicting the method used to quantify of polarization. Golgi apparatus (green) position with respect to the nucleus (magenta) was used to classify EC polarization as toward either the saphenous (black bars) or muscular branch (white bars) arteries. Golgi that were circum-nuclear and without preferential orientation were classified as dispersed C) Bar graph of HUVEC planar polarization at 2 hours after simulated FAL. $(n=3)$. D) Bar graph of HUVEC planar polarization at 6 hours after simulated FAL. E) Representative images of perinuclear position of the MTOC with respect to pre-conditioning shear direction to confirm HUVEC repolarization with reversed shear stress. F) Bar graph showing degree of polarization at 6 hours after simulated FAL $(n=3-4)$. ${ }^{*} p<0.05$ between upstream and downstream polarization within the given flow condition. Data are mean \pm SEM. 
A

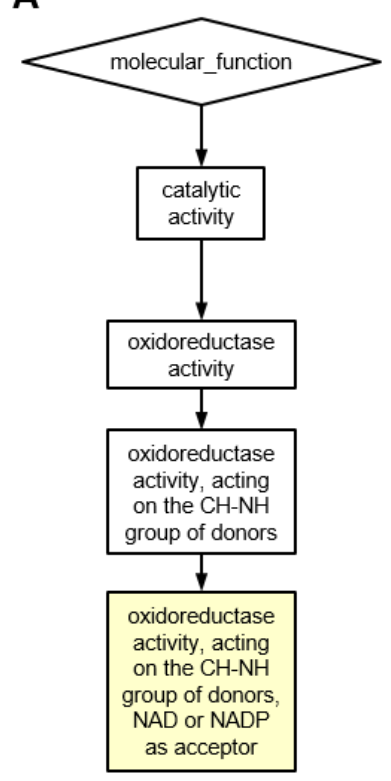

B

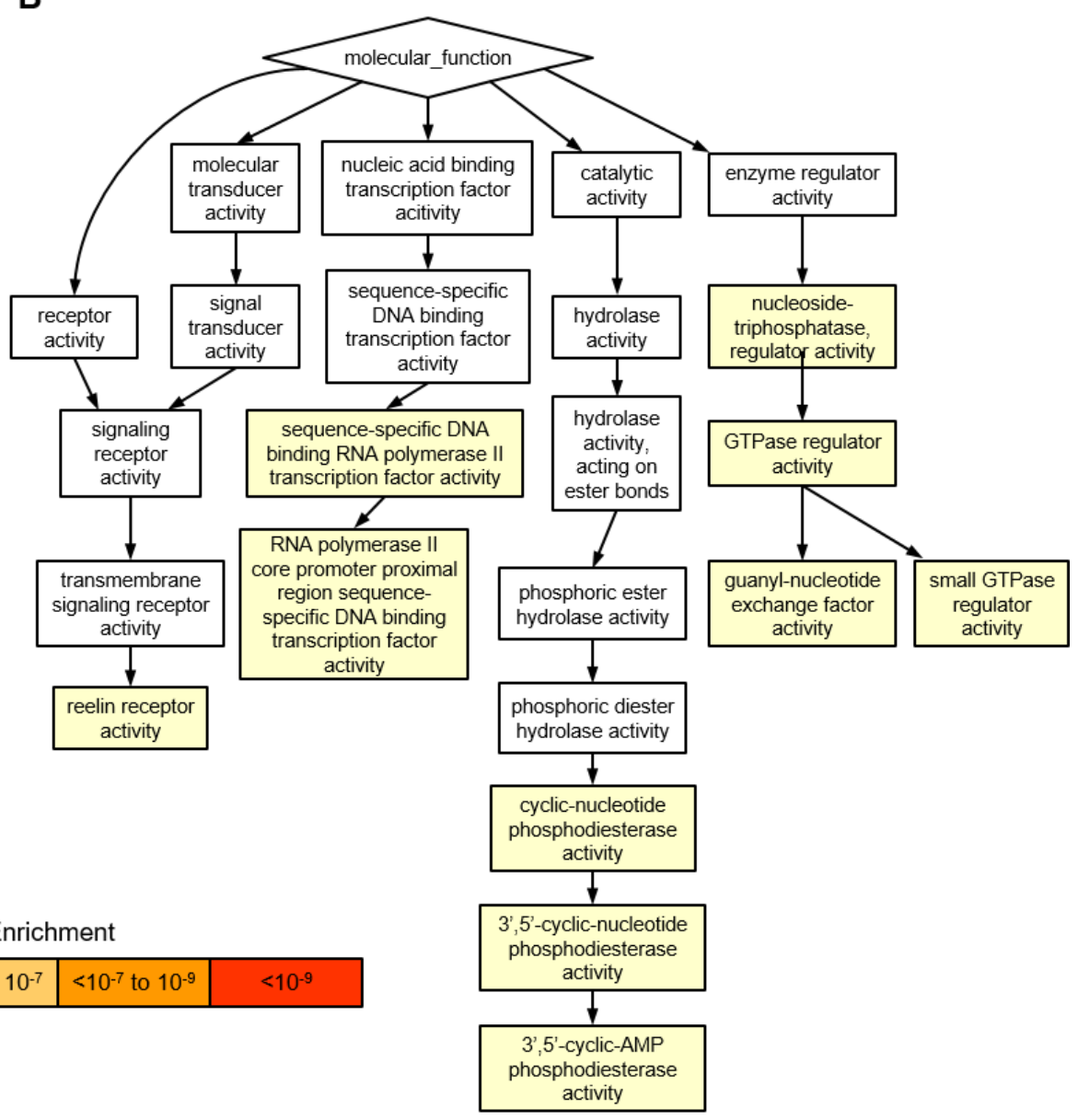

Supplemental Figure 4.5. Gene ontology clustering architecture of microarray expression in nonreversed and reversed flow waveforms.

Clustering of over-represented molecular functions for the 500 top transcripts as ranked by $p$-value was performed. A) Visual depiction of the changes seen with non-reversed increase in shear stress compared to control after 6 hours after simulated FAL Only one significant molecular function is over-represented. B) Visual depiction of the changes seen with an increase in shear stress plus reversed direction compared to control after 6 hours after simulated FAL. Numerous pathways are over-represented. Of particular interest, CAMP PDE activity suggests involvement of PKA and Akt signaling, while the increased activity in small GTPases suggests involvement of cytoskeletal reorganization. 
A

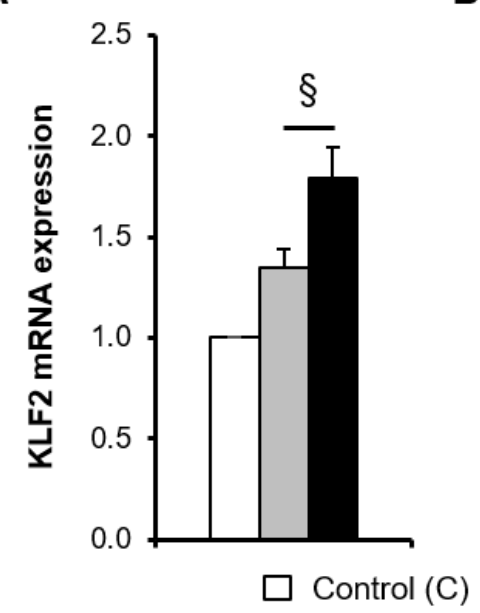

B

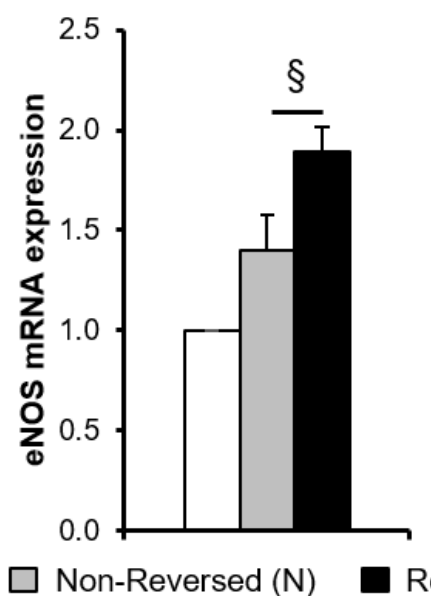

C
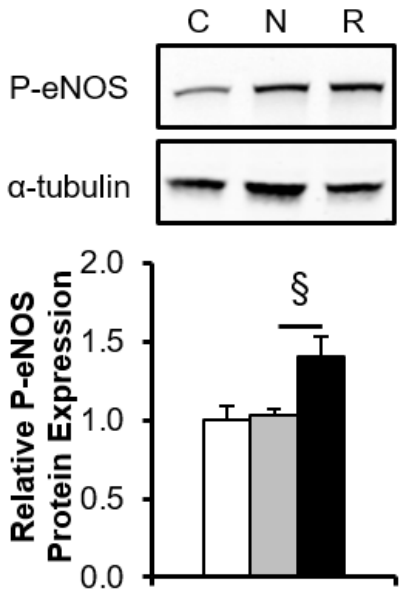

Reversed (R)

D

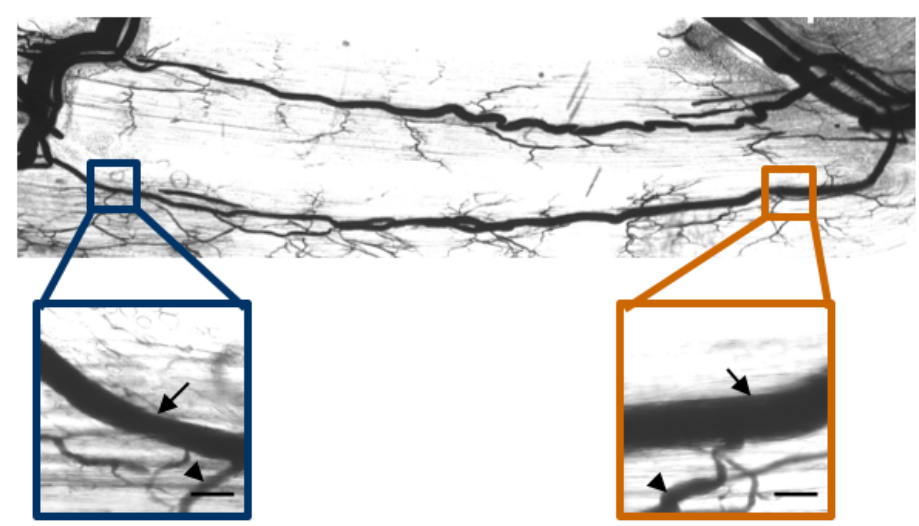

G

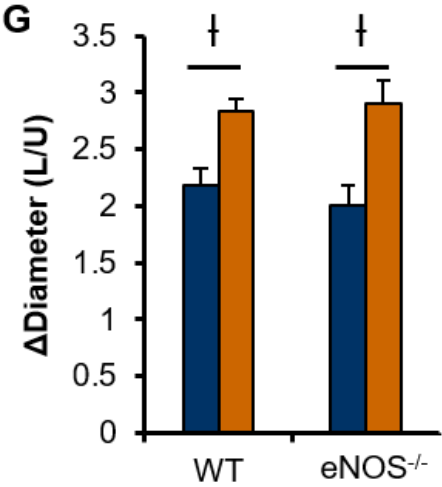

$\mathbf{E}$

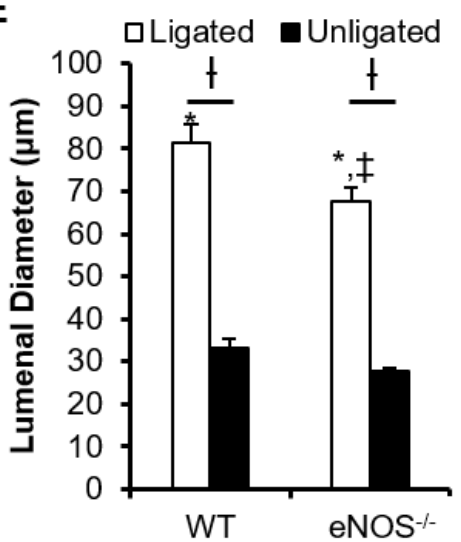

$\mathbf{F}$

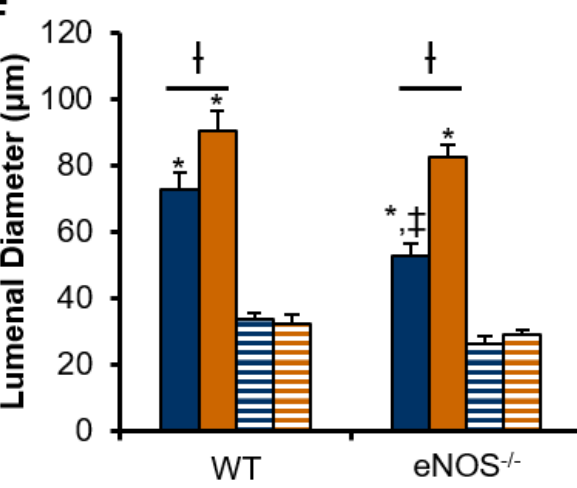

Muscular Ligated (Non-reversed)

Saphenous Ligated (Reversed)

曰 Muscular Unligated

Saphenous Unligated

Supplemental Figure 4.6. Amplified arteriogenesis in flow reversed segments does not require eNOS.

A,B) KLF2 and eNOS mRNA expression by RT-PCR $(n=8)$ and C) phosphorylated eNOS (Ser1177) protein expression $(n=4)$ relative to control in HUVECs exposed to reversed or non-reversed shear conditions 6 hours after simulated FAL. D) Representative vascular cast image from gracilis collateral arteries of eNOS1- mice 14 days post-FAL, with insets from both muscular and saphenous regions. Arrows indicate measured collateral artery while arrowheads indicate transverse arterioles. Inset scale bar $=100$ microns. E-G) Bar graphs of binned (all regions) lumenal diameter, regional lumenal diameter, and fold change of ligated diameter (L) from unligated control $(U)$ for both wild-type (WT) and eNOS ${ }^{-1-}$ mice $(n=6)$. ${ }^{*} p<0.05$ between ligated and unligated; $t p<0.05$ between saphenous and muscular regions; $\ddagger p<0.05$ between WT and eNOS1. $\$ p<0.05$ between reversed $(R)$ and non-reversed $(N)$ conditions in vitro. Data are mean \pm SEM. 


\subsection{Supplemental Tables}

\section{Supplemental Table 4.1. Gene ontology analysis for over-representation of molecular function}

\begin{tabular}{|c|c|c|c|c|}
\hline Description & $P$-value & $\begin{array}{l}\text { FDR } \\
\text { q-value }\end{array}$ & $\begin{array}{l}\text { Enrichment } \\
\text { Score }\end{array}$ & Enriched Genes in Pathway \\
\hline $\begin{array}{l}\text { A. Non-reversed v Control } \\
\text { oxidoreductase activity, acting on } \\
\text { the CH-NH group of donors, } \\
\text { NAD or NADP as acceptor }\end{array}$ & $6.66 \mathrm{E}-04$ & $1.00 \mathrm{E}+00$ & 9.57 & AASS, MTHFR, PYCR1, MTHFD2 \\
\hline $\begin{array}{l}\text { B. Reversed v Control } \\
\text { 3',5'-cyclic-AMP } \\
\text { phosphodiesterase activity }\end{array}$ & 8.29E-05 & $3.22 \mathrm{E}-01$ & 15.42 & PDE4D, PDE4B, PDE3A, PDE7B \\
\hline $\begin{array}{l}\text { nucleoside-triphosphatase } \\
\text { regulator activity }\end{array}$ & $1.07 \mathrm{E}-04$ & 2.07E-01 & 2.25 & $\begin{array}{l}\text { ARHGAP18, RAPGEF5, CYTH1, } \\
\text { TBC1D2, GRPEL1, PREX2, } \\
\text { ARHGAP29, TBC1D22B, KIAA1244, } \\
\text { HTR2B, SYDE2, STARD8, } \\
\text { SIPA1L3, PLEKHG5, RGL1, TBCK, } \\
\text { RASA4, PLEKHG1, PSD3, } \\
\text { ARHGAP28, DNMBP, RASGEF1B, } \\
\text { ARHGEF37, SH2D3C, TBXA2R, } \\
\text { DOCK11 }\end{array}$ \\
\hline GTPase regulator activity & $1.73 \mathrm{E}-04$ & 2.25E-01 & 2.23 & $\begin{array}{l}\text { ARHGAP18, RAPGEF5, CYTH1, } \\
\text { TBC1D2, PREX2, ARHGAP29, } \\
\text { TBC1D22B, KIAA1244, HTR2B, } \\
\text { SYDE2, STARD8, SIPA1L3, } \\
\text { PLEKHG5, RGL1, TBCK, RASA4, } \\
\text { PLEKHG1, PSD3, ARHGAP28, } \\
\text { DNMBP, RASGEF1B, ARHGEF37, } \\
\text { SH2D3C, TBXA2R, DOCK11 }\end{array}$ \\
\hline small GTPase regulator activity & 2.07E-04 & 2.01E-01 & 2.53 & $\begin{array}{l}\text { RASA4, RAPGEF5, PLEKHG1, } \\
\text { CYTH1, TBC1D2, PSD3, PREX2, } \\
\text { ARHGAP29, TBC1D22B, } \\
\text { KIAA1244, RASGEF1B, DNMBP, } \\
\text { HTR2B, ARHGEF37, SYDE2, } \\
\text { TBCK, PLEKHG5, RGL1, DOCK11 }\end{array}$ \\
\hline $\begin{array}{l}\text { guanyl-nucleotide exchange } \\
\text { factor activity }\end{array}$ & $2.45 \mathrm{E}-04$ & $1.91 \mathrm{E}-01$ & 3.01 & $\begin{array}{l}\text { RAPGEF5, CYTH1, PLEKHG1, } \\
\text { PSD3, PREX2, KIAA1244, DNMBP, } \\
\text { RASGEF1B, ARHGEF37, SH2D3C, } \\
\text { TBXA2R, RGL1, PLEKHG5, } \\
\text { DOCK11 }\end{array}$ \\
\hline $\begin{array}{l}\text { 3',5'-cyclic-nucleotide } \\
\text { phosphodiesterase activity }\end{array}$ & $3.25 E-04$ & $2.10 \mathrm{E}-01$ & 8.03 & $\begin{array}{l}\text { PDE4D, PDE4B, PDE3A, PDE2A, } \\
\text { PDE7B }\end{array}$ \\
\hline $\begin{array}{l}\text { RNA polymerase II core } \\
\text { promoter proximal region } \\
\text { sequence-specific DNA binding } \\
\text { transcription factor activity }\end{array}$ & 3.53E-04 & $1.96 \mathrm{E}-01$ & 4.08 & $\begin{array}{l}\text { RCOR1, NR4A1, CREB5, PLSCR1, } \\
\text { KLF4, DACH1, TCF4, SOX4, } \\
\text { ARNTL }\end{array}$ \\
\hline $\begin{array}{l}\text { cyclic-nucleotide } \\
\text { phosphodiesterase activity }\end{array}$ & 3.97E-04 & $1.93 \mathrm{E}-01$ & 7.71 & $\begin{array}{l}\text { PDE4D, PDE4B, PDE3A, PDE2A, } \\
\text { PDE7B }\end{array}$ \\
\hline $\begin{array}{l}\text { sequence-specific DNA binding } \\
\text { RNA polymerase II transcription } \\
\text { factor activity }\end{array}$ & $5.28 \mathrm{E}-04$ & $2.28 \mathrm{E}-01$ & 2.58 & $\begin{array}{l}\text { RCOR1, NR4A1, RARB, DACH1, } \\
\text { TCF4, NR1D2, KLF11, ARNTL, } \\
\text { STAT1, CREB5, PLSCR1, FOXN2, } \\
\text { KLF4, SOX4, MEF2A, FOXN3 }\end{array}$ \\
\hline reelin receptor activity & $6.72 \mathrm{E}-04$ & $2.61 \mathrm{E}-01$ & 38.55 & VLDLR, LRP8 \\
\hline
\end{tabular}

Represents gene ontology clustering from top 500 genes from each group as ranked by $p$-value 


\section{Supplemental Table 4.2. Additional predicted upstream regulators of gene expression patterns seen with reversed and non-reversed flow waveforms}

\begin{tabular}{|c|c|c|c|c|}
\hline \multirow[b]{2}{*}{ Upstream Regulator } & \multicolumn{2}{|c|}{ Reversed v Control } & \multicolumn{2}{|c|}{ Non-reversed v Control } \\
\hline & Activation Z-Score & $p$-value overlap & Activation Z-Score & $p$-value overlap \\
\hline \multicolumn{5}{|l|}{ A. MAPK Pathways } \\
\hline Mek (group) & 2.215 & $3.50 \mathrm{E}-02$ & & \\
\hline RAF1 & 2.037 & 2.62E-05 & & $9.31 \mathrm{E}-04$ \\
\hline ERK1/2 & 1.912 & 3.05E-02 & & \\
\hline MAPK14 & 1.793 & $2.50 \mathrm{E}-02$ & & 3.38E-02 \\
\hline MAP3K1 & 1.513 & $1.65 E-02$ & & $6.70 \mathrm{E}-03$ \\
\hline ERK & 1.418 & $5.74 \mathrm{E}-03$ & & \\
\hline P38 MAPK & 1.403 & 2.72E-04 & & \\
\hline $\begin{array}{l}\text { U0126 } \\
\text { (inhibitor of MEK 1/2) }\end{array}$ & -2.336 & $2.24 \mathrm{E}-05$ & & \\
\hline $\begin{array}{l}\text { PD98059 } \\
\text { (inhibitor of MEK 1) }\end{array}$ & -3.215 & $6.65 \mathrm{E}-05$ & -0.577 & 1.91E-02 \\
\hline \multicolumn{5}{|l|}{ B. Growth Factors } \\
\hline HGF & 5.243 & $1.87 \mathrm{E}-13$ & & \\
\hline Vegf (group) & 4.955 & $1.81 \mathrm{E}-15$ & & \\
\hline EGF & 2.407 & 7.47E-05 & & \\
\hline PDGF BB & 2.33 & $1.78 \mathrm{E}-02$ & & $1.76 \mathrm{E}-02$ \\
\hline FGF1 & 2.18 & 8.10E-03 & & \\
\hline EGR2 & 2.178 & $1.41 \mathrm{E}-02$ & & \\
\hline FGF2 & 2.11 & $1.68 \mathrm{E}-05$ & & \\
\hline TGFBR2 & 1.969 & 3.76E-02 & & \\
\hline ERBB2 & 1.849 & $1.55 \mathrm{E}-03$ & & \\
\hline VEGFA & 1.84 & $1.74 \mathrm{E}-04$ & & \\
\hline TGFB3 & 1.134 & $5.48 \mathrm{E}-03$ & & \\
\hline Tgf beta (group) & 1.07 & $9.46 \mathrm{E}-03$ & & \\
\hline MET & 1 & 4.26E-03 & & \\
\hline $\begin{array}{l}\text { tyrphostin AG } 1478 \\
\text { (inhibitor of EGFR1) }\end{array}$ & -1.04 & 1.40E-02 & & \\
\hline CTGF & -1.134 & 2.39E-03 & & 1.01E-02 \\
\hline \multicolumn{5}{|c|}{ C. PIЗK/AKT Pathway } \\
\hline Akt & 1.941 & $3.40 \mathrm{E}-02$ & & \\
\hline PI3K (complex) & 1.714 & 1.43E-02 & & \\
\hline $\mathrm{Ca} 2+$ & 1.191 & 4.45E-07 & & \\
\hline $\begin{array}{l}\text { LY294002 } \\
\text { (inhibitor of PI3K) }\end{array}$ & -1.12 & $2.25 \mathrm{E}-04$ & & 4.99E-02 \\
\hline $\begin{array}{l}\text { wortmannin } \\
\text { (inhibitor of PI3K) }\end{array}$ & -2.007 & 8.01E-02 & & \\
\hline \multicolumn{5}{|l|}{ D. Cytokines } \\
\hline IL13 & 2.493 & $1.46 \mathrm{E}-01$ & & \\
\hline CXCL12 & 1.542 & 2.26E-02 & & \\
\hline IL3 & 1.342 & 2.20E-02 & & \\
\hline Ifn (group) & 1.305 & 1.19E-03 & & \\
\hline IL1RN & 1.195 & 1.47E-02 & & \\
\hline IL1A & 1.115 & 2.82E-04 & & \\
\hline TNF & 0.062 & $1.03 E-10$ & 1.236 & 8.23E-05 \\
\hline IFNA1/IFNA13 & -1 & $1.52 \mathrm{E}-02$ & & \\
\hline Tnf (family) & -1.089 & $5.67 \mathrm{E}-03$ & & \\
\hline IL27 & -1.277 & 8.09E-03 & & \\
\hline IFNA2 & -1.564 & $1.20 \mathrm{E}-02$ & & \\
\hline IFNB1 & -1.665 & 2.75E-03 & & \\
\hline TNFSF10 & -1.715 & 2.22E-02 & & \\
\hline
\end{tabular}




\section{Supplemental Table 4.3. Ingenuity Pathways Analysis significant canonical pathways}

\begin{tabular}{|c|c|c|c|}
\hline Pathway & P-value & Ratio & Molecules \\
\hline \multicolumn{4}{|l|}{ A. Reversed v Control } \\
\hline Cellular Effects of Sildenafil (Viagra) & 1.17E-03 & $7.8 \mathrm{E}-02$ & $\begin{array}{l}\text { PRKACB, PDE2A, KCNN2, GUCY1A3, } \\
\text { GPR37, PDE3A, ACTA2, ITPR3, } \\
\text { PDE4B, NOS3, PDE4D }\end{array}$ \\
\hline Corticotropin Releasing Hormone Signaling & $1.45 \mathrm{E}-03$ & 7.46E-02 & $\begin{array}{l}\text { PRKACB, SHH, GUCY1A3, BDNF, } \\
\text { NPR1, ITPR3, MEF2A, NR4A1, CREB5, } \\
\text { NOS3 }\end{array}$ \\
\hline tRNA Splicing & $2.45 \mathrm{E}-03$ & 1.19E-01 & $\begin{array}{l}\text { PDE2A, PDE7B, PDE3A, PDE4B, } \\
\text { PDE4D }\end{array}$ \\
\hline Protein Kinase A Signaling & $5.37 \mathrm{E}-03$ & $5.29 \mathrm{E}-02$ & $\begin{array}{l}\text { PRKACB, AKAP12, SHH, TCF4, } \\
\text { PDE2A, H3F3A/H3F3B, YWHAH, } \\
\text { PDE3A, PDE4B, CREB5, NOS3, } \\
\text { PDE4D, DHH, DUSP5, ADD3, PDE7B, } \\
\text { PTPRJ, DUSP1, ITPR3, EBI3 }\end{array}$ \\
\hline cAMP-mediated signaling & $6.17 \mathrm{E}-03$ & $6.36 \mathrm{E}-02$ & $\begin{array}{l}\text { AKAP12, PRKACB, SRC, GPR17, } \\
\text { PDE2A, PDE7B, DUSP1, PDE3A, } \\
\text { TBXA2R, HTR1D, PDE4B, PDE4D, } \\
\text { CREB5, PKIG }\end{array}$ \\
\hline p38 MAPK Signaling & $6.46 \mathrm{E}-03$ & 7.89E-02 & $\begin{array}{l}\text { H3F3A/H3F3B, TIFA, DUSP1, } \\
\text { PLA2G4C, MEF2A, IL1R1, CREB5, } \\
\text { STAT1, FAS }\end{array}$ \\
\hline Gap Junction Signaling & $1.55 \mathrm{E}-02$ & $5.85 \mathrm{E}-02$ & $\begin{array}{l}\text { PRKACB, SRC, HTR2B, NOV, } \\
\text { GUCY1A3, NPR1, ACTA2, TUBB2A, } \\
\text { ITPR3, TUBA4A }\end{array}$ \\
\hline Sertoli Cell-Sertoli Cell Junction Signaling & $1.58 \mathrm{E}-02$ & 5.7E-02 & $\begin{array}{l}\text { PRKACB, SRC, EPB41, CLDN12, } \\
\text { GUCY1A3, CLDN1, ACTA2, TUBB2A, } \\
\text { TUBA4A, NOS3, MAP3K3 }\end{array}$ \\
\hline Relaxin Signaling & 1.91E-02 & 5.77E-02 & $\begin{array}{l}\text { PRKACB, PDE2A, PDE7B, GUCY1A3, } \\
\text { PDE3A, NPR1, PDE4B, NOS3, PDE4D }\end{array}$ \\
\hline$\beta$-alanine Degradation I & 3.09E-02 & $1.11 \mathrm{E}-01$ & ALDH6A1 \\
\hline All-trans-decaprenyl Diphosphate Biosynthesis & 3.09E-02 & $2 \mathrm{E}-01$ & PDSS1 \\
\hline Epithelial Adherens Junction Signaling & 3.31E-02 & $6.16 \mathrm{E}-02$ & $\begin{array}{l}\text { SRC, DLL1, TCF4, MAG11, LMO7, } \\
\text { FGFR1, ACTA2, TUBB2A, TUBA4A }\end{array}$ \\
\hline ERK5 Signaling & $3.55 \mathrm{E}-02$ & 7.94E-02 & $\begin{array}{l}\text { SRC, YWHAH, MEF2A, CREB5, } \\
\text { MAP3K3 }\end{array}$ \\
\hline FGF Signaling & $3.98 \mathrm{E}-02$ & $6.82 \mathrm{E}-02$ & $\begin{array}{l}\text { FGFR3, FGF16, FGF18, FGFR1, } \\
\text { FGF12, CREB5 }\end{array}$ \\
\hline G-Protein Coupled Receptor Signaling & 4.37E-02 & 4.89E-02 & $\begin{array}{l}\text { PRKACB, SRC, GPR17, PDE2A, } \\
\text { HTR2B, PDE7B, DUSP1, PDE3A, } \\
\text { TBXA2R, HTR1D, PDE4B, PDE4D, } \\
\text { CREB5 }\end{array}$ \\
\hline Cardiac $\beta$-adrenergic Signaling & 4.47E-02 & 5.33E-02 & $\begin{array}{l}\text { PRKACB, AKAP12, PDE2A, PDE7B, } \\
\text { PDE3A, PDE4B, PDE4D, PKIG }\end{array}$ \\
\hline Aryl Hydrocarbon Receptor Signaling & 4.57E-02 & $5.03 \mathrm{E}-02$ & $\begin{array}{l}\text { SRC, NCOA7, CCND3, NFIA, RARB, } \\
\text { ALDH6A1, FAS, MCM7 }\end{array}$ \\
\hline ERK/MAPK Signaling & 4.68E-02 & $5.03 \mathrm{E}-02$ & $\begin{array}{l}\text { PRKACB, ETS1, SRC, ELF4, } \\
\text { H3F3A/H3F3B, YWHAH, DUSP1, } \\
\text { PLA2G4C, CREB5, STAT1 }\end{array}$ \\
\hline \multicolumn{4}{|l|}{ B. Non-reversed v Control } \\
\hline UDP-D-xylose and UDP-D-glucuronate Biosynthesis & $5.13 \mathrm{E}-03$ & 1.43E-01 & UGDH \\
\hline Sphingomyelin Metabolism & $1.51 \mathrm{E}-02$ & 7.14E-02 & SGMS2 \\
\hline VDR/RXR Activation & $1.70 \mathrm{E}-02$ & 2.47E-02 & MXD1, THBD \\
\hline G-Protein Coupled Receptor Signaling & $2.45 \mathrm{E}-02$ & 1.13E-02 & HTR2B, DUSP1, PDE4D \\
\hline Hematopoiesis from Multipotent Stem Cells & $3.02 \mathrm{E}-02$ & 8.33E-02 & KITLG \\
\hline Colanic Acid Building Blocks Biosynthesis & $3.02 \mathrm{E}-02$ & 2.94E-02 & UGDH \\
\hline p38 MAPK Signaling & 3.09E-02 & $1.75 \mathrm{E}-02$ & DUSP1, PLA2G4C \\
\hline Leukotriene Biosynthesis & 3.31E-02 & 4E-02 & MGST2 \\
\hline The Visual Cycle & $3.55 \mathrm{E}-02$ & 3.7E-02 & DHRS3 \\
\hline Glutathione Redox Reactions I & $3.80 \mathrm{E}-02$ & 4.55E-02 & MGST2 \\
\hline
\end{tabular}


CDP-diacylglycerol Biosynthesis I

Extrinsic Prothrombin Activation Pathway

Phosphatidylglycerol Biosynthesis II (Non-plastidic)

Aldosterone Signaling in Epithelial Cells

$\begin{array}{ccl}3.80 \mathrm{E}-02 & 3.7 \mathrm{E}-02 & \text { AGPAT9 } \\ 4.07 \mathrm{E}-02 & 5 \mathrm{E}-02 & \text { THBD } \\ 4.07 \mathrm{E}-02 & 3.03 \mathrm{E}-02 & \text { AGPAT9 } \\ 4.90 \mathrm{E}-02 & 1.27 \mathrm{E}-02 & \text { DUSP1, DNAJB9 }\end{array}$


Supplemental Table 4.4. All differentially regulated genes between reversed versus control conditions with FDR $<0.10$

\begin{tabular}{|c|c|c|c|c|c|}
\hline Gene Name & Entrez ID & Symbol & $\overline{\log _{2} F C}$ & $\pm 995 \%$ C.I. & 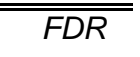 \\
\hline \multicolumn{6}{|l|}{ A. Upregulated Genes } \\
\hline neutrophil cytosolic factor 2 & 4688 & NCF2 & 1.157 & 0.293 & 7.72E-03 \\
\hline $\begin{array}{l}\text { semaphorin 7A, GPI membrane anchor (John Milton Hagen } \\
\text { blood group) }\end{array}$ & 8482 & SEMA7A & 1.133 & 0.221 & $1.96 \mathrm{E}-03$ \\
\hline thrombomodulin & 7056 & THBD & 1.002 & 0.127 & $1.91 \mathrm{E}-04$ \\
\hline 1-acylglycerol-3-phosphate O-acyltransferase 9 & 84803 & AGPAT9 & 0.971 & 0.184 & $1.96 \mathrm{E}-03$ \\
\hline parathyroid hormone-like hormone & 5744 & PTHLH & 0.914 & 0.216 & $6.37 \mathrm{E}-03$ \\
\hline chemokine (C-C motif) ligand 20 & 6364 & CCL20 & 0.870 & 0.353 & 4.73E-02 \\
\hline chemokine-like receptor 1 & 1240 & CMKLR1 & 0.860 & 0.292 & $2.58 \mathrm{E}-02$ \\
\hline ADAM metallopeptidase with thrombospondin type 1 motif, 4 & 9507 & ADAMTS4 & 0.836 & 0.204 & $6.37 \mathrm{E}-03$ \\
\hline ADAM metallopeptidase with thrombospondin type 1 motif, 9 & 56999 & ADAMTS9 & 0.820 & 0.217 & $9.70 \mathrm{E}-03$ \\
\hline serpin peptidase inhibitor, clade B (ovalbumin), member 2 & 5055 & SERPINB2 & 0.810 & 0.356 & $6.22 \mathrm{E}-02$ \\
\hline RasGEF domain family, member 1B & 153020 & RASGEF1B & 0.803 & 0.372 & 7.46E-02 \\
\hline stanniocalcin 1 & 6781 & STC1 & 0.765 & 0.306 & 4.43E-02 \\
\hline nephroblastoma overexpressed & 4856 & NOV & 0.756 & 0.129 & $1.40 \mathrm{E}-03$ \\
\hline kelch repeat and BTB (POZ) domain containing 8 & 84541 & KBTBD8 & 0.737 & 0.114 & 7.39E-04 \\
\hline KIT ligand & 4254 & KITLG & 0.718 & 0.205 & $1.12 \mathrm{E}-02$ \\
\hline fermitin family member 3 & 83706 & FERMT3 & 0.695 & 0.316 & 7.04E-02 \\
\hline diacylglycerol lipase, alpha & 747 & DAGLA & 0.682 & 0.198 & $1.21 \mathrm{E}-02$ \\
\hline $\begin{array}{l}\text { sema domain, immunoglobulin domain (Ig), transmembrane } \\
\text { domain (TM) and short cytoplasmic domain, (semaphorin) 4B }\end{array}$ & 10509 & SEMA4B & 0.679 & 0.125 & $1.96 \mathrm{E}-03$ \\
\hline RCAN family member 3 & 11123 & RCAN3 & 0.675 & 0.338 & $9.89 \mathrm{E}-02$ \\
\hline ras homolog family member $\mathrm{F}$ (in filopodia) & 54509 & RHOF & 0.671 & 0.240 & $3.10 \mathrm{E}-02$ \\
\hline insulin induced gene 1 & 3638 & INSIG1 & 0.660 & 0.164 & 7.23E-03 \\
\hline heparanase & 10855 & HPSE & 0.635 & 0.255 & 4.50E-02 \\
\hline leucine rich repeat (in FLII) interacting protein 1 & 9208 & LRRFIP1 & 0.629 & 0.267 & 5.57E-02 \\
\hline ADAM metallopeptidase with thrombospondin type 1 motif, 1 & 9510 & ADAMTS1 & 0.629 & 0.265 & $5.51 \mathrm{E}-02$ \\
\hline dual specificity phosphatase 5 & 1847 & DUSP5 & 0.616 & 0.139 & $5.04 \mathrm{E}-03$ \\
\hline angiopoietin-like 4 & 51129 & ANGPTL4 & 0.610 & 0.276 & $6.94 \mathrm{E}-02$ \\
\hline paraneoplastic Ma antigen 2 & 10687 & PNMA2 & 0.603 & 0.255 & $5.54 \mathrm{E}-02$ \\
\hline carbonic anhydrase XIII & 377677 & CA13 & 0.601 & 0.213 & $3.10 \mathrm{E}-02$ \\
\hline dedicator of cytokinesis 11 & 139818 & DOCK11 & 0.585 & 0.223 & 3.69E-02 \\
\hline
\end{tabular}




\begin{tabular}{|c|c|c|c|c|c|}
\hline G protein-coupled receptor, family C, group 5, member A & 9052 & GPRC5A & 0.583 & 0.253 & $5.95 \mathrm{E}-02$ \\
\hline plasminogen activator, urokinase receptor & 5329 & PLAUR & 0.583 & 0.147 & 7.72E-03 \\
\hline FH2 domain containing 1 & 85462 & FHDC1 & 0.568 & 0.138 & 6.37E-03 \\
\hline 5-hydroxytryptamine (serotonin) receptor 1D, G protein-coupled & 3352 & HTR1D & 0.563 & 0.271 & 8.71E-02 \\
\hline G protein-coupled receptor 37 (endothelin receptor type B-like) & 2861 & GPR37 & 0.562 & 0.241 & 5.71E-02 \\
\hline very low density lipoprotein receptor & 7436 & VLDLR & 0.547 & 0.261 & $8.48 \mathrm{E}-02$ \\
\hline grainyhead-like 1 (Drosophila) & 29841 & GRHL1 & 0.547 & 0.129 & 6.37E-03 \\
\hline $\begin{array}{l}\text { UDP-N-acetyl-alpha-D-galactosamine:polypeptide N- } \\
\text { acetylgalactosaminyltransferase } 12 \text { (GalNAc-T12) }\end{array}$ & 79695 & GALNT12 & 0.545 & 0.260 & $8.48 \mathrm{E}-02$ \\
\hline neurotensin receptor 1 (high affinity) & 4923 & NTSR1 & 0.544 & 0.130 & 6.37E-03 \\
\hline Rap guanine nucleotide exchange factor (GEF) 5 & 9771 & RAPGEF5 & 0.541 & 0.139 & 7.72E-03 \\
\hline desert hedgehog & 50846 & $\mathrm{DHH}$ & 0.531 & 0.170 & 1.97E-02 \\
\hline $\begin{array}{l}\text { pleckstrin homology domain containing, family G (with RhoGef } \\
\text { domain) member } 1\end{array}$ & 57480 & PLEKHG1 & 0.523 & 0.117 & 4.79E-03 \\
\hline fibroblast growth factor 18 & 8817 & FGF18 & 0.523 & 0.185 & $3.10 \mathrm{E}-02$ \\
\hline $\begin{array}{l}\text { Ras association (RalGDS/AF-6) and pleckstrin homology } \\
\text { domains } 1\end{array}$ & 65059 & RAPH1 & 0.522 & 0.149 & $1.14 \mathrm{E}-02$ \\
\hline fibroblast growth factor 16 & 8823 & FGF16 & 0.520 & 0.200 & 3.77E-02 \\
\hline protocadherin 12 & 51294 & $\mathrm{PCDH} 12$ & 0.508 & 0.163 & 1.97E-02 \\
\hline solute carrier family 35, member $\mathrm{E} 4$ & 339665 & SLC35E4 & 0.506 & 0.144 & $1.12 \mathrm{E}-02$ \\
\hline carbohydrate (chondroitin 6) sulfotransferase 3 & 9469 & CHST3 & 0.503 & 0.213 & $5.57 \mathrm{E}-02$ \\
\hline $\begin{array}{l}\text { natriuretic peptide receptor } \mathrm{A} / \text { guanylate cyclase } \mathrm{A} \\
\text { (atrionatriuretic peptide receptor } \mathrm{A} \text { ) }\end{array}$ & 4881 & NPR1 & 0.503 & 0.188 & 3.37E-02 \\
\hline chromosome 3 open reading frame 55 & 152078 & C3orf55 & 0.498 & 0.141 & $1.12 \mathrm{E}-02$ \\
\hline TSC22 domain family, member 3 & 1831 & TSC22D3 & 0.489 & 0.171 & 2.93E-02 \\
\hline family with sequence similarity 134 , member $B$ & 54463 & FAM134B & 0.487 & 0.181 & 3.23E-02 \\
\hline TBC1 domain family, member 2 & 55357 & TBC1D2 & 0.485 & 0.161 & $2.28 \mathrm{E}-02$ \\
\hline solute carrier organic anion transporter family, member $4 \mathrm{C} 1$ & 353189 & SLCO4C1 & 0.485 & 0.193 & $4.28 \mathrm{E}-02$ \\
\hline small nucleolar RNA, H/ACA box 1 & 677792 & SNORA1 & 0.482 & 0.237 & $9.51 \mathrm{E}-02$ \\
\hline LIM domain 7 & 4008 & LMO7 & 0.477 & 0.171 & $3.10 \mathrm{E}-02$ \\
\hline ADAM metallopeptidase domain 19 & 8728 & ADAM19 & 0.476 & 0.139 & $1.21 \mathrm{E}-02$ \\
\hline nitric oxide synthase 3 (endothelial cell) & 4846 & NOS3 & 0.474 & 0.131 & $1.08 \mathrm{E}-02$ \\
\hline Kruppel-like factor 4 (gut) & 9314 & KLF4 & 0.473 & 0.129 & $1.02 \mathrm{E}-02$ \\
\hline small cell adhesion glycoprotein & 57228 & SMAGP & 0.469 & 0.113 & 6.37E-03 \\
\hline StAR-related lipid transfer (START) domain containing 8 & 9754 & STARD8 & 0.465 & 0.126 & $1.02 \mathrm{E}-02$ \\
\hline interleukin 11 & 3589 & $\mathrm{IL} 11$ & 0.456 & 0.153 & $2.48 \mathrm{E}-02$ \\
\hline
\end{tabular}




\begin{tabular}{|c|c|c|c|c|c|}
\hline cytochrome b5 reductase 2 & 51700 & CYB5R2 & 0.456 & 0.221 & $8.98 \mathrm{E}-02$ \\
\hline CASK interacting protein 2 & 57513 & CASKIN2 & 0.455 & 0.128 & $1.12 \mathrm{E}-02$ \\
\hline $\begin{array}{l}\text { solute carrier family } 7 \text { (amino acid transporter light chain, L } \\
\text { system), member } 5\end{array}$ & 8140 & SLC7A5 & 0.454 & 0.215 & 8.17E-02 \\
\hline GTP cyclohydrolase 1 & 2643 & $\mathrm{GCH} 1$ & 0.454 & 0.224 & $9.51 \mathrm{E}-02$ \\
\hline ephrin-B1 & 1947 & EFNB1 & 0.453 & 0.178 & 4.15E-02 \\
\hline olfactory receptor, family 2 , subfamily A, member 9 pseudogene & 441295 & OR2A9P & 0.444 & 0.207 & 7.57E-02 \\
\hline odz, odd Oz/ten-m homolog 3 (Drosophila) & 55714 & ODZ3 & 0.441 & 0.143 & 2.02E-02 \\
\hline platelet-derived growth factor alpha polypeptide & 5154 & PDGFA & 0.441 & 0.162 & $3.22 \mathrm{E}-02$ \\
\hline myelin protein zero-like 3 & 196264 & MPZL3 & 0.436 & 0.157 & $3.14 \mathrm{E}-02$ \\
\hline OTU domain, ubiquitin aldehyde binding 2 & 78990 & OTUB2 & 0.434 & 0.140 & $2.02 \mathrm{E}-02$ \\
\hline claudin 1 & 9076 & CLDN1 & 0.433 & 0.217 & $9.84 \mathrm{E}-02$ \\
\hline versican & 1462 & VCAN & 0.431 & 0.195 & $6.89 \mathrm{E}-02$ \\
\hline sprouty homolog 4 (Drosophila) & 81848 & SPRY4 & 0.430 & 0.200 & 7.47E-02 \\
\hline tumor necrosis factor receptor superfamily, member 9 & 3604 & TNFRSF9 & 0.427 & 0.133 & 1.74E-02 \\
\hline KIAA1522 & 57648 & KIAA1522 & 0.426 & 0.116 & $1.02 \mathrm{E}-02$ \\
\hline sonic hedgehog & 6469 & $\mathrm{SHH}$ & 0.424 & 0.177 & $5.45 \mathrm{E}-02$ \\
\hline cAMP responsive element binding protein 5 & 9586 & CREB5 & 0.424 & 0.134 & $1.90 \mathrm{E}-02$ \\
\hline meningioma (disrupted in balanced translocation) 1 & 4330 & MN1 & 0.423 & 0.139 & $2.22 \mathrm{E}-02$ \\
\hline potassium channel, subfamily $\mathrm{K}$, member 1 & 3775 & KCNK1 & 0.417 & 0.144 & 2.72E-02 \\
\hline epithelial mitogen homolog (mouse) & 255324 & EPGN & 0.411 & 0.163 & 4.31E-02 \\
\hline BCL2-antagonist/killer 1 & 578 & BAK1 & 0.408 & 0.186 & 7.04E-02 \\
\hline protein tyrosine phosphatase, receptor type, $\mathrm{J}$ & 5795 & PTPRJ & 0.407 & 0.137 & $2.48 \mathrm{E}-02$ \\
\hline LY6/PLAUR domain containing 5 & 284348 & LYPD5 & 0.406 & 0.149 & $3.21 \mathrm{E}-02$ \\
\hline Kruppel-like factor 8 & 11279 & KLF8 & 0.404 & 0.197 & $9.01 \mathrm{E}-02$ \\
\hline $\begin{array}{l}\text { methylenetetrahydrofolate dehydrogenase (NADP+ dependent) } \\
2 \text {, methenyltetrahydrofolate cyclohydrolase }\end{array}$ & 10797 & MTHFD2 & 0.404 & 0.153 & $3.51 \mathrm{E}-02$ \\
\hline basic, immunoglobulin-like variable motif containing & 54841 & BIVM & 0.403 & 0.174 & $5.88 \mathrm{E}-02$ \\
\hline UDP-GlcNAc:betaGal beta-1,3-N-acetylglucosaminyltransferase & 10678 & B3GNT2 & 0.400 & 0.152 & $3.56 \mathrm{E}-02$ \\
\hline zinc finger protein 467 & 168544 & ZNF467 & 0.397 & 0.156 & 4.13E-02 \\
\hline delta-like 1 (Drosophila) & 28514 & DLL1 & 0.396 & 0.156 & 4.15E-02 \\
\hline cell division cycle 6 homolog (S. cerevisiae) & 990 & CDC6 & 0.393 & 0.143 & 3.14E-02 \\
\hline interleukin 1 receptor, type I & 3554 & IL1R1 & 0.392 & 0.171 & $6.01 \mathrm{E}-02$ \\
\hline tensin 1 & 7145 & TNS1 & 0.391 & 0.152 & 4.05E-02 \\
\hline delta/notch-like EGF repeat containing & 92737 & DNER & 0.388 & 0.187 & 8.76E-02 \\
\hline
\end{tabular}




\begin{tabular}{|c|c|c|c|c|c|}
\hline mixed lineage kinase domain-like & 197259 & MLKL & 0.385 & 0.182 & 8.22E-02 \\
\hline small nucleolar RNA, C/D box 50B & 692088 & SNORD50B & 0.383 & 0.189 & $9.54 \mathrm{E}-02$ \\
\hline gasdermin $\mathrm{C}$ & 56169 & GSDMC & 0.383 & 0.178 & 7.47E-02 \\
\hline chemokine ( $\mathrm{C}-\mathrm{C}$ motif) receptor-like 2 & 9034 & CCRL2 & 0.383 & 0.170 & $6.43 \mathrm{E}-02$ \\
\hline plasminogen activator, urokinase & 5328 & PLAU & 0.381 & 0.160 & 5.51E-02 \\
\hline Kruppel-like factor 11 & 8462 & KLF11 & 0.380 & 0.107 & $1.12 \mathrm{E}-02$ \\
\hline coagulation factor II (thrombin) receptor-like 3 & 9002 & F2RL3 & 0.377 & 0.135 & 3.10E-02 \\
\hline Epstein-Barr virus induced 3 & 10148 & EBI3 & 0.376 & 0.169 & $6.75 \mathrm{E}-02$ \\
\hline microtubule-associated protein $1 \mathrm{~A}$ & 4130 & MAP1A & 0.374 & 0.135 & 3.14E-02 \\
\hline DnaJ (Hsp40) homolog, subfamily B, member 9 & 4189 & DNAJB9 & 0.374 & 0.131 & 2.93E-02 \\
\hline zinc finger protein 589 & 51385 & ZNF589 & 0.373 & 0.144 & 3.86E-02 \\
\hline zinc finger protein 643 & 65243 & ZNF643 & 0.373 & 0.178 & $8.45 \mathrm{E}-02$ \\
\hline ankyrin repeat domain 20 family, member $\mathrm{A} 3$ & 441425 & ANKRD20A3 & 0.371 & 0.159 & $5.66 \mathrm{E}-02$ \\
\hline $\begin{array}{l}\text { low density lipoprotein receptor-related protein } 8 \text {, apolipoprotein } \\
\text { e receptor }\end{array}$ & 7804 & LRP8 & 0.369 & 0.164 & $6.59 \mathrm{E}-02$ \\
\hline chromosome 14 open reading frame 43 & 91748 & C14orf43 & 0.368 & 0.175 & 8.43E-02 \\
\hline pantothenate kinase 2 & 80025 & PANK2 & 0.368 & 0.171 & 7.47E-02 \\
\hline KIAA1199 & 57214 & KIAA1199 & 0.367 & 0.132 & 3.14E-02 \\
\hline proline rich 5 like & 79899 & PRR5L & 0.366 & 0.139 & 3.56E-02 \\
\hline ganglioside induced differentiation associated protein 1 & 54332 & GDAP1 & 0.365 & 0.166 & 7.04E-02 \\
\hline EPH receptor A4 & 2043 & EPHA4 & 0.364 & 0.134 & 3.22E-02 \\
\hline tribbles homolog 1 (Drosophila) & 10221 & TRIB1 & 0.363 & 0.131 & 3.14E-02 \\
\hline fibroblast growth factor 12 & 2257 & FGF12 & 0.360 & 0.152 & $5.51 \mathrm{E}-02$ \\
\hline HEG homolog 1 (zebrafish) & 57493 & HEG1 & 0.359 & 0.125 & $2.90 \mathrm{E}-02$ \\
\hline sphingomyelin synthase 2 & 166929 & SGMS2 & 0.359 & 0.100 & $1.12 \mathrm{E}-02$ \\
\hline spectrin repeat containing, nuclear envelope family member 3 & 161176 & SYNE3 & 0.358 & 0.139 & 3.92E-02 \\
\hline FERM domain containing 3 & 257019 & FRMD3 & 0.358 & 0.126 & 3.03E-02 \\
\hline CXXC finger protein 5 & 51523 & CXXC5 & 0.357 & 0.137 & $3.70 \mathrm{E}-02$ \\
\hline NEDD4 binding protein 2 & 55728 & N4BP2 & 0.356 & 0.161 & $6.89 \mathrm{E}-02$ \\
\hline chromosome $\mathrm{X}$ open reading frame 23 & 256643 & CXorf23 & 0.354 & 0.160 & $6.89 \mathrm{E}-02$ \\
\hline thromboxane $A 2$ receptor & 6915 & TBXA2R & 0.354 & 0.143 & 4.57E-02 \\
\hline Kruppel-like factor 13 & 51621 & KLF13 & 0.354 & 0.159 & 6.87E-02 \\
\hline zinc finger, MIZ-type containing 1 & 57178 & ZMIZ1 & 0.353 & 0.113 & 1.97E-02 \\
\hline chromosome 15 open reading frame 26 & 161502 & C15orf26 & 0.352 & 0.126 & $3.10 \mathrm{E}-02$ \\
\hline laminin, alpha 5 & 3911 & LAMA5 & 0.352 & 0.131 & 3.36E-02 \\
\hline
\end{tabular}




\begin{tabular}{|c|c|c|c|c|c|}
\hline inositol 1,4,5-trisphosphate receptor, type 3 & 3710 & ITPR3 & 0.351 & 0.110 & $1.81 \mathrm{E}-02$ \\
\hline endonuclease domain containing 1 & 23052 & ENDOD1 & 0.349 & 0.151 & 5.89E-02 \\
\hline sushi domain containing 1 & 64420 & SUSD1 & 0.346 & 0.144 & $5.22 \mathrm{E}-02$ \\
\hline heat shock $70 \mathrm{kD}$ protein $12 \mathrm{~B}$ & 116835 & HSPA12B & 0.346 & 0.139 & 4.50E-02 \\
\hline Niemann-Pick disease, type $\mathrm{C} 1$ & 4864 & NPC1 & 0.344 & 0.137 & 4.26E-02 \\
\hline FIC domain containing & 11153 & FICD & 0.344 & 0.150 & 6.01E-02 \\
\hline sprouty-related, EVH1 domain containing 1 & 161742 & SPRED1 & 0.343 & 0.169 & $9.61 \mathrm{E}-02$ \\
\hline fibroblast growth factor receptor 3 & 2261 & FGFR3 & 0.340 & 0.145 & 5.66E-02 \\
\hline lysophosphatidic acid receptor 5 & 57121 & LPAR5 & 0.340 & 0.119 & $3.00 \mathrm{E}-02$ \\
\hline ring finger protein 125 , E3 ubiquitin protein ligase & 54941 & RNF125 & 0.340 & 0.149 & 6.29E-02 \\
\hline smoothelin & 6525 & SMTN & 0.339 & 0.099 & 1.21E-02 \\
\hline 4 solute carrier family 36 (proton/amino acid symporter), member & 120103 & SLC36A4 & 0.339 & 0.170 & 9.97E-02 \\
\hline leucine rich repeat (in FLII) interacting protein 1 & 9208 & LRRFIP1 & 0.339 & 0.134 & 4.19E-02 \\
\hline zinc finger, $\mathrm{AN} 1$-type domain $2 \mathrm{~A}$ & 90637 & ZFAND2A & 0.338 & 0.096 & 1.12E-02 \\
\hline $\begin{array}{l}\text { mucosa associated lymphoid tissue lymphoma translocation } \\
\text { gene } 1\end{array}$ & 10892 & MALT1 & 0.338 & 0.124 & 3.22E-02 \\
\hline lipocalin 6 & 158062 & LCN6 & 0.336 & 0.157 & $7.58 \mathrm{E}-02$ \\
\hline calicin & 881 & CCIN & 0.335 & 0.144 & $5.71 \mathrm{E}-02$ \\
\hline tubulin, beta $2 \mathrm{~A}$ class Ila & 7280 & TUBB2A & 0.335 & 0.157 & 7.67E-02 \\
\hline glucoside xylosyltransferase 1 & 283464 & GXYLT1 & 0.331 & 0.122 & 3.23E-02 \\
\hline long intergenic non-protein coding RNA 341 & 79686 & LINC00341 & 0.329 & 0.112 & 2.59E-02 \\
\hline Kruppel-like factor 2 (lung) & 10365 & KLF2 & 0.328 & 0.112 & $2.58 \mathrm{E}-02$ \\
\hline alkaline ceramidase 3 & 55331 & ACER3 & 0.327 & 0.121 & $3.23 E-02$ \\
\hline apolipoprotein L domain containing 1 & 81575 & APOLD1 & 0.324 & 0.130 & 4.39E-02 \\
\hline non imprinted in Prader-Willi/Angelman syndrome 1 & 123606 & NIPA1 & 0.324 & 0.089 & $1.02 \mathrm{E}-02$ \\
\hline $\begin{array}{l}\text { TATA box binding protein (TBP)-associated factor, RNA } \\
\text { polymerase I, A, } 48 \mathrm{kDa}\end{array}$ & 9015 & TAF1A & 0.324 & 0.128 & 4.21E-02 \\
\hline $\begin{array}{l}\text { UDP-N-acetyl-alpha-D-galactosamine:polypeptide N- } \\
\text { acetylgalactosaminyltransferase } 4 \text { (GalNAc-T4) }\end{array}$ & 8693 & GALNT4 & 0.323 & 0.107 & $2.28 \mathrm{E}-02$ \\
\hline zinc finger protein 185 (LIM domain) & 7739 & ZNF185 & 0.323 & 0.148 & 7.17E-02 \\
\hline G protein-coupled receptor 132 & 29933 & GPR132 & 0.322 & 0.121 & 3.38E-02 \\
\hline chromosome 5 open reading frame 48 & 389320 & C5orf48 & 0.322 & 0.115 & $3.10 \mathrm{E}-02$ \\
\hline chromosome 8 open reading frame 48 & 157773 & C8orf48 & 0.319 & 0.128 & 4.43E-02 \\
\hline Fas (TNF receptor superfamily, member 6) & 355 & FAS & 0.319 & 0.118 & 3.23E-02 \\
\hline ubiquitin carboxyl-terminal esterase L3 (ubiquitin thiolesterase) & 7347 & UCHL3 & 0.318 & 0.115 & 3.14E-02 \\
\hline
\end{tabular}




\begin{tabular}{|c|c|c|c|c|c|}
\hline G protein-coupled receptor 4 & 2828 & GPR4 & 0.316 & 0.137 & $5.90 \mathrm{E}-02$ \\
\hline solute carrier family 41 , member 2 & 84102 & SLC41A2 & 0.315 & 0.115 & 3.14E-02 \\
\hline EF-hand calcium binding domain 4B & 84766 & EFCAB4B & 0.315 & 0.143 & $6.95 \mathrm{E}-02$ \\
\hline cryptochrome 1 (photolyase-like) & 1407 & CRY1 & 0.314 & 0.128 & $4.86 \mathrm{E}-02$ \\
\hline claudin 12 & 9069 & CLDN12 & 0.312 & 0.144 & 7.46E-02 \\
\hline ubiquitin specific peptidase 12 & 219333 & USP12 & 0.312 & 0.149 & 8.49E-02 \\
\hline Rho family GTPase 3 & 390 & RND3 & 0.310 & 0.130 & 5.47E-02 \\
\hline SRY (sex determining region Y)-box 13 & 9580 & SOX13 & 0.310 & 0.114 & $3.22 \mathrm{E}-02$ \\
\hline $\begin{array}{l}\text { pleckstrin homology domain containing, family H (with MyTH4 } \\
\text { domain) member } 2\end{array}$ & 130271 & PLEKHH2 & 0.308 & 0.110 & $3.10 \mathrm{E}-02$ \\
\hline pleckstrin homology domain containing, family A member 6 & 22874 & PLEKHA6 & 0.308 & 0.118 & $3.68 \mathrm{E}-02$ \\
\hline heat shock protein 70kDa family, member 13 & 6782 & HSPA13 & 0.308 & 0.139 & $6.89 \mathrm{E}-02$ \\
\hline signal peptide, CUB domain, EGF-like 1 & 80274 & SCUBE1 & 0.307 & 0.143 & 7.47E-02 \\
\hline $\mathrm{SH} 2$ domain containing $3 \mathrm{C}$ & 10044 & SH2D3C & 0.305 & 0.121 & 4.21E-02 \\
\hline uncharacterized LOC388022 & 388022 & LOC388022 & 0.305 & 0.097 & 1.97E-02 \\
\hline glutamine-fructose-6-phosphate transaminase 2 & 9945 & GFPT2 & 0.303 & 0.146 & $8.76 \mathrm{E}-02$ \\
\hline A kinase (PRKA) anchor protein 12 & 9590 & AKAP12 & 0.301 & 0.138 & 7.06E-02 \\
\hline $\begin{array}{l}\text { pleckstrin homology domain containing, family M (with RUN } \\
\text { domain) member } 1\end{array}$ & 9842 & PLEKHM1 & 0.300 & 0.139 & $7.46 \mathrm{E}-02$ \\
\hline fibronectin type III and SPRY domain containing 1-like & 83856 & FSD1L & 0.299 & 0.114 & 3.69E-02 \\
\hline small nucleolar RNA, H/ACA box 45 & 677826 & SNORA45 & 0.297 & 0.137 & $7.32 \mathrm{E}-02$ \\
\hline G protein-coupled receptor 83 & 10888 & GPR83 & 0.294 & 0.125 & 5.57E-02 \\
\hline aquaporin 3 (Gill blood group) & 360 & AQP3 & 0.293 & 0.120 & $4.90 \mathrm{E}-02$ \\
\hline ACAH3104 & $1 \mathrm{E}+08$ & LOC100128816 & 0.292 & 0.139 & $8.38 \mathrm{E}-02$ \\
\hline retinoblastoma binding protein 8 & 5932 & RBBP8 & 0.291 & 0.122 & $5.48 \mathrm{E}-02$ \\
\hline discoidin domain receptor tyrosine kinase 2 & 4921 & DDR2 & 0.291 & 0.126 & $5.89 \mathrm{E}-02$ \\
\hline small nucleolar RNA, C/D box 14E & 85391 & SNORD14E & 0.289 & 0.139 & $8.71 \mathrm{E}-02$ \\
\hline OAF homolog (Drosophila) & 220323 & OAF & 0.288 & 0.136 & $8.22 \mathrm{E}-02$ \\
\hline $\begin{array}{l}\text { v-src sarcoma (Schmidt-Ruppin A-2) viral oncogene homolog } \\
\text { (avian) }\end{array}$ & 6714 & SRC & 0.288 & 0.116 & $4.50 \mathrm{E}-02$ \\
\hline intermediate filament family orphan 2 & 126917 & IFFO2 & 0.287 & 0.137 & 8.38E-02 \\
\hline family with sequence similarity 214, member $B$ & 80256 & FAM214B & 0.286 & 0.139 & $9.01 \mathrm{E}-02$ \\
\hline PDZ and LIM domain 4 & 8572 & PDLIM4 & 0.285 & 0.120 & $5.48 \mathrm{E}-02$ \\
\hline homer homolog 1 (Drosophila) & 9456 & HOMER1 & 0.285 & 0.102 & 3.14E-02 \\
\hline PDZ and LIM domain 3 & 27295 & PDLIM3 & 0.284 & 0.111 & 4.13E-02 \\
\hline ABI family, member 3 & 51225 & $\mathrm{ABI} 3$ & 0.284 & 0.123 & 5.89E-02 \\
\hline
\end{tabular}




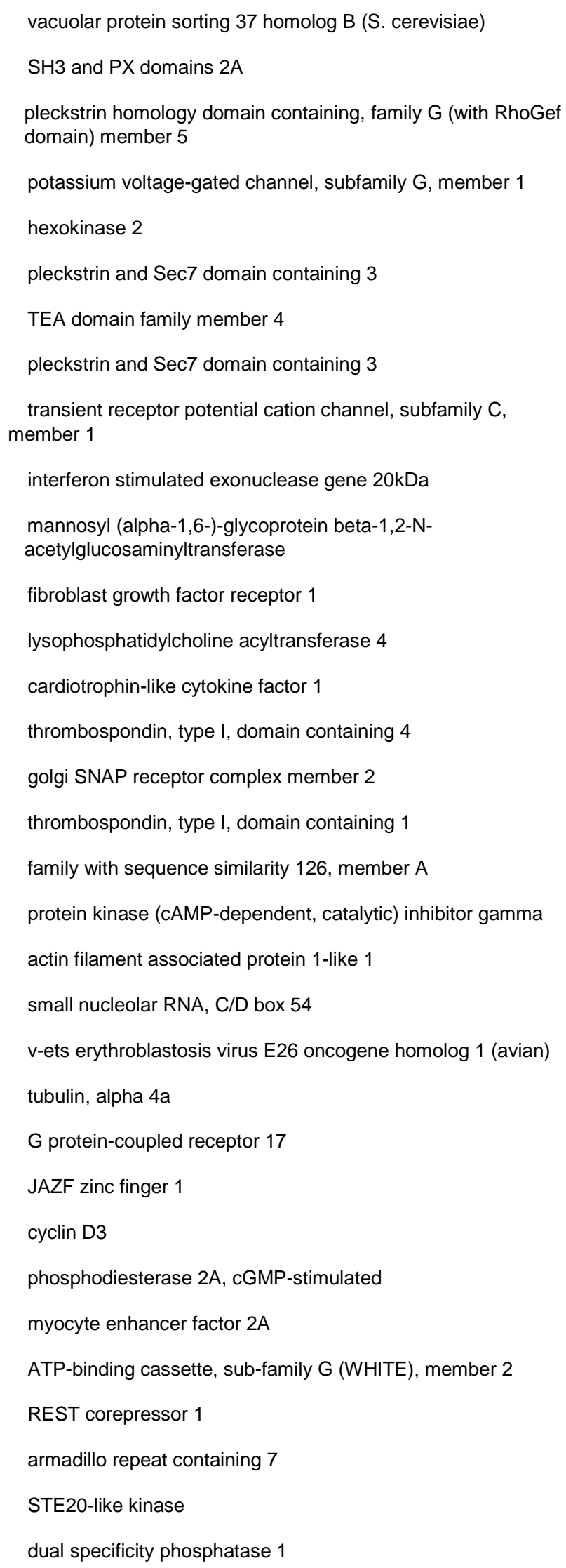

\begin{tabular}{|c|c|c|c|c|}
\hline 79720 & VPS37B & 0.283 & 0.130 & 7.28E-02 \\
\hline 9644 & SH3PXD2A & 0.283 & 0.141 & $9.73 \mathrm{E}-02$ \\
\hline 57449 & PLEKHG5 & 0.283 & 0.123 & $5.95 \mathrm{E}-02$ \\
\hline 3755 & KCNG1 & 0.283 & 0.129 & 7.05E-02 \\
\hline 3099 & HK2 & 0.282 & 0.122 & 5.82E-02 \\
\hline 23362 & PSD3 & 0.281 & 0.106 & $3.45 \mathrm{E}-02$ \\
\hline 7004 & TEAD4 & 0.280 & 0.134 & 8.53E-02 \\
\hline 23362 & PSD3 & 0.279 & 0.124 & $6.59 \mathrm{E}-02$ \\
\hline 7220 & TRPC1 & 0.278 & 0.095 & $2.58 \mathrm{E}-02$ \\
\hline 3669 & ISG20 & 0.276 & 0.102 & $3.22 \mathrm{E}-02$ \\
\hline 4247 & MGAT2 & 0.276 & 0.136 & $9.51 \mathrm{E}-02$ \\
\hline 2260 & FGFR1 & 0.275 & 0.114 & 5.22E-02 \\
\hline 254531 & LPCAT4 & 0.274 & 0.135 & $9.44 \mathrm{E}-02$ \\
\hline 23529 & CLCF1 & 0.271 & 0.125 & 7.25E-02 \\
\hline 79875 & THSD4 & 0.271 & 0.120 & $6.43 \mathrm{E}-02$ \\
\hline 9570 & GOSR2 & 0.268 & 0.098 & 3.17E-02 \\
\hline 55901 & THSD1 & 0.267 & 0.112 & $5.51 \mathrm{E}-02$ \\
\hline 84668 & FAM126A & 0.266 & 0.114 & 5.78E-02 \\
\hline 11142 & PKIG & 0.266 & 0.116 & $6.01 \mathrm{E}-02$ \\
\hline 134265 & AFAP1L1 & 0.265 & 0.117 & $6.43 \mathrm{E}-02$ \\
\hline 26795 & SNORD54 & 0.263 & 0.119 & $6.89 \mathrm{E}-02$ \\
\hline 2113 & ETS1 & 0.262 & 0.132 & 9.97E-02 \\
\hline 7277 & TUBA4A & 0.262 & 0.119 & 6.97E-02 \\
\hline 2840 & GPR17 & 0.260 & 0.110 & 5.57E-02 \\
\hline 221895 & JAZF1 & 0.258 & 0.128 & $9.73 \mathrm{E}-02$ \\
\hline 896 & CCND3 & 0.257 & 0.110 & 5.78E-02 \\
\hline 5138 & PDE2A & 0.255 & 0.110 & $5.88 \mathrm{E}-02$ \\
\hline 4205 & MEF2A & 0.253 & 0.113 & $6.78 \mathrm{E}-02$ \\
\hline 9429 & ABCG2 & 0.252 & 0.110 & 5.94E-02 \\
\hline 23186 & RCOR1 & 0.252 & 0.106 & 5.51E-02 \\
\hline 79637 & ARMC7 & 0.250 & 0.125 & $9.94 \mathrm{E}-02$ \\
\hline 9748 & SLK & 0.250 & 0.093 & $3.23 \mathrm{E}-02$ \\
\hline 1843 & DUSP1 & 0.250 & 0.103 & 5.19E-02 \\
\hline
\end{tabular}




\begin{tabular}{|c|c|c|c|c|c|}
\hline chromodomain helicase DNA binding protein 9 & 80205 & CHD9 & 0.250 & 0.124 & $9.62 \mathrm{E}-02$ \\
\hline 5 UDP-GIcNAc:betaGal beta-1,3-N-acetylglucosaminyltransferase & 84002 & B3GNT5 & 0.249 & 0.114 & 7.06E-02 \\
\hline nuclear receptor subfamily 4 , group $A$, member 1 & 3164 & NR4A1 & 0.247 & 0.101 & 4.86E-02 \\
\hline prenyl (decaprenyl) diphosphate synthase, subunit 1 & 23590 & PDSS1 & 0.246 & 0.118 & 8.53E-02 \\
\hline ATPase, $\mathrm{Ca}++$ transporting, plasma membrane 4 & 493 & ATP2B4 & 0.246 & 0.122 & 9.63E-02 \\
\hline family with sequence similarity 40 , member $B$ & 57464 & FAM40B & 0.243 & 0.113 & 7.47E-02 \\
\hline lysophosphatidylcholine acyltransferase 1 & 79888 & LPCAT1 & 0.242 & 0.104 & $5.80 \mathrm{E}-02$ \\
\hline GrpE-like 1, mitochondrial (E. coli) & 80273 & GRPEL1 & 0.242 & 0.101 & $5.48 \mathrm{E}-02$ \\
\hline ST3 beta-galactoside alpha-2,3-sialyltransferase 1 & 6482 & ST3GAL1 & 0.241 & 0.105 & $5.98 \mathrm{E}-02$ \\
\hline epithelial membrane protein 3 & 2014 & EMP3 & 0.241 & 0.116 & 8.78E-02 \\
\hline dual specificity phosphatase 7 & 1849 & DUSP7 & 0.241 & 0.096 & 4.37E-02 \\
\hline 3 human immunodeficiency virus type I enhancer binding protein & 59269 & HIVEP3 & 0.237 & 0.101 & 5.57E-02 \\
\hline E74-like factor 4 (ets domain transcription factor) & 2000 & ELF4 & 0.235 & 0.111 & 8.23E-02 \\
\hline aryl hydrocarbon receptor nuclear translocator-like & 406 & ARNTL & 0.234 & 0.100 & $5.75 \mathrm{E}-02$ \\
\hline $\begin{array}{l}\text { mannosyl (alpha-1,3-)-glycoprotein beta-1,4-N- } \\
\text { acetylglucosaminyltransferase, isozyme } \mathrm{A}\end{array}$ & 11320 & MGAT4A & 0.231 & 0.104 & 6.89E-02 \\
\hline Ras interacting protein 1 & 54922 & RASIP1 & 0.231 & 0.115 & 9.77E-02 \\
\hline fem-1 homolog b (C. elegans) & 10116 & FEM1B & 0.228 & 0.109 & 8.31E-02 \\
\hline $\mathrm{N}$-myc downstream regulated 1 & 10397 & NDRG1 & 0.228 & 0.111 & 8.96E-02 \\
\hline kinesin family member $\mathrm{C} 1$ & 3833 & KIFC1 & 0.226 & 0.102 & $6.88 \mathrm{E}-02$ \\
\hline ATPase type 13 A3 & 79572 & ATP13A3 & 0.226 & 0.095 & $5.51 \mathrm{E}-02$ \\
\hline solute carrier family 33 (acetyl-CoA transporter), member 1 & 9197 & SLC33A1 & 0.226 & 0.112 & $9.73 \mathrm{E}-02$ \\
\hline MAX dimerization protein 1 & 4084 & MXD1 & 0.225 & 0.108 & 8.49E-02 \\
\hline Ras-like without CAAX 1 & 6016 & RIT1 & 0.225 & 0.095 & $5.51 \mathrm{E}-02$ \\
\hline family with sequence similarity 65 , member $A$ & 79567 & FAM65A & 0.224 & 0.107 & 8.38E-02 \\
\hline $\begin{array}{l}\text { pleckstrin homology domain containing, family M (with RUN } \\
\text { domain) member } 1 \text { pseudogene }\end{array}$ & 440456 & PLEKHM1P & 0.223 & 0.096 & $5.82 \mathrm{E}-02$ \\
\hline suppressor of cytokine signaling 1 & 8651 & SOCS1 & 0.223 & 0.099 & $6.53 \mathrm{E}-02$ \\
\hline ring finger protein 149 & 284996 & RNF149 & 0.223 & 0.092 & $5.20 \mathrm{E}-02$ \\
\hline collagen, type XIII, alpha 1 & 1305 & COL13A1 & 0.220 & 0.110 & 9.77E-02 \\
\hline G protein-coupled receptor kinase 5 & 2869 & GRK5 & 0.220 & 0.100 & 7.04E-02 \\
\hline signal-induced proliferation-associated 1 like 3 & 23094 & SIPA1L3 & 0.220 & 0.108 & $9.31 \mathrm{E}-02$ \\
\hline fibrillin 1 & 2200 & FBN1 & 0.219 & 0.100 & 7.06E-02 \\
\hline RAS p21 protein activator 4 & 10156 & RASA4 & 0.218 & 0.100 & 7.10E-02 \\
\hline
\end{tabular}




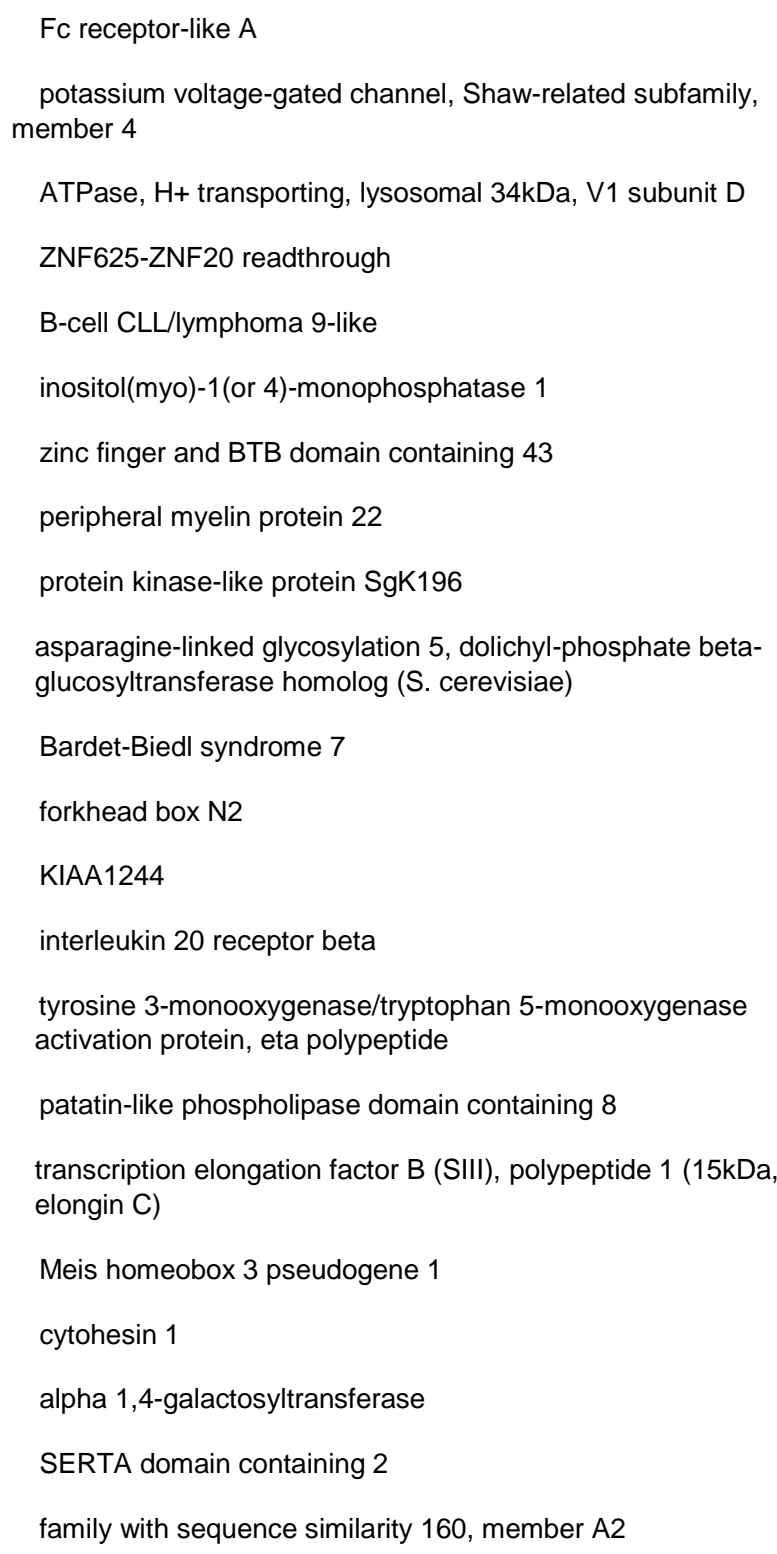

\section{B. Downregulated Genes}

ubiquitin $\mathrm{D}$

ADP-ribosyltransferase 4 (Dombrock blood group)

microRNA 146a

selectin $\mathrm{E}$

endothelin 1

retinoic acid receptor, beta

guanylate binding protein 4

chromosome 15 open reading frame 54

ets variant 1

\begin{tabular}{|c|c|c|c|c|}
\hline 84824 & FCRLA & 0.217 & 0.108 & $9.61 \mathrm{E}-02$ \\
\hline 3749 & KCNC4 & 0.215 & 0.092 & 5.66E-02 \\
\hline 51382 & ATP6V1D & 0.213 & 0.103 & 8.78E-02 \\
\hline $1.01 \mathrm{E}+08$ & ZNF625-ZNF20 & 0.213 & 0.107 & $9.94 \mathrm{E}-02$ \\
\hline 283149 & BCL9L & 0.212 & 0.098 & 7.46E-02 \\
\hline 3612 & IMPA1 & 0.211 & 0.104 & $9.45 \mathrm{E}-02$ \\
\hline 23099 & ZBTB43 & 0.210 & 0.102 & 8.90E-02 \\
\hline 5376 & PMP22 & 0.209 & 0.099 & 8.38E-02 \\
\hline 84197 & SGK196 & 0.208 & 0.104 & $9.89 \mathrm{E}-02$ \\
\hline 29880 & ALG5 & 0.204 & 0.096 & 7.84E-02 \\
\hline 55212 & BBS7 & 0.203 & 0.101 & $9.61 \mathrm{E}-02$ \\
\hline 3344 & FOXN2 & 0.202 & 0.094 & 7.47E-02 \\
\hline 57221 & KIAA1244 & 0.202 & 0.098 & $9.01 \mathrm{E}-02$ \\
\hline 53833 & IL20RB & 0.201 & 0.089 & $6.43 \mathrm{E}-02$ \\
\hline 7533 & YWHAH & 0.201 & 0.092 & 7.06E-02 \\
\hline 50640 & PNPLA8 & 0.196 & 0.096 & $9.30 \mathrm{E}-02$ \\
\hline 6921 & TCEB1 & 0.196 & 0.097 & $9.75 \mathrm{E}-02$ \\
\hline 4213 & MEIS3P1 & 0.193 & 0.093 & 8.82E-02 \\
\hline 9267 & CYTH1 & 0.190 & 0.088 & $7.40 \mathrm{E}-02$ \\
\hline 53947 & A4GALT & 0.187 & 0.092 & $9.44 \mathrm{E}-02$ \\
\hline 9792 & SERTAD2 & 0.185 & 0.090 & $9.21 \mathrm{E}-02$ \\
\hline 84067 & FAM160A2 & 0.184 & 0.092 & 9.77E-02 \\
\hline
\end{tabular}

$\begin{array}{ccccc}10537 & \text { UBD } & -0.967 & 0.478 & 9.61 \mathrm{E}-02 \\ 420 & \text { ART4 } & -0.951 & 0.185 & 1.96 \mathrm{E}-03 \\ 406938 & \text { MIR146A } & -0.945 & 0.252 & 1.00 \mathrm{E}-02 \\ 6401 & \text { SELE } & -0.861 & 0.319 & 3.23 \mathrm{E}-02 \\ 1906 & \text { EDN1 } & -0.816 & 0.231 & 1.12 \mathrm{E}-02 \\ 5915 & \text { RARB } & -0.791 & 0.298 & 3.47 \mathrm{E}-02 \\ 115361 & \text { GBP4 } & -0.783 & 0.239 & 1.52 \mathrm{E}-02 \\ 400360 & \text { C15orf54 } & -0.764 & 0.294 & 3.77 \mathrm{E}-02 \\ 2115 & \text { ETV1 } & -0.760 & 0.205 & 1.02 \mathrm{E}-02\end{array}$




\begin{tabular}{|c|c|c|c|c|c|}
\hline 5-hydroxytryptamine (serotonin) receptor $2 \mathrm{~B}, \mathrm{G}$ protein-coupled & 3357 & HTR2B & -0.724 & 0.292 & 4.56E-02 \\
\hline multiple C2 domains, transmembrane 1 & 79772 & MCTP1 & -0.696 & 0.201 & 1.17E-02 \\
\hline guanylate binding protein 2 , interferon-inducible & 2634 & GBP2 & -0.691 & 0.195 & $1.12 \mathrm{E}-02$ \\
\hline raftlin family member 2 & 130132 & RFTN2 & -0.689 & 0.177 & $7.72 \mathrm{E}-03$ \\
\hline $\begin{array}{l}\text { phosphatidylinositol-3,4,5-trisphosphate-dependent Rac } \\
\text { exchange factor } 2\end{array}$ & 80243 & PREX2 & -0.661 & 0.200 & $1.52 \mathrm{E}-02$ \\
\hline sestrin 3 & 143686 & SESN3 & -0.652 & 0.155 & 6.37E-03 \\
\hline chemokine (C-X-C motif) receptor 4 & 7852 & CXCR4 & -0.614 & 0.245 & 4.36E-02 \\
\hline Rho GTPase activating protein 28 & 79822 & ARHGAP28 & -0.613 & 0.200 & 2.16E-02 \\
\hline tumor necrosis factor (ligand) superfamily, member 10 & 8743 & TNFSF10 & -0.613 & 0.219 & $3.10 \mathrm{E}-02$ \\
\hline Rho GTPase activating protein 18 & 93663 & ARHGAP18 & -0.612 & 0.165 & $1.02 \mathrm{E}-02$ \\
\hline epidermal growth factor receptor pathway substrate 8 & 2059 & EPS8 & -0.595 & 0.152 & 7.72E-03 \\
\hline NADPH oxidase 4 & 50507 & NOX4 & -0.581 & 0.191 & $2.22 \mathrm{E}-02$ \\
\hline leucine-rich repeats and immunoglobulin-like domains 3 & 121227 & LRIG3 & -0.570 & 0.223 & 4.13E-02 \\
\hline protein phosphatase 1 , regulatory subunit $16 \mathrm{~B}$ & 26051 & PPP1R16B & -0.561 & 0.141 & $7.68 \mathrm{E}-03$ \\
\hline GTPase, IMAP family member 4 & 55303 & GIMAP4 & -0.558 & 0.188 & $2.48 \mathrm{E}-02$ \\
\hline dachshund homolog 1 (Drosophila) & 1602 & $\mathrm{DACH} 1$ & -0.555 & 0.116 & $2.95 \mathrm{E}-03$ \\
\hline Rho family GTPase 1 & 27289 & RND1 & -0.552 & 0.175 & $1.90 \mathrm{E}-02$ \\
\hline histone cluster 1, H2ai & 8329 & HIST1H2AI & -0.551 & 0.116 & $2.95 \mathrm{E}-03$ \\
\hline microtubule associated tumor suppressor 1 & 57509 & MTUS1 & -0.533 & 0.156 & $1.21 \mathrm{E}-02$ \\
\hline microRNA 100 & 406892 & MIR100 & -0.532 & 0.197 & 3.23E-02 \\
\hline NUAK family, SNF1-like kinase, 1 & 9891 & NUAK1 & -0.526 & 0.198 & 3.47E-02 \\
\hline BCL2-like 11 (apoptosis facilitator) & 10018 & BCL2L11 & -0.518 & 0.148 & $1.12 \mathrm{E}-02$ \\
\hline solute carrier family 40 (iron-regulated transporter), member 1 & 30061 & SLC40A1 & -0.517 & 0.155 & $1.40 \mathrm{E}-02$ \\
\hline TRAF-interacting protein with forkhead-associated domain & 92610 & TIFA & -0.515 & 0.187 & $3.14 \mathrm{E}-02$ \\
\hline chromosome 11 open reading frame 82 & 220042 & C11orf82 & -0.512 & 0.219 & $5.66 \mathrm{E}-02$ \\
\hline growth differentiation factor 6 & 392255 & GDF6 & -0.482 & 0.213 & $6.34 \mathrm{E}-02$ \\
\hline family with sequence similarity 117 , member $B$ & 150864 & FAM117B & -0.479 & 0.100 & $2.95 \mathrm{E}-03$ \\
\hline phosphodiesterase 7B & 27115 & PDE7B & -0.476 & 0.169 & $3.10 \mathrm{E}-02$ \\
\hline Cdk5 and Abl enzyme substrate 1 & 91768 & CABLES1 & -0.474 & 0.144 & $1.52 \mathrm{E}-02$ \\
\hline microRNA 199a-2 & 406977 & MIR199A2 & -0.473 & 0.140 & $1.30 \mathrm{E}-02$ \\
\hline SH3-domain GRB2-like (endophilin) interacting protein 1 & 84251 & SGIP1 & -0.471 & 0.168 & $3.10 \mathrm{E}-02$ \\
\hline microRNA 34a & 407040 & MIR34A & -0.469 & 0.170 & 3.14E-02 \\
\hline phosphoinositide-3-kinase interacting protein 1 & 113791 & PIK3IP1 & -0.469 & 0.227 & 8.87E-02 \\
\hline KAT8 regulatory NSL complex subunit 1-like & 151050 & KANSL1L & -0.462 & 0.213 & 7.33E-02 \\
\hline
\end{tabular}




\begin{tabular}{|c|c|c|c|c|c|}
\hline programmed cell death 4 (neoplastic transformation inhibitor) & 27250 & PDCD4 & -0.462 & 0.089 & $1.96 \mathrm{E}-03$ \\
\hline butyrophilin, subfamily 3 , member $\mathrm{A} 1$ & 11119 & BTN3A1 & -0.460 & 0.122 & $9.92 \mathrm{E}-03$ \\
\hline dehydrogenase/reductase (SDR family) member 3 & 9249 & DHRS3 & -0.457 & 0.154 & $2.48 \mathrm{E}-02$ \\
\hline $\mathrm{H} 3$ histone, family $3 \mathrm{~A}$ & 3020 & H3F3A & -0.452 & 0.169 & 3.34E-02 \\
\hline aminoadipate-semialdehyde synthase & 10157 & AASS & -0.451 & 0.177 & 4.15E-02 \\
\hline R-spondin 3 & 84870 & RSPO3 & -0.447 & 0.137 & 1.53E-02 \\
\hline zinc finger protein 704 & 619279 & ZNF704 & -0.440 & 0.195 & $6.43 \mathrm{E}-02$ \\
\hline microRNA $216 a$ & 406998 & MIR216A & -0.438 & 0.184 & $5.48 \mathrm{E}-02$ \\
\hline histone cluster $1, \mathrm{H} 2 \mathrm{bg}$ & 8339 & HIST1H2BG & -0.437 & 0.133 & 1.52E-02 \\
\hline brain-derived neurotrophic factor & 627 & BDNF & -0.436 & 0.142 & 2.05E-02 \\
\hline interferon induced with helicase $\mathrm{C}$ domain 1 & 64135 & $\mathrm{IFIH} 1$ & -0.434 & 0.156 & $3.14 \mathrm{E}-02$ \\
\hline myotubularin related protein 4 & 9110 & MTMR4 & -0.434 & 0.121 & $1.12 \mathrm{E}-02$ \\
\hline SATB homeobox 1 & 6304 & SATB1 & -0.432 & 0.177 & 4.90E-02 \\
\hline transmembrane protein 144 & 55314 & TMEM144 & -0.431 & 0.178 & $5.14 \mathrm{E}-02$ \\
\hline apolipoprotein L, 3 & 80833 & APOL3 & -0.429 & 0.145 & $2.54 \mathrm{E}-02$ \\
\hline $\begin{array}{l}\text { potassium intermediate/small conductance calcium-activated } \\
\text { channel, subfamily N, member } 2\end{array}$ & 3781 & KCNN2 & -0.429 & 0.214 & $9.84 \mathrm{E}-02$ \\
\hline nuclear receptor subfamily 1 , group D, member 2 & 9975 & NR1D2 & -0.429 & 0.124 & $1.18 \mathrm{E}-02$ \\
\hline latexin & 56925 & LXN & -0.425 & 0.190 & $6.72 \mathrm{E}-02$ \\
\hline histone cluster $1, \mathrm{H} 3 \mathrm{e}$ & 8353 & HIST1H3E & -0.421 & 0.127 & $1.50 \mathrm{E}-02$ \\
\hline phosphodiesterase 3A, cGMP-inhibited & 5139 & PDE3A & -0.419 & 0.207 & $9.61 \mathrm{E}-02$ \\
\hline nuclear factor I/A & 4774 & NFIA & -0.418 & 0.164 & 4.13E-02 \\
\hline chromosome 5 open reading frame 4 & 10826 & C5orf4 & -0.417 & 0.168 & $4.50 \mathrm{E}-02$ \\
\hline Sp4 transcription factor & 6671 & SP4 & -0.417 & 0.141 & $2.48 \mathrm{E}-02$ \\
\hline nuclear receptor coactivator 7 & 135112 & NCOA7 & -0.414 & 0.145 & $3.00 \mathrm{E}-02$ \\
\hline DnaJ (Hsp40) homolog, subfamily B, member 4 & 11080 & DNAJB4 & -0.413 & 0.123 & $1.31 \mathrm{E}-02$ \\
\hline $\begin{array}{l}\text { apolipoprotein B mRNA editing enzyme, catalytic polypeptide- } \\
\text { like } 3 F\end{array}$ & 200316 & APOBEC3F & -0.413 & 0.145 & $3.00 \mathrm{E}-02$ \\
\hline phospholipid scramblase 4 & 57088 & PLSCR4 & -0.412 & 0.130 & $1.90 \mathrm{E}-02$ \\
\hline butyrophilin, subfamily 3 , member $A 3$ & 10384 & BTN3A3 & -0.410 & 0.150 & $3.21 \mathrm{E}-02$ \\
\hline cell division cycle associated 7-like & 55536 & CDCA7L & -0.408 & 0.172 & $5.51 \mathrm{E}-02$ \\
\hline caspase recruitment domain family, member 16 & 114769 & CARD16 & -0.407 & 0.171 & $5.48 \mathrm{E}-02$ \\
\hline pre-B-cell leukemia homeobox 1 & 5087 & PBX1 & -0.407 & 0.185 & 7.04E-02 \\
\hline KIAA1958 & 158405 & KIAA1958 & -0.406 & 0.190 & 7.74E-02 \\
\hline tropomodulin 1 & 7111 & TMOD1 & -0.406 & 0.184 & $6.89 \mathrm{E}-02$ \\
\hline
\end{tabular}


inositol polyphosphate-4-phosphatase, type II, 105kDa

cysteine-rich, angiogenic inducer, 61

F-box protein 32

butyrophilin, subfamily 3 , member $\mathrm{A} 2$

regulatory factor X, 2 (influences HLA class II expression)

GTPase, IMAP family member 8

yippee-like 1 (Drosophila)

actin, alpha 2, smooth muscle, aorta

protein phosphatase 1 , regulatory subunit $3 \mathrm{~B}$

guanylate binding protein 7

yippee-like 3 (Drosophila)

SLAIN motif family, member 1

GATS protein-like 1

transmembrane protein 140

protein phosphatase 1 , regulatory subunit $9 \mathrm{~A}$

dimethylarginine dimethylaminohydrolase 1

exocyst complex component 6

mastermind-like 2 (Drosophila)

KIAA0922

membrane associated guanylate kinase, WW and PDZ domain containing 1

serum deprivation response

histone cluster $1, \mathrm{H} 2 \mathrm{bf}$

C-type lectin domain family 14 , member A

pyruvate dehydrogenase kinase, isozyme 4

neuronal regeneration related protein homolog (rat)

Cbl proto-oncogene, E3 ubiquitin protein ligase B

myeloid/lymphoid or mixed-lineage leukemia (trithorax homolog, Drosophila); translocated to, 3

zinc finger protein 48

guanosine monophosphate reductase

DLGAP1 antisense RNA 1

FK506 binding protein 5

zinc finger, $\mathrm{CCHC}$ domain containing 18

transcription factor Dp-2 (E2F dimerization partner 2)

\begin{tabular}{|c|c|c|c|c|}
\hline 8821 & INPP4B & -0.406 & 0.184 & 6.97E-02 \\
\hline 3491 & CYR61 & -0.399 & 0.142 & $3.10 \mathrm{E}-02$ \\
\hline 114907 & FBXO32 & -0.399 & 0.142 & $3.10 \mathrm{E}-02$ \\
\hline 11118 & BTN3A2 & -0.399 & 0.101 & 7.72E-03 \\
\hline 5990 & RFX2 & -0.398 & 0.161 & 4.66E-02 \\
\hline 155038 & GIMAP8 & -0.398 & 0.176 & $6.43 E-02$ \\
\hline 29799 & YPEL1 & -0.394 & 0.154 & 4.07E-02 \\
\hline 59 & ACTA2 & -0.393 & 0.111 & 1.12E-02 \\
\hline 79660 & PPP1R3B & -0.391 & 0.133 & $2.58 \mathrm{E}-02$ \\
\hline 388646 & GBP7 & -0.390 & 0.126 & 2.01E-02 \\
\hline 83719 & YPEL3 & -0.387 & 0.130 & $2.48 \mathrm{E}-02$ \\
\hline 122060 & SLAIN1 & -0.385 & 0.186 & 8.78E-02 \\
\hline 389523 & GATSL1 & -0.385 & 0.123 & 1.97E-02 \\
\hline 55281 & TMEM140 & -0.385 & 0.117 & $1.52 \mathrm{E}-02$ \\
\hline 55607 & PPP1R9A & -0.384 & 0.113 & 1.23E-02 \\
\hline 23576 & DDAH1 & -0.384 & 0.137 & $3.10 \mathrm{E}-02$ \\
\hline 54536 & EXOC6 & -0.383 & 0.163 & 5.57E-02 \\
\hline 84441 & MAML2 & -0.383 & 0.133 & 2.84E-02 \\
\hline 23240 & KIAA0922 & -0.380 & 0.143 & $3.45 \mathrm{E}-02$ \\
\hline 9223 & MAGI1 & -0.380 & 0.145 & 3.73E-02 \\
\hline 8436 & SDPR & -0.379 & 0.128 & $2.48 \mathrm{E}-02$ \\
\hline 8343 & HIST1H2BF & -0.372 & 0.158 & 5.57E-02 \\
\hline 161198 & CLEC14A & -0.371 & 0.120 & 2.02E-02 \\
\hline 5166 & PDK4 & -0.370 & 0.164 & $6.43 \mathrm{E}-02$ \\
\hline 9315 & NREP & -0.367 & 0.167 & 7.06E-02 \\
\hline 868 & CBLB & -0.365 & 0.117 & 2.01E-02 \\
\hline 4300 & MLLT3 & -0.363 & 0.141 & 3.98E-02 \\
\hline 197407 & ZNF48 & -0.361 & 0.104 & 1.14E-02 \\
\hline 2766 & GMPR & -0.359 & 0.098 & $1.02 \mathrm{E}-02$ \\
\hline 649446 & DLGAP1-AS1 & -0.358 & 0.149 & 5.39E-02 \\
\hline 2289 & FKBP5 & -0.352 & 0.136 & 3.97E-02 \\
\hline 644353 & ZCCHC18 & -0.351 & 0.152 & 5.94E-02 \\
\hline 7029 & TFDP2 & -0.351 & 0.085 & 6.37E-03 \\
\hline
\end{tabular}




\begin{tabular}{|c|c|c|c|c|c|}
\hline homeobox A6 & 3203 & HOXA6 & -0.344 & 0.151 & $6.10 \mathrm{E}-02$ \\
\hline LYR motif containing 1 & 57149 & LYRM1 & -0.342 & 0.135 & 4.15E-02 \\
\hline SRY (sex determining region Y)-box 4 & 6659 & SOX4 & -0.341 & 0.152 & 6.66E-02 \\
\hline GA binding protein transcription factor, beta subunit 1 & 2553 & GABPB1 & -0.341 & 0.124 & 3.14E-02 \\
\hline TRIM6-TRIM34 readthrough & 445372 & TRIM6-TRIM34 & -0.338 & 0.169 & $9.89 \mathrm{E}-02$ \\
\hline guanylate binding protein 1 , interferon-inducible & 2633 & GBP1 & -0.336 & 0.132 & 4.15E-02 \\
\hline ribonucleoprotein, PTB-binding 2 & 55225 & RAVER2 & -0.335 & 0.162 & 8.96E-02 \\
\hline SMAD family member 1 & 4086 & SMAD1 & -0.333 & 0.142 & $5.66 \mathrm{E}-02$ \\
\hline lysophosphatidic acid receptor 6 & 10161 & LPAR6 & -0.329 & 0.157 & $8.49 \mathrm{E}-02$ \\
\hline kinesin family member $18 \mathrm{~A}$ & 81930 & KIF18A & -0.328 & 0.164 & 9.77E-02 \\
\hline CCR4-NOT transcription complex, subunit 6-like & 246175 & CNOT6L & -0.327 & 0.151 & 7.36E-02 \\
\hline MDS1 and EVI1 complex locus & 2122 & MECOM & -0.326 & 0.134 & 4.91E-02 \\
\hline sterile alpha motif domain containing 13 & 148418 & SAMD13 & -0.325 & 0.137 & $5.51 \mathrm{E}-02$ \\
\hline aldehyde dehydrogenase 6 family, member $A 1$ & 4329 & ALDH6A1 & -0.322 & 0.116 & 3.14E-02 \\
\hline solute carrier family 25 , member 30 & 253512 & SLC25A30 & -0.321 & 0.117 & 3.14E-02 \\
\hline phospholipase A2, group IVC (cytosolic, calcium-independent) & 8605 & PLA2G4C & -0.320 & 0.100 & $1.81 \mathrm{E}-02$ \\
\hline kelch-like 13 (Drosophila) & 90293 & KLHL13 & -0.319 & 0.132 & $5.21 \mathrm{E}-02$ \\
\hline TBC1 domain family, member 22B & 55633 & TBC1D22B & -0.313 & 0.141 & $6.81 \mathrm{E}-02$ \\
\hline family with sequence similarity 84 , member $B$ & 157638 & FAM84B & -0.312 & 0.144 & 7.33E-02 \\
\hline vestigial like 3 (Drosophila) & 389136 & VGLL3 & -0.312 & 0.155 & 9.77E-02 \\
\hline centrosomal protein $19 \mathrm{kDa}$ & 84984 & CEP19 & -0.311 & 0.156 & $9.94 \mathrm{E}-02$ \\
\hline protein kinase, cAMP-dependent, catalytic, beta & 5567 & PRKACB & -0.308 & 0.121 & 4.15E-02 \\
\hline $\begin{array}{l}\text { solute carrier family } 2 \text { (facilitated glucose transporter), member } \\
\end{array}$ & 154091 & SLC2A12 & -0.307 & 0.148 & $8.71 \mathrm{E}-02$ \\
\hline mitochondria-localized glutamic acid-rich protein & 84709 & MGARP & -0.305 & 0.111 & $3.14 \mathrm{E}-02$ \\
\hline golgi phosphoprotein 3-like & 55204 & GOLPH3L & -0.305 & 0.135 & $6.43 E-02$ \\
\hline DIS3 mitotic control homolog (S. cerevisiae)-like & 115752 & DIS3L & -0.305 & 0.111 & 3.17E-02 \\
\hline zinc finger protein 610 & 162963 & ZNF610 & -0.303 & 0.097 & 1.97E-02 \\
\hline carnitine O-octanoyltransferase & 54677 & CROT & -0.303 & 0.142 & 7.86E-02 \\
\hline Rho GTPase activating protein 29 & 9411 & ARHGAP29 & -0.301 & 0.121 & $4.50 \mathrm{E}-02$ \\
\hline Rho guanine nucleotide exchange factor (GEF) 37 & 389337 & ARHGEF37 & -0.301 & 0.124 & $5.20 \mathrm{E}-02$ \\
\hline regulatory factor X, 5 (influences HLA class II expression) & 5993 & RFX5 & -0.300 & 0.109 & 3.14E-02 \\
\hline family with sequence similarity 43 , member A & 131583 & FAM43A & -0.300 & 0.127 & 5.57E-02 \\
\hline family with sequence similarity 149, member $B 1$ & 317662 & FAM149B1 & -0.296 & 0.133 & $6.80 \mathrm{E}-02$ \\
\hline phosphodiesterase 4D, cAMP-specific & 5144 & PDE4D & -0.296 & 0.117 & $4.20 \mathrm{E}-02$ \\
\hline
\end{tabular}




\begin{tabular}{|c|c|c|c|c|c|}
\hline G protein-coupled receptor 125 & 166647 & GPR125 & -0.294 & 0.122 & 5.19E-02 \\
\hline sideroflexin 2 & 118980 & SFXN2 & -0.292 & 0.135 & 7.46E-02 \\
\hline phospholipid scramblase 1 & 5359 & PLSCR1 & -0.291 & 0.095 & 2.22E-02 \\
\hline chromosome 10 open reading frame 114 & 399726 & C10orf114 & -0.290 & 0.106 & 3.17E-02 \\
\hline proline-rich nuclear receptor coactivator 1 & 10957 & PNRC1 & -0.290 & 0.107 & 3.23E-02 \\
\hline ral guanine nucleotide dissociation stimulator-like 1 & 23179 & RGL1 & -0.288 & 0.107 & 3.23E-02 \\
\hline zinc finger and SCAN domain containing 16 & 80345 & ZSCAN16 & -0.288 & 0.129 & $6.64 \mathrm{E}-02$ \\
\hline forkhead box N3 & 1112 & FOXN3 & -0.288 & 0.109 & 3.56E-02 \\
\hline signal transducer and activator of transcription $1,91 \mathrm{kDa}$ & 6772 & STAT1 & -0.287 & 0.116 & 4.50E-02 \\
\hline ER membrane protein complex subunit 2 & 9694 & EMC2 & -0.287 & 0.121 & 5.51E-02 \\
\hline KIAA1107 & 23285 & KIAA1107 & -0.287 & 0.113 & 4.17E-02 \\
\hline long intergenic non-protein coding RNA 478 & 388815 & LINC00478 & -0.287 & 0.119 & $5.22 \mathrm{E}-02$ \\
\hline homeobox A4 & 3201 & HOXA4 & -0.285 & 0.143 & $9.97 \mathrm{E}-02$ \\
\hline G protein-coupled receptor 146 & 115330 & GPR146 & -0.285 & 0.121 & 5.57E-02 \\
\hline SH3 domain containing 19 & 152503 & SH3D19 & -0.285 & 0.119 & $5.45 \mathrm{E}-02$ \\
\hline adducin 3 (gamma) & 120 & ADD3 & -0.285 & 0.124 & $6.01 E-02$ \\
\hline Rho-related BTB domain containing 3 & 22836 & RHOBTB3 & -0.285 & 0.141 & $9.62 \mathrm{E}-02$ \\
\hline ST6 beta-galactosamide alpha-2,6-sialyltranferase 1 & 6480 & ST6GAL1 & -0.285 & 0.109 & $3.68 \mathrm{E}-02$ \\
\hline uracil-DNA glycosylase & 7374 & UNG & -0.284 & 0.126 & $6.43 \mathrm{E}-02$ \\
\hline dynamin binding protein & 23268 & DNMBP & -0.284 & 0.097 & 2.64E-02 \\
\hline hydroxysteroid (17-beta) dehydrogenase 7 & 51478 & HSD17B7 & -0.284 & 0.112 & 4.17E-02 \\
\hline WD repeat domain, phosphoinositide interacting 1 & 55062 & WIPI1 & -0.283 & 0.132 & 7.47E-02 \\
\hline histone cluster $1, \mathrm{H} 3 \mathrm{~h}$ & 8357 & HIST1H3H & -0.283 & 0.105 & $3.23 E-02$ \\
\hline methylcrotonoyl-CoA carboxylase 1 (alpha) & 56922 & MCCC1 & -0.282 & 0.093 & $2.22 \mathrm{E}-02$ \\
\hline $\begin{array}{l}\text { NADH dehydrogenase (ubiquinone) } 1 \text { alpha subcomplex, } 2 \text {, } \\
8 \mathrm{kDa}\end{array}$ & 4695 & NDUFA2 & -0.280 & 0.126 & $6.89 \mathrm{E}-02$ \\
\hline leucine-rich repeats and immunoglobulin-like domains 1 & 26018 & LRIG1 & -0.280 & 0.100 & $3.10 \mathrm{E}-02$ \\
\hline WW domain binding protein 1 -like & 54838 & WBP1L & -0.279 & 0.101 & $3.14 \mathrm{E}-02$ \\
\hline ubiquitin specific peptidase 28 & 57646 & USP28 & -0.279 & 0.109 & 4.15E-02 \\
\hline syntaxin binding protein 4 & 252983 & STXBP4 & -0.278 & 0.132 & $8.29 \mathrm{E}-02$ \\
\hline $\begin{array}{l}\text { translocase of inner mitochondrial membrane } 8 \text { homolog B } \\
\text { (yeast) }\end{array}$ & 26521 & TIMM8B & -0.276 & 0.135 & $9.20 \mathrm{E}-02$ \\
\hline $\begin{array}{l}\text { erythrocyte membrane protein band } 4.1 \text { (elliptocytosis } 1, \mathrm{RH} \text { - } \\
\text { linked) }\end{array}$ & 2035 & EPB41 & -0.276 & 0.135 & 9.39E-02 \\
\hline chromosome 6 open reading frame 141 & 135398 & C6orf141 & -0.275 & 0.132 & 8.61E-02 \\
\hline caspase 2 , apoptosis-related cysteine peptidase & 835 & CASP2 & -0.274 & 0.125 & 7.04E-02 \\
\hline
\end{tabular}




\begin{tabular}{|c|c|c|c|c|c|}
\hline histone cluster $1, \mathrm{H} 4 \mathrm{~d}$ & 8360 & HIST1H4D & -0.274 & 0.121 & 6.43E-02 \\
\hline guanylate cyclase 1 , soluble, alpha 3 & 2982 & GUCY1A3 & -0.271 & 0.118 & 5.97E-02 \\
\hline histone cluster $1, \mathrm{H} 3 \mathrm{~d}$ & 8351 & HIST1H3D & -0.270 & 0.110 & 4.86E-02 \\
\hline zinc finger and SCAN domain containing 2 & 54993 & ZSCAN2 & -0.269 & 0.095 & $3.10 \mathrm{E}-02$ \\
\hline Bcl2 modifying factor & 90427 & BMF & -0.269 & 0.117 & $6.01 \mathrm{E}-02$ \\
\hline cyclin M3 & 26505 & CNNM3 & -0.266 & 0.104 & 4.13E-02 \\
\hline kinesin family member $13 \mathrm{~A}$ & 63971 & KIF13A & -0.265 & 0.102 & $3.76 \mathrm{E}-02$ \\
\hline Rho guanine nucleotide exchange factor (GEF) 28 & 64283 & ARHGEF28 & -0.263 & 0.102 & $3.88 \mathrm{E}-02$ \\
\hline OMA1 zinc metallopeptidase homolog (S. cerevisiae) & 115209 & OMA1 & -0.262 & 0.120 & 7.06E-02 \\
\hline t-complex 11 (mouse)-like 2 & 255394 & TCP11L2 & -0.261 & 0.102 & 4.13E-02 \\
\hline tumor protein p53 inducible nuclear protein 1 & 94241 & TP53INP1 & -0.260 & 0.118 & $7.00 \mathrm{E}-02$ \\
\hline KIAA1328 & 57536 & KIAA1328 & -0.258 & 0.118 & 7.06E-02 \\
\hline family with sequence similarity 63 , member $A$ & 55793 & FAM63A & -0.256 & 0.119 & 7.47E-02 \\
\hline TBC1 domain containing kinase & 93627 & TBCK & -0.256 & 0.093 & $3.14 \mathrm{E}-02$ \\
\hline CREB3 regulatory factor & 153222 & CREBRF & -0.255 & 0.110 & $5.80 \mathrm{E}-02$ \\
\hline RAB30, member RAS oncogene family & 27314 & RAB30 & -0.254 & 0.121 & 8.29E-02 \\
\hline integrator complex subunit 8 & 55656 & INTS8 & -0.254 & 0.104 & $5.00 \mathrm{E}-02$ \\
\hline family with sequence similarity 198, member $B$ & 51313 & FAM198B & -0.253 & 0.110 & $5.90 \mathrm{E}-02$ \\
\hline chromosome 17 open reading frame 28 & 283987 & C17orf28 & -0.250 & 0.123 & $9.53 \mathrm{E}-02$ \\
\hline microRNA 93 & 407050 & MIR93 & -0.250 & 0.111 & $6.43 \mathrm{E}-02$ \\
\hline transmembrane protein 117 & 84216 & TMEM117 & -0.249 & 0.118 & $8.18 \mathrm{E}-02$ \\
\hline minichromosome maintenance complex component 7 & 4176 & MCM7 & -0.249 & 0.092 & $3.23 E-02$ \\
\hline leucine rich repeat containing 8 family, member B & 23507 & LRRC8B & -0.249 & 0.102 & 4.93E-02 \\
\hline $\begin{array}{l}\text { apolipoprotein B mRNA editing enzyme, catalytic polypeptide- } \\
\text { like 3F }\end{array}$ & 200316 & APOBECЗF & -0.248 & 0.113 & 7.05E-02 \\
\hline DnaJ (Hsp40) homolog, subfamily C, member 6 & 9829 & DNAJC6 & -0.243 & 0.102 & $5.51 \mathrm{E}-02$ \\
\hline TRAF family member-associated NFKB activator & 10010 & TANK & -0.241 & 0.120 & 9.77E-02 \\
\hline phosphodiesterase 4B, cAMP-specific & 5142 & PDE4B & -0.241 & 0.115 & 8.54E-02 \\
\hline ring finger protein 44 & 22838 & RNF44 & -0.240 & 0.120 & 9.77E-02 \\
\hline phosphofructokinase, muscle & 5213 & PFKM & -0.240 & 0.108 & $6.80 \mathrm{E}-02$ \\
\hline opsin 3 & 23596 & OPN3 & -0.240 & 0.090 & $3.46 \mathrm{E}-02$ \\
\hline solute carrier family 35 , member E2B & 728661 & SLC35E2B & -0.240 & 0.120 & 9.97E-02 \\
\hline HMG-box transcription factor 1 & 26959 & HBP1 & -0.239 & 0.104 & 5.97E-02 \\
\hline dystrophin & 1756 & DMD & -0.237 & 0.100 & 5.51E-02 \\
\hline tripartite motif containing 5 & 85363 & TRIM5 & -0.237 & 0.086 & $3.14 \mathrm{E}-02$ \\
\hline
\end{tabular}


CTD (carboxy-terminal domain, RNA polymerase II, polypeptide A) small phosphatase 2

histone cluster 1, H2ag

filamin A interacting protein 1

XRCC6 binding protein 1

solute carrier family 1 (neuronal/epithelial high affinity glutamate transporter, system Xag), member 1

ankyrin repeat domain 50

zinc finger protein 254

family with sequence similarity 111 , member $A$

LIM and senescent cell antigen-like domains 3

kelch-like 23 (Drosophila)

uridine phosphorylase 1

NMDA receptor regulated 2

polymerase (DNA directed), beta

synovial sarcoma, $\mathrm{X}$ breakpoint 2

transcription factor 4

PDZ and LIM domain 5

sestrin 1

SEC14 and spectrin domains 1

stearoyl-CoA desaturase 5

myeloid/lymphoid or mixed-lineage leukemia (trithorax homolog, Drosophila); translocated to, 1

methionine adenosyltransferase II, beta

synapse defective 1, Rho GTPase, homolog 2 (C. elegans)

zinc finger protein 260

chromosome 2 open reading frame 27A

eukaryotic translation initiation factor 2 alpha kinase 4

mitogen-activated protein kinase 3

synuclein, alpha (non A4 component of amyloid precursor)

centrosomal protein $41 \mathrm{kDa}$

mex-3 homolog B (C. elegans)

ubiquitin specific peptidase 49

SEC14-like 1 (S. cerevisiae)

nuclear mitotic apparatus protein 1

uncharacterized LOC441204

\begin{tabular}{|c|c|c|c|c|}
\hline 10106 & CTDSP2 & -0.236 & 0.115 & $9.01 \mathrm{E}-02$ \\
\hline 8969 & HIST1H2AG & -0.236 & 0.116 & 9.32E-02 \\
\hline 27145 & FILIP1 & -0.235 & 0.109 & $7.42 \mathrm{E}-02$ \\
\hline 91419 & XRCC6BP1 & -0.234 & 0.115 & $9.20 \mathrm{E}-02$ \\
\hline 6505 & SLC1A1 & -0.234 & 0.118 & $9.98 \mathrm{E}-02$ \\
\hline 57182 & ANKRD50 & -0.232 & 0.099 & 5.66E-02 \\
\hline 9534 & ZNF254 & -0.231 & 0.111 & 8.49E-02 \\
\hline 63901 & FAM111A & -0.230 & 0.110 & 8.49E-02 \\
\hline 96626 & LIMS3 & -0.230 & 0.108 & 7.99E-02 \\
\hline 151230 & KLHL23 & -0.229 & 0.112 & $9.20 \mathrm{E}-02$ \\
\hline 7378 & UPP1 & -0.229 & 0.099 & 5.81E-02 \\
\hline 79664 & NARG2 & -0.229 & 0.103 & 6.89E-02 \\
\hline 5423 & POLB & -0.228 & 0.097 & 5.57E-02 \\
\hline 6757 & SSX2 & -0.227 & 0.097 & 5.59E-02 \\
\hline 6925 & TCF4 & -0.224 & 0.105 & 7.92E-02 \\
\hline 10611 & PDLIM5 & -0.224 & 0.104 & 7.47E-02 \\
\hline 27244 & SESN1 & -0.223 & 0.093 & 5.45E-02 \\
\hline 91404 & SESTD1 & -0.222 & 0.090 & 4.63E-02 \\
\hline 79966 & SCD5 & -0.221 & 0.102 & 7.26E-02 \\
\hline 4298 & MLLT1 & -0.221 & 0.110 & $9.72 \mathrm{E}-02$ \\
\hline 27430 & MAT2B & -0.221 & 0.106 & 8.50E-02 \\
\hline 84144 & SYDE2 & -0.220 & 0.104 & 7.97E-02 \\
\hline 339324 & ZNF260 & -0.220 & 0.110 & 9.84E-02 \\
\hline 29798 & C2orf27A & -0.219 & 0.108 & $9.71 \mathrm{E}-02$ \\
\hline 440275 & EIF2AK4 & -0.216 & 0.094 & 6.01E-02 \\
\hline 4215 & MAP3КЗ & -0.215 & 0.106 & 9.44E-02 \\
\hline 6622 & SNCA & -0.211 & 0.097 & 7.17E-02 \\
\hline 95681 & CEP41 & -0.209 & 0.096 & 7.06E-02 \\
\hline 84206 & MEX3B & -0.209 & 0.094 & $6.80 \mathrm{E}-02$ \\
\hline 25862 & USP49 & -0.206 & 0.092 & 6.75E-02 \\
\hline 6397 & SEC14L1 & -0.202 & 0.100 & 9.69E-02 \\
\hline 4926 & NUMA1 & -0.201 & 0.098 & 9.01E-02 \\
\hline 441204 & LOC441204 & -0.199 & 0.099 & 9.77 \\
\hline
\end{tabular}


ATP/GTP binding protein-like 5

family with sequence similarity 115 , member $C$

hematopoietic prostaglandin D synthase

Hermansky-Pudlak syndrome 3

cysteine conjugate-beta lyase, cytoplasmic

adaptor-related protein complex 3 , mu 2 subunit

GRAM domain containing $1 \mathrm{~A}$

damage-specific DNA binding protein 2, 48kDa

\begin{tabular}{ccccc}
60509 & AGBL5 & -0.198 & 0.090 & $7.06 \mathrm{E}-02$ \\
285966 & FAM115C & -0.197 & 0.089 & $6.78 \mathrm{E}-02$ \\
27306 & HPGDS & -0.194 & 0.097 & $9.77 \mathrm{E}-02$ \\
84343 & HPS3 & -0.193 & 0.093 & $8.85 \mathrm{E}-02$ \\
883 & CCBL1 & -0.190 & 0.088 & $7.47 \mathrm{E}-02$ \\
10947 & AP3M2 & -0.190 & 0.092 & $8.96 \mathrm{E}-02$ \\
57655 & GRAMD1A & -0.185 & 0.090 & $9.20 \mathrm{E}-02$ \\
1643 & DDB2 & -0.184 & 0.090 & $9.08 \mathrm{E}-02$ \\
\hline
\end{tabular}




\section{Supplemental Table 4.5. All differentially regulated genes between non-reversed versus control conditions with FDR $<0.10$}

Gene Name

\section{A. Upregulated Genes}

1-acylglycerol-3-phosphate O-acyltransferase 9

thrombomodulin

carbonic anhydrase XIII

KIT ligand

kelch repeat and BTB (POZ) domain containing 8

cell division cycle 6 homolog (S. cerevisiae)

nephroblastoma overexpressed

sema domain, immunoglobulin domain (lg), transmembrane domain (TM) and short cytoplasmic domain, (semaphorin) 4B

odz, odd Oz/ten-m homolog 3 (Drosophila)

DnaJ (Hsp40) homolog, subfamily B, member 9

Rap guanine nucleotide exchange factor (GEF) 5

MAX dimerization protein 1

pleckstrin homology domain containing, family $\mathrm{G}$ (with RhoGef domain) member 1

pentraxin 3, long

forkhead box F1

smoothelin

UDP-glucose 6-dehydrogenase

small cell adhesion glycoprotein

KIAA1024

sphingomyelin synthase 2

dual specificity phosphatase 1

\section{B. Downregulated Genes}

5-hydroxytryptamine (serotonin) receptor $2 \mathrm{~B}, \mathrm{G}$ protein-coupled microRNA 519a-2

guanylate binding protein 4

guanylate binding protein 2, interferon-inducible

ADP-ribosyltransferase 4 (Dombrock blood group)

dehydrogenase/reductase (SDR family) member 3

sestrin 3
Entrez ID $\quad$ Symbol $\quad \log _{2} F C \pm 95 \%$ C.I. $\quad$ FDR

\begin{tabular}{|c|c|c|c|c|}
\hline 84803 & AGPAT9 & 0.652 & 0.184 & 6.61E-02 \\
\hline 7056 & THBD & 0.606 & 0.127 & 1.63E-02 \\
\hline 377677 & CA13 & 0.588 & 0.213 & 9.97E-02 \\
\hline 4254 & KITLG & 0.581 & 0.205 & 9.97E-02 \\
\hline 84541 & KBTBD8 & 0.571 & 0.114 & 1.63E-02 \\
\hline 990 & CDC6 & 0.549 & 0.143 & $6.13 \mathrm{E}-02$ \\
\hline 4856 & NOV & 0.478 & 0.129 & $6.13 \mathrm{E}-02$ \\
\hline 10509 & SEMA4B & 0.429 & 0.125 & 6.61E-02 \\
\hline 55714 & ODZ3 & 0.394 & 0.143 & 9.97E-02 \\
\hline 4189 & DNAJB9 & 0.388 & 0.131 & 9.97E-02 \\
\hline 9771 & RAPGEF5 & 0.385 & 0.139 & 9.97E-02 \\
\hline 4084 & MXD1 & 0.380 & 0.108 & 6.61E-02 \\
\hline 57480 & PLEKHG1 & 0.368 & 0.117 & 7.90E-02 \\
\hline 5806 & PTX3 & 0.362 & 0.129 & 9.97E-02 \\
\hline 2294 & FOXF1 & 0.346 & 0.124 & 9.97E-02 \\
\hline 6525 & SMTN & 0.333 & 0.099 & $6.61 \mathrm{E}-02$ \\
\hline 7358 & UGDH & 0.327 & 0.118 & 9.97E-02 \\
\hline 57228 & SMAGP & 0.317 & 0.113 & 9.97E-02 \\
\hline 23251 & KIAA1024 & 0.307 & 0.097 & 7.90E-02 \\
\hline 166929 & SGMS2 & 0.291 & 0.100 & $9.97 \mathrm{E}-02$ \\
\hline 1843 & DUSP1 & 0.290 & 0.103 & 9.97E-02 \\
\hline
\end{tabular}

3357 HTR2B $\quad-0.823 \quad 0.292 \quad 9.97 E-02$

$\begin{array}{lllll}574500 & \text { MIR519A2 } & -0.717 & 0.215 & 6.61 \mathrm{E}-02\end{array}$

$\begin{array}{lllll}115361 & \text { GBP4 } & -0.712 & 0.239 & 9.97 \mathrm{E}-02\end{array}$

$2634 \quad$ GBP2 $\quad-0.651 \quad 0.195 \quad 6.61 \mathrm{E}-02$

$\begin{array}{lllll}420 & \text { ART4 } & -0.570 & 0.185 & 8.69 \mathrm{E}-02\end{array}$

$9249 \quad$ DHRS3 $\quad-0.508 \quad 0.154 \quad 6.70 \mathrm{E}-02$

143686 SESN3 $-0.492 \quad 0.155 \quad 7.90 \mathrm{E}-02$ 


\begin{tabular}{|c|c|c|c|c|c|}
\hline Rho family GTPase 1 & 27289 & RND1 & -0.483 & 0.175 & 9.97E-02 \\
\hline microRNA 199a-2 & 406977 & MIR199A2 & -0.477 & 0.140 & 6.61E-02 \\
\hline butyrophilin, subfamily 3 , member $\mathrm{A} 1$ & 11119 & BTN3A1 & -0.453 & 0.122 & 6.13E-02 \\
\hline DLGAP1 antisense RNA 1 & 649446 & DLGAP1-AS1 & -0.419 & 0.149 & 9.97E-02 \\
\hline apolipoprotein L, 3 & 80833 & APOL3 & -0.411 & 0.145 & 9.97E-02 \\
\hline G protein-coupled receptor 146 & 115330 & GPR146 & -0.375 & 0.121 & 8.69E-02 \\
\hline microsomal glutathione S-transferase 2 & 4258 & MGST2 & -0.373 & 0.134 & 9.97E-02 \\
\hline transmembrane protein 140 & 55281 & TMEM140 & -0.369 & 0.117 & $7.90 \mathrm{E}-02$ \\
\hline guanylate binding protein 7 & 388646 & GBP7 & -0.367 & 0.126 & 9.97E-02 \\
\hline histone cluster 1, H2ai & 8329 & $\mathrm{HIST} 1 \mathrm{H} 2 \mathrm{Al}$ & -0.343 & 0.116 & 9.97E-02 \\
\hline protein phosphatase 1 , regulatory subunit $9 \mathrm{~A}$ & 55607 & PPP1R9A & -0.335 & 0.113 & 9.97E-02 \\
\hline KIAA1107 & 23285 & KIAA1107 & -0.334 & 0.113 & 9.97E-02 \\
\hline phosphodiesterase 4D, cAMP-specific & 5144 & PDE4D & -0.333 & 0.117 & 9.97E-02 \\
\hline zinc finger protein 610 & 162963 & ZNF610 & -0.326 & 0.097 & $6.61 \mathrm{E}-02$ \\
\hline transcription factor Dp-2 (E2F dimerization partner 2) & 7029 & TFDP2 & -0.319 & 0.085 & $6.13 \mathrm{E}-02$ \\
\hline phospholipase A2, group IVC (cytosolic, calcium-independent) & 8605 & PLA2G4C & -0.278 & 0.100 & 9.97E-02 \\
\hline guanosine monophosphate reductase & 2766 & GMPR & -0.272 & 0.098 & 9.97E-02 \\
\hline sestrin 1 & 27244 & SESN1 & -0.265 & 0.093 & 9.97E-02 \\
\hline
\end{tabular}




\section{DNMT1-dependent DNA hypermethylation constrains arteriogenesis by augmenting shear stress set-point}

Authors: Joshua L. Heuslein, Catherine M. Gorick, Ji Song, Richard J. Price. In preparation.

\subsection{Abstract}

Objective: Collateral arteriogenesis is initiated by increased shear stress and is thought to continue until shear stress is returned to its original "set-point". It follows that shear stress setpoint is critical in determining arteriogenic capacity. Nonetheless, the molecular mechanism(s) through which shear stress set-point is established by endothelial cells (ECs) are essentially unstudied. Here, we tested the hypothesis that DNMT1-dependent EC DNA methylation regulates arteriogenic capacity via adjustments to shear stress set-point.

Approach and Results: Previously, we reported that collateral artery segments exposed to a non-reversed increase in shear stress magnitude display limited arteriogenic capacity when compared to segments exposed to reversed flow direction. Here, using 5-mC immunolabeling, we first demonstrated that these non-reversed collateral segments exhibit a $70 \%$ increase in global DNA methylation in-vivo. Then, we applied flow waveforms, biomimetic of those leading to either amplified arteriogenic capacity (i.e. reversed flow) or constrained arteriogenic capacity (i.e. non-reversed flow) in-vivo, to ECs in-vitro, and performed both reduced representation bisulfite sequencing (RRBS) and mRNA-sequencing. ECs exposed to the non-reversed waveform exhibited increased DNMT1 expression, genome-wide hypermethylation of significantly regulated gene promoters $(>70 \%)$, and a DNMT1-dependent reduction in pro-arteriogenic monocyte adhesion. This led us to test whether DNMT1 regulates arteriogenic capacity in-vivo. We found that, in non-reversed collateral artery segments, DNMT1 inhibition rescued arteriogenic capacity $(70.9 \pm 7.9 \mu \mathrm{m}$ vs. $50.1 \pm 3.4 \mu \mathrm{m}, \mathrm{p} \leq 0.05, \mathrm{n}=6)$ and returned shear stress back to its original set point. 
Conclusions: We conclude that, although shear stress initiates arteriogenesis, it also elicits an EC DNA hypermethylation response that inhibits endothelial mechanosensing (i.e. it augments shear stress set-point). In turn, this augmented shear stress set-point constrains the arteriogenic capacity of the vessel. This epigenetic effect could have important bearing on both endogenous collateralization and therapeutic arteriogenesis in peripheral arterial disease (PAD) patients.

\subsection{Abbreviations}

PAD peripheral arterial disease

FAL femoral arterial ligation

EC endothelial cell

HUVEC human umbilical vein endothelial cell

DNMT1 DNA methyltransferase 1

RRBS reduced representation bisulfite sequencing

DMR differential methylated region

5AZA 5-Aza-2'-deoxycytidine

HRM high resolution melting

LSF laser speckle flowmetry

\subsection{Introduction}

Collateral arteriogenesis, the growth of existing arterial vessels to a larger diameter, is a fundamental adaptive response that is often critical for the perfusion and survival of tissues downstream of chronic arterial occlusion(s). Arterial occlusion(s) create steep pressure gradients and increased flow along collateral arterial pathways bypassing the occlusion(s). The resulting increase in shear stress acting on the endothelium initiates a highly coordinated signaling cascade, ultimately resulting in the outward growth of the collateral vessel $\left.\right|^{37,39}$. Outward lumenal 
growth is hypothesized to continue until normalization to the original shear stress level (i.e. the shear stress "set-point") has been achieved ${ }^{39,41,80,81}$.

However, in addition to shear stress magnitude, other hemodynamic factors can also influence arteriogenesis. Indeed, we have recently demonstrated that collateral artery segments exposed to both a 2 -fold increase in shear stress magnitude and reversed flow direction (i.e. "reversed" flow) following femoral arterial ligation (FAL) exhibit amplified arteriogenesis, whereas segments experiencing just a 2-fold increase in shear stress magnitude (i.e. "non-reversed") exhibit a more constrained extent of arteriogenesis ${ }^{237}$. Furthermore, this difference in arteriogenic capacity was maintained up to 12-weeks following FAL. Importantly, these collateral artery segments start at the same basal diameter and shear stress magnitude prior to FAL, suggesting that shear stress set-point may be altered due to differential hemodynamics post-FAL.

Though critical in determining ultimate arteriogenic capacity, the molecular mechanism(s) involved in establishing and maintaining the shear stress set-point remain unknown. Epigenetic mechanisms, such as DNA methylation, histone modifications, and noncoding RNA regulation, could be a way for local hemodynamics to regulate long-term gene expression changes ${ }^{102}$. Of these epigenetic mechanisms, DNA methylation is considered the most stable ${ }^{100,103}$. DNA is methylated at a cysteine base pair, most often at a CpG dinucleotide ( $\mathrm{CpG}$ site $)^{103}$. Methylation of $\mathrm{CpG}$ sites in the promoter region of a gene is commonly associated with repression of gene expression ${ }^{110-112}$. DNA methylation occurs through the activity of DNA methyltransferases (DNMTs), particularly DNMT1 post-development ${ }^{98,104,105}$. Recently, DNA methylation has been shown to differentially regulate flow-mediated endothelial gene expression through DNMT1 ${ }^{238-241}$, though the role of DNA methylation in the regulation of arteriogenesis has not been explored. Here, using our previous observations of differential arteriogenic capacity within collateral artery segments in-vivo, we tested the central hypothesis that an augmented shear stress set-point constrains arteriogenic capacity via DNMT1-dependent endothelial cell DNA hypermethylation. 


\subsection{Materials and Methods}

\subsubsection{Mice.}

All animal protocols were approved by the Institutional Animal Care and Use Committee at the University of Virginia (Protocol 3814) and conformed to all regulations for animal use outlined in the American Heart Association Guidelines for the Use of Animals in Research. Male C57BL/6 mice and Balb/c were purchased from Charles River Laboratory (Wilmington, MA). All animals were housed in the animal facilities at the University of Virginia. C57BL/6 mice were used for all studies unless otherwise noted.

\subsubsection{Femoral arterial ligation model.}

We used a previously detailed femoral artery ligation (FAL) scheme ${ }^{167,237,242}$ that produces consistent arteriogenesis in the collateral arteries of the gracilis adductor muscles $29,30,177-180,237$, along with minimal heterogeneity in the baseline collateral structure and known changes in flow direction from baseline. Male mice, (10-12 and 20-21 weeks of age for C57BL/6 and Balb/c, respectively), were anesthetized (i.p $120 \mathrm{mg} / \mathrm{kg}$ ketamine, $12 \mathrm{mg} / \mathrm{kg}$ xylazine, and $0.08 \mathrm{mg} / \mathrm{kg}$ atropine), depilated, and prepped for aseptic surgery. On the left leg, an incision was made directly above and along the femoral artery, which was gently dissected from the femoral vein and nerve between the bifurcation of the superior epigastric artery and popliteal artery. Two 6.0 silk sutures were placed immediately distal to the epigastric artery, which served as the origin of the muscular branch artery in all mice, and the artery segment between the two ligatures was then severed with micro dissecting scissors. The surgical site was then closed with 5.0 prolene sutures. A sham surgery, wherein the femoral artery was exposed but not ligated, was performed on the right hindlimb (i.e. on the other leg). Animals received one injection of buprenorphine for analgesia at the time of surgery and a second dose 8-12 hours later. 


\subsubsection{AZA treatment.}

5-aza-2'-deoxycytidine (5AZA) (Sigma, \#A3656) was reconstituted in DMSO to a stock concentration of $0.25 \mathrm{mg} / \mu \mathrm{l}$. Each day immediately before use, stock $5 A Z A$ was diluted to $1.25 \mu \mathrm{g} / \mu \mathrm{l}$ and DMSO to $0.01 \%$ DMSO in sterile saline. Both solutions were then passed through a $0.22 \mu \mathrm{m}$ sterile syringe filter. Mice were treated daily with an i.p. injection of $0.1 \mathrm{mg} / \mathrm{kg} 5 A Z A$ in sterile saline or $0.01 \%$ DMSO in a total volume of $100 \mu$.

\subsubsection{Quantification of global DNA methylation by high resolution melting (HRM).} Methylation of genomic repeat elements, such as LINE1, have been used as markers of global gDNA methylation ${ }^{100,243,244}$. LINE1 methylation in the peripheral blood was therefore used as an indicator of the efficacy of our 5AZA treatment protocol on DNA methylation in-vivo. Peripheral

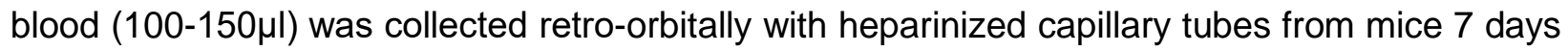
after beginning daily 5AZA or DMSO i.p. injections. Genomic DNA was immediately extracted using the Quick-gDNA MiniPrep kit (Zymo Research, \#D3006) and gDNA (80ng) underwent bisulfite conversion using the EZ-DNA Methylation-Gold kit (Zymo Research, \#D5005) according to manufacturer's instructions. PCR and HRM analysis were adapted from a previously determined protocol ${ }^{243}$. Briefly, modified DNA was diluted to $10 \mathrm{ng} / \mu \mathrm{l}$ with nuclease-free water. A $20 \mu$ l reaction mix of 20 ng bisulfite modified DNA and a final concentration of $1 x$ EpiTect HRM

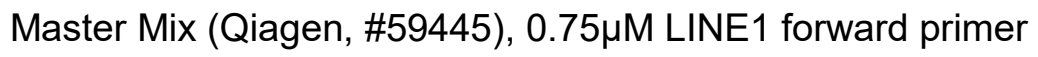

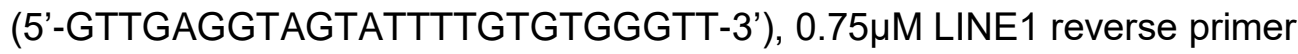

(5'-TCCAAAAACTATCAAATTCTCTAACAC-3'), and nuclease-free water. PCR cycling conditions for LINE 1 were $95^{\circ} \mathrm{C}$ for 5 minutes followed by 40 cycles of $95^{\circ} \mathrm{C}$ for 20 s then $55^{\circ} \mathrm{C}$ for 30 s then $72^{\circ} \mathrm{C}$ for 20 s. Melt analysis occurred from $60-90^{\circ} \mathrm{C}$ rising by $0.1^{\circ} \mathrm{C} / 5 \mathrm{~s}$. PCR and HRM was performed on a CFX96 Real Time Detection System (Biorad) and each sample was run in duplicate. 
Differences in DNA methylation as detected by HRM were quantified by the Net Temperature Shift (NTS) as previously calculated ${ }^{243}$. Briefly, the NTS was calculated as average distance between the normalized melt curves of experimental samples from a universal methylated positive control (Zymo Research, \#D5012) where a more negative NTS indicates a less methylated sample. For LINE1, the two normalization regions used were between $72-73^{\circ} \mathrm{C}$ and $82-83^{\circ} \mathrm{C}$.

\subsubsection{Tissue harvesting for whole mount vascular casting and cross sectional analysis.}

For analysis of lumenal diameters in the gracilis collateral arteries and to enable sectioning at specific regions, vascular casting was performed using an opaque polymer that allows for accurate lumenal diameter measurements ${ }^{179}$. After femoral artery ligation, mice were anesthetized (i.p $120 \mathrm{mg} / \mathrm{kg}$ ketamine, $12 \mathrm{mg} / \mathrm{kg}$ xylazine, and $0.08 \mathrm{mg} / \mathrm{kg}$ atropine), euthanized via an overdose of pentobarbital, and then the abdominal aorta was cannulated. The lower body was then perfused with $7 \mathrm{~mL}$ of $2 \%$ heparinized saline with $2 \mathrm{mmol} / \mathrm{L}$ adenosine $(16404$, Fisher Scientific, Pittsburg, PA) and 0.1 mmol/L papaverine (P3510, Sigma Aldrich, St Louis, MO) to clear and vasodilate the downstream vasculature at a constant rate of $1 \mathrm{~mL} / \mathrm{min}$ (PHD2000, Harvard Apparatus). Once perfused, we waited 5 minutes to enable vasodilation. Tissues were then perfused with $3 \mathrm{~mL}$ of $4 \%$ paraformaldehyde solution (19943, Affymetrix, Cleveland, OH) at $1 \mathrm{~mL} / \mathrm{min}$ and allowed to fix for 10 minutes. The lower body was then perfused with $0.8 \mathrm{~mL}$ of Microfil ${ }_{B}$ casting agent (FlowTech, Inc, Carver, Massachusetts) at a constant pressure of $100 \mathrm{mmHg}$. Viscosity of Microfilß was adjusted to minimize transport across capillaries. After curing for 1.5 hours at room temperature, gracilis muscles were dissected free and then cleared in $50 \%$ glycerol in phosphate buffered saline (PBS) overnight. Cleared tissues were mounted between two coverslips using $500 \mu \mathrm{m}$ thick spacers (645501, Grace Bio-Labs Inc) to keep constant thickness between muscles. Muscles were imaged using transmitted light at $4 \mathrm{x}$ 
magnification on a Nikon TE200 inverted microscope with a CCD camera (Quantifier, Optronics Inc). Individual fields of view were montaged together (Photoshop CS2, Adobe Systems Inc).

For analysis of lumenal diameters from intact gracilis collateral whole mounts (i.e. vascular casting), collateral entrance regions were defined according to the following method. A cropped portion $(560 \mu \mathrm{m} \times 560 \mu \mathrm{m})$ of the montaged image (previously randomized and de-identified) was taken of the collateral artery at the first visible branch point of a terminal arteriole from the primary collateral as it extended from either the muscular branch or saphenous artery as previously described $^{237}$. After each cropped image region was taken, all images were randomized and deidentified. The mean diameter was then taken from 4-5 separate diameter measurements along the length of cropped portion of the collateral artery.

After imaging, muscles were rehydrated, cut, and then paraffin embedded for cross sectional analysis at the muscular branch and saphenous artery entrance regions to the collateral arteries. Resulting cross sections were re-hydrated and immunolabeled for 5-methylcytosine (84 days post-FAL) or H\&E stained for collateral artery structure analysis (day 28 post-FAL).

\subsubsection{Immunofluorescence labeling of 5-methylcytidine.}

Sections ( $5 \mu \mathrm{m}$ thickness) of paraffin embedded muscle from the muscular and saphenous regions were rehydrated and subjected to heat mediated antigen retrieval for 20 minutes in a citrate based antigen retrieval buffer (Vector Laboratories, Burlingame, $\mathrm{CA} ; \mathrm{H}-3300)$. After cooling, tissues were encircled with a hydrophobic barrier pen and blocked with PBS $+0.1 \%$ saponin $+2 \%$ bovine serum albumin (Jackson Immunoresearch) for 1 hour at room temperature. Tissues were then incubated overnight at $4^{\circ} \mathrm{C}$ with anti-5-methylcytidine (1:100, BI-MECY, Eurogentec) and rat anti-CD31 (1:75, SZ31, Dianova). Following primary antibody incubation, slides were washed in PBS then

incubated with DRAQ5 (a nuclear marker), a donkey-anti-mouse Cy3 $F_{a b}$ (1:200, Jackson Immunoresearch), and a goat-anti-rat-488 secondary antibody (1:200, Jackson Immunoresearch) for 1 hour at room temperature. Following incubation, slides were washed again in PBS, mounted 
with Prolong Gold (Life Technologies) to minimize photobleaching, allowed to cure overnight, and imaged using a Nikon TE2000 C1 laser scanning confocal microscope with a 20x oil objective. All settings were held constant throughout imaging. Cropped fields of view $(200 \mu \mathrm{m} \times 200 \mu \mathrm{m})$ encompassing the collaterals in each region were randomized and de-identified. The collateral diameter, collateral cross-sectional area, and the integrated density of 5-methylcytidine with an individual cross-section were determined in $\mathrm{Fiji}^{181}$. For each mouse, mean collateral diameter, cross-sectional area, and integrated density was calculated from the average of the two primary gracilis collateral arteries, with two immunolabeled sections per collateral artery for a total of 4 images per mouse.

\subsubsection{Cross sectional analysis of collateral artery structure.}

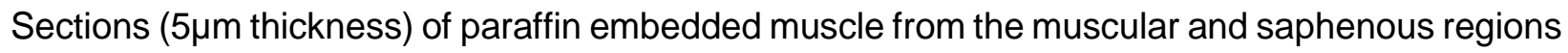
were labeled for H\&E. Individual fields of view encompassing the collateral vessels were imaged with a 40x water objective on a Zeiss inverted microscope (Zeiss Axioskop, Thornwood, NY) with a CCD camera (Quantifier, Optronics Inc). All images were randomized and de-identified prior to analysis. Lumenal diameter, wall area, and wall thickness were determined using $\mathrm{Fiji}^{181}$.

\subsubsection{Human umbilical vein endothelial cell culture.}

Human umbilical vein endothelial cells (HUVECs) purchased from VEC Technologies Inc. (Rensselaer, NY) were thawed and maintained on $0.1 \%$ gelatin coated flasks in M-199 medium (Lonza, Basel, Switzerland), supplemented with 10\% fetal bovine serum (Life Technologies Inc, Grand Island, NY), $\quad 100 \mathrm{U} / \mathrm{mL}$ penicillin-G $+100 \mathrm{ug} / \mathrm{ml}$ streptomyocin (Life Technologies Inc), 2mmol/L L-glutamine (Life Technologies Inc), 5ug/ml endothelial cell growth supplement (Biomedical Technologies, Stoughton, MA), and 10ug/ml heparin (Sigma Aldrich, St. Louis, MO).

For each set of experimental comparisons, cells were used from the same cell line between subculture passages 2-3. 


\subsubsection{In vitro exposure of endothelial cells to biomimetic shear stress waveforms.}

HUVECs were plated on cell culture grade plastic dishes coated with $0.1 \%$ gelatin and grown to confluence. A cone and plate flow apparatus ${ }^{187}$, which maintains cells at $5 \% \mathrm{CO}_{2}$ and $37^{\circ} \mathrm{C}$, was used to induce a shear stress protocol. The applied shear stress protocol consisted of a 24 hour preconditioning period at a steady of $15 \mathrm{dyne} / \mathrm{cm}^{2}$, which was then either increased to 30 dynes $/ \mathrm{cm}^{2}$ (non-reversed flow) or increased to 30 dynes $/ \mathrm{cm}^{2}$ and reversed in direction (reversed flow) to simulate relative hemodynamics previously quantified in our in-vivo FAL mode ${ }^{176}$. Fresh culture media consisting of M199 with 4\% dextran from Leuconostoc spp (Sigma Aldrich, $\mathrm{Mr}_{\mathrm{r}}$ 500,000), $2 \%$ fetal bovine serum, $100 \mathrm{U} / \mathrm{mL}$ penicillin-G $+100 \mathrm{ug} / \mathrm{ml}$ streptomyocin, $2 \mathrm{mmol} / \mathrm{L} \mathrm{L-}$ glutamine, $5 \mathrm{ug} / \mathrm{ml}$ endothelial cell growth supplement, and $10 \mathrm{ug} / \mathrm{ml}$ heparin was added to cells before exposure to shear stress and was continuously exchanged throughout the duration in the cone and plate apparatus.

\subsubsection{HUVEC RNA isolation and qRT-PCR.}

Total RNA was extracted using the PureLink total RNA purification system using the on-column DNase protocol (Life Technologies Inc) according to manufacturer's instructions. RNA concentration and purity was determined with a NanoDrop spectrophotometer in duplicate. For quantitative reverse transcriptase PCR (qRT-PCR), 500ng of total RNA was reverse transcribed using the iScript cNDA synthesis kit (Bio-Rad, Hercules, CA). A reaction mixture of $12.5 \mathrm{ng}$ of reverse transcribed CDNA, DNMT1 forward primer (TGCCAGCTGAGCGTGGTGGT), DNMT1 reverse primer (GCATGCGGGCAGCCACCAAT), and FastStart SYBR Green (Roche Applied Sciences) underwent qRT-PCR on a CFX96 Real Time Detection System (Biorad). Expression was normalized to $\beta 2$-microglobulin (forward 5'-AGCATTCGGGCCGAGATGTCT-3', reverse 5'CTGCTGGATGACGTGAGTAAACCT-3') which is endogenously expressed and is not altered by many stimuli including shear stress ${ }^{44}$. Normalized expression was quantified using the comparative $2^{\Delta \Delta C t}$ method. 


\subsubsection{Reduced representation bisulfite sequencing (RRBS) and mRNA sequencing.}

Total gDNA and total RNA were extracted from flow-exposed HUVECs using the Quick-gDNA MiniPrep kit (Zymo Research, Irvine, CA, \#D3006) and the Quick-RNA MiniPrep kit (Zymo Research, \#R1054) according to manufacturer's instructions. Total gDNA and total RNA concentration and purity were determined with a NanoDrop spectrophotometer in duplicate. Both gDNA and RNA were isolated from the same plate of cells for each condition within an experiment. Purified genomic DNA and purified total RNA isolated from flow-exposed HUVECs were pooled from 2 independent flow experiments. Pooled gDNA samples were sent to Zymo Research where DNA fragmentation, library preparation, bisulfite conversion, next-generation sequencing, and bioinformatics were performed. Pooled RNA samples were also sent to Zymo Research where they performed mRNA sequencing (mRNA-Seq). HiSeq 50bp singleton reads from RNA-Seq were first adaptor trimmed and then analyzed using the TopHat and Cufflinks software. TopHat (v2.2.0) was used for alignment of short read to the human genome hg19. Cufflinks (v2.2.0) was used to transcript assembly and differential expression. CommeRbund (v2.0.0) was used for visualization of differential analysis. Default parameters were used in all instances.

\subsubsection{RRBS Analysis.}

RRBS next-generation sequencing reads were mapped to the "Feb. 2009 (GRCh37/hg19)" genome assembly by Zymo Research. The \%CpG methylation was calculated as the percent of methylated CpG sites per total CpG sites in a given differentially methylated region (DMR) with 210x CpG coverage in a given DMR. Significance was determined using a Fisher's exact test then applying a Benjamini-Hochberg procedure to find false discovery rate (FDR). DMRs with 210x CpG coverage in their promoter regions (transcription start site $\pm 1 \mathrm{~kb}$ ) were considered significant if FDR $<0.1$ and the absolute value of $\% \mathrm{CpG}$ methylation of a DMR in $\mathrm{N}$ minus the $\%$ CpG methylation of a DMR in R was $\geq 10 \%$ (Supplemental Table 5.3). We then compared this 
list of significant DMRs with our mRNA-Seq dataset to determine genes with relative gene expression changes that correspond to their methylation status between shear stress conditions, i.e. identify genes up-regulated in $\mathrm{N}$ conditions that also have a significantly hypomethylated promoter region as well as genes that are down-regulated in $\mathrm{N}$ conditions that have hypermethylated promoter regions compared to in R conditions (Supplemental Table 5.4 \& Supplemental Table 5.5). Only genes demonstrating this relative gene expression-methylation correlation were used for gene ontology analysis using $\mathrm{MSigDB}^{245}$ from the Broad Institute.

\subsubsection{Transillumination laser speckle imaging and shear stress analysis.}

Transillumination laser speckle imaging was performed as previously described ${ }^{176}$. Briefly, 28days after femoral arterial ligation, mice were anesthetized (i.p $120 \mathrm{mg} / \mathrm{kg}$ ketamine, $12 \mathrm{mg} / \mathrm{kg}$ xylazine, and $0.08 \mathrm{mg} / \mathrm{kg}$ atropine), depilated, and prepped for aseptic surgery. On the left leg (ligated leg), an incision was made above and along the femoral artery such that a window of skin was dissected free and retracted directly above the superficial adductor muscles. Exposed tissue was superfused throughout the procedure and during imaging with a warmed solution of Tris$\mathrm{CaCl}_{2}\left(0.1 \mathrm{~g} / \mathrm{L} \mathrm{CaCl}_{2}\right)$ with $2 \mathrm{mmol} / \mathrm{L}$ adenosine (16404, Fisher Scientific) and $0.1 \mathrm{mmol} / \mathrm{L}$ papaverine (P3510, Sigma Aldrich). To image the gracilis muscle, the mouse was placed supine on an intravital microscope stage (Zeiss Axioskop). A 30mW, 658nm laser diode (LPM658-30, Newport Corporation, Irvine, CA) was coupled to a fiber optic cable and placed beneath the mouse in a trans-illumination orientation. A cooled, monochrome CCD camera (Optonics Quantifier, Goleta, CA) was used to acquire the raw speckle images using a 4x air objective (Zeiss Acroplan

LD NA=0.1). The objective and camera were chosen to ensure satisfaction of the Nyquist sampling criteria of at least 2 pixels per individual speckle. ${ }^{29}$ An objective mounted fiber optic light guide allowed for brightfield imaging to enable lumenal diameter measurements (A08650, Schott Inc., Elmsford, NY). For each field of view, a sequence of 20 12-bit raw speckle images was acquired with a $5 \mathrm{~ms}$ exposure time to capture average velocity over multiple cardiac cycles. 
All processing of raw speckle images was performed using Fiji ${ }^{181}$ as described previously ${ }^{176}$. Briefly, raw speckle images were converted to laser speckle flow index maps, removing any images with excessive motion artifact. To then account for the influence of whole background tissue variations, the processed flow images were normalized to median background intensity. Individual flow images were then merged into larger two-dimensional maps using Adobe Photoshop (CS2, Adobe Systems Inc., San Jose, CA). Finally, to allow for comparison of velocity change across experiments vessel speckle intensity (SI) was normalized to the background tissue according to Eq. 1 to obtain the normalized speckle index (NSI).

$$
\mathrm{NSI}=\frac{\mathrm{SI}_{\text {vessel }}}{\mathrm{SI}_{\text {background }}}-1
$$

Blood velocity analysis of laser speckle images was limited to defined muscular branch and saphenous collateral artery regions and assumed Poiseuille flow. The mean speckle shear rate (SSR) in each region was calculated using the normalized speckle index and vessel diameter (D) according to Eq. 2:

$$
\mathrm{SSR} \propto \frac{\mathrm{NSI}}{\mathrm{D}}
$$

\subsubsection{4 siRNA transfection in HUVECs.}

Twenty-four hours prior to exposure of HUVECs to flow conditions, HUVECs were plated without antibiotics on $0.1 \%$ gelatin coated plates in serum-free M199 (Life Technologies) supplemented with $10 \%$ fetal bovine serum, $2 \mathrm{mmol} / \mathrm{L} \mathrm{L-glutamine,} 5 \mathrm{ug} / \mathrm{ml}$ endothelial cell growth supplement (Biomedical Technologies, Stoughton, MA), and 10ug/ml heparin (Sigma Aldrich Inc). After cells were allowed to adhere for 2 hours after plating, cells were transfected with either $120 \mathrm{pmol}$ of 
ON-TARGETplus SMARTpool human DNMT1 siRNA (GE Dharmacon, Lafayette, CO, L-004605$00-0005$,) or $120 \mathrm{pmol}$ of ON-TARGETplus non-targeting siRNA (GE Dharmacon, D-001810-1005 ) in $52 \mu \mathrm{L}$ of Oligofectamine transfection reagent (Life Technologies) and $6.8 \mathrm{~mL}$ Opti-MEM media (Life Technologies) for 5 hours at $37^{\circ} \mathrm{C}$. After 5 hours, plates were flooded with $8 \mathrm{~mL}$ of M199 media without antibiotics supplemented with 10\% fetal bovine serum + L-glutamine + $5 \mathrm{ug} / \mathrm{ml}$ endothelial cell growth supplement (Biomedical Technologies, Stoughton, MA), and 10ug/ml heparin (Sigma Aldrich Inc). 24 hours post-transfection this solution was aspirated off and normal flow media was applied. Validation of transfection was performed on HUVEC plates (54 hours post-transfection) via DNMT1 western blotting.

\subsubsection{Western blot analysis.}

HUVECs were lysed in RIPA buffer (Sigma-Aldrich, \#R0278) with protease inhibitor (Sigma, 1:50, \#P8340). Samples were then cleared for 30 minutes at $4^{\circ} \mathrm{C}$ under constant agitation. Samples were centrifuged for 1 minute at 10,000g, the supernatant was collected, and a Pierce BCA assay (ThermoFisher Scientific, \#23225) was used to determine total protein concentration. Samples were diluted 1:1 in 2x Laemmli sample buffer (Biorad, \#1610737) with $\beta$-mercaptoethanol (1:200) and boiled for 10 minutes. Equal protein was loaded onto a 10\% SDS-PAGE gel and blotted on a nitrocellulose membrane. After transfer, membranes were blocked for 1 hour at room temperature with Odyssey Blocking Buffer (LICOR, Lincoln, NB, \#927-40000) and then incubated with primary antibodies overnight at $4^{\circ} \mathrm{C}$. Western blots were performed by using primary antibodies directed against DNMT1 (Abcam, 1:1000, ab92314) and GAPDH (EMD Millipore,

$0.0625 \mu \mathrm{g} / \mathrm{mL}$, \#AB2302). Secondary antibodies were purchased from LICOR and used at a 1:10,000 dilution. A LICOR Odyssey imager was used for blot image acquisition and densitometry analysis. 


\subsubsection{Monocyte adhesion functional assay.}

Human derived monocytes (THP-1 cell line) were purchased from the ATCC. Monocytes were unthawed and maintained in RPMI 1640 (Life Technologies, \#11875-093) + 10\% fetal bovine serum (Life Technologies Inc) $+0.05 \mathrm{mM} \beta$-mercaptoethanol per ATCC culture instructions. Monocytes sub-cultured once cell density approached 800,000 cells $/ \mathrm{mL}$. Cells were used between passages $2-6$.

Prior to the adhesion assay, cells were counted to obtain $3,000,000$ cells / plate of HUVECs. Cells were pelleted, washed with PBS, pelleted, and then re-suspended in serum-free RPMI media at 1,000,000 cells $/ \mathrm{mL}$. Thawed calcein AM was added at $1 \mathrm{ug} / \mathrm{ml}$ and incubated with cells for 15 minutes at $37^{\circ} \mathrm{C}$. After 15 minutes, the reaction was stopped by adding excess serum-free RPMI to the cell solution then pelleted. Cells were washed once with serum-free M199 media, pelleted, and then re-suspended in serum-free M199 at 500,000 cells/mL. Immediately following completion of flow exposure to HUVECs, flow media was removed by aspiration. HUVECs were quickly washed with serum-free M199 media. This media was then aspirated off and $6 \mathrm{~mL}$ of serum-free M199 + monocytes (3,000,000 / plate) were added to and incubated with HUVECs for 30 minutes at $37^{\circ} \mathrm{C}$. Following the 30 minutes, cells were washed twice with PBS to remove unbound monocytes. Adhered monocytes and HUVECs were fixed with 4\% PFA for 10 minutes followed by two washes with PBS. Cover slips were mounted with Prolong Gold (Life Technologies). Plates were then imaged using a Nikon TE2000 C1 laser scanning confocal microscope. Randomly selected FOVs (8-9) per condition for 3 independent experiments were obtained. Images were then deidentified and randomized in MATLAB. Images were converted to 8-bit images, set to an equivalent threshold, and bound monocytes were quantified using Fijis "Analyze Particles" tool $\left(20 \mu \mathrm{m}^{2}\right.$ minimum particle size). Results were centered on the mean of all conditions within each independent experiment. 


\subsubsection{Statistical Analyses.}

All results are reported as mean \pm standard error of the mean (SEM), unless otherwise noted. All data were first tested for normality and equal variance. Statistical significance was then assessed by a Student's $t$ test or a two-way ANOVA followed by a Holm-Sidak multiple comparisons test, unless otherwise noted (SigmaStat 3.5, Systat Inc). Significance was assessed at $p<0.05$.

\subsection{Results}

\subsubsection{Collateral artery segments exhibiting limited arteriogenic capacity exhibit hypermethylated DNA 12-weeks after FAL.}

Using an FAL model identical to that employed previously by our group to demonstrate differential arteriogenesis at either end of gracilis collateral arteries (Figure 5.1A), we first sought to determine if these two collateral artery regions displayed differential EC DNA methylation. To this end, we immunolabeled collateral artery cross-sections for 5methylcytodine, a marker of DNA methylation, along with endothelial cell (CD31) and nuclear (DRAQ5) counter-labels (Figure 5.1B). These cross sections confirmed our previous findings ${ }^{237}$ that muscular (non-reversed) collateral artery regions experience limited arteriogenesis when compared to saphenous (reversed) regions 12-weeks post-FAL (Figure 5.1C-D). We also found there was a $>1.7$-fold increase in circumference-normalized 5-methylcytodine expression in muscular (non-reversed) segments (Figure 5.1B, F). These results demonstrate that collateral segments exposed to a non-reversed increase in shear stress magnitude exhibit DNA hypermethylation which correlates to limited long-term arteriogenic capacity. 
A.

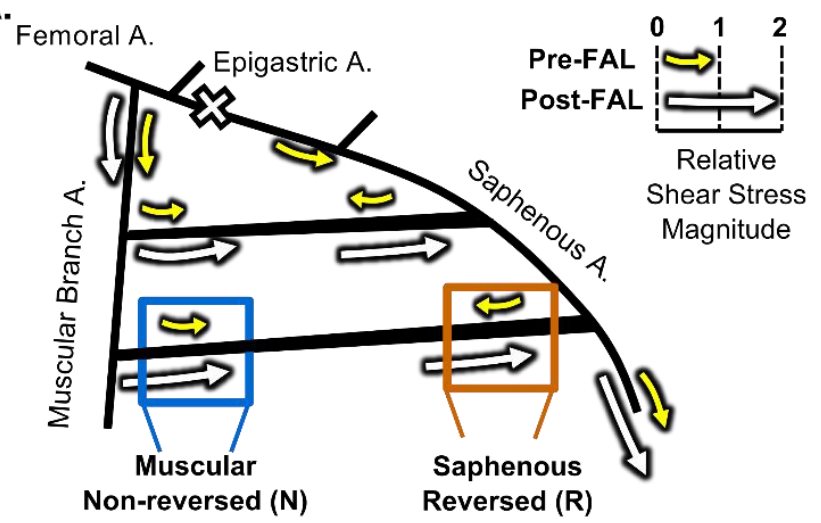

B.

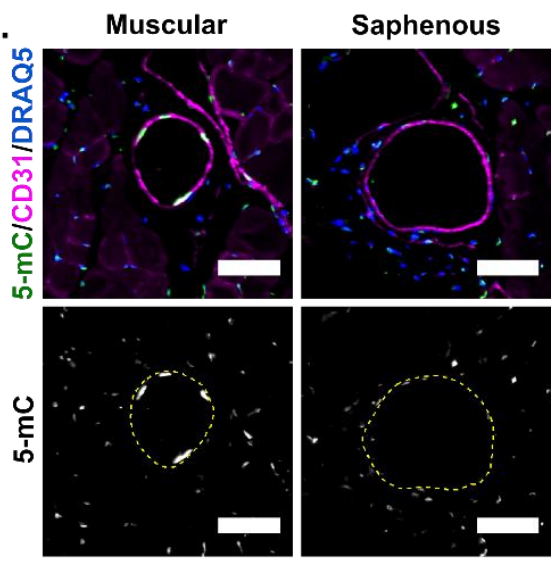

E.

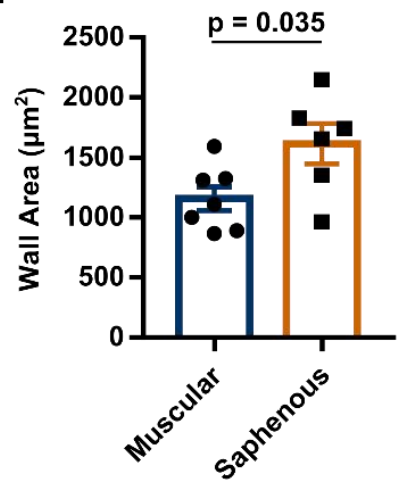

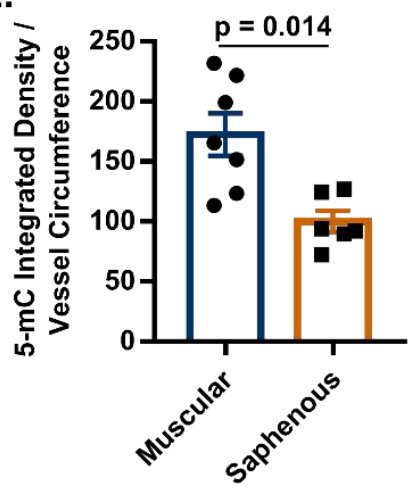

Figure 5.1. Gracilis collateral arteries exposed to a non-reversed increase in shear stress magnitude exhibit limited arteriogenic capacity and hypermethylated DNA.

A) Schematic of the primary gracilis adductor collateral flow pathways after femoral arterial ligation (FAL). Arrows indicate the direction and magnitude of blood flow pre- (yellow) and post- (white) FAL. The femoral artery is ligated just distal to the epigastric artery such that some collateral segments ("muscular") experience a 2-fold increase in shear stress magnitude ("non-reversed" flow), while other segments ("saphenous") are exposed to both a 2-fold increase in shear stress magnitude and reversed flow direction ("reversed" flow). B) Representative cross-sections of gracilis collateral regions in C57BL/6 mice 12 weeks post-FAL immunolabeled with 5-methylcytodine (5-mC, green), CD31 (ECs, magenta), and DRAQ5 (nuclei, blue) (Scale bar $=50 \mu \mathrm{m}$ ). Dotted yellow line indicates vessel wall. C-E) Bar graphs of lumenal diameter, collateral wall area, and 5-mC vessel density (total 5-mC per vessel circumference) for both muscular (nonreversed, blue) and saphenous (reversed, orange) regions 12 weeks post-FAL $(n=6-7) . p<0.05$, Student's $t$ test. Data are mean \pm SEM.

\subsubsection{ECs exposed to a non-reversed increase in shear stress magnitude exhibit}

\section{augmented DNMT1 expression}

To further investigate the influence of these FAL-elicited hemodynamic changes on endothelial cell (EC) DNA methylation, human umbilical vein endothelial cells (HUVECs) were exposed to flow waveforms biomimetic of those experienced by collateral arteries following FAL in-vivo ${ }^{176}$

(Figure 5.2A). Briefly, ECs were preconditioned for 24 hours at 15 dynes $/ \mathrm{cm}^{2}$ to establish basal 
endothelial cell alignment and planar cell polarity, thereby mimicking the in-vivo baseline state. A femoral arterial ligation (FAL) was then simulated by a step-wise $100 \%$ increase in shear stress, in either the same direction or in the opposite direction, to mimic shear stress changes occurring in the muscular branch (non-reversed flow) and saphenous artery (reversed flow) entrance regions, respectively (Figure 5.2A). We examined DNMT1 mRNA expression by qRT-PCR 1hour and 6-hours after our simulated FAL, finding it was transiently increased by $\sim 25 \%$ after 1 hour in HUVECs exposed to non-reversed flow, but was unchanged in reversed flow conditions (Figure 5.2B). DNMT1 mRNA expression returned to basal level by 6-hours after simulated FAL (Figure 5.2B).

\subsubsection{Altered genome-wide DNA methylation patterns in ECs exposed to a arteriogenic shear stress waveforms in-vitro}

To then determine how these biomimetic waveforms affect global DNA methylation patterns, we exposed HUVECs to these same flow waveforms. Six hours after simulated FAL, we isolated both genomic DNA (gDNA) and total RNA and performed both reduced representation bisulfite sequencing (RRBS) and mRNA-sequencing (mRNA-seq) on these samples, respectively. Both data sets were mapped to the hg19 human genome assembly (GRCh37/hg19, NCBI, Feb. 2009) and showed a similar degree of coverage between our non-reversed and reversed datasets (Supplemental Table 5.1 and Supplemental Table 5.2). Additionally, there was a similar degree of total $\mathrm{CpG}$ and promoter $\mathrm{CpG}$ coverage in both non-reversed and reversed datasets (Supplemental Figure 5.1). 
A.

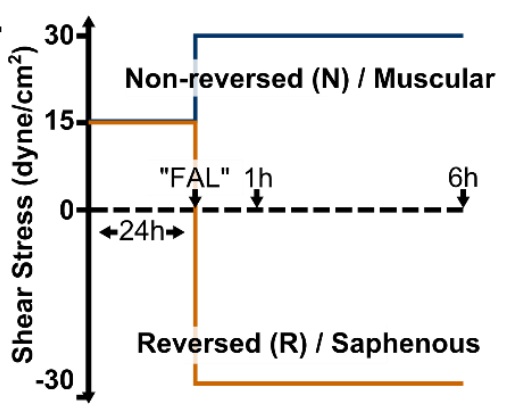

C.

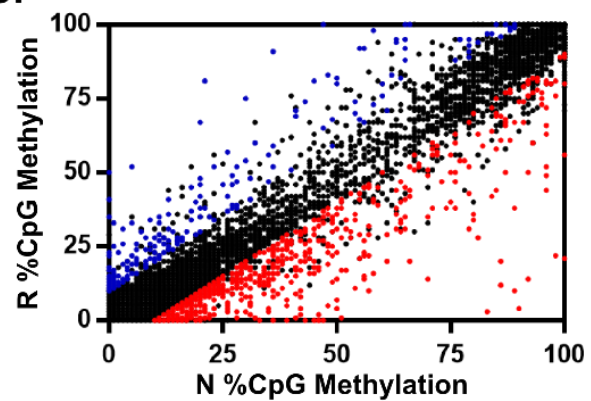

E.

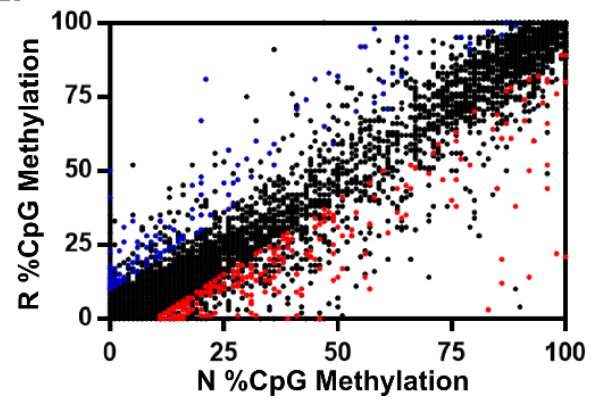

B.

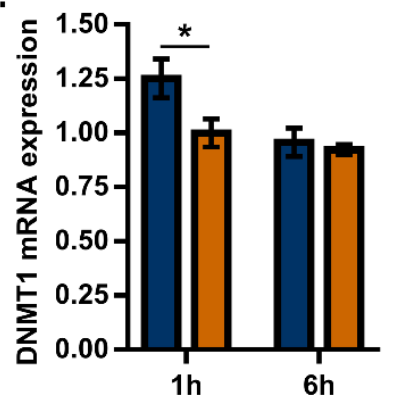

D.

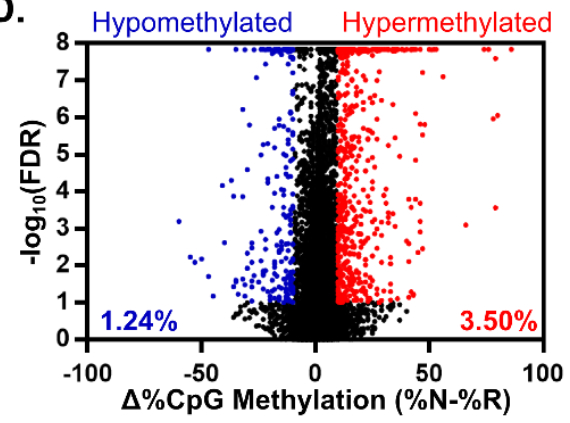

F.

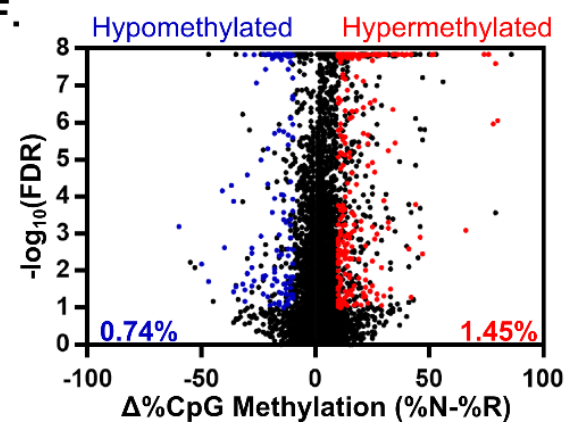

Figure 5.2. ECs exposed to a non-reversed increase in shear stress magnitude exhibit augmented DNMT1 expression and altered genome-wide DNA methylation patterns in-vitro.

A) Schematic depicting biomimetic shear stress conditions applied to HUVECs to simulate nonreversed/muscular ( $\mathrm{N}$, blue) and reversed/saphenous ( $\mathrm{R}$, orange) regions. $\mathrm{B}$ ) Bar graph of DNMT1 mRNA expression in each flow condition 1-hour or 6-hours post simulated FAL ( $n=8$ for $1 \mathrm{hr}$ and $n=6$ for $6 \mathrm{hr}$ ). ${ }^{*} p<0.05$ between reversed and non-reversed within a time-point, Student's t-test. Data are mean \pm SEM. C-D) Scatter plot and Volcano plot of all DMRs in a gene promoter region (17,227 total). Significant DMRs were designated as hypomethylated (blue, 213 total) or hypermethylated (red, 603 total) with respect to the non-reversed condition (Supplemental Table 5.3). FDR = false discovery rate. E-F) Using our mRNA-Seq dataset, we further filtered this list of significantly hyper- and hypomethylated genes (816 total genes) to contain only those with gene expression changes between non-reversed and reversed conditions in the expected direction based on their change in promoter methylation [i.e. genes that were down-regulated and had a hypermethylated promoter (red, 250 genes) or were up-regulated and had a hypomethylated promoter (blue, 127 genes) in non-reversed versus reversed conditions (Supplemental Table 5.4, Supplemental Table 5.5). 
To characterize global DNA methylation changes between non-reversed $(\mathrm{N})$ and reversed (R) data sets in genomic regions, we analyzed the CpG methylation in promoter (transcription start site $\pm 1 \mathrm{~kb}$ ), exon, and intron regions. From our RRBS analysis, only read regions with at least 10x CpG read coverage (henceforth termed differentially methylated regions or DMRs) and mRNA expression in both non-reversed and reversed datasets were selected for further analysis. Intron regions displayed a higher degree of $\mathrm{CpG}$ methylation compared to exon or promoter regions; however, exon regions displayed the highest mean methylation density within a DMR, consistent with a previous study ${ }^{241}$ (Supplemental Table 5.2A-B). Average methylation across gene regions was similar for both non-reversed and reversed data sets. However, when we considered only significantly different $(F D R<0.1)$ DMRs, we observed global hypermethylation in non-reversed conditions compared to reversed conditions, across all gene regions (Supplemental Table 5.2C-D). Expectedly, we observed that the degree of promoter methylation inversely correlated with raw gene expression levels on a global scale (Supplemental Table 5.3).

As numerous studies have shown that DNA methylation in the promoter region regulates transcription ${ }^{109}$, we focused on DNA methylation differences within gene promoters. We found that $4.74 \%(816 / 17,227)$ of DMRs in promoter regions have $a \geq|10 \%|$ difference in CpG methylation and an FDR $<0.1$ between non-reversed and reversed conditions (Supplemental Table 5.3). Of these 816 genes, $73.9 \%$ (603/816) were hypermethylated in non-reversed compared to reversed conditions (Figure 5.2C-D, red). To determine which mechanosensitive genes demonstrate a correlation between relative gene expression and promoter DNA methylation status, we further filtered this list of 816 genes to contain only genes with expression changes in the expected direction based on their change on promoter methylation [i.e. genes that were down-regulated and had a hypermethylated promoter in non-reversed vs. reversed conditions and vice versa] (Supplemental Table 5.4). We found that $66.3 \%$ of these genes (250/377) were hypermethylated and down-regulated compared to $33.7 \%(127 / 377)$ that were upregulated and hypomethylated in non-reversed vs. reversed conditions (Figure 5.2E-F, 
Supplemental Table 5.4 \& Supplemental Table 5.5). We then performed gene ontology analysis on these 377 genes to identify overrepresented pathways (Supplemental Table 5.6). Cellular metabolism, nucleic acid metabolism, and transcription processes were among the most significantly regulated pathways but a number of additional pathways were overrepresented including protein metabolism, MAPK signaling, apoptosis, cellular localization and transport, and signal transduction

\subsubsection{DNMT1 regulates the adhesion of monocytes to endothelial cells exposed to a non-reversed increase in shear stress magnitude in-vitro.}

We next sought to determine whether DNMT1 regulates monocyte adhesion to endothelial cells, which is a required step in the arteriogenesis cascade $52,56,58,60,62,246$. HUVECs were transfected with DNMT1 siRNA (siDNMT1) or non-targeting control (siC) and subjected to the biomimetic shear stress waveforms. DNMT1 expression was increased $>40 \%$ in HUVECs exposed to nonreversed compared to reversed shear stress waveforms in siC-treated conditions (Figure 5.3A). This corresponded to a $60 \%$ reduction in monocyte adhesion $(p=0.023)$ to HUVECs exposed to the non-reversed shear stress waveform (Figure 5.3B-C). Knockdown of DNMT1 significantly $(p=0.002)$ increased monocyte adhesion to HUVECs exposed to the non-reversed waveform compared to siC, while there was no significant effect on HUVECs exposed to the reversed waveform (Figure 5.3B-C). 
A.

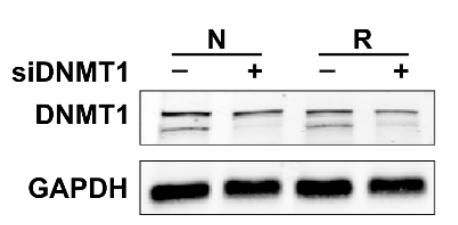

B.

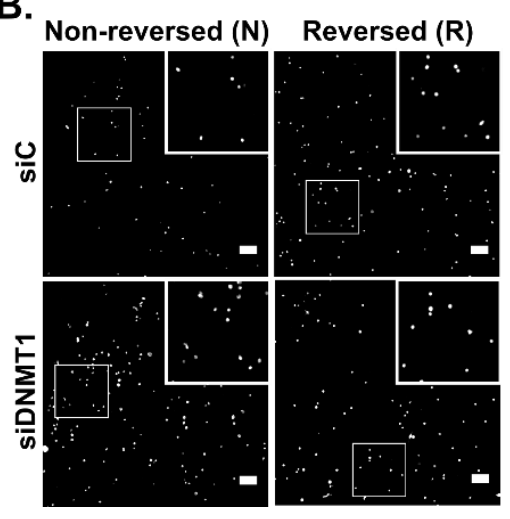

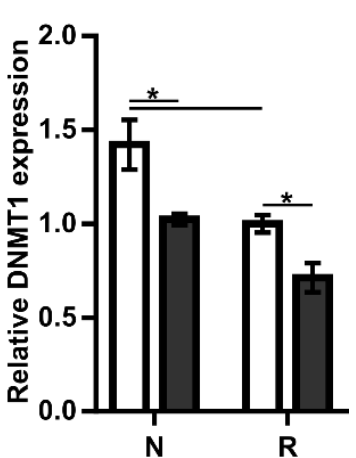

C.

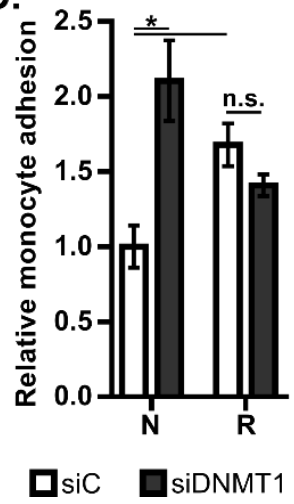

Figure 5.3. DNMT1 regulates the adhesion of monocytes to endothelial cells exposed to a non-reversed increase in shear stress magnitude in-vitro.

A) Relative DNMT1 protein expression, normalized to GAPDH, in HUVECs transfected with either DNMT1 siRNA (siDNMT1) or nontargeting control (siC) and exposed to the nonreversed $(N)$ or reversed $(R)$ biomimetic shear stress waveforms in Figure 5.2, 6-hours post simulated FAL $(n=4)$. * $p<0.05$, two-way ANOVA followed by a Holm-Sidak multiple comparisons test. B) Representative confocal microscopy images of fluorescently labeled THP-1 monocytes (white) adhered to flow-exposed HUVECs (Scale bar $=100 \mu \mathrm{m}$ ). Insets are the magnified $300 \mu \mathrm{m} \times 300 \mu \mathrm{m}$ regions outlined by white boxes. C) Bar graph of the relative number of adhered monocytes in each condition $(n=3)$. ${ }^{*} p<0.05$, two-way ANOVA followed by a HolmSidak multiple comparisons test. Data are mean \pm SEM.

\subsubsection{The arteriogenic capacity of non-reversed collateral artery segments is restored by DNMT1 inhibition.}

Our observations led us to test the hypothesis that arteriogenic capacity can be rescued in nonreversed collateral artery segments by reversing DNA hypermethylation through DNMT1 inhibition. As outlined in Figure 5.4A, we performed FALs on C57BL/6 mice and allowed them to recover for two weeks, which is sufficient time for collaterals to achieve steady-state diameters in this model ${ }^{237}$. We then treated mice with daily i.p. injections of $0.1 \mathrm{mg} / \mathrm{kg} 5$-Aza-2'-deoxycytidine (5AZA) or $0.1 \%$ DMSO vehicle control for an additional two weeks (Figure $5.4 A$ ). $5 \mathrm{AZA}$ is a nucleoside analog that preferentially targets DNMT1 via ubiquitin-dependent proteasomal degradation ${ }^{247} .5 A Z A$ treatment was shown to be effective in reducing global DNA methylation after only one week by high resolution melting (HRM) (Figure 5.4B). 
A.

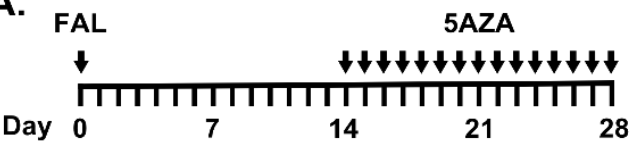

C.
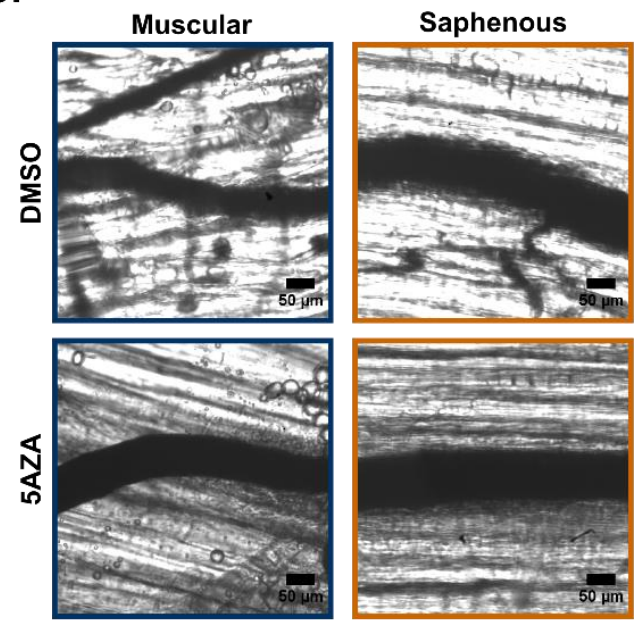

E.

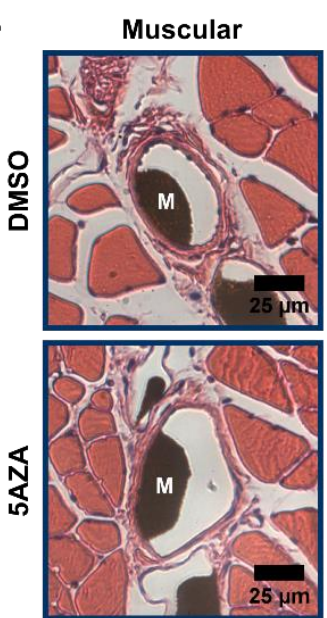

B.

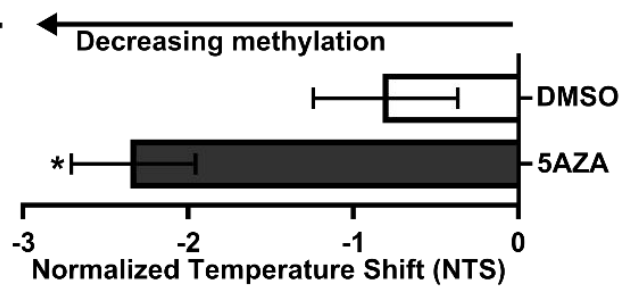

D.

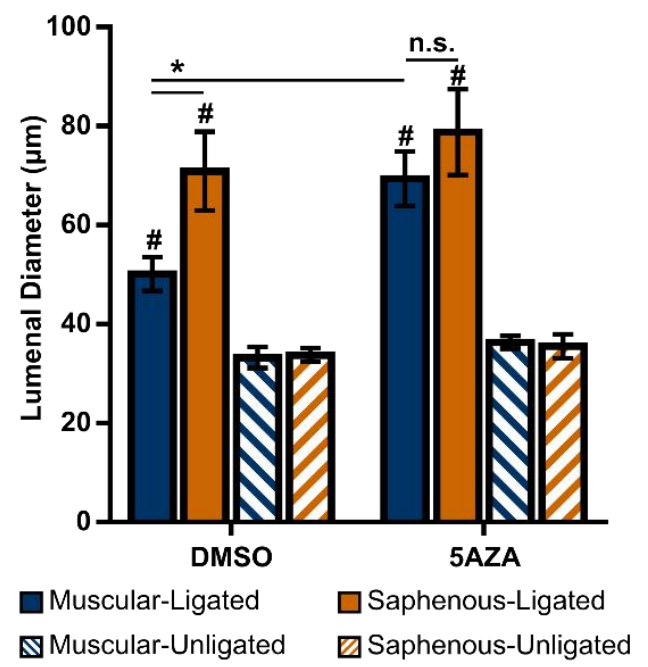

F.

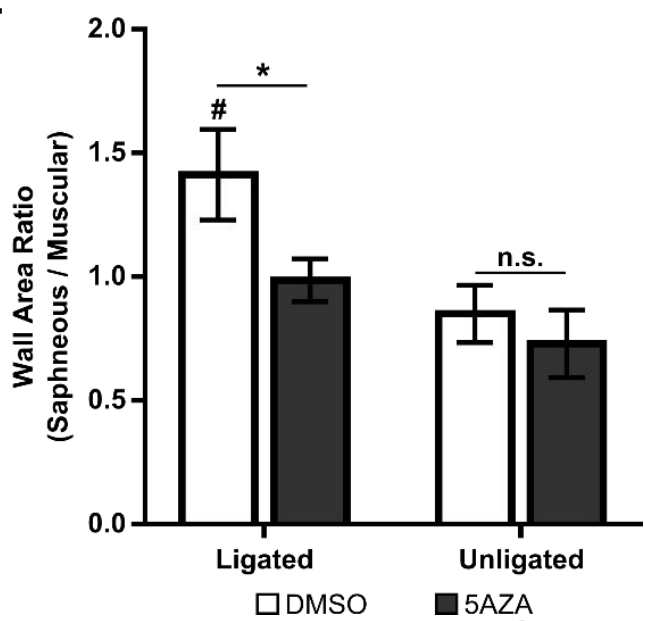

Figure 5.4. The arteriogenic capacity of non-reversed collateral artery segments is restored by DNMT1 inhibition.

A) Experimental treatment time-course. Femoral arterial ligation (FAL) is performed on Day 0. On day 14, mice begin receiving daily i.p. injections of DMSO or 5-Aza-2'-deoxycytidine (5AZA) until day 28. B) Bar graph of normalized temperature shift (NTS) determined by high resolution melting (HRM) of LINE1 repeat elements after 7 days treatment $(n=6)$. ${ }^{*} p<0.05$ between DMSO and 5AZA, Student's t-test. C) Representative vascular cast images from muscular (non-reversed) and saphenous (reversed) collateral artery regions 28 days post-FAL from C57BL/6 mice treated according to $(B)($ Scale Bar $=50 \mu \mathrm{m})$. D) Bar graph of regional lumenal diameter in DMSO or 5 AZA treated mice. ${ }^{*} p<0.05 ; \# p<0.01$ between ligated and unligated within the given region. Two-way ANOVA followed by a Holm-Sidak multiple comparisons test. E) Representative H\&E stained cross-sections of gracilis collateral artery regions in DMSO and 5AZA treated mice at day 28 post-FAL (Scale bar $=25 \mu \mathrm{m}$ ). Microfil casting material $(\mathrm{M})$ is evident in artery lumen. F) Bar graph of wall area ratio of saphenous:muscular collateral regions. \#p<0.01 between ligated and unligated for a given treatment; ${ }^{*} \mathrm{p}<0.05$ between DMSO and 5AZA treated mice; Two-way ANOVA followed by a Holm-Sidak multiple comparisons test. Data are mean \pm SEM. 
Vascular casting was used to determine collateral artery diameter in both muscular (nonreversed) and saphenous (reversed) regions 28 days post-FAL. Consistent with previous results (Figure 5.1C and Heuslein et al. ${ }^{237}$ ), we observed limited arteriogenesis in muscular (nonreversed) compared to saphenous (reversed) collateral artery segments in DMSO-treated vehicle control mice. However, DNMT1 inhibition increased the arteriogenic capacity of nonreversed collateral segments by $>40 \%$, while there was no significant $(p=0.33)$ effect on reversed segments (Figure 5.4C-D). Cross-sections were used to determine collateral wall area, a metric that further indicated that the differential arteriogenic capacity along the collateral length in DMSO-treated mice was normalized by DNMT1 inhibition (Figure 5.4E-F). Of note, we observed similar results in FAL-treated Balb/c mice (Supplemental Figure 5.6). Here, DNMT1 inhibition increased arteriogenic capacity by $\sim 44 \%$ in non-reversed segments, while there was no effect on reversed segments $(p=0.163)$, indicating that this response is not limited to the C57BL/6 strain.

\subsubsection{Shear stress set-point is re-established in non-reversed collateral artery segments by DNMT1 inhibition}

Finally, we sought to determine if DNMT1-dependent DNA hypermethylation alters non-reversed collateral artery shear stress set-point. Mice were treated with 5AZA or DMSO according to Figure 5.4A. Relative changes in collateral artery shear rates were then determined by transillumination laser speckle flowmetry (LSF) ${ }^{176} 28$ days post-FAL. Interestingly, in DMSO-treated vehicle control mice, shear stress remained elevated ( 2.5-fold) in muscular (non-reversed) collateral segments, while it was restored to pre-FAL levels in saphenous (reversed) segments (Figure 5.5). DNMT1 inhibition restored shear stress in non-reversed segments to pre-FAL levels, whereas there was no significant effect on reversed segments (Figure 5.5). 
A.

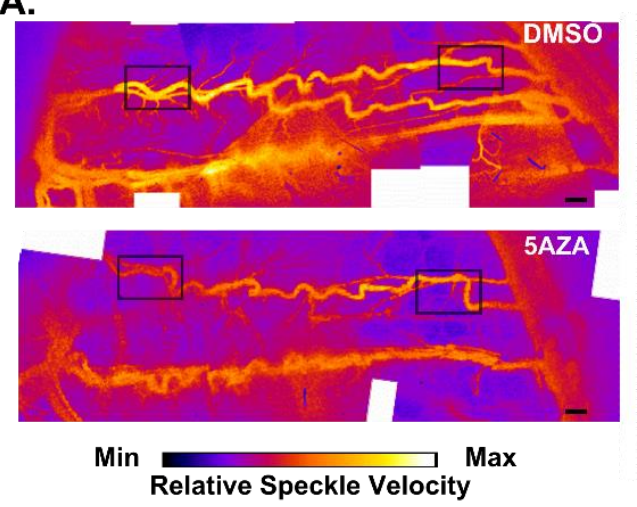

B.

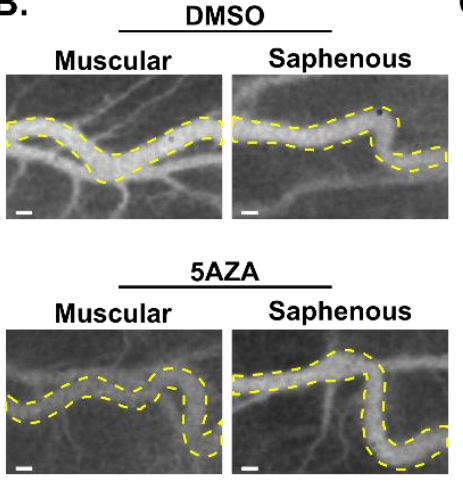

C.

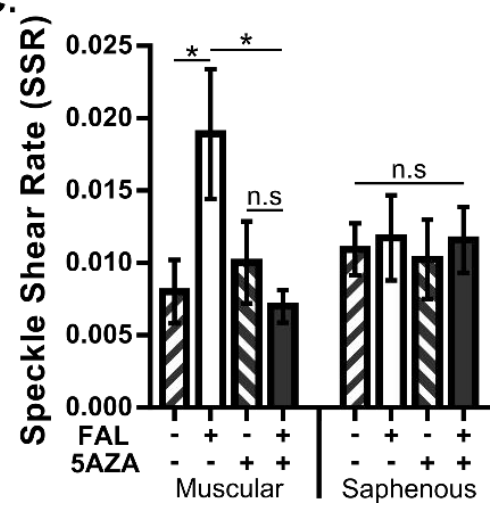

Figure 5.5. Shear stress set-point in non-reversed collateral artery segments is restored by DNMT1 inhibition.

A) Representative relative speckle velocity maps across the primary gracilis muscle collateral pathways 28 days post-FAL in C57BL/6 mice treated with DMSO and 5AZA according to Figure 5.4A. (Scale bar = $500 \mu \mathrm{m})$. B) Greyscale images of the 300pixel X 200pixel boxed regions shown in (A) for both the muscular (non-reversed, left) and saphenous (reversed, right) collateral regions. (Scale bar $=100 \mu \mathrm{m}$ ). Dotted line indicates collateral artery ROI used for analysis. C) Bar graph of regional speckle shear rate in DMSO and $5 A Z A$ treated mice $(n=6$ for DMSO, $n=5$ for $5 A Z A)$. ${ }^{*} p<0.05$, n.s. $=$ not significant. Student's t-test. Data are mean \pm SEM.

\subsection{Discussion}

In this study, we tested the hypothesis that DNMT1-dependent EC DNA methylation regulates arteriogenic capacity via adjustments to shear stress set-point. Previously, we demonstrated that collateral artery segments exposed to a non-reversed increase in shear stress magnitude display limited arteriogenic capacity when compared to segments exposed to reversed flow direction. Here, we first determined that these non-reversed collateral segments exhibit global DNA hypermethylation in-vivo. We then applied flow waveforms, biomimetic of those leading to either amplified arteriogenic capacity (i.e. reversed flow) or constrained arteriogenic capacity (i.e. nonreversed flow) in-vivo, to ECs in-vitro, and performed both RRBS and mRNA-sequencing. ECs exposed to the non-reversed waveform exhibited increased DNMT1 expression, genome-wide hypermethylation of significantly regulated gene promoters, and a DNMT1-dependent reduction in pro-arteriogenic monocyte adhesion. Together, this led us to next test whether DNMT1 regulates arteriogenic capacity in-vivo. We ascertained that, in non-reversed collateral artery segments, DNMT1 inhibition rescued arteriogenic capacity and returned the elevated shear stress 
in these segments back to its original set point. Collectively, these results demonstrate that DNMT1-dependent DNA hypermethylation constrains arteriogenesis by dampening EC mechanosensing, which effectively augments shear stress set-point. The epigenetic regulation of shear stress set-point may therefore have critical impact on both endogenous and therapeutic arteriogenesis in patients with PAD.

\subsubsection{Mapping the EC mechanosensitive DNA methylation to differential arteriogenic capacity}

The significance of epigenetics in vascular biology, with roles as regulators of molecular signaling known to drive physiology and as potential therapeutic targets to treat disease, is now wellrecognized $^{98,99,103}$. Both histone modifications and microRNAs regulate flow-mediated EC gene expression $^{248-254}$ and arteriogenesis ${ }^{144,151,155,162,163}$; however, DNA methylation has only recently been shown to regulate flow-mediated EC gene expression in any context ${ }^{238-241}$. Moreover, to our knowledge, the role of DNMT1-mediated DNA methylation in arteriogenesis has not been previously studied.

Our study directly maps EC mechanosensitive DNA methylation to differential, sustained arteriogenesis responses. Additionally, by using both RRBS and mRNA-sequencing, we were able to determine mechanosensitive genes whose expression correlated to gene promoter DNA methylation status. Gene ontology analysis of these genes identified a number of pathways crucial for EC mechanotransduction and arteriogenesis, including several metabolism, transcription, MAPK signaling, and cell transport pathways ${ }^{43,73}$. Of note, SIRT4 was involved in a number of these significantly overrepresented pathways (Supplemental Figure 5.5). SIRT4 has been shown to disrupt the NFKB pathway whereby overexpression of SIRT4 in ECs abrogates NFKB nuclear translocation and decreases expression of pro-inflammatory cytokines (IL-1 $\beta$, IL-6, and IL-8), MMP-9, and ICAM-1255. As we also previously reported ECs exposed to non-reversed shear stress waveforms exhibited decreased NFKB-ICAM-1 activity ${ }^{237}$ and because the NFKB-ICAM-1 
pathway is crucial for arteriogenesis ${ }^{56,237,256}$, flow-dependent regulation of SIRT4 could be of particular interest.

Additionally, earlier studies examining flow-mediated EC DNA methylation identified Homeobox transcription factors (e.g. HOXA5) as differentially regulated in atheroprone conditions $^{239,241}$. HOX transcription factors are considered "master regulators" as they regulate EC proliferation, migration, differentiation, morphogenesis, and permeability during development and vascular remodeling processes ${ }^{257}$. Interestingly, we found HOXB3 to be among the genes down-regulated (decreased $25 \%)$ and hypermethylated (20\% vs. $0.1 \%$ methylation) in nonreversed compared to reversed conditions (Supplemental Table 5.4). As HOXB3 regulates EC activation and promotes angiogenesis ${ }^{258}$, our results are consistent with the hypothesis that hypermethylation of the HOXB3 promoter decreases its expression, thereby limiting EC activation and arteriogenic potential of collateral artery segments.

\subsubsection{The role of DNMT1 in flow-mediated endothelial inflammation is dependent on hemodynamic context}

Monocyte adhesion to an activated endothelium is required for collateral artery

growth $52,56,58,60,62,246,68$. Here, we used a monocyte adhesion assay, which has been previously used to examine flow-mediated EC function $237,239,259,260$, to determine the role of endothelial DNMT1 expression in regulating this essential step in arteriogenesis. Our results showed increased DNMT1 expression and limited monocyte adhesion to ECs exposed to the nonreversed shear stress waveform. Monocyte adhesion to ECs under non-reversed conditions was enhanced with DNMT1 inhibition but not under reversed conditions, indicating an antiinflammatory role for DNMT1 that is dependent on hemodynamic context. In contrast, DNMT1 has been shown to also promote EC inflammation in HUVECs exposed to atheroprone flow conditions demonstrated by a DNMT1-dependent increase in monocyte adhesion ${ }^{239}$. However, our pro-arteriogenic flow conditions, which include a laminar flow pre-conditioning phase, are very 
different from oscillatory, atheroprone conditions ${ }^{239}$, further supporting the idea that DNMT1's role in endothelial inflammation is dependent on hemodynamic context. Furthermore, exposure to atheroprone conditions led to a chronic increase in DNMT1 expression ${ }^{239}$, whereas our results suggest a transient increase in DNMT1 expression. This adaptive, instead of chronic, response may contribute to context-dependent role of DNMT1 on monocyte adhesion to ECs.

\subsubsection{Molecular regulation of collateral artery shear stress set-point}

Finally, we have determined that DNMT1-dependent DNA methylation regulates, at least in part, long-term arteriogenic capacity and shear stress set-point. The concept of an arterial homeostatic wall shear stress magnitude (i.e. shear stress set-point) at which vessels maintain a steady-state lumenal diameter ${ }^{85}$ arises from Murray's principle of minimum work ${ }^{86}$. Outward collateral artery growth is therefore hypothesized to stop once normalization to the shear stress set-point has been achieved $39,41,80,81$. Premature normalization to the shear stress set-point has been a predominant rationalization for the failure of collateral arteries to realize full arteriogenic capacity, frequently reaching only $30-40 \%$ of the maximal conductance ${ }^{49}$. However, our results indicate that shear stress actually remains elevated in collateral artery segments exhibiting limited arteriogenic capacity. In essence, EC DNA hypermethylation prevents these collaterals from continuing to increase in diameter; therefore, shear stress remains chronically elevated. Yet, when DNMT1-dependent DNA methylation is inhibited, these collaterals become re-sensitized to their elevated shear stress and are able to resume the arteriogenic process until the original setpoint is achieved (Figure 5.6). This response appears to require a basal level of DNA methylation

as there was no effect of DNMT1 inhibition on collateral artery segments that were not hypermethylated. 
DNA hypermethylation

(i.e. Non-reversed flow segments)

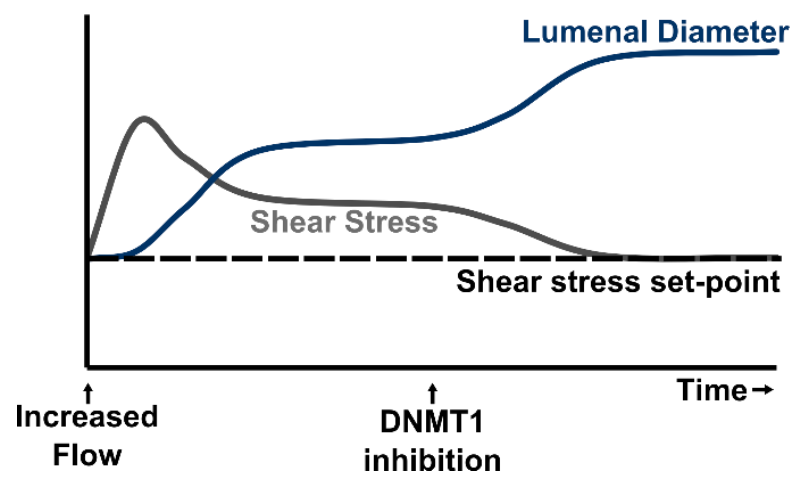

DNA hypomethylation

(i.e. Reversed flow segments)

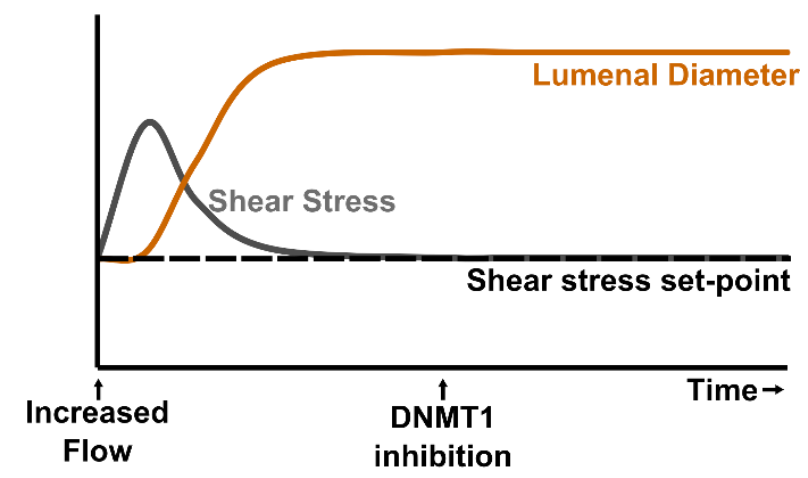

Figure 5.6. Summary of DNMT1-dependent regulation of arteriogenic capacity and shear stress setpoint in gracilis adductor collateral arteries following FAL.

There are several other studies that have reported an altered set-point following arterial adaptation. To this end, a left-right carotid anastomosis was used to induce an acute increase in blood flow in the common carotid artery mature and weanling rabbits. Two months later, shear stress remained augmented in mature rabbits due to a lack of compensatory arterial enlargement, whereas weanling rabbits exhibited significant diameter enlargement, enabling for shear stress to normalize to the set-point ${ }^{83}$. This age-dependent remodeling was also observed in rats in which ligation of the left internal and external carotid arteries increased right carotid blood flow by $46 \%$. After 4-weeks, the right carotid outer diameter increased and shear stress returned to initial values in juvenile but not adult rats ${ }^{94}$. The constrained arterial remodeling and augmented shear stress set-point of aged animals is strikingly similar to the phenotype we observed in non-reversed collateral segments. Given that age alters DNA methylation ${ }^{123}$, together, these results would be consistent with the hypothesis that acutely increased shear stress yields incomplete arterial remodeling and augmented shear stress set-point due to DNA hypermethylation.

\subsubsection{Clinical perspective}

Given our results, modulation of the shear stress set-point by DNMT1 inhibition could represent a therapeutic strategy for treating arterial occlusive diseases. By focusing on the molecular mechanisms regulating the maturation stage of arteriogenesis, as opposed to initiation and growth 
stages, such an approach could better account for the chronic nature of arterial occlusive diseases in humans. To this end, we didn't begin DNMT1 inhibition until 2-weeks after FAL. Though a few previous studies have demonstrated increased arteriogenic capacity after such a delayed treatment ${ }^{58,66,261}$, ours is the first to demonstrate an epigenetic mechanism. DNMT1 inhibition may also be clinically advantageous because it appears to avoid the so-called "Janus phenomenon", which refers to the conundrum created by the fact that pro-arteriogenic therapies also tend to promote atherosclerosis ${ }^{262}$. To this point, DNMT1 inhibition with 5AZA reverses DNA hypermethylation induced by atheroprone shear stress ${ }^{239,240}$ and reduces atherosclerotic plaque size $^{239}$. Ultimately, because shear-stress induced changes in DNMT1 expression markedly affect both atherosclerosis and arteriogenic capacity, DNMT1 may represent an interesting target for PAD therapy.

\subsection{Acknowledgements}

The authors would like to thank the University of Virginia Research Histology Core (under the direction of Sheri VanHoose) for histological tissue processing.

\subsection{Sources of Funding}

Supported by NIH R03 EB017927 and R01 EB020147. JLH was supported by National Science Foundation Graduate Research Fellowship Program Grant No. NSF DGE-1315231. 


\subsection{Supplemental Figures}

A.

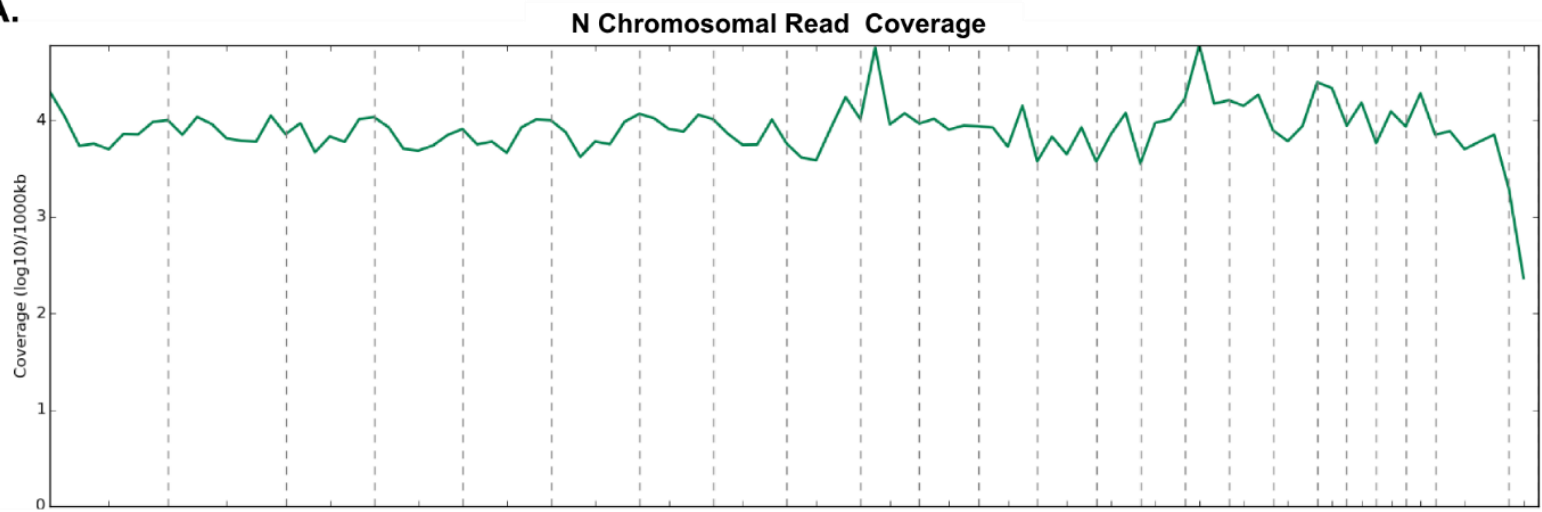

B.

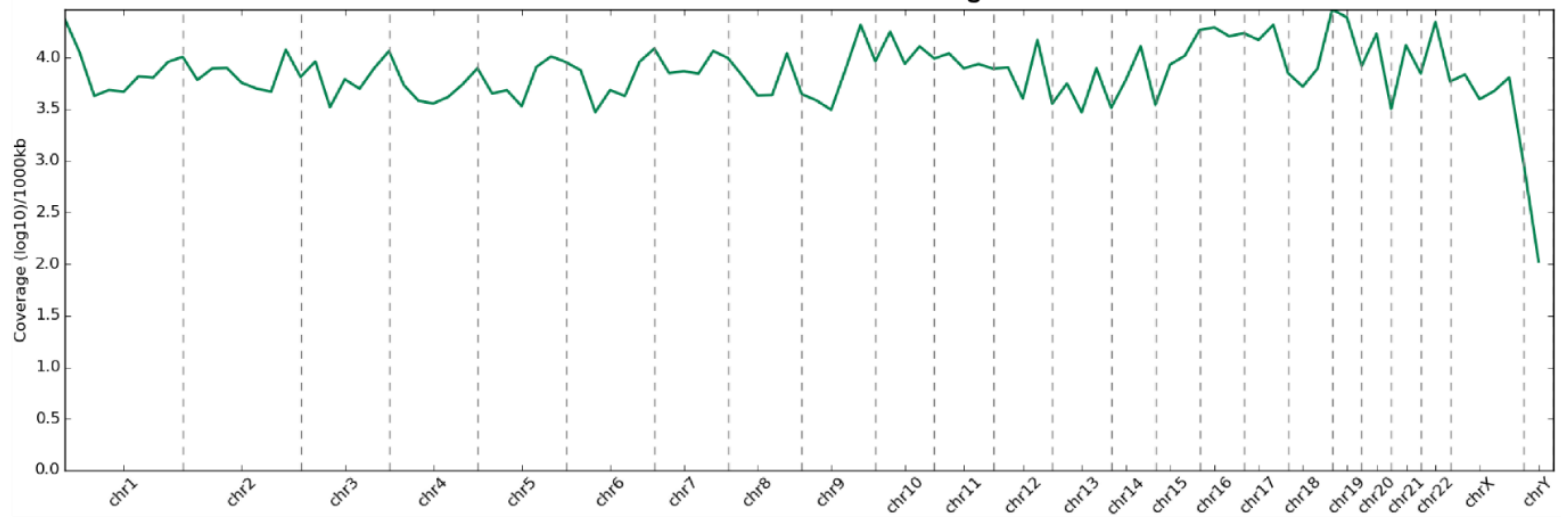

C.

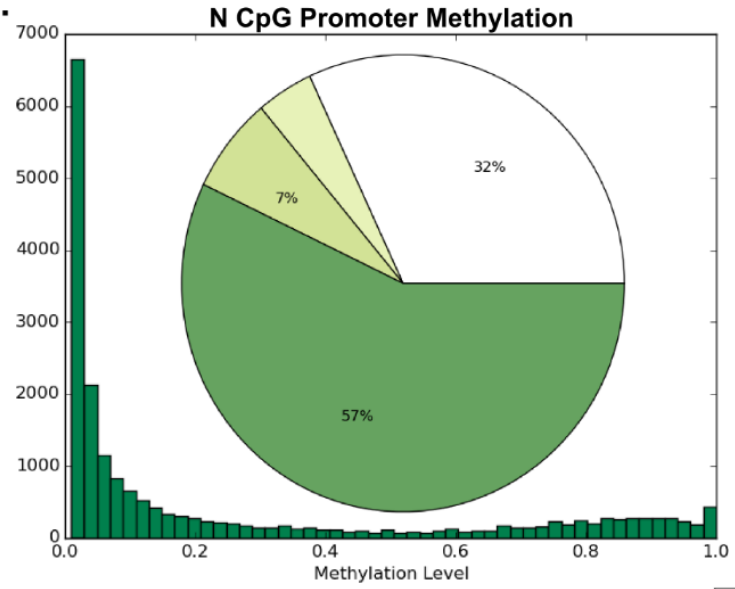

D.

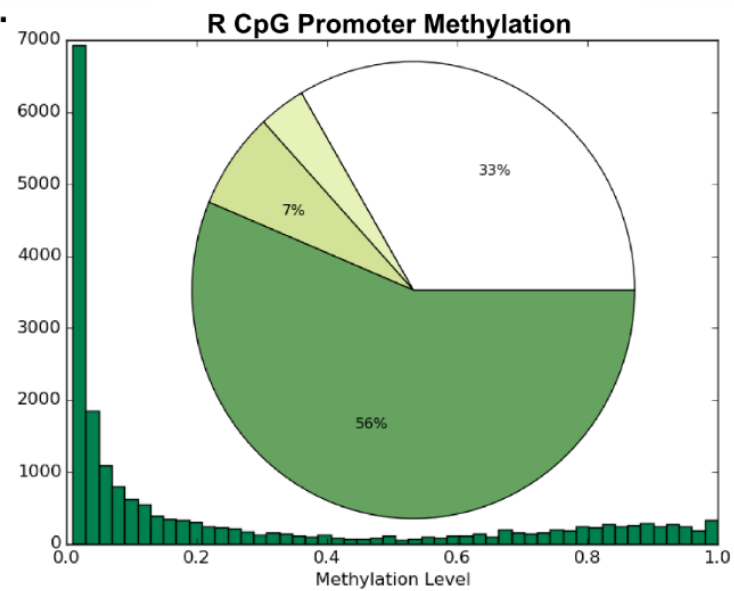

$$
\begin{array}{|l|l|}
\hline \text { No covered } \\
\square \text { Less than 10x } \\
\text { 10-49x } \\
\text { More than 50x } \\
\text { CpG Promoter Coverage }
\end{array}
$$

Supplemental Figure 5.1. Similar degree of methylation coverage in HUVECs exposed to biomimetic shear stress waveforms.

A-B) Representation of methylation read coverage by chromosome across the genome for HUVECs exposed to non-reversed $(N)$ and reversed (R) shear stress waveforms $C-D$ ) Pie charts of CpG site coverage and histograms of methylation level in gene promoter region in HUVECs exposed to non-reversed $(\mathrm{N})$ and reversed $(\mathrm{R})$ shear stress waveforms. 
A.

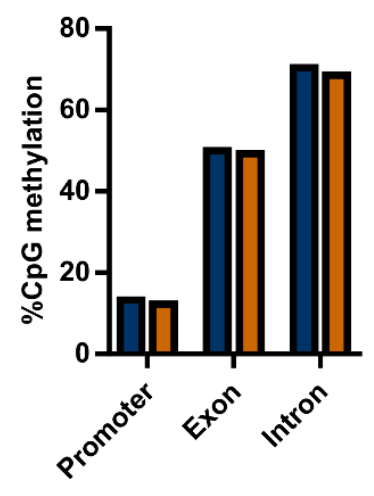

C.

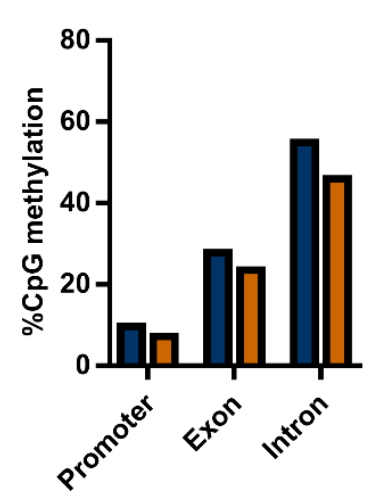

B.

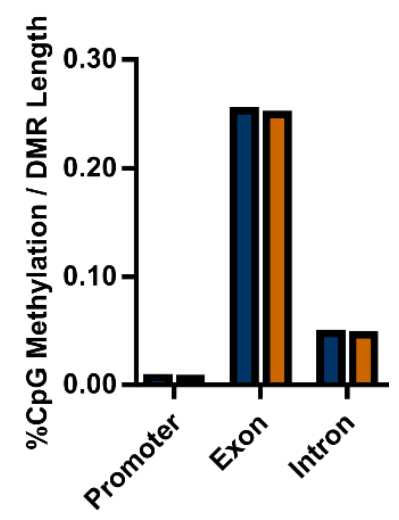

D.

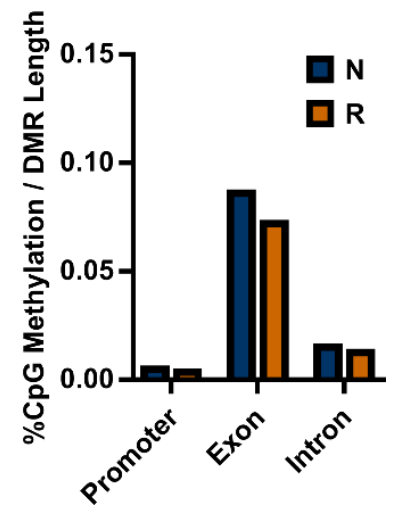

Supplemental Figure 5.2. DNA methylation across different gene regions.

A-B) Mean \% CpG methylation (\#methylated CpG sites / total CpG sites in given DMR) and mean \%CpG methylation normalized by DMR length in promoter, exon, and intron gene regions for all DMRs with $\geq 10 x$ CpG coverage in a given DMR. C-D) Mean \%CpG methylation and mean \%CpG methylation normalized to DMR length in promoter, exon, and intron gene regions for all DMRs with $\geq 10 \times \mathrm{CpG}$ coverage in a given DMR and FDR $<0.1$ between HUVECs exposed to non-reversed (blue) and reversed (orange) shear stress waveforms. 

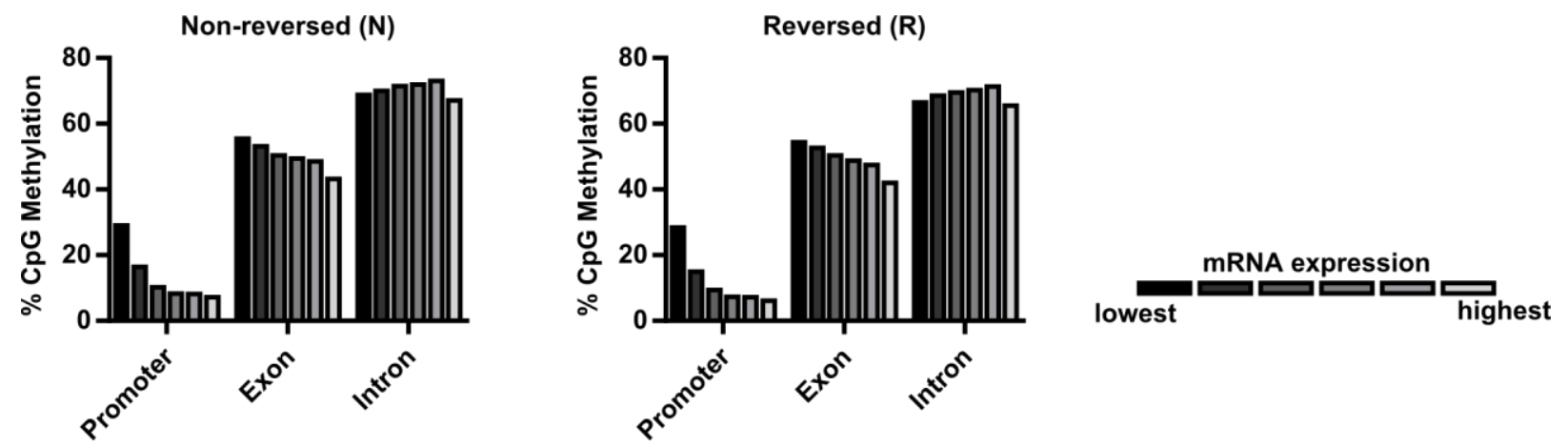

Supplemental Figure 5.3. DNA methylation negatively correlates to mRNA expression in gene promoter regions.

Raw mRNA expression (FPKM) from HUVECs exposed to either non-reversed/N or reversed/R shear stress waveforms was ordered from lowest mRNA expression to highest and divided into six, equal sized bins. Mean \%CpG methylation was determined for each bin within the promoter, exon, and intron gene regions. Only DMRs with $\geq 10 \times \mathrm{CpG}$ coverage were included in the analysis. FPKM = fragments per kilobase of transcript per million mapped reads. 
A.

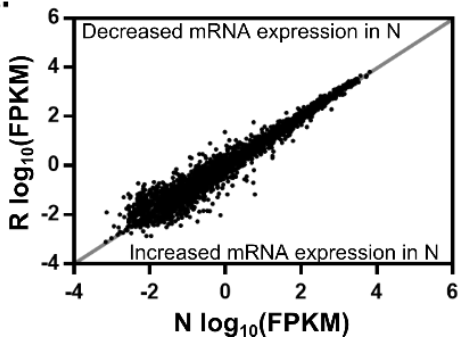

B.

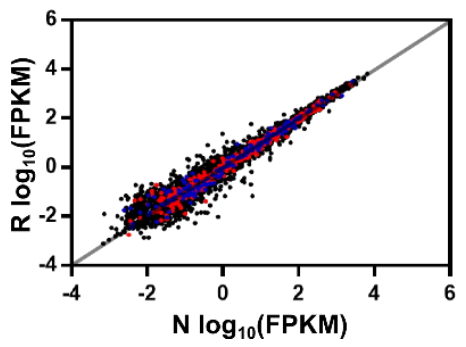

C.

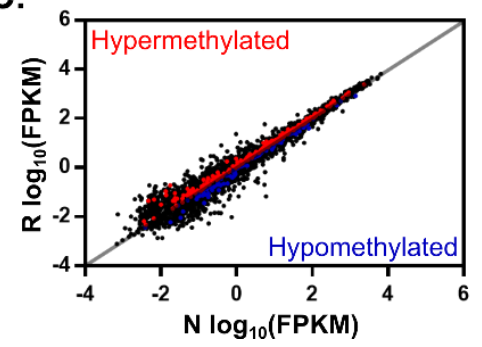

Supplemental Figure 5.4. Genome-wide mRNA expression patterns in HUVECs exposed to arteriogenesis biomimetic shear stress waveforms.

A-C) HUVECs were exposed to biomimetic shear stress waveforms, RNA was isolated 6-hours post-FAL, and genome-wide mRNA expression was determined by mRNA-sequencing. Scatter plots indicate relative expression of each gene represented in both non-reversed $(N)$ and reversed $(R)$ conditions and contained in our RRBS dataset (17,227 total). A) Indicates all genes corresponding to promoter regions of genes with a methylation ratio difference $\geq|0.10|$ and FDR $<0.1$ between non-reversed and reversed conditions. B) Contains all 17,227 genes, where genes with significantly hypermethylated promoters (in $\mathrm{N}$ relative to $\mathrm{R}$ ) are red and hypomethylated genes are blue, corresponding to Supplemental Table 5.3. C) All 17,227 genes, where significantly hyper- or hypomethylated genes are also downregulated or upregulated, respectively, in N relative to R, corresponding to Supplemental Table 5.4 and Supplemental Table 5.5. $\mathrm{FPKM}=$ fragments per kilobase of transcript per million mapped reads. 


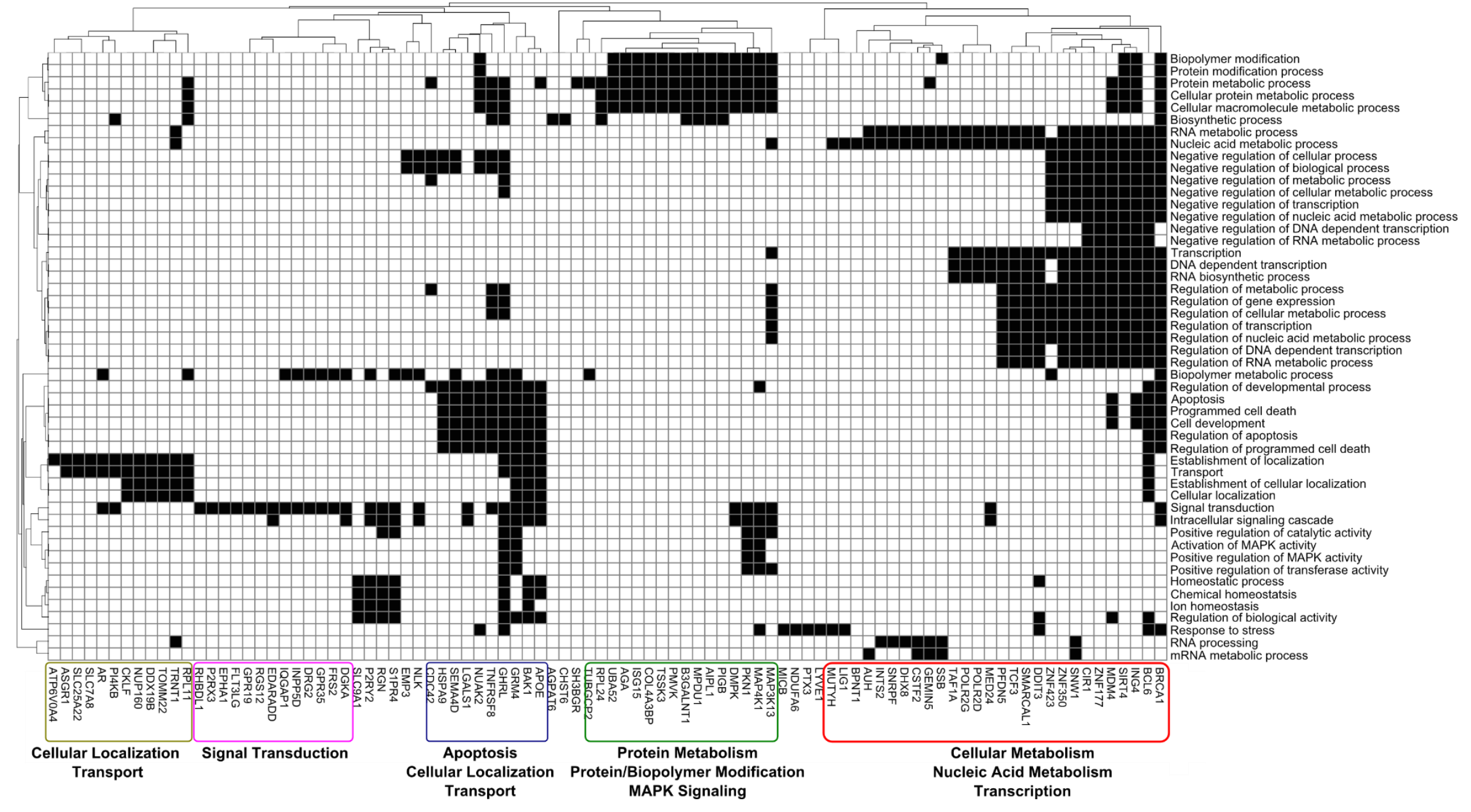

Supplemental Figure 5.5. Top 50 most significantly overrepresented gene ontology (GO) biological processes.

Heat map of the top 50 significantly overrepresented biological process GO terms (columns) for genes with differential changes in expression corresponding to significantly different $\mathrm{CpG}$ promoter methylation in non-reversed vs. reversed conditions (i.e. genes that were down-regulated and had a hypermethylated promoter or were up-regulated and had a hypomethylated promoter in non-reversed versus reversed conditions) as listed in Supplemental Table 5.4 and Supplemental Table 5.5. Black indicates the presence of a gene in a GO process whereas white indicates its absence. Only genes present in at least one GO process are shown. 
A.

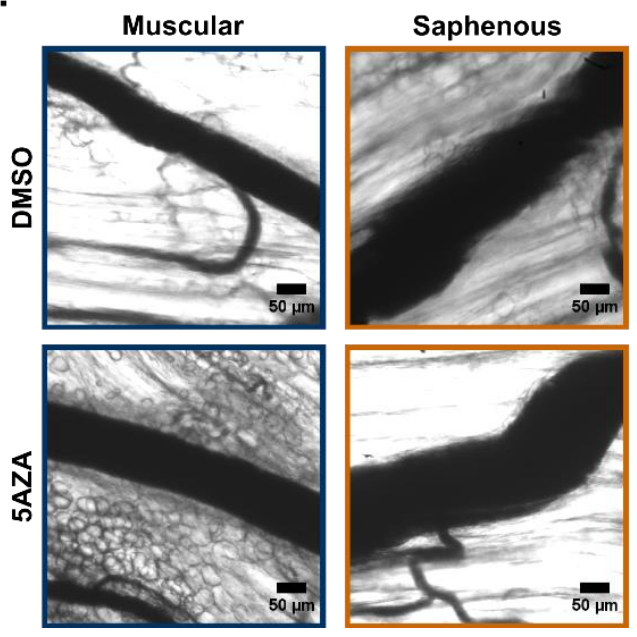

B.

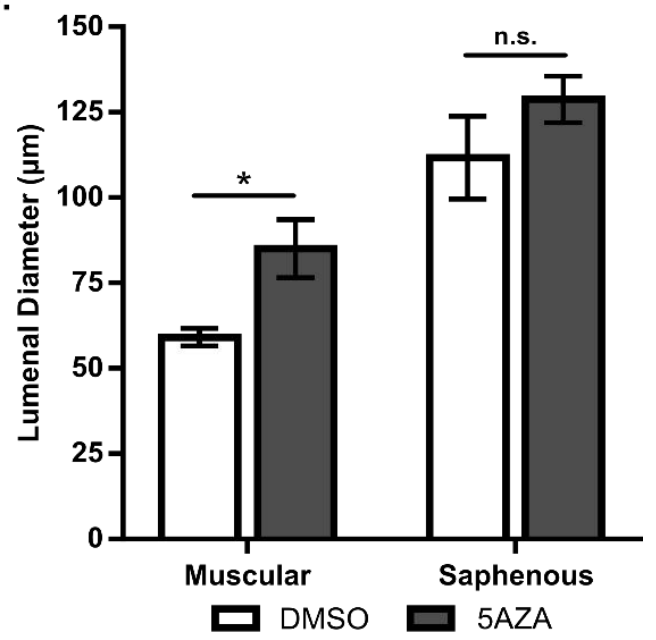

Supplemental Figure 5.6. DNMT1 inhibition also restores the arteriogenic capacity of non-reversed collateral artery segments in Balb/c mice

A) Representative vascular cast images from muscular (non-reversed) and saphenous (reversed) collateral artery regions 28 days post-FAL from Balb/c mice treated according to Figure $5.4 A$. (Scale Bar $=50 \mu \mathrm{m}$ ). B) Bar graph of regional lumenal diameter in DMSO or 5AZA treated mice. ${ }^{*} p<0.05$ between DMSO and 5AZA treated mice; Two-way ANOVA followed by a Holm-Sidak multiple comparisons test. Data are mean \pm SEM. 


\subsection{Supplemental Tables}

Supplemental Table 5.1. Summary of total number of reads, mapping ratio, and CpG coverage in RRBS datasets.

\begin{tabular}{ccccccccc}
\hline \hline Label & Species & $\begin{array}{c}\text { Seq } \\
\text { Type }\end{array}$ & $\begin{array}{c}\text { Total Read } \\
\#\end{array}$ & $\begin{array}{c}\text { Mapped } \\
\text { Read \# }\end{array}$ & $\begin{array}{c}\text { Mapping } \\
\text { Ratio }\end{array}$ & $\begin{array}{c}\text { Unique } \\
\text { CpG }\end{array}$ & $\begin{array}{c}\text { CpG } \\
\text { Coverage } \\
(\mathrm{X})\end{array}$ & $\begin{array}{c}\text { Bisulfite } \\
\text { Conversion } \\
\text { Rate }\end{array}$ \\
\hline $\mathrm{N}$ & $\begin{array}{c}\text { human } \\
\text { (hg19) }\end{array}$ & MiniSeq & $34,206,095$ & $15,411,220$ & $45.05 \%$ & $7,126,531$ & 7 & $98.24 \%$ \\
& $\begin{array}{c}\text { human } \\
\text { (hg19) }\end{array}$ & MiniSeq & $23,777,663$ & $13,438,821$ & $56.52 \%$ & $7,269,847$ & 7 & $99.21 \%$
\end{tabular}


Supplemental Table 5.2. Summary of total number of reads, mapped reads, and mapping ratio for mRNA-seq datasets.

\begin{tabular}{ccccccc}
\hline \hline Label & Species & Seq Type & $\begin{array}{c}\text { Total Read } \\
\text { Count }\end{array}$ & $\begin{array}{c}\text { Total Read } \\
\text { Count After } \\
\text { QC }\end{array}$ & $\begin{array}{c}\text { Mapped } \\
\text { Read } \\
\text { Count }\end{array}$ & $\begin{array}{c}\text { Mapping } \\
\text { Ratio }\end{array}$ \\
\hline $\mathrm{N}$ & $\begin{array}{c}\text { human } \\
\text { (hg19) }\end{array}$ & mRNASeq & $93,600,141$ & $91,395,543$ & $86,700,087$ & $94.90 \%$ \\
R & $\begin{array}{c}\text { human } \\
\text { (hg19) }\end{array}$ & mRNASeq & $85,738,933$ & $83,771,202$ & $79,624,282$ & $95.00 \%$
\end{tabular}


Supplemental Table 5.3. All DMRs corresponding to the promoter regions of genes with a methylation ratio difference $\geq|0.10|$ and FDR $<0.1$ between non-reversed and reversed conditions

\begin{tabular}{|c|c|c|c|c|c|c|c|}
\hline gene name & $\begin{array}{c}\mathrm{R} \\
\text { methylation } \\
\%\end{array}$ & $\begin{array}{c}\mathrm{N} \\
\text { methylation } \\
\%\end{array}$ & $\begin{array}{c}\text { methylation } \\
\% \\
\text { difference } \\
(\mathrm{N}-\mathrm{R})\end{array}$ & $\begin{array}{l}\text { methylation } \\
\text { adjusted } \\
\text { Pvalue }\end{array}$ & $\begin{array}{c}\text { mRNASeq } \\
\text { Nvalue }\end{array}$ & $\begin{array}{l}\text { mRNASeq } \\
\text { Rvalue }\end{array}$ & $\begin{array}{c}\text { mRNASeq } \\
\text { Log2FC }\end{array}$ \\
\hline EPS8L3 & 81 & 21 & -60 & 6.33E-04 & 0.03 & 0.01 & 1.90 \\
\hline DSCR8 & 91 & 36 & -55 & 5.92E-03 & 0.22 & 0.28 & -0.36 \\
\hline MAB21L2 & 100 & 47 & -53 & 8.13E-03 & 0.01 & 0.07 & -2.72 \\
\hline EGFLAM & 50 & 0 & -50 & 6.61E-03 & 0.77 & 0.39 & 0.98 \\
\hline C8orf74 & 52 & 5 & -47 & 1.44E-08 & 0.01 & 0.03 & -1.55 \\
\hline MICB & 67 & 20 & -47 & 1.94E-02 & 8.63 & 5.20 & 0.73 \\
\hline AQP4-AS1 & 75 & 30 & -45 & $6.69 \mathrm{E}-02$ & 0.13 & 0.16 & -0.38 \\
\hline BPNT1 & 41 & 0 & -41 & $6.95 \mathrm{E}-05$ & 27.27 & 24.52 & 0.15 \\
\hline POM121L9P & 98 & 58 & -40 & $2.38 \mathrm{E}-03$ & 0.02 & 0.01 & 1.59 \\
\hline ITGBL1 & 92 & 55 & -37 & $4.98 \mathrm{E}-05$ & 0.89 & 0.65 & 0.47 \\
\hline PLCH2 & 81 & 45 & -36 & 1.33E-04 & 0.04 & 0.02 & 1.30 \\
\hline SYNPO2L & 92 & 56 & -36 & 3.69E-02 & 0.16 & 0.13 & 0.36 \\
\hline PMEPA1 & 35 & 0 & -35 & $1.44 \mathrm{E}-08$ & 14.44 & 17.10 & -0.24 \\
\hline BTBD16 & 100 & 65 & -35 & $2.46 \mathrm{E}-02$ & 0.11 & 0.41 & -1.90 \\
\hline CSAD & 83 & 48 & -35 & $2.73 E-02$ & 9.61 & 7.18 & 0.42 \\
\hline NRG1 & 100 & 66 & -34 & 5.92E-02 & 29.29 & 34.46 & -0.23 \\
\hline SLC22A15 & 33 & 1 & -32 & $5.96 \mathrm{E}-07$ & 0.01 & 0.01 & -1.18 \\
\hline POTEF & 95 & 63 & -32 & $1.38 \mathrm{E}-04$ & 0.02 & 0.02 & -0.51 \\
\hline АCOT2 & 82 & 50 & -32 & $2.29 \mathrm{E}-02$ & 0.87 & 0.46 & 0.91 \\
\hline $\begin{array}{l}\text { ACP5 } \\
\text { CCDC74B- }\end{array}$ & 57 & 26 & -31 & $1.48 \mathrm{E}-08$ & 46.28 & 30.30 & 0.61 \\
\hline AS1 & 94 & 63 & -31 & 3.27E-02 & 0.12 & 0.11 & 0.11 \\
\hline C9orf153 & 74 & 43 & -31 & 6.69E-02 & 0.09 & 0.07 & 0.39 \\
\hline CD79B & 71 & 41 & -30 & $2.58 \mathrm{E}-05$ & 0.38 & 0.34 & 0.16 \\
\hline KIAA1549L & 80 & 50 & -30 & 4.65E-03 & 4.04 & 4.36 & -0.11 \\
\hline LAMB3 & 95 & 65 & -30 & 1.18E-02 & 10.22 & 8.45 & 0.27 \\
\hline LAMB3 & 95 & 65 & -30 & $1.18 \mathrm{E}-02$ & 10.22 & 8.45 & 0.27 \\
\hline PAPOLB & 52 & 23 & -29 & $1.60 \mathrm{E}-06$ & 0.00 & 0.02 & -2.59 \\
\hline METTL5 & 48 & 20 & -28 & $2.43 E-03$ & 66.99 & 63.77 & 0.07 \\
\hline ZNF503-AS1 & 33 & 5 & -28 & $3.35 \mathrm{E}-03$ & 0.38 & 0.20 & 0.93 \\
\hline RGS12 & 31 & 3 & -28 & 8.09E-03 & 29.65 & 28.00 & 0.08 \\
\hline PLEKHB1 & 60 & 32 & -28 & $2.61 \mathrm{E}-02$ & 2.43 & 2.29 & 0.09 \\
\hline KCNK2 & 69 & 41 & -28 & $3.75 \mathrm{E}-02$ & 0.09 & 0.13 & -0.49 \\
\hline PDGFB & 41 & 13 & -28 & $9.14 \mathrm{E}-02$ & 3.77 & 4.11 & -0.13 \\
\hline $\mathrm{EZH} 1$ & 45 & 18 & -27 & 1.47E-08 & 13.01 & 10.64 & 0.29 \\
\hline ZNF423 & 92 & 65 & -27 & 1.89E-02 & 2.56 & 2.41 & 0.08 \\
\hline CCDC152 & 46 & 20 & -26 & 8.49E-08 & 1.33 & 1.11 & 0.26 \\
\hline GGT1 & 34 & 9 & -25 & $2.79 E-02$ & 74.25 & 78.80 & -0.09 \\
\hline SLC13A3 & 25 & 1 & -24 & $1.44 \mathrm{E}-08$ & 0.07 & 0.09 & -0.34 \\
\hline MPDU1 & 52 & 28 & -24 & $1.02 \mathrm{E}-05$ & 44.96 & 44.47 & 0.02 \\
\hline PRKACA & 54 & 30 & -24 & $1.18 \mathrm{E}-03$ & 67.05 & 61.07 & 0.13 \\
\hline FGL2 & 35 & 11 & -24 & 7.45E-03 & 0.04 & 0.05 & -0.21 \\
\hline TOMM22 & 23 & 0 & -23 & $1.44 \mathrm{E}-08$ & 75.48 & 58.83 & 0.36 \\
\hline PLA2G4C & 36 & 13 & -23 & 5.39E-06 & 18.51 & 39.81 & -1.11 \\
\hline LINC00672 & 88 & 65 & -23 & 1.42E-02 & 0.32 & 0.25 & 0.34 \\
\hline KLK10 & 26 & 3 & -23 & 1.61E-02 & 0.37 & 0.32 & 0.22 \\
\hline GGT1 & 30 & 7 & -23 & 1.85E-02 & 74.25 & 78.80 & -0.09 \\
\hline
\end{tabular}




\begin{tabular}{|c|c|c|c|c|c|c|c|}
\hline DAND5 & 59 & 36 & -23 & 4.23E-02 & 0.31 & 0.20 & 0.67 \\
\hline ZNF597 & 49 & 27 & -22 & $1.48 \mathrm{E}-08$ & 1.27 & 1.51 & -0.25 \\
\hline DRG2 & 33 & 11 & -22 & $3.61 \mathrm{E}-08$ & 10.53 & 10.52 & 0.00 \\
\hline ERAL1 & 22 & 0 & -22 & 1.97E-03 & 41.46 & 49.11 & -0.24 \\
\hline CPVL & 33 & 11 & -22 & $3.62 \mathrm{E}-03$ & 0.07 & 0.11 & -0.76 \\
\hline SPIN3 & 42 & 20 & -22 & 1.37E-02 & 1.37 & 1.01 & 0.44 \\
\hline CSTF2T & 23 & 2 & -21 & $1.44 \mathrm{E}-08$ & 9.50 & 8.92 & 0.09 \\
\hline INPP5D & 23 & 2 & -21 & 1.44E-08 & 60.83 & 48.65 & 0.32 \\
\hline HELB & 46 & 25 & -21 & $5.02 \mathrm{E}-06$ & 1.03 & 0.93 & 0.14 \\
\hline RAX2 & 83 & 62 & -21 & $4.50 \mathrm{E}-05$ & 0.03 & 0.03 & 0.01 \\
\hline MSL3 & 40 & 19 & -21 & $5.74 \mathrm{E}-05$ & 86.41 & 96.78 & -0.16 \\
\hline PKN1 & 56 & 35 & -21 & $5.04 \mathrm{E}-02$ & 59.22 & 41.39 & 0.52 \\
\hline RNF166 & 28 & 8 & -20 & $1.44 \mathrm{E}-08$ & 16.37 & 15.28 & 0.10 \\
\hline CLASRP & 26 & 6 & -20 & $1.44 \mathrm{E}-08$ & 27.58 & 25.50 & 0.11 \\
\hline ITGAM & 74 & 54 & -20 & 6.76E-02 & 0.03 & 0.09 & -1.51 \\
\hline BPIFB1 & 80 & 60 & -20 & 8.40E-02 & 0.11 & 0.11 & 0.03 \\
\hline NAA60 & 46 & 27 & -19 & 1.87E-08 & 105.11 & 95.31 & 0.14 \\
\hline SIRT4 & 47 & 28 & -19 & 2.69E-04 & 0.79 & 0.68 & 0.21 \\
\hline RBMX2 & 37 & 18 & -19 & $2.48 \mathrm{E}-02$ & 22.84 & 22.56 & 0.02 \\
\hline NLRP11 & 68 & 49 & -19 & 4.81E-02 & 0.00 & 0.00 & -0.23 \\
\hline $\mathrm{ZC} 3 \mathrm{H} 4$ & 18 & 0 & -18 & $1.44 \mathrm{E}-08$ & 15.21 & 10.06 & 0.60 \\
\hline ATHL1 & 31 & 13 & -18 & $1.65 \mathrm{E}-08$ & 3.98 & 3.19 & 0.32 \\
\hline S1PR4 & 24 & 6 & -18 & 1.94E-06 & 0.23 & 0.12 & 0.90 \\
\hline HPRT1 & 38 & 20 & -18 & $6.50 \mathrm{E}-06$ & 31.01 & 35.05 & -0.18 \\
\hline RGN & 65 & 47 & -18 & $1.12 \mathrm{E}-04$ & 0.18 & 0.06 & 1.51 \\
\hline COMMD6 & 29 & 11 & -18 & 7.77E-03 & 98.08 & 105.99 & -0.11 \\
\hline $\mathrm{RCN} 3$ & 95 & 77 & -18 & 8.90E-03 & 82.51 & 39.28 & 1.07 \\
\hline SLC7A8 & 28 & 10 & -18 & $1.12 \mathrm{E}-02$ & 2.60 & 2.49 & 0.06 \\
\hline SPC24 & 31 & 13 & -18 & 1.67E-02 & 6.08 & 5.49 & 0.15 \\
\hline SLC6A10P & 95 & 77 & -18 & 4.42E-02 & 0.00 & 0.02 & -2.87 \\
\hline SERPINA4 & 82 & 64 & -18 & 7.75E-02 & 0.10 & 0.06 & 0.58 \\
\hline STYX & 17 & 0 & -17 & $1.44 \mathrm{E}-08$ & 26.56 & 25.30 & 0.07 \\
\hline MED1 & 22 & 5 & -17 & 3.94E-05 & 19.87 & 20.22 & -0.03 \\
\hline SPON2 & 38 & 21 & -17 & $1.30 \mathrm{E}-03$ & 0.19 & 0.28 & -0.60 \\
\hline MAP4K1 & 26 & 9 & -17 & 2.84E-03 & 0.93 & 0.54 & 0.79 \\
\hline PCDHB3 & 48 & 31 & -17 & 1.91E-02 & 0.04 & 0.02 & 1.26 \\
\hline C8orf44 & 17 & 1 & -16 & $1.44 \mathrm{E}-08$ & 14.52 & 12.54 & 0.21 \\
\hline PHF14 & 20 & 4 & -16 & $1.45 \mathrm{E}-08$ & 20.98 & 21.22 & -0.02 \\
\hline HEXB & 17 & 1 & -16 & 1.47E-08 & 410.11 & 529.01 & -0.37 \\
\hline TTC38 & 19 & 3 & -16 & $1.52 \mathrm{E}-08$ & 12.85 & 12.93 & -0.01 \\
\hline PLEKHG6 & 30 & 14 & -16 & $1.55 \mathrm{E}-08$ & 0.34 & 0.12 & 1.47 \\
\hline PLEKHG6 & 30 & 14 & -16 & $1.55 \mathrm{E}-08$ & 0.34 & 0.12 & 1.47 \\
\hline SPAG4 & 24 & 8 & -16 & 1.67E-08 & 1.89 & 1.20 & 0.66 \\
\hline RGMA & 28 & 12 & -16 & 1.12E-06 & 0.01 & 0.01 & -0.28 \\
\hline APOE & 17 & 1 & -16 & 1.63E-06 & 4.60 & 4.09 & 0.17 \\
\hline TMEM209 & 52 & 36 & -16 & $1.07 \mathrm{E}-03$ & 1.92 & 1.17 & 0.71 \\
\hline TCF3 & 34 & 18 & -16 & $3.92 \mathrm{E}-03$ & 71.64 & 55.68 & 0.36 \\
\hline DBNDD2 & 95 & 79 & -16 & 4.39E-03 & 14.96 & 17.62 & -0.24 \\
\hline C1orf86 & 27 & 11 & -16 & 3.81E-02 & 4.82 & 4.10 & 0.24 \\
\hline PKD1L1 & 76 & 60 & -16 & 7.03E-02 & 1.48 & 1.20 & 0.30 \\
\hline AMPD2 & 70 & 54 & -16 & 7.53E-02 & 41.20 & 35.57 & 0.21 \\
\hline CALD1 & 42 & 26 & -16 & 8.38E-02 & 571.85 & 513.31 & 0.16 \\
\hline PPID & 16 & 1 & -15 & $1.52 \mathrm{E}-08$ & 47.81 & 64.65 & -0.44 \\
\hline ZNF493 & 15 & 0 & -15 & 2.03E-08 & 5.54 & 5.51 & 0.01 \\
\hline
\end{tabular}




\begin{tabular}{|c|c|c|c|c|c|c|c|}
\hline BAK1 & 21 & 6 & -15 & 8.76E-05 & 3.54 & 2.95 & 0.27 \\
\hline BDKRB2 & 25 & 10 & -15 & 2.31E-03 & 0.03 & 0.13 & -2.21 \\
\hline RPL38 & 24 & 9 & -15 & $2.94 \mathrm{E}-03$ & 2612.63 & 3092.61 & -0.24 \\
\hline ANKRD33 & 35 & 20 & -15 & $3.71 \mathrm{E}-03$ & 0.09 & 0.07 & 0.42 \\
\hline SLCO1A2 & 100 & 85 & -15 & 7.77E-03 & 0.10 & 0.24 & -1.30 \\
\hline ASGR1 & 73 & 58 & -15 & 4.08E-02 & 3.04 & 2.98 & 0.03 \\
\hline LPIN2 & 15 & 1 & -14 & $1.44 \mathrm{E}-08$ & 2.38 & 8.63 & -1.86 \\
\hline TAB1 & 14 & 0 & -14 & 1.44E-08 & 5.50 & 8.00 & -0.54 \\
\hline DKKL1 & 19 & 5 & -14 & 1.47E-08 & 0.17 & 0.12 & 0.46 \\
\hline DKKL1 & 19 & 5 & -14 & 1.47E-08 & 0.17 & 0.12 & 0.46 \\
\hline BRCA1 & 18 & 4 & -14 & $1.74 \mathrm{E}-06$ & 5.17 & 5.14 & 0.01 \\
\hline ZNF207 & 27 & 13 & -14 & $9.74 \mathrm{E}-06$ & 168.27 & 171.22 & -0.03 \\
\hline PHF17 & 14 & 0 & -14 & $9.24 \mathrm{E}-05$ & 13.84 & 12.17 & 0.19 \\
\hline MBNL3 & 26 & 12 & -14 & $1.25 \mathrm{E}-04$ & 0.67 & 0.73 & -0.12 \\
\hline MED24 & 14 & 0 & -14 & $2.69 \mathrm{E}-04$ & 52.08 & 47.89 & 0.12 \\
\hline OCRL & 20 & 6 & -14 & $4.18 \mathrm{E}-04$ & 16.60 & 13.84 & 0.26 \\
\hline SYCE1L & 97 & 83 & -14 & 2.29E-02 & 16.68 & 14.18 & 0.23 \\
\hline C12orf36 & 93 & 79 & -14 & $9.90 \mathrm{E}-02$ & 0.14 & 0.04 & 1.94 \\
\hline AGPAT6 & 13 & 0 & -13 & $1.44 \mathrm{E}-08$ & 68.63 & 66.13 & 0.05 \\
\hline DGCR14 & 15 & 2 & -13 & $1.44 \mathrm{E}-08$ & 4.17 & 4.07 & 0.04 \\
\hline IDUA & 13 & 0 & -13 & $1.52 \mathrm{E}-08$ & 8.72 & 5.44 & 0.68 \\
\hline SNX29 & 17 & 4 & -13 & 1.07E-05 & 5.30 & 5.94 & -0.17 \\
\hline USP16 & 28 & 15 & -13 & 2.24E-05 & 15.29 & 15.39 & -0.01 \\
\hline ADAM32 & 30 & 17 & -13 & 3.37E-05 & 1.50 & 1.52 & -0.0 \\
\hline DMPK & 23 & 10 & -13 & 4.92E-04 & 39.58 & 37.02 & 0.10 \\
\hline TCEB3B & 97 & 84 & -13 & $2.64 \mathrm{E}-03$ & 0.01 & 0.01 & -0.06 \\
\hline PCYT1B & 19 & 6 & -13 & $6.38 \mathrm{E}-03$ & 0.07 & 0.10 & -0.46 \\
\hline SLC5A4 & 100 & 87 & -13 & $1.42 \mathrm{E}-02$ & 0.10 & 0.08 & 0.3 \\
\hline DMKN & 14 & 1 & -13 & 2.24E-02 & 0.09 & 0.05 & 0.7 \\
\hline C1QTNF1 & 35 & 22 & -13 & 3.09E-02 & 0.46 & 0.22 & 1.0 \\
\hline MUTYH & 13 & 0 & -13 & 4.32E-02 & 4.19 & 4.03 & 0.0 \\
\hline TOE1 & 13 & 0 & -13 & 4.32E-02 & 3.62 & 3.14 & 0.2 \\
\hline NRN1L & 68 & 55 & -13 & $5.14 \mathrm{E}-02$ & 34.26 & 35.66 & -0.06 \\
\hline FTLP10 & 94 & 81 & -13 & $6.78 \mathrm{E}-02$ & 0.04 & 0.11 & -1.5 \\
\hline LINC00574 & 13 & 0 & -13 & 8.23E-02 & 0.13 & 0.12 & 0.1 \\
\hline RFESD & 12 & 0 & -12 & $1.44 \mathrm{E}-08$ & 0.57 & 0.22 & 1.3 \\
\hline RFESD & 12 & 0 & -12 & $1.44 \mathrm{E}-08$ & 0.57 & 0.22 & 1.35 \\
\hline PI4KB & 13 & 1 & -12 & 1.47E-08 & 31.10 & 26.02 & 0.26 \\
\hline PI4KB & 13 & 1 & -12 & 1.47E-08 & 31.10 & 26.02 & 0.26 \\
\hline TSSK3 & 20 & 8 & -12 & 1.67E-08 & 0.53 & 0.48 & 0.16 \\
\hline EDARADD & 12 & 0 & -12 & 3.66E-08 & 0.20 & 0.16 & 0.29 \\
\hline SUMO2 & 28 & 16 & -12 & $2.15 \mathrm{E}-06$ & 441.13 & 338.71 & 0.38 \\
\hline RHBDL1 & 27 & 15 & -12 & 4.01E-06 & 0.13 & 0.10 & 0.50 \\
\hline SUGT1P3 & 25 & 13 & -12 & $5.12 \mathrm{E}-06$ & 1.39 & 2.47 & -0.83 \\
\hline MARS & 47 & 35 & -12 & 4.05E-05 & 90.69 & 97.27 & -0.10 \\
\hline SNAPC5 & 21 & 9 & -12 & $4.51 \mathrm{E}-04$ & 30.90 & 31.57 & -0.03 \\
\hline MRPL28 & 20 & 8 & -12 & $2.57 \mathrm{E}-03$ & 39.19 & 34.56 & 0.18 \\
\hline KBTBD7 & 39 & 27 & -12 & 3.96E-03 & 2.99 & 2.59 & 0.21 \\
\hline FXYD2 & 88 & 76 & -12 & 1.89E-02 & 15.81 & 24.14 & -0.61 \\
\hline MORN3 & 91 & 79 & -12 & $6.08 \mathrm{E}-02$ & 0.67 & 0.45 & 0.58 \\
\hline APOBEC3D & 12 & 0 & -12 & 7.81E-02 & 11.13 & 9.90 & 0.17 \\
\hline C19orf33 & 47 & 35 & -12 & 8.20E-02 & 39.79 & 40.11 & -0.01 \\
\hline CKS1B & 12 & 0 & -12 & 8.87E-02 & 5.51 & 5.85 & -0.09 \\
\hline $\mathrm{SHC} 1$ & 12 & 0 & -12 & 8.87E-02 & 202.71 & 208.14 & -0.04 \\
\hline
\end{tabular}




\begin{tabular}{|c|c|c|c|c|c|c|c|}
\hline ASL & 14 & 3 & -11 & $1.44 \mathrm{E}-08$ & 70.34 & 67.45 & 0.06 \\
\hline ERICH1 & 13 & 2 & -11 & $1.51 \mathrm{E}-08$ & 37.35 & 23.08 & 0.69 \\
\hline TUBGCP2 & 22 & 11 & -11 & $1.55 E-08$ & 15.68 & 12.74 & 0.30 \\
\hline GP1BB & 16 & 5 & -11 & 1.57E-08 & 8.91 & 9.43 & -0.08 \\
\hline ARC & 21 & 10 & -11 & 1.64E-08 & 0.02 & 0.06 & -2.00 \\
\hline CRISPLD2 & 11 & 0 & -11 & 2.32E-08 & 2.74 & 2.11 & 0.38 \\
\hline RASSF1 & 12 & 1 & -11 & 7.16E-07 & 5.10 & 5.37 & -0.07 \\
\hline P2RY2 & 16 & 5 & -11 & 7.63E-07 & 1.54 & 1.44 & 0.10 \\
\hline P2RY2 & 16 & 5 & -11 & 7.63E-07 & 1.54 & 1.44 & 0.10 \\
\hline RGPD2 & 100 & 89 & -11 & $1.51 \mathrm{E}-05$ & 0.08 & 0.09 & -0.24 \\
\hline PMVK & 13 & 2 & -11 & 3.89E-05 & 7.59 & 6.70 & 0.18 \\
\hline MSL3 & 38 & 27 & -11 & $3.12 \mathrm{E}-04$ & 86.41 & 96.78 & -0.16 \\
\hline RBP5 & 23 & 12 & -11 & $4.10 \mathrm{E}-04$ & 0.87 & 0.87 & 0.00 \\
\hline FLT3LG & 22 & 11 & -11 & $5.74 \mathrm{E}-04$ & 782.26 & 935.32 & -0.26 \\
\hline ZC4H2 & 26 & 15 & -11 & 5.96E-04 & 8.73 & 8.31 & 0.07 \\
\hline C20orf196 & 15 & 4 & -11 & 7.96E-04 & 2.89 & 2.91 & -0.01 \\
\hline MSL3 & 39 & 28 & -11 & 8.02E-04 & 86.41 & 96.78 & -0.16 \\
\hline MGAT5B & 21 & 10 & -11 & 1.27E-03 & 0.15 & 0.08 & 0.91 \\
\hline SPON2 & 24 & 13 & -11 & $1.34 \mathrm{E}-03$ & 0.19 & 0.28 & -0.60 \\
\hline KCNH2 & 34 & 23 & -11 & 8.19E-03 & 0.02 & 0.03 & -0.34 \\
\hline ZNF177 & 21 & 10 & -11 & $1.51 \mathrm{E}-02$ & 10.72 & 9.81 & 0.13 \\
\hline MYADM & 22 & 11 & -11 & 1.86E-02 & 32.83 & 34.40 & -0.07 \\
\hline ZNF682 & 37 & 26 & -11 & 2.03E-02 & 1.07 & 0.90 & 0.26 \\
\hline HYPK & 19 & 8 & -11 & 2.37E-02 & 304.92 & 347.00 & -0.19 \\
\hline AR & 24 & 13 & -11 & 2.86E-02 & 2.56 & 2.19 & 0.23 \\
\hline MYO1H & 99 & 88 & -11 & $3.22 \mathrm{E}-02$ & 0.09 & 0.30 & -1.79 \\
\hline DNAJC8 & 51 & 40 & -11 & 3.70E-02 & 113.66 & 116.81 & -0.04 \\
\hline NUP160 & 27 & 16 & -11 & 4.44E-02 & 37.20 & 35.05 & 0.09 \\
\hline BDNF & 13 & 2 & -11 & 4.61E-02 & 2.82 & 3.05 & -0.11 \\
\hline LYNX1 & 67 & 56 & -11 & $9.05 \mathrm{E}-02$ & 4.97 & 5.21 & -0.07 \\
\hline EIF4G1 & 12 & 2 & -10 & $1.44 \mathrm{E}-08$ & 193.74 & 209.84 & -0.12 \\
\hline GRM4 & 13 & 3 & -10 & $1.51 \mathrm{E}-08$ & 0.00 & 0.00 & 0.08 \\
\hline HMBS & 12 & 2 & -10 & 2.40E-08 & 5.93 & 6.03 & -0.02 \\
\hline IRF7 & 20 & 10 & -10 & $2.64 \mathrm{E}-08$ & 1.87 & 2.87 & -0.62 \\
\hline LGALS1 & 10 & 0 & -10 & $6.26 \mathrm{E}-08$ & 1405.19 & 820.91 & 0.78 \\
\hline LZTS2 & 11 & 1 & -10 & 1.93E-07 & 10.24 & 10.10 & 0.02 \\
\hline USP39 & 16 & 6 & -10 & 2.29E-07 & 55.11 & 72.89 & -0.40 \\
\hline C2orf68 & 16 & 6 & -10 & 2.29E-07 & 7.45 & 6.91 & 0.11 \\
\hline CREB3L1 & 12 & 2 & -10 & $1.10 \mathrm{E}-06$ & 1.10 & 1.01 & 0.12 \\
\hline ASL & 18 & 8 & -10 & $2.56 \mathrm{E}-06$ & 70.34 & 67.45 & 0.06 \\
\hline FAM57B & 11 & 1 & -10 & 2.91E-06 & 0.11 & 0.14 & -0.42 \\
\hline TNK1 & 14 & 4 & -10 & 7.13E-06 & 0.16 & 0.56 & -1.84 \\
\hline RGMA & 18 & 8 & -10 & 1.33E-05 & 0.01 & 0.01 & -0.28 \\
\hline NOP2 & 12 & 2 & -10 & 1.17E-04 & 24.14 & 22.62 & 0.09 \\
\hline CCDC78 & 21 & 11 & -10 & $2.50 \mathrm{E}-04$ & 0.10 & 0.07 & 0.57 \\
\hline THUMPD1 & 20 & 10 & -10 & 2.81E-04 & 29.93 & 34.48 & -0.20 \\
\hline NFRKB & 18 & 8 & -10 & $2.62 \mathrm{E}-03$ & 5.75 & 6.45 & -0.17 \\
\hline POC5 & 24 & 14 & -10 & 2.71E-03 & 6.05 & 4.78 & 0.34 \\
\hline TTC25 & 30 & 20 & -10 & $6.50 \mathrm{E}-03$ & 0.13 & 0.07 & 0.78 \\
\hline NUP210L & 41 & 31 & -10 & 7.33E-03 & 0.04 & 0.04 & -0.03 \\
\hline WDR38 & 29 & 19 & -10 & $1.48 \mathrm{E}-02$ & 0.05 & 0.05 & 0.10 \\
\hline DDX43 & 96 & 86 & -10 & $1.49 \mathrm{E}-02$ & 0.26 & 0.17 & 0.63 \\
\hline JDP2 & 98 & 88 & -10 & 2.33E-02 & 3.98 & 5.75 & -0.53 \\
\hline POM121L10P & 89 & 79 & -10 & 3.81E-02 & 0.06 & 0.06 & -0.15 \\
\hline
\end{tabular}




\begin{tabular}{|c|c|c|c|c|c|c|c|}
\hline SEMA4D & 99 & 89 & -10 & 4.39E-02 & 4.05 & 3.11 & 0.38 \\
\hline TRIM72 & 41 & 31 & -10 & 4.91E-02 & 0.04 & 0.04 & -0.23 \\
\hline CD320 & 71 & 61 & -10 & $5.49 \mathrm{E}-02$ & 25.89 & 23.20 & 0.16 \\
\hline PKP3 & 59 & 49 & -10 & $5.49 \mathrm{E}-02$ & 0.18 & 0.09 & 1.04 \\
\hline WBSCR27 & 38 & 28 & -10 & $9.71 \mathrm{E}-02$ & 0.13 & 0.19 & -0.50 \\
\hline ADPGK-AS1 & 4 & 14 & 10 & $1.44 \mathrm{E}-08$ & 1.01 & 1.25 & -0.31 \\
\hline PDLIM5 & 3 & 13 & 10 & $1.44 \mathrm{E}-08$ & 46.85 & 54.30 & -0.21 \\
\hline KIAA1598 & 1 & 11 & 10 & $1.44 \mathrm{E}-08$ & 1.42 & 1.52 & -0.10 \\
\hline KLC4 & 1 & 11 & 10 & $1.44 \mathrm{E}-08$ & 11.20 & 11.40 & -0.03 \\
\hline KLC4 & 1 & 11 & 10 & $1.44 \mathrm{E}-08$ & 11.20 & 11.40 & -0.03 \\
\hline POLR3H & 1 & 11 & 10 & $1.44 \mathrm{E}-08$ & 10.20 & 9.57 & 0.09 \\
\hline POLR3H & 1 & 11 & 10 & $1.44 \mathrm{E}-08$ & 10.20 & 9.57 & 0.09 \\
\hline MRPL2 & 1 & 11 & 10 & $1.44 \mathrm{E}-08$ & 30.42 & 27.23 & 0.16 \\
\hline DEGS1 & 6 & 16 & 10 & $1.44 \mathrm{E}-08$ & 147.47 & 120.36 & 0.29 \\
\hline SLC12A6 & 4 & 14 & 10 & $1.44 \mathrm{E}-08$ & 20.84 & 23.38 & -0.17 \\
\hline TRNT1 & 6 & 16 & 10 & $1.46 \mathrm{E}-08$ & 26.46 & 31.82 & -0.27 \\
\hline PITPNA & 5 & 15 & 10 & 1.47E-08 & 106.60 & 108.69 & -0.03 \\
\hline WDR85 & 7 & 17 & 10 & $1.48 \mathrm{E}-08$ & 8.45 & 6.47 & 0.39 \\
\hline VKORC1 & 9 & 19 & 10 & $1.52 \mathrm{E}-08$ & 55.52 & 42.57 & 0.38 \\
\hline ZNF707 & 8 & 18 & 10 & 1.57E-08 & 5.34 & 4.73 & 0.17 \\
\hline SSB & 2 & 12 & 10 & 1.57E-08 & 654.63 & 681.75 & -0.06 \\
\hline NLK & 7 & 17 & 10 & $1.60 \mathrm{E}-08$ & 18.02 & 18.78 & -0.06 \\
\hline B3GALNT1 & 9 & 19 & 10 & 1.67E-08 & 7.45 & 8.31 & -0.16 \\
\hline CXXC1 & 6 & 16 & 10 & $1.76 \mathrm{E}-08$ & 28.92 & 22.11 & 0.39 \\
\hline TSPAN15 & 3 & 13 & 10 & 8.05E-08 & 34.23 & 30.63 & 0.16 \\
\hline C12orf29 & 2 & 12 & 10 & $9.87 \mathrm{E}-08$ & 10.01 & 9.63 & 0.06 \\
\hline UBA52 & 9 & 19 & 10 & 2.17E-07 & 365.84 & 531.97 & -0.54 \\
\hline SDHC & 12 & 22 & 10 & 2.99E-07 & 42.94 & 46.05 & -0.10 \\
\hline C11orf92 & 6 & 16 & 10 & 1.63E-06 & 0.08 & 0.09 & -0.29 \\
\hline SNRNP35 & 15 & 25 & 10 & 2.32E-06 & 10.38 & 5.57 & 0.90 \\
\hline HCG11 & 8 & 18 & 10 & 7.14E-06 & 2.54 & 2.32 & 0.13 \\
\hline ZFR & 7 & 17 & 10 & 8.39E-06 & 42.36 & 47.72 & -0.17 \\
\hline C9orf3 & 10 & 20 & 10 & $1.28 \mathrm{E}-05$ & 44.26 & 43.18 & 0.04 \\
\hline CCDC103 & 3 & 13 & 10 & 6.69E-05 & 6.99 & 5.36 & 0.3 \\
\hline PTGDS & 8 & 18 & 10 & $6.81 \mathrm{E}-05$ & 18.31 & 13.32 & 0.46 \\
\hline CORO1B & 18 & 28 & 10 & 1.19E-04 & 51.78 & 37.39 & 0.47 \\
\hline STIL & 9 & 19 & 10 & $1.24 \mathrm{E}-04$ & 2.96 & 2.74 & 0.1 \\
\hline PPP1R14A & 13 & 23 & 10 & $1.75 \mathrm{E}-04$ & 6.36 & 7.54 & -0.25 \\
\hline ZFYVE26 & 8 & 18 & 10 & $2.62 \mathrm{E}-04$ & 18.97 & 15.73 & 0.27 \\
\hline KCNJ11 & 12 & 22 & 10 & 3.43E-04 & 0.11 & 0.06 & 0.84 \\
\hline STX6 & 10 & 20 & 10 & $5.54 \mathrm{E}-04$ & 28.34 & 26.31 & 0.11 \\
\hline ZNF552 & 18 & 28 & 10 & 5.73E-04 & 0.24 & 0.46 & -0.95 \\
\hline PAIP2 & 10 & 20 & 10 & $6.26 \mathrm{E}-04$ & 200.32 & 160.85 & 0.32 \\
\hline GYPC & 12 & 22 & 10 & $9.27 \mathrm{E}-04$ & 1.17 & 1.08 & 0.11 \\
\hline MAPK15 & 11 & 21 & 10 & $1.08 \mathrm{E}-03$ & 0.05 & 0.02 & 1.49 \\
\hline RPL11 & 15 & 25 & 10 & $1.36 \mathrm{E}-03$ & 917.46 & 985.09 & -0.10 \\
\hline TRAPPC12 & 12 & 22 & 10 & $1.50 \mathrm{E}-03$ & 17.57 & 18.98 & -0.11 \\
\hline KLHL12 & 37 & 47 & 10 & $1.74 \mathrm{E}-03$ & 11.53 & 11.68 & -0.02 \\
\hline ZNF391 & 13 & 23 & 10 & 1.95E-03 & 1.04 & 0.44 & 1.25 \\
\hline MAР3К13 & 11 & 21 & 10 & 2.04E-03 & 9.80 & 10.21 & -0.06 \\
\hline FAM215A & 18 & 28 & 10 & $2.74 \mathrm{E}-03$ & 0.07 & 0.14 & -0.98 \\
\hline LHB & 20 & 30 & 10 & 2.89E-03 & 0.03 & 0.03 & 0.16 \\
\hline KCNJ11 & 23 & 33 & 10 & 3.04E-03 & 0.11 & 0.06 & 0.84 \\
\hline BTBD2 & 29 & 39 & 10 & $3.62 \mathrm{E}-03$ & 23.13 & 35.63 & -0.62 \\
\hline
\end{tabular}




\begin{tabular}{|c|c|c|c|c|c|c|c|}
\hline SYNE4 & 19 & 29 & 10 & 4.06E-03 & 4.57 & 3.64 & 0.33 \\
\hline DHX8 & 23 & 33 & 10 & 4.26E-03 & 15.44 & 15.56 & -0.01 \\
\hline FBXW4P1 & 77 & 87 & 10 & $5.56 \mathrm{E}-03$ & 0.23 & 0.28 & -0.26 \\
\hline PDHA1 & 22 & 32 & 10 & $5.85 \mathrm{E}-03$ & 155.70 & 162.93 & -0.07 \\
\hline CWF19L1 & 18 & 28 & 10 & $6.65 \mathrm{E}-03$ & 11.89 & 11.96 & -0.01 \\
\hline ELAC1 & 11 & 21 & 10 & 7.78E-03 & 79.22 & 69.68 & 0.19 \\
\hline OIP5-AS1 & 12 & 22 & 10 & 7.78E-03 & 28.34 & 17.94 & 0.66 \\
\hline AIPL1 & 70 & 80 & 10 & 1.02E-02 & 0.11 & 0.12 & -0.03 \\
\hline RIBC2 & 41 & 51 & 10 & $1.48 \mathrm{E}-02$ & 0.34 & 0.47 & -0.48 \\
\hline SMC1B & 41 & 51 & 10 & $1.48 \mathrm{E}-02$ & 0.02 & 0.02 & -0.03 \\
\hline FGFBP3 & 0 & 10 & 10 & $1.67 \mathrm{E}-02$ & 0.18 & 0.07 & 1.30 \\
\hline LAPTM5 & 3 & 13 & 10 & $1.75 \mathrm{E}-02$ & 59.38 & 53.75 & 0.14 \\
\hline PAFAH2 & 2 & 12 & 10 & 2.73E-02 & 6.94 & 3.98 & 0.80 \\
\hline CPLX3 & 14 & 24 & 10 & $2.79 \mathrm{E}-02$ & 0.02 & 0.04 & -0.93 \\
\hline PCDHGC4 & 38 & 48 & 10 & $2.80 \mathrm{E}-02$ & 44.63 & 44.34 & 0.01 \\
\hline EXOC7 & 50 & 60 & 10 & 2.85E-02 & 44.66 & 54.64 & -0.29 \\
\hline KIF20B & 6 & 16 & 10 & 3.22E-02 & 4.68 & 3.57 & 0.3 \\
\hline ZNF165 & 18 & 28 & 10 & 3.33E-02 & 0.22 & 0.29 & -0.3 \\
\hline GHITM & 22 & 32 & 10 & $4.18 \mathrm{E}-02$ & 117.28 & 115.52 & 0.0 \\
\hline PTP4A3 & 89 & 99 & 10 & $4.48 \mathrm{E}-02$ & 2.51 & 2.57 & -0.0 \\
\hline SPESP1 & 75 & 85 & 10 & 4.92E-02 & 3.15 & 2.05 & 0.6 \\
\hline NOX5 & 75 & 85 & 10 & 4.92E-02 & 3.15 & 2.05 & 0.62 \\
\hline TBPL2 & 43 & 53 & 10 & 5.75E-02 & 0.76 & 0.48 & 0.67 \\
\hline ISLR & 78 & 88 & 10 & $6.20 \mathrm{E}-02$ & 0.03 & 0.03 & -0.0 \\
\hline ZDHHC9 & 28 & 38 & 10 & 7.65E-02 & 14.97 & 18.54 & -0.3 \\
\hline LIMS2 & 9 & 19 & 10 & 8.73E-02 & 18.41 & 16.73 & 0.1 \\
\hline MED11 & 7 & 17 & 10 & $9.21 \mathrm{E}-02$ & 4.32 & 5.14 & -0.2 \\
\hline RP1L1 & 90 & 100 & 10 & 9.46E-02 & 0.44 & 0.26 & 0.7 \\
\hline ZNF808 & 2 & 13 & 11 & $1.44 \mathrm{E}-08$ & 16.48 & 25.19 & -0.6 \\
\hline MIS18BP1 & 0 & 11 & 11 & $1.44 \mathrm{E}-08$ & 14.50 & 20.73 & -0.5 \\
\hline IQGAP1 & 3 & 14 & 11 & $1.44 \mathrm{E}-08$ & 248.25 & 307.74 & -0.3 \\
\hline FLT3LG & 0 & 11 & 11 & $1.44 \mathrm{E}-08$ & 782.26 & 935.32 & -0.2 \\
\hline GMNN & 0 & 11 & 11 & $1.44 \mathrm{E}-08$ & 22.50 & 17.67 & 0.3 \\
\hline MCU & 2 & 13 & 11 & $1.47 \mathrm{E}-08$ & 18.85 & 19.38 & -0.04 \\
\hline AUH & 4 & 15 & 11 & 1.47E-08 & 6.69 & 6.75 & -0.01 \\
\hline VPS11 & 8 & 19 & 11 & $1.55 \mathrm{E}-08$ & 27.65 & 24.21 & 0.19 \\
\hline LAMP1 & 9 & 20 & 11 & $1.90 \mathrm{E}-08$ & 258.24 & 234.38 & 0.14 \\
\hline XPO4 & 5 & 16 & 11 & 1.95E-08 & 6.71 & 6.76 & -0.01 \\
\hline CHST7 & 26 & 37 & 11 & $2.07 \mathrm{E}-08$ & 4.57 & 4.10 & 0.15 \\
\hline ANKRD30BL & 27 & 38 & 11 & $2.34 \mathrm{E}-08$ & 0.04 & 0.07 & -0.91 \\
\hline FGFRL1 & 13 & 24 & 11 & $2.41 \mathrm{E}-08$ & 4.36 & 3.94 & 0.15 \\
\hline IKBKE & 0 & 11 & 11 & $5.88 \mathrm{E}-08$ & 10.37 & 7.64 & 0.44 \\
\hline CDC42 & 4 & 15 & 11 & $2.51 \mathrm{E}-07$ & 185.16 & 192.43 & -0.06 \\
\hline ZSWIM3 & 2 & 13 & 11 & $1.09 \mathrm{E}-06$ & 1.08 & 1.18 & -0.12 \\
\hline C3orf55 & 9 & 20 & 11 & $1.47 \mathrm{E}-06$ & 9.52 & 9.75 & -0.03 \\
\hline EMR2 & 2 & 13 & 11 & $2.21 \mathrm{E}-06$ & 0.18 & 0.22 & -0.34 \\
\hline USP51 & 19 & 30 & 11 & 4.29E-06 & 1.23 & 0.92 & 0.41 \\
\hline PIH1D3 & 24 & 35 & 11 & $6.35 \mathrm{E}-06$ & 0.08 & 0.09 & -0.23 \\
\hline ZNF560 & 18 & 29 & 11 & 1.77E-05 & 0.00 & 0.00 & -0.22 \\
\hline GGACT & 6 & 17 & 11 & $2.08 \mathrm{E}-05$ & 1.56 & 1.20 & 0.38 \\
\hline NLRX1 & 8 & 19 & 11 & $6.05 \mathrm{E}-05$ & 1.27 & 0.97 & 0.39 \\
\hline PSMB4 & 14 & 25 & 11 & 1.23E-04 & 89.62 & 80.22 & 0.16 \\
\hline CEND1 & 10 & 21 & 11 & 1.33E-04 & 0.28 & 0.19 & 0.60 \\
\hline TMEM218 & 10 & 21 & 11 & $1.63 \mathrm{E}-04$ & 18.41 & 13.72 & 0.42 \\
\hline
\end{tabular}




\begin{tabular}{|c|c|c|c|c|c|c|c|}
\hline TMEM218 & 10 & 21 & 11 & 1.63E-04 & 18.41 & 13.72 & 0.42 \\
\hline RNF8 & 7 & 18 & 11 & 2.32E-04 & 29.41 & 30.97 & -0.07 \\
\hline TGDS & 13 & 24 & 11 & $2.55 \mathrm{E}-04$ & 4.97 & 4.86 & 0.03 \\
\hline RGAG4 & 23 & 34 & 11 & $2.72 \mathrm{E}-04$ & 0.52 & 0.43 & 0.28 \\
\hline FOXO4 & 8 & 19 & 11 & 3.07E-04 & 4.45 & 3.31 & 0.42 \\
\hline CDK20 & 7 & 18 & 11 & 3.99E-04 & 0.41 & 0.44 & -0.09 \\
\hline CCM2 & 4 & 15 & 11 & 4.77E-04 & 41.58 & 30.78 & 0.43 \\
\hline AMMECR1 & 16 & 27 & 11 & $5.11 \mathrm{E}-04$ & 5.61 & 5.56 & 0.02 \\
\hline MSN & 24 & 35 & 11 & $8.21 \mathrm{E}-04$ & 283.85 & 304.74 & -0.10 \\
\hline AGA & 6 & 17 & 11 & $9.90 \mathrm{E}-04$ & 12.45 & 15.11 & -0.28 \\
\hline PTPLAD2 & 27 & 38 & 11 & $1.10 \mathrm{E}-03$ & 4.87 & 5.90 & -0.28 \\
\hline ZMYND10 & 19 & 30 & 11 & $1.47 \mathrm{E}-03$ & 0.07 & 0.06 & 0.33 \\
\hline SNRPF & 11 & 22 & 11 & 2.89E-03 & 91.74 & 114.33 & -0.32 \\
\hline TLE2 & 12 & 23 & 11 & $3.43 E-03$ & 22.37 & 19.69 & 0.18 \\
\hline RAD51D & 27 & 38 & 11 & $5.73 E-03$ & 8.49 & 9.87 & -0.22 \\
\hline RAD9A & 40 & 51 & 11 & $1.25 \mathrm{E}-02$ & 3.72 & 2.38 & 0.64 \\
\hline PIWIL1 & 60 & 71 & 11 & 1.33E-02 & 0.06 & 0.04 & 0.74 \\
\hline ZNF551 & 30 & 41 & 11 & 1.38E-02 & 8.81 & 7.22 & 0.29 \\
\hline LPIN3 & 11 & 22 & 11 & 1.66E-02 & 0.14 & 0.17 & -0.25 \\
\hline PPAPDC3 & 56 & 67 & 11 & $1.74 \mathrm{E}-02$ & 0.36 & 0.31 & 0.19 \\
\hline FAM9C & 81 & 92 & 11 & 2.02E-02 & 0.14 & 0.16 & -0.19 \\
\hline GLUD2 & 18 & 29 & 11 & 2.42E-02 & 0.09 & 0.12 & -0.3 \\
\hline TCTEX1D4 & 77 & 88 & 11 & 2.79E-02 & 0.20 & 0.16 & 0.32 \\
\hline ASIC2 & 46 & 57 & 11 & $5.88 \mathrm{E}-02$ & 0.00 & 0.04 & -3.2 \\
\hline ZNF492 & 45 & 56 & 11 & $6.60 \mathrm{E}-02$ & 0.08 & 0.07 & 0.18 \\
\hline DNAH6 & 16 & 27 & 11 & 7.47E-02 & 0.01 & 0.05 & -2.9 \\
\hline GON4L & 34 & 45 & 11 & 8.31E-02 & 14.46 & 17.91 & -0.31 \\
\hline PIGA & 25 & 36 & 11 & $8.45 \mathrm{E}-02$ & 3.85 & 2.88 & 0.42 \\
\hline MS4A10 & 82 & 93 & 11 & 8.49E-02 & 0.18 & 0.15 & 0.29 \\
\hline ADTRP & 89 & 100 & 11 & $9.37 \mathrm{E}-02$ & 14.26 & 14.84 & -0.06 \\
\hline BCL2L14 & 81 & 92 & 11 & $9.88 \mathrm{E}-02$ & 0.32 & 0.63 & -0.9 \\
\hline PIWIL2 & 46 & 57 & 11 & 9.96E-02 & 0.13 & 0.13 & -0.05 \\
\hline RNASEH 1 & 7 & 19 & 12 & $1.44 \mathrm{E}-08$ & 18.38 & 17.32 & $0.0 \varsigma$ \\
\hline SNX33 & 4 & 16 & 12 & $1.44 \mathrm{E}-08$ & 4.38 & 3.92 & 0.16 \\
\hline SRP14 & 1 & 13 & 12 & $1.44 \mathrm{E}-08$ & 1089.93 & 942.41 & 0.2 \\
\hline ZNF771 & 1 & 13 & 12 & $1.44 \mathrm{E}-08$ & 1.15 & 0.90 & 0.35 \\
\hline METTL13 & 6 & 18 & 12 & $1.44 \mathrm{E}-08$ & 15.91 & 11.91 & 0.42 \\
\hline C6orf57 & 5 & 17 & 12 & $1.44 \mathrm{E}-08$ & 5.46 & 3.87 & 0.50 \\
\hline ZNHIT6 & 2 & 14 & 12 & $1.44 \mathrm{E}-08$ & 15.33 & 12.92 & 0.25 \\
\hline POLR3F & 5 & 17 & 12 & 1.47E-08 & 11.01 & 12.78 & -0.22 \\
\hline SNAPC1 & 10 & 22 & 12 & 1.57E-08 & 406.34 & 404.96 & 0.00 \\
\hline MAP7D3 & 17 & 29 & 12 & 1.60E-08 & 22.27 & 16.64 & 0.42 \\
\hline ACOT4 & 10 & 22 & 12 & 1.73E-08 & 0.03 & 0.03 & -0.16 \\
\hline GNAS & 31 & 43 & 12 & $2.51 \mathrm{E}-08$ & 458.16 & 442.50 & 0.05 \\
\hline MCM5 & 27 & 39 & 12 & 3.67E-08 & 12.56 & 9.31 & 0.43 \\
\hline TMEM62 & 8 & 20 & 12 & 4.94E-08 & 30.43 & 35.77 & -0.23 \\
\hline RHPN2 & 11 & 23 & 12 & $6.11 \mathrm{E}-08$ & 1.55 & 1.56 & -0.01 \\
\hline TCTN3 & 12 & 24 & 12 & 1.67E-06 & 56.15 & 52.02 & 0.11 \\
\hline EIF1AX & 24 & 36 & 12 & 2.43E-06 & 56.38 & 47.36 & 0.25 \\
\hline RUFY1 & 5 & 17 & 12 & 2.95E-06 & 109.75 & 111.85 & -0.03 \\
\hline MRPS25 & 7 & 19 & 12 & 7.02E-06 & 33.83 & 29.94 & 0.18 \\
\hline BLVRB & 11 & 23 & 12 & 7.04E-06 & 12.40 & 14.19 & -0.20 \\
\hline NHLRC1 & 18 & 30 & 12 & 7.90E-06 & 0.41 & 0.29 & 0.49 \\
\hline PHF16 & 16 & 28 & 12 & $1.42 \mathrm{E}-05$ & 5.93 & 8.25 & -0.48 \\
\hline
\end{tabular}




\begin{tabular}{|c|c|c|c|c|c|c|c|}
\hline PHF16 & 16 & 28 & 12 & $1.42 \mathrm{E}-05$ & 5.93 & 8.25 & -0.48 \\
\hline C16orf80 & 35 & 47 & 12 & 1.69E-04 & 68.93 & 72.29 & -0.07 \\
\hline SMARCAL1 & 8 & 20 & 12 & 2.02E-04 & 13.36 & 13.73 & -0.04 \\
\hline SMARCAL1 & 8 & 20 & 12 & 2.02E-04 & 13.36 & 13.73 & -0.04 \\
\hline C1GALT1C1 & 36 & 48 & 12 & 2.36E-04 & 33.64 & 38.22 & -0.18 \\
\hline MMADHC & 0 & 12 & 12 & 3.32E-04 & 65.47 & 73.95 & -0.18 \\
\hline CSTF2 & 36 & 48 & 12 & $6.15 \mathrm{E}-04$ & 6.05 & 7.68 & -0.35 \\
\hline KCNN3 & 9 & 21 & 12 & 8.37E-04 & 6.89 & 6.08 & 0.18 \\
\hline C14orf2 & 17 & 29 & 12 & $9.41 \mathrm{E}-04$ & 283.38 & 220.43 & 0.36 \\
\hline SYCE2 & 43 & 55 & 12 & $1.10 \mathrm{E}-03$ & 0.25 & 0.12 & 1.04 \\
\hline MAOA & 30 & 42 & 12 & $1.15 \mathrm{E}-03$ & 3.31 & 2.91 & 0.19 \\
\hline C2orf74 & 82 & 94 & 12 & 1.63E-03 & 40.17 & 44.10 & -0.13 \\
\hline MDM4 & 27 & 39 & 12 & $3.48 \mathrm{E}-03$ & 11.11 & 11.93 & -0.10 \\
\hline BCL6 & 4 & 16 & 12 & $3.71 \mathrm{E}-03$ & 6.69 & 6.92 & -0.05 \\
\hline LY6K & 67 & 79 & 12 & 9.63E-03 & 0.83 & 0.71 & 0.23 \\
\hline MED16 & 16 & 28 & 12 & 1.73E-02 & 10.24 & 7.71 & 0.41 \\
\hline DNAJC19 & 38 & 50 & 12 & 1.73E-02 & 30.36 & 23.15 & 0.39 \\
\hline DNAJC19 & 34 & 46 & 12 & 1.87E-02 & 30.36 & 23.15 & 0.39 \\
\hline CDHR5 & 72 & 84 & 12 & 2.84E-02 & 0.02 & 0.02 & 0.20 \\
\hline C1D & 12 & 24 & 12 & 2.88E-02 & 16.36 & 13.16 & 0.31 \\
\hline TNFRSF25 & 45 & 57 & 12 & 3.60E-02 & 29.69 & 24.12 & 0.30 \\
\hline RHBDL2 & 9 & 21 & 12 & $6.21 \mathrm{E}-02$ & 1.74 & 2.10 & -0.27 \\
\hline PEX19 & 31 & 43 & 12 & $8.24 \mathrm{E}-02$ & 96.70 & 79.61 & 0.28 \\
\hline DIO1 & 82 & 94 & 12 & 8.31E-02 & 0.06 & 0.07 & -0.11 \\
\hline ALDH3B1 & 80 & 92 & 12 & 8.56E-02 & 0.29 & 0.18 & 0.71 \\
\hline EVI5L & 79 & 91 & 12 & 9.86E-02 & 23.04 & 21.63 & 0.09 \\
\hline UBE2L6 & 0 & 13 & 13 & $1.44 \mathrm{E}-08$ & 19.57 & 22.84 & -0.22 \\
\hline MLH3 & 1 & 14 & 13 & $1.44 \mathrm{E}-08$ & 5.17 & 5.83 & -0.17 \\
\hline ING4 & 1 & 14 & 13 & $1.44 \mathrm{E}-08$ & 33.56 & 35.69 & -0.09 \\
\hline COA6 & 3 & 16 & 13 & $1.44 \mathrm{E}-08$ & 28.20 & 29.70 & -0.07 \\
\hline TBCCD1 & 1 & 14 & 13 & $1.44 \mathrm{E}-08$ & 9.27 & 9.72 & -0.07 \\
\hline C11orf48 & 2 & 15 & 13 & $1.44 \mathrm{E}-08$ & 306.26 & 300.07 & 0.03 \\
\hline MKS1 & 0 & 13 & 13 & 1.44E-08 & 7.54 & 6.41 & 0.24 \\
\hline FOXRED2 & 29 & 42 & 13 & 1.91E-08 & 3.21 & 3.15 & 0.03 \\
\hline FOXRED2 & 30 & 43 & 13 & 2.30E-08 & 3.21 & 3.15 & 0.03 \\
\hline AMZ2P1 & 9 & 22 & 13 & 5.41E-08 & 6.21 & 5.71 & 0.12 \\
\hline CHST6 & 12 & 25 & 13 & 6.54E-08 & 0.06 & 0.11 & -0.93 \\
\hline WIF1 & 23 & 36 & 13 & 2.36E-07 & 0.01 & 0.01 & 0.03 \\
\hline DNAJC15 & 8 & 21 & 13 & 2.46E-07 & 5.56 & 5.21 & 0.09 \\
\hline SERF2 & 15 & 28 & 13 & 4.74E-07 & 304.92 & 347.00 & -0.19 \\
\hline IFFO1 & 14 & 27 & 13 & $6.39 \mathrm{E}-07$ & 11.83 & 10.79 & 0.13 \\
\hline UBQLN2 & 16 & 29 & 13 & $1.13 \mathrm{E}-06$ & 11.33 & 10.28 & 0.14 \\
\hline ANGEL2 & 9 & 22 & 13 & $2.44 \mathrm{E}-06$ & 16.92 & 17.08 & -0.01 \\
\hline TNFSF11 & 3 & 16 & 13 & $3.65 \mathrm{E}-06$ & 0.01 & 0.01 & -0.41 \\
\hline GANC & 3 & 16 & 13 & 7.39E-06 & 13.30 & 13.94 & -0.07 \\
\hline ZNF695 & 7 & 20 & 13 & 8.06E-06 & 2.38 & 2.13 & 0.16 \\
\hline$A B C C 10$ & 9 & 22 & 13 & 8.90E-06 & 12.79 & 11.98 & 0.09 \\
\hline CCND3 & 9 & 22 & 13 & 1.16E-05 & 32.18 & 30.73 & 0.07 \\
\hline RINT1 & 10 & 23 & 13 & $2.48 \mathrm{E}-05$ & 30.19 & 29.98 & 0.01 \\
\hline SSTR5 & 9 & 22 & 13 & 2.91E-05 & 0.10 & 0.01 & 2.97 \\
\hline TGFB1I1 & 5 & 18 & 13 & 4.61E-05 & 10.45 & 10.33 & 0.02 \\
\hline TGFB1I1 & 5 & 18 & 13 & 4.61E-05 & 10.45 & 10.33 & 0.02 \\
\hline TGFB1I1 & 5 & 18 & 13 & $6.50 \mathrm{E}-05$ & 10.45 & 10.33 & 0.02 \\
\hline TAF1A & 23 & 36 & 13 & 1.02E-04 & 2.19 & 3.27 & -0.5 \\
\hline
\end{tabular}




\begin{tabular}{|c|c|c|c|c|c|c|c|}
\hline CYP11A1 & 8 & 21 & 13 & 1.40E-04 & 0.05 & 0.03 & 0.53 \\
\hline ZNF331 & 38 & 51 & 13 & $1.93 \mathrm{E}-04$ & 3.57 & 2.98 & 0.26 \\
\hline USP18 & 9 & 22 & 13 & $2.21 \mathrm{E}-04$ & 5.00 & 5.69 & -0.19 \\
\hline C15orf26 & 5 & 18 & 13 & 2.59E-04 & 1.50 & 0.93 & 0.69 \\
\hline MAP2К3 & 1 & 14 & 13 & 4.89E-04 & 12.12 & 10.05 & 0.27 \\
\hline SFR1 & 0 & 13 & 13 & 5.81E-04 & 22.46 & 23.37 & -0.06 \\
\hline SFR1 & 0 & 13 & 13 & $5.81 \mathrm{E}-04$ & 22.46 & 23.37 & -0.06 \\
\hline PEX14 & 18 & 31 & 13 & $9.82 \mathrm{E}-04$ & 12.49 & 11.03 & 0.18 \\
\hline IZUMO1 & 27 & 40 & 13 & 1.22E-03 & 0.29 & 0.41 & -0.52 \\
\hline DNALI1 & 18 & 31 & 13 & $2.12 \mathrm{E}-03$ & 4.45 & 5.35 & -0.27 \\
\hline RSPH10B & 14 & 27 & 13 & $2.87 \mathrm{E}-03$ & 0.18 & 0.38 & -1.10 \\
\hline RSPH10B2 & 14 & 27 & 13 & 2.87E-03 & 0.04 & 0.06 & -0.58 \\
\hline MIR202 & 63 & 76 & 13 & $3.90 \mathrm{E}-03$ & 0.37 & 0.42 & -0.20 \\
\hline NGFRAP1 & 26 & 39 & 13 & $4.76 \mathrm{E}-03$ & 151.93 & 159.11 & -0.07 \\
\hline PCDHGA2 & 24 & 37 & 13 & $8.40 \mathrm{E}-03$ & 44.63 & 44.34 & 0.01 \\
\hline CAMKK2 & 58 & 71 & 13 & $9.04 \mathrm{E}-03$ & 12.21 & 11.78 & 0.05 \\
\hline DDIT3 & 11 & 24 & 13 & $1.23 \mathrm{E}-02$ & 5.17 & 5.66 & -0.13 \\
\hline MFAP2 & 9 & 22 & 13 & 3.66E-02 & 114.38 & 150.38 & -0.39 \\
\hline KLK6 & 59 & 72 & 13 & $5.26 \mathrm{E}-02$ & 1.88 & 2.03 & -0.11 \\
\hline CT45A6 & 81 & 94 & 13 & $6.60 \mathrm{E}-02$ & 0.06 & 0.04 & 0.81 \\
\hline DAB2IP & 24 & 37 & 13 & 7.52E-02 & 17.74 & 19.02 & -0.10 \\
\hline LRRC36 & 3 & 17 & 14 & 1.44E-08 & 0.23 & 0.32 & -0.47 \\
\hline CIR1 & 0 & 14 & 14 & 1.44E-08 & 32.00 & 38.12 & -0.25 \\
\hline SCRN3 & 0 & 14 & 14 & 1.44E-08 & 23.96 & 27.89 & -0.22 \\
\hline FRS2 & 1 & 15 & 14 & 1.44E-08 & 10.88 & 12.61 & -0.21 \\
\hline PNPO & 1 & 15 & 14 & 1.44E-08 & 47.87 & 55.37 & -0.21 \\
\hline CARS & 1 & 15 & 14 & 1.44E-08 & 15.79 & 17.60 & -0.16 \\
\hline SNW1 & 5 & 19 & 14 & 1.44E-08 & 80.71 & 86.15 & -0.09 \\
\hline SCAP & 6 & 20 & 14 & 1.44E-08 & 11.47 & 11.46 & 0.00 \\
\hline MRP63 & 1 & 15 & 14 & 1.44E-08 & 7.54 & 7.52 & 0.00 \\
\hline RUFY2 & 1 & 15 & 14 & 1.44E-08 & 32.12 & 31.90 & 0.01 \\
\hline RUFY2 & 1 & 15 & 14 & 1.44E-08 & 32.12 & 31.90 & 0.01 \\
\hline HN1L & 1 & 15 & 14 & 1.44E-08 & 171.21 & 156.30 & 0.13 \\
\hline PPT1 & 0 & 14 & 14 & 1.44E-08 & 99.58 & 80.88 & 0.30 \\
\hline PSMC1 & 1 & 15 & 14 & 1.44E-08 & 83.29 & 65.49 & 0.35 \\
\hline XKR9 & 0 & 14 & 14 & 1.44E-08 & 0.43 & 0.34 & 0.35 \\
\hline COPS3 & 4 & 18 & 14 & 1.44E-08 & 136.84 & 100.11 & 0.45 \\
\hline KCTD19 & 3 & 17 & 14 & 1.44E-08 & 1.31 & 0.88 & 0.59 \\
\hline LACTB2 & 0 & 14 & 14 & 1.44E-08 & 1.95 & 0.71 & 1.46 \\
\hline NDUFA6 & 5 & 19 & 14 & $1.52 \mathrm{E}-08$ & 56.97 & 73.19 & -0.36 \\
\hline COX8A & 14 & 28 & 14 & $1.52 \mathrm{E}-08$ & 49.36 & 45.00 & 0.13 \\
\hline PLK4 & 11 & 25 & 14 & 1.55E-08 & 4.44 & 2.41 & 0.88 \\
\hline CDC25B & 8 & 22 & 14 & 1.63E-08 & 241.43 & 191.42 & 0.33 \\
\hline FAM71E1 & 10 & 24 & 14 & $1.79 \mathrm{E}-08$ & 0.12 & 0.15 & -0.30 \\
\hline EMC10 & 10 & 24 & 14 & 1.79E-08 & 48.33 & 17.51 & 1.46 \\
\hline LONRF3 & 24 & 38 & 14 & $2.21 \mathrm{E}-08$ & 4.25 & 4.17 & 0.03 \\
\hline LRFN4 & 26 & 40 & 14 & $3.12 \mathrm{E}-08$ & 2.48 & 2.21 & 0.17 \\
\hline FAM83D & 13 & 27 & 14 & $3.90 \mathrm{E}-08$ & 8.08 & 5.55 & 0.54 \\
\hline GATA2 & 33 & 47 & 14 & 4.12E-08 & 13.84 & 13.07 & 0.08 \\
\hline RNF113A & 31 & 45 & 14 & $6.46 \mathrm{E}-08$ & 6.27 & 5.73 & 0.13 \\
\hline NDUFA1 & 31 & 45 & 14 & 1.33E-07 & 70.69 & 78.72 & -0.16 \\
\hline TCOF1 & 9 & 23 & 14 & 2.19E-07 & 19.21 & 18.95 & 0.02 \\
\hline KIAA1919 & 13 & 27 & 14 & 8.61E-07 & 2.65 & 2.60 & 0.03 \\
\hline GNAS & 33 & 47 & 14 & 4.93E-06 & 458.16 & 442.50 & 0.05 \\
\hline
\end{tabular}




\begin{tabular}{|c|c|c|c|c|c|c|c|}
\hline TRIP4 & 13 & 27 & 14 & 5.73E-06 & 36.61 & 27.87 & 0.39 \\
\hline OXGR1 & 8 & 22 & 14 & $1.22 \mathrm{E}-05$ & 0.05 & 0.02 & 1.11 \\
\hline RPSAP58 & 9 & 23 & 14 & 3.78E-05 & 0.75 & 0.74 & 0.00 \\
\hline NCMAP & 8 & 22 & 14 & 4.37E-05 & 0.05 & 0.08 & -0.65 \\
\hline SUMF1 & 25 & 39 & 14 & $3.60 \mathrm{E}-04$ & 22.44 & 20.47 & 0.13 \\
\hline ALDH1L2 & 25 & 39 & 14 & $4.11 \mathrm{E}-04$ & 0.34 & 0.29 & 0.22 \\
\hline EPHA1 & 33 & 47 & 14 & 1.06E-03 & 1.04 & 1.67 & -0.68 \\
\hline MTIF2 & 4 & 18 & 14 & 1.23E-03 & 16.40 & 16.32 & 0.01 \\
\hline ZMAT1 & 32 & 46 & 14 & $1.85 \mathrm{E}-03$ & 2.42 & 3.10 & -0.36 \\
\hline ZCCHC4 & 22 & 36 & 14 & $3.13 \mathrm{E}-03$ & 4.57 & 5.04 & -0.14 \\
\hline PFDN5 & 52 & 66 & 14 & 7.59E-03 & 165.38 & 213.81 & -0.37 \\
\hline GABRB3 & 45 & 59 & 14 & 7.66E-03 & 1.19 & 1.16 & 0.04 \\
\hline NEURL3 & 41 & 55 & 14 & 8.07E-03 & 0.03 & 0.01 & 2.08 \\
\hline PCDHA9 & 13 & 27 & 14 & $1.31 \mathrm{E}-02$ & 0.88 & 0.67 & 0.39 \\
\hline PYCRL & 36 & 50 & 14 & 2.15E-02 & 0.48 & 0.48 & 0.00 \\
\hline ANO4 & 17 & 31 & 14 & 4.60E-02 & 0.56 & 1.79 & -1.69 \\
\hline PRND & 35 & 49 & 14 & 8.78E-02 & 0.06 & 0.09 & -0.54 \\
\hline PIGB & 1 & 16 & 15 & $1.44 \mathrm{E}-08$ & 9.07 & 10.53 & -0.22 \\
\hline ZNF497 & 5 & 20 & 15 & $1.44 \mathrm{E}-08$ & 1.91 & 1.49 & 0.36 \\
\hline FAM127B & 13 & 28 & 15 & $5.65 \mathrm{E}-07$ & 33.00 & 30.29 & 0.12 \\
\hline TOP1MT & 24 & 39 & 15 & 5.95E-07 & 9.47 & 8.28 & 0.19 \\
\hline DGKA & 10 & 25 & 15 & 7.82E-07 & 198.25 & 199.67 & -0.01 \\
\hline GPR19 & 0 & 15 & 15 & $6.35 \mathrm{E}-06$ & 0.13 & 0.32 & -1.25 \\
\hline C9orf129 & 20 & 35 & 15 & 8.63E-06 & 0.05 & 0.14 & -1.43 \\
\hline CALHM2 & 2 & 17 & 15 & 3.33E-05 & 35.25 & 30.30 & 0.22 \\
\hline MGARP & 34 & 49 & 15 & 7.52E-05 & 62.54 & 63.24 & -0.02 \\
\hline MTL5 & 27 & 42 & 15 & $1.86 \mathrm{E}-04$ & 0.72 & 0.70 & 0.05 \\
\hline RUSC1 & 20 & 35 & 15 & 1.07E-03 & 7.78 & 7.27 & 0.10 \\
\hline KLC4 & 14 & 29 & 15 & $1.08 \mathrm{E}-03$ & 11.20 & 11.40 & -0.03 \\
\hline EID3 & 24 & 39 & 15 & 1.33E-03 & 1.30 & 1.95 & -0.58 \\
\hline ZMAT4 & 9 & 24 & 15 & $4.69 \mathrm{E}-03$ & 0.17 & 0.27 & -0.64 \\
\hline C2orf50 & 56 & 71 & 15 & $4.90 \mathrm{E}-03$ & 0.07 & 0.10 & -0.54 \\
\hline MRVI1 & 70 & 85 & 15 & $1.14 \mathrm{E}-02$ & 0.52 & 0.48 & 0.12 \\
\hline C1orf64 & 81 & 96 & 15 & 1.32E-02 & 0.08 & 0.10 & -0.34 \\
\hline ZNF235 & 3 & 18 & 15 & $1.54 \mathrm{E}-02$ & 5.76 & 5.89 & -0.03 \\
\hline CARD14 & 56 & 71 & 15 & 2.64E-02 & 0.51 & 0.41 & 0.30 \\
\hline C19orf38 & 57 & 72 & 15 & 3.30E-02 & 0.17 & 0.14 & 0.22 \\
\hline LIG1 & 2 & 17 & 15 & 3.50E-02 & 0.97 & 2.31 & -1.25 \\
\hline AQP7 & 53 & 68 & 15 & $6.55 \mathrm{E}-02$ & 0.14 & 0.09 & 0.70 \\
\hline C11orf83 & 0 & 16 & 16 & $1.44 \mathrm{E}-08$ & 2.48 & 3.10 & -0.32 \\
\hline UFSP2 & 0 & 16 & 16 & $1.44 \mathrm{E}-08$ & 71.39 & 84.81 & -0.25 \\
\hline TMEM219 & 13 & 29 & 16 & $1.44 \mathrm{E}-08$ & 28.20 & 28.97 & -0.04 \\
\hline GINS1 & 1 & 17 & 16 & $1.44 \mathrm{E}-08$ & 4.54 & 3.91 & 0.21 \\
\hline WDR66 & 5 & 21 & 16 & $1.44 \mathrm{E}-08$ & 23.62 & 19.81 & 0.25 \\
\hline KPNA6 & 9 & 25 & 16 & $1.52 \mathrm{E}-08$ & 28.25 & 21.96 & 0.36 \\
\hline C11orf70 & 7 & 23 & 16 & $1.52 \mathrm{E}-08$ & 0.33 & 0.17 & 0.93 \\
\hline SET & 2 & 18 & 16 & $1.60 \mathrm{E}-08$ & 275.42 & 222.70 & 0.31 \\
\hline ZNF414 & 11 & 27 & 16 & 1.63E-08 & 3.94 & 3.86 & 0.03 \\
\hline ZNF726 & 21 & 37 & 16 & $1.84 \mathrm{E}-08$ & 0.95 & 0.99 & -0.07 \\
\hline SEPSECS & 9 & 25 & 16 & $9.29 \mathrm{E}-08$ & 3.84 & 3.66 & 0.07 \\
\hline RBMXL2 & 51 & 67 & 16 & $9.89 E-08$ & 0.17 & 0.16 & 0.07 \\
\hline PKD2L2 & 11 & 27 & 16 & 4.90E-07 & 0.65 & 0.82 & -0.34 \\
\hline ZNF350 & 14 & 30 & 16 & 7.06E-07 & 6.13 & 11.48 & -0.90 \\
\hline SMUG1 & 18 & 34 & 16 & $3.41 \mathrm{E}-06$ & 6.35 & 5.63 & 0.17 \\
\hline
\end{tabular}




\begin{tabular}{|c|c|c|c|c|c|c|c|}
\hline UFC1 & 15 & 31 & 16 & $3.90 \mathrm{E}-06$ & 120.85 & 88.13 & 0.46 \\
\hline HSDL2 & 3 & 19 & 16 & 5.77E-06 & 19.85 & 18.99 & 0.06 \\
\hline DYNLT3 & 16 & 32 & 16 & 7.12E-06 & 33.88 & 32.82 & 0.05 \\
\hline DUS3L & 15 & 31 & 16 & 1.09E-05 & 6.30 & 4.87 & 0.37 \\
\hline MFSD5 & 5 & 21 & 16 & 1.09E-05 & 21.51 & 18.82 & 0.19 \\
\hline SYCE1 & 56 & 72 & 16 & $2.25 \mathrm{E}-05$ & 0.57 & 0.25 & 1.20 \\
\hline HOXB8 & 12 & 28 & 16 & $2.28 \mathrm{E}-05$ & 4.92 & 5.01 & -0.02 \\
\hline ANO7 & 76 & 92 & 16 & $2.59 \mathrm{E}-04$ & 0.70 & 0.57 & 0.30 \\
\hline KIAA1598 & 60 & 76 & 16 & $2.71 \mathrm{E}-04$ & 1.42 & 1.52 & -0.10 \\
\hline HEMK1 & 15 & 31 & 16 & 2.73E-04 & 1.37 & 1.44 & -0.07 \\
\hline KCNE3 & 33 & 49 & 16 & $9.06 \mathrm{E}-04$ & 0.06 & 0.10 & -0.83 \\
\hline TOR1AIP2 & 10 & 26 & 16 & $1.28 \mathrm{E}-03$ & 51.44 & 46.67 & 0.14 \\
\hline QPRT & 69 & 85 & 16 & $2.23 \mathrm{E}-03$ & 0.50 & 0.50 & 0.00 \\
\hline IGF2 & 77 & 93 & 16 & $3.13 \mathrm{E}-03$ & 8.78 & 7.78 & 0.17 \\
\hline ISG15 & 51 & 67 & 16 & $4.76 \mathrm{E}-03$ & 10.67 & 13.13 & -0.30 \\
\hline RASAL3 & 52 & 68 & 16 & 1.64E-02 & 0.34 & 0.24 & 0.49 \\
\hline NEK8 & 29 & 45 & 16 & $1.84 \mathrm{E}-02$ & 0.68 & 0.48 & 0.52 \\
\hline PAK6 & 22 & 38 & 16 & $2.05 \mathrm{E}-02$ & 17.78 & 9.87 & 0.85 \\
\hline CAPN12 & 74 & 90 & 16 & $2.26 \mathrm{E}-02$ & 13.78 & 12.26 & 0.17 \\
\hline SNAPC4 & 76 & 92 & 16 & $2.53 \mathrm{E}-02$ & 2.77 & 1.86 & 0.57 \\
\hline SLC23A1 & 78 & 94 & 16 & $3.23 \mathrm{E}-02$ & 0.15 & 0.11 & 0.34 \\
\hline IDI2-AS1 & 80 & 96 & 16 & 3.32E-02 & 0.04 & 0.04 & -0.04 \\
\hline SLC38A7 & 13 & 29 & 16 & 4.59E-02 & 17.72 & 18.57 & -0.07 \\
\hline MTO1 & 11 & 28 & 17 & 1.44E-08 & 21.26 & 26.76 & -0.33 \\
\hline ATL3 & 0 & 17 & 17 & 1.44E-08 & 42.26 & 39.60 & 0.09 \\
\hline ZNF440 & 1 & 18 & 17 & 1.44E-08 & 4.24 & 3.89 & 0.12 \\
\hline NINL & 2 & 19 & 17 & 1.44E-08 & 12.26 & 9.74 & 0.33 \\
\hline AKR1E2 & 17 & 34 & 17 & 1.46E-08 & 1.94 & 1.50 & 0.37 \\
\hline DLG3 & 19 & 36 & 17 & 1.52E-08 & 1.70 & 1.94 & -0.19 \\
\hline SMUG1 & 17 & 34 & 17 & 5.07E-07 & 6.35 & 5.63 & 0.17 \\
\hline SYTL4 & 20 & 37 & 17 & 3.32E-06 & 356.86 & 227.69 & 0.65 \\
\hline SYTL4 & 20 & 37 & 17 & 3.32E-06 & 356.86 & 227.69 & 0.65 \\
\hline SOX30 & 27 & 44 & 17 & $6.21 \mathrm{E}-06$ & 0.04 & 0.03 & 0.29 \\
\hline TGIF1 & 8 & 25 & 17 & 6.61E-06 & 30.13 & 24.18 & 0.32 \\
\hline BBS2 & 26 & 43 & 17 & $1.31 \mathrm{E}-04$ & 90.58 & 81.61 & 0.15 \\
\hline POLR2G & 41 & 58 & 17 & $3.21 \mathrm{E}-04$ & 36.69 & 40.07 & -0.13 \\
\hline EXOC7 & 23 & 40 & 17 & 5.72E-04 & 44.66 & 54.64 & -0.29 \\
\hline CCDC58 & 14 & 31 & 17 & 8.36E-04 & 24.64 & 25.73 & -0.06 \\
\hline JAK3 & 19 & 36 & 17 & 2.89E-03 & 0.20 & 0.15 & 0.47 \\
\hline IVD & 4 & 21 & 17 & 3.17E-03 & 17.42 & 16.32 & 0.09 \\
\hline KCNE1L & 47 & 64 & 17 & 4.83E-03 & 35.93 & 35.28 & 0.03 \\
\hline POGZ & 81 & 98 & 17 & 2.27E-02 & 43.20 & 30.12 & 0.52 \\
\hline MORF4L2 & 3 & 20 & 17 & 2.55E-02 & 301.86 & 347.28 & -0.20 \\
\hline C1orf168 & 69 & 86 & 17 & $5.04 \mathrm{E}-02$ & 0.17 & 0.13 & 0.42 \\
\hline CNN1 & 26 & 43 & 17 & 6.16E-02 & 0.39 & 0.25 & 0.68 \\
\hline CCDC73 & 74 & 91 & 17 & $8.45 \mathrm{E}-02$ & 3.61 & 4.21 & -0.22 \\
\hline LCT & 80 & 97 & 17 & 8.59E-02 & 0.00 & 0.00 & 0.87 \\
\hline SERF2 & 16 & 34 & 18 & $1.57 \mathrm{E}-08$ & 304.92 & 347.00 & -0.19 \\
\hline LARS & 15 & 33 & 18 & 5.69E-08 & 122.66 & 129.09 & -0.07 \\
\hline DNM1P46 & 12 & 30 & 18 & $1.52 \mathrm{E}-07$ & 0.16 & 0.06 & 1.43 \\
\hline ZNF331 & 34 & 52 & 18 & $2.15 \mathrm{E}-07$ & 3.57 & 2.98 & 0.26 \\
\hline EBPL & 21 & 39 & 18 & $2.49 \mathrm{E}-07$ & 18.18 & 17.40 & 0.06 \\
\hline POLR2D & 20 & 38 & 18 & $4.74 \mathrm{E}-07$ & 17.62 & 24.88 & -0.50 \\
\hline C5orf63 & 9 & 27 & 18 & $1.48 \mathrm{E}-05$ & 0.48 & 0.24 & 1.01 \\
\hline
\end{tabular}




\begin{tabular}{|c|c|c|c|c|c|c|c|}
\hline NHLRC4 & 40 & 58 & 18 & 2.85E-05 & 7.35 & 6.73 & 0.13 \\
\hline COX7A1 & 20 & 38 & 18 & 4.67E-05 & 5.18 & 10.00 & -0.95 \\
\hline CECR1 & 11 & 29 & 18 & 4.67E-04 & 5.87 & 4.76 & 0.30 \\
\hline CLNS1A & 15 & 33 & 18 & $6.44 \mathrm{E}-04$ & 198.48 & 174.26 & 0.19 \\
\hline MKLN1 & 3 & 21 & 18 & $1.39 \mathrm{E}-02$ & 18.39 & 17.39 & 0.08 \\
\hline MCTS1 & 18 & 36 & 18 & $1.55 \mathrm{E}-02$ & 30.71 & 26.45 & 0.22 \\
\hline ZNF439 & 77 & 95 & 18 & $5.01 \mathrm{E}-02$ & 0.40 & 0.35 & 0.17 \\
\hline NAP1L5 & 36 & 54 & 18 & 7.74E-02 & 4.00 & 4.13 & -0.05 \\
\hline SATL1 & 63 & 81 & 18 & 8.61E-02 & 2.29 & 2.10 & 0.13 \\
\hline STARD8 & 13 & 32 & 19 & $1.44 \mathrm{E}-08$ & 11.86 & 12.08 & -0.03 \\
\hline ECSIT & 21 & 40 & 19 & $1.47 \mathrm{E}-08$ & 26.08 & 12.80 & 1.03 \\
\hline TCP11 & 30 & 49 & 19 & 1.52E-08 & 0.02 & 0.01 & 0.74 \\
\hline MAFG & 68 & 87 & 19 & 1.57E-08 & 17.53 & 12.47 & 0.49 \\
\hline SSR4P1 & 31 & 50 & 19 & $1.62 \mathrm{E}-08$ & 0.14 & 0.21 & -0.58 \\
\hline RBM5 & 14 & 33 & 19 & $1.64 \mathrm{E}-08$ & 312.40 & 296.50 & 0.08 \\
\hline FAM217B & 27 & 46 & 19 & $1.87 \mathrm{E}-08$ & 4.34 & 4.88 & -0.17 \\
\hline CCDC19 & 15 & 34 & 19 & 3.38E-06 & 0.62 & 0.47 & 0.40 \\
\hline LRRC37A6P & 19 & 38 & 19 & 4.49E-06 & 0.17 & 0.24 & -0.48 \\
\hline REEP2 & 4 & 23 & 19 & $1.10 \mathrm{E}-05$ & 2.15 & 1.32 & 0.70 \\
\hline ACBD4 & 0 & 19 & 19 & 1.97E-04 & 1.18 & 0.84 & 0.49 \\
\hline PLA2G6 & 22 & 41 & 19 & 1.37E-03 & 4.90 & 2.67 & 0.87 \\
\hline PLA2G6 & 22 & 41 & 19 & 1.37E-03 & 4.90 & 2.67 & 0.87 \\
\hline ZNF331 & 51 & 70 & 19 & $1.41 \mathrm{E}-03$ & 3.57 & 2.98 & 0.26 \\
\hline ZNF331 & 51 & 70 & 19 & $1.41 \mathrm{E}-03$ & 3.57 & 2.98 & 0.26 \\
\hline HOXB3 & 1 & 20 & 19 & $4.90 \mathrm{E}-03$ & 5.12 & 6.78 & -0.41 \\
\hline ZNF711 & 16 & 35 & 19 & $5.80 \mathrm{E}-03$ & 7.58 & 6.58 & 0.20 \\
\hline SH3BGR & 44 & 63 & 19 & $1.59 \mathrm{E}-02$ & 2.03 & 2.06 & -0.02 \\
\hline $\mathrm{CHM}$ & 18 & 37 & 19 & 2.19E-02 & 17.05 & 16.25 & 0.07 \\
\hline CACNA2D4 & 56 & 75 & 19 & 3.02E-02 & 0.08 & 0.02 & 1.82 \\
\hline CEP85 & 13 & 33 & 20 & $1.44 \mathrm{E}-08$ & 4.58 & 4.28 & 0.10 \\
\hline MYSM1 & 2 & 22 & 20 & $1.44 \mathrm{E}-08$ & 15.49 & 13.92 & 0.15 \\
\hline TBL2 & 6 & 26 & 20 & $1.44 \mathrm{E}-08$ & 12.16 & 10.49 & 0.21 \\
\hline RPS24 & 11 & 31 & 20 & $1.50 \mathrm{E}-08$ & 2446.97 & 2193.85 & 0.16 \\
\hline ECSIT & 24 & 44 & 20 & $1.51 \mathrm{E}-08$ & 26.08 & 12.80 & 1.03 \\
\hline ZRSR2 & 9 & 29 & 20 & $1.52 \mathrm{E}-08$ & 10.87 & 10.98 & -0.01 \\
\hline COL4A3BP & 1 & 21 & 20 & $1.52 \mathrm{E}-08$ & 15.96 & 16.99 & -0.09 \\
\hline ZXDB & 23 & 43 & 20 & $1.55 \mathrm{E}-08$ & 2.26 & 1.85 & 0.29 \\
\hline TTLL10 & 34 & 54 & 20 & 1.67E-08 & 0.30 & 0.12 & 1.33 \\
\hline CABYR & 16 & 36 & 20 & 2.00E-07 & 2.47 & 1.91 & 0.37 \\
\hline HOXB5 & 39 & 59 & 20 & 7.20E-06 & 63.50 & 50.37 & 0.33 \\
\hline GPR35 & 64 & 84 & 20 & 4.56E-04 & 2.99 & 3.07 & -0.04 \\
\hline INPP5F & 45 & 65 & 20 & $7.75 \mathrm{E}-04$ & 13.34 & 14.35 & -0.11 \\
\hline $\mathrm{RDH} 13$ & 27 & 47 & 20 & $1.41 \mathrm{E}-03$ & 0.77 & 0.43 & 0.85 \\
\hline CREBL2 & 0 & 20 & 20 & 2.22E-03 & 18.61 & 15.84 & 0.23 \\
\hline ZNF749 & 0 & 20 & 20 & $3.15 \mathrm{E}-03$ & 13.20 & 12.87 & 0.04 \\
\hline COMT & 67 & 87 & 20 & $2.84 \mathrm{E}-02$ & 98.34 & 72.18 & 0.45 \\
\hline TSSC4 & 75 & 95 & 20 & 4.71E-02 & 6.96 & 4.30 & 0.70 \\
\hline SIPA1L1 & 80 & 100 & 20 & 8.53E-02 & 9.01 & 9.36 & -0.06 \\
\hline TOMM20 & 5 & 26 & 21 & $1.44 \mathrm{E}-08$ & 56.21 & 49.11 & 0.19 \\
\hline VILL & 12 & 33 & 21 & $1.44 \mathrm{E}-08$ & 1.75 & 1.43 & 0.29 \\
\hline GCAT & 1 & 22 & 21 & $1.44 \mathrm{E}-08$ & 1.93 & 1.16 & 0.74 \\
\hline MRPS22 & 16 & 37 & 21 & $1.52 \mathrm{E}-08$ & 70.84 & 99.59 & -0.49 \\
\hline STPG1 & 22 & 43 & 21 & $1.55 \mathrm{E}-08$ & 8.50 & 12.38 & -0.54 \\
\hline PSTK & 1 & 22 & 21 & $1.55 \mathrm{E}-08$ & 1.88 & 1.82 & 0.05 \\
\hline
\end{tabular}




\begin{tabular}{|c|c|c|c|c|c|c|c|}
\hline CCDC8 & 23 & 44 & 21 & $1.60 \mathrm{E}-08$ & 0.03 & 0.03 & 0.00 \\
\hline FAM96A & 13 & 34 & 21 & 7.54E-08 & 82.61 & 87.34 & -0.08 \\
\hline CUEDC2 & 1 & 22 & 21 & 3.96E-07 & 27.78 & 31.40 & -0.18 \\
\hline CABYR & 22 & 43 & 21 & $6.00 \mathrm{E}-07$ & 2.47 & 1.91 & 0.37 \\
\hline CBWD2 & 2 & 23 & 21 & 1.97E-06 & 5.73 & 6.26 & -0.13 \\
\hline UBE2L3 & 14 & 35 & 21 & $9.09 \mathrm{E}-05$ & 101.13 & 76.80 & 0.40 \\
\hline PAK6 & 13 & 34 & 21 & $3.10 \mathrm{E}-04$ & 17.78 & 9.87 & 0.85 \\
\hline CLK2 & 31 & 52 & 21 & $2.53 \mathrm{E}-03$ & 25.67 & 22.67 & 0.18 \\
\hline GHRL & 49 & 70 & 21 & 3.94E-02 & 97.76 & 108.06 & -0.14 \\
\hline SUSD2 & 66 & 87 & 21 & 5.03E-02 & 0.03 & 0.06 & -1.03 \\
\hline C1orf198 & 7 & 29 & 22 & $1.44 \mathrm{E}-08$ & 7.65 & 5.78 & 0.40 \\
\hline PUM1 & 7 & 29 & 22 & $1.44 \mathrm{E}-08$ & 110.62 & 80.78 & 0.45 \\
\hline IL10RA & 13 & 35 & 22 & 1.47E-08 & 2.31 & 3.39 & -0.55 \\
\hline STOM & 8 & 30 & 22 & $1.60 \mathrm{E}-08$ & 301.47 & 266.40 & 0.18 \\
\hline PIGU & 20 & 42 & 22 & $9.81 \mathrm{E}-08$ & 27.67 & 26.12 & 0.08 \\
\hline LDLRAD4 & 26 & 48 & 22 & $2.78 \mathrm{E}-05$ & 1.20 & 1.04 & 0.21 \\
\hline LDLRAD4 & 26 & 48 & 22 & 2.78E-05 & 1.20 & 1.04 & 0.21 \\
\hline ELN & 12 & 34 & 22 & $3.41 \mathrm{E}-04$ & 9.47 & 6.93 & 0.45 \\
\hline RGPD2 & 44 & 66 & 22 & 2.13E-03 & 0.08 & 0.09 & -0.24 \\
\hline RGS4 & 4 & 26 & 22 & 6.93E-03 & 55.64 & 51.60 & 0.11 \\
\hline RGS4 & 4 & 26 & 22 & 6.93E-03 & 55.64 & 51.60 & 0.11 \\
\hline RGS4 & 4 & 26 & 22 & 6.93E-03 & 55.64 & 51.60 & 0.11 \\
\hline ZSCAN23 & 76 & 98 & 22 & $9.68 \mathrm{E}-03$ & 0.23 & 0.43 & -0.88 \\
\hline HMSD & 14 & 36 & 22 & 1.79E-02 & 32.21 & 33.50 & -0.06 \\
\hline KRT8 & 73 & 95 & 22 & 2.79E-02 & 84.31 & 101.27 & -0.26 \\
\hline ABAT & 8 & 30 & 22 & 4.97E-02 & 0.32 & 0.37 & -0.18 \\
\hline TMPRSS6 & 67 & 89 & 22 & 5.98E-02 & 0.02 & 0.01 & 1.78 \\
\hline NSA2 & 8 & 31 & 23 & 1.47E-08 & 176.72 & 195.11 & -0.14 \\
\hline ZMAT3 & 3 & 26 & 23 & $1.52 \mathrm{E}-08$ & 62.84 & 52.54 & 0.26 \\
\hline VMA21 & 20 & 43 & 23 & 1.68E-08 & 24.56 & 22.91 & 0.10 \\
\hline GEMIN5 & 28 & 51 & 23 & $2.13 \mathrm{E}-06$ & 6.55 & 7.35 & -0.17 \\
\hline SPARC & 21 & 44 & 23 & 3.67E-06 & 2342.55 & 2385.35 & -0.03 \\
\hline UBE2L3 & 12 & 35 & 23 & 3.87E-06 & 101.13 & 76.80 & 0.40 \\
\hline ETV4 & 30 & 53 & 23 & 1.47E-05 & 3.25 & 4.36 & -0.42 \\
\hline MGAT1 & 25 & 48 & 23 & $1.36 \mathrm{E}-04$ & 36.02 & 28.16 & 0.36 \\
\hline MCTS1 & 18 & 41 & 23 & 1.81E-03 & 30.71 & 26.45 & 0.22 \\
\hline OC90 & 54 & 77 & 23 & 7.60E-02 & 0.80 & 1.13 & -0.50 \\
\hline LINC00320 & 59 & 82 & 23 & $9.29 \mathrm{E}-02$ & 0.07 & 0.03 & 1.36 \\
\hline PLEC & 15 & 39 & 24 & 1.56E-08 & 35.07 & 27.76 & 0.34 \\
\hline ATP5J2 & 25 & 49 & 24 & 2.99E-07 & 389.08 & 433.24 & -0.16 \\
\hline AARS & 15 & 39 & 24 & 2.33E-06 & 52.17 & 48.55 & 0.10 \\
\hline HSD3B7 & 41 & 65 & 24 & $1.98 \mathrm{E}-04$ & 0.78 & 0.66 & 0.24 \\
\hline RAPGEF1 & 72 & 96 & 24 & 3.49E-04 & 56.37 & 51.44 & 0.13 \\
\hline SCG5 & 42 & 66 & 24 & 2.10E-02 & 0.68 & 0.51 & 0.40 \\
\hline INS-IGF2 & 54 & 78 & 24 & 3.47E-02 & 8.78 & 7.78 & 0.17 \\
\hline INS & 54 & 78 & 24 & 3.47E-02 & 8.78 & 7.78 & 0.17 \\
\hline P2RX3 & 67 & 91 & 24 & 3.57E-02 & 0.02 & 0.19 & -3.44 \\
\hline MR1 & 40 & 64 & 24 & 3.64E-02 & 2.24 & 2.22 & 0.01 \\
\hline IFITM10 & 17 & 41 & 24 & 7.83E-02 & 61.13 & 46.32 & 0.40 \\
\hline ATF5 & 3 & 28 & 25 & $1.44 \mathrm{E}-08$ & 12.77 & 8.74 & 0.55 \\
\hline ATF5 & 3 & 28 & 25 & 1.44E-08 & 12.77 & 8.74 & 0.55 \\
\hline TASP1 & 9 & 34 & 25 & 1.47E-08 & 2.91 & 3.38 & -0.22 \\
\hline STPG1 & 32 & 57 & 25 & $2.08 \mathrm{E}-08$ & 8.50 & 12.38 & -0.54 \\
\hline MIR503 & 23 & 48 & 25 & 4.06E-07 & 3.89 & 3.39 & 0.20 \\
\hline
\end{tabular}




\begin{tabular}{|c|c|c|c|c|c|c|c|}
\hline MIR424 & 23 & 48 & 25 & 4.06E-07 & 3.89 & 3.39 & 0.20 \\
\hline XKRX & 38 & 63 & 25 & $2.58 \mathrm{E}-06$ & 0.03 & 0.01 & 1.56 \\
\hline RPL24 & 20 & 45 & 25 & $1.21 \mathrm{E}-05$ & 913.12 & 1152.83 & -0.34 \\
\hline LSG1 & 17 & 42 & 25 & $5.52 \mathrm{E}-05$ & 51.45 & 50.61 & 0.02 \\
\hline FAM74A3 & 50 & 75 & 25 & $8.40 \mathrm{E}-05$ & 0.35 & 0.04 & 3.10 \\
\hline SV2A & 6 & 31 & 25 & $6.56 \mathrm{E}-04$ & 0.16 & 0.20 & -0.29 \\
\hline GPATCH2 & 28 & 53 & 25 & $5.50 \mathrm{E}-03$ & 6.47 & 10.20 & -0.66 \\
\hline SPATA17 & 28 & 53 & 25 & $5.50 \mathrm{E}-03$ & 0.34 & 0.49 & -0.51 \\
\hline RPAP1 & 61 & 86 & 25 & $2.52 \mathrm{E}-02$ & 8.67 & 9.04 & -0.06 \\
\hline SEC61A2 & 10 & 36 & 26 & $1.44 \mathrm{E}-08$ & 7.47 & 10.53 & -0.50 \\
\hline MRPS17 & 29 & 55 & 26 & $1.44 \mathrm{E}-08$ & 63.91 & 55.15 & 0.21 \\
\hline ALKBH7 & 5 & 31 & 26 & 2.49E-07 & 3.86 & 3.92 & -0.02 \\
\hline ZNF468 & 15 & 41 & 26 & 2.16E-05 & 5.89 & 6.96 & -0.24 \\
\hline FAM101A & 50 & 76 & 26 & $5.65 \mathrm{E}-04$ & 55.48 & 48.24 & 0.20 \\
\hline C19orf21 & 47 & 73 & 26 & $1.40 \mathrm{E}-03$ & 0.00 & 0.01 & -0.88 \\
\hline HSPA9 & 22 & 49 & 27 & $1.44 \mathrm{E}-08$ & 318.67 & 415.42 & -0.38 \\
\hline DCAF8 & 3 & 30 & 27 & $1.44 \mathrm{E}-08$ & 96.70 & 79.61 & 0.28 \\
\hline CAPS2 & 1 & 28 & 27 & 1.47E-08 & 0.49 & 0.54 & -0.14 \\
\hline COIL & 18 & 45 & 27 & 1.57E-08 & 11.05 & 9.17 & 0.27 \\
\hline PALM2 & 11 & 38 & 27 & $1.01 \mathrm{E}-07$ & 284.69 & 216.88 & 0.39 \\
\hline NDN & 23 & 50 & 27 & $1.12 \mathrm{E}-06$ & 15.12 & 13.74 & 0.14 \\
\hline CFD & 58 & 85 & 27 & $3.84 \mathrm{E}-06$ & 0.10 & 0.10 & 0.00 \\
\hline LAS1L & 52 & 79 & 27 & $1.01 \mathrm{E}-03$ & 4.84 & 5.32 & -0.14 \\
\hline RBBP9 & 13 & 40 & 27 & $3.45 \mathrm{E}-03$ & 7.35 & 5.78 & 0.35 \\
\hline PPP1R2P3 & 36 & 63 & 27 & 4.26E-02 & 0.06 & 0.09 & -0.68 \\
\hline MYT1L & 61 & 88 & 27 & 5.91E-02 & 0.12 & 0.17 & -0.55 \\
\hline CCDC106 & 16 & 44 & 28 & $1.44 \mathrm{E}-08$ & 30.48 & 27.18 & 0.17 \\
\hline SMU1 & 9 & 37 & 28 & $1.55 \mathrm{E}-08$ & 54.58 & 55.96 & -0.04 \\
\hline GBAP1 & 8 & 36 & 28 & $5.85 \mathrm{E}-08$ & 11.40 & 10.46 & 0.12 \\
\hline IFITM1 & 29 & 57 & 28 & $9.88 \mathrm{E}-02$ & 96.29 & 88.64 & 0.12 \\
\hline BMPR1B & 18 & 46 & 28 & 9.97E-02 & 0.68 & 0.64 & 0.10 \\
\hline GRAMD1C & 2 & 31 & 29 & $1.44 \mathrm{E}-08$ & 6.13 & 6.35 & -0.05 \\
\hline CDKL3 & 27 & 56 & 29 & 3.39E-07 & 771.93 & 681.71 & 0.18 \\
\hline AMBRA1 & 16 & 45 & 29 & $6.63 \mathrm{E}-04$ & 8.10 & 6.58 & 0.30 \\
\hline GFI1B & 19 & 48 & 29 & $1.54 \mathrm{E}-03$ & 0.10 & 0.07 & 0.64 \\
\hline TNFRSF8 & 47 & 76 & 29 & $8.20 \mathrm{E}-03$ & 0.01 & 0.03 & -1.63 \\
\hline ATP6V0A4 & 17 & 46 & 29 & 8.70E-02 & 0.02 & 0.09 & -1.93 \\
\hline C1orf141 & 34 & 64 & 30 & $1.44 \mathrm{E}-08$ & 0.02 & 0.04 & -1.10 \\
\hline CKLF-CMTM1 & 35 & 65 & 30 & $1.28 \mathrm{E}-04$ & 30.75 & 34.38 & -0.16 \\
\hline CKLF & 35 & 65 & 30 & $1.28 \mathrm{E}-04$ & 30.75 & 34.38 & -0.16 \\
\hline HHIPL2 & 66 & 96 & 30 & 4.79E-02 & 0.02 & 0.06 & -1.2 \\
\hline GCFC2 & 1 & 32 & 31 & $1.44 \mathrm{E}-08$ & 1.10 & 1.19 & -0.12 \\
\hline APC2 & 4 & 35 & 31 & 8.53E-05 & 0.41 & 0.32 & 0.36 \\
\hline TMPRSS12 & 33 & 64 & 31 & $2.08 \mathrm{E}-04$ & 0.05 & 0.03 & 0.71 \\
\hline DCAF8 & 3 & 35 & 32 & $1.44 \mathrm{E}-08$ & 96.70 & 79.61 & 0.28 \\
\hline DCAF8 & 3 & 35 & 32 & $1.44 \mathrm{E}-08$ & 96.70 & 79.61 & 0.28 \\
\hline DVL3 & 7 & 39 & 32 & $1.48 \mathrm{E}-08$ & 24.04 & 21.47 & 0.16 \\
\hline C10orf11 & 9 & 41 & 32 & 5.61E-06 & 10.04 & 11.32 & -0.17 \\
\hline TNFRSF1A & 31 & 63 & 32 & $3.68 \mathrm{E}-04$ & 210.02 & 172.32 & 0.29 \\
\hline MFSD4 & 10 & 42 & 32 & 4.90E-04 & 0.60 & 0.70 & -0.22 \\
\hline FBXL22 & 29 & 61 & 32 & 7.10E-04 & 0.08 & 0.09 & -0.07 \\
\hline PSMF1 & 63 & 96 & 33 & 5.96E-03 & 49.90 & 47.08 & 0.08 \\
\hline GBA & 0 & 33 & 33 & 8.91E-03 & 103.23 & 93.11 & 0.15 \\
\hline GBA & 0 & 33 & 33 & 8.91E-03 & 103.23 & 93.11 & 0.15 \\
\hline
\end{tabular}




\begin{tabular}{|c|c|c|c|c|c|c|c|}
\hline C12orf71 & 60 & 93 & 33 & $3.16 \mathrm{E}-02$ & 0.01 & 0.08 & -2.45 \\
\hline $\mathrm{PC}$ & 38 & 71 & 33 & $5.08 \mathrm{E}-02$ & 14.53 & 11.54 & 0.33 \\
\hline MYL4 & 23 & 56 & 33 & 8.42E-02 & 1.55 & 1.22 & 0.35 \\
\hline ANKRD20A5P & 17 & 51 & 34 & $1.44 \mathrm{E}-08$ & 0.14 & 0.19 & -0.47 \\
\hline EXOSC5 & 8 & 42 & 34 & $1.44 \mathrm{E}-08$ & 1.92 & 2.63 & -0.46 \\
\hline FMO5 & 31 & 65 & 34 & 1.44E-08 & 1.33 & 1.32 & 0.01 \\
\hline ZNF878 & 21 & 55 & 34 & $1.44 \mathrm{E}-08$ & 1.65 & 1.38 & 0.26 \\
\hline HOOK2 & 8 & 42 & 34 & 1.44E-08 & 9.77 & 7.90 & 0.31 \\
\hline MEG3 & 30 & 64 & 34 & $1.60 \mathrm{E}-08$ & 40.71 & 33.61 & 0.28 \\
\hline MEG3 & 30 & 64 & 34 & $1.60 \mathrm{E}-08$ & 40.71 & 33.61 & 0.28 \\
\hline DDX19B & 22 & 56 & 34 & $4.40 \mathrm{E}-07$ & 44.14 & 44.31 & -0.01 \\
\hline HIF3A & 5 & 39 & 34 & $4.00 \mathrm{E}-04$ & 1.25 & 0.71 & 0.82 \\
\hline SETDB1 & 17 & 52 & 35 & 1.44E-08 & 21.03 & 20.94 & 0.01 \\
\hline DND1 & 35 & 70 & 35 & 1.57E-08 & 45.60 & 37.72 & 0.27 \\
\hline LYPD3 & 40 & 75 & 35 & $3.52 \mathrm{E}-06$ & 0.01 & 0.11 & -3.00 \\
\hline LIMK1 & 22 & 57 & 35 & 8.77E-04 & 11.62 & 9.79 & 0.25 \\
\hline KPTN & 14 & 50 & 36 & 1.44E-08 & 0.47 & 0.58 & -0.31 \\
\hline NAPA-AS1 & 14 & 50 & 36 & 1.44E-08 & 0.30 & 0.32 & -0.08 \\
\hline ZNF70 & 13 & 49 & 36 & $1.44 \mathrm{E}-08$ & 1.78 & 1.86 & -0.06 \\
\hline ZNF615 & 0 & 36 & 36 & 1.44E-08 & 4.13 & 3.05 & 0.44 \\
\hline BSG & 38 & 74 & 36 & 1.47E-08 & 256.20 & 194.09 & 0.40 \\
\hline CFP & 43 & 79 & 36 & 7.96E-02 & 1.11 & 0.88 & 0.34 \\
\hline GMFG & 50 & 87 & 37 & $1.12 \mathrm{E}-05$ & 12.75 & 10.41 & 0.29 \\
\hline SLC22A17 & 38 & 76 & 38 & 1.44E-08 & 1.15 & 1.16 & -0.01 \\
\hline TJP2 & 4 & 42 & 38 & 1.44E-08 & 46.09 & 42.79 & 0.11 \\
\hline RBM34 & 0 & 39 & 39 & 1.51E-08 & 123.18 & 128.23 & -0.06 \\
\hline DDX1 & 1 & 41 & 40 & 1.44E-08 & 124.08 & 159.39 & -0.36 \\
\hline KIAA1755 & 2 & 42 & 40 & $2.16 \mathrm{E}-03$ & 0.04 & 0.03 & 0.62 \\
\hline RAB17 & 15 & 56 & 41 & 1.62E-04 & 0.77 & 0.39 & 1.00 \\
\hline ALG1L2 & 44 & 85 & 41 & $2.59 \mathrm{E}-03$ & 0.18 & 0.35 & -0.98 \\
\hline SNORA69 & 44 & 85 & 41 & 7.76E-03 & 7.84 & 3.33 & 1.24 \\
\hline GPR141 & 20 & 61 & 41 & 7.22E-02 & 0.18 & 0.10 & 0.87 \\
\hline HCCS & 14 & 56 & 42 & 1.44E-08 & 30.54 & 34.21 & -0.16 \\
\hline AIMP1 & 16 & 58 & 42 & 1.47E-08 & 38.94 & 35.05 & 0.15 \\
\hline AIMP1 & 16 & 58 & 42 & 1.47E-08 & 38.94 & 35.05 & 0.15 \\
\hline SLC1A5 & 42 & 84 & 42 & $2.64 \mathrm{E}-04$ & 22.59 & 21.97 & 0.04 \\
\hline CLK3 & 50 & 92 & 42 & 5.19E-02 & 63.88 & 63.92 & 0.00 \\
\hline MSTO1 & 5 & 48 & 43 & $1.44 \mathrm{E}-08$ & 5.02 & 4.72 & 0.09 \\
\hline GABPB2 & 11 & 54 & 43 & 1.44E-08 & 2.67 & 2.16 & 0.30 \\
\hline SELPLG & 7 & 50 & 43 & 5.92E-02 & 0.78 & 0.74 & 0.08 \\
\hline ARHGAP6 & 0 & 43 & 43 & 6.18E-02 & 0.74 & 0.68 & 0.11 \\
\hline SLC19A3 & 36 & 80 & 44 & $1.44 \mathrm{E}-08$ & 0.13 & 0.08 & 0.62 \\
\hline МАT2B & 2 & 46 & 44 & $7.75 \mathrm{E}-07$ & 112.95 & 110.35 & 0.03 \\
\hline CECR1 & 56 & 100 & 44 & $1.43 \mathrm{E}-05$ & 5.87 & 4.76 & 0.30 \\
\hline ZFYVE27 & 52 & 96 & 44 & $1.62 E-04$ & 6.01 & 6.34 & -0.08 \\
\hline GZF1 & 0 & 45 & 45 & 4.43E-03 & 5.19 & 5.05 & 0.04 \\
\hline RNASEH2A & 20 & 66 & 46 & $1.44 \mathrm{E}-08$ & 8.73 & 8.43 & 0.05 \\
\hline IFT172 & 34 & 80 & 46 & $1.50 \mathrm{E}-06$ & 12.97 & 12.61 & 0.04 \\
\hline NR1H3 & 0 & 46 & 46 & $2.05 E-04$ & 2.64 & 2.35 & 0.17 \\
\hline WNT2B & 4 & 50 & 46 & $6.40 \mathrm{E}-04$ & 2.47 & 2.42 & 0.03 \\
\hline LYVE1 & 0 & 46 & 46 & 1.23E-03 & 107.15 & 114.96 & -0.10 \\
\hline NCR1 & 49 & 96 & 47 & $6.15 \mathrm{E}-08$ & 0.07 & 0.07 & 0.10 \\
\hline TRIM5 & 0 & 47 & 47 & 2.92E-06 & 72.47 & 66.35 & 0.13 \\
\hline CCM2L & 10 & 57 & 47 & 3.50E-03 & 11.59 & 12.04 & -0.05 \\
\hline
\end{tabular}




$\begin{array}{lccccccc}\text { PARP6 } & 31 & 79 & 48 & 1.53 \mathrm{E}-06 & 58.49 & 55.67 & 0.07 \\ \text { TMEM177 } & 1 & 51 & 50 & 1.44 \mathrm{E}-08 & 1.44 & 1.27 & 0.18 \\ \text { INTS2 } & 38 & 89 & 51 & 1.47 \mathrm{E}-08 & 9.14 & 12.60 & -0.46 \\ \text { PTX3 } & 44 & 96 & 52 & 1.44 \mathrm{E}-08 & 107.71 & 114.02 & -0.08 \\ \text { RNF125 } & 28 & 81 & 53 & 1.44 \mathrm{E}-08 & 47.96 & 47.68 & 0.01 \\ \text { ABCB9 } & 14 & 70 & 56 & 7.88 \mathrm{E}-08 & 2.64 & 1.98 & 0.42 \\ \text { C9orf117 } & 20 & 86 & 66 & 8.11 \mathrm{E}-04 & 0.64 & 0.69 & -0.11 \\ \text { SLC9A1 } & 12 & 86 & 74 & 1.44 \mathrm{E}-08 & 25.16 & 29.17 & -0.21 \\ \text { IQCD } & 22 & 98 & 76 & 1.44 \mathrm{E}-08 & 0.19 & 0.21 & -0.17 \\ \text { SLC25A22 } & 14 & 92 & 78 & 1.08 \mathrm{E}-06 & 3.14 & 3.31 & -0.08 \\ \text { NUAK2 } & 21 & 100 & 79 & 2.54 \mathrm{E}-08 & 0.29 & 0.42 & -0.54 \\ \text { BTC } & 10 & 89 & 79 & 2.76 \mathrm{E}-04 & 0.22 & 0.17 & 0.39 \\ \text { EMP3 } & 3 & 83 & 80 & 8.78 \mathrm{E}-07 & 848.00 & 949.45 & -0.16 \\ \text { FOLH1 } & 4 & 90 & 86 & 1.44 \mathrm{E}-08 & 1.06 & 0.96 & 0.15\end{array}$


Supplemental Table 5.4. All significantly hypermethylated promoter regions corresponding to genes that are down-regulated in non-reversed conditions compared to reversed conditions

\begin{tabular}{|c|c|c|c|c|c|c|c|}
\hline gene name & $\begin{array}{c}\mathrm{R} \\
\text { methylation } \\
\%\end{array}$ & $\begin{array}{c}\mathrm{N} \\
\text { methylation } \\
\%\end{array}$ & $\begin{array}{c}\text { methylation } \\
\% \text { difference } \\
(\mathrm{N}-\mathrm{R})\end{array}$ & $\begin{array}{l}\text { methylation } \\
\text { adjusted } \\
\text { Pvalue }\end{array}$ & $\begin{array}{c}\text { mRNASeq } \\
\text { Nvalue }\end{array}$ & $\begin{array}{l}\text { mRNASeq } \\
\text { Rvalue }\end{array}$ & $\begin{array}{c}\text { mRNASeq } \\
\text { Log2FC }\end{array}$ \\
\hline EMP3 & 3 & 83 & 80 & 8.78E-07 & 848.00 & 949.45 & -0.16 \\
\hline NUAK2 & 21 & 100 & 79 & $2.54 \mathrm{E}-08$ & 0.29 & 0.42 & -0.54 \\
\hline SLC25A22 & 14 & 92 & 78 & $1.08 \mathrm{E}-06$ & 3.14 & 3.31 & -0.08 \\
\hline IQCD & 22 & 98 & 76 & $1.44 \mathrm{E}-08$ & 0.19 & 0.21 & -0.17 \\
\hline SLC9A1 & 12 & 86 & 74 & $1.44 \mathrm{E}-08$ & 25.16 & 29.17 & -0.21 \\
\hline C9orf117 & 20 & 86 & 66 & $8.11 \mathrm{E}-04$ & 0.64 & 0.69 & -0.11 \\
\hline PTX3 & 44 & 96 & 52 & $1.44 \mathrm{E}-08$ & 107.71 & 114.02 & -0.08 \\
\hline INTS2 & 38 & 89 & 51 & $1.47 \mathrm{E}-08$ & 9.14 & 12.60 & -0.46 \\
\hline CCM2L & 10 & 57 & 47 & $3.50 \mathrm{E}-03$ & 11.59 & 12.04 & -0.05 \\
\hline LYVE1 & 0 & 46 & 46 & $1.23 \mathrm{E}-03$ & 107.15 & 114.96 & -0.10 \\
\hline ZFYVE27 & 52 & 96 & 44 & $1.62 \mathrm{E}-04$ & 6.01 & 6.34 & -0.08 \\
\hline HCCS & 14 & 56 & 42 & $1.44 \mathrm{E}-08$ & 30.54 & 34.21 & -0.16 \\
\hline CLK3 & 50 & 92 & 42 & $5.19 \mathrm{E}-02$ & 63.88 & 63.92 & 0.00 \\
\hline ALG1L2 & 44 & 85 & 41 & $2.59 \mathrm{E}-03$ & 0.18 & 0.35 & -0.98 \\
\hline DDX1 & 1 & 41 & 40 & $1.44 \mathrm{E}-08$ & 124.08 & 159.39 & -0.36 \\
\hline RBM34 & 0 & 39 & 39 & $1.51 \mathrm{E}-08$ & 123.18 & 128.23 & -0.06 \\
\hline SLC22A17 & 38 & 76 & 38 & $1.44 \mathrm{E}-08$ & 1.15 & 1.16 & -0.01 \\
\hline KPTN & 14 & 50 & 36 & $1.44 \mathrm{E}-08$ & 0.47 & 0.58 & -0.31 \\
\hline NAPA-AS1 & 14 & 50 & 36 & $1.44 \mathrm{E}-08$ & 0.30 & 0.32 & -0.08 \\
\hline ZNF70 & 13 & 49 & 36 & $1.44 \mathrm{E}-08$ & 1.78 & 1.86 & -0.06 \\
\hline LYPD3 & 40 & 75 & 35 & 3.52E-06 & 0.01 & 0.11 & -3.00 \\
\hline ANKRD20A5P & 17 & 51 & 34 & $1.44 \mathrm{E}-08$ & 0.14 & 0.19 & -0.47 \\
\hline EXOSC5 & 8 & 42 & 34 & $1.44 \mathrm{E}-08$ & 1.92 & 2.63 & -0.46 \\
\hline DDX19B & 22 & 56 & 34 & 4.40E-07 & 44.14 & 44.31 & -0.01 \\
\hline C12orf71 & 60 & 93 & 33 & 3.16E-02 & 0.01 & 0.08 & -2.45 \\
\hline MFSD4 & 10 & 42 & 32 & 4.90E-04 & 0.60 & 0.70 & -0.22 \\
\hline C10orf11 & 9 & 41 & 32 & $5.61 \mathrm{E}-06$ & 10.04 & 11.32 & -0.17 \\
\hline FBXL22 & 29 & 61 & 32 & 7.10E-04 & 0.08 & 0.09 & -0.07 \\
\hline GCFC2 & 1 & 32 & 31 & $1.44 \mathrm{E}-08$ & 1.10 & 1.19 & -0.12 \\
\hline HHIPL2 & 66 & 96 & 30 & 4.79E-02 & 0.02 & 0.06 & -1.25 \\
\hline C1orf141 & 34 & 64 & 30 & $1.44 \mathrm{E}-08$ & 0.02 & 0.04 & -1.10 \\
\hline CKLF-CMTM1 & 35 & 65 & 30 & $1.28 \mathrm{E}-04$ & 30.75 & 34.38 & -0.16 \\
\hline CKLF & 35 & 65 & 30 & $1.28 \mathrm{E}-04$ & 30.75 & 34.38 & -0.16 \\
\hline ATP6V0A4 & 17 & 46 & 29 & 8.70E-02 & 0.02 & 0.09 & -1.93 \\
\hline TNFRSF8 & 47 & 76 & 29 & $8.20 \mathrm{E}-03$ & 0.01 & 0.03 & -1.63 \\
\hline GRAMD1C & 2 & 31 & 29 & $1.44 \mathrm{E}-08$ & 6.13 & 6.35 & -0.05 \\
\hline SMU1 & 9 & 37 & 28 & $1.55 \mathrm{E}-08$ & 54.58 & 55.96 & -0.04 \\
\hline PPP1R2P3 & 36 & 63 & 27 & 4.26E-02 & 0.06 & 0.09 & -0.68 \\
\hline MYT1L & 61 & 88 & 27 & 5.91E-02 & 0.12 & 0.17 & -0.55 \\
\hline HSPA9 & 22 & 49 & 27 & $1.44 \mathrm{E}-08$ & 318.67 & 415.42 & -0.38 \\
\hline CAPS2 & 1 & 28 & 27 & 1.47E-08 & 0.49 & 0.54 & -0.14 \\
\hline LAS1L & 52 & 79 & 27 & $1.01 \mathrm{E}-03$ & 4.84 & 5.32 & -0.14 \\
\hline C19orf21 & 47 & 73 & 26 & $1.40 \mathrm{E}-03$ & 0.00 & 0.01 & -0.88 \\
\hline SEC61A2 & 10 & 36 & 26 & $1.44 \mathrm{E}-08$ & 7.47 & 10.53 & -0.50 \\
\hline ZNF468 & 15 & 41 & 26 & 2.16E-05 & 5.89 & 6.96 & -0.24 \\
\hline ALKBH7 & 5 & 31 & 26 & $2.49 \mathrm{E}-07$ & 3.86 & 3.92 & -0.02 \\
\hline
\end{tabular}




\begin{tabular}{|c|c|c|c|c|c|c|c|}
\hline GPATCH2 & 28 & 53 & 25 & $5.50 \mathrm{E}-03$ & 6.47 & 10.20 & -0.66 \\
\hline STPG1 & 32 & 57 & 25 & 2.08E-08 & 8.50 & 12.38 & -0.54 \\
\hline SPATA17 & 28 & 53 & 25 & 5.50E-03 & 0.34 & 0.49 & -0.51 \\
\hline RPL24 & 20 & 45 & 25 & $1.21 \mathrm{E}-05$ & 913.12 & 1152.83 & -0.34 \\
\hline SV2A & 6 & 31 & 25 & 6.56E-04 & 0.16 & 0.20 & -0.29 \\
\hline TASP1 & 9 & 34 & 25 & 1.47E-08 & 2.91 & 3.38 & -0.22 \\
\hline RPAP1 & 61 & 86 & 25 & 2.52E-02 & 8.67 & 9.04 & -0.06 \\
\hline P2RX3 & 67 & 91 & 24 & 3.57E-02 & 0.02 & 0.19 & -3.44 \\
\hline ATP5J2 & 25 & 49 & 24 & 2.99E-07 & 389.08 & 433.24 & -0.16 \\
\hline OC90 & 54 & 77 & 23 & 7.60E-02 & 0.80 & 1.13 & -0.50 \\
\hline ETV4 & 30 & 53 & 23 & 1.47E-05 & 3.25 & 4.36 & -0.42 \\
\hline GEMIN5 & 28 & 51 & 23 & $2.13 E-06$ & 6.55 & 7.35 & -0.17 \\
\hline NSA2 & 8 & 31 & 23 & 1.47E-08 & 176.72 & 195.11 & -0.14 \\
\hline SPARC & 21 & 44 & 23 & 3.67E-06 & 2342.55 & 2385.35 & -0.03 \\
\hline ZSCAN23 & 76 & 98 & 22 & $9.68 \mathrm{E}-03$ & 0.23 & 0.43 & -0.88 \\
\hline IL10RA & 13 & 35 & 22 & 1.47E-08 & 2.31 & 3.39 & -0.55 \\
\hline KRT8 & 73 & 95 & 22 & 2.79E-02 & 84.31 & 101.27 & -0.26 \\
\hline RGPD2 & 44 & 66 & 22 & 2.13E-03 & 0.08 & 0.09 & -0.24 \\
\hline ABAT & 8 & 30 & 22 & 4.97E-02 & 0.32 & 0.37 & -0.18 \\
\hline HMSD & 14 & 36 & 22 & 1.79E-02 & 32.21 & 33.50 & -0.06 \\
\hline SUSD2 & 66 & 87 & 21 & 5.03E-02 & 0.03 & 0.06 & -1.03 \\
\hline STPG1 & 22 & 43 & 21 & $1.55 \mathrm{E}-08$ & 8.50 & 12.38 & -0.54 \\
\hline MRPS22 & 16 & 37 & 21 & $1.52 \mathrm{E}-08$ & 70.84 & 99.59 & -0.49 \\
\hline CUEDC2 & 1 & 22 & 21 & 3.96E-07 & 27.78 & 31.40 & -0.18 \\
\hline GHRL & 49 & 70 & 21 & 3.94E-02 & 97.76 & 108.06 & -0.14 \\
\hline CBWD2 & 2 & 23 & 21 & 1.97E-06 & 5.73 & 6.26 & -0.13 \\
\hline FAM96A & 13 & 34 & 21 & 7.54E-08 & 82.61 & 87.34 & -0.08 \\
\hline CCDC8 & 23 & 44 & 21 & $1.60 \mathrm{E}-08$ & 0.03 & 0.03 & 0.00 \\
\hline INPP5F & 45 & 65 & 20 & 7.75E-04 & 13.34 & 14.35 & -0.11 \\
\hline COL4A3BP & 1 & 21 & 20 & $1.52 \mathrm{E}-08$ & 15.96 & 16.99 & -0.09 \\
\hline SIPA1L1 & 80 & 100 & 20 & 8.53E-02 & 9.01 & 9.36 & -0.06 \\
\hline GPR35 & 64 & 84 & 20 & 4.56E-04 & 2.99 & 3.07 & -0.04 \\
\hline ZRSR2 & 9 & 29 & 20 & $1.52 \mathrm{E}-08$ & 10.87 & 10.98 & -0.01 \\
\hline SSR4P1 & 31 & 50 & 19 & 1.62E-08 & 0.14 & 0.21 & -0.58 \\
\hline LRRC37A6P & 19 & 38 & 19 & 4.49E-06 & 0.17 & 0.24 & -0.48 \\
\hline HOXB3 & 1 & 20 & 19 & $4.90 \mathrm{E}-03$ & 5.12 & 6.78 & -0.41 \\
\hline FAM217B & 27 & 46 & 19 & 1.87E-08 & 4.34 & 4.88 & -0.17 \\
\hline STARD8 & 13 & 32 & 19 & 1.44E-08 & 11.86 & 12.08 & -0.03 \\
\hline SH3BGR & 44 & 63 & 19 & 1.59E-02 & 2.03 & 2.06 & -0.02 \\
\hline COX7A1 & 20 & 38 & 18 & 4.67E-05 & 5.18 & 10.00 & -0.95 \\
\hline POLR2D & 20 & 38 & 18 & $4.74 \mathrm{E}-07$ & 17.62 & 24.88 & -0.50 \\
\hline SERF2 & 16 & 34 & 18 & 1.57E-08 & 304.92 & 347.00 & -0.19 \\
\hline LARS & 15 & 33 & 18 & $5.69 \mathrm{E}-08$ & 122.66 & 129.09 & -0.07 \\
\hline NAP1L5 & 36 & 54 & 18 & 7.74E-02 & 4.00 & 4.13 & -0.05 \\
\hline MTO1 & 11 & 28 & 17 & 1.44E-08 & 21.26 & 26.76 & -0.33 \\
\hline EXOC7 & 23 & 40 & 17 & $5.72 \mathrm{E}-04$ & 44.66 & 54.64 & -0.29 \\
\hline CCDC73 & 74 & 91 & 17 & 8.45E-02 & 3.61 & 4.21 & -0.22 \\
\hline MORF4L2 & 3 & 20 & 17 & 2.55E-02 & 301.86 & 347.28 & -0.20 \\
\hline DLG3 & 19 & 36 & 17 & $1.52 \mathrm{E}-08$ & 1.70 & 1.94 & -0.19 \\
\hline POLR2G & 41 & 58 & 17 & $3.21 \mathrm{E}-04$ & 36.69 & 40.07 & -0.13 \\
\hline CCDC58 & 14 & 31 & 17 & 8.36E-04 & 24.64 & 25.73 & -0.06 \\
\hline ZNF350 & 14 & 30 & 16 & 7.06E-07 & 6.13 & 11.48 & -0.90 \\
\hline KCNE3 & 33 & 49 & 16 & 9.06E-04 & 0.06 & 0.10 & -0.83 \\
\hline PKD2L2 & 11 & 27 & 16 & $4.90 \mathrm{E}-07$ & 0.65 & 0.82 & -0.34 \\
\hline
\end{tabular}




\begin{tabular}{|c|c|c|c|c|c|c|c|}
\hline C11orf83 & 0 & 16 & 16 & $1.44 \mathrm{E}-08$ & 2.48 & 3.10 & -0.32 \\
\hline ISG15 & 51 & 67 & 16 & 4.76E-03 & 10.67 & 13.13 & -0.30 \\
\hline UFSP2 & 0 & 16 & 16 & $1.44 \mathrm{E}-08$ & 71.39 & 84.81 & -0.25 \\
\hline KIAA1598 & 60 & 76 & 16 & $2.71 \mathrm{E}-04$ & 1.42 & 1.52 & -0.10 \\
\hline ZNF726 & 21 & 37 & 16 & $1.84 \mathrm{E}-08$ & 0.95 & 0.99 & -0.07 \\
\hline HEMK1 & 15 & 31 & 16 & 2.73E-04 & 1.37 & 1.44 & -0.07 \\
\hline SLC38A7 & 13 & 29 & 16 & 4.59E-02 & 17.72 & 18.57 & -0.07 \\
\hline IDI2-AS1 & 80 & 96 & 16 & 3.32E-02 & 0.04 & 0.04 & -0.04 \\
\hline TMEM219 & 13 & 29 & 16 & $1.44 \mathrm{E}-08$ & 28.20 & 28.97 & -0.04 \\
\hline HOXB8 & 12 & 28 & 16 & 2.28E-05 & 4.92 & 5.01 & -0.02 \\
\hline QPRT & 69 & 85 & 16 & 2.23E-03 & 0.50 & 0.50 & 0.00 \\
\hline C9orf129 & 20 & 35 & 15 & 8.63E-06 & 0.05 & 0.14 & -1.43 \\
\hline GPR19 & 0 & 15 & 15 & 6.35E-06 & 0.13 & 0.32 & -1.25 \\
\hline LIG1 & 2 & 17 & 15 & 3.50E-02 & 0.97 & 2.31 & -1.25 \\
\hline ZMAT4 & 9 & 24 & 15 & 4.69E-03 & 0.17 & 0.27 & -0.64 \\
\hline EID3 & 24 & 39 & 15 & 1.33E-03 & 1.30 & 1.95 & -0.58 \\
\hline C2orf50 & 56 & 71 & 15 & $4.90 \mathrm{E}-03$ & 0.07 & 0.10 & -0.54 \\
\hline C1orf64 & 81 & 96 & 15 & 1.32E-02 & 0.08 & 0.10 & -0.34 \\
\hline PIGB & 1 & 16 & 15 & 1.44E-08 & 9.07 & 10.53 & -0.22 \\
\hline ZNF235 & 3 & 18 & 15 & 1.54E-02 & 5.76 & 5.89 & -0.03 \\
\hline KLC4 & 14 & 29 & 15 & $1.08 \mathrm{E}-03$ & 11.20 & 11.40 & -0.03 \\
\hline MGARP & 34 & 49 & 15 & 7.52E-05 & 62.54 & 63.24 & -0.02 \\
\hline DGKA & 10 & 25 & 15 & 7.82E-07 & 198.25 & 199.67 & -0.01 \\
\hline ANO4 & 17 & 31 & 14 & 4.60E-02 & 0.56 & 1.79 & -1.69 \\
\hline EPHA1 & 33 & 47 & 14 & 1.06E-03 & 1.04 & 1.67 & -0.68 \\
\hline NCMAP & 8 & 22 & 14 & 4.37E-05 & 0.05 & 0.08 & -0.65 \\
\hline PRND & 35 & 49 & 14 & 8.78E-02 & 0.06 & 0.09 & -0.54 \\
\hline LRRC36 & 3 & 17 & 14 & 1.44E-08 & 0.23 & 0.32 & -0.47 \\
\hline PFDN5 & 52 & 66 & 14 & 7.59E-03 & 165.38 & 213.81 & -0.37 \\
\hline NDUFA6 & 5 & 19 & 14 & 1.52E-08 & 56.97 & 73.19 & -0.36 \\
\hline ZMAT1 & 32 & 46 & 14 & $1.85 \mathrm{E}-03$ & 2.42 & 3.10 & -0.36 \\
\hline FAM71E1 & 10 & 24 & 14 & 1.79E-08 & 0.12 & 0.15 & -0.30 \\
\hline CIR1 & 0 & 14 & 14 & $1.44 \mathrm{E}-08$ & 32.00 & 38.12 & -0.25 \\
\hline SCRN3 & 0 & 14 & 14 & $1.44 \mathrm{E}-08$ & 23.96 & 27.89 & -0.22 \\
\hline FRS2 & 1 & 15 & 14 & 1.44E-08 & 10.88 & 12.61 & -0.21 \\
\hline PNPO & 1 & 15 & 14 & 1.44E-08 & 47.87 & 55.37 & -0.21 \\
\hline CARS & 1 & 15 & 14 & 1.44E-08 & 15.79 & 17.60 & -0.16 \\
\hline NDUFA1 & 31 & 45 & 14 & 1.33E-07 & 70.69 & 78.72 & -0.16 \\
\hline ZCCHC4 & 22 & 36 & 14 & 3.13E-03 & 4.57 & 5.04 & -0.14 \\
\hline SNW1 & 5 & 19 & 14 & $1.44 \mathrm{E}-08$ & 80.71 & 86.15 & -0.09 \\
\hline RSPH10B & 14 & 27 & 13 & 2.87E-03 & 0.18 & 0.38 & -1.10 \\
\hline CHST6 & 12 & 25 & 13 & $6.54 \mathrm{E}-08$ & 0.06 & 0.11 & -0.93 \\
\hline RSPH10B2 & 14 & 27 & 13 & 2.87E-03 & 0.04 & 0.06 & -0.58 \\
\hline TAF1A & 23 & 36 & 13 & 1.02E-04 & 2.19 & 3.27 & -0.58 \\
\hline IZUMO1 & 27 & 40 & 13 & $1.22 \mathrm{E}-03$ & 0.29 & 0.41 & -0.52 \\
\hline TNFSF11 & 3 & 16 & 13 & $3.65 \mathrm{E}-06$ & 0.01 & 0.01 & -0.41 \\
\hline MFAP2 & 9 & 22 & 13 & 3.66E-02 & 114.38 & 150.38 & -0.39 \\
\hline DNALI1 & 18 & 31 & 13 & 2.12E-03 & 4.45 & 5.35 & -0.27 \\
\hline UBE2L6 & 0 & 13 & 13 & 1.44E-08 & 19.57 & 22.84 & -0.22 \\
\hline MIR202 & 63 & 76 & 13 & $3.90 \mathrm{E}-03$ & 0.37 & 0.42 & -0.20 \\
\hline USP18 & 9 & 22 & 13 & $2.21 \mathrm{E}-04$ & 5.00 & 5.69 & -0.19 \\
\hline SERF2 & 15 & 28 & 13 & $4.74 \mathrm{E}-07$ & 304.92 & 347.00 & -0.19 \\
\hline MLH3 & 1 & 14 & 13 & 1.44E-08 & 5.17 & 5.83 & -0.17 \\
\hline DDIT3 & 11 & 24 & 13 & 1.23E-02 & 5.17 & 5.66 & -0.13 \\
\hline
\end{tabular}




\begin{tabular}{|c|c|c|c|c|c|c|c|}
\hline KLK6 & 59 & 72 & 13 & $5.26 \mathrm{E}-02$ & 1.88 & 2.03 & -0.11 \\
\hline DAB2IP & 24 & 37 & 13 & 7.52E-02 & 17.74 & 19.02 & -0.10 \\
\hline ING4 & 1 & 14 & 13 & $1.44 \mathrm{E}-08$ & 33.56 & 35.69 & -0.09 \\
\hline COA6 & 3 & 16 & 13 & $1.44 \mathrm{E}-08$ & 28.20 & 29.70 & -0.07 \\
\hline TBCCD1 & 1 & 14 & 13 & $1.44 \mathrm{E}-08$ & 9.27 & 9.72 & -0.07 \\
\hline GANC & 3 & 16 & 13 & 7.39E-06 & 13.30 & 13.94 & -0.07 \\
\hline NGFRAP1 & 26 & 39 & 13 & 4.76E-03 & 151.93 & 159.11 & -0.07 \\
\hline SFR1 & 0 & 13 & 13 & $5.81 \mathrm{E}-04$ & 22.46 & 23.37 & -0.06 \\
\hline SFR1 & 0 & 13 & 13 & 5.81E-04 & 22.46 & 23.37 & -0.06 \\
\hline ANGEL2 & 9 & 22 & 13 & 2.44E-06 & 16.92 & 17.08 & -0.01 \\
\hline PHF16 & 16 & 28 & 12 & 1.42E-05 & 5.93 & 8.25 & -0.48 \\
\hline PHF16 & 16 & 28 & 12 & $1.42 \mathrm{E}-05$ & 5.93 & 8.25 & -0.48 \\
\hline CSTF2 & 36 & 48 & 12 & $6.15 \mathrm{E}-04$ & 6.05 & 7.68 & -0.35 \\
\hline RHBDL2 & 9 & 21 & 12 & $6.21 \mathrm{E}-02$ & 1.74 & 2.10 & -0.27 \\
\hline TMEM62 & 8 & 20 & 12 & 4.94E-08 & 30.43 & 35.77 & -0.23 \\
\hline POLR3F & 5 & 17 & 12 & 1.47E-08 & 11.01 & 12.78 & -0.22 \\
\hline BLVRB & 11 & 23 & 12 & 7.04E-06 & 12.40 & 14.19 & -0.20 \\
\hline C1GALT1C1 & 36 & 48 & 12 & 2.36E-04 & 33.64 & 38.22 & -0.18 \\
\hline MMADHC & 0 & 12 & 12 & 3.32E-04 & 65.47 & 73.95 & -0.18 \\
\hline ACOT4 & 10 & 22 & 12 & 1.73E-08 & 0.03 & 0.03 & -0.16 \\
\hline C2orf74 & 82 & 94 & 12 & 1.63E-03 & 40.17 & 44.10 & -0.13 \\
\hline DIO1 & 82 & 94 & 12 & 8.31E-02 & 0.06 & 0.07 & -0.11 \\
\hline MDM4 & 27 & 39 & 12 & $3.48 \mathrm{E}-03$ & 11.11 & 11.93 & -0.10 \\
\hline C16orf80 & 35 & 47 & 12 & $1.69 \mathrm{E}-04$ & 68.93 & 72.29 & -0.07 \\
\hline BCL6 & 4 & 16 & 12 & $3.71 \mathrm{E}-03$ & 6.69 & 6.92 & -0.05 \\
\hline SMARCAL1 & 8 & 20 & 12 & 2.02E-04 & 13.36 & 13.73 & -0.04 \\
\hline SMARCAL1 & 8 & 20 & 12 & 2.02E-04 & 13.36 & 13.73 & -0.04 \\
\hline RUFY1 & 5 & 17 & 12 & $2.95 \mathrm{E}-06$ & 109.75 & 111.85 & -0.03 \\
\hline RHPN2 & 11 & 23 & 12 & 6.11E-08 & 1.55 & 1.56 & -0.01 \\
\hline ASIC2 & 46 & 57 & 11 & 5.88E-02 & 0.00 & 0.04 & -3.22 \\
\hline DNAH6 & 16 & 27 & 11 & 7.47E-02 & 0.01 & 0.05 & -2.92 \\
\hline BCL2L14 & 81 & 92 & 11 & 9.88E-02 & 0.32 & 0.63 & -0.96 \\
\hline ANKRD30BL & 27 & 38 & 11 & 2.34E-08 & 0.04 & 0.07 & -0.91 \\
\hline ZNF808 & 2 & 13 & 11 & 1.44E-08 & 16.48 & 25.19 & -0.61 \\
\hline MIS18BP1 & 0 & 11 & 11 & $1.44 \mathrm{E}-08$ & 14.50 & 20.73 & -0.52 \\
\hline GLUD2 & 18 & 29 & 11 & 2.42E-02 & 0.09 & 0.12 & -0.38 \\
\hline EMR2 & 2 & 13 & 11 & 2.21E-06 & 0.18 & 0.22 & -0.34 \\
\hline SNRPF & 11 & 22 & 11 & 2.89E-03 & 91.74 & 114.33 & -0.32 \\
\hline IQGAP1 & 3 & 14 & 11 & $1.44 \mathrm{E}-08$ & 248.25 & 307.74 & -0.31 \\
\hline GON4L & 34 & 45 & 11 & 8.31E-02 & 14.46 & 17.91 & -0.31 \\
\hline AGA & 6 & 17 & 11 & $9.90 \mathrm{E}-04$ & 12.45 & 15.11 & -0.28 \\
\hline PTPLAD2 & 27 & 38 & 11 & $1.10 \mathrm{E}-03$ & 4.87 & 5.90 & -0.28 \\
\hline FLT3LG & 0 & 11 & 11 & 1.44E-08 & 782.26 & 935.32 & -0.26 \\
\hline LPIN3 & 11 & 22 & 11 & 1.66E-02 & 0.14 & 0.17 & -0.25 \\
\hline PIH1D3 & 24 & 35 & 11 & 6.35E-06 & 0.08 & 0.09 & -0.23 \\
\hline RAD51D & 27 & 38 & 11 & 5.73E-03 & 8.49 & 9.87 & -0.22 \\
\hline ZNF560 & 18 & 29 & 11 & 1.77E-05 & 0.00 & 0.00 & -0.22 \\
\hline FAM9C & 81 & 92 & 11 & 2.02E-02 & 0.14 & 0.16 & -0.19 \\
\hline ZSWIM3 & 2 & 13 & 11 & 1.09E-06 & 1.08 & 1.18 & -0.12 \\
\hline MSN & 24 & 35 & 11 & $8.21 \mathrm{E}-04$ & 283.85 & 304.74 & -0.10 \\
\hline CDK20 & 7 & 18 & 11 & 3.99E-04 & 0.41 & 0.44 & -0.09 \\
\hline RNF8 & 7 & 18 & 11 & 2.32E-04 & 29.41 & 30.97 & -0.07 \\
\hline ADTRP & 89 & 100 & 11 & 9.37E-02 & 14.26 & 14.84 & -0.06 \\
\hline CDC42 & 4 & 15 & 11 & $2.51 \mathrm{E}-07$ & 185.16 & 192.43 & -0.06 \\
\hline
\end{tabular}




\begin{tabular}{|c|c|c|c|c|c|c|c|}
\hline PIWIL2 & 46 & 57 & 11 & 9.96E-02 & 0.13 & 0.13 & -0.05 \\
\hline MCU & 2 & 13 & 11 & $1.47 \mathrm{E}-08$ & 18.85 & 19.38 & -0.04 \\
\hline C3orf55 & 9 & 20 & 11 & 1.47E-06 & 9.52 & 9.75 & -0.03 \\
\hline $\mathrm{AUH}$ & 4 & 15 & 11 & 1.47E-08 & 6.69 & 6.75 & -0.01 \\
\hline XPO4 & 5 & 16 & 11 & 1.95E-08 & 6.71 & 6.76 & -0.01 \\
\hline FAM215A & 18 & 28 & 10 & $2.74 \mathrm{E}-03$ & 0.07 & 0.14 & -0.98 \\
\hline ZNF552 & 18 & 28 & 10 & 5.73E-04 & 0.24 & 0.46 & -0.95 \\
\hline CPLX3 & 14 & 24 & 10 & 2.79E-02 & 0.02 & 0.04 & -0.93 \\
\hline BTBD2 & 29 & 39 & 10 & $3.62 \mathrm{E}-03$ & 23.13 & 35.63 & -0.62 \\
\hline UBA52 & 9 & 19 & 10 & 2.17E-07 & 365.84 & 531.97 & -0.54 \\
\hline RIBC2 & 41 & 51 & 10 & $1.48 \mathrm{E}-02$ & 0.34 & 0.47 & -0.48 \\
\hline ZNF165 & 18 & 28 & 10 & 3.33E-02 & 0.22 & 0.29 & -0.35 \\
\hline ZDHHC9 & 28 & 38 & 10 & 7.65E-02 & 14.97 & 18.54 & -0.31 \\
\hline ADPGK-AS1 & 4 & 14 & 10 & $1.44 \mathrm{E}-08$ & 1.01 & 1.25 & -0.31 \\
\hline C11orf92 & 6 & 16 & 10 & 1.63E-06 & 0.08 & 0.09 & -0.29 \\
\hline EXOC7 & 50 & 60 & 10 & $2.85 \mathrm{E}-02$ & 44.66 & 54.64 & -0.29 \\
\hline TRNT1 & 6 & 16 & 10 & $1.46 \mathrm{E}-08$ & 26.46 & 31.82 & -0.27 \\
\hline FBXW4P1 & 77 & 87 & 10 & $5.56 \mathrm{E}-03$ & 0.23 & 0.28 & -0.26 \\
\hline MED11 & 7 & 17 & 10 & $9.21 \mathrm{E}-02$ & 4.32 & 5.14 & -0.25 \\
\hline PPP1R14A & 13 & 23 & 10 & $1.75 \mathrm{E}-04$ & 6.36 & 7.54 & -0.25 \\
\hline PDLIM5 & 3 & 13 & 10 & $1.44 \mathrm{E}-08$ & 46.85 & 54.30 & -0.21 \\
\hline ZFR & 7 & 17 & 10 & 8.39E-06 & 42.36 & 47.72 & -0.17 \\
\hline SLC12A6 & 4 & 14 & 10 & $1.44 \mathrm{E}-08$ & 20.84 & 23.38 & -0.17 \\
\hline B3GALNT1 & 9 & 19 & 10 & 1.67E-08 & 7.45 & 8.31 & -0.16 \\
\hline TRAPPC12 & 12 & 22 & 10 & $1.50 \mathrm{E}-03$ & 17.57 & 18.98 & -0.11 \\
\hline RPL11 & 15 & 25 & 10 & 1.36E-03 & 917.46 & 985.09 & -0.10 \\
\hline SDHC & 12 & 22 & 10 & 2.99E-07 & 42.94 & 46.05 & -0.10 \\
\hline KIAA1598 & 1 & 11 & 10 & $1.44 \mathrm{E}-08$ & 1.42 & 1.52 & -0.10 \\
\hline PDHA1 & 22 & 32 & 10 & $5.85 \mathrm{E}-03$ & 155.70 & 162.93 & -0.07 \\
\hline NLK & 7 & 17 & 10 & $1.60 \mathrm{E}-08$ & 18.02 & 18.78 & -0.06 \\
\hline SSB & 2 & 12 & 10 & $1.57 \mathrm{E}-08$ & 654.63 & 681.75 & -0.06 \\
\hline MAP3К13 & 11 & 21 & 10 & $2.04 \mathrm{E}-03$ & 9.80 & 10.21 & -0.06 \\
\hline ISLR & 78 & 88 & 10 & $6.20 \mathrm{E}-02$ & 0.03 & 0.03 & -0.05 \\
\hline PTP4A3 & 89 & 99 & 10 & $4.48 \mathrm{E}-02$ & 2.51 & 2.57 & -0.03 \\
\hline PITPNA & 5 & 15 & 10 & 1.47E-08 & 106.60 & 108.69 & -0.03 \\
\hline AIPL1 & 70 & 80 & 10 & 1.02E-02 & 0.11 & 0.12 & -0.03 \\
\hline SMC1B & 41 & 51 & 10 & 1.48E-02 & 0.02 & 0.02 & -0.03 \\
\hline KLC4 & 1 & 11 & 10 & $1.44 \mathrm{E}-08$ & 11.20 & 11.40 & -0.03 \\
\hline KLC4 & 1 & 11 & 10 & $1.44 \mathrm{E}-08$ & 11.20 & 11.40 & -0.03 \\
\hline KLHL12 & 37 & 47 & 10 & $1.74 \mathrm{E}-03$ & 11.53 & 11.68 & -0.02 \\
\hline DHX8 & 23 & 33 & 10 & 4.26E-03 & 15.44 & 15.56 & -0.01 \\
\hline CWF19L1 & 18 & 28 & 10 & $6.65 \mathrm{E}-03$ & 11.89 & 11.96 & -0.01 \\
\hline BLVRB & 11 & 23 & 12 & 7.04E-06 & 12.40 & 14.19 & -0.20 \\
\hline DLG3 & 19 & 36 & 17 & 1.52E-08 & 1.70 & 1.94 & -0.19 \\
\hline USP18 & 9 & 22 & 13 & 2.21E-04 & 5.00 & 5.69 & -0.19 \\
\hline SERF2 & 15 & 28 & 13 & 4.74E-07 & 304.92 & 347.00 & -0.19 \\
\hline SERF2 & 16 & 34 & 18 & $1.57 \mathrm{E}-08$ & 304.92 & 347.00 & -0.19 \\
\hline FAM9C & 81 & 92 & 11 & $2.02 \mathrm{E}-02$ & 0.14 & 0.16 & -0.19 \\
\hline C1GALT1C1 & 36 & 48 & 12 & $2.36 \mathrm{E}-04$ & 33.64 & 38.22 & -0.18 \\
\hline CUEDC2 & 1 & 22 & 21 & 3.96E-07 & 27.78 & 31.40 & -0.18 \\
\hline MMADHC & 0 & 12 & 12 & $3.32 \mathrm{E}-04$ & 65.47 & 73.95 & -0.18 \\
\hline ABAT & 8 & 30 & 22 & 4.97E-02 & 0.32 & 0.37 & -0.18 \\
\hline IQCD & 22 & 98 & 76 & 1.44E-08 & 0.19 & 0.21 & -0.17 \\
\hline C10orf11 & 9 & 41 & 32 & $5.61 \mathrm{E}-06$ & 10.04 & 11.32 & -0.17 \\
\hline
\end{tabular}




\begin{tabular}{|c|c|c|c|c|c|c|c|}
\hline MLH3 & 1 & 14 & 13 & $1.44 \mathrm{E}-08$ & 5.17 & 5.83 & -0.17 \\
\hline ZFR & 7 & 17 & 10 & 8.39E-06 & 42.36 & 47.72 & -0.17 \\
\hline FAM217B & 27 & 46 & 19 & 1.87E-08 & 4.34 & 4.88 & -0.17 \\
\hline GEMIN5 & 28 & 51 & 23 & 2.13E-06 & 6.55 & 7.35 & -0.17 \\
\hline SLC12A6 & 4 & 14 & 10 & 1.44E-08 & 20.84 & 23.38 & -0.17 \\
\hline HCCS & 14 & 56 & 42 & 1.44E-08 & 30.54 & 34.21 & -0.16 \\
\hline EMP3 & 3 & 83 & 80 & 8.78E-07 & 848.00 & 949.45 & -0.16 \\
\hline CKLF-CMTM1 & 35 & 65 & 30 & $1.28 \mathrm{E}-04$ & 30.75 & 34.38 & -0.16 \\
\hline CKLF & 35 & 65 & 30 & $1.28 \mathrm{E}-04$ & 30.75 & 34.38 & -0.16 \\
\hline B3GALNT1 & 9 & 19 & 10 & 1.67E-08 & 7.45 & 8.31 & -0.16 \\
\hline ACOT4 & 10 & 22 & 12 & 1.73E-08 & 0.03 & 0.03 & -0.16 \\
\hline CARS & 1 & 15 & 14 & 1.44E-08 & 15.79 & 17.60 & -0.16 \\
\hline NDUFA1 & 31 & 45 & 14 & 1.33E-07 & 70.69 & 78.72 & -0.16 \\
\hline ATP5J2 & 25 & 49 & 24 & 2.99E-07 & 389.08 & 433.24 & -0.16 \\
\hline GHRL & 49 & 70 & 21 & 3.94E-02 & 97.76 & 108.06 & -0.14 \\
\hline NSA2 & 8 & 31 & 23 & 1.47E-08 & 176.72 & 195.11 & -0.14 \\
\hline ZCCHC4 & 22 & 36 & 14 & 3.13E-03 & 4.57 & 5.04 & -0.14 \\
\hline CAPS2 & 1 & 28 & 27 & 1.47E-08 & 0.49 & 0.54 & -0.14 \\
\hline LAS1L & 52 & 79 & 27 & 1.01E-03 & 4.84 & 5.32 & -0.14 \\
\hline C2orf74 & 82 & 94 & 12 & 1.63E-03 & 40.17 & 44.10 & -0.13 \\
\hline DDIT3 & 11 & 24 & 13 & 1.23E-02 & 5.17 & 5.66 & -0.13 \\
\hline POLR2G & 41 & 58 & 17 & 3.21E-04 & 36.69 & 40.07 & -0.13 \\
\hline CBWD2 & 2 & 23 & 21 & 1.97E-06 & 5.73 & 6.26 & -0.13 \\
\hline ZSWIM3 & 2 & 13 & 11 & 1.09E-06 & 1.08 & 1.18 & -0.12 \\
\hline GCFC2 & 1 & 32 & 31 & 1.44E-08 & 1.10 & 1.19 & -0.12 \\
\hline KLK6 & 59 & 72 & 13 & $5.26 \mathrm{E}-02$ & 1.88 & 2.03 & -0.11 \\
\hline C9orf117 & 20 & 86 & 66 & $8.11 \mathrm{E}-04$ & 0.64 & 0.69 & -0.11 \\
\hline TRAPPC12 & 12 & 22 & 10 & $1.50 \mathrm{E}-03$ & 17.57 & 18.98 & -0.11 \\
\hline DIO1 & 82 & 94 & 12 & 8.31E-02 & 0.06 & 0.07 & -0.11 \\
\hline INPP5F & 45 & 65 & 20 & 7.75E-04 & 13.34 & 14.35 & -0.11 \\
\hline MDM4 & 27 & 39 & 12 & $3.48 \mathrm{E}-03$ & 11.11 & 11.93 & -0.10 \\
\hline RPL11 & 15 & 25 & 10 & 1.36E-03 & 917.46 & 985.09 & -0.10 \\
\hline MSN & 24 & 35 & 11 & $8.21 \mathrm{E}-04$ & 283.85 & 304.74 & -0.10 \\
\hline LYVE1 & 0 & 46 & 46 & 1.23E-03 & 107.15 & 114.96 & -0.10 \\
\hline SDHC & 12 & 22 & 10 & 2.99E-07 & 42.94 & 46.05 & -0.10 \\
\hline DAB2IP & 24 & 37 & 13 & 7.52E-02 & 17.74 & 19.02 & -0.10 \\
\hline KIAA1598 & 1 & 11 & 10 & 1.44E-08 & 1.42 & 1.52 & -0.10 \\
\hline KIAA1598 & 60 & 76 & 16 & 2.71E-04 & 1.42 & 1.52 & -0.10 \\
\hline CDK20 & 7 & 18 & 11 & 3.99E-04 & 0.41 & 0.44 & -0.09 \\
\hline SNW1 & 5 & 19 & 14 & 1.44E-08 & 80.71 & 86.15 & -0.09 \\
\hline COL4A3BP & 1 & 21 & 20 & 1.52E-08 & 15.96 & 16.99 & -0.09 \\
\hline ING4 & 1 & 14 & 13 & 1.44E-08 & 33.56 & 35.69 & -0.09 \\
\hline PTX3 & 44 & 96 & 52 & 1.44E-08 & 107.71 & 114.02 & -0.08 \\
\hline FAM96A & 13 & 34 & 21 & 7.54E-08 & 82.61 & 87.34 & -0.08 \\
\hline ZFYVE27 & 52 & 96 & 44 & 1.62E-04 & 6.01 & 6.34 & -0.08 \\
\hline NAPA-AS1 & 14 & 50 & 36 & 1.44E-08 & 0.30 & 0.32 & -0.08 \\
\hline SLC25A22 & 14 & 92 & 78 & 1.08E-06 & 3.14 & 3.31 & -0.08 \\
\hline COA6 & 3 & 16 & 13 & 1.44E-08 & 28.20 & 29.70 & -0.07 \\
\hline RNF8 & 7 & 18 & 11 & 2.32E-04 & 29.41 & 30.97 & -0.07 \\
\hline LARS & 15 & 33 & 18 & 5.69E-08 & 122.66 & 129.09 & -0.07 \\
\hline ZNF726 & 21 & 37 & 16 & 1.84E-08 & 0.95 & 0.99 & -0.07 \\
\hline TBCCD1 & 1 & 14 & 13 & 1.44E-08 & 9.27 & 9.72 & -0.07 \\
\hline C16orf80 & 35 & 47 & 12 & 1.69E-04 & 68.93 & 72.29 & -0.07 \\
\hline HEMK1 & 15 & 31 & 16 & 2.73E-04 & 1.37 & 1.44 & -0.07 \\
\hline
\end{tabular}




\begin{tabular}{|c|c|c|c|c|c|c|c|}
\hline GANC & 3 & 16 & 13 & 7.39E-06 & 13.30 & 13.94 & -0.07 \\
\hline SLC38A7 & 13 & 29 & 16 & 4.59E-02 & 17.72 & 18.57 & -0.07 \\
\hline FBXL22 & 29 & 61 & 32 & 7.10E-04 & 0.08 & 0.09 & -0.07 \\
\hline NGFRAP1 & 26 & 39 & 13 & 4.76E-03 & 151.93 & 159.11 & -0.07 \\
\hline PDHA1 & 22 & 32 & 10 & $5.85 \mathrm{E}-03$ & 155.70 & 162.93 & -0.07 \\
\hline ZNF70 & 13 & 49 & 36 & $1.44 \mathrm{E}-08$ & 1.78 & 1.86 & -0.06 \\
\hline CCDC58 & 14 & 31 & 17 & 8.36E-04 & 24.64 & 25.73 & -0.06 \\
\hline RPAP1 & 61 & 86 & 25 & 2.52E-02 & 8.67 & 9.04 & -0.06 \\
\hline NLK & 7 & 17 & 10 & 1.60E-08 & 18.02 & 18.78 & -0.06 \\
\hline SSB & 2 & 12 & 10 & 1.57E-08 & 654.63 & 681.75 & -0.06 \\
\hline MAP3К13 & 11 & 21 & 10 & 2.04E-03 & 9.80 & 10.21 & -0.06 \\
\hline ADTRP & 89 & 100 & 11 & 9.37E-02 & 14.26 & 14.84 & -0.06 \\
\hline RBM34 & 0 & 39 & 39 & 1.51E-08 & 123.18 & 128.23 & -0.06 \\
\hline SFR1 & 0 & 13 & 13 & $5.81 \mathrm{E}-04$ & 22.46 & 23.37 & -0.06 \\
\hline SFR1 & 0 & 13 & 13 & 5.81E-04 & 22.46 & 23.37 & -0.06 \\
\hline HMSD & 14 & 36 & 22 & 1.79E-02 & 32.21 & 33.50 & -0.06 \\
\hline SIPA1L1 & 80 & 100 & 20 & 8.53E-02 & 9.01 & 9.36 & -0.06 \\
\hline CDC42 & 4 & 15 & 11 & $2.51 \mathrm{E}-07$ & 185.16 & 192.43 & -0.06 \\
\hline CCM2L & 10 & 57 & 47 & 3.50E-03 & 11.59 & 12.04 & -0.05 \\
\hline PIWIL2 & 46 & 57 & 11 & 9.96E-02 & 0.13 & 0.13 & -0.05 \\
\hline GRAMD1C & 2 & 31 & 29 & $1.44 \mathrm{E}-08$ & 6.13 & 6.35 & -0.05 \\
\hline BCL6 & 4 & 16 & 12 & 3.71E-03 & 6.69 & 6.92 & -0.05 \\
\hline ISLR & 78 & 88 & 10 & 6.20E-02 & 0.03 & 0.03 & -0.05 \\
\hline NAP1L5 & 36 & 54 & 18 & 7.74E-02 & 4.00 & 4.13 & -0.05 \\
\hline IDI2-AS1 & 80 & 96 & 16 & 3.32E-02 & 0.04 & 0.04 & -0.04 \\
\hline MCU & 2 & 13 & 11 & 1.47E-08 & 18.85 & 19.38 & -0.04 \\
\hline SMARCAL1 & 8 & 20 & 12 & 2.02E-04 & 13.36 & 13.73 & -0.04 \\
\hline SMARCAL1 & 8 & 20 & 12 & 2.02E-04 & 13.36 & 13.73 & -0.04 \\
\hline TMEM219 & 13 & 29 & 16 & $1.44 \mathrm{E}-08$ & 28.20 & 28.97 & -0.04 \\
\hline SMU1 & 9 & 37 & 28 & $1.55 \mathrm{E}-08$ & 54.58 & 55.96 & -0.04 \\
\hline GPR35 & 64 & 84 & 20 & 4.56E-04 & 2.99 & 3.07 & -0.04 \\
\hline C3orf55 & 9 & 20 & 11 & $1.47 \mathrm{E}-06$ & 9.52 & 9.75 & -0.03 \\
\hline PTP4A3 & 89 & 99 & 10 & 4.48E-02 & 2.51 & 2.57 & -0.03 \\
\hline ZNF235 & 3 & 18 & 15 & $1.54 \mathrm{E}-02$ & 5.76 & 5.89 & -0.03 \\
\hline PITPNA & 5 & 15 & 10 & 1.47E-08 & 106.60 & 108.69 & -0.03 \\
\hline RUFY1 & 5 & 17 & 12 & 2.95E-06 & 109.75 & 111.85 & -0.03 \\
\hline STARD8 & 13 & 32 & 19 & $1.44 \mathrm{E}-08$ & 11.86 & 12.08 & -0.03 \\
\hline AIPL1 & 70 & 80 & 10 & $1.02 \mathrm{E}-02$ & 0.11 & 0.12 & -0.03 \\
\hline SPARC & 21 & 44 & 23 & 3.67E-06 & 2342.55 & 2385.35 & -0.03 \\
\hline SMC1B & 41 & 51 & 10 & 1.48E-02 & 0.02 & 0.02 & -0.03 \\
\hline KLC4 & 1 & 11 & 10 & 1.44E-08 & 11.20 & 11.40 & -0.03 \\
\hline KLC4 & 1 & 11 & 10 & $1.44 \mathrm{E}-08$ & 11.20 & 11.40 & -0.03 \\
\hline KLC4 & 14 & 29 & 15 & 1.08E-03 & 11.20 & 11.40 & -0.03 \\
\hline HOXB8 & 12 & 28 & 16 & 2.28E-05 & 4.92 & 5.01 & -0.02 \\
\hline ALKBH7 & 5 & 31 & 26 & 2.49E-07 & 3.86 & 3.92 & -0.02 \\
\hline SH3BGR & 44 & 63 & 19 & 1.59E-02 & 2.03 & 2.06 & -0.02 \\
\hline KLHL12 & 37 & 47 & 10 & $1.74 \mathrm{E}-03$ & 11.53 & 11.68 & -0.02 \\
\hline MGARP & 34 & 49 & 15 & 7.52E-05 & 62.54 & 63.24 & -0.02 \\
\hline ZRSR2 & 9 & 29 & 20 & 1.52E-08 & 10.87 & 10.98 & -0.01 \\
\hline ANGEL2 & 9 & 22 & 13 & 2.44E-06 & 16.92 & 17.08 & -0.01 \\
\hline $\mathrm{AUH}$ & 4 & 15 & 11 & 1.47E-08 & 6.69 & 6.75 & -0.01 \\
\hline SLC22A17 & 38 & 76 & 38 & 1.44E-08 & 1.15 & 1.16 & -0.01 \\
\hline DHX8 & 23 & 33 & 10 & 4.26E-03 & 15.44 & 15.56 & -0.01 \\
\hline DGKA & 10 & 25 & 15 & 7.82E-07 & 198.25 & 199.67 & -0.01 \\
\hline
\end{tabular}




$\begin{array}{lccccccc}\text { XPO4 } & 5 & 16 & 11 & 1.95 \mathrm{E}-08 & 6.71 & 6.76 & -0.01 \\ \text { RHPN2 } & 11 & 23 & 12 & 6.11 \mathrm{E}-08 & 1.55 & 1.56 & -0.01 \\ \text { CWF19L1 } & 18 & 28 & 10 & 6.65 \mathrm{E}-03 & 11.89 & 11.96 & -0.01 \\ \text { DDX19B } & 22 & 56 & 34 & 4.40 \mathrm{E}-07 & 44.14 & 44.31 & -0.01 \\ \text { QPRT } & 69 & 85 & 16 & 2.23 \mathrm{E}-03 & 0.50 & 0.50 & 0.00 \\ \text { CCDC8 } & 23 & 44 & 21 & 1.60 \mathrm{E}-08 & 0.03 & 0.03 & 0.00 \\ \text { CLK3 } & 50 & 92 & 42 & 5.19 \mathrm{E}-02 & 63.88 & 63.92 & 0.00\end{array}$


Supplemental Table 5.5. All significantly hypomethylated promoter regions corresponding to genes that are up-regulated in non-reversed conditions compared to reversed conditions

\begin{tabular}{|c|c|c|c|c|c|c|c|}
\hline gene name & $\begin{array}{c}\mathrm{R} \\
\text { methylation } \\
\%\end{array}$ & $\begin{array}{c}\mathrm{N} \\
\text { methylation } \\
\%\end{array}$ & $\begin{array}{c}\text { methylation } \\
\% \\
\text { difference } \\
(\mathrm{N}-\mathrm{R})\end{array}$ & $\begin{array}{l}\text { methylation } \\
\text { adjusted } \\
\text { Pvalue }\end{array}$ & $\begin{array}{l}\text { mRNASeq } \\
\text { Nvalue }\end{array}$ & $\begin{array}{l}\text { mRNASeq } \\
\text { Rvalue }\end{array}$ & $\begin{array}{c}\text { mRNASeq } \\
\text { Log2FC }\end{array}$ \\
\hline EPS8L3 & 81 & 21 & -60 & 0.000633 & 0.03 & 0.01 & 1.90 \\
\hline EGFLAM & 50 & 0 & -50 & 0.006606 & 0.77 & 0.39 & 0.98 \\
\hline MICB & 67 & 20 & -47 & 0.01945 & 8.63 & 5.20 & 0.73 \\
\hline BPNT1 & 41 & 0 & -41 & $6.95 \mathrm{E}-05$ & 27.27 & 24.52 & 0.15 \\
\hline POM121L9P & 98 & 58 & -40 & 0.002384 & 0.02 & 0.01 & 1.59 \\
\hline ITGBL1 & 92 & 55 & -37 & $4.98 \mathrm{E}-05$ & 0.89 & 0.65 & 0.47 \\
\hline SYNPO2L & 92 & 56 & -36 & 0.036867 & 0.16 & 0.13 & 0.36 \\
\hline $\mathrm{PLCH} 2$ & 81 & 45 & -36 & 0.000133 & 0.04 & 0.02 & 1.30 \\
\hline CSAD & 83 & 48 & -35 & 0.02734 & 9.61 & 7.18 & 0.42 \\
\hline $\begin{array}{l}\text { ACOT2 } \\
\text { CCDC74B- }\end{array}$ & 82 & 50 & -32 & 0.022889 & 0.87 & 0.46 & 0.91 \\
\hline AS1 & 94 & 63 & -31 & 0.032704 & 0.12 & 0.11 & 0.11 \\
\hline C9orf153 & 74 & 43 & -31 & 0.066878 & 0.09 & 0.07 & 0.39 \\
\hline ACP5 & 57 & 26 & -31 & $1.48 \mathrm{E}-08$ & 46.28 & 30.30 & 0.61 \\
\hline CD79B & 71 & 41 & -30 & 2.58E-05 & 0.38 & 0.34 & 0.16 \\
\hline LAMB3 & 95 & 65 & -30 & 0.011814 & 10.22 & 8.45 & 0.27 \\
\hline LAMB3 & 95 & 65 & -30 & 0.011814 & 10.22 & 8.45 & 0.27 \\
\hline METTL5 & 48 & 20 & -28 & 0.002432 & 66.99 & 63.77 & 0.07 \\
\hline RGS12 & 31 & 3 & -28 & 0.008089 & 29.65 & 28.00 & 0.08 \\
\hline PLEKHB1 & 60 & 32 & -28 & 0.026128 & 2.43 & 2.29 & 0.09 \\
\hline ZNF503-AS1 & 33 & 5 & -28 & 0.003345 & 0.38 & 0.20 & 0.93 \\
\hline ZNF423 & 92 & 65 & -27 & 0.018858 & 2.56 & 2.41 & 0.08 \\
\hline $\mathrm{EZH} 1$ & 45 & 18 & -27 & 1.47E-08 & 13.01 & 10.64 & 0.29 \\
\hline CCDC152 & 46 & 20 & -26 & 8.49E-08 & 1.33 & 1.11 & 0.26 \\
\hline MPDU1 & 52 & 28 & -24 & 1.02E-05 & 44.96 & 44.47 & 0.02 \\
\hline PRKACA & 54 & 30 & -24 & 0.00118 & 67.05 & 61.07 & 0.13 \\
\hline KLK10 & 26 & 3 & -23 & 0.016083 & 0.37 & 0.32 & 0.22 \\
\hline LINC00672 & 88 & 65 & -23 & 0.01419 & 0.32 & 0.25 & 0.34 \\
\hline TOMM22 & 23 & 0 & -23 & 1.44E-08 & 75.48 & 58.83 & 0.36 \\
\hline DAND5 & 59 & 36 & -23 & 0.042271 & 0.31 & 0.20 & 0.67 \\
\hline DRG2 & 33 & 11 & -22 & 3.61E-08 & 10.53 & 10.52 & 0.00 \\
\hline SPIN3 & 42 & 20 & -22 & 0.01372 & 1.37 & 1.01 & 0.44 \\
\hline RAX2 & 83 & 62 & -21 & 4.50E-05 & 0.03 & 0.03 & 0.01 \\
\hline CSTF2T & 23 & 2 & -21 & 1.44E-08 & 9.50 & 8.92 & 0.09 \\
\hline HELB & 46 & 25 & -21 & 5.02E-06 & 1.03 & 0.93 & 0.14 \\
\hline INPP5D & 23 & 2 & -21 & 1.44E-08 & 60.83 & 48.65 & 0.32 \\
\hline PKN1 & 56 & 35 & -21 & 0.05044 & 59.22 & 41.39 & 0.52 \\
\hline BPIFB1 & 80 & 60 & -20 & 0.084018 & 0.11 & 0.11 & 0.03 \\
\hline RNF166 & 28 & 8 & -20 & 1.44E-08 & 16.37 & 15.28 & 0.10 \\
\hline CLASRP & 26 & 6 & -20 & 1.44E-08 & 27.58 & 25.50 & 0.11 \\
\hline RBMX2 & 37 & 18 & -19 & 0.024771 & 22.84 & 22.56 & 0.02 \\
\hline NAA60 & 46 & 27 & -19 & 1.87E-08 & 105.11 & 95.31 & 0.14 \\
\hline SIRT4 & 47 & 28 & -19 & 0.000269 & 0.79 & 0.68 & 0.21 \\
\hline SLC7A8 & 28 & 10 & -18 & 0.011235 & 2.60 & 2.49 & 0.06 \\
\hline SPC24 & 31 & 13 & -18 & 0.016683 & 6.08 & 5.49 & 0.15 \\
\hline ATHL1 & 31 & 13 & -18 & 1.65E-08 & 3.98 & 3.19 & 0.32 \\
\hline SERPINA4 & 82 & 64 & -18 & 0.077535 & 0.10 & 0.06 & 0.58 \\
\hline
\end{tabular}




\begin{tabular}{|c|c|c|c|c|c|c|c|}
\hline $\mathrm{ZC} 3 \mathrm{H} 4$ & 18 & 0 & -18 & 1.44E-08 & 15.21 & 10.06 & 0.60 \\
\hline S1PR4 & 24 & 6 & -18 & $1.94 \mathrm{E}-06$ & 0.23 & 0.12 & 0.90 \\
\hline RCN3 & 95 & 77 & -18 & 0.008897 & 82.51 & 39.28 & 1.07 \\
\hline RGN & 65 & 47 & -18 & 0.000112 & 0.18 & 0.06 & 1.51 \\
\hline STYX & 17 & 0 & -17 & $1.44 \mathrm{E}-08$ & 26.56 & 25.30 & 0.07 \\
\hline MAP4K1 & 26 & 9 & -17 & 0.002836 & 0.93 & 0.54 & 0.79 \\
\hline PCDHB3 & 48 & 31 & -17 & 0.019056 & 0.04 & 0.02 & 1.26 \\
\hline CALD1 & 42 & 26 & -16 & 0.083803 & 571.85 & 513.31 & 0.16 \\
\hline APOE & 17 & 1 & -16 & 1.63E-06 & 4.60 & 4.09 & 0.17 \\
\hline C8orf44 & 17 & 1 & -16 & $1.44 \mathrm{E}-08$ & 14.52 & 12.54 & 0.21 \\
\hline AMPD2 & 70 & 54 & -16 & 0.075339 & 41.20 & 35.57 & 0.21 \\
\hline C1orf86 & 27 & 11 & -16 & 0.03815 & 4.82 & 4.10 & 0.24 \\
\hline PKD1L1 & 76 & 60 & -16 & 0.07031 & 1.48 & 1.20 & 0.30 \\
\hline TCF3 & 34 & 18 & -16 & 0.003922 & 71.64 & 55.68 & 0.36 \\
\hline SPAG4 & 24 & 8 & -16 & 1.67E-08 & 1.89 & 1.20 & 0.66 \\
\hline TMEM209 & 52 & 36 & -16 & 0.001065 & 1.92 & 1.17 & 0.71 \\
\hline PLEKHG6 & 30 & 14 & -16 & $1.55 \mathrm{E}-08$ & 0.34 & 0.12 & 1.47 \\
\hline PLEKHG6 & 30 & 14 & -16 & $1.55 \mathrm{E}-08$ & 0.34 & 0.12 & 1.47 \\
\hline ZNF493 & 15 & 0 & -15 & 2.03E-08 & 5.54 & 5.51 & 0.01 \\
\hline ASGR1 & 73 & 58 & -15 & 0.040817 & 3.04 & 2.98 & 0.03 \\
\hline BAK1 & 21 & 6 & -15 & 8.76E-05 & 3.54 & 2.95 & 0.27 \\
\hline ANKRD33 & 35 & 20 & -15 & 0.003708 & 0.09 & 0.07 & 0.42 \\
\hline BRCA1 & 18 & 4 & -14 & $1.74 \mathrm{E}-06$ & 5.17 & 5.14 & 0.01 \\
\hline MED24 & 14 & 0 & -14 & 0.000269 & 52.08 & 47.89 & 0.12 \\
\hline PHF17 & 14 & 0 & -14 & $9.24 \mathrm{E}-05$ & 13.84 & 12.17 & 0.19 \\
\hline SYCE1L & 97 & 83 & -14 & 0.022915 & 16.68 & 14.18 & 0.23 \\
\hline OCRL & 20 & 6 & -14 & 0.000418 & 16.60 & 13.84 & 0.26 \\
\hline DKKL1 & 19 & 5 & -14 & $1.47 \mathrm{E}-08$ & 0.17 & 0.12 & 0.46 \\
\hline DKKL1 & 19 & 5 & -14 & 1.47E-08 & 0.17 & 0.12 & 0.46 \\
\hline C12orf36 & 93 & 79 & -14 & 0.098991 & 0.14 & 0.04 & 1.94 \\
\hline DGCR14 & 15 & 2 & -13 & $1.44 \mathrm{E}-08$ & 4.17 & 4.07 & 0.04 \\
\hline AGPAT6 & 13 & 0 & -13 & $1.44 \mathrm{E}-08$ & 68.63 & 66.13 & 0.05 \\
\hline MUTYH & 13 & 0 & -13 & 0.043238 & 4.19 & 4.03 & 0.06 \\
\hline DMPK & 23 & 10 & -13 & 0.000492 & 39.58 & 37.02 & 0.10 \\
\hline LINC00574 & 13 & 0 & -13 & 0.082325 & 0.13 & 0.12 & 0.10 \\
\hline TOE1 & 13 & 0 & -13 & 0.043238 & 3.62 & 3.14 & 0.21 \\
\hline SLC5A4 & 100 & 87 & -13 & 0.014153 & 0.10 & 0.08 & 0.33 \\
\hline IDUA & 13 & 0 & -13 & $1.52 \mathrm{E}-08$ & 8.72 & 5.44 & 0.68 \\
\hline DMKN & 14 & 1 & -13 & 0.022411 & 0.09 & 0.05 & 0.78 \\
\hline C1QTNF1 & 35 & 22 & -13 & 0.030878 & 0.46 & 0.22 & 1.06 \\
\hline TSSK3 & 20 & 8 & -12 & 1.67E-08 & 0.53 & 0.48 & 0.16 \\
\hline APOBEC3D & 12 & 0 & -12 & 0.078091 & 11.13 & 9.90 & 0.17 \\
\hline MRPL28 & 20 & 8 & -12 & 0.002575 & 39.19 & 34.56 & 0.18 \\
\hline KBTBD7 & 39 & 27 & -12 & 0.003955 & 2.99 & 2.59 & 0.21 \\
\hline PI4KB & 13 & 1 & -12 & 1.47E-08 & 31.10 & 26.02 & 0.26 \\
\hline PI4KB & 13 & 1 & -12 & 1.47E-08 & 31.10 & 26.02 & 0.26 \\
\hline EDARADD & 12 & 0 & -12 & 3.66E-08 & 0.20 & 0.16 & 0.29 \\
\hline SUMO2 & 28 & 16 & -12 & $2.15 \mathrm{E}-06$ & 441.13 & 338.71 & 0.38 \\
\hline RHBDL1 & 27 & 15 & -12 & 4.01E-06 & 0.13 & 0.10 & 0.50 \\
\hline MORN3 & 91 & 79 & -12 & 0.06079 & 0.67 & 0.45 & 0.58 \\
\hline RFESD & 12 & 0 & -12 & $1.44 \mathrm{E}-08$ & 0.57 & 0.22 & 1.35 \\
\hline RFESD & 12 & 0 & -12 & 1.44E-08 & 0.57 & 0.22 & 1.35 \\
\hline RBP5 & 23 & 12 & -11 & 0.00041 & 0.87 & 0.87 & 0.00 \\
\hline ASL & 14 & 3 & -11 & $1.44 \mathrm{E}-08$ & 70.34 & 67.45 & 0.06 \\
\hline
\end{tabular}




$\begin{array}{lccccccc}\text { ZC4H2 } & 26 & 15 & -11 & 0.000596 & 8.73 & 8.31 & 0.07 \\ \text { NUP160 } & 27 & 16 & -11 & 0.044358 & 37.20 & 35.05 & 0.09 \\ \text { P2RY2 } & 16 & 5 & -11 & 7.63 \mathrm{E}-07 & 1.54 & 1.44 & 0.10 \\ \text { P2RY2 } & 16 & 5 & -11 & 7.63 \mathrm{E}-07 & 1.54 & 1.44 & 0.10 \\ \text { ZNF177 } & 21 & 10 & -11 & 0.015138 & 10.72 & 9.81 & 0.13 \\ \text { PMVK } & 13 & 2 & -11 & 3.89 \mathrm{E}-05 & 7.59 & 6.70 & 0.18 \\ \text { AR } & 24 & 13 & -11 & 0.02855 & 2.56 & 2.19 & 0.23 \\ \text { ZNF682 } & 37 & 26 & -11 & 0.020253 & 1.07 & 0.90 & 0.26 \\ \text { TUBGCP2 } & 22 & 11 & -11 & 1.55 \mathrm{E}-08 & 15.68 & 12.74 & 0.30 \\ \text { CRISPLD2 } & 11 & 0 & -11 & 2.32 \mathrm{E}-08 & 2.74 & 2.11 & 0.38 \\ \text { ERICH1 } & 13 & 2 & -11 & 1.51 \mathrm{E}-08 & 37.35 & 23.08 & 0.69 \\ \text { MGAT5B } & 21 & 10 & -11 & 0.001269 & 0.15 & 0.08 & 0.91 \\ \text { LZTS2 } & 11 & 1 & -10 & 1.93 \mathrm{E}-07 & 10.24 & 10.10 & 0.02 \\ \text { ASL } & 18 & 8 & -10 & 2.56 \mathrm{E}-06 & 70.34 & 67.45 & 0.06 \\ \text { GRM4 } & 13 & 3 & -10 & 1.51 \mathrm{E}-08 & 0.00 & 0.00 & 0.08 \\ \text { NOP2 } & 12 & 2 & -10 & 0.000117 & 24.14 & 22.62 & 0.09 \\ \text { WDR38 } & 29 & 19 & -10 & 0.014801 & 0.05 & 0.05 & 0.10 \\ \text { C2orf68 } & 16 & 6 & -10 & 2.29 \mathrm{E}-07 & 7.45 & 6.91 & 0.11 \\ \text { CREB3L1 } & 12 & 2 & -10 & 1.10 \mathrm{E}-06 & 1.10 & 1.01 & 0.12 \\ \text { CD320 } & 71 & 61 & -10 & 0.054866 & 25.89 & 23.20 & 0.16 \\ \text { POC5 } & 24 & 14 & -10 & 0.002707 & 6.05 & 4.78 & 0.34 \\ \text { SEMA4D } & 99 & 89 & -10 & 0.043859 & 4.05 & 3.11 & 0.38 \\ \text { CCDC78 } & 21 & 11 & -10 & 0.00025 & 0.10 & 0.07 & 0.57 \\ \text { DDX43 } & 96 & 86 & -10 & 0.014869 & 0.26 & 0.17 & 0.63 \\ \text { LGALS1 } & 10 & 0 & -10 & 6.26 \mathrm{E}-08 & 1405.19 & 820.91 & 0.78 \\ \text { TTC25 } & 30 & 20 & -10 & 0.006495 & 0.13 & 0.07 & 0.78 \\ \text { PKP3 } & 59 & 49 & -10 & 0.054925 & 0.18 & 0.09 & 1.04 \\ & & & & & & & \end{array}$


Supplemental Table 5.6. Top 50 significantly overrepresented biological process GO terms for genes with expression patterns corresponding to differential promoter methylation under arteriogenic shear stress waveforms

\begin{tabular}{|c|c|c|c|c|c|}
\hline Gene Set Name & $\begin{array}{l}\text { \# Genes } \\
\text { in Gene } \\
\text { Set (K) }\end{array}$ & $\begin{array}{c}\text { \# Genes } \\
\text { in } \\
\text { Overlap } \\
\text { (k) }\end{array}$ & $\mathrm{k} / \mathrm{K}$ & p-value & FDR \\
\hline BIOPOLYMER_METABOLIC_PROCESS & 1684 & 98 & 0.06 & $2.52 E-29$ & $2.08 \mathrm{E}-26$ \\
\hline $\begin{array}{l}\text { NUCLEOBASENUCLEOSIDENUCLEOTIDE_AND_ } \\
\text { NUCLEIC_ACID_METABOLIC_PROCESS }\end{array}$ & 1244 & 71 & 0.06 & $6.79 E-21$ & $2.80 \mathrm{E}-18$ \\
\hline RNA_METABOLIC_PROCESS & 841 & 54 & 0.06 & $2.57 \mathrm{E}-18$ & $7.08 \mathrm{E}-16$ \\
\hline PROTEIN_METABOLIC_PROCESS & 1231 & 65 & 0.05 & $1.62 \mathrm{E}-17$ & 3.35E-15 \\
\hline TRANSCRIPTION & 753 & 45 & 0.06 & $2.44 \mathrm{E}-14$ & $4.02 \mathrm{E}-12$ \\
\hline CELLULAR_PROTEIN_METABOLIC_PROCESS & 1117 & 55 & 0.05 & $9.61 \mathrm{E}-14$ & $1.32 \mathrm{E}-11$ \\
\hline TRANSCRIPTION_DNA_DEPENDENT & 636 & 40 & 0.06 & $1.36 \mathrm{E}-13$ & $1.45 \mathrm{E}-11$ \\
\hline RNA_BIOSYNTHETIC_PROCESS & 638 & 40 & 0.06 & $1.51 \mathrm{E}-13$ & $1.45 \mathrm{E}-11$ \\
\hline CELLULAR_MACROMOLECULE_METABOLIC_PROCESS & 1131 & 55 & 0.05 & $1.58 \mathrm{E}-13$ & $1.45 \mathrm{E}-11$ \\
\hline REGULATION_OF_METABOLIC_PROCESS & 799 & 44 & 0.06 & $7.76 \mathrm{E}-13$ & $6.41 \mathrm{E}-11$ \\
\hline SIGNAL_TRANSDUCTION & 1634 & 66 & 0.04 & 2.63E-12 & $1.97 \mathrm{E}-10$ \\
\hline BIOPOLYMER_MODIFICATION & 650 & 37 & 0.06 & 2.07E-11 & 1.43E-09 \\
\hline BIOSYNTHETIC_PROCESS & 470 & 31 & 0.07 & $2.38 \mathrm{E}-11$ & $1.51 \mathrm{E}-09$ \\
\hline REGULATION_OF_CELLULAR_METABOLIC_PROCESS & 787 & 41 & 0.05 & 2.66E-11 & $1.57 \mathrm{E}-09$ \\
\hline PROTEIN_MODIFICATION_PROCESS & 631 & 36 & 0.06 & $3.65 \mathrm{E}-11$ & 2.01E-09 \\
\hline NEGATIVE_REGULATION_OF_BIOLOGICAL_PROCESS & 677 & 37 & 0.05 & $6.58 \mathrm{E}-11$ & 3.39E-09 \\
\hline NEGATIVE_REGULATION_OF_METABOLIC_PROCESS & 262 & 22 & 0.08 & $2.13 \mathrm{E}-10$ & $9.84 \mathrm{E}-09$ \\
\hline REGULATION_OF_GENE_EXPRESSION & 673 & 36 & 0.05 & $2.15 \mathrm{E}-10$ & $9.84 \mathrm{E}-09$ \\
\hline $\begin{array}{l}\text { TRANSCRIPTION_FROM_RNA_POLYMERASE_II_PROMO } \\
\text { TER }\end{array}$ & 457 & 29 & 0.06 & $2.59 \mathrm{E}-10$ & $1.12 \mathrm{E}-08$ \\
\hline NEGATIVE_REGULATION_OF_CELLULAR_PROCESS & 646 & 35 & 0.05 & 2.73E-10 & 1.13E-08 \\
\hline INTRACELLULAR_SIGNALING_CASCADE & 667 & 35 & 0.05 & $6.35 \mathrm{E}-10$ & $2.50 \mathrm{E}-08$ \\
\hline $\begin{array}{l}\text { REGULATION_OF } \\
\text { NUCLEOBASENUC̄LEOSIDENUCLEOTIDE_AND_NUCLEIC } \\
\text { _ACID_METABOLIC_PROCESS }\end{array}$ & 618 & 33 & 0.05 & 1.27E-09 & $4.78 \mathrm{E}-08$ \\
\hline $\begin{array}{l}\text { NEGATIVE_REGULATION_OF_CELLULAR_METABOLIC_ } \\
\text { PROCESS }\end{array}$ & 259 & 20 & 0.08 & 5.94E-09 & 2.13E-07 \\
\hline $\begin{array}{l}\text { NEGATIVE_REGULATION_OF } \\
\text { NUCLEOBASENUCLEOSIDENŪCLEOTIDE_AND_NUCLEIC } \\
\text { _ACID_METABOLIC_PROCESS }\end{array}$ & 211 & 18 & 0.09 & 7.23E-09 & $2.48 \mathrm{E}-07$ \\
\hline REGULATION_OF_TRANSCRIPTION & 566 & 30 & 0.05 & $8.50 \mathrm{E}-09$ & 2.80E-07 \\
\hline
\end{tabular}




\begin{tabular}{|c|c|c|c|c|c|}
\hline TRANSPORT & 795 & 36 & 0.05 & $1.70 \mathrm{E}-08$ & $5.38 \mathrm{E}-07$ \\
\hline REGULATION_OF_TRANSCRIPTIONDNA_DEPENDENT & 461 & 26 & 0.06 & 2.43E-08 & 7.43E-07 \\
\hline REGULATION_OF_RNA_METABOLIC_PROCESS & 471 & 26 & 0.06 & 3.73E-08 & $1.10 \mathrm{E}-06$ \\
\hline CELLULAR_BIOSYNTHETIC_PROCESS & 321 & 21 & 0.07 & $4.50 \mathrm{E}-08$ & $1.24 \mathrm{E}-06$ \\
\hline MACROMOLECULE_BIOSYNTHETIC_PROCESS & 321 & 21 & 0.07 & $4.50 \mathrm{E}-08$ & $1.24 \mathrm{E}-06$ \\
\hline ESTABLISHMENT_OF_LOCALIZATION & 870 & 37 & 0.04 & $5.33 \mathrm{E}-08$ & $1.42 \mathrm{E}-06$ \\
\hline CELLULAR_COMPONENT_ASSEMBLY & 298 & 20 & 0.07 & $6.19 \mathrm{E}-08$ & $1.60 \mathrm{E}-06$ \\
\hline PROTEIN_AMINO_ACID_PHOSPHORYLATION & 279 & 19 & 0.07 & $1.05 \mathrm{E}-07$ & $2.62 \mathrm{E}-06$ \\
\hline MACROMOLECULAR_COMPLEX_ASSEMBLY & 280 & 19 & 0.07 & $1.11 \mathrm{E}-07$ & $2.68 \mathrm{E}-06$ \\
\hline POSITIVE_REGULATION_OF_BIOLOGICAL_PROCESS & 709 & 32 & 0.05 & $1.14 \mathrm{E}-07$ & $2.68 \mathrm{E}-06$ \\
\hline POST_TRANSLATIONAL_PROTEIN_MODIFICATION & 476 & 25 & 0.05 & $1.75 \mathrm{E}-07$ & $4.00 \mathrm{E}-06$ \\
\hline LIPID_METABOLIC_PROCESS & 325 & 20 & 0.06 & $2.51 \mathrm{E}-07$ & $5.60 \mathrm{E}-06$ \\
\hline NEGATIVE_REGULATION_OF_TRANSCRIPTION & 188 & 15 & 0.08 & 3.06E-07 & $6.64 \mathrm{E}-06$ \\
\hline POSITIVE_REGULATION_OF_CELLULAR_PROCESS & 668 & 30 & 0.04 & $3.14 \mathrm{E}-07$ & $6.64 \mathrm{E}-06$ \\
\hline LIPID_BIOSYNTHETIC_PROCESS & 97 & 11 & 0.11 & 3.55E-07 & 7.32E-06 \\
\hline PHOSPHORYLATION & 313 & 19 & 0.06 & $6.12 \mathrm{E}-07$ & $1.23 \mathrm{E}-05$ \\
\hline CELLULAR_LIPID_METABOLIC_PROCESS & 255 & 17 & 0.07 & $6.55 \mathrm{E}-07$ & $1.29 \mathrm{E}-05$ \\
\hline CARBOXYLIC_ACID_METABOLIC_PROCESS & 178 & 14 & 0.08 & 8.97E-07 & $1.72 \mathrm{E}-05$ \\
\hline ORGANIC_ACID_METABOLIC_PROCESS & 180 & 14 & 0.08 & $1.03 \mathrm{E}-06$ & $1.92 \mathrm{E}-05$ \\
\hline REGULATION_OF_MOLECULAR_FUNCTION & 324 & 18 & 0.06 & 4.18E-06 & 7.67E-05 \\
\hline MAPKKK_CASCADE_GO_0000165 & 104 & 10 & 0.10 & $5.48 \mathrm{E}-06$ & 9.83E-05 \\
\hline REGULATION_OF_PROTEIN_KINASE_ACTIVITY & 155 & 12 & 0.08 & $6.30 \mathrm{E}-06$ & $1.11 \mathrm{E}-04$ \\
\hline REGULATION_OF_KINASE_ACTIVITY & 157 & 12 & 0.08 & 7.18E-06 & $1.23 \mathrm{E}-04$ \\
\hline POSITIVE_REGULATION_OF_TRANSFERASE_ACTIVITY & 86 & 9 & 0.10 & 8.04E-06 & $1.35 \mathrm{E}-04$ \\
\hline REGULATION_OF_CATALYTIC_ACTIVITY & 276 & 16 & 0.06 & 8.31E-06 & $1.35 \mathrm{E}-04$ \\
\hline
\end{tabular}




\title{
6 Mechanosensitive microRNA-199a regulates perfusion
}

\section{recovery and collateral arteriogenesis in Balb/c mice}

\section{following femoral arterial ligation}

\author{
Authors: Joshua L. Heuslein, Ji Song, Richard J. Price. In preparation
}

\subsection{Abstract}

Objective: The lumenal growth of collateral arteries that bypass arterial occlusions (i.e. arteriogenesis) is a fundamental shear stress-induced adaptation that may provide important clues for the treatment of peripheral arterial disease (PAD). MicroRNAs are key regulators of gene expression in vascular biology, but the explicit role of mechanosensitive miRNAs in regulating collateral arteriogenesis has not been studied. Here, we tested the hypothesis that a mechanosensitive microRNA, miR-199a, regulates perfusion recovery and collateral arteriogenesis following femoral arterial ligation (FAL) via control of monocyte recruitment and pro-arteriogenic gene expression.

Approach and Results: Previously, we reported that collateral artery segments exhibit either "moderate" or "amplified" arteriogenesis depending on the hemodynamics to which they are exposed post-FAL. Using a genome-wide approach, we examined comparative microRNA expression in endothelial cells (ECs) exposed to shear stress waveforms biomimetic of those leading to either a moderate or amplified arteriogenic response, in-vitro. We determined that microRNA-199a-5p (miR-199a), which was downregulated by $\sim 40 \%$ in ECs exposed to the amplified arteriogenesis waveform, regulated the expression of genes (CCND1, CD44, IKK $\beta$ ) crucial for the pro-arteriogenic functions of EC proliferation and monocyte adhesion. We then tested whether miR-199a regulates arteriogenesis in-vivo. Following FAL in Balb/c mice, overexpression of miR-199a impaired both foot perfusion and arteriogenesis. In contrast, miR199a inhibition elicited complete foot perfusion recovery, markedly augmented collateral 
arteriogenesis (a $36 \%$ increase in diameter corresponding to a $>3.4$-fold increase in segment conductance), and improved gastrocnemius muscle tissue composition 21 days post-FAL. Furthermore, miR-199a-modulated pro-arteriogenic macrophage recruitment in proportion to the degree of collateral artery growth.

Conclusions: We have identified mechanosensitive miR-199a as a potent regulator of perfusion recovery and arteriogenesis after arterial occlusion. MicroRNA-199a may therefore represent a new target for the therapeutic stimulation of arteriogenesis and treatment of PAD.

\subsection{Abbreviations}

PAD peripheral arterial disease

FAL femoral arterial ligation

EC endothelial cell

HUVEC human umbilical vein endothelial cell

miRNA microRNA

\subsection{Introduction}

Peripheral arterial disease (PAD) has become a global problem - it is estimated that over 202 million people worldwide have $\mathrm{PAD}^{3}$. PAD arises when atherosclerotic plaques block arteries in the lower limbs, thereby limiting blood flow to the distal tissue, ultimately leading to intermittent claudication or critical limb ischemia in severe cases. Many PAD patients are either not amenable to surgical intervention or receive little long term benefit from surgery ${ }^{4}$. Revascularization strategies to stimulate the growth of new capillaries from preexisting vessels (i.e. angiogenesis) or lumenal expansion of pre-existing arteries (i.e. arteriogenesis) remain promising therapeutic options, despite their limited success to date ${ }^{39}$. The stimulation of angiogenesis is important in PAD, as capillary density is reduced in these patients ${ }^{4,25,28}$; however, it is also imperative to restore 
the driving pressure to the distal tissue via lumenal expansion (i.e. arteriogenesis) of collateral arteries bypassing the occlusion(s) ${ }^{26,29,30}$.

We recently reported that a subset of collateral segments in the mouse hindlimb display remarkably permanent, amplified arteriogenesis after femoral arterial ligation (FAL) ${ }^{237}$.

Specifically, we showed that collateral artery segments exposed to both a 2 -fold increase in shear stress magnitude and reversed flow direction ("reversed" flow) following FAL exhibit a $\sim 30 \%$ increase in lumenal diameter 12 -weeks post-FAL compared to segments experiencing just a 2-fold increase in shear stress magnitude ("non-reversed” flow) (Figure 6.1 $A)^{176}$. Moreover, by applying shear stress waveforms biomimetic of these in-vivo hemodynamics to endothelial cells (ECs) in-vitro, we were able to generate a direct, comprehensive mapping of EC mechanosensitive signaling to differential arteriogenesis responses ${ }^{176}$. Comparative analysis of EC mechano-signaling corresponding to these differential responses may enable for the discovery of novel regulators of arteriogenesis.

To this end, mature microRNAs (miRNAs) are now well-recognized as key regulators of vascular remodeling 263,264 . MicroRNAs are $\sim 22$ nucleotide, non-coding RNAs that are endogenous regulators of gene expression ${ }^{117}$. Mature miRNA incorporates into the RNA-induced silencing complex (RISC) and then binds to a target mRNA, usually in the 3' untranslated region (UTR) of the mRNA ${ }^{118}$. MicroRNA binding acts to suppress target gene expression by inhibiting mRNA translation to protein or by promoting mRNA degradation ${ }^{119}$, depending on miRNA-target complementarity ${ }^{120}$. Additionally, miRNAs are attractive as potential therapeutic targets as they are short, highly conserved, and can negatively regulate gene expression of multiple mRNA targets $^{265}$. Several miRNAs have been implicated as regulators vascular growth, primarily angiogenesis in ischemic tissue, in response to arterial occlusion ${ }^{143,150,151,154,155,159,161,162,164}$. However, the explicit role of miRNAs in collateral arteriogenesis remains unstudied. Here, we tested the hypothesis that mechanosensitive microRNA-199a regulates perfusion recovery and 
collateral arteriogenesis following femoral arterial ligation (FAL) via control of monocyte recruitment and pro-arteriogenic gene expression.

\subsection{Materials and Methods}

\subsubsection{Human umbilical vein endothelial cell culture.}

Human umbilical vein endothelial cells (HUVECs) purchased from VEC Technologies Inc. (Rensselaer, NY) were thawed and maintained on $0.1 \%$ gelatin coated flasks in $\mathrm{M}-199$ medium (Lonza, Basel, Switzerland), supplemented with 10\% fetal bovine serum (Life Technologies Inc, Grand Island, NY), 100U/mL penicillin-G + 100ug/ml streptomyocin (Life Technologies Inc), 2mmol/L L-glutamine (Life Technologies Inc), $5 \mathrm{ug} / \mathrm{ml}$ endothelial cell growth supplement (Biomedical Technologies, Stoughton, MA), and 10ug/ml heparin (Sigma Aldrich, St. Louis, MO). For each set of experimental comparisons, cells were used from the same cell line between subculture passages 2-3.

\subsubsection{In vitro exposure of endothelial cells to biomimetic shear stress waveforms.}

HUVECs were plated on cell culture grade plastic dishes coated with $0.1 \%$ gelatin and grown to confluence. A cone and plate flow apparatus ${ }^{187}$, which maintains cells at $5 \% \mathrm{CO}_{2}$ and $37^{\circ} \mathrm{C}$, was used to induce a shear stress protocol. The applied shear stress protocol consisted of a 24 hour preconditioning period at a steady of $15 \mathrm{dyne} / \mathrm{cm}^{2}$, which was then either increased to 30 dynes $/ \mathrm{cm}^{2}$ (non-reversed flow) or increased to 30 dynes $/ \mathrm{cm}^{2}$ and reversed in direction (reversed flow) to simulate relative hemodynamics previously quantified in our in-vivo FAL model ${ }^{176}$. Fresh culture media consisting of M199 with 4\% dextran from Leuconostoc spp (Sigma Aldrich, $\mathrm{Mr}_{\mathrm{r}}$ 500,000), $2 \%$ fetal bovine serum, $100 \mathrm{U} / \mathrm{mL}$ penicillin-G $+100 \mathrm{ug} / \mathrm{ml}$ streptomyocin, $2 \mathrm{mmol} / \mathrm{L} \mathrm{L}-$ glutamine, $5 \mathrm{ug} / \mathrm{ml}$ endothelial cell growth supplement, and $10 \mathrm{ug} / \mathrm{ml}$ heparin was added to cells before exposure to shear stress and was continuously exchanged throughout the duration in the cone and plate apparatus. 


\subsubsection{HUVEC microarray gene expression profiling.}

Microarray analysis was performed as previously described and is publically available at GEO (http://www.ncbi.nlm.nih.gov/geo/ (GSE46248)). Volcano plots were generated using the expression change and false discovery rates (FDR) from the RvN and RvC datasets, respectively.

\subsubsection{Transfection of miRNA antagomirs and mimics in HUVECs.}

Twenty-four hours prior to exposure of HUVECs to flow conditions, HUVECs were plated without antibiotics on $0.1 \%$ gelatin coated plates in serum-free M199 (Life Technologies Inc) supplemented with $10 \%$ fetal bovine serum, $2 \mathrm{mmol} / \mathrm{L} \mathrm{L-glutamine,} 5 \mathrm{ug} / \mathrm{ml}$ endothelial cell growth supplement (Biomedical Technologies, Stoughton, MA), and 10ug/ml heparin (Sigma Aldrich Inc). After cells were allowed to adhere for 2 hours after plating, cells were transfected using Lipofectamine RNAiMax (Invitrogen, Carlsbad, CA) according to manufacturer's instructions. For inhibitor experiments, HUVECs were transfected with 20nM control or miR-199a-5p lockednucleic acid (LNA) inhibitors (Power Inhibitors, Exiqon, Vedbaek, Denmark). For over-expression experiments, HUVECs were transfected with 20nM control or miR-199a-5p locked-nucleic acid (LNA) mimic (miRCURY LNA mimics, Exiqon).

\subsubsection{HUVEC RNA isolation and qRT-PCR.}

Total RNA was extracted using the PureLink total RNA purification system (Life Technologies Inc) using the on-column DNase protocol (Life Technologies Inc) according to manufacturer's instructions. RNA concentration and purity was determined with a NanoDrop spectrophotometer in duplicate.

For quantitative reverse transcriptase PCR (qRT-PCR), 500ng of total RNA was reverse transcribed using the miScript II reverse transcription kit (Qiagen, Hilden, Germany, \#218160) with HiFlex buffer mix according to manufacturer's instructions. Following reverse transcription, RT-PCR was performed on 20ng cDNA using miScript SYBR Green PCR kit (Qiagen, \#218073) and miScript Primer Assays (Qiagen) for U6 (\#MS00033740), miR-199a-5p (\#MS00006741), 
miR-146a-5p (\#MS00003535), and miR-29a (\#MS00003262) on a CFX96 Real Time Detection System (Biorad). Normalized expression to U6 was quantified using the comparative $2^{\Delta \Delta \mathrm{Ct}}$ method.

Target mRNA expression was assessed by qRT-PCR on 12.5ng reverse transcribed cDNA with FastStart SYBR Green (Roche Applied Sciences, Penzberg, Germany) on a CFX96 Real Time Detection System (Biorad, Hercules, CA). Primers used were as follows: CD44 (forward 5'GCAGCCAACTTCCGAGGCAGC-3', reverse 5'-CGGAGGACGGGACGAGGATGG-3'), CCND1 (forward 5'-AACTACCTGGACCGCTTC-3', reverse 5'-GAGTTGTCGGTGTAGATGC-3'), IKKß (forward 5'-GCCTGGGAAATGAAAGAGCG-3', reverse 5'-ATCTGCTCACCTGTTTCCTGA-3'), Expression was normalized to $\beta 2$-microglobulin (forward 5'-AGCATTCGGGCCGAGATGTCT-3', reverse 5'-CTGCTGGATGACGTGAGTAAACCT-3') which is endogenously expressed and is not altered by many stimuli including shear stress ${ }^{44}$. Normalized expression was quantified using the comparative $2^{\Delta \Delta \mathrm{Ct}}$ method.

\subsubsection{Monocyte adhesion functional assay}

Human derived monocytes (THP-1 cell line) were purchased from the ATCC. Monocytes were unthawed and maintained in RPMI 1640 (11875-093, Life Technologies Inc) + 10\% fetal bovine serum (Life Technologies Inc) + 0.05mM $\beta$-mercaptoethanol per ATCC culture instructions. Monocytes sub-cultured once cell density approached 800,000 cells $/ \mathrm{mL}$. Cells were used between passages $2-6$.

Prior to the adhesion assay, cells were counted to obtain 3,000,000 cells / plate of HUVECs. Cells were pelleted, washed with PBS, pelleted, and then re-suspended in serum-free RPMI media at $1,000,000$ cells $/ \mathrm{mL}$. Thawed calcein AM was added at $1 \mathrm{ug} / \mathrm{ml}$ and incubated with cells for 15 minutes at $37^{\circ} \mathrm{C}$. After 15 minutes, the reaction was stopped by adding excess serum-free RPMI to the cell solution then pelleted. Cells were washed once with serum-free M199 media, pelleted, and then re-suspended in serum-free M199 at 500,000 cells/mL. Immediately following 
completion of flow exposure to HUVECs, flow media was removed by aspiration. HUVECs were quickly washed with serum-free M199 media. This media was then aspirated off and $6 \mathrm{~mL}$ of serum-free M199 + monocytes (3,000,000 / plate) were added to and incubated with HUVECs for 30 minutes at $37^{\circ} \mathrm{C}$. Following the 30 minutes, cells were washed twice with PBS to remove unbound monocytes. Adhered monocytes and HUVECs were fixed with 4\% PFA for 10 minutes followed by two washes with PBS. Cover slips were mounted with Prolong Gold (Life Technologies Inc). Plates were then imaged using a Nikon TE2000 C1 laser scanning confocal microscope. Nine randomly selected FOVs per condition for 4 independent experiments were obtained. Images were de-identified and randomized in MATLAB. Images were converted to 8bit images, set to an equivalent threshold, and bound monocytes were quantified using Fiji's "Analyze Particles" tool $\left(20 \mu \mathrm{m}^{2}\right.$ minimum particle size). Results were centered on the mean of all conditions within each independent experiment.

\subsubsection{Mice.}

All animal protocols were approved by the Institutional Animal Care and Use Committee at the University of Virginia (Protocol 3814) and conformed to all regulations for animal use outlined in the American Heart Association Guidelines for the Use of Animals in Research. Balb/c mice were purchased from Charles River Laboratory (Wilmington, MA). All animals were housed in the animal facilities at the University of Virginia.

\subsubsection{Femoral arterial ligation model.}

We used a previously detailed femoral artery ligation (FAL) scheme s67,237,242 $^{\text {that }}$ produces consistent arteriogenesis in the collateral arteries of the gracilis adductor muscles ${ }^{29,30,177-180,237}$, along with minimal heterogeneity in the baseline collateral structure and with known changes in flow direction from baseline. Male mice, 10-12 weeks of age, were anesthetized (i.p $120 \mathrm{mg} / \mathrm{kg}$ ketamine, $12 \mathrm{mg} / \mathrm{kg}$ xylazine, and $0.08 \mathrm{mg} / \mathrm{kg}$ atropine), depilated, and prepped for aseptic surgery. On the left leg, an incision was made directly above and along the femoral artery, which 
was gently dissected from the femoral vein and nerve between the bifurcation of the superior epigastric artery and popliteal artery. Two 6.0 silk sutures were placed immediately distal to the epigastric artery, which served as the origin of the muscular branch artery in all mice, and the artery segment between the two ligatures was then severed with micro dissecting scissors. The surgical site was then closed with 5.0 prolene sutures. A sham surgery, wherein the femoral artery was exposed but not ligated, was performed on the right hindlimb (i.e. on the other leg). Animals received one injection of buprenorphine for analgesia at the time of surgery and a second dose 8-12 hours later.

\subsubsection{In vivo miR-199a antagomir treatment.}

Anti-miR-199a-5p (5'-TAGTCTGAACACTGGG-3') and non-targeting control (5'ACGTCTATACGCCCA-3') locked nucleic acid (LNA) oligonucleotides were purchased from Exiqon. Oligonucleotides were reconstituted in sterile TE buffer and stored at $1.2 \mathrm{nmol} / \mu \mathrm{l}$ at $-20^{\circ} \mathrm{C}$. Prior to use, aliquots of oligonucleotides were thawed and diluted in sterile saline to a final concentration of $0.25 \mathrm{nmol} / \mu \mathrm{l}$. Immediately following FAL, $7.5 \mathrm{nmol}$ of oligonucleotide was injected into each (ligated and sham-operated) gracilis muscles.

\subsubsection{In vivo miR-199a mimic treatment.}

In-vivo ready miRVana miR-199a-5p (\#4464070, MC10893) mimic and non-targeting control (\#4464061) mimic were purchased from Ambion. Mimics (250nmol) were reconstituted in sterile nuclease-free water and stored at $0.5 \mathrm{nmol} / \mu \mathrm{l}$ at $-20^{\circ} \mathrm{C}$. Prior to use, aliquots of oligonucleotides were thawed and diluted in sterile saline to a final concentration of $0.25 \mathrm{nmol} / \mu \mathrm{l}$. Immediately following FAL, 7.5nmol of oligonucleotide was injected into both ligated and sham-operated gracilis muscles.

\subsubsection{Laser Doppler perfusion imaging.}

For monitoring blood flow recovery and post-surgical ischemia, mice were anesthetized via 1.5\% isoflurane under constant oxygen. Mice were placed in a prone position and the soles of the feet 
were scanned (PeriCam PSI, PeriMed, Stockholm, Sweden). Mean foot perfusion was used to calculate relative perfusion ratio (ligated/unligated).

\subsubsection{Tissue harvesting for miRNA and target expression.}

Seven days after femoral artery ligation, mice were anesthetized (i.p $120 \mathrm{mg} / \mathrm{kg}$ ketamine, 12 $\mathrm{mg} / \mathrm{kg}$ xylazine, and $0.08 \mathrm{mg} / \mathrm{kg}$ atropine) and euthanized via an overdose of pentobarbital. The left ventricle was cannulated with a 23-gauge catheter (right ventricle was carefully opened to act as a sink for perfusate) and the entire body was perfused with $7 \mathrm{~mL}$ of $T$ ris- $\mathrm{CaCl}_{2}(0.1 \mathrm{~g} / \mathrm{L} \mathrm{CaCl})$ containing $2 \%$ heparin, 2mmol/L adenosine (16404, Fisher Scientific, Pittsburg, PA) and $0.1 \mathrm{mmol} / \mathrm{L}$ papaverine (P3510, Sigma Aldrich, St Louis, MO) to clear and vasodilate the downstream vasculature at a constant rate of $1.5 \mathrm{~mL} / \mathrm{min}$ (PHD2000, Harvard Apparatus). Once perfused, we waited 5 minutes to enable vasodilation. The entire body was then perfused with $14 \mathrm{~mL}$ Tris- $\mathrm{CaCl}_{2}$ and both gracilis muscles dissected free, placed in RNAlater (Ambion), and stored at $-20^{\circ} \mathrm{C}$.

\subsubsection{RNA isolation from tissues and qRT-PCR.}

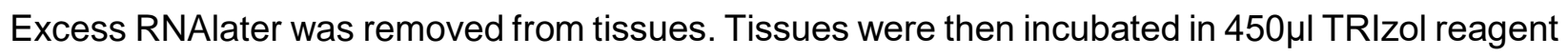
for 5 minutes at room temperature. Tissues were then placed on ice and homogenized using a power homogenizer (Omni International, Kennesaw, GA) in short bursts to avoid overheating.

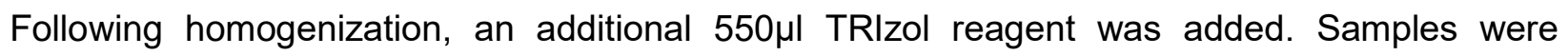
incubated for another 5 minutes at room temperature to ensure complete lysis. $200 \mu \mathrm{ll}$ of chloroform was added to each sample. Samples were then shaken vigorously for 15 seconds and incubated for 3 minutes at room temperature. Following this incubation, samples were centrifuged for 10 minutes at $12,000 \mathrm{~g}$ at $4^{\circ} \mathrm{C}$. The resulting aqueous layer was carefully removed, place in a new RNA/DNAse-free tube, and an equal volume of $70 \%$ ethanol was added to the save aqueous layer. RNA isolation then proceeded using the PureLink total RNA purification system (Life Technologies Inc) with the on-column DNase protocol (Life Technologies Inc) according to 
manufacturer's instructions. RNA concentration and purity was determined with a NanoDrop spectrophotometer in duplicate.

For reverse transcription (Applied Biosystems, \#4366596) and miR-199a-5p detection, TaqMan MicroRNA assays (Applied Biosystems, \#4427975) were used. Quantitative real-time PCRs were done on an ABI Prism 7900 HT detection system (Applied Biosystems). Gene expression was normalized to RNU6 (Applied Biosystems, \#4427975) and the relative expression was determined with the comparative $2^{\Delta \Delta C t}$ method.

\subsubsection{Tissue harvesting for whole mount vascular casting and cross sectional analysis.}

For analysis of lumenal diameters in the gracilis collateral arteries and to enable sectioning at specific regions, vascular casting was performed using an opaque polymer that allows for accurate lumenal diameter measurements ${ }^{179}$. Twenty-one days after femoral artery ligation, mice were anesthetized (i.p $120 \mathrm{mg} / \mathrm{kg}$ ketamine, $12 \mathrm{mg} / \mathrm{kg}$ xylazine, and $0.08 \mathrm{mg} / \mathrm{kg}$ atropine), euthanized via an overdose of pentobarbital, and then the abdominal aorta was cannulated. The lower body was then perfused with $7 \mathrm{~mL}$ of $2 \%$ heparinized saline with $2 \mathrm{mmol} / \mathrm{L}$ adenosine (16404, Fisher Scientific) and $0.1 \mathrm{mmol} / \mathrm{L}$ papaverine (P3510, Sigma Aldrich) to clear and vasodilate the downstream vasculature at a constant rate of $1 \mathrm{~mL} / \mathrm{min}$ (PHD2000, Harvard Apparatus). Once perfused, we waited 5 minutes to enable vasodilation. Tissues were then perfused with $3 \mathrm{~mL}$ of 4\% paraformaldehyde solution (19943, Affymetrix, Cleveland, $\mathrm{OH}$ ) at $1 \mathrm{~mL} / \mathrm{min}$ and allowed to fix for 10 minutes. The lower body was then perfused with $0.8 \mathrm{~mL}$ Microfil@ casting agent (FlowTech, Inc, Carver, Massachusetts) at a constant pressure of $100 \mathrm{mmHg}$. The viscosity of Microfil@ was adjusted to minimize transport across capillaries. After curing for 1.5 hours at room temperature, gracilis muscles were dissected free and then cleared in $50 \%$ glycerol in phosphate buffered saline (PBS) overnight. Cleared tissues were mounted between two coverslips using $500 \mu \mathrm{m}$ thick spacers (645501, Grace Bio-Labs Inc) to keep constant thickness between muscles. Muscles 
were imaged using transmitted light at 4x magnification on a Nikon TE200 inverted microscope with a CCD camera (Quantifier, Optronics Inc). Individual fields of view were montaged together (Photoshop CS2, Adobe Systems Inc).

For analysis of lumenal diameters from intact gracilis collateral whole mounts (i.e. vascular casting), collateral entrance regions were defined according to the following method. A cropped portion $(560 \mu \mathrm{m} \times 560 \mu \mathrm{m})$ of the montaged image (previously randomized and de-identified) was taken of the collateral artery at the first visible branch point of a terminal arteriole from the primary collateral as it extended from either the muscular branch or saphenous artery as previously described $^{237}$. After each cropped image region was taken, all images were randomized and deidentified. The mean diameter was then taken from 4-5 separate diameter measurements along the length of cropped portion of the collateral artery.

After imaging, muscles were rehydrated, cut, and then paraffin embedded for cross sectional analysis at the muscular branch and saphenous artery entrance regions to the collateral arteries. Resulting cross sections were re-hydrated and immunolabeled for the macrophage marker Mac3 (day 7 post-FAL) and H\&E stained for collateral artery structure analysis (day 21 post-FAL).

\subsubsection{Cross sectional analysis of collateral artery structure.}

Sections (5 $5 \mathrm{~m}$ thickness) of paraffin embedded muscle from the muscular and saphenous regions were labeled for H\&E. Individual fields of view encompassing the collateral vessels were imaged with a 40x water objective on a Zeiss inverted microscope (Zeiss Axioskop, Thornwood, NY) with a CCD camera (Quantifier, Optronics Inc). All images were randomized and de-identified prior to analysis. Lumenal diameter, wall area, and wall thickness were determined using Fiji ${ }^{181}$.

\subsubsection{Immunofluorescence labeling of pericollateral Mac3 ${ }^{+}$cells.}

Sections ( $5 \mu \mathrm{m}$ thickness) of paraffin embedded muscle from the muscular and saphenous regions were rehydrated and subjected to heat mediated antigen retrieval for 10 minutes in a citrate based antigen retrieval buffer (Vector Laboratories, Burlingame, CA; H-3300). Slides were then 
quenched of endogenous peroxidase activity with a 30 minute incubation in $3 \% \mathrm{H}_{2} \mathrm{O}_{2}$, blocked, and labeled with rat-anti-Mac3 (1:100, M3/4 clone, 550292; BD Biosciences, San Jose, CA) overnight at $4^{\circ} \mathrm{C}$. Slides were washed and incubated with a biotinylated sheep-anti-rat secondary antibody (Abcam, Cambridge, MA, ab6851, 1:500) for 1 hour at room temperature. Slides were washed and incubated with an avidin-biotin complex (Vectastain ABC solution, Vector Laboratories) for 30 minutes at room temperature. Slides were washed and incubated with a Tyramide Signal Amplification (TSA) reagent (Perkin Elmer, Waltham, MA; 1:50) for 10 minutes at room temperature. Slides were washed and incubated with streptavidin-488 (Life Technologies Inc, 1:500), Cy3-anti-SMA (1A4 clone, Sigma, 1:1000) and DRAQ5 (Thermo Scientific Inc, 1:1000). Slides were then mounted with Prolong Gold (Life Technologies Inc) to minimize photobleaching, allowed to cure overnight, and imaged using a Nikon TE2000 C1 laser scanning confocal microscope with a 20x oil objective. Cropped fields of view (512x512 pixels) encompassing the collaterals in each region were randomized and de-identified. The pericollateral region was outlined ( 25 microns around the vessel) and pericollateral Mac3 ${ }^{+}$nuclei were counted in Fiji ${ }^{181}$.

\subsubsection{Cross sectional analysis for gastrocnemius muscle morphology.}

Sections ( $5 \mu \mathrm{m}$ thickness) of paraffin embedded muscle from the gastrocnemius muscle were H\&E labeled. Individual fields of view were imaged with a 10x objective on a Zeiss inverted microscope (Zeiss Axioskop) with a CCD camera (Quantifier, Optronics Inc). Individual fields of view were montaged together (Photoshop CS2, Adobe Systems Inc). All montaged images were randomized and de-identified prior to analysis. Muscle areas were manually outlined using Fiji ${ }^{181}$. Tissue composition was classified into viable and non-viable, which were defined as:

1. Viable: fibers are present and have centrally located nuclei (regenerating) or fibers are comparable in size, organization, and structure to unligated control with peripheral nuclei $(\text { mature })^{30}$ 
2. Non-viable: fibers lack nuclei, are rounded and dilated in appearance, have weak eosinphillic cytoplasm (necrotic) or where there is minimal presence of myoblasts and is dominated by fibrous matrix and adipose tissue (fibroadipose) $)^{30}$

\subsubsection{Cross sectional analysis for gastrocnemius capillary density.}

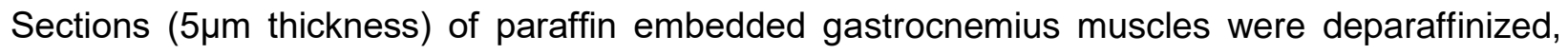
rehydrated, then blocked in Carbofree blocking solution (1:10, Vector Labs). Slides were then incubated with fluorophore conjugated primary antibody (isolectin-IB4-AlexFluor-647, 1:200, Life Technologies Inc) overnight at $4{ }^{\circ} \mathrm{C}$. Nuclei were counterstained with Sytox green (500uM, Life Technologies Inc). Slides were washed and sealed with Prolong Gold (Life Technologies Inc) to minimize photobleaching. Individual fields of view were imaged with a Nikon TE2000 C1 laser scanning confocal microscope with a 10x objective and the same imaging parameters for all fields of view. Fields of view were then montaged together using Photoshop CS2 (Adobe Systems Inc). Muscle areas were manually outlined using Fiji ${ }^{181}$ and classified as either viable or non-viable tissue. The number of capillaries (Isolectin-B4+ vessels $<25 \mu \mathrm{m}^{2}$ in diameter) and muscle area (identified from autofluorescence) were determined in each montaged image view using a semiautomated Fiji image analysis.

\subsubsection{Statistical analyses.}

All results are reported as mean \pm standard error of the mean (SEM), unless otherwise noted. All data were first tested for normality and equal variance. Statistical significance was then assessed by a Student's $t$ test or a two-way ANOVA followed by a Holm-Sidak multiple comparisons test, unless otherwise noted (SigmaStat 3.5, Systat Inc). Significance was assessed at $p<0.05$. 


\subsection{Results}

\subsubsection{Endothelial cell miRNA expression is regulated by shear stress waveforms biomimetic of collateral artery segments exhibiting differential arteriogenic responses.}

To identify candidate miRNAs, we examined previously generated genome-wide cDNA microarray data (GSE46248) ${ }^{237}$ from ECs exposed to shear stress waveforms biomimetic of those measured in-vivo ${ }^{176}$ in collateral artery segments exhibiting either moderate (muscular) or amplified (saphenous) arteriogenesis responses (Figure 6.1A). Briefly, human umbilical vein endothelial cells (HUVECs) were preconditioned for 24 hours at a baseline arterial shear stress (15 dynes $\left./ \mathrm{cm}^{2}\right)^{190}$ to establish endothelial cell alignment, planar cell polarity, and steady-state signaling, thereby mimicking the in-vivo baseline state. A femoral arterial ligation $(F A L)$ was then simulated by a step-wise $100 \%$ increase in shear stress, in either the same direction or in the opposite direction, to mimic shear stress changes occurring in the muscular branch (nonreversed flow) and saphenous artery (reversed flow) entrance regions, respectively (Figure 6.1A-B). Six hours after our simulated FAL, we isolated total RNA and performed genome-wide analysis using Affymetrix CDNA 1.0 ST microarrays. Genome-wide analysis identified a small subset of miRNA genes differentially regulated between reversed and non-reversed flow conditions (Figure 6.1C, Supplemental Table 6.1). The expression of several candidate miRNAs (miR-199a-5p, miR-146a-5p, and miR-29a) was assessed by RT-qPCR both at 1-hour and 6-hours after simulated FAL to confirm microarray results. We found all these candidate miRNAs to be significantly downregulated ( 40\%) in HUVECs exposed to the reversed flow waveform 6-hours after simulated FAL (Figure 6.1D-F). 

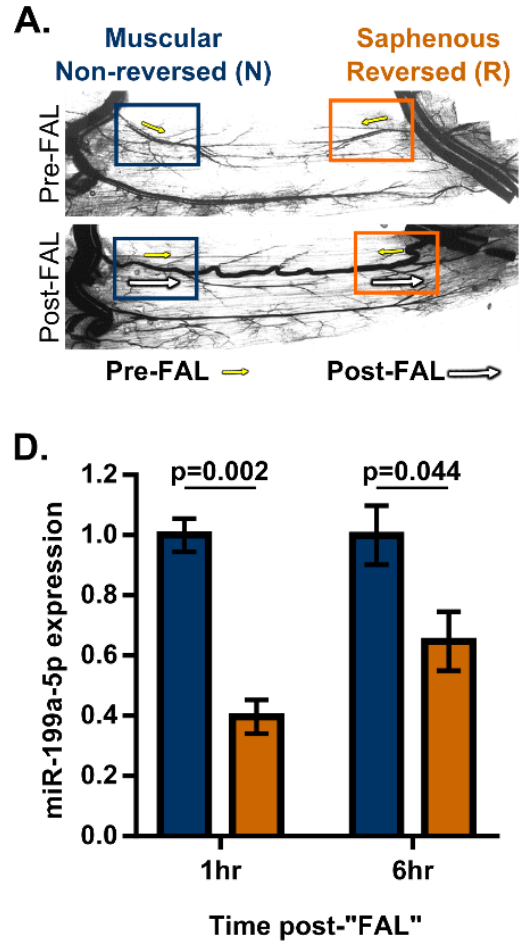

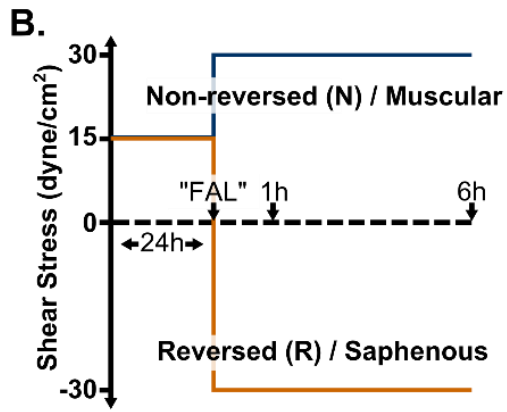

E.

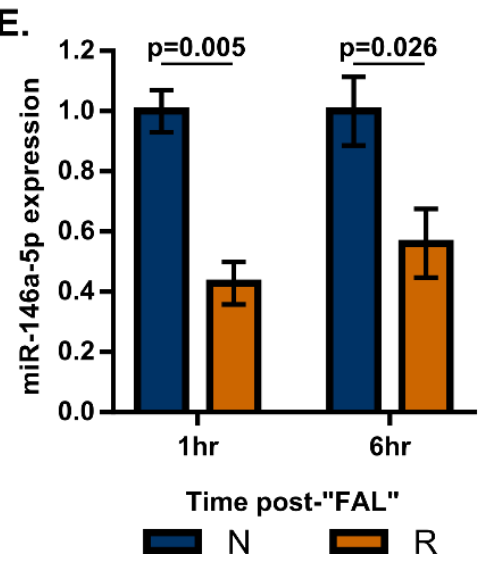

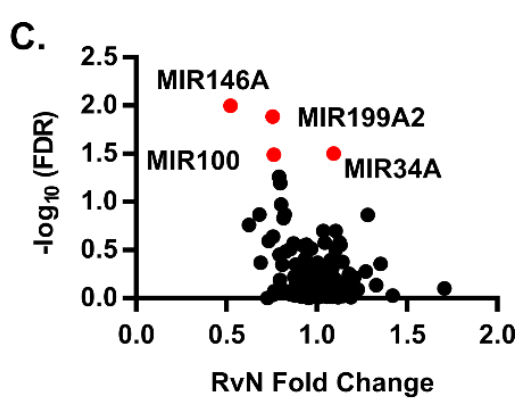

F.

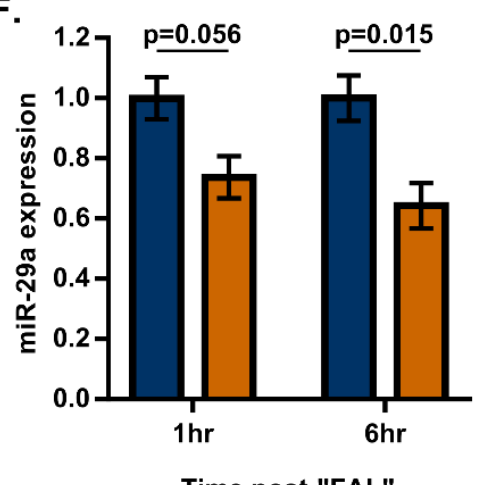

Time post-"FAL"

Figure 6.1. Endothelial cell miRNA expression is differentially regulated by shear stress waveforms biomimetic of collateral artery segments exhibiting varied arteriogenic responses.

A) Representative vascular cast images of gracilis adductor collaterals in Balb/c mice taken from unligated sham control (top) and day 21 post-femoral arterial ligation (FAL, bottom) groups. Arrows indicate direction and magnitude of blood flow pre- (yellow) and post- (white) FAL. The femoral artery is ligated just distal to the epigastric artery such that some collateral segments ("muscular") experience a 2-fold increase in shear stress magnitude ("non-reversed" flow) while other segments ("saphenous") are exposed to both a 2-fold increase in shear stress magnitude and reversed flow direction ("reversed" flow). Arteriogenesis is amplified in the flow-reversed, saphenous regions of collateral arteries. B) Schematic depicting biomimetic waveforms applied to HUVECs to simulate saphenous (reversed flow, R) and muscular (non-reversed flow, $\mathrm{N})$ regions. C) Volcano plot of all microRNAs (miRNAs) on the Affymetrix ST 1.0 human microarray dataset $(n=4)$ from Heuslein et al. ${ }^{237}$ HUVECs were exposed to the flow waveforms in (B). Gene expression was determined at 6 hours after simulated FAL. False discovery rate $=$ FDR. Red dots indicate miRNAs with FDR<0.05. D-F) Bar graphs of mature miR-199a-5p, miR-146a-5p, and miR-29a expression in HUVECs exposed to biomimetic shear stress conditions from $(B)(n=3-5)$. Student's $t$-test. Data are mean \pm SEM.

\subsubsection{MicroRNA-199a negatively regulates pro-arteriogenic endothelial gene} expression and monocyte adhesion to flow-exposed ECs in-vitro.

Of these candidate miRNAs, miR-199a-5p (henceforth denoted as miR-199a) was of particular interest as it is known to regulate mRNAs of several pathways necessary for arteriogenesis, including $C D 44^{266,267}, C C D N 1^{268}$, and IKK $\beta^{269}$. We therefore sought to determine if miR-199a 
regulates the expression of these pro-arteriogenic genes in ECs exposed to the biomimetic shear stress waveforms. HUVECs were transfected with 20nM miR-199a mimic, anti-miR-199a locked nucleic acid oligonucleotides, or respective non-targeting controls, 24 hours prior to shear stress exposure.

A.

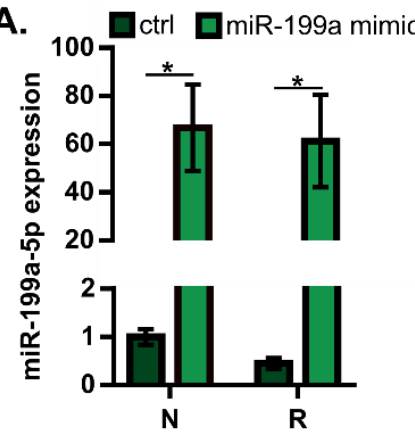

E.

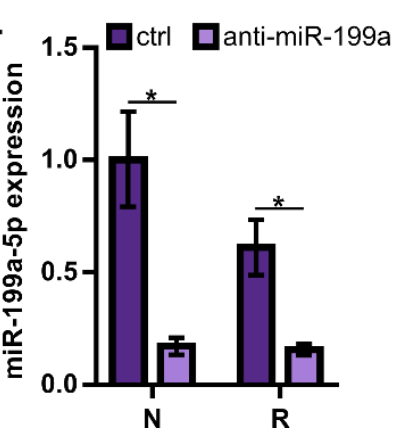

B.

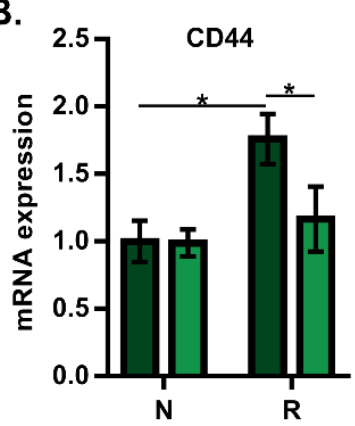

F.

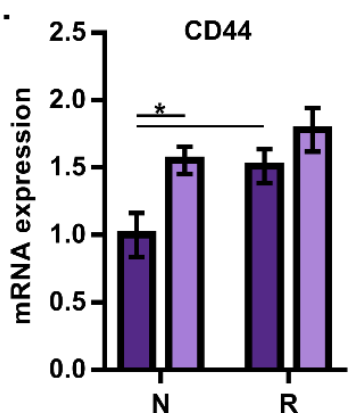

C.

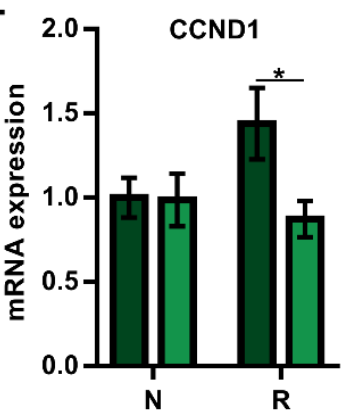

G.

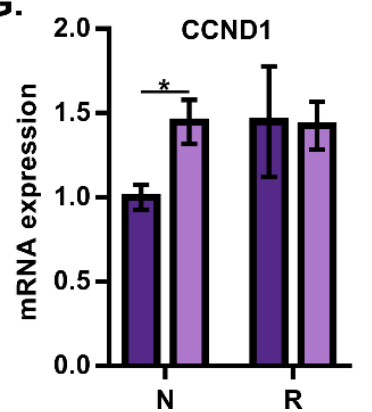

D.

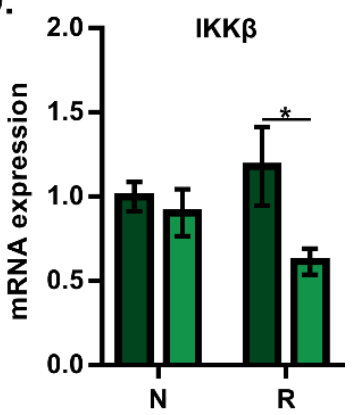

H.

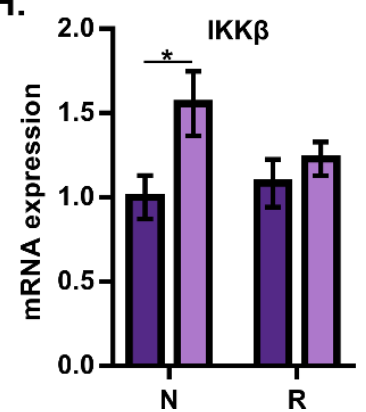

Figure 6.2. MicroRNA-199a negatively regulates the expression of CD44, CCND1, and IKK $\beta$ in endothelial cells exposed to biomimetic shear stress waveforms in-vitro.

A) Bar graph of relative miR-199a expression 6-hours after simulated FAL in HUVECs transfected with miR-199a or non-targeting control (ctrl) mimics and subjected to non-reversed $(N)$ or reversed $(R)$ flow waveforms as outlined in Figure $6.1 B(n=4) .{ }^{*} p<0.05$, Student's $t$-test. B-D) Bar graphs of relative CD44, CCND1, and IKK $\beta$ mRNA expression in each condition $(n=4)$. ${ }^{*} p<0.05$, two-way ANOVA followed by a Holm-Sidak test for multiple comparisons. E) Bar graph of relative miR-199a expression 6-hours after simulated FAL in HUVECs transfected with anti-miR-199a or non-targeting control (ctrl) locked nucleic acid oligonucleotides ( $n=4)$. * $p<0.05$, Student's $t$-test. F-H) Bar graphs of relative CD44, CCND1, and IKK $\beta$ mRNA expression in each condition $(n=4)$. ${ }^{*} p<0.05$, two-way ANOVA followed by a Holm-Sidak test for multiple comparisons. Data are mean \pm SEM.

Transfection with miR-199a mimics increased relative miR-199a expression >60-fold (Figure 6.2A) and anti-miR-199a reduced miR-199a expression by $\sim 80 \%$ (Figure $6.2 E$ ) compared to their respective non-targeting controls in HUVECs exposed to our biomimetic waveforms. Six-hours after simulated FAL, CD44 expression was significantly upregulated 
between non-reversed and reversed flow waveforms in HUVECs under non-targeting controltreated conditions (Figure 6.2B, F). However, transfection with miR-199a mimic significantly decreased the relative expression of CD44, CCND1, and IKK $\beta$ in HUVECs exposed to reversed flow conditions (Figure 6.2B-D). Inhibition of miR-199a led to an upregulation in CD44, CCND1, and IKK $\beta$ expression in HUVECs exposed to a non-reversed waveform, while there was no significant effect under reversed flow conditions (Figure 6.2F-H).

A.

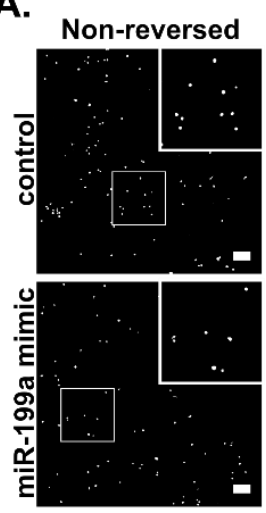

Reversed

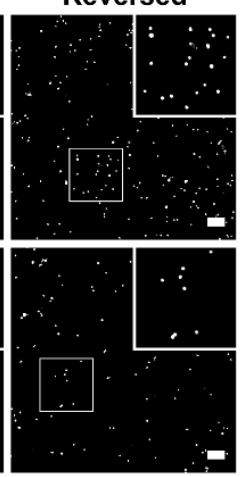

C.
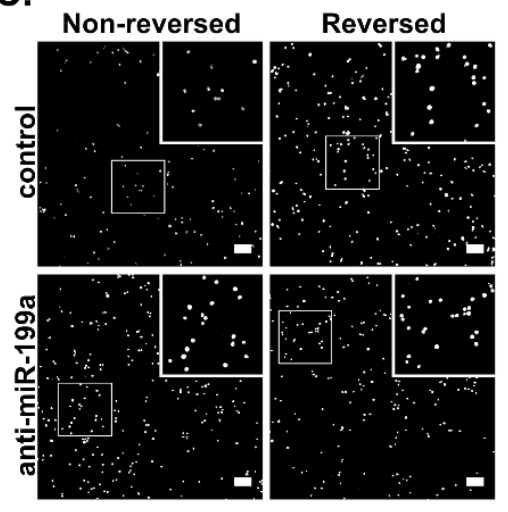

B.

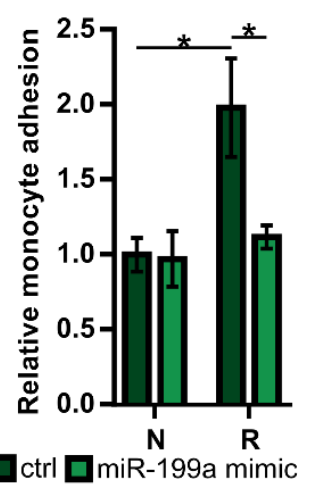

D.

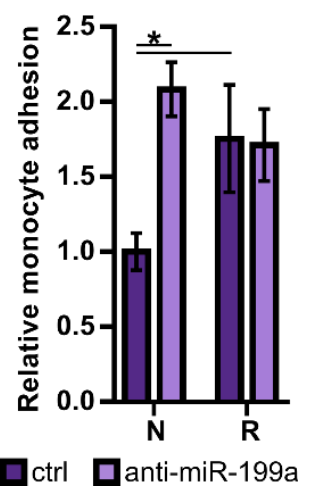

Figure 6.3. Monocyte adhesion to HUVECs exposed to shear stress waveforms biomimetic of arteriogenic collaterals is modulated by miR-199a.

A) Representative confocal microscopy images of fluorescently labeled THP-1 monocytes adhered to HUVECs transfected with miR-199a or nontargeting control (ctrl) mimic then subjected to non-reversed $(\mathrm{N})$ or reversed $(\mathrm{R})$ flow waveforms outlined in Figure 6.1B, 6-hours post-simulated FAL (Scale bar $=100 \mu \mathrm{m})$. Insets are the magnified $300 \mu \mathrm{m} \times 300 \mu \mathrm{m}$ regions outlined by white boxes. B) Bar graph quantifying relative number of adhered monocytes in each condition $(n=4) .{ }^{*} p<0.05$, two-way ANOVA followed by a Holm-Sidak multiple comparisons test. Representative images of fluorescently labeled THP-1 monocytes adhered to flow exposed HUVECs transfected with anti-miR-199a or nontargeting control (ctrl) locked nucleic acid oligonucleotides, 6-hours after simulated FAL (Scale bar $=100 \mu \mathrm{m})$. D) Bar graph quantifying relative number of adhered monocytes in each condition $(n=4) . \quad{ }^{*} p<0.05$, two-way ANOVA followed by a Holm-Sidak multiple comparisons test. Data are mean \pm SEM.

Monocyte adhesion to activated endothelium is a required step in the arteriogenesis cascade $52,56,58,60,62,246$. We sought to determine whether altered miR-199a expression modulates this critical process. In control-treated conditions, HUVECs exposed to the reversed flow waveform experienced increased monocyte adhesion compared to those exposed to the nonreversed waveform (Figure 6.3), consistent with previous results ${ }^{237}$. Overexpression of miR-199a attenuated this enhanced functional response, leading to a $44 \%$ decrease in monocyte adhesion 
in reversed flow conditions (Figure 6.3A-B). In non-reversed flow conditions, miR-199a overexpression did not further reduce monocyte adhesion. Conversely, inhibition of miR-199a led to enhanced pro-arteriogenic function in-vitro as seen by a $90 \%$ increase in monocyte adhesion to non-reversed flow-exposed HUVECs, while there was no further increase with reversed flow (Figure 6.3C-D).

\subsubsection{Overexpression of miR-199a limits foot reperfusion following FAL while miR- 199a inhibition elicits complete perfusion recovery in Balb/c mice.}

To test the hypothesis that miR-199a regulates perfusion recovery and/or arteriogenesis in-vivo, we performed FALs on Balb/c mice and modulated miR-199a expression via intramuscular injection of miR-199a mimic, anti-miR-199a locked nucleic acid oligonucleotides, or respective non-targeting control, directly into the gracilis muscle immediately following FAL. Local intramuscular injection of miR-199a mimic led to a $\sim 5$-fold increase in relative miR-199a expression (Figure 6.4A), while anti-miR-199a decreased miR-199a expression 2.6-fold in the gracilis muscle 7 days post-FAL (Figure 6.4B). Perfusion measurements of the plantar surface of the foot indicated moderate ischemia immediately post-FAL, followed by an incomplete perfusion recovery in the control-treated mice of both overexpression $(0.92 \pm 0.02$, day 21 post-FAL) and inhibition (0.88 \pm 0.02 , day 21 post-FAL) studies (Figure 6.4C- $F$ ). When miR-199a was overexpressed, the reperfusion response was further dampened as early as 4 days post-FAL and only reached $80 \%$ reperfusion 21 days post-FAL (Figure 6.4C, E). However, anti-miR-199a treated mice fully recovered foot perfusion by day 21 post-FAL (Figure 6.4D, $F$ ). 
A.

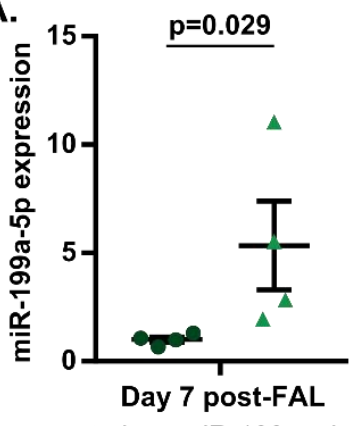

- control $\Delta$ miR-199a mimic
B.

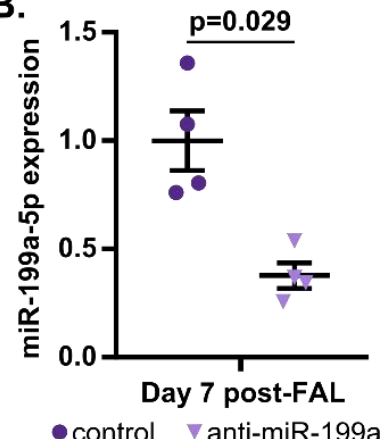

C.

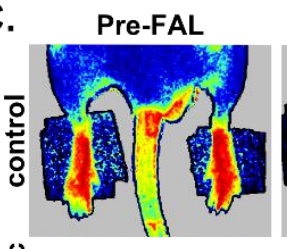

Post-FAL

Day 21 post-FAL
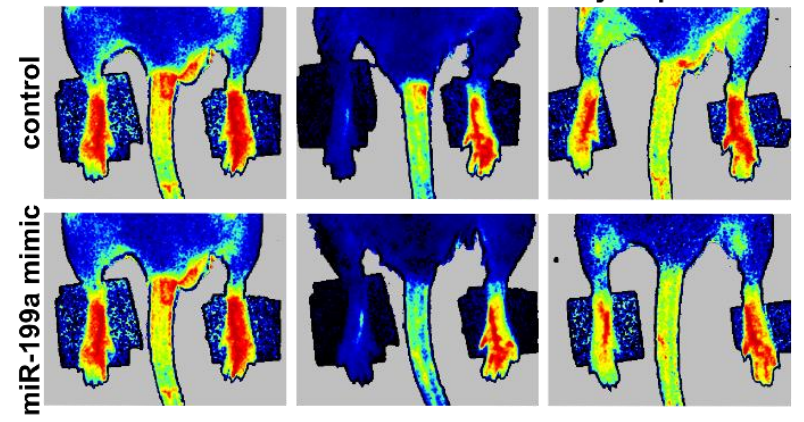

D.
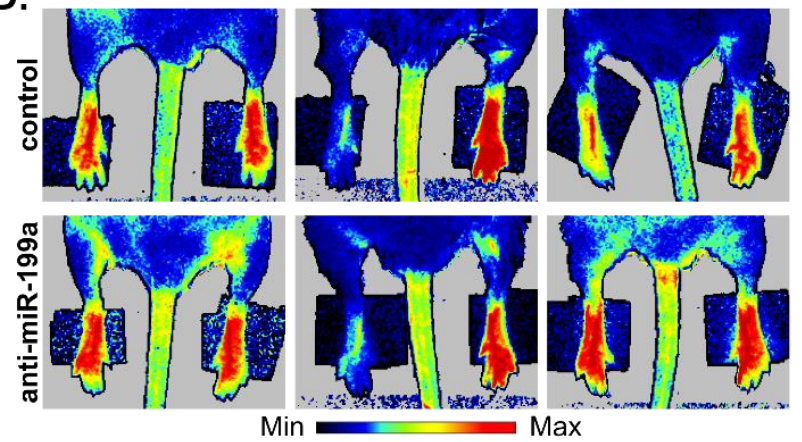

E.

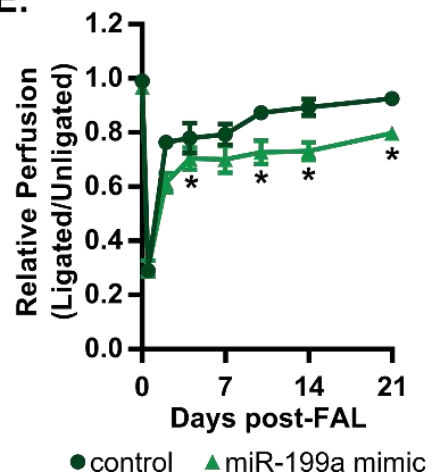

F.

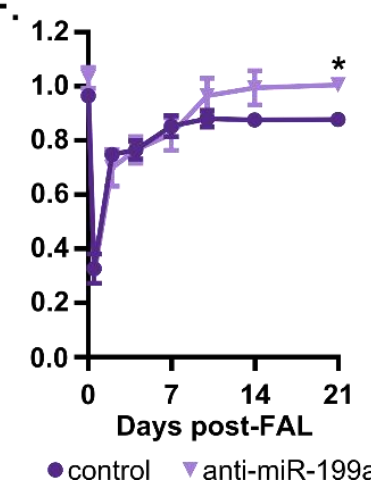

Figure 6.4. Overexpression of miR-199a limits foot reperfusion following FAL while miR-199a inhibition leads to complete perfusion recovery in Balb/c mice.

A-B) Relative miR-199a-5p expression in the gracilis muscle 7 days post-FAL in Balb/c mice. Mice were treated with a single i.m. injection of $7.5 \mathrm{nmol}$ miR-199a mimic, anti-miR-199a, or respective non-targeting control oligonucleotides immediately post-FAL $(n=4)$. Mann-Whitney $U$ test. C-F) Post-FAL foot perfusion recovery as assessed by laser Doppler perfusion imaging $(n=6) .{ }^{*} p<0.05$ vs. control, two-way ANOVA with repeated measures followed by Holm-Sidak test for multiple comparisons. Data are mean \pm SEM. 
A.
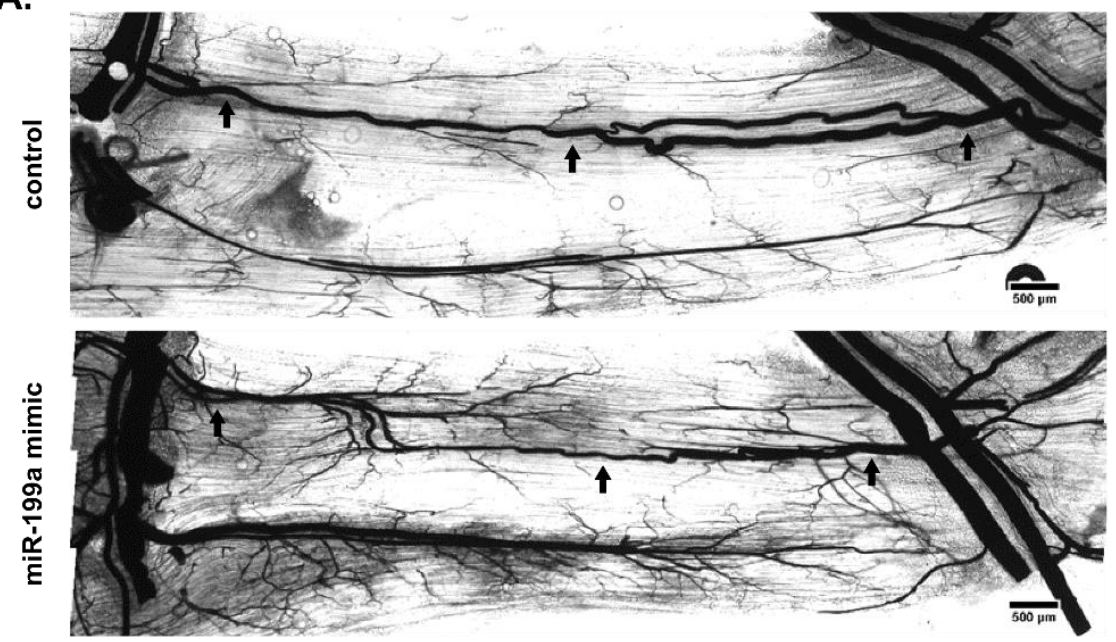

B.

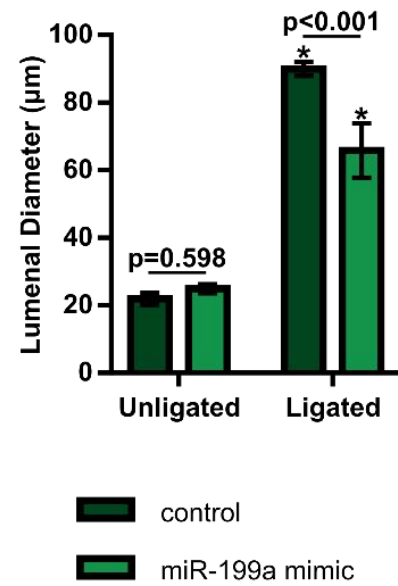

$\mathbf{F}$.

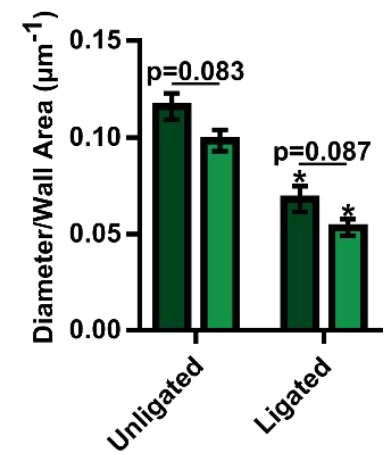

Figure 6.5. Overexpression of miR-199a inhibits arteriogenesis in Balb/c mice.

A) Representative whole mount vascular cast images of gracilis collateral arteries 21 days post-FAL in nontargeting control (top) or miR-199a mimic (bottom) treated Balb/c mice. B) Bar graph of mean lumenal diameter along collateral artery length for ligated and unligated limbs of miR-199a mimic and control treated mice ( $n=5-6$ for mimic and control groups respectively). ${ }^{*} p<0.001$ vs. unligated, two-way ANOVA followed by Holm-Sidak test for multiple comparisons C) Representative H\&E stained cross-sections of collateral arteries from ligated and unligated limbs. D-F) Bar graphs of lumenal diameter, wall area, and diameter per wall area from H\&E stained cross-sections ( $n=5-6$ for mimic and control groups respectively). ${ }^{*} p<0.001$ vs. unligated, two-way ANOVA followed by Holm-Sidak test for multiple comparisons. Arrows indicate the primary collateral artery in $(A)$ and $(C)$. Data are mean \pm SEM.

\subsubsection{Overexpression of miR-199a inhibits arteriogenesis in Balb/c mice.}

We next examined whether overexpression of miR-199a expression affects arteriogenesis by measuring the lumenal diameter of gracilis collateral arteries 21 days post-FAL (Figure 6.5A, 
Figure 6.6A). While both control and miR-199a mimic treated mice experienced significant $(p<0.0001)$ arteriogenesis in their ligated limbs compared to sham-operated controls, treatment with a miR-199a mimic significantly $(p=0.0008)$ reduced collateral artery growth by $>25 \%$ (Figure 6.5B). Moreover, this reduction in arteriogenesis occurred at both the muscular (nonreversed) and saphenous (reversed) collateral artery regions (Supplemental Figure 6.1). Cross-sectional analysis of these collateral arteries was used to confirm whole-mount diameter measurements and to determine collateral wall area (Figure 6.5C). Mice treated with miR-199a mimic experienced a lesser degree of enhancement in lumenal diameter (Figure 6.5D) and wall area (Figure 6.5E) than controls, while there was no significant difference $(p=0.087)$ in the diameter to wall area ratio, 21 days after FAL.

\subsubsection{Inhibition of miR-199a amplifies arteriogenesis in Balb/c mice following FAL}

In contrast, when we administered a single intramuscular injection of an anti-miR-199a lockednucleic acid oligonucleotide to mice immediately after FAL, arteriogenesis was significantly amplified (Figure 6.6A). Inhibition of miR-199a generated a $36 \%$ increase in collateral lumenal diameter 21 days post-FAL ( $p<0.0001$ ) (Figure 6.6B). Again, cross-sectional analysis was used to confirm whole-mount diameter measurements and to determine wall area (Figure 6.6C). Following FAL, intramuscular anti-miR-199a treatment produced increased collateral artery lumenal diameter (Figure 6.6D) and wall area (Figure 6.6E) compared to control-treated Balb/c mice, while there was no significant difference in the diameter to wall area ratio (Figure 6.6F). The enhancement in lumenal diameter and wall area in response to miR-199a inhibition was evident along the collateral at both the muscular (non-reversed) and saphenous (reversed) regions (Supplemental Figure 6.2). 
A.
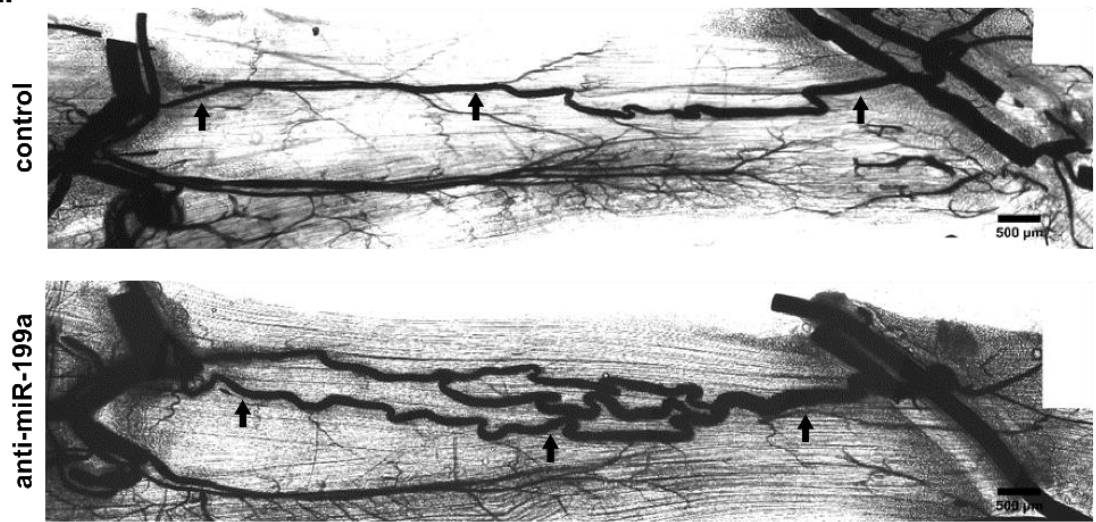

B.

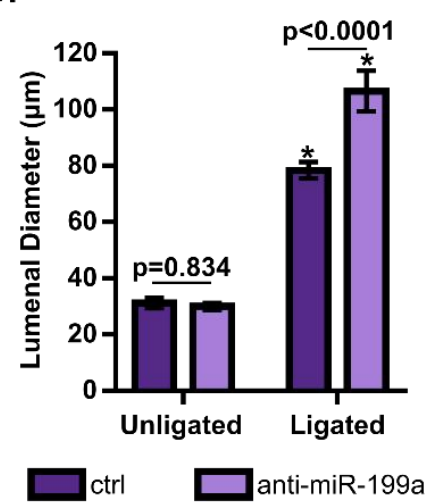

C.

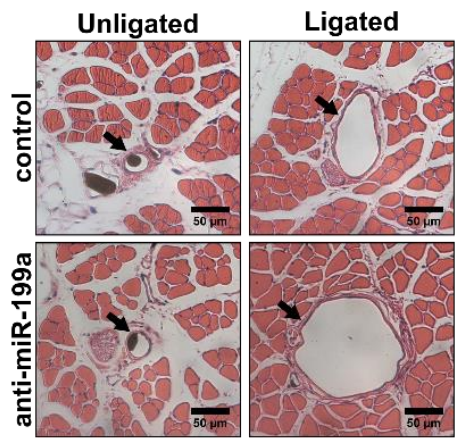

D.

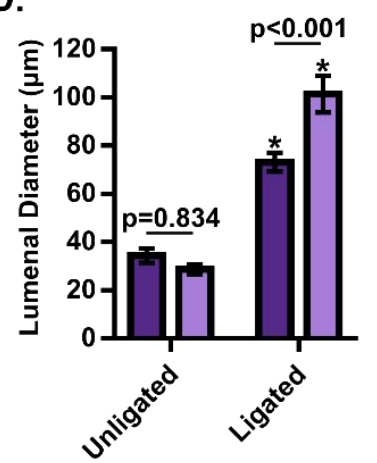

E.

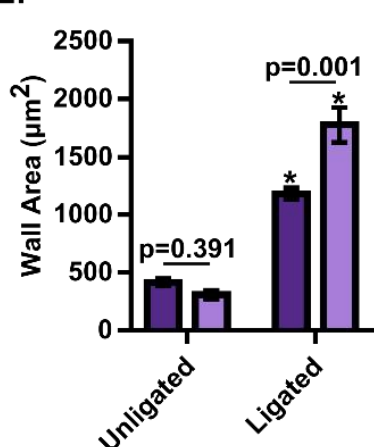

F.

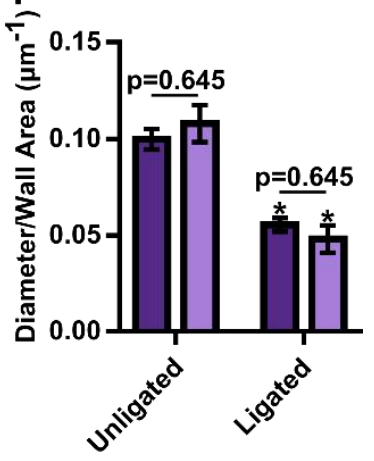

Figure 6.6. Inhibition of miR-199a amplifies arteriogenesis in Balb/c mice following FAL.

A) Representative whole mount vascular cast images of gracilis collateral arteries 21 days post-FAL in nontargeting control (top) or anti-miR-199a (bottom) treated Balb/c mice. B) Bar graph of mean lumenal diameter along collateral artery length for ligated and unligated limbs of anti-miR-199a and control treated mice $(n=6)$. ${ }^{*} p<0.001$ vs. unligated, two-way ANOVA followed by Holm-Sidak test for multiple comparisons. C) Representative H\&E stained cross-sections of collateral arteries from ligated and unligated limbs. D-F) Bar graphs of lumenal diameter, wall area, and diameter per wall area from H\&E stained cross-sections $(n=6) .{ }^{*} p<0.001$ vs. unligated, two-way ANOVA followed by Holm-Sidak test for multiple comparisons. Arrows indicate the primary collateral artery in $(A)$ and $(C)$. Data are mean $\pm S E M$.

\subsubsection{Pericollateral macrophage recruitment is modulated by miR-199a.}

We next sought to determine whether modulating miR-199a expression altered macrophage recruitment, a necessary component of collateral artery growth ${ }^{52,56,58,60,62,246,68}$, in-vivo. We determined that miR-199a overexpression caused to a $36 \%$ decrease in pericollateral Mac3 ${ }^{+}$ macrophages 7 days post-FAL in Balb/c mice, whereas miR-199a inhibition caused a 43\% increase in pericollateral macrophage recruitment (Figure 6.7). This trend was also observed when the muscular (non-reversed) and saphenous (reversed) collateral artery regions were assessed individually (Supplemental Figure 6.3). 
A.
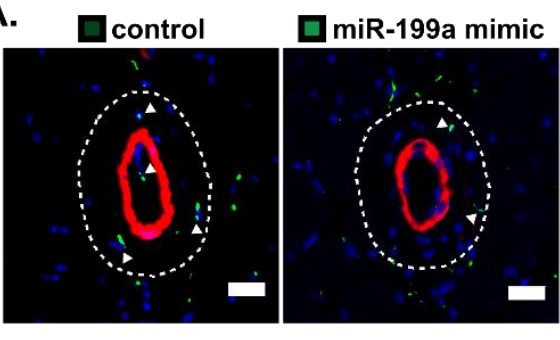

C.

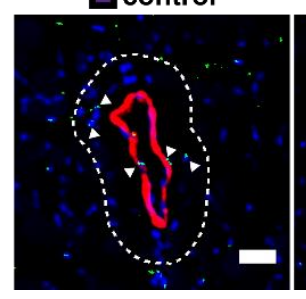

口miR-199a mimic

B.

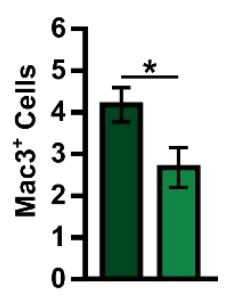

D.
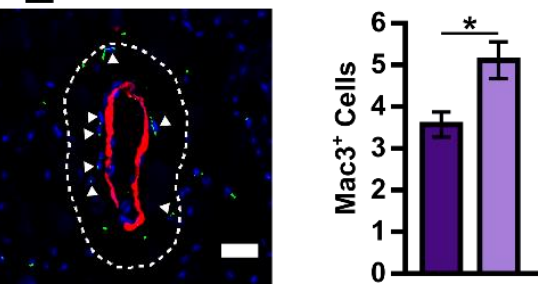

Figure 6.7. Pericollateral macrophage recruitment is modulated by miR-199a.

A) Representative cross-sections of gracilis collateral artery regions immunolabeled for macrophage marker, Mac3 (green), smooth muscle alpha actin (SMaA, red), and nuclei (DRAQ5, blue) in Balb/c mice treated with miR-199a mimic or non-targeting control mimic 7 days post-FAL. Dotted line indicates the pericollateral region $\left(25 \mu \mathrm{m}\right.$ from vessel wall) used for quantification. Arrowheads indicate Mac $3^{+}$cells

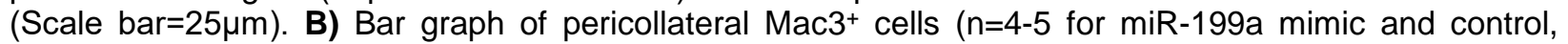
respectively). ${ }^{*} \mathrm{p}<0.05$, Student's t-test. C) Immunolabeled gracilis collateral artery regions, as in (A), in Balb/c mice treated with anti-miR-199a or non-targeting control oligonucleotide 7 days post-FAL. D) Bar graph of pericollateral Mac3+ cells $(n=3) .{ }^{*} p<0.05$, Student's t-test. Data are mean \pm SEM.

\subsubsection{MicroRNA-199a inhibition improves gastrocnemius muscle composition in} FAL-operated Balb/c mice.

Finally, we examined the effect of altered miR-199a expression on the composition of ischemic muscle tissue downstream of the femoral artery occlusion. Gastrocnemius muscle tissue was categorized as viable (mature and regenerating fibers) or non-viable (necrotic and fibro-adipose tissue) by histological analysis 21 days after FAL (Figure 6.8A-B). Though muscle composition was not significantly altered in miR-199a mimic treated mice (Supplemental Figure 6.4), inhibition of miR-199a resulted in a greater than 34\% reduction in the percentage of non-viable tissue in the gastrocnemius muscle (Figure 6.8C). 
A.
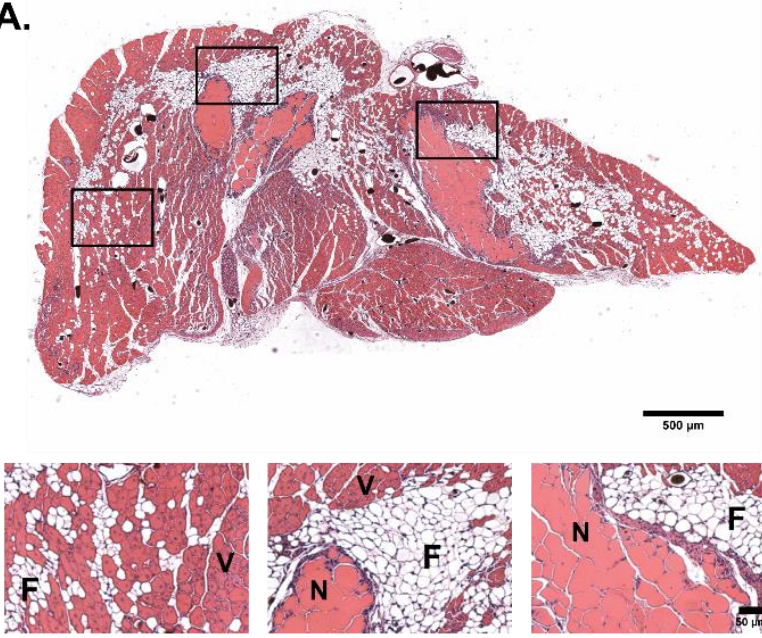
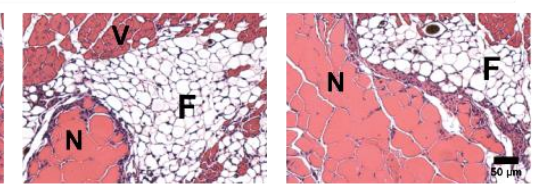

B.

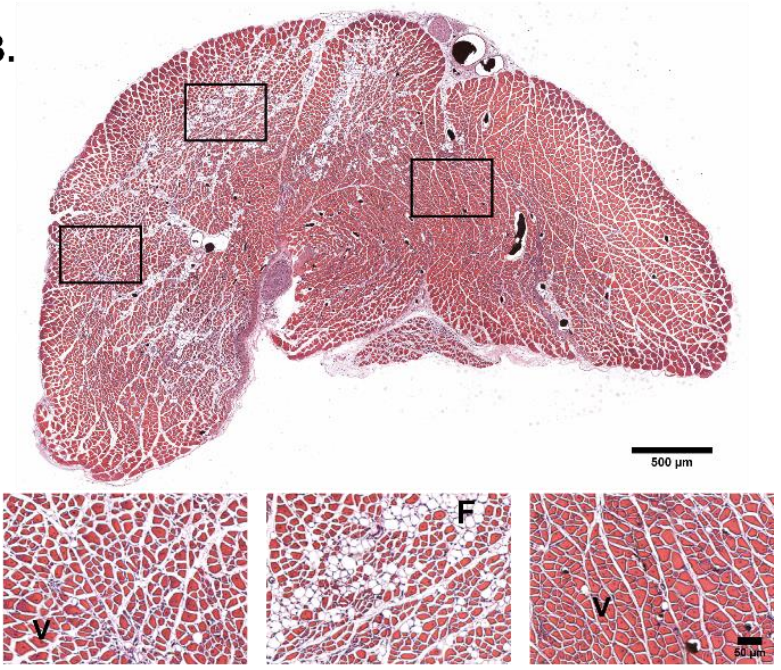

C.

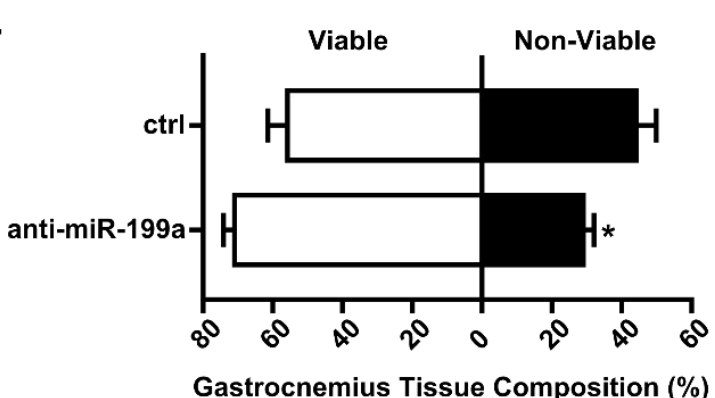

Figure 6.8. MicroRNA-199a inhibition improves gastrocnemius muscle composition in FALoperated Balb/c mice.

A-B) Representative images of H\&E staining of gastrocnemius muscle for ligated limb of Balb/c mice treated with non-targeting control (ctrl) (A) or anti-miR-199a (B) locked nucleic acid oligonucleotide immediately after FAL (Scale bar $=500 \mu \mathrm{m}$, inset scale bar $=50 \mu \mathrm{m})$. $\mathrm{V}=$ viable muscle, $\mathrm{N}=$ necrotic tissue, and $\mathrm{F}=$ fibro-adipose tissue. C) Bar graph of the percentage of gastrocnemius muscle that is viable (white) or non-viable (black) at day 21 post-FAL in each group $(n=5) .{ }^{*} p<0.05$ vs. control, Student's t-test. Data are mean \pm SEM.

\subsection{Discussion}

The primary goal of this study was to determine whether mechanosensitive miRNAs regulate endogenous collateral artery growth and perfusion recovery following femoral arterial occlusion. First, we identified candidate regulators of arteriogenesis by comparing differential miRNA expression in ECs exposed to shear stress waveforms corresponding to moderate and amplified arteriogenesis responses in-vivo. Among these candidate miRNAs, miR-199a was significantly downregulated by the amplified arteriogenesis (reversed flow) waveform and shown to regulate 
pro-arteriogenic gene (CD44, IKKß, and CCND1) expression and monocyte adhesion to shear stress-exposed endothelium. In-vivo, overexpression of miR-199a limited foot perfusion, arteriogenesis, and monocyte recruitment in the mouse FAL model. In contrast, inhibition of miR199a elicited complete foot perfusion recovery, substantially enhanced collateral arteriogenesis via increased pericollateral macrophage recruitment, and considerably improved gastrocnemius muscle tissue composition. We have therefore identified miR-199a as a novel mechanosensitive miRNA that regulates perfusion recovery and arteriogenesis. MicroRNA-199a may represent a new target for the therapeutic stimulation of arteriogenesis and the treatment of peripheral arterial disease.

\subsubsection{Identification of microRNA-199a as a novel mechanosensitive regulator of pro-arteriogenic gene expression}

Our strategy of interrogating differential mechano-signaling in ECs exposed to a biomimetic "amplified arteriogenesis" waveform represents a unique approach for identifying miRNA regulators of vascular growth and perfusion recovery in response to arterial occlusion(s). This strategy was enabled by our group's previous development of a transillumination laser speckle flowmetry method customized for measuring blood velocities in the collateral arteries of the mouse gracilis adductor muscle ${ }^{176}$. Using this method, we were able to determine segment-tosegment hemodynamic changes induced by $\mathrm{FAL}^{176}$, correlate these to variations in arteriogenesis $^{237}$, and directly apply these flow waveforms to ECs in-vitro. The utility of this approach is supported by our present results, as well as by our approach's identification of miR100 as another candidate miRNA (Figure 6.1C). Inhibition of miR-100 has been shown previously to enhance perfusion recovery following $F A L^{161}$. When considered in light of our findings, we postulate that the enhancement of shear stress-mediated collateral arteriogenesis may be a probable component of perfusion recovery with anti-miR-100 treatment. 
We also identified miR-34a and miR-146a as potential mechanosensitive regulators of arteriogenesis that could be targeted for therapeutic arteriogenesis (Figure 6.1). However, based on their known downstream targets and functions, we postulate that these miRNAs could be subject to the so-called "Janus phenomenon", wherein pro-arteriogenic therapies also tend to promote atherosclerosis ${ }^{262}$. To this end, miR-146a $\mathrm{a}^{-/}$Ldlr $^{-/-}$mice on a high-cholesterol diet display an increased number of atherosclerotic plaques $^{270}$. Moreover, while miR-34a is significantly down-regulated in the peripheral blood of a subpopulation of PAD patients ${ }^{140}$, it has been found to be significantly up-regulated in human atherosclerotic plaques ${ }^{271}$.

To date, miR-199a has been mostly studied for its roles in cardiac hypertrophy ${ }^{272,273}$, smooth muscle proliferation 274 , angiogenesis ${ }^{275-280}$, and as a tumor suppressor in a variety of cancers $^{269,276,278,281-285}$. However, miR-199a also regulates several genes important for arteriogenesis via direct seed sequence binding to target mRNAs. To this end, it has been shown that miR-199a negatively regulates NFKB, which is necessary for arteriogenesis ${ }^{256}$, by directly targeting IKK ${ }^{269}$. Another verified miR-199a target known to be necessary for arteriogenesis is CD44266,267. CD44 is a glycoprotein involved in leukocyte adhesion, cell growth, survival, and differentiation by regulating the activity of several pro-arteriogenic growth factors, including FGF and HGF ${ }^{286}$. Additionally, miR-199a indirectly moderates cyclin D1 (CCDN1) expression ${ }^{268}$, a key regulator of cell cycle and proliferation ${ }^{287}$. Here, we found that miR-199a alters both IKK $\beta$ and CD44 expression in ECs exposed to our biomimetic shear stress waveforms, suggesting potential pathways by which altered miR-199a is able to regulate arteriogenesis. Indeed, in-vitro, CD44 was significantly altered between reversed and non-reversed conditions in control-treated ECs, suggesting possible endogenous regulation by miR-199a. Furthermore, through both loss- and gain-of-function studies, we found miR-199a to regulate EC proliferation and monocyte adhesion, both key aspects of arteriogenesis. Altogether, this made miR-199a a compelling candidate miRNA to study in the context of arteriogenesis in-vivo. 
Our experimental approaches also allowed us to examine whether miR-199a regulated functions depend on hemodynamic context. In-vitro, anti-miR-199a interventions primarily affected ECs exposed to the non-reversed flow waveform, while miR-199a mimic interventions primarily affected ECs exposed to the reversed flow waveform. This response pattern appears to be dependent on the relative miR-199a expression in ECs rather than the hemodynamic context, per se (Supplemental Figure 6.5). Moreover, modulation of miR-199a expression invivo led to similar relative changes in collateral growth responses and macrophage recruitment in both muscular (non-reversed) and saphenous (reversed) collateral regions (Supplemental Figure 6.1 - Supplemental Figure 6.3).

\subsubsection{Previous studies implicating a role of miRNA-regulated arteriogenesis}

Despite a number of previous reports indicating miRNA regulation of perfusion recovery in hindlimb ischemia models ${ }^{143,150,151,154,155,159,161,162,164}$, only a few of these microRNAs (e.g. miR155, miR-17 92a, miR-487b) have been suggested to have a role in arteriogenesis per se. Inhibition of miR-155 attenuates blood flow recovery and leukocyte recruitment, despite promoting angiogenesis in the ischemic tissue of FAL-treated mice, thereby indirectly implying a role for miR-155 in arteriogenesis. However, collateral diameters were not explicitly reported ${ }^{162}$. In another study, endothelial-specific deletion of the miR-17 92 cluster increased foot perfusion 14 days following FAL as well as arteriole density in FAL-operated limbs. However, arteriole diameters were not significantly greater in these mice, indicating increased perfusion was due to an increase in arteriole density, rather than endogenous collateral artery growth ${ }^{151}$. Finally, miR329, miR-487b, miR-494, and miR-495 inhibition all led to a $25-40 \%$ increase in blood flow recovery following FAL, though only miR-487b inhibition increased vessel diameters from PBStreated controls ${ }^{155}$. While this study further corroborates microRNA-mediated regulation of arteriogenesis, it is difficult to directly compare our results with this study due to differences in 
oligonucleotide dosing, the extent of ischemia in FAL models, and methods for assessing $\operatorname{arteriogenesis}^{155}$.

\subsubsection{MicroRNA-199a as a regulator of arteriogenesis leading to enhanced perfusion recovery}

In testing our hypothesis that miR-199a negatively regulates perfusion recovery and arteriogenesis in-vivo, we chose to perform our FAL model in Balb/c mice to enable for the necessary dynamic range for both loss and gain-of function studies, as these mice exhibit a more blunted arteriogenic response compared to C57BL/6 ${ }^{165,168}$. Following FAL, control treated mice exhibited incomplete perfusion recovery as expected, though they did recover $80-90 \%$ perfusion in ligated limbs compared to the $\sim 50-60 \%$ perfusion recovery seen in previous studies ${ }^{165}$. This greater than expected recovery was likely due to the more mild nature of our ligation scheme; nonetheless, we were still able to observe significant changes in foot perfusion with modulation of miR-199a expression, similar to those seen by previous miRNA studies ${ }^{161,164,288}$.

Corresponding to the observed changes in foot perfusion, we found that miR-199a negatively regulates arteriogenesis. Our results indicating miR-199a modulates monocyte/macrophage recruitment are consistent with the known necessary role for leukocyte recruitment in arteriogenesis ${ }^{56,60,62,289}$ and suggest that this is the mechanism through which miR199a regulates arteriogenesis following FAL. Critical in determining the reperfusion response, alterations to endogenous collateral growth can lead to substantial changes in collateral conductance. To this end, miR-199a overexpression attenuated collateral artery growth 25\% ( 2.4-fold decrease in conductance) and miR-199a inhibition enhanced arteriogenesis by 36\% (>3.4-fold conductance increase). Of particular importance for potential clinical translation, collateral arteries in anti-miR-199a treated mice were morphologically normal as there was no difference in the lumenal diameter to wall area ratio when miR-199a expression was altered. 
In addition to arteriogenesis, perfusion recovery following FAL can also be dependent upon angiogenesis ${ }^{29}$ and/or tissue clearance and skeletal muscle regeneration ${ }^{30}$. Here, we did not observe any significant differences in gastrocnemius capillary density in anti-miR-199a treated mice at day 21 post-FAL (Supplemental Figure 6.6). However, we did find that a single bolus of anti-miR-199a oligonucleotide was sufficient to improve gastrocnemius muscle tissue composition in FAL-operated mice (Figure 6.8), though there was no significant change in muscle composition with miR-199a overexpression (Supplemental Figure 6.4). While it is probable that this improvement in muscle composition is a consequence of enhanced downstream tissue perfusion due to amplified arteriogenesis with miR-199a inhibition, miR-199a may also play a direct role in tissue clearance and regeneration. Indeed, miR-199a has been implicated in tissue fibrosis $^{290}$, myoblast proliferation and differentiation ${ }^{268}$, and adipocyte differentiation ${ }^{291}$, all of which are consistent with the hypothesis that miR-199a can directly regulate skeletal muscle proliferation and/or differentiation in a hypoxic environment.

\subsubsection{Clinical perspective}

Based on both our results and previous studies, we postulate that miRNA-199a inhibition represents a potentially promising treatment for arterial occlusive diseases. MicroRNA-199a is up-regulated in patients with coronary artery disease (CAD) versus matched controls ${ }^{292}$. Additionally, the expression of CD44, which is directly regulated by miR-199a, correlates to the degree of vascular remodeling in CAD patients. Specifically, low levels of CD44 expression in peripheral blood monocytes correlate to poor collateralization ${ }^{266}$. Furthermore, our results indicate that the inhibition of miR-199a improves limb perfusion by enhancing arteriogenesis and reverses the fibro-adipose composition of ischemic muscle, which is often found in PAD patients ${ }^{293-296}$.

\subsection{Acknowledgements}

The authors would like to thank the University of Virginia Research Histology Core (under the direction of Sheri VanHoose) for histological tissue processing and the DNA Sciences Core 
(under the direction of Yongde Bao), which is supported by the University Of Virginia School Of Medicine, for running TaqMan miRNA qRT-PCR plates.

\subsection{Sources of Funding}

Supported by NIH R03 EB017927 and R01 EB020147. JLH was also supported by National Science Foundation Graduate Research Fellowship Program Grant No. NSF DGE-1315231. 


\subsection{Supplemental Figures}

A.

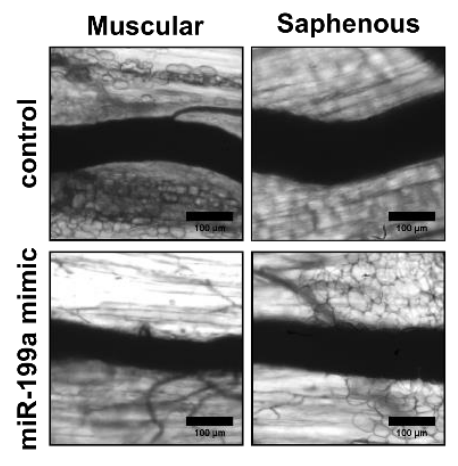

C.

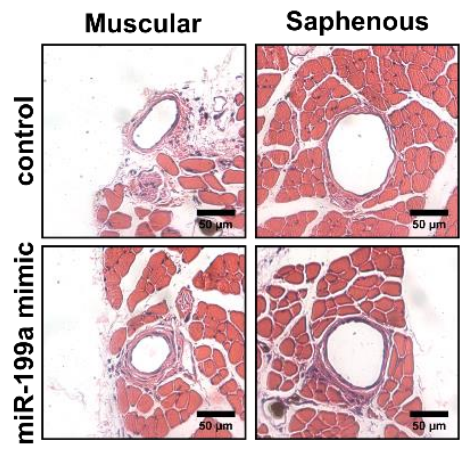

B.

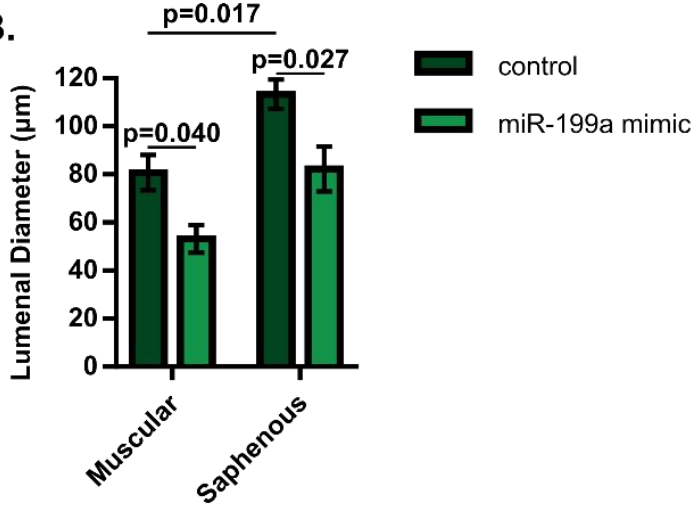

D.

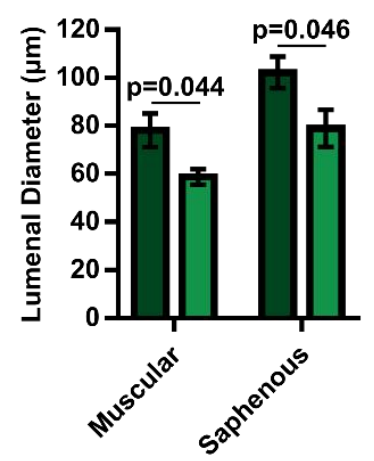

E.

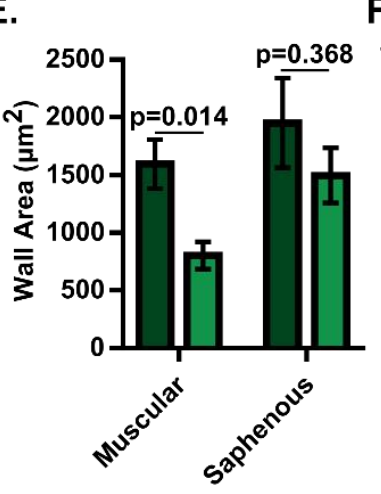

$\mathbf{F}$.

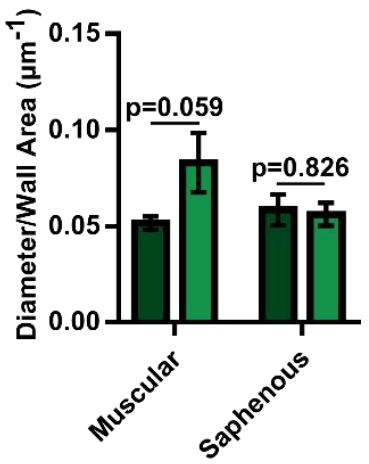

Supplemental Figure 6.1. Regional analysis of Balb/c mice treated with miR-199a mimic after FAL. A) Representative whole mount vascular cast images of collateral artery regions in non-targeting control or miR-199a mimic treated Balb/c mice 21 days post-FAL (Scale bar $=100 \mu \mathrm{m}$ ). B) Bar graph of lumenal diameter for each group. ${ }^{*} \mathrm{p}<0.001$ vs. unligated, two-way ANOVA followed by Holm-Sidak test for multiple comparisons. C) Representative H\&E stained cross-sections of collateral arteries regions ( $n=5-6$ for mimic and control groups respectively). D-F) Bar graphs of lumenal diameter, wall area, and diameter per wall area ratio from H\&E stained cross-sections ( $n=5-6$ for mimic and control groups respectively); Student's ttest. Data are mean \pm SEM. 
A.

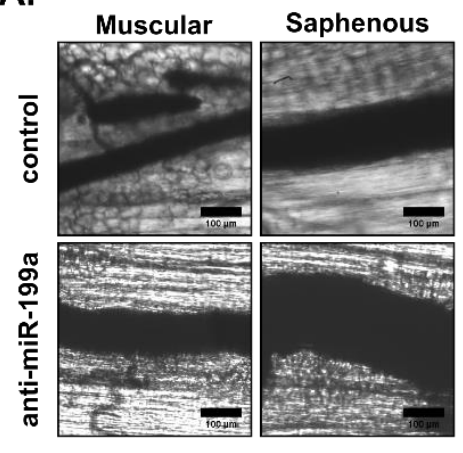

C.

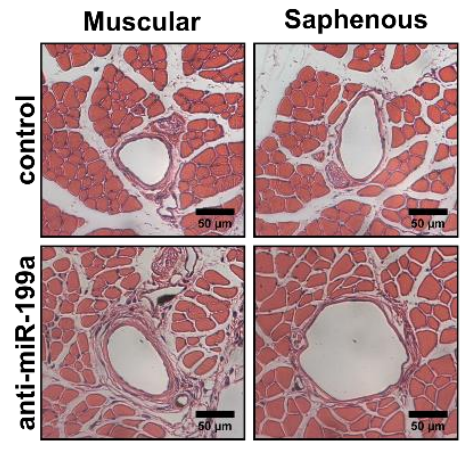

B.

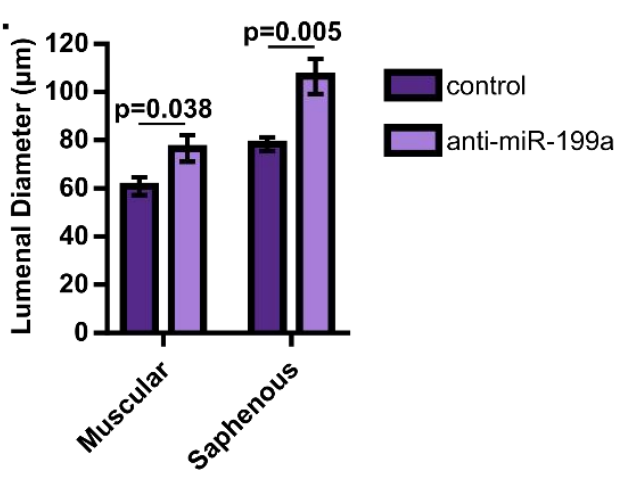

D.

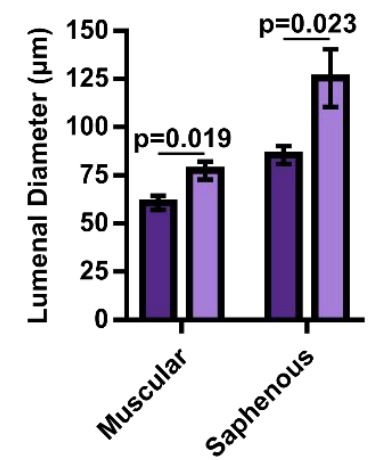

E.

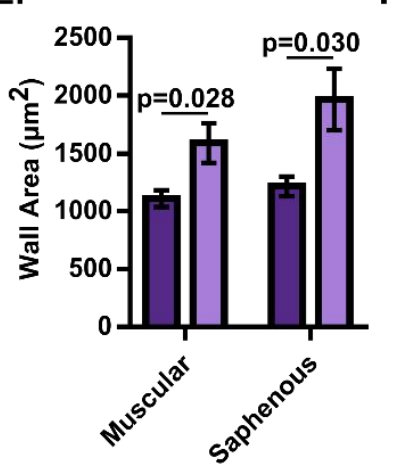

F.

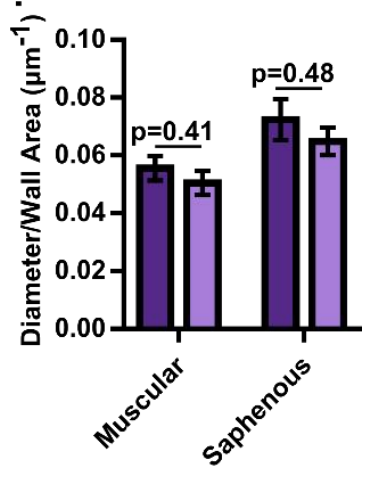

Supplemental Figure 6.2. Regional analysis of Balb/c mice treated with anti-miR-199a after FAL.

A) Representative whole mount vascular cast images of collateral artery regions in non-targeting control or anti-miR-199a treated Balb/c mice 21 days post-FAL (Scale bar=100 $\mu \mathrm{m}$ ). B) Bar graph of lumenal diameter for each group $(n=6)$. ${ }^{*} p<0.001$ vs. unligated, two-way ANOVA followed by Holm-Sidak test for multiple comparisons. C) Representative H\&E stained cross-sections of collateral arteries regions $(n=6)$. D-F) Bar graphs of lumenal diameter, wall area, and diameter per wall area ratio from H\&E stained crosssections $(n=6)$; Student's t-test. Data are mean \pm SEM. 
A.
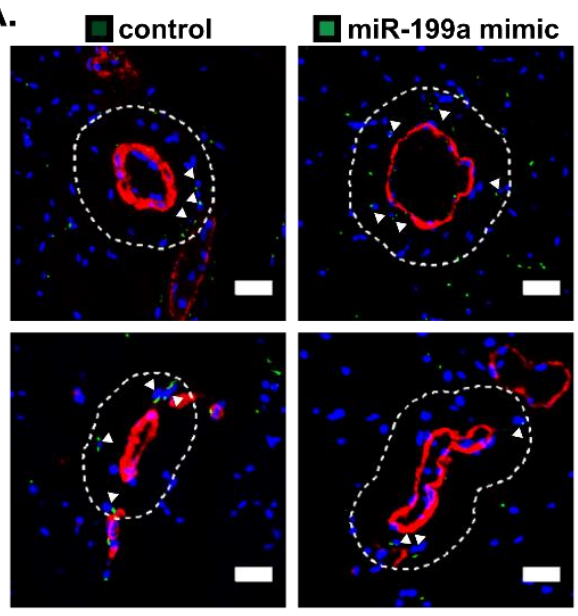

C.
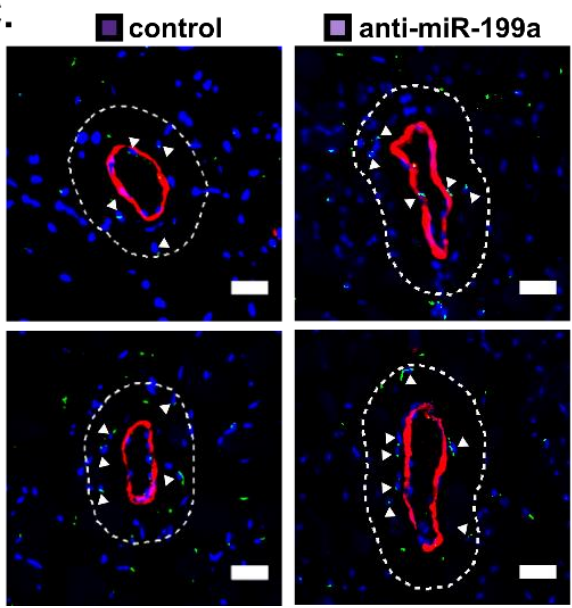

B.
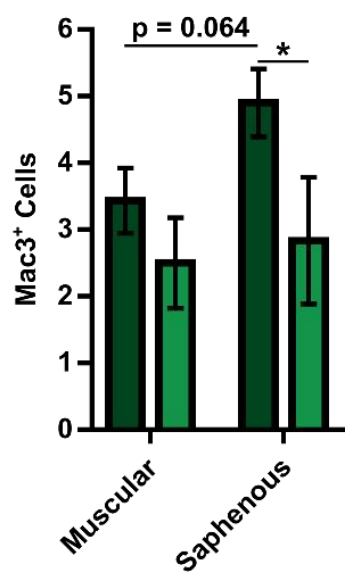

D.

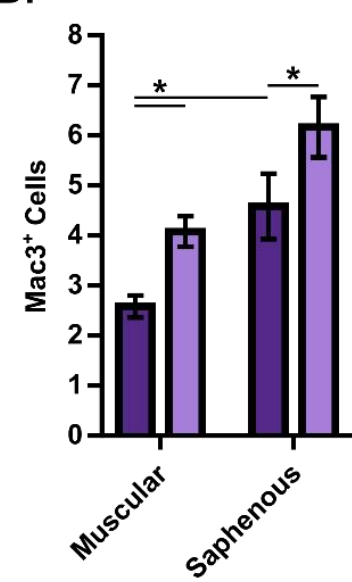

Supplemental Figure 6.3. Regional pericollateral macrophage recruitment is modulated by miR199a.

A) Representative cross-sections of gracilis collateral artery regions 7 days post-FAL immunolabeled for macrophage marker, Mac3 (green), smooth muscle alpha actin (SMaA, red), and nuclei (DRAQ5, blue) in Balb/c mice treated with miR-199a mimic or non-targeting control mimic. Dotted line indicates the pericollateral region $(25 \mu \mathrm{m}$ from vessel wall) used for quantification. Arrowheads indicate Mac3+ cells (Scale bar $=25 \mu \mathrm{m})$. B) Bar graph of pericollateral Mac3+ cells $(n=4-5$ for miR-199a mimic and control, respectively). ${ }^{*} p<0.05$, two-way ANOVA followed by a Holm-Sidak test for multiple comparisons. C) Immunolabeled gracilis collateral artery regions, as in (A), in Balb/c mice treated with anti-miR-199a or nontargeting control oligonucleotide. D) Bar graph of pericollateral Mac3 ${ }^{+}$cells $(n=3)$. ${ }^{*} p<0.05$, two-way ANOVA followed by a Holm-Sidak test for multiple comparisons. Data are mean \pm SEM. 
A.

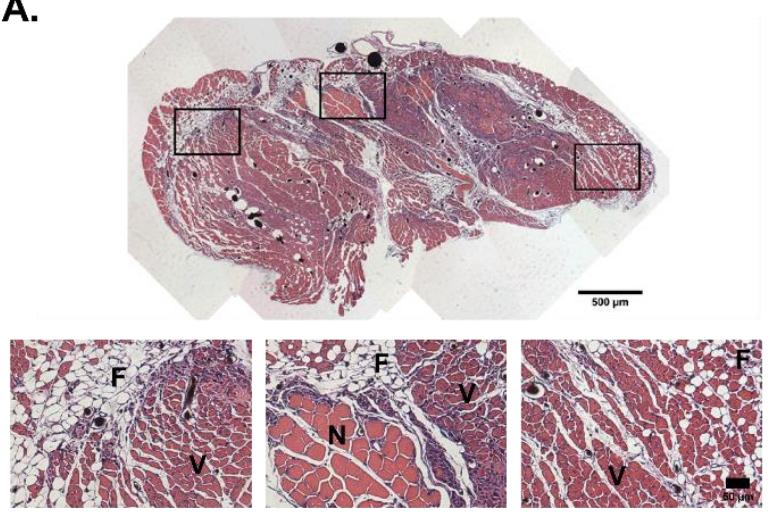

B.

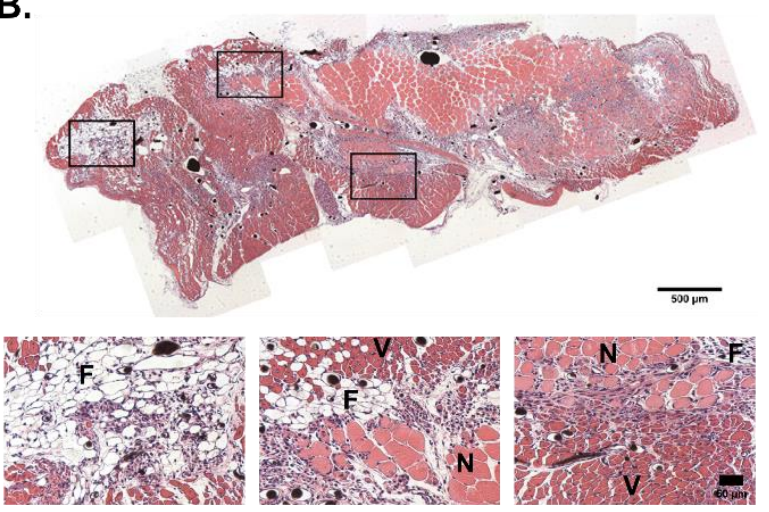

C.

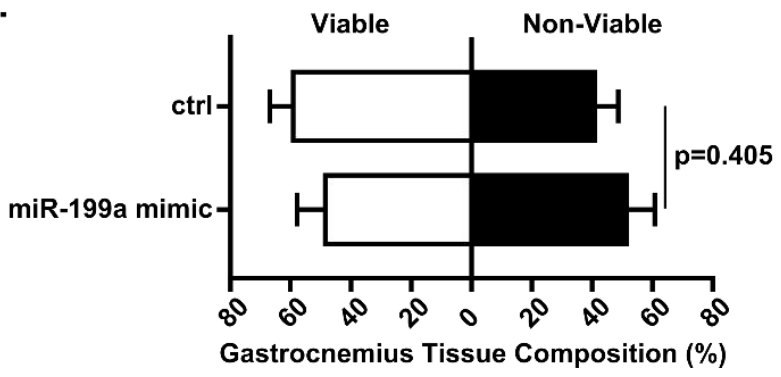

Supplemental Figure 6.4. Gastrocnemius muscle composition is not further impaired with miR-199a overexpression following FAL

A-B) Representative images of $\mathrm{H} \& \mathrm{E}$ staining of gastrocnemius muscle for ligated limb of Balb/c mice treated with non-targeting control (ctrl) (A) or miR-199a mimic (B) (Scale bar $=500 \mu m$, inset scale bar=50 $\mu \mathrm{m})$. $V=$ viable muscle, $\mathrm{N}=$ necrotic tissue, and $\mathrm{F}=$ fibro-adipose tissue. C) Bar graph of the percentage of gastrocnemius muscle that is viable (white) or non-viable (black) at day 21 post-FAL in each group ( $n=6-5$ for ctrl and miR-199a mimic, respectively). Student's t-test. Data are mean \pm SEM. 
A.

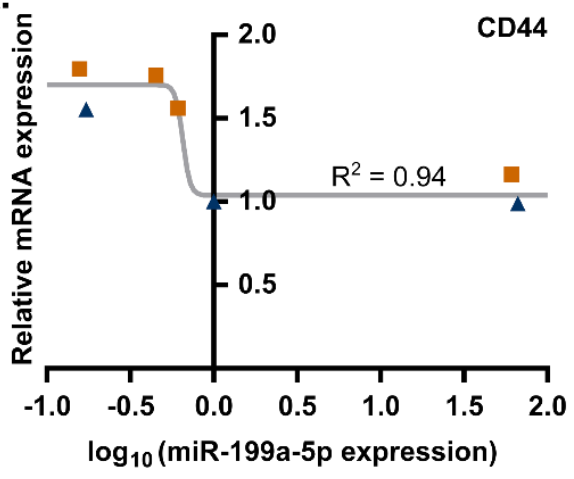

C.

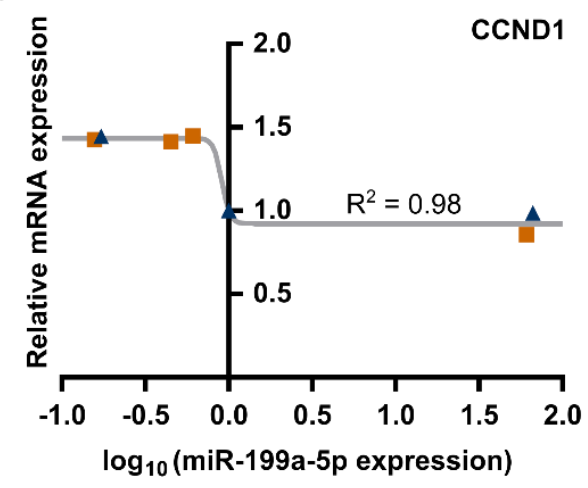

B.

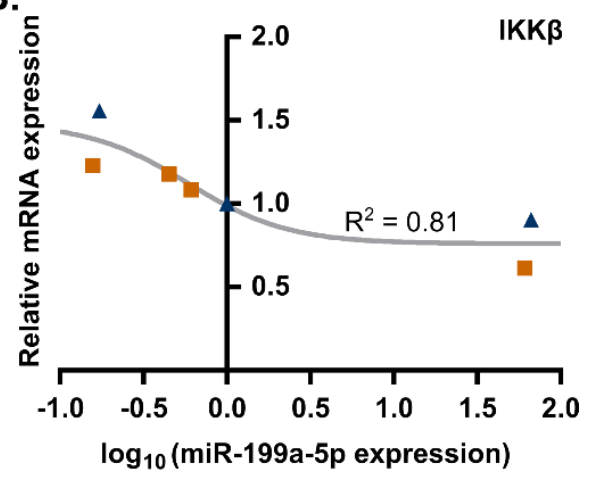

D.

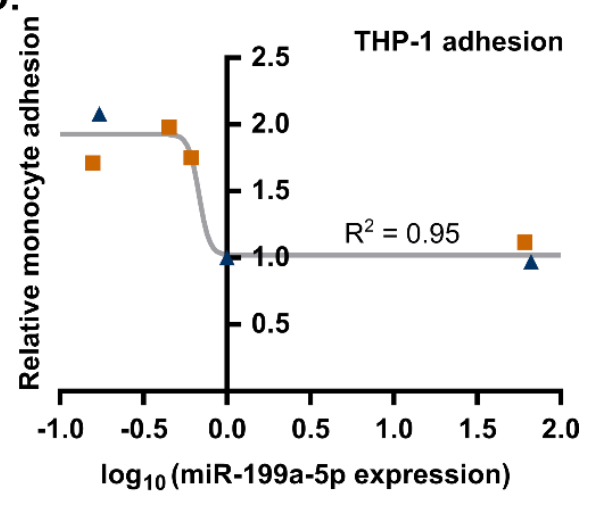

$\Delta$ Non-reversed $\quad$ Reversed

Supplemental Figure 6.5. Sigmoidal response of CD44, IKK $\beta$, CCND1 mRNA expression and THP-1 monocyte adhesion to alterations in miR-199a expression in endothelial cells exposed to biomimetic shear stress waveforms in-vitro.

A-D) Scatter plots of mean relative CD44, IKK 3 , CCND1 mRNA expression and THP-1 monocyte adhesion plotted against $\log _{10}$ miR-199a expression. Data points are from both miR-199a overexpression and inhibition studies in HUVECs subjected to non-reversed (blue) or reversed (orange) flow waveforms. Data was fitted to a logistic function (gray line) of the general form: $y(x)=\frac{a}{b+c e^{-d(f-x)}}+t$, where $\mathrm{x}$ is the $\log _{10}$ (miR-199a expression). 

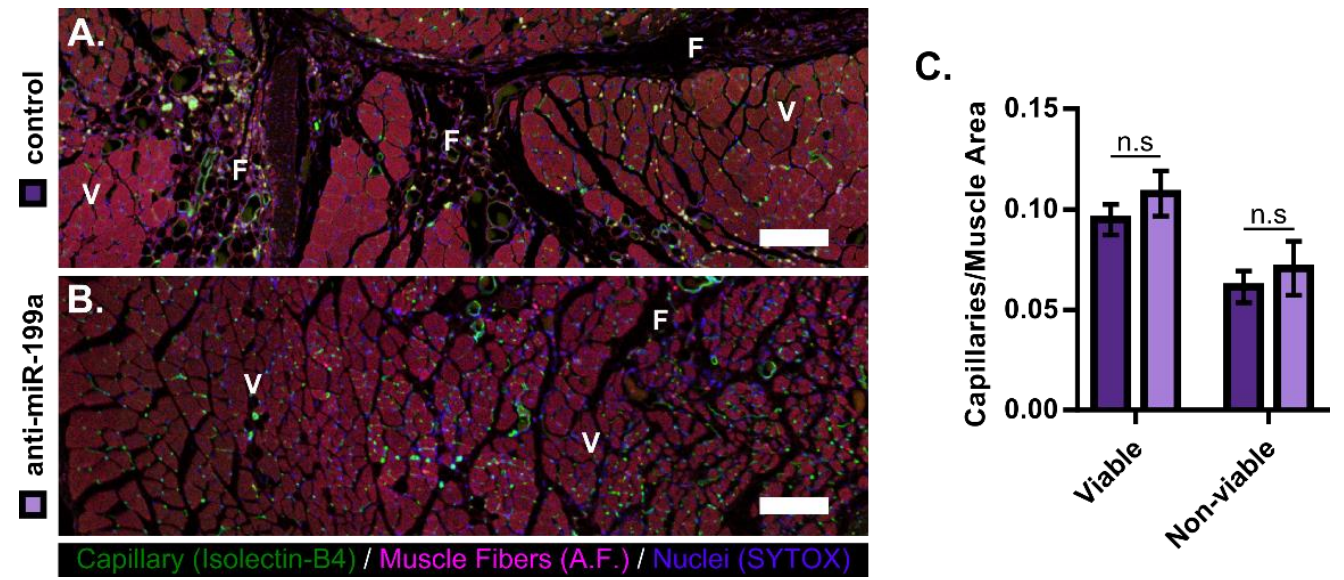

Supplemental Figure 6.6. MicroRNA-199a inhibition does not affect capillary density in the gastrocnemius muscle of FAL-operated Balb/c mice.

A-B) Representative photomerged images of gastrocnemius muscle for ligated limb of Balb/c mice treated with non-targeting control (ctrl) (A) or anti-miR-199a (B) locked nucleic acid oligonucleotides immediately after FAL (Scale bar=500 $\mu \mathrm{m}$ ). Tissues are immunolabeled for capillaries (green, isolectin-B4), muscle fibers (red, autofluorescence [A.F]), and nuclei (blue, SYTOX). C) Capillary density was determined for both groups in viable tissue (mature and regenerating muscle, $\mathrm{V}$ ) and non-viable tissue (necrotic and fibroadipose [F]) ( $n=4-5$, for ctrl and anti-miR-199a, respectively). n.s = not significant, Student's t-test. Data are mean \pm SEM. 
6.10 Supplemental Tables

Supplemental Table 6.1. Differential microRNA gene expression in ECs exposed to arteriogenesisbiomimetic shear stress waveforms.

\begin{tabular}{|c|c|c|c|c|c|}
\hline Symbol & Name & "NvC FC & RvC FC & "RvN FC & $\log _{10}$ (FDR) \\
\hline MIR146A & microRNA 146a & 0.99 & 0.52 & 0.52 & 2.00 \\
\hline MIR155 & microRNA 155 & 1.23 & 0.77 & 0.63 & 0.76 \\
\hline MIR224 & microRNA 224 & 1.07 & 0.73 & 0.68 & 0.87 \\
\hline MIR29A & microRNA 29a & 1.13 & 0.78 & 0.69 & 0.37 \\
\hline MIR34A & microRNA 34a & 0.99 & 0.72 & 0.73 & 0.00 \\
\hline MIR21 & microRNA 21 & 1.05 & 0.77 & 0.73 & 0.59 \\
\hline MIR199A2 & microRNA 199a-2 & 0.95 & 0.72 & 0.76 & 1.89 \\
\hline MIR32 & microRNA 32 & 1.00 & 0.76 & 0.76 & 0.64 \\
\hline MIR425 & microRNA 425 & 1.28 & 0.97 & 0.76 & 0.03 \\
\hline MIR100 & microRNA 100 & 0.91 & 0.69 & 0.76 & 1.49 \\
\hline MIR223 & microRNA 223 & 1.27 & 0.97 & 0.77 & 0.07 \\
\hline MIR216A & microRNA 216a & 0.93 & 0.74 & 0.79 & 1.26 \\
\hline MIR199A1 & microRNA 199a-1 & 1.12 & 0.89 & 0.79 & 0.45 \\
\hline MIR24-1 & microRNA 24-1 & 1.14 & 0.90 & 0.80 & 0.19 \\
\hline MIR93 & microRNA 93 & 1.05 & 0.84 & 0.80 & 1.19 \\
\hline MIR17HG & miR-17-92 cluster host gene & 1.01 & 0.81 & 0.80 & 0.97 \\
\hline MIR29B2 & microRNA 29b-2 & 1.07 & 0.86 & 0.81 & 0.35 \\
\hline MIR181B1 & microRNA 181b-1 & 0.99 & 0.81 & 0.82 & 0.83 \\
\hline MIR126 & microRNA 126 & 0.85 & 0.70 & 0.82 & 0.87 \\
\hline MIR495 & microRNA 495 & 1.16 & 0.96 & 0.83 & 0.07 \\
\hline MIR23A & microRNA 23a & 1.02 & 0.84 & 0.83 & 0.48 \\
\hline MIR600HG & MIR600 host gene & 1.14 & 0.96 & 0.85 & 0.07 \\
\hline MIRLET7C & microRNA let-7c & 0.96 & 0.82 & 0.85 & 0.51 \\
\hline MIR504 & microRNA 504 & 1.20 & 1.03 & 0.86 & 0.04 \\
\hline MIR181B2 & microRNA 181b-2 & 0.96 & 0.84 & 0.87 & 0.57 \\
\hline MIR129-2 & microRNA 129-2 & 1.11 & 0.97 & 0.87 & 0.04 \\
\hline MIR195 & microRNA 195 & 1.06 & 0.93 & 0.88 & 0.22 \\
\hline MIR92A2 & microRNA 92a-2 & 1.12 & 0.99 & 0.88 & 0.03 \\
\hline MIR137 & microRNA 137 & 1.18 & 1.04 & 0.88 & 0.07 \\
\hline MIR143 & microRNA 143 & 1.05 & 0.92 & 0.88 & 0.35 \\
\hline MIR210 & microRNA 210 & 1.07 & 0.95 & 0.89 & 0.18 \\
\hline MIR301A & microRNA 301a & 1.09 & 0.96 & 0.89 & 0.06 \\
\hline MIR30C1 & microRNA 30c-1 & 1.00 & 0.89 & 0.90 & 0.32 \\
\hline MIR217 & microRNA 217 & 0.98 & 0.88 & 0.90 & 0.10 \\
\hline MIR302B & microRNA 302b & 1.11 & 1.01 & 0.91 & 0.01 \\
\hline MIR503 & microRNA 503 & 1.07 & 0.97 & 0.91 & 0.05 \\
\hline MIRLET7A3 & microRNA let-7a-3 & 1.02 & 0.93 & 0.91 & 0.10 \\
\hline MIR520C & microRNA 520c & 0.99 & 0.91 & 0.91 & 0.16 \\
\hline MIR29C & microRNA 29c & 1.20 & 1.10 & 0.92 & 0.13 \\
\hline MIR125B2 & microRNA 125b-2 & 1.08 & 0.99 & 0.92 & 0.01 \\
\hline MIR148B & microRNA 148b & 1.05 & 0.97 & 0.92 & 0.03 \\
\hline MIR181C & microRNA 181c & 1.10 & 1.01 & 0.92 & 0.01 \\
\hline MIR519A2 & microRNA 519a-2 & 1.00 & 0.92 & 0.92 & 0.08 \\
\hline MIR516B1 & microRNA 516b-1 & 1.06 & 0.98 & 0.92 & 0.02 \\
\hline MIRLET7A1 & microRNA let-7a-1 & 1.01 & 0.93 & 0.93 & 0.13 \\
\hline MIR1-2 & microRNA 1-2 & 1.06 & 0.98 & 0.93 & 0.02 \\
\hline MIR487A & microRNA 487a & 1.12 & 1.04 & 0.93 & 0.06 \\
\hline MIR127 & microRNA 127 & 0.96 & 0.90 & 0.93 & 0.54 \\
\hline MIR124-3 & microRNA 124-3 & 1.03 & 0.95 & 0.93 & 0.06 \\
\hline
\end{tabular}




\begin{tabular}{|c|c|c|c|c|c|}
\hline MIR30C2 & microRNA 30c-2 & 0.99 & 0.92 & 0.93 & 0.07 \\
\hline MIRLET7A2 & microRNA let-7a-2 & 1.03 & 0.96 & 0.93 & 0.04 \\
\hline MIR181A1 & microRNA 181a-1 & 0.99 & 0.92 & 0.93 & 0.23 \\
\hline MIR106B & microRNA 106b & 0.98 & 0.91 & 0.94 & 0.41 \\
\hline MIR135A1 & microRNA 135a-1 & 1.00 & 0.93 & 0.94 & 0.12 \\
\hline MIR184 & microRNA 184 & 0.99 & 0.94 & 0.94 & 0.17 \\
\hline MIR30E & microRNA 30e & 0.93 & 0.88 & 0.94 & 0.56 \\
\hline MIR423 & microRNA 423 & 1.04 & 0.98 & 0.94 & 0.02 \\
\hline MIR153-1 & microRNA 153-1 & 1.01 & 0.95 & 0.94 & 0.11 \\
\hline MIR222 & microRNA 222 & 1.03 & 0.98 & 0.95 & 0.02 \\
\hline MIR124-2 & microRNA 124-2 & 1.00 & 0.95 & 0.95 & 0.25 \\
\hline MIR24-2 & microRNA 24-2 & 0.93 & 0.88 & 0.95 & 0.29 \\
\hline MIR188 & microRNA 188 & 1.08 & 1.03 & 0.95 & 0.09 \\
\hline MIR26A1 & microRNA 26a-1 & 0.94 & 0.90 & 0.96 & 0.26 \\
\hline MIR98 & microRNA 98 & 1.05 & 1.00 & 0.96 & 0.00 \\
\hline MIR99B & microRNA 99b & 0.96 & 0.93 & 0.96 & 0.21 \\
\hline MIR519A2 & microRNA 519a-2 & 1.00 & 0.96 & 0.96 & 0.10 \\
\hline MIR153-2 & microRNA 153-2 & 1.01 & 0.98 & 0.97 & 0.05 \\
\hline MIR200B & microRNA 200b & 1.03 & 1.00 & 0.97 & 0.01 \\
\hline MIR141 & microRNA 141 & 1.01 & 0.98 & 0.97 & 0.02 \\
\hline MIRLET7B & microRNA let-7b & 1.00 & 0.97 & 0.97 & 0.06 \\
\hline MIR10A & microRNA 10a & 0.81 & 0.78 & 0.97 & 0.51 \\
\hline MIR183 & microRNA 183 & 0.98 & 0.95 & 0.97 & 0.11 \\
\hline MIR30B & microRNA 30b & 1.06 & 1.03 & 0.97 & 0.03 \\
\hline MIRLET7BHG & MIRLET7B host gene & 1.01 & 0.98 & 0.97 & 0.03 \\
\hline MIR138-1 & microRNA 138-1 & 1.05 & 1.02 & 0.97 & 0.02 \\
\hline MIR516B2 & microRNA 516b-2 & 0.97 & 0.94 & 0.97 & 0.07 \\
\hline MIR320C1 & microRNA 320c-1 & 1.11 & 1.08 & 0.97 & 0.25 \\
\hline MIR122 & microRNA 122 & 1.09 & 1.07 & 0.97 & 0.21 \\
\hline MIR191 & microRNA 191 & 1.01 & 0.99 & 0.97 & 0.02 \\
\hline MIR192 & microRNA 192 & 1.06 & 1.03 & 0.97 & 0.03 \\
\hline MIR185 & microRNA 185 & 0.98 & 0.96 & 0.98 & 0.09 \\
\hline MIR323A & microRNA 323a & 1.08 & 1.06 & 0.98 & 0.14 \\
\hline MIR133B & microRNA 133b & 1.04 & 1.02 & 0.98 & 0.03 \\
\hline MIR140 & microRNA 140 & 0.98 & 0.97 & 0.98 & 0.06 \\
\hline MIR410 & microRNA 410 & 0.96 & 0.95 & 0.99 & 0.09 \\
\hline MIR107 & microRNA 107 & 1.02 & 1.00 & 0.99 & 0.00 \\
\hline MIR31 & microRNA 31 & 1.03 & 1.02 & 0.99 & 0.01 \\
\hline MIR9-1 & microRNA 9-1 & 1.02 & 1.01 & 0.99 & 0.01 \\
\hline MIR105-1 & microRNA 105-1 & 1.24 & 1.24 & 0.99 & 0.17 \\
\hline MIR330 & microRNA 330 & 1.01 & 1.01 & 1.00 & 0.01 \\
\hline MIR214 & microRNA 214 & 0.93 & 0.93 & 1.00 & 0.24 \\
\hline MIR33A & microRNA 33a & 0.93 & 0.93 & 1.00 & 0.09 \\
\hline MIR7-3 & microRNA 7-3 & 0.97 & 0.97 & 1.00 & 0.05 \\
\hline MIR187 & microRNA 187 & 0.96 & 0.97 & 1.00 & 0.06 \\
\hline MIR10B & microRNA 10b & 1.01 & 1.01 & 1.00 & 0.01 \\
\hline MIR124-1 & microRNA 124-1 & 1.04 & 1.05 & 1.00 & 0.07 \\
\hline MIR494 & microRNA 494 & 1.00 & 1.00 & 1.00 & 0.00 \\
\hline MIR27A & microRNA 27a & 0.85 & 0.85 & 1.01 & 0.37 \\
\hline MIR16-2 & microRNA 16-2 & 0.95 & 0.96 & 1.01 & 0.08 \\
\hline MIR296 & microRNA 296 & 1.00 & 1.01 & 1.01 & 0.01 \\
\hline MIR339 & microRNA 339 & 1.03 & 1.04 & 1.01 & 0.06 \\
\hline MIR331 & microRNA 331 & 1.03 & 1.04 & 1.01 & 0.05 \\
\hline MIR205 & microRNA 205 & 0.99 & 1.00 & 1.01 & 0.00 \\
\hline
\end{tabular}




\begin{tabular}{|c|c|c|c|c|c|}
\hline MIR26A2 & microRNA 26a-2 & 1.04 & 1.06 & 1.01 & 0.06 \\
\hline MIR150 & microRNA 150 & 0.99 & 1.01 & 1.01 & 0.01 \\
\hline MIR200C & microRNA 200c & 0.99 & 1.01 & 1.01 & 0.01 \\
\hline MIR509-1 & microRNA 509-1 & 1.02 & 1.03 & 1.01 & 0.06 \\
\hline MIR26B & microRNA 26b & 0.94 & 0.96 & 1.01 & 0.13 \\
\hline MIR106A & microRNA 106a & 0.99 & 1.01 & 1.02 & 0.01 \\
\hline MIR31HG & MIR31 host gene & 0.99 & 1.01 & 1.02 & 0.01 \\
\hline MIR139 & microRNA 139 & 1.02 & 1.04 & 1.02 & 0.09 \\
\hline MIR129-1 & microRNA 129-1 & 0.97 & 0.99 & 1.02 & 0.01 \\
\hline MIR99A & microRNA 99a & 0.93 & 0.95 & 1.02 & 0.10 \\
\hline MIR197 & microRNA 197 & 0.93 & 0.95 & 1.02 & 0.09 \\
\hline MIR125B1 & microRNA 125b-1 & 0.97 & 0.99 & 1.02 & 0.01 \\
\hline MIR206 & microRNA 206 & 1.04 & 1.06 & 1.02 & 0.24 \\
\hline MIR101-2 & microRNA 101-2 & 1.03 & 1.05 & 1.02 & 0.15 \\
\hline MIR302A & microRNA 302a & 0.94 & 0.97 & 1.02 & 0.08 \\
\hline MIR128-2 & microRNA 128-2 & 0.97 & 0.99 & 1.03 & 0.00 \\
\hline MIR152 & microRNA 152 & 0.98 & 1.01 & 1.03 & 0.01 \\
\hline MIR1247 & microRNA 1247 & 0.95 & 0.98 & 1.03 & 0.05 \\
\hline MIR135A2 & microRNA 135a-2 & 0.96 & 0.98 & 1.03 & 0.02 \\
\hline MIR130B & microRNA 130b & 1.00 & 1.03 & 1.03 & 0.05 \\
\hline MIR103A1 & microRNA 103a-1 & 0.87 & 0.90 & 1.04 & 0.70 \\
\hline MIR412 & microRNA 412 & 1.01 & 1.05 & 1.04 & 0.09 \\
\hline MIR455 & microRNA 455 & 0.95 & 0.98 & 1.04 & 0.03 \\
\hline MIR196A2 & microRNA 196a-2 & 0.98 & 1.01 & 1.04 & 0.02 \\
\hline MIR202 & microRNA 202 & 1.00 & 1.04 & 1.04 & 0.06 \\
\hline MIRLET7E & microRNA let-7e & 0.89 & 0.93 & 1.04 & 0.08 \\
\hline MIR134 & microRNA 134 & 1.00 & 1.04 & 1.04 & 0.07 \\
\hline MIR194-2 & microRNA 194-2 & 0.98 & 1.02 & 1.04 & 0.03 \\
\hline MIR130A & microRNA 130a & 0.98 & 1.02 & 1.04 & 0.02 \\
\hline MIR136 & microRNA 136 & 1.03 & 1.08 & 1.04 & 0.12 \\
\hline MIR492 & microRNA 492 & 1.03 & 1.08 & 1.05 & 0.15 \\
\hline MIR142 & microRNA 142 & 0.96 & 1.01 & 1.05 & 0.01 \\
\hline MIR22HG & MIR22 host gene & 1.07 & 1.12 & 1.05 & 0.58 \\
\hline MIR128-1 & microRNA 128-1 & 0.98 & 1.02 & 1.05 & 0.06 \\
\hline MIR451A & microRNA 451a & 0.98 & 1.03 & 1.05 & 0.06 \\
\hline MIR95 & microRNA 95 & 0.97 & 1.02 & 1.05 & 0.03 \\
\hline MIR9-3 & microRNA 9-3 & 0.91 & 0.96 & 1.05 & 0.07 \\
\hline MIR182 & microRNA 182 & 0.92 & 0.96 & 1.05 & 0.06 \\
\hline MIR1-1 & microRNA 1-1 & 0.98 & 1.03 & 1.06 & 0.06 \\
\hline MIR375 & microRNA 375 & 0.96 & 1.01 & 1.06 & 0.01 \\
\hline MIR132 & microRNA 132 & 0.92 & 0.97 & 1.06 & 0.03 \\
\hline MIR199B & microRNA 199b & 0.87 & 0.93 & 1.06 & 0.11 \\
\hline MIR7-3HG & MIR7-3 host gene & 0.89 & 0.95 & 1.06 & 0.12 \\
\hline MIR218-1 & microRNA 218-1 & 0.85 & 0.91 & 1.07 & 0.16 \\
\hline MIR96 & microRNA 96 & 0.98 & 1.05 & 1.07 & 0.08 \\
\hline MIR9-2 & microRNA 9-2 & 1.00 & 1.07 & 1.07 & 0.31 \\
\hline MIR125A & microRNA 125a & 0.90 & 0.97 & 1.07 & 0.04 \\
\hline MIR212 & microRNA 212 & 0.90 & 0.97 & 1.07 & 0.06 \\
\hline MIR23B & microRNA 23b & 0.85 & 0.91 & 1.07 & 0.16 \\
\hline MIRLET7I & microRNA let-7i & 1.03 & 1.11 & 1.08 & 0.39 \\
\hline MIR137HG & MIR137 host gene & 1.00 & 1.08 & 1.08 & 0.20 \\
\hline MIR28 & microRNA 28 & 1.01 & 1.09 & 1.08 & 0.14 \\
\hline MIRLET7G & microRNA let-7g & 0.91 & 0.98 & 1.08 & 0.01 \\
\hline MIR34C & microRNA 34c & 0.86 & 0.93 & 1.08 & 0.24 \\
\hline
\end{tabular}




\begin{tabular}{|c|c|c|c|c|c|}
\hline MIR25 & microRNA 25 & 0.85 & 0.91 & 1.08 & 0.26 \\
\hline MIR204 & microRNA 204 & 0.96 & 1.03 & 1.08 & 0.04 \\
\hline MIR215 & microRNA 215 & 0.94 & 1.02 & 1.08 & 0.03 \\
\hline MIR138-2 & microRNA 138-2 & 0.93 & 1.01 & 1.08 & 0.01 \\
\hline MIR193A & microRNA 193a & 0.96 & 1.04 & 1.08 & 0.07 \\
\hline MIR181A2 & microRNA 181a-2 & 0.86 & 0.93 & 1.09 & 0.08 \\
\hline MIRLET7F1 & microRNA let-7f-1 & 1.07 & 1.16 & 1.09 & 0.30 \\
\hline MIR218-2 & microRNA 218-2 & 0.88 & 0.95 & 1.09 & 0.07 \\
\hline MIR196A1 & microRNA 196a-1 & 0.93 & 1.01 & 1.09 & 0.01 \\
\hline MIR208A & microRNA 208a & 0.97 & 1.06 & 1.09 & 0.11 \\
\hline MIRLET7F2 & microRNA let-7f-2 & 1.00 & 1.08 & 1.09 & 0.09 \\
\hline MIR203 & microRNA 203 & 0.94 & 1.03 & 1.09 & 0.06 \\
\hline MIR34A & microRNA 34a & 0.92 & 1.00 & 1.09 & 1.50 \\
\hline MIR200A & microRNA 200a & 0.98 & 1.08 & 1.10 & 0.14 \\
\hline MIR27B & microRNA 27b & 0.94 & 1.03 & 1.10 & 0.02 \\
\hline MIR377 & microRNA 377 & 0.98 & 1.07 & 1.10 & 0.20 \\
\hline MIR15A & microRNA $15 a$ & 0.80 & 0.87 & 1.10 & 0.16 \\
\hline MIR30D & microRNA 30d & 0.95 & 1.04 & 1.10 & 0.08 \\
\hline MIR34B & microRNA 34b & 0.94 & 1.03 & 1.10 & 0.04 \\
\hline MIR105-2 & microRNA 105-2 & 1.12 & 1.23 & 1.10 & 0.69 \\
\hline MIR29B1 & microRNA 29b-1 & 0.91 & 1.00 & 1.10 & 0.00 \\
\hline MIR429 & microRNA 429 & 1.01 & 1.11 & 1.11 & 0.50 \\
\hline MIR145 & microRNA 145 & 1.07 & 1.18 & 1.11 & 0.70 \\
\hline MIR299 & microRNA 299 & 0.91 & 1.00 & 1.11 & 0.00 \\
\hline MIRLET7D & microRNA let-7d & 0.94 & 1.04 & 1.11 & 0.07 \\
\hline MIR101-1 & microRNA 101-1 & 0.89 & 0.99 & 1.12 & 0.01 \\
\hline MIR219-1 & microRNA 219-1 & 0.96 & 1.07 & 1.12 & 0.11 \\
\hline MIR148A & microRNA 148a & 0.92 & 1.03 & 1.12 & 0.05 \\
\hline MIR219-2 & microRNA 219-2 & 0.97 & 1.10 & 1.13 & 0.58 \\
\hline MIR485 & microRNA 485 & 0.99 & 1.12 & 1.13 & 0.55 \\
\hline MIR190A & microRNA 190a & 0.90 & 1.02 & 1.14 & 0.04 \\
\hline MIR133A1 & microRNA 133a-1 & 0.97 & 1.11 & 1.14 & 0.38 \\
\hline MIR149 & microRNA 149 & 0.89 & 1.02 & 1.14 & 0.02 \\
\hline MIR320A & microRNA 320a & 0.92 & 1.06 & 1.15 & 0.14 \\
\hline MIR376C & microRNA 376c & 0.92 & 1.05 & 1.15 & 0.16 \\
\hline MIR144 & microRNA 144 & 0.91 & 1.05 & 1.16 & 0.10 \\
\hline MIR30A & microRNA 30a & 0.91 & 1.06 & 1.16 & 0.07 \\
\hline MIR16-1 & microRNA 16-1 & 0.82 & 0.96 & 1.17 & 0.13 \\
\hline MIR365A & microRNA 365a & 0.78 & 0.91 & 1.18 & 0.24 \\
\hline MIR186 & microRNA 186 & 0.94 & 1.11 & 1.18 & 0.24 \\
\hline MIR15B & microRNA 15b & 0.77 & 0.91 & 1.18 & 0.25 \\
\hline MIR326 & microRNA 326 & 0.85 & 1.01 & 1.19 & 0.01 \\
\hline MIR194-1 & microRNA 194-1 & 0.92 & 1.10 & 1.20 & 0.20 \\
\hline MIR221 & microRNA 221 & 0.95 & 1.14 & 1.21 & 0.22 \\
\hline MIR103A2 & microRNA 103a-2 & 0.87 & 1.05 & 1.21 & 0.06 \\
\hline MIR211 & microRNA 211 & 0.77 & 0.94 & 1.23 & 0.09 \\
\hline MIR154 & microRNA 154 & 0.97 & 1.24 & 1.27 & 0.28 \\
\hline MIR382 & microRNA 382 & 0.95 & 1.22 & 1.28 & 0.87 \\
\hline MIR147A & microRNA 147a & 0.71 & 0.94 & 1.33 & 0.14 \\
\hline MIR622 & microRNA 622 & 0.90 & 1.21 & 1.36 & 0.36 \\
\hline MIR19B2 & microRNA 19b-2 & 0.72 & 1.02 & 1.42 & 0.03 \\
\hline MIR7-2 & microRNA 7-2 & 0.61 & 1.04 & 1.71 & 0.10 \\
\hline
\end{tabular}

${ }^{*} \mathrm{FC}=$ fold change. FDR = false discovery rate of RvC dataset. from Heuslein and Meisner et al. 


\section{Discussion and future directions}

\subsection{Summary}

In this thesis, we have described several significant contributions toward improving our understanding of each stage (i.e. initiation, growth, and maturation) of arteriogenesis.

In Chapter 4, we discovered that collateral artery segments in the mouse hindlimb exhibit either "moderate" or "amplified" arteriogenesis, depending on the initiating hemodynamics to which they are exposed (i.e. non-reversed or reversed flow waveforms, respectively) following femoral arterial ligation (FAL). We determined that this reversed flow-mediated amplification of collateral artery growth to be dependent on ICAM-1 mediated macrophage recruitment. Moreover, enabled by our lab's ability to quantitatively measure the relative hemodynamics in these collateral segments in-vivo and apply them to ECs in-vitro, we were able to utilize genome-wide analyses to, for the first time, comprehensively map EC mechanosensitive signaling to sustained, differential arteriogenesis responses.

In Chapter 5, we used this unique case of differential collateral growth as a model system to uncover molecular regulators of arteriogenic capacity and collateral artery maturation. We determined that ECs exposed to a non-reversed flow waveform exhibit increased DNMT1 expression and DNA hypermethylation. Moreover, we determined that DNMT1-dependent EC DNA hypermethylation regulates arteriogenic capacity via adjustments to shear stress set-point in-vivo, identifying a role for DNA methylation in arteriogenesis for the first time.

Finally, in Chapter 6, we interrogated our genome-wide analysis of EC mechanosensitive signaling mapped to differential arteriogenesis responses to identify potentially novel microRNA regulators of arteriogenesis. Using this unique approach, we identified microRNA-199a as a potent mechanosensitive regulator of perfusion recovery and arteriogenesis after arterial occlusion. 
Overall, these studies, stemming from an initial observation of differential collateral artery growth in-vivo, demonstrate the importance of EC signaling and epigenetics in regulating shear stress-mediated arteriogenesis. These results may also have significant implications for therapeutically stimulating arteriogenesis in patients with arterial occlusive diseases.

\subsection{Thoughts and future studies: DNMT1, DNA methylation, and regulation to the shear stress set-point}

In Chapter 5, though the pharmacological DNMT1-inhibitor, 5AZA, enabled us to determine a role for DNMT1-dependent DNA methylation in regulating arteriogenic capacity via shear stress set-point alteration, it likely affected more than endothelial DNA methylation. Future studies could seek to determine the significance of cell-specific loss of DNMT1 expression on the regulation of arteriogenic capacity. To do this, we could generate EC- and/or SMC-specific inducible DNMT1 KO mice by breeding floxed DNMT1 knockout mice (DNMT1 $\left.1^{210 \times 10 x}\right)^{297}$ with tamoxifen-inducible, EC-specific, Cre-recombinase mice (VECad-Cre-ER $\left.{ }^{\text {T2}}\right)^{298}$ or tamoxifen-inducible, SMC-specific, Cre-recombinase mice (Myh11-Cre-ER $\left.{ }^{\text {T2}}\right)^{299}$ mice. As an additional or alternative approach, future work could also develop a platform for local delivery of and transfection of ECs and/or SMCs with shDNMT1 plasmids. Techniques for gene-specific editing of DNA methylation are beginning to be reported ${ }^{300-302}$. As these gene-editing techniques, such as the CRISPR-Cas9 system, continue to develop, we could investigate the contribution of gene-specific DNA methylation to arteriogenic capacity and shear stress set-point.

Future studies could also seek to determine if DNMT1-dependent DNA methylation regulates shear stress set-point in other vascular networks. Selective ligations of mesenteric arteries can induce different levels of shear stress in collateral vessels that correspond to differential growth ${ }^{46}$. The nearly two-dimensional nature of these mesenteric windows would enable for us to simultaneously measure collateral diameter and relative hemodynamic changes. Additionally, we could potentially use the dorsal skinfold window chamber ${ }^{167}$ or ear artery 
ligation ${ }^{303,304}$ models followed by non-invasive imaging (e.g. LSF, photoacoustic microscopy) to enable for longitudinal study of the same vascular network over-time. Each of these experimental models would enable us to simultaneously determine DNMT1-dependent alterations in collateral artery growth, sensitivity, and shear stress set-point.

As several early studies investigated the shear stress set-point hypothesis in the carotid artery, it would be interesting to also determine the role of DNMT1-dependent DNA methylation in these arteries. Interestingly, shear stress set-point was shown to be augmented in the common carotid artery experiencing the greatest relative flow increases in a canine arteriovenous-shunt model ${ }^{47}$. This is similar to the phenotype we observed in non-reversed collateral segments following FAL, indicating that DNA hypermethylation may also be regulating the set-point in the carotid. Moreover, despite an initial increase in flow, several studies have shown that aged animals exhibit impaired diameter enlargement of the carotid, thereby leading to an elevated shear set-point ${ }^{83,94}$. Young, healthy animals, however, exhibit significant diameter enlargement

and are able to restore shear stress to the original set-point ${ }^{83,94}$. The phenotype seen in aged animals is again similar to the constrained collateral artery growth and shear stress set-point augmentation due to DNA hypermethylation that we observed in Chapter 5. Given that age alters DNA methylation ${ }^{123}$, we would hypothesize that the carotid arteries of age animals undergo DNA hypermethylation, which in turn, constrains remodeling capacity by augmenting shear stress setpoint.

\subsection{Thoughts and future directions: $\mathrm{miR}-199 \mathrm{a}$ and mechanosensitive miRNA as regulators of arteriogenesis and as therapeutic targets}

There are a number of potential future studies stemming from our work on mechanosensitive miRNAs in Chapter 6. Though we showed miR-199a regulates the expression of several direct binding targets (e.g. IKK 3 , and CD44) previously validated in other studies ${ }^{269,281}$, we did not explicitly determine miR-199a to directly bind to these targets in our system. We could therefore 
perform luciferase reporter assays to test if these mRNAs are indeed direct seed-sequence binding targets. Future studies could also seek to further establish the clinical relevance of miR199a. To this end, we can determine if miR-199a-5p expression and/or target(s) are differentially expressed in muscle biopsies of PAD patients and if expression is correlated to disease outcomes. Additionally, it may be worthwhile to determine if anti-miR-199a improves functional outcome (i.e. perfusion, vascular growth, muscle composition, exercise capacity) in more preclinical models of PAD. While the FAL model used here is an excellent tool for studying endogenous arteriogenesis, it is not a model of PAD per se. Pre-clinical models could incorporate more severe ischemia, mouse strains with co-morbidities typically associated with PAD (i.e. hyperlipidemia and diabetes), and delayed treatment of anti-miRs to better account for the chronic nature of PAD. Moreover, though a single, direct intramuscular injection of anti-miR-199a showed some therapeutic promise, there is still significant non-localized delivery. This highlights a critical need to develop a more optimized, targeted delivery method. To this end, our lab has successfully used an ultrasound-microbubble system for the targeted delivery of gene-carrying nanoparticles to the skeletal muscle ${ }^{305}$. We could utilize a similar approach for the delivery of nanoparticles carrying miR-targeted plasmids or by electrostatic conjugation of anti-miRs to microbubbles.

\subsection{Thoughts and future studies: arteriogenesis, angiogenesis, and beyond}

\subsubsection{Promise for pro-arteriogenic and pro-angiogenic combination therapy}

Therapeutic arteriogenesis is promising for restoring blood flow to the distal tissue because of its efficiency. Only a few collaterals are needed to protect against ischemia ${ }^{306}$ and small changes in diameter lead to large changes in conductance ${ }^{307}$. However, the stimulation of angiogenesis is also important as capillary density is reduced in PAD patients ${ }^{4,25,28}$. Exercise, which has been shown to improve arteriole ${ }^{26}$ and capillary density ${ }^{25}$, improves peak treadmill walking time to a greater extent than stent revascularization in PAD patients with claudication, further highlighting 
the need to restore the underlying microvasculature to improve patient outcome (CLEVER study $)^{308}$.

It is likely necessary to stimulate both arteriogenic and angiogenic adaptive responses to arterial occlusion(s) ${ }^{29,39,309}$. Our recent results using a computational arteriolar network model to estimate perfusion in PAD patients after exercise and/or percutaneous interventions emphasize the importance of designing therapeutics to restore normal microvascular structure and function in combination with restoration of upstream perfusion pressure (via revascularization or arteriogenesis $)^{26}$. These computational results are supported by the recent results of the ERASE study which demonstrate that, at 12-months post-treatment, a combined treatment of endovascular revascularization followed by supervised exercise improved maximal walking distance, pain-free walking distance, and patient-reported quality of life compared to supervised exercise only ${ }^{310}$. Though it would be beneficial to have one clinical trial directly comparing PAD patients receiving revascularization, supervised exercise, or a combination therapy, together, these clinical trials support the hypothesis that while microvascular expansion and revascularization individually provide a similar, modest clinical benefit, a combination therapy would be an "optimal" treatment.

\subsubsection{Need to consider muscle composition and metabolism}

Tissue clearance, composition, and metabolism are critical, but often over-looked, responses to an upstream arterial occlusion ${ }^{30}$. PAD patients exhibit a number of abnormalities and impairments in the muscles of their lower limbs including fatty tissues ${ }^{18,293}$, smaller calf muscles ${ }^{18,293}$, increased apoptosis $^{295}$, abnormal fiber distribution ${ }^{293,311,312}$, denervation ${ }^{293}$, and inflammation ${ }^{20}$. Moreover, the Lsq-1 locus was shown to be responsible for the strain-dependent, differential skeletal muscle responses to ischemia in a pre-clinical, murine hindlimb ischemia model ${ }^{313}$.

These abnormalities indicate that targeting muscle fiber composition and metabolism, likely in concert with stimulation of arteriogenesis and angiogenesis, may be a therapeutically beneficial 
approach. To this end, miR-199a inhibition was sufficient to improve gastrocnemius muscle tissue composition in FAL-operated mice and has been implicated in tissue fibrosis ${ }^{290}$, myoblast proliferation and differentiation ${ }^{268}$, and adipocyte differentiation ${ }^{291}$. It may therefore be interesting to determine if miR-199a regulates proliferation, regeneration, and/or differentiation of ischemic skeletal muscle.

Additionally, recent studies in humans have highlighted that the reduction in blood flow alone does not fully account for the exercise limitations of PAD patients ${ }^{20}$. To this point, there appears to be an uncoupling of calf muscle perfusion and muscle metabolism in PAD patients ${ }^{314}$. Acylcarnitine, a metabolite that reflects the metabolic state/capacity of the muscle, has been found to be elevated in PAD patients and to be associated with reduced peak treadmill exercise performance ${ }^{315,316}$. Furthermore, PAD patients exhibit abnormal mitochondria function ${ }^{317}$ and reduced activity of complex III of the mitochondrial electron transport chain ${ }^{318}$, leading to significant oxidative stress ${ }^{317}$. Collectively, this emphasizes that treatments for PAD are faced with two fundamental transport problems: (1) getting enough oxygenated blood to the ischemic muscle and (2) ensuring that oxygen is functionally available for proper mitochondrial usage. To design effective therapeutics, we need to determine how these issues are inter-related - i.e. if we can increase the vascular supply (via stenting, arteriogenesis, angiogenesis, etc), do we mitigate mitochondrial dysfunction and increase $\mathrm{O}_{2}$ bioavailability? Alternatively, if we can repair the mitochondria, do we prevent capillary and arteriolar rarefaction and maintain an intact vascular supply? Which is a more effective strategy?

A potentially interesting idea would be to determine if oxygen delivery, independent of the vasculature, could provide therapeutic benefit. For example, delivery of oxygen-generating biomaterials ${ }^{319-321}$ may be able to provide sufficient oxygen to areas with an insufficient vascular supply due to microvascular defects. This approach could help to test the hypothesis whether an oxygen supply is sufficient for restoring mitochondrial and skeletal muscle function. Additionally, it could be used in concert with other therapies, such as exercise, whereby a short-lived "dose" of 
supplemental oxygen to ischemic limbs (via oxygen-generating biomaterials) enhances a patient's exercise capacity, which in turn, restores microvascular and metabolic function.

\subsection{Thoughts and future directions: applications to other disease models}

Our results identifying mechanosensitive epigenetic regulators of arteriogenesis may also have broader implications outside of PAD. Adequate collateral development and growth is also critical for patient outcomes in $\mathrm{CAD}^{322}$ and ischemic stroke ${ }^{323}$. Additionally, arteriogenesis is the predominant vascular adaptation in the surgical "flap delay" strategy for autologous transplantation for reconstructive plastic surgery ${ }^{324}$. Future studies could determine if inhibition of miR-199a or DNMT1 enhances arteriogenesis and functional outcomes in pre-clinical models of these clinical scenarios. In contrast, other future studies could seek to inhibit arteriogenesis (e.g. via ICAM-1 inhibition, miR-199a overexpression) to elicit therapeutic benefit. To this end, recombinant parathyroid hormone (rPTH) therapy promotes scarless healing of critical bone defects via inhibition of arteriogenesis and mast cell recruitment. However, given that $\mathrm{rPTH}$ therapy is contraindicated for cancer patients, which constitutes a large percentage of patients with critical bone defects, alternate strategies for limiting mast cell recruitment and arteriogenesis are needed $^{325}$. Moreover, though largely unstudied, there is some evidence of lumenal expansion of feeder arterioles during tumor growth ${ }^{326,327}$. Additionally, both the inherent heterogeneity of the tumor vasculature and surgical excision treatments may alter flow pathways such that "tumor collaterals" develop. Targeting collateral artery growth may therefore be a novel anti-cancer therapeutic strategy. 


\section{References}

1. Norgren L, Hiatt WR, Dormandy JA, Nehler MR, Harris KA, Fowkes FGR. Inter-society consensus for the management of peripheral arterial disease (TASC II). Eur $J$ Vasc Endovasc Surg. 2007;33:S1-S75.

2. Norgren L, Hiatt WR, Dormandy JA, Hirsch AT, Jaff MR, Diehm C, Baumgartner I, Belch JJF. The next 10 years in the management of peripheral artery disease: Perspectives from the "PAD 2009" conference. Eur J Vasc Endovasc Surg. 2010;40:375-380.

3. Fowkes FGR, Rudan D, Rudan I, Aboyans V, Denenberg JO, McDermott MM, Norman PE, Sampson UKA, Williams LJ, Mensah GA, Criqui MH. Comparison of global estimates of prevalence and risk factors for peripheral artery disease in 2000 and 2010: a systematic review and analysis. Lancet. 2013;382:1329-40.

4. Annex BH. Therapeutic angiogenesis for critical limb ischaemia. Nat Rev Cardiol. 2013;10:387-96.

5. Hiatt WR, Hoag S, Hamman RF. Effect of diagnostic criteria on the prevalence of peripheral arterial disease: The San Luis Valley diabetes study. Circulation. 1995;91:1472-1479.

6. Selvin E, Erlinger TP. Prevalence of and risk factors for peripheral arterial disease in the United States: Results from the National Health and Nutrition Examination Survey, 19992000. Circulation. 2004;110:738-743.

7. Belch JJF, Topol EJ, Agnelli G, Bertrand M, Califf RM, Clement DL, Creager M a, Easton JD, Gavin JR, Greenland P, Hankey G, Hanrath P, Hirsch AT, Meyer J, Smith SC, Sullivan F, Weber MA. Critical issues in peripheral arterial disease detection and management: a call to action. Arch Intern Med. 2003;163:884-92.

8. Gandhi S, Weinberg I, Margey R, Jaff MR. Comprehensive medical management of peripheral arterial disease. Prog Cardiovasc Dis. 2011;54:2-13.

9. Criqui $\mathrm{MH}$, Fronek A, Barrett-Connor E, Goodman D. The prevalence of peripheral arterial disease in defined population. Circulation. 1985;71:510-515.

10. Beckman JA, Creager MA, Libby P. Diabetes and atherosclerosis: epidemiology, pathophysiology, and management. JAMA. 2002;287:2570-2581.

11. Lau JF, Weinberg MD, Olin JW. Peripheral artery disease. Part 1: clinical evaluation and noninvasive diagnosis. Nat Rev Cardiol. 2011;8:405-18.

12. Hirsch AT, Criqui MH, Treat-Jacobson D, Regensteiner JG, Creager M a, Olin JW, Krook $\mathrm{SH}$, Hunninghake DB, Comerota AJ, Walsh ME, McDermott MM, Hiatt WR. Peripheral arterial disease detection, awareness, and treatment in primary care. JAMA. 2001;286:1317-24.

13. McDermott MM, Liu K, Criqui MH, Ruth K, Goff D, Saad MF, Wu C, Homma S, Sharrett AR. Ankle-brachial index and subclinical cardiac and carotid disease: The multi-ethnic study of atherosclerosis. Am J Epidemiol. 2005;162:33-41.

14. Jude E, Chalmers N, Oyibo S, Boulton A. Peripheral arterial disease in diabetic and nondiabetic patients. Diabetes Care. 2001;24:1433-1437.

15. Kullo IJ, Leeper NJ. The genetic basis of peripheral arterial disease: current knowledge, challenges, and future directions. Circ Res. 2015;116:1551-60. 
16. Ankle Brachial Index Collaboration. Ankle brachial index combined with Framingham Risk Score to predict cardiovascular events and mortality: a meta-analysis. JAMA. 2008;300:197-208.

17. Lau JF, Weinberg MD, Olin JW. Peripheral artery disease. Part 1: clinical evaluation and noninvasive diagnosis. Nat Rev Cardiol. 2011;8:405-18.

18. McDermott MM, Guralnik JM, Ferrucci L, Tian L, Liu K, Liao Y, Green D, Sufit R, Hoff F, Nishida T, Sharma L, Pearce WH, Schneider JR, Criqui MH. Asymptomatic peripheral arterial disease is associated with more adverse lower extremity characteristics than intermittent claudication. Circulation. 2008;117:2484-2491.

19. Criqui MH, Langer R, Fronek A, Feigelson H, Klauber M, McCann TJ, Browner D. Mortality over a period of 10 years in patients with peripheral arterial disease. $N$ Engl $J$ Med. 1992;326:381-386.

20. Hiatt WR, Armstrong EJ, Larson CJ, Brass EP. Pathogenesis of the limb manifestations and exercise limitations in peripheral artery disease. Circ Res. 2015;116:1527-1539.

21. Tabas I, García-Cardeña G, Owens GK. Recent insights into the cellular biology of atherosclerosis. J Cell Biol. 2015;209:13-22.

22. Lusis AJ. Atherosclerosis. Nature. 2000;407:233-241.

23. Liao JK, Bettmann M a, Sandor T, Tucker JI, Coleman SM, Creager MA. Differential impairment of vasodilator responsiveness of peripheral resistance and conduit vessels in humans with atherosclerosis. Circ Res. 1991;68:1027-34.

24. Schellong SM, Boger RH, Burchert W, Bode-Boger SM, Galland A, Frolich JC, Hundeshagen $\mathrm{H}$, Alexander K. Dose-related effect of intravenous L-arginine on muscular blood flow of the calf in patients with peripheral vascular disease: a $\mathrm{H} 2150$ positron emission tomography study. Clin Sci (Lond). 1997;93:159-165.

25. Robbins JL, Jones WS, Duscha BD, Allen JD, Kraus WE, Regensteiner JG, Hiatt WR, Annex $\mathrm{BH}$. Relationship between leg muscle capillary density and peak hyperemic blood flow with endurance capacity in peripheral artery disease. J Appl Physiol. 2011;111:8186.

26. Heuslein JL, Li X, Murrell KP, Annex BH, Peirce SM, Price RJ. Computational network model prediction of hemodynamic alterations due to arteriolar rarefaction and estimation of skeletal muscle perfusion in peripheral arterial disease. Microcirculation. 2015;22:360 369.

27. Berceli SA, Hevelone ND, Lipsitz SR, Bandyk DF, Clowes AW, Moneta GL, Conte MS. Surgical and endovascular revision of infrainguinal vein bypass grafts: analysis of midterm outcomes from the PREVENT III trial. J Vasc Surg. 2007;46:1173-1179.

28. Duscha BD, Robbins JL, Jones WS, Kraus WE, Lye RJ, Sanders JM, Allen JD, Regensteiner JG, Hiatt WR, Annex BH. Angiogenesis in skeletal muscle precede improvements in peak oxygen uptake in peripheral artery disease patients. Arterioscler Thromb Vasc Biol. 2011;31:2742-8.

29. Meisner JK, Song J, Annex BH, Price RJ. Myoglobin overexpression inhibits reperfusion in the ischemic mouse hindlimb through impaired angiogenesis but not arteriogenesis. Am J Pathol. 2013;183:1-10.

30. Meisner JK, Annex BH, Price RJ. Despite normal arteriogenic and angiogenic responses, hind limb perfusion recovery and necrotic and fibroadipose tissue clearance are impaired 
in matrix metalloproteinase 9-deficient mice. J Vasc Surg. 2014;61:1583-1594.

31. Seiler C, Pohl T, Wustmann K, Hutter D, Nicolet P a, Windecker S, Eberli FR, Meier B. Promotion of collateral growth by granulocyte-macrophage colony-stimulating factor in patients with coronary artery disease: a randomized, double-blind, placebo-controlled study. Circulation. 2001;104:2012-7.

32. van Royen N, Schirmer SH, Atasever B, Behrens CYH, Ubbink D, Buschmann EE, Voskuil M, Bot P, Hoefer I, Schlingemann RO, Biemond BJ, Tijssen JG, Bode C, Schaper W, Oskam J, Legemate DA, Piek JJ, Buschmann I. START Trial: a pilot study on STimulation of ARTeriogenesis using subcutaneous application of granulocytemacrophage colony-stimulating factor as a new treatment for peripheral vascular disease. Circulation. 2005;112:1040-6.

33. Zbinden S, Zbinden R, Meier P, Windecker S, Seiler C. Safety and efficacy of subcutaneous-only granulocyte-macrophage colony-stimulating factor for collateral growth promotion in patients with coronary artery disease. J Am Coll Cardiol. 2005;46:1636-42.

34. Ripa RS, Jørgensen E, Wang Y, Thune JJ, Nilsson JC, Søndergaard L, Johnsen HE, Køber L, Grande P, Kastrup J. Stem cell mobilization induced by subcutaneous granulocyte-colony stimulating factor to improve cardiac regeneration after acute STelevation myocardial infarction: result of the double-blind, randomized, placebo-controlled stem cells in myocardial infarc. Circulation. 2006;113:1983-92.

35. Subramaniyam V, Waller EK, Murrow JR, Manatunga A, Lonial S, Kasirajan K, Sutcliffe D, Harris W, Taylor WR, Alexander RW, Quyyumi AA. Bone marrow mobilization with granulocyte macrophage colony-stimulating factor improves endothelial dysfunction and exercise capacity in patients with peripheral arterial disease. Am Hear J. 2009;158:5360.

36. Ziegler MA, Distasi MR, Bills RG, Miller SJ, Alloosh M, Murphy MP, Akingba AG, Sturek M, Dalsing MC, Unthank JL. Marvels, mysteries, and misconceptions of vascular compensation to peripheral artery occlusion. Microcirculation. 2010;17:3-20.

37. Schaper W. Collateral circulation: past and present. Basic Res Cardiol. 2009;104:5-21.

38. Liebeskind DS. Collateral circulation. Stroke. 2003;34:2279-2284.

39. Meisner JK, Price RJ. Spatial and temporal coordination of bone marrow-derived cell activity during arteriogenesis: regulation of the endogenous response and therapeutic implications. Microcirculation. 2010;17:583-599.

40. Scholz D, Schaper W. Preconditioning of arteriogenesis. Cardiovasc Res. 2005;65:51323.

41. Langille BL, O'Donnell F. Reductions in arterial diameter produced by chronic decreases in blood flow are endothelium-dependent. Science (80- ). 1986;231:405-407.

42. Moraes F, Paye J, Mac Gabhann F, Zhuang ZW, Zhang J, Lanahan AA, Simons M. Endothelial cell-dependent regulation of arteriogenesis. Circ Res. 2013;113:1076-86.

43. Davies PF. Flow-mediated endothelial mechanotransduction. Physiol Rev. 1995;75:519560.

44. Garcia-Cardeña G, Comander J, Anderson KR, Blackman BR, Gimbrone MA. Biomechanical activation of vascular endothelium as a determinant of its functional phenotype. Proc Natl Acad Sci U S A. 2001;98:4478-85. 
45. Topper JN, Gimbrone MA. Blood flow and vascular gene expression: fluid shear stress as a modulator of endothelial phenotype. Mol Med Today. 1999;5:40-6.

46. Tuttle JL, Nachreiner RD, Bhuller AS, Condict KW, Connors BA, Herring BP, Dalsing MC, Unthank JL. Shear level influences resistance artery remodeling : wall dimensions, cell density, and eNOS expression. Am J Physiol Hear Circ Physiol. 2001;H1380-H1389.

47. Kamiya A, Togawa T. Adaptive regulation of wall shear stress to flow change in the canine carotid artery. Am J Physiol Hear Circ Physiol. 1980;239:H14-H21.

48. Zarins CK, Zatina MA, Giddens DP, Ku DN, Glagov S. Shear stress regulation of artery lumen diameter in experimental atherogenesis. J Vasc Surg. 1987;5:413-420.

49. Eitenmüller I, Volger O, Kluge A, Troidl K, Barancik M, Cai W-J, Heil M, Pipp F, Fischer S, Horrevoets AJG, Schmitz-Rixen T, Schaper W. The range of adaptation by collateral vessels after femoral artery occlusion. Circ Res. 2006;99:656-62.

50. Tzima E, Irani-Tehrani M, Kiosses WB, Dejana E, Schultz DA, Engelhardt B, Cao G, DeLisser H, Schwartz MA. A mechanosensory complex that mediates the endothelial cell response to fluid shear stress. Nature. 2005;437:426-31.

51. Chen Z, Rubin J, Tzima E. Role of PECAM-1 in arteriogenesis and specification of preexisting collaterals. Circ Res. 2010;107:1355-63.

52. Scholz D, Ito W, Fleming I, Deindl E, Sauer A, Wiesnet M, Busse R, Schaper J, Schaper W. Ultrastructure and molecular histology of rabbit hind-limb collateral artery growth (arteriogenesis). Virchows Arch. 2000;436:257-70.

53. Lee CW, Stabile E, Kinnaird T, Shou M, Devaney JM, Epstein SE, Burnett MS. Temporal patterns of gene expression after acute hindlimb ischemia in mice: Insights into the genomic program for collateral vessel development. J Am Coll Cardiol. 2004;43:474-482.

54. Pipp F, Boehm S, Cai W-J, Adili F, Ziegler B, Karanovic G, Ritter R, Balzer J, Scheler C, Schaper W, Schmitz-Rixen T. Elevated fluid shear stress enhances postocclusive collateral artery growth and gene expression in the pig hind limb. Arterioscler Thromb Vasc Biol. 2004;24:1664-8.

55. Nagel T, Resnick N, Atkinson WJ, Dewey CF, Gimbrone MA. Shear stress selectively upregulates intercellular adhesion molecule-1 expression in cultured human vascular endothelial cells. J Clin Invest. 1994;94:885-891.

56. Hoefer IE, van Royen N, Rectenwald JE, Deindl E, Hua J, Jost M, Grundmann S, Voskuil M, Ozaki CK, Piek JJ, Buschmann IR. Arteriogenesis proceeds via ICAM-1/Mac-1mediated mechanisms. Circ Res. 2004;94:1179-85.

57. Shireman P. The Chemokine System in Arteriogenesis and Hind Limb Ischemia. $J$ Vasc Surg. 2007;45:A48-A56.

58. Buschmann IR, Hoefer IE, van Royen N, Katzer E, Braun-Dulleaus R, Heil M, Kostin S, Bode C, Schaper W. GM-CSF: a strong arteriogenic factor acting by amplification of monocyte function. Atherosclerosis. 2001;159:343-56.

59. Vries MHM, Wagenaar A, Post MJ. CXCL1 promotes arteriogenesis through enhanced monocyte recruitment into the peri-collateral space. Angiogenesis. 2015;18:163-71.

60. Bergmann CE, Hoefer IE, Meder B, Roth H, Royen N Van, Breit SM, Jost MM, Aharinejad S, Hartmann S, Buschmann IR. Arteriogenesis depends on circulating monocytes and macrophage accumulation and is severely depressed in op / op mice. J Leukoc Biol. 2006;80:59-65. 
61. Pipp F, Heil M, Issbrücker K, Ziegelhoeffer T, Martin S, van den Heuvel J, Weich H, Fernandez B, Golomb G, Carmeliet P, Schaper W, Clauss M. VEGFR-1-selective VEGF homologue PIGF is arteriogenic: evidence for a monocyte-mediated mechanism. Circ Res. 2003;92:378-85.

62. Arras M, Ito WD, Scholz D, Winkler B, Schaper J, Schaper W. Monocyte activation in angiogenesis and collateral growth in the rabbit hindlimb. J Clin Invest. 1998;101:40-50.

63. Heil M, Ziegelhoeffer T, Pipp F, Kostin S, Martin S, Clauss M, Schaper W. Blood monocyte concentration is critical for enhancement of collateral artery growth. Am J Physiol Hear Circ Physiol. 2002;283:H2411-9.

64. Francke A, Weinert S, Strasser RH, Braun-Dullaeus RC, Herold J. Transplantation of bone marrow derived monocytes: a novel approach for augmentation of arteriogenesis in a murine model of femoral artery ligation. Am J Transl Res. 2013;5:155-69.

65. Troidl C, Jung G, Troidl K, Hoffmann J, Mollmann H, Nef H, Schaper W, Hamm CW, Schmitz-Rixen T. The temporal and spatial distribution of macrophage subpopulations during arteriogenesis. Curr Vasc Pharmacol. 2013;11:5-12.

66. Awojoodu AO, Ogle ME, Sefcik LS, Bowers DT, Martin K, Brayman KL, Lynch KR, Peirce-Cottler SM, Botchwey E. Sphingosine 1-phosphate receptor 3 regulates recruitment of anti-inflammatory monocytes to microvessels during implant arteriogenesis. Proc Natl Acad Sci U S A. 2013;110:13785-90.

67. Heil M, Ziegelhoeffer T, Wagner S, Fernández B, Helisch A, Martin S, Tribulova S, Kuziel W a, Bachmann G, Schaper W. Collateral artery growth (arteriogenesis) after experimental arterial occlusion is impaired in mice lacking CC-chemokine receptor-2. Circ Res. 2004;94:671-7.

68. Bruce AC, Kelly-Goss MR, Heuslein JL, Meisner JK, Price RJ, Peirce SM. Monocytes are recruited from venules during arteriogenesis in the murine spinotrapezius ligation model. Arterioscler Thromb Vasc Biol. 2014;34:2012-22.

69. Schirmer SH, Bot PT, Fledderus JO, Van Der Laan AM, Volger OL, Laufs U, Böhm M, De Vries CJM, Horrevoets AJG, Piek JJ, Hoefer IE, Van Royen N. Blocking interferon $\beta$ stimulates vascular smooth muscle cell proliferation and arteriogenesis. J Biol Chem. 2010;285:34677-34685.

70. Hoefer IE, Royen N Van, Rectenwald JE, Bray EJ, Abouhamze Z, Moldawer LL, Voskuil M, Piek JJ, Buschmann IR, Ozaki CK. Direct evidence for tumor necrosis factor-alpha signaling in arteriogenesis. Circulation. 2002;105:1639-1641.

71. van Royen N, Hoefer I, Buschmann I, Heil M, Kostin S, Deindl E, Vogel S, Korff T, Augustin $\mathrm{H}$, Bode $\mathrm{C}$, Piek JJ, Schaper W. Exogenous application of transforming growth factor beta 1 stimulates arteriogenesis in the peripheral circulation. FASEB J. 2002;16:432-4.

72. Deindl E, Hoefer IE, Fernandez B, Barancik M, Heil M, Strniskova M, Schaper W. Involvement of the fibroblast growth factor system in adaptive and chemokine-induced arteriogenesis. Circ Res. 2003;92:561-568.

73. Heil M, Schaper W. Influence of mechanical, cellular, and molecular factors on collateral artery growth (arteriogenesis). Circ Res. 2004;95:449-58.

74. Ziegelhoeffer T, Fernandez B, Kostin S, Heil M, Voswinckel R, Helisch A, Schaper W. Bone marrow-derived cells do not incorporate into the adult growing vasculature. Circ Res. 2004;94:230-238. 
75. Kinnaird T, Burnett ES, Shou M, Lee CW, Barr S, Fuchs S, Epstein SE. Local delivery of marrow-derived stromal cells augments collateral perfusion through paracrine mechanisms. Circulation. 2004;109:1543-1549.

76. Cai W, Vosschulte R, Afsah-Hedjri a, Koltai S, Kocsis E, Scholz D, Kostin S, Schaper W, Schaper J. Altered balance between extracellular proteolysis and antiproteolysis is associated with adaptive coronary arteriogenesis. J Mol Cell Cardiol. 2000;32:997-1011.

77. Cai W-J, Kocsis E, Wu X, Rodrigues M, Luo X, Schaper W, Schaper J. Remodeling of the vascular tunica media is essential for development of collateral vessels in the canine heart. Mol Cell Biochem. 2004;264:201-210.

78. Hacking WJ, Vanbavel E, Spaan JA. Shear stress is not sufficient to control growth of vascular networks : a model study. Am J Physiol Hear Circ Physiol. 1996;270:H364H375.

79. Longland CJ. The collateral circulation of the limb. Ann R Coll Surg Engl. 1953;13:161176.

80. Heil M, Schaper W. Pathophysiology of collateral development. Coron Artery Dis. 2004;15:373-8.

81. Unthank JL, Fath SW, Burkhart HM, Miller SC, Dalsing MC. Wall remodeling during luminal expansion of mesenteric arterial collaterals in the rat. Circ Res. 1996;79:10151023.

82. Langille BL, Bendeck MP, Keeley FW. Adaptations of carotid arteries of young and mature rabbits to reduced carotid blood flow. Am J Physiol. 1989;256:H931-H939.

83. Brownlee RD, Langille BL. Arterial adaptations to altered blood flow. Can J Physiol Pharmacol. 1991;69:978-983.

84. Rodbard S. Vascular Caliber. Cardiology. 1975;60:4-49.

85. Kassab GS, Fung Y-CB. The pattern of coronary arteriolar bifurcations and the uniform shear hypothesis. Ann Biomed Eng. 1995;23:13-20.

86. Murray CD. The physiological principle of minimum work: I. The vascular system and the cost of blood volume. Proc Natl Acad Sci U S A. 1926;12:207-214.

87. Zamir M. Shear forces and blood vessel radii in the cardiovascular system. J Gen Physiol. 1977;69:449-61.

88. Cheng C, Helderman F, Tempel D, Segers D, Hierck B, Poelmann R, Tol A Van, Duncker DJ, Robbers-visser D, Ursem NTC, Haperen R Van, Wentzel JJ, Gijsen F, Steen AFW Van Der, Crom R De, Krams R. Large variations in absolute wall shear stress levels within one species and between species. Atherosclerosis. 2007;195:225-235.

89. Reneman RS, Arts T, Hoeks APG. Wall shear stress--an important determinant of endothelial cell function and structure--in the arterial system in vivo. $J$ Vasc Res. 2006;43:251-69.

90. Mayrovitz HN, Roy J. Microvascular blood flow: evidence indicating a cubic dependence on arteriolar diameter. Am J Physiol Hear Circ Physiol. 1983;H1031-H1038.

91. Kamiya A, Bukhari R, Togawa $T$. Adaptive regulation of wall shear stress optimizing vascular tree function. Bull Math Biol. 1984;46:127-137.

92. LaBarbera M. Principles of design of fluid transport systems in zoology. Science (80- ). 1990;249:992-1000. 
93. Kassab GS, Gregersen H, Nielsen SL, Lu X, Tanko LB, Falk E. Remodelling of the left anterior descending artery in a porcine model of supravalvular aortic stenosis. $J$ Hypertens. 2002;20:2429-37.

94. Miyashiro JK, Poppa V, Berk BC. Flow-induced vascular remodeling in the rat carotid artery diminishes with age. Circ Res. 1997;81:311-319.

95. Baeyens N, Nicoli S, Coon BG, Ross TD, Dries K Van Den, Han J, Lauridsen HM, Mejean CO, Eichmann A, Thomas J-L, Humphrey JD, Schwartz MA. Vascular remodeling is governed by a VEGFR3-dependent fluid shear stress set point. Elife. 2015;

96. Baccarelli A, Rienstra M, Benjamin EJ. Cardiovascular epigenetics: basic concepts and results from animal and human studies. Circ Cardiovasc Genet. 2010;3:567-73.

97. Ordovas JM, Smith CE. Epigenetics and cardiovascular disease. Nat Rev Cardiol. 2010;7:510-519.

98. Matouk CC, Marsden PA. Epigenetic regulation of vascular endothelial gene expression. Circ Res. 2008;102:873-87.

99. Chen L-J, Wei S-Y, Chiu J-J. Mechanical regulation of epigenetics in vascular biology and pathobiology. J Cell Mol Med. 2013;17:437-448.

100. Dunn J, Thabet S, Jo H. Flow-dependent epigenetic DNA methylation in endothelial gene expression and atherosclerosis. Arterioscler Thromb Vasc Biol. 2015;1-9.

101. Jiang Y-Z, Manduchi E, Jimenez JM, Davies PF. Endothelial epigenetics in biomechanical stress: disturbed flow-mediated epigenomic plasticity in vivo and in vitro. Arterioscler Thromb Vasc Biol. 2015;35:1317-1326.

102. Goldberg AD, Allis CD, Bernstein E. Epigenetics: A landscape takes shape. Cell. 2007;128:635-638.

103. Yan MS, Marsden PA. Epigenetics in the vascular endothelium. Arterioscler Thromb Vasc Biol. 2015;25:ATVBAHA.115.305043.

104. Bestor TH. The DNA methyltransferases of mammals. Hum Mol Genet. 2000;9:23952402.

105. Handy DE, Castro R, Loscalzo J. Epigenetic modifications: basic mechanisms and role in cardiovascular disease. Circulation. 2011;123:2145-56.

106. Ito S, Shen L, Dai Q, Wu SC, Collins LB, Swenberg JA, He C, Zhang Y. Tet proteins can convert 5-methylcytosine to 5-formylcytosine and 5-carboxylcytosine. Science (80- ). 2011;333:1300-3.

107. Hill PWS, Amouroux R, Hajkova P. DNA demethylation, Tet proteins and 5hydroxymethylcytosine in epigenetic reprogramming: An emerging complex story. Genomics. 2014;104:324-333.

108. Miranda TB, Jones PA. DNA methylation: The nuts and bolts of repression. J. Cell. Physiol. 2007;213:384-390.

109. Jones PA. Functions of DNA methylation: islands, start sites, gene bodies and beyond. Nat Rev Genet. 2012;13:484-92.

110. Song F, Smith JF, Kimura MT, Morrow AD, Matsuyama T, Nagase H, Held WA. Association of tissue-specific differentially methylated regions (TDMs) with differential gene expression. Proc Natl Acad Sci U S A. 2005;102:3336. 
111. Grunau C, Hindermann W, Rosenthal A. Large-scale methylation analysis of human genomic DNA reveals tissue-specific differences between the methylation profiles of genes and pseudogenes. Hum Mol Genet. 2000;9:2651-2663.

112. Weber M, Davies JJ, Wittig D, Oakeley EJ, Haase M, Lam WL, Schübeler D. Chromosome-wide and promoter-specific analyses identify sites of differential DNA methylation in normal and transformed human cells. Nat Genet. 2005;37:853-862.

113. Kamakaka RT, Biggins S. Histone variants: Deviants? Genes Dev. 2005;19:295-310.

114. Bernstein BE, Meissner A, Lander ES. The mammalian epigenome. Cell. 2007;128:66981.

115. Uchida S, Dimmeler S. Long noncoding RNAs in cardiovascular diseases. Circ Res. 2015;116:737-50.

116. Mercer TR, Mattick JS. Structure and function of long noncoding RNAs in epigenetic regulation. Nat Struct Mol Biol. 2013;20:300-7.

117. Bartel DP. MicroRNAs: genomics, biogenesis, mechanism, and function. Cell. 2004;116:281-297.

118. Ha M, Kim VN. Regulation of microRNA biogenesis. Nat Rev Mol Cell Biol. 2014;15:509_ 524.

119. Jonas S, Izaurralde E. Towards a molecular understanding of microRNA-mediated gene silencing. Nat Rev Genet. 2015;16:421-433.

120. Lewis BP, Shih IH, Jones-Rhoades MW, Bartel DP, Burge CB. Prediction of mammalian microRNA targets. Cell. 2003;115:787-798.

121. Khaleghi M, Isseh IN, Bailey KR, Kullo IJ. Family history as a risk factor for peripheral arterial disease. Am J Cardiol. 2014;114:928-932.

122. Wahlgren CM, Magnusson PKE. Genetic influences on peripheral arterial disease in a twin population. Arterioscler Thromb Vasc Biol. 2011;31:678-682.

123. Pal S, Tyler JK. Epigenetics and aging. Sci Adv. 2016;2:e1600584.

124. Breitling LP. Current genetics and epigenetics of smoking/tobacco-related cardiovascular disease. Arterioscler Thromb Vasc Biol. 2013;33:1468-72.

125. Schones DE, Leung A, Natarajan R. Chromatin modifications associated with diabetes and obesity. Arterioscler Thromb Vasc Biol. 2015;35:1557-1561.

126. Joehanes R, Just AC, Marioni RE, Pilling LC, Reynolds LM, Mandaviya PR, Guan W, Xu T, Elks CE, Aslibekyan S, Moreno-Macias H, Smith JA, Brody JA, Dhingra R, Yousefi P, Pankow JS, Kunze S, Shah SH, McRae AF, Lohman K, Sha J, Absher DM, Ferrucci L, Zhao W, Demerath EW, Bressler J, Grove ML, Huan T, Liu C, Mendelson MM, Yao C, Kiel DP, Peters A, Wang-Sattler R, Visscher PM, Wray NR, Starr JM, Ding J, Rodriguez CJ, Wareham NJ, Irvin MR, Zhi D, Barrdahl M, Vineis P, Ambatipudi S, Uitterlinden AG, Hofman A, Schwartz J, Colicino E, Hou L, Vokonas PS, Hernandez DG, Singleton AB, Bandinelli S, Turner ST, Ware EB, Smith AK, Klengel T, Binder EB, Psaty BM, Taylor KD, Gharib SA, Swenson BR, Liang L, DeMeo DL, O'Connor GT, Herceg Z, Ressler KJ, Conneely KN, Sotoodehnia N, Kardia SLR, Melzer D, Baccarelli AA, van Meurs JBJ, Romieu I, Arnett DK, Ong KK, Liu Y, Waldenberger M, Deary IJ, Fornage M, Levy D, London SJ. Epigenetic signatures of cigarette smoking. Circ Cardiovasc Genet. 2016;9:436-447. 
127. Wan ES, Qiu W, Baccarelli A, Carey VJ, Bacherman H, Rennard SI, Agusti A, Anderson W, Lomas DA, Demeo DL. Cigarette smoking behaviors and time since quitting are associated with differential DNA methylation across the human genome. Hum Mol Genet. 2012;21:3073-82.

128. Breitling LP, Yang R, Korn B, Burwinkel B, Brenner H. Tobacco-smoking-related differential DNA methylation: 27K discovery and replication. Am J Hum Genet. $2011 ; 88: 450-457$.

129. Ling C, Groop L. Epigenetics: a molecular link between environmental factors and type 2 diabetes. Diabetes. 2009;58:2718-25.

130. Sharma P, Kumar J, Garg G, Kumar A, Patowary A, Karthikeyan G, Ramakrishnan L, Brahmachari $\mathrm{V}$, Sengupta $\mathrm{S}$. Detection of altered global DNA methylation in coronary artery disease patients. DNA Cell Biol. 2008;27:357-365.

131. Mandaviya PR, Stolk L, Heil SG. Homocysteine and DNA methylation: A review of animal and human literature. Mol Genet Metab. 2014;113:243-252.

132. Khandanpour N, Loke YK, Meyer FJ, Jennings B, Armon MP. Homocysteine and peripheral arterial disease: systematic review and meta-analysis. Eur J Vasc Endovasc Surg. 2009;38:316-322.

133. Brunelli S, Fusco A, Iosa M, Ricciardi E, Traballesi M. Functional outcome after lower limb amputation: Is hyperhomocysteinemia a predictive factor?: An observational study. Medicine (Baltimore). 2015;94:e2167.

134. Heneghan HM, Sultan S. Homocysteine, the cholesterol of the 21st century. Impact of hyperhomocysteinemia on patency and amputation-free survival after intervention for critical limb ischemia. J Endovasc Ther. 2008;15:399-407.

135. Yan T-T, Li Q, Zhang X-H, Wu W-K, Sun J, Li L, Zhang Q, Tan H-M. Homocysteine impaired endothelial function through compromised vascular endothelial growth factor/Akt/endothelial nitric oxide synthase signaling. Clin Exp Pharmacol Physiol. 2010;37:1071-7.

136. Jiang X, Yang F, Tan H, Liao D, Bryan RM, Randhawa JK, Rumbaut RE, Durante W, Schafer Al, Yang X, Wang $\mathrm{H}$. Hyperhomocystinemia impairs endothelial function and eNOS activity via PKC activation. Arter Thromb Vasc Biol. 2005;25:2515-21.

137. Bosch-Marcé M, Pola R, Wecker AB, Silver M, Weber A, Luedemann C, Curry C, Murayama T, Kearny M, Yoon Y, Malinow MR, Asahara T, Isner JM, Losordo DW. Hyperhomocyst(e)inemia impairs angiogenesis in a murine model of limb ischemia. Vasc Med. 2005;10:15-22.

138. Veeranki S, Givvimani S, Pushpakumar S, Tyagi SC. Hyperhomocysteinemia attenuates angiogenesis through reduction of HIF-1 $\alpha$ and PGC-1 $\alpha$ levels in muscle fibers during hindlimb ischemia. Am J Physiol Heart Circ Physiol. 2014;306:H1116-27.

139. Duan J, Murohara T, Ikeda H, Sasaki K -i., Shintani S, Akita T, Shimada T, Imaizumi T. Hyperhomocysteinemia impairs angiogenesis in response to hindlimb ischemia. Arterioscler Thromb Vasc Biol. 2000;20:2579-2585.

140. Stather PW, Sylvius N, Wild JB, Choke E, Sayers RD, Bown MJ. Differential microRNA expression profiles in peripheral arterial disease. Circ Cardiovasc Genet. 2013;6:490_ 497.

141. Zampetaki A, Kiechl S, Drozdov I, Willeit P, Mayr U, Prokopi M, Mayr A, Weger S, 
Oberhollenzer F, Bonora E, Shah A, Willeit J, Mayr M. Plasma microRNA profiling reveals loss of endothelial miR-126 and other microRNAs in type 2 diabetes. Circ Res.

2010;107:810-7.

142. Spinetti G, Fortunato O, Caporali A, Shantikumar S, Marchetti M, Meloni M, Descamps B, Floris I, Sangalli E, Vono R, Faglia E, Specchia C, Pintus G, Madeddu P, Emanueli C. MicroRNA-15a and microRNA-16 impair human circulating proangiogenic cell functions and are increased in the proangiogenic cells and serum of patients with critical limb ischemia. Circ Res. 2013;112:335-46.

143. Caporali A, Meloni M, Völlenkle C, Bonci D, Sala-Newby GB, Addis R, Spinetti G, Losa S, Masson R, Baker AH, Agami R, Le Sage C, Condorelli G, Madeddu P, Martelli F, Emanueli C. Deregulation of microRNA-503 contributes to diabetes mellitus-induced impairment of endothelial function and reparative angiogenesis after limb ischemia. Circulation. 2011;123:282-291.

144. Bastiaansen AJNM, Ewing MM, De Boer HC, Van Der Pouw Kraan TC, De Vries MR, Peters EAB, Welten SMJ, Arens R, Moore SM, Faber JE, Jukema JW, Hamming JF, Nossent AY, Quax PHA. Lysine acetyltransferase PCAF is a key regulator of arteriogenesis. Arterioscler Thromb Vasc Biol. 2013;33:1902-1910.

145. Krishna SM, Trollope AF, Golledge J. The relevance of epigenetics to occlusive cerebral and peripheral arterial disease. Clin Sci. 2015;128:537-558.

146. Kaluza D, Kroll J, Gesierich S, Manavski Y, Boeckel J-N, Doebele C, Zelent A, Rössig L, Zeiher AM, Augustin HG, Urbich C, Dimmeler S. Histone deacetylase 9 promotes angiogenesis by targeting the antiangiogenic microRNA-17-92 cluster in endothelial cells. Arterioscler Thromb Vasc Biol. 2013;33:533-43.

147. Fatemi M, Wade $\mathrm{P}$ a. MBD family proteins: reading the epigenetic code. J Cell Sci. 2006;119:3033-3037.

148. Volkmann I, Kumarswamy R, Pfaff N, Fiedler J, Dangwal S, Holzmann A, Batkai S, Geffers R, Lother A, Hein L, Thum T. MicroRNA-mediated epigenetic silencing of sirtuin1 contributes to impaired angiogenic responses. Circ Res. 2013;113:997-1003.

149. Rao X, Zhong J, Zhang S, Zhang Y, Yu Q, Yang P, Wang MH, Fulton DJ, Shi H, Dong Z, Wang D, Wang CY. Loss of methyl-CpG-binding domain protein 2 enhances endothelial angiogenesis and protects mice against hind-limb ischemic injury. Circulation. 2011;123:2964-2974.

150. Bonauer A, Carmona G, Iwasaki M, Mione M, Koyanagi M, Fischer A, Burchfield J, Fox H, Doebele C, Ohtani K, Chavakis E, Potente M, Tjwa M, Urbich C, Zeiher AM, Dimmeler S. MicroRNA-92a controls angiogenesis and functional recovery of ischemic tissues in mice. Science (80- ). 2009;324:1710-1713.

151. Landskroner-Eiger S, Qiu C, Perrotta P, Siragusa M, Lee MY, Ulrich V, Luciano AK, Zhuang ZW, Corti F, Simons M, Montgomery RL, Wu D, Yu J, Sessa WC. Endothelial miR-17 92 cluster negatively regulates arteriogenesis via miRNA-19 repression of WNT signaling. Proc Natl Acad Sci U S A. 2015;112:12812-12817.

152. Shi L, Fisslthaler B, Zippel N, Frömel T, Hu J, Elgheznawy A, Heide H, Popp R, Fleming I. MicroRNA-223 antagonizes angiogenesis by targeting $\beta 1$ integrin and preventing growth factor signaling in endothelial cells. Circ Res. 2013;113:1320-30.

153. Yin KJ, Deng Z, Hamblin M, Xiang Y, Huang $H$, Zhang J, Jiang X, Wang Y, Chen YE. Peroxisome proliferator-activated receptor delta regulation of miR-15a in ischemia- 
induced cerebral vascular endothelial injury. J Neurosci. 2010;30:6398-6408.

154. Yin KJ, Olsen K, Hamblin M, Zhang J, Schwendeman SP, Chen YE. Vascular endothelial cell-specific microRNA-15a inhibits angiogenesis in hindlimb ischemia. J Biol Chem. 2012;287:27055-27064.

155. Welten SM, Bastiaansen AJ, de Jong R, de Vries MR, Peters EH, Boonstra M, Sheikh SP, La Monica N, Kandimalla ER, Quax PH, Nossent AY. Inhibition of 14q32 microRNAs miR-329, miR-487b, miR-494 and miR-495 increases neovascularization and blood flow recovery after ischemia. Circ Res. 2014;115:696-708.

156. van Solingen C, Seghers L, Bijkerk R, Duijs JMGJ, Roeten MK, van Oeveren-Rietdijk AM, Baelde HJ, Monge M, Vos JB, de Boer HC, Quax PHA, Rabelink TJ, van Zonneveld AJ. Antagomir-mediated silencing of endothelial cell specific microRNA-126 impairs ischemia-induced angiogenesis. J Cell Mol Med. 2009;13:1577-85.

157. Wang S, Aurora AB, Johnson B a., Qi X, McAnally J, Hill J a., Richardson J a., BasselDuby R, Olson EN. The endothelial-epecific microRNA miR-126 governs vascular integrity and angiogenesis. Dev Cell. 2008;15:261-271.

158. Fish JE, Santoro MM, Morton SU, Yu S, Yeh R-F, Wythe JD, Ivey KN, Bruneau BG, Stainier DYR, Srivastava D. miR-126 regulates angiogenic signaling and vascular integrity. Dev Cell. 2008;15:272-284.

159. Cao WJ, Rosenblat JD, Roth NC, Kuliszewski M a., Matkar PN, Rudenko D, Liao C, Lee $\mathrm{PJH}$, Leong-Poi H. Therapeutic angiogenesis by ultrasound-mediated microRNA-126-3p delivery. Arterioscler Thromb Vasc Biol. 2015;35:2401-2411.

160. Semo J, Sharir R, Afek A, Avivi C, Barshack I, Maysel-Auslender S, Krelin Y, Kain D, Entin-Meer M, Keren G, George J. The 106b 25 microRNA cluster is essential for neovascularization after hindlimb ischaemia in mice. Eur Heart J. 2014;35:3212-3223.

161. Grundmann S, Hans FP, Kinniry S, Heinke J, Helbing T, Bluhm F, Sluijter JPG, Hoefer I, Pasterkamp G, Bode C, Moser M. MicroRNA-100 regulates neovascularization by suppression of mammalian target of rapamycin in endothelial and vascular smooth muscle cells. Circulation. 2011;123:999-1009.

162. Pankratz F, Bemtgen X, Zeiser R, Leonhardt F, Kreuzaler S, Hilgendorf I, Smolka C, Helbing T, Hoefer I, Esser JS, Kustermann M, Moser M, Bode C, Grundmann S. MicroRNA-155 exerts cell-specific antiangiogenic but proarteriogenic effects during adaptive neovascularization. Circulation. 2015;131:1575-1589.

163. Lei Z, van Mil A, Brandt MM, Grundmann S, Hoefer I, Smits M, El Azzouzi H, Fukao T, Cheng C, Doevendans PA, Sluijter JPG. MicroRNA-132/212 family enhances arteriogenesis after hindlimb ischaemia through modulation of the Ras-MAPK pathway. $J$ Cell Mol Med. 2015;20:1-12.

164. Hazarika S, Farber CR, Dokun AO, Pitsillides AN, Wang T, Lye RJ, Annex BH. MicroRNA-93 controls perfusion recovery following hind-limb ischemia by modulating expression of multiple genes in the cell cycle pathway. Circulation. 2013;127:1818-1828.

165. Helisch A, Wagner S, Khan N, Drinane M, Wolfram S, Heil M, Ziegelhoeffer T, Brandt U, Pearlman JD, Swartz HM, Schaper W. Impact of mouse strain differences in innate hindlimb collateral vasculature. Arterioscler Thromb Vasc Biol. 2006;26:520-6.

166. Mac Gabhann F, Peirce SM. Collateral capillary arterialization following arteriolar ligation in murine skeletal muscle. Microcirculation. 2010;17:333-47. 
167. Meisner JK, Sumer S, Murrell KP, Higgins TJ, Price RJ. Laser speckle flowmetry method for measuring spatial and temporal hemodynamic alterations throughout large microvascular networks. Microcirculation. 2012;19:619-31.

168. Scholz D, Ziegelhoeffer T, Helisch A, Wagner S, Friedrich C, Podzuweit T, Schaper W. Contribution of arteriogenesis and angiogenesis to postocclusive hindlimb perfusion in mice. J Mol Cell Cardiol. 2002;34:775-87.

169. Li Q, Hu B, Hu G, Chen C, Niu X, Liu J, Zhou S, Zhang C, Wang Y, Deng Z-F. tRNAderived small non-coding RNAs in response to ischemia inhibit angiogenesis. Sci Rep. 2016;6:20850.

170. Voellenkle C, Garcia-Manteiga JM, Pedrotti S, Perfetti A, De Toma I, Da Silva D, Maimone B, Greco S, Fasanaro P, Creo P, Zaccagnini G, Gaetano C, Martelli F. Implication of Long noncoding RNAs in the endothelial cell response to hypoxia revealed by RNA-sequencing. Sci Rep. 2016;6:24141.

171. Michalik KM, You X, Manavski Y, Doddaballapur A, Zörnig M, Braun T, John D, Ponomareva Y, Chen W, Uchida S, Boon RA, Dimmeler S. Long noncoding RNA MALAT1 regulates endothelial cell function and vessel growth. Circ Res. 2014;114:138997.

172. van Royen N, Piek JJ, Schaper W, Fulton WF. A critical review of clinical arteriogenesis research. J Am Coll Cardiol. 2009;55:17-25.

173. Simons M, Annex BH, Laham RJ, Kleiman N, Henry T, Dauerman H, Udelson JE, Gervino E V., Pike MP, Whitehouse M., Moon T, Chronos NA. Pharmacological treatment of coronary artery disease with recombinant fibroblast growth factor-2: double-blind, randomized, controlled clinical trial. Circulation. 2002;105:788-793.

174. Kusumanto $\mathrm{YH}$, van Weel $\mathrm{V}$, Mulder $\mathrm{NH}$, Smit AJ, van den Dungen JJAM, Hooymans JMM, Sluiter WJ, Tio RA, Quax PHA, Gans ROB, Dullaart RPF, Hospers GAP. Treatment with intramuscular vascular endothelial growth factor gene compared with placebo for patients with diabetes mellitus and critical limb ischemia: a double-blind randomized trial. Hum Gene Ther. 2006;17:683-91.

175. Schierling W, Troidl K, Mueller C, Troidl C, Wustrack H, Bachmann G, Kasprzak PM, Schaper W, Schmitz-Rixen T. Increased intravascular flow rate triggers cerebral arteriogenesis. J Cereb blood flow Metab. 2009;29:726-37.

176. Meisner JK, Niu J, Sumer S, Price RJ. Trans-illuminated laser speckle imaging of collateral artery blood flow in ischemic mouse hindlimb. J Biomed Opt. 2013;18:96011.

177. Chappell JC, Song J, Burke CW, Klibanov AL, Price RJ. Targeted delivery of nanoparticles bearing fibroblast growth factor-2 by ultrasonic microbubble destruction for therapeutic arteriogenesis. Small. 2008;4:1769-1777.

178. Nickerson MM, Burke CW, Meisner JK, Shuptrine CW, Song J, Price RJ. Capillary arterialization requires the bone-marrow-derived cell (BMC)-specific expression of chemokine ( $\mathrm{C}-\mathrm{C}$ motif) receptor-2, but BMCs do not transdifferentiate into microvascular smooth muscle. Angiogenesis. 2009;12:355-63.

179. Distasi MR, Case J, Ziegler MA, Dinauer MC, Yoder MC, Haneline LS, Dalsing MC, Miller SJ, Labarrere CA, Murphy MP, Ingram DA, Unthank JL. Suppressed hindlimb perfusion in Rac2-/- and Nox2-/- mice does not result from impaired collateral growth. Am J Physiol Hear Circ Physiol. 2009;296:H877-86.

180. Dai X, Faber JE. Endothelial nitric oxide synthase deficiency causes collateral vessel 
rarefaction and impairs activation of a cell cycle gene network during arteriogenesis. Circ Res. 2010;106:1870-81.

181. Schindelin J, Arganda-Carreras I, Frise E, Kaynig V, Longair M, Pietzsch T, Preibisch S, Rueden C, Saalfeld S, Schmid B, Tinevez J-Y, White DJ, Hartenstein V, Eliceiri K, Tomancak P, Cardona A. Fiji: an open-source platform for biological-image analysis. Nat Methods. 2012;9:676-82.

182. McCue S, Dajnowiec D, Xu F, Zhang M, Jackson MR, Langille BL. Shear stress regulates forward and reverse planar cell polarity of vascular endothelium in vivo and in vitro. Circ Res. 2006;98:939-46.

183. Tzima E, Kiosses WB, del Pozo M a, Schwartz MA. Localized cdc42 activation, detected using a novel assay, mediates microtubule organizing center positioning in endothelial cells in response to fluid shear stress. J Biol Chem. 2003;278:31020-3.

184. Rogers KA, McKee NH, Kalnins VI. Preferential orientation of centrioles toward the heart in endothelial cells of major blood vessels is reestablished after reversal of a segment. Proc Natl Acad Sci U S A. 1985;82:3272-6.

185. Allan VJ, Thompson HM, McNiven MA. Motoring around the Golgi. Nat Cell Biol. 2002;4:E236-42.

186. Coan DE, Wechezak AR, Viggers RF, Sauvage LR. Effect of shear stress upon localization of the Golgi apparatus and microtubule organizing center in isolated cultured endothelial cells. J Cell Sci. 1993;104:1145-53.

187. Blackman BR, García-Cardeña G, Gimbrone MA. A new in vitro model to evaluate differential responses of endothelial cells to simulated arterial shear stress waveforms. $J$ Biomech Eng. 2002;124:397.

188. Irizarry RA, Bolstad BM, Collin F, Cope LM, Hobbs B, Speed TP. Summaries of Affymetrix GeneChip probe level data. Nucleic Acids Res. 2003;31:e15.

189. Eden E, Navon R, Steinfeld I, Lipson D, Yakhini Z. GOrilla: a tool for discovery and visualization of enriched GO terms in ranked gene lists. BMC Bioinformatics. 2009;10:48.

190. Chiu J-J, Chien S. Effects of disturbed flow on vascular endothelium: pathophysiological basis and clinical perspectives. Physiol Rev. 2011;91:327-87.

191. Zhang J, Friedman MH. Adaptive response of vascular endothelial cells to an acute increase in shear stress magnitude. Am J Physiol Hear Circ Physiol. 2012;302:H983-991.

192. Mack PJ, Zhang Y, Chung S, Vickerman V, Kamm RD, García-Cardeña G. Biomechanical regulation of endothelium-dependent events critical for adaptive remodeling. J Biol Chem. 2009;284:8412-20.

193. Morigi BM, Zoja C, Figliuzzi M, Foppolo M, Micheletti G, Bontempelli M, Saronni M, Remuzzi G, Remuzzi A. Fluid shear stress modulates surface expression of adhesion molecules by endothelial cells. Blood. 1995;85:1696-1703.

194. Malek AM, Jiang L, Lee I, Sessa WC, Izumo S, Alper SL. Induction of nitric oxide synthase mRNA by shear stress requires intracellular calcium and G-protein signals and is modulated by PI 3 kinase. Biochem Biophys Res Commun. 1999;254:231-42.

195. Davis ME, Grumbach IM, Fukai T, Cutchins A, Harrison DG. Shear stress regulates endothelial nitric-oxide synthase promoter activity through nuclear factor kappaB binding. J Biol Chem. 2004;279:163-8. 
196. Boo YC, Sorescu G, Boyd N, Shiojima I, Walsh K, Du J, Jo H. Shear stress stimulates phosphorylation of endothelial nitric-oxide synthase at Ser1179 by Akt-independent mechanisms: role of protein kinase A. J Biol Chem. 2002;277:3388-96.

197. Dekker RJ, Boon RA, Rondaij MG, Kragt A, Volger OL, Elderkamp YW, Meijers JCM, Voorberg J, Pannekoek H, Horrevoets AJG. KLF2 provokes a gene expression pattern that establishes functional quiescent differentiation of the endothelium. Blood. 2006;107:4354-63.

198. Dekker RJ, van Thienen J V, Rohlena J, de Jager SC, Elderkamp YW, Seppen J, de Vries CJM, Biessen EAL, van Berkel TJC, Pannekoek H, Horrevoets AJG. Endothelial KLF2 links local arterial shear stress levels to the expression of vascular tone-regulating genes. Am J Pathol. 2005;167:609-18.

199. Fledderus JO, van Thienen J V, Boon RA, Dekker RJ, Rohlena J, Volger OL, Bijnens APJJ, Daemen MJAP, Kuiper J, van Berkel TJC, Pannekoek H, Horrevoets AJG. Prolonged shear stress and KLF2 suppress constitutive proinflammatory transcription through inhibition of ATF2. Blood. 2007;109:4249-57.

200. Boon RA, Leyen TA, Fontijn RD, Fledderus JO, Baggen JMC, Volger OL, van Nieuw Amerongen GP, Horrevoets AJG. KLF2-induced actin shear fibers control both alignment to flow and JNK signaling in vascular endothelium. Blood. 2010;115:2533-42.

201. Parmar KM, Nambudiri V, Dai G, Larman HB, Gimbrone MA, García-Cardeña G. Statins exert endothelial atheroprotective effects via the KLF2 transcription factor. J Biol Chem. 2005;280:26714-9.

202. van Keulen JK, Timmers L, van Kuijk LP, Retnam L, Hoefer IE, Pasterkamp G, Lim SK, de Kleijn DP V. The Nuclear Factor-kappa B p50 subunit is involved in flow-induced outward arterial remodeling. Atherosclerosis. 2009;202:424-30.

203. Castier Y, Ramkhelawon B, Riou S, Tedgui A, Lehoux S. Role of NF-kappaB in flowinduced vascular remodeling. Antioxid Redox Signal. 2009;11:1641-9.

204. Tirziu D, Jaba IM, Yu P, Larrivée B, Coon BG, Cristofaro B, Zhuang ZW, Lanahan AA, Schwartz MA, Eichmann A, Simons M. Endothelial nuclear factor-kB-dependent regulation of arteriogenesis and branching. Circulation. 2012;126:2589-600.

205. Toyota E, Warltier DC, Brock T, Ritman E, Kolz C, O’Malley P, Rocic P, Focardi M, Chilian WM. Vascular endothelial growth factor is required for coronary collateral growth in the rat. Circulation. 2005;112:2108-13.

206. Babiak A, Schumm A-M, Wangler C, Loukas M, Wu J, Dombrowski S, Matuschek C, Kotzerke J, Dehio C, Waltenberger J. Coordinated activation of VEGFR-1 and VEGFR-2 is a potent arteriogenic stimulus leading to enhancement of regional perfusion. Cardiovasc Res. 2004;61:789-95.

207. Hayashi S, Morishita R, Nakamura S, Yamamoto K, Moriguchi A, Nagano T, Taiji M, Noguchi H, Matsumoto K, Nakamura T, Higaki J, Ogihara T. Potential role of hepatocyte growth factor, a novel sngiogenic growth factor, in peripheral arterial disease: downregulation of HGF in response to hypoxia in vascular cells. Circulation. 1999;100:II301-308.

208. Taniyama Y, Morishita R, Aoki M, Nakagami H, Yamamoto K, Yamazaki K, Matsumoto K, Nakamura T, Kaneda Y, Ogihara T. Therapeutic angiogenesis induced by human hepatocyte growth factor gene in rat and rabbit hindlimb ischemia models: preclinical study for treatment of peripheral arterial disease. Gene Ther. 2001;8:181-9. 
209. Masaki I, Yonemitsu Y, Yamashita A, Sata S, Tanii M, Komori K, Nakagawa K, Hou X, Nagai Y, Hasegawa M, Sugimachi K, Sueishi K. Angiogenic gene therapy for experimental critical limb ischemia: acceleration of limb loss by overexpression of vascular endothelial growth factor 165 but not of fibroblast growth factor-2. Circ Res. 2002;90:966-73.

210. Fujii T, Yonemitsu Y, Onimaru M, Tanii M, Nakano T, Egashira K, Takehara T, Inoue M, Hasegawa M, Kuwano H, Sueishi K. Nonendothelial mesenchymal cell-derived MCP-1 is required for FGF-2-mediated therapeutic neovascularization: critical role of the inflammatory/arteriogenic pathway. Arterioscler Thromb Vasc Biol. 2006;26:2483-9.

211. Ren B, Deng Y, Mukhopadhyay A, Lanahan AA, Zhuang ZW, Moodie KL, Mulligan-Kehoe MJ, Byzova T V, Peterson RT, Simons M. ERK1/2-Akt1 crosstalk regulates arteriogenesis in mice and zebrafish. J Clin Invest. 2010;120:1217-28.

212. Sarateanu CS, Retuerto MA, Beckmann JT, McGregor L, Carbray J, Patejunas G, Nayak L, Milbrandt J, Rosengart TK. An Egr-1 master switch for arteriogenesis: studies in Egr-1 homozygous negative and wild-type animals. J Thorac Cardiovasc Surg. 2006;131:13845.

213. Kinnaird T, Stabile E, Zbinden S, Burnett MS, Epstein SE. Cardiovascular risk factors impair native collateral development and may impair efficacy of therapeutic interventions. Cardiovasc Res. 2008;78:257-64.

214. Senthilkumar A, Smith RD, Khitha J, Arora N, Veerareddy S, Langston W, Chidlow JH, Barlow SC, Teng X, Patel RP, Lefer DJ, Kevil CG. Sildenafil promotes ischemia-induced angiogenesis through a PKG-dependent pathway. Arterioscler Thromb Vasc Biol. 2007;27:1947-54.

215. Bir SC, Xiong Y, Kevil CG, Luo J. Emerging role of PKA/eNOS pathway in therapeutic angiogenesis for ischaemic tissue diseases. Cardiovasc Res. 2012;95:7-18.

216. Venkatesh PK, Pattillo CB, Branch B, Hood J, Thoma S, Illum S, Pardue S, Teng X, Patel $\mathrm{RP}$, Kevil CG. Dipyridamole enhances ischaemia-induced arteriogenesis through an endocrine nitrite/nitric oxide-dependent pathway. Cardiovasc Res. 2010;85:661-70.

217. Sweet DT, Chen Z, Givens CS, Owens AP, Rojas M, Tzima E. Endothelial Shc regulates arteriogenesis through dual control of arterial specification and inflammation via the Notch and NF-kB pathways. Circ Res. 2013;113:32-39.

218. Lan Q, Mercurius KO, Davies PF. Stimulation of transcription factors NFkB and AP1 in ECs subjected to shear stress. Biochem Biophys Res Commun. 1994;201:950-956.

219. Gruionu G, Hoying JB, Pries AR, Secomb TW. Structural remodeling of mouse gracilis artery after chronic alteration in blood supply. Am J Physiol Hear Circ Physiol. 2005;288:2047-2054.

220. Gruionu G, Hoying JB, Pries AR, Secomb TW. Structural remodeling of the mouse gracilis artery: coordinated changes in diameter and medial area maintain circumferential stress. Microcirculation. 2012;19:610-618.

221. Johnson C, Sung H-J, Lessner SM, Fini ME, Galis ZS. Matrix metalloproteinase-9 is required for adequate angiogenic revascularization of ischemic tissues: potential role in capillary branching. Circ Res. 2004;94:262-8.

222. Deindl E, Buschmann I, Hoefer IE, Podzuweit T, Boengler K, Vogel S, van Royen N, Fernandez B, Schaper W. Role of ischemia and of hypoxia-inducible genes in arteriogenesis after femoral artery occlusion in the rabbit. Circ Res. 2001;89:779-786. 
223. Ito WD, Arras M, Scholz D, Winkler B, Htun P, Schaper W. Angiogenesis but not collateral growth is associated with ischemia after femoral artery occlusion. Am J Physiol Hear Circ Physiol. 1997;273:H1255-H1265.

224. Adamson RH, Sarai RK, Altangerel A, Clark JF, Weinbaum S, Curry F-RE. Microvascular permeability to water is independent of shear stress, but dependent on flow direction. $A m$ J Physiol Hear Circ Physiol. 2013;304:H1077-1084.

225. Melchior B, Frangos JA. Shear-induced endothelial cell-cell junction inclination. Am J Physiol Cell Physiol. 2010;299:C621-9.

226. Passerini AG, Milsted A, Rittgers SE. Shear stress magnitude and directionality modulate growth factor gene expression in preconditioned vascular endothelial cells. J Vasc Surg. 2003;37:182-90.

227. Wang C, Baker BM, Chen CS, Schwartz MA. Endothelial cell sensing of flow direction. Arter Thromb Vasc Biol. 2013;33:2130-2136.

228. Tzima E. Role of small GTPases in endothelial cytoskeletal dynamics and the shear stress response. Circ Res. 2006;98:176-85.

229. Tzima E, Del Pozo MA, Kiosses WB, Mohamed SA, Li S, Chien S, Schwartz MA. Activation of Rac1 by shear stress in endothelial cells mediates both cytoskeletal reorganization and effects on gene expression. EMBO J. 2002;21:6791-800.

230. Imberti B, Morigi M, Zoja C, Angioletti S, Abbate M, Remuzzi a, Remuzzi G. Shear stress-induced cytoskeleton rearrangement mediates NF-kappaB-dependent endothelial expression of ICAM-1. Microvasc Res. 2000;60:182-8.

231. Troidl K, Rüding I, Cai W-J, Mücke Y, Grossekettler L, Piotrowska I, Apfelbeck H, Schierling W, Volger OL, Horrevoets AJ, Grote K, Schmitz-Rixen T, Schaper W, Troidl C. Actin-binding rho activating protein (Abra) is essential for fluid shear stress-induced arteriogenesis. Arterioscler Thromb Vasc Biol. 2009;29:2093-101.

232. Yu J, deMuinck ED, Zhuang Z, Drinane M, Kauser K, Rubanyi GM, Qian HS, Murata T, Escalante B, Sessa WC. Endothelial nitric oxide synthase is critical for ischemic remodeling, mural cell recruitment, and blood flow reserve. Proc Natl Acad Sci U S A. 2005;102:10999-1004.

233. Park B, Hoffman A, Yang Y, Yan J, Tie G, Bagshahi H, Nowicki PT, Messina LM. eNOS affects both early and late collateral arterial adaptation and blood flow recovery after induction of hindlimb ischemia in mice. J Vasc Surg. 2010;51:1-17.

234. Lloyd PG, Yang HT, Terjung RL. Arteriogenesis and angiogenesis in rat ischemic hindlimb: role of nitric oxide. Am J Physiol Hear Circ Physiol. 2001;281:H2528-38.

235. Mees B, Wagner S, Ninci E, Tribulova S, Martin S, van Haperen R, Kostin S, Heil M, de Crom R, Schaper W. Endothelial nitric oxide synthase activity is essential for vasodilation during blood flow recovery but not for arteriogenesis. Arterioscler Thromb Vasc Biol. 2007;27:1926-33.

236. Kassab GS. Scaling laws of vascular trees : of form and function. Am J Physiol Hear Circ Physiol. 2005;290:894-903.

237. Heuslein JL, Meisner JK, Li X, Song J, Vincentelli H, Leiphart RJ, Ames EG, Blackman $\mathrm{BR}$, Price RJ. Mechanisms of amplified arteriogenesis in collateral artery segments exposed to reversed flow direction. Arterioscler Thromb Vasc Biol. 2015;35:2354-2365.

238. Jiang Y-Z, Jiménez JM, Ou K, McCormick ME, Zhang L-D, Davies PF. Hemodynamic 
disturbed flow induces differential DNA methylation of endothelial Kruppel-Like Factor 4 promoter in vitro and in vivo. Circ Res. 2014;115:32-43.

239. Dunn J, Qiu H, Kim S, Jjingo D, Hoffman R, Kim CW, Jang I, Son DJ, Kim D, Pan C, Fan Y, Jordan IK, Jo H. Flow-dependent epigenetic DNA methylation regulates endothelial gene expression and atherosclerosis. J Clin Invest. 2014;124:3187-3199.

240. Zhou J, Li Y-S, Chien S. Epigenetic mechanism in regulation of endothelial function by disturbed flow: Induction of DNA hypermethylation by DNMT1. Cell Mol Bioeng. 2014;7:218-224.

241. Jiang Y-Z, Manduchi E, Stoeckert CJ, Davies PF. Arterial endothelial methylome: differential DNA methylation in athero-susceptible disturbed flow regions in vivo. BMC Genomics. 2015;16:506.

242. Heuslein JL, Murrell KP, Leiphart RJ, Llewellyn RA, Meisner JK, Price RJ. Vascular growth responses to chronic arterial occlusion are unaffected by myeloid specific focal adhesion kinase (FAK) deletion. Sci Rep. 2016;6:27029.

243. Newman MR, Blyth BJ, Hussey DJ, Jardine D, Sykes PJ, Ormsby RJ. Sensitive quantitative analysis of murine LINE1 DNA methylation using high resolution melt analysis. Epigenetics. 2012;7:92-105.

244. Tse MY, Ashbury JE, Zwingerman N, King WD, Taylor SA, Pang SC. A refined, rapid and reproducible high resolution melt (HRM)-based method suitable for quantification of global LINE-1 repetitive element methylation. BMC Res Notes. 2011;4:565.

245. Subramanian A, Tamayo P, Mootha VK, Mukherjee S, Ebert BL. Gene set enrichment analysis : A knowledge-based approach for interpreting genome-wide. Proc Natl Acad Sci U S A. 2005;102:15545-15550.

246. Hoefer IE, Grundmann S, van Royen N, Voskuil M, Schirmer SH, Ulusans S, Bode C, Buschmann IR, Piek JJ. Leukocyte subpopulations and arteriogenesis: specific role of monocytes, lymphocytes and granulocytes. Atherosclerosis. 2005;181:285-93.

247. Ghoshal K, Datta J, Majumder S, Bai S, Kutay H, Motiwala T, Jacob ST. 5-Azadeoxycytidine induces selective degradation of DNA methyltransferase 1 by a proteasomal pathway that requires the KEN box, bromo-adjacent homology domain, and nuclear localization signal. Mol Cell Biol. 2005;25:4727-4741.

248. Illi B, Nanni S, Scopece A, Farsetti A, Biglioli P, Capogrossi MC, Gaetano C. Shear stress-mediated chromatin remodeling provides molecular basis for flow-dependent regulation of gene expression. Circ Res. 2003;93:155-61.

249. Chen W, Bacanamwo M, Harrison DG. Activation of p300 histone acetyltransferase activity is an early endothelial response to laminar shear stress and is essential for stimulation of endothelial nitric-oxide synthase mRNA transcription. J Biol Chem. 2008;283:16293-16298.

250. Lee D-Y, Lee C-I, Lin T-E, Lim SH, Zhou J, Tseng Y-C, Chien S, Chiu J-J. Role of histone deacetylases in transcription factor regulation and cell cycle modulation in endothelial cells in response to disturbed flow. Proc Natl Acad Sci U S A. 2012;109:1967-72.

251. Chen L-J, Chuang L, Huang Y-H, Zhou J, Lim SH, Lee C-I, Lin W-W, Lin T-E, Wang W-L, Chen L, Chien S, Chiu J-J. MicroRNA mediation of endothelial inflammatory response to smooth muscle cells and its inhibition by atheroprotective shear stress. Circ Res. 2015;116:1157-1169. 
252. Fang Y, Shi C, Manduchi E, Civelek M, Davies PF. MicroRNA-10a regulation of proinflammatory phenotype in athero-susceptible endothelium in vivo and in vitro. Proc Natl Acad Sci U S A. 2010;107:13450-13455.

253. Ni C-W, Qiu H, Jo H. MicroRNA-663 upregulated by oscillatory shear stress plays a role in inflammatory response of endothelial cells. Am J Physiol Heart Circ Physiol. 2011;300:H1762-9.

254. Qin X, Wang X, Wang Y, Tang Z, Cui Q, Xi J, Li Y-SJ, Chien S, Wang N. MicroRNA-19a mediates the suppressive effect of laminar flow on cyclin D1 expression in human umbilical vein endothelial cells. Proc Natl Acad Sci U S A. 2010;107:3240-3244.

255. Tao Y, Huang C, Huang Y, Hong L, Wang H, Zhou Z, Qiu Y. SIRT4 suppresses inflammatory responses in human hmbilical vein endothelial cells. Cardiovasc Toxicol. 2015;15:217-223.

256. Tirziu D, Jaba IM, Yu P, Larrivée B, Coon BG, Cristofaro B, Zhuang ZW, Lanahan AA, Schwartz MA, Eichmann A, Simons M. Endothelial nuclear factor-kB-dependent regulation of arteriogenesis and branching. Circulation. 2012;126:2589-600.

257. Gorski $\mathrm{DH}$, Walsh $\mathrm{K}$. The role of homeobox genes in vascular remodeling and angiogenesis. Circ Res. 2000;87:865-872.

258. Myers C, Charboneau A, Boudreau N. Homeobox B3 promotes capillary morphogenesis and angiogenesis. J Cell Biol. 2000;148:343-351.

259. Hastings NE, Feaver RE, Lee MY, Wamhoff BR, Blackman BR. Human IL-8 regulates smooth muscle cell VCAM-1 expression in response to endothelial cells exposed to atheroprone flow. Arterioscler Thromb Vasc Biol. 2009;29:725-31.

260. Chiu JJ, Wung BS, Shyy JYJ, Hsieh HJ, Wang DL. Reactive oxygen species are involved in shear stress-induced intercellular adhesion molecule-1 expression in endothelial cells. Arterioscler Thromb Vasc Biol. 1997;17:3570-3577.

261. Herold J, Pipp F, Fernandez B, Xing Z, Heil M, Tillmanns H, Braun-Dullaeus RC. Transplantation of monocytes: a novel strategy for in vivo augmentation of collateral vessel growth. Hum Gene Ther. 2004;15:1-12.

262. Epstein SE, Stabile E, Kinnaird T, Lee CW, Clavijo L, Burnett MS. Janus phenomenon: the interrelated tradeoffs inherent in therapies designed to enhance collateral formation and those designed to inhibit atherogenesis. Circulation. 2004;109:2826-31.

263. Neth P, Nazari-Jahantigh M, Schober A, Weber C. MicroRNAs in flow-dependent vascular remodelling. Cardiovasc Res. 2013;

264. Urbich C, Kuehbacher A, Dimmeler S. Role of microRNAs in vascular diseases, inflammation, and angiogenesis. Cardiovasc Res. 2008;79:581-8.

265. van Rooij E. The art of microRNA research. Circ Res. 2011;108:219-34.

266. van Royen N. CD44 regulates arteriogenesis in mice and is differentially expressed in patients with poor and good collateralization. Circulation. 2004;109:1647-1652.

267. Bot PT, Grundmann S, van Royen N, Joosten SPJ, Schirmer SH, de Kleijn DP V, Pals ST, Pasterkamp G, Piek JJ, Hoefer IE. Distinct CD44 splice variants differentially affect collateral artery growth. Curr Vasc Pharmacol. 2013;11:13-20.

268. Alexander MS, Kawahara G, Motohashi N, Casar JC, Eisenberg I, Myers J a, Gasperini MJ, Estrella EA, Kho AT, Mitsuhashi S, Shapiro F, Kang PB, Kunkel LM. MicroRNA-199a 
is induced in dystrophic muscle and affects WNT signaling, cell proliferation, and myogenic differentiation. Cell Death Differ. 2013;20:1194-208.

269. Chen R, Alvero AB, Silasi D a, Kelly MG, Fest S, Visintin I, Leiser A, Schwartz PE, Rutherford T, Mor G. Regulation of IKKbeta by miR-199a affects NF-kappaB activity in ovarian cancer cells. Oncogene. 2008;27:4712-23.

270. Cheng HS, Siu A, Cybulsky MI, Fish JE. Abstract 640: Role of microRNA-146a in vascular inflammation and atherosclerosis. Arterioscler Thromb Vasc Biol. 2014;34:A640.

271. Raitoharju E, Lyytikäinen L-P, Levula M, Oksala N, Mennander A, Tarkka M, Klopp N, Illig T, Kähönen M, Karhunen PJ, Laaksonen R, Lehtimäki T. miR-21, miR-210, miR-34a, and $\mathrm{miR}-146 \mathrm{a} / \mathrm{b}$ are up-regulated in human atherosclerotic plaques in the Tampere Vascular Study. Atherosclerosis. 2011;219:211-7.

272. Song XW, Li Q, Lin LI, Wang XC, Li DF, Wang GK, Ren ANJ, Wang YR, Qin YW, Yuan WJ, Jing Q. MicroRNAs are dynamically regulated in hypertrophic hearts, and miR-199a is essential for the maintenance of cell size in cardiomyocytes. $J$ Cell Physiol. 2010;225:437-443.

273. Li Z, Song Y, Liu L, Hou N, An X, Zhan D, Li Y, Zhou L, Li P, Yu L, Xia J, Zhang Y, Wang J, Yang X. miR-199a impairs autophagy and induces cardiac hypertrophy through mTOR activation. Cell Death Differ. 2015;63895937:1-9.

274. Gheinani AH, Burkhard FC, Rehrauer H, Fournier CA, Monastyrskaya K. MicroRNA MiR199a-5p regulates smooth muscle cell proliferation and morphology by targeting WNT2 signaling pathway. J Biol Chem. 2015;290:7067-7086.

275. Chan YC, Roy S, Huang Y, Khanna S, Sen CK. The microRNA miR-199a-5p downregulation switches on wound angiogenesis by derepressing the v-ets erythroblastosis virus E26 oncogene homolog 1-matrix metalloproteinase-1 pathway. J Biol Chem. 2012;287:41032-43.

276. Ding G, Huang G, Liu HD, Liang HX, Ni YF, Ding ZH, Ni GY, Hua HW. MiR-199a suppresses the hypoxia-induced proliferation of non-small cell lung cancer cells through targeting HIF1alpha. Mol Cell Biochem. 2013;384:173-180.

277. He J, Jing Y, Li W, Qian X, Xu Q, Li FS, Liu LZ, Jiang BH, Jiang Y. Roles and mechanism of miR-199a and miR-125b in tumor angiogenesis. PLoS One. 2013;8:e56647.

278. Raimondi L, Amodio N, Teresa M, Martino D, Altomare E, Leotta M, Caracciolo D, Gullà A, Neri A, Aquila PD, Alessandro R, Giordano A, Tassone P. Targeting of multiple myeloma-related angiogenesis by miR-199a-5p mimics : in vitro and in vivo anti-tumor activity. Oncotarget. 2014;5:3039-3054.

279. Dai L, Lou W, Zhu J, Zhou X, Di W. MiR-199a inhibits the angiogenic potential of endometrial stromal cells under hypoxia by targeting HIF-1a / VEGF pathway. Int $J$ Clin Exp Pathol. 2015;8:4735-4744.

280. Liu G-T, Huang Y-L, Tzeng H-E, Tsai C-H, Wang S-W, Tang C-H. CCL5 promotes vascular endothelial growth factor expression and induces angiogenesis by downregulating miR-199a in human chondrosarcoma cells. Cancer Lett. 2015;357:476-487.

281. Cheng W, Liu T, Wan X, Gao Y, Wang H. MicroRNA-199a targets CD44 to suppress the tumorigenicity and multidrug resistance of ovarian cancer-initiating cells. FEBS J. 2012;279:2047-59.

282. Song J, Gao L, Yang G, Tang S, Xie H, Wang Y, Wang J, Zhang Y, Jin J, Gou Y, Yang Z, 
Chen Z, Wu K, Liu J, Fan D. MiR-199a regulates cell proliferation and survival by targeting FZD7. PLoS One. 2014;9:e110074.

283. Zhang L, Lou J, Lu M, Gao C, Zhao S, Li B. Suppression of miR-199a maturation by HuR is crucial for hypoxia-induced glycolytic switch in hepatocellular carcinoma. EMBO J. 2015;34:2671-2685.

284. Gui R, Huang R, Zhang J-H, Wen X-H, Nie X-M. MicroRNA-199a-5p inhibits VEGFinduced tumorigenesis through targeting oxidored-nitro domain-containing protein 1 in human HepG2 cells. Oncol Rep. 2016;35:2216-2222.

285. Yang X, Lei S, Long J, Liu X, Wu Q. MicroRNA-199a-5p inhibits tumor proliferation in melanoma by mediating HIF-1a. Mol Med Rep. 2016;13:5241-5247.

286. Ponta H, Sherman L, Herrlich PA. CD44: from adhesion molecules to signalling regulators. Nat Rev Mol Cell Biol. 2003;4:33-45.

287. Stacey DW. Cyclin D1 serves as a cell cycle regulatory switch in actively proliferating cells. Curr Opin Cell Biol. 2003;15:158-163.

288. Van Solingen C, Seghers L, Bijkerk R, Duijs JMGJ, Roeten MK, Van Oeveren-Rietdijk AM, Baelde HJ, Monge M, Vos JB, De Boer HC, Quax PHA, Rabelink TJ, Van Zonneveld AJ. Antagomir-mediated silencing of endothelial cell specific microRNA-126 impairs ischemia-induced angiogenesis. J Cell Mol Med. 2009;13:1577-1585.

289. Heil M, Ziegelhoeffer T, Pipp F, Kostin S, Martin S, Clauss M, Schaper W. Blood monocyte concentration is critical for enhancement of collateral artery growth. $\mathrm{Am} \mathrm{J}$ Physiol Heart Circ Physiol. 2002;283:H2411-9.

290. Lino Cardenas CL, Henaoui IS, Courcot E, Roderburg C, Cauffiez C, Aubert S, Copin MC, Wallaert B, Glowacki F, Dewaeles E, Milosevic J, Maurizio J, Tedrow J, Marcet B, Lo-Guidice JM, Kaminski N, Barbry P, Luedde T, Perrais M, Mari B, Pottier N. miR-199a$5 p$ is upregulated during fibrogenic response to tissue injury and mediates TGFbetainduced lung fibroblast activation by targeting caveolin-1. PLoS Genet. 2013;9:e1003291.

291. Oskowitz AZ, Lu J, Penfornis P, Ylostalo J, McBride J, Flemington EK, Prockop DJ, Pochampally R. Human multipotent stromal cells from bone marrow and microRNA: regulation of differentiation and leukemia inhibitory factor expression. Proc Natl Acad Sci U S A. 2008;105:18372-7.

292. D'Alessandra Y, Carena MC, Spazzafumo L, Martinelli F, Bassetti B, Devanna P, Rubino M, Marenzi G, Colombo GI, Achilli F, Maggiolini S, Capogrossi MC, Pompilio G.

Diagnostic potential of plasmatic microRNA signatures in stable and unstable angina. PLoS One. 2013;8:e80345.

293. Regensteiner JG, Wolfel EE, Brass EP, Carry MR, Ringel SP, Hargarten ME, Stamm ER, Hiatt WR. Chronic changes in skeletal muscle histology and function in peripheral arterial disease. Circulation. 1993;87:413-421.

294. Hedberg B, Angquist K-A, Henriksson-Larsen K, Sjöström M. Fibre Loss and distibution in skeletal muscle from patients with severe peripheral arterial insufficiency. Eur $J$ Vasc Surg. 1989;3:315-322.

295. Mitchell RG, Duscha BD, Robbins JL, Redfern SI, Chung J, Bensimhon DR, Kraus WE, Hiatt WR, Regensteiner JG, Annex BH. Increased levels of apoptosis in gastrocnemius skeletal muscle in patients with peripheral arterial disease. Vasc Med. 2007;12:285-290.

296. Koutakis P, Myers SA, Cluff K, Ha DM, Haynatzki G, McComb RD, Uchida K, Miserlis D, 
Papoutsi E, Johanning JM, Casale GP, Pipinos II. Abnormal myofiber morphology and limb dysfunction in claudication. J Surg Res. 2015;196:172-179.

297. Fan G, Beard C, Chen RZ, Csankovszki G, Sun Y, Siniaia M, Biniszkiewicz D, Bates B, Lee PP, Kuhn R, Trumpp a, Poon C, Wilson CB, Jaenisch R. DNA hypomethylation perturbs the function and survival of CNS neurons in postnatal animals. J Neurosci. $2001 ; 21: 788-797$.

298. Monvoisin A, Alva J a., Hofmann JJ, Zovein AC, Lane TF, Iruela-Arispe ML. VE-cadherinCreERT2 transgenic mouse: A model for inducible recombination in the endothelium. Dev Dyn. 2006;235:3413-3422.

299. Shankman LS, Gomez D, Cherepanova O a, Salmon M, Alencar GF, Haskins RM, Swiatlowska P, Newman A a C, Greene ES, Straub AC, Isakson B, Randolph GJ, Owens GK. KLF4-dependent phenotypic modulation of smooth muscle cells has a key role in atherosclerotic plaque pathogenesis. Nat Med. 2015;21:628-37.

300. Xu X, Tao Y, Gao X, Zhang L, Li X, Zou W, Ruan K, Wang F, Xu G-L, Hu R. A CRISPRbased approach for targeted DNA demethylation. Cell Discov. 2016;2:16009.

301. Maeder ML, Angstman JF, Richardson ME, Linder SJ, Cascio VM, Tsai SQ, Ho QH, Sander JD, Reyon D, Bernstein BE, Costello JF, Wilkinson MF, Joung JK. Targeted DNA demethylation and activation of endogenous genes using programmable TALE-TET1 fusion proteins. Nat Biotechnol. 2013;31:1137-1142.

302. Vojta A, Dobrinić $P$, Tadić V, Bočkor L, Korać $P$, Julg B, Klasić M, Zoldoš V. Repurposing the CRISPR-Cas9 system for targeted DNA methylation. Nucleic Acids Res. 2016;44:5615-28.

303. Demicheva E, Hecker M, Korff T. Stretch-induced activation of the transcription factor activator protein-1 controls monocyte chemoattractant protein-1 expression during arteriogenesis. Circ Res. 2008;103:477-84.

304. Billaud M, Ross JA, Greyson MA, Bruce AC, Seaman SA, Heberlein KR, Han J, Best AK, Peirce SM, Isakson BE. A new method for in vivo visualization of vessel remodeling using a near-infrared dye. Microcirculation. 2011;18:163-71.

305. Burke CW, Soo J, Kim AJ, Hsiang YJ, Klibanov AL, Hanes J, Price RJ. Markedly enhanced skeletal muscle transfection achieved by the ultrasound-targeted delivery of non-viral gene nanocarriers with microbubbles. J Control Release. 2012;162:414-421.

306. Mac Gabhann F, Peirce SM. Collateral capillary arterialization following arteriolar ligation in murine skeletal muscle. Microcirculation. 2010;17:333-47.

307. Scholz D. Contribution of arteriogenesis and angiogenesis to postocclusive hindlimb perfusion in mice. J Mol Cell Cardiol. 2002;34:775-787.

308. Murphy TP, Cutlip DE, Regensteiner JG, Mohler ER, Cohen DJ, Reynolds MR, Massaro JM, Lewis B a, Cerezo J, Oldenburg NC, Thum CC, Goldberg S, Jaff MR, Steffes MW, Comerota AJ, Ehrman J, Treat-Jacobson D, Walsh ME, Collins T, Badenhop DT, Bronas U, Hirsch AT. Supervised exercise versus primary stenting for claudication resulting from aortoiliac peripheral artery disease: six-month outcomes from the claudication: exercise versus endoluminal revascularization (CLEVER) study. Circulation. 2012;125:130-9.

309. van Royen N, Piek JJ, Buschmann I, Hoefer I, Voskuil M, Schaper W. Stimulation of arteriogenesis; a new concept for the treatment of arterial occlusive disease. Cardiovasc Res. 2001;49:543-53. 
310. Fakhry F, Spronk S, van der Laan L, Wever JJ, Teijink JAW, Hoffmann WH, Smits TM, van Brussel JP, Stultiens GNM, Derom A, den Hoed PT, Ho GH, van Dijk LC, Verhofstad N, Orsini M, van Petersen A, Woltman K, Hulst I, van Sambeek MRHM, Rizopoulos D, Rouwet E V., Hunink MGM. Endovascular revascularization and supervised exercise for peripheral artery disease and intermittent claudication. Jama. 2015;314:1936.

311. Teravainen $\mathrm{H}$, Makitie J. Striated muscle ultrastructure in intermittent claudication. Arch Pathol Lab Med. 1977;101:230-35.

312. Makitie J, Teravainen $\mathrm{H}$. Histochemical changes in striated muscle in patients with intermittent claudication. Arch Pathol Lab Med. 1977;101:658-63.

313. McClung JM, McCord TJ, Keum S, Johnson S, Annex BH, Marchuk DA, Kontos CD. Skeletal muscle-specific genetic determinants contribute to the differential straindependent effects of hindlimb ischemia in mice. Am J Pathol. 2012;180:2156-69.

314. Anderson JD, Epstein $\mathrm{FH}$, Meyer $\mathrm{CH}$, Hagspiel KD, Wang $\mathrm{H}$, Berr SS, Harthun NL, Weltman A, Dimaria JM, West AM, Kramer CM. Mutltifactorial determinants of functional capacity in peripheral arterial disease: uncoupling of calf muscle perfusion and metabolism. J Am Coll Cardiol. 2009;54:628-635.

315. Hiatt WR, Nawaz D, Brass EP. Carnitine metabolism during exercise in patients with peripheral vascular disease. J Appl Physiol. 1987;62:2383-7.

316. Hiatt WR, Wolfel EE, Regensteiner JG, Brass EP. Skeletal muscle carnitine metabolism in patients with unilateral peripheral arterial disease. J Appl Physiol. 1992;73:346-353.

317. Pipinos II, Judge AR, Zhu Z, Selsby JT, Swanson SA, Johanning JM, Baxter BT, Lynch TG, Dodd SL. Mitochondrial defects and oxidative damage in patients with peripheral arterial disease. Free Radic Biol Med. 2006;41:262-269.

318. Brass EP, Hiatt WR, Gardner AW, Hoppel CL. Decreased NADH dehydrogenase and ubiquinol-cytochrome c oxidoreductase in peripheral arterial disease. Am J Physiol Hear Circ Physiol. 2001;280:H603-9.

319. Pedraza E, Coronel MM, Fraker C a., Ricordi C, Stabler CL. Preventing hypoxia-induced cell death in beta cells and islets via hydrolytically activated, oxygen-generating biomaterials. Proc Natl Acad Sci U S A. 2012;109:4245-4250.

320. Harrison BS, Eberli D, Lee SJ, Atala A, Yoo JJ. Oxygen producing biomaterials for tissue regeneration. Biomaterials. 2007;28:4628-4634.

321. Ward CL, Corona BT, Yoo JJ, Harrison BS, Christ GJ. Oxygen generating biomaterials preserve skeletal muscle homeostasis under hypoxic and ischemic conditions. PLoS One. 2013;8:e72485.

322. van Royen N, Piek JJ, Schaper W, Fulton WF. A critical review of clinical arteriogenesis research. J Am Coll Cardiol. 2009;55:17-25.

323. Nishijima Y, Akamatsu Y, Weinstein PR, Liu J. Collaterals: implications in cerebral ischemic diseases and therapeutic interventions. Brain Res. 2015;1-12.

324. Seaman SA, Cao Y, Campbell CA, Peirce SM. Macrophage recruitment and polarization during collateral vessel remodeling in murine adipose tissue. Microcirculation. 2016;23:75-87.

325. Antebi B, Zhang L, Sheyn D, Pelled G, Zhang X, Gazit Z, Schwarz E, Gazit D. Controlling arteriogenesis and mast cells are central to bioengineering solutions for critical bone defect repair using allografts. Bioengineering. 2016;3:6. 
326. Yu JL, Rak JW. Host microenvironment in breast cancer development: inflammatory and immune cells in tumour angiogenesis and arteriogenesis. Breast Cancer Res. 2003;5:8388.

327. Muto J, Shirabe K, Yoshizumi T, Ikegami T, Aishima S, Ishigami K, Yonemitsu Y, Ikeda T, Soejima Y, Maehara Y. The apelin-APJ system induces tumor arteriogenesis in hepatocellular carcinoma. Anticancer Res. 2014;34:5313-5320. 